

\section{Thermophysical Properties of Helium-4 from 4 to $3000 R$ with Pressures to 15000 PSIA}

U. S.

PARTMENT

OF

OMMERCE

National

Rureau

QC of

QC ds

00

5753

.622

972 
The National Bureau of Standards ${ }^{1}$ was established by an act of Congress March 3 , 1901. The Bureau's overall goal is to strengthen and advance the Nation's science and technology and facilitate their effective application for public benefit. To this end, the Bureau conducts research and provides: (1) a basis for the Nation's physical measurement system, (2) scientific and technological services for industry and government, (3) a technical basis for equity in trade, and (4) technical services to promote public safety. The Bureau consists of the Institute for Basic Standards, the Institute for Materials Research, the Institute for Applied Technology, the Center for Computer Sciences and Technology, and the Office for Information Programs.

THE INSTITUTE FOR BASIC STANDARDS provides the central basis within the United States of a complete and consistent system of physical measurement; coordinates that system with measurement systems of other nations; and furnishes essential services leading to accurate and uniform physical measurements throughout the Nation's scientific community, industry, and commerce. The Institute consists of a Center for Radiation Research, an Office of Measurement Services and the following divisions:

Applied Mathematics-Electricity-Heat-Mechanics-Optical Physics-Linac Radiation ${ }^{2}$ - Nuclear Radiation²-Applied Radiation ${ }^{2}$ - Quantum Electronics ${ }^{3}$ Electromagnetics ${ }^{3}$ - Time and Frequency ${ }^{3}$ - Laboratory Astrophysics ${ }^{3}$-Cryogenics ${ }^{3}$.

THE INSTITUTE FOR MATERIALS RESEARCH conducts materials research leading to improved methods of measurement, standards, and data on the properties of well-characterized materials needed by industry, commerce, educational institutions, and Government; provides advisory and research services to other Government agencies; and develops, produces, and distributes standard reference materials. The Institute consists of the Office of Standard Reference Materials and the following divisions:

Analytical Chemistry-Polymers-Metallurgy-Inorganic Materials-Reactor Radiation-Physical Chemistry.

THE INSTITUTE FOR APPLIED TECHNOLOGY provides technical services to promote the use of available technology and to facilitate technological innovation in industry and Government; cooperates with public and private organizations leading to the development of technological standards (including mandatory safety standards), codes and methods of test; and provides technical advice and services to Government agencies upon request. The Institute also monitors NBS engineering standards activities and provides liaison between NBS and national and international engineering standards bodies. The Institute consists of the following divisions and offices:

Engineering Standards Services-Weights and Measures-Invention and Innovation-Product Evaluation Technology-Building Research-Electronic Technology-Technical Analysis-Measurement Engineering-Office of Fire Programs.

THE CENTER FOR COMPUTER SCIENCES AND TECHNOLOGY conducts research and provides technical services designed to aid Government agencies in improving cost effectiveness in the conduct of their programs through the selection, acquisition, and effective utilization of automatic data processing equipment; and serves as the principal focus within the executive branch for the development of Federal standards for automatic data processing equipment, techniques, and computer languages. The Center consists of the following offices and divisions:

Information Processing Standards-Computer Information-Computer Services -Systems Development-Information Processing Technology.

THE OFFICE FOR INFORMATION PROGRAMS promotes optimum dissemination and accessibility of scientific information generated within NBS and other agencies of the Federal Government; promotes the development of the National Standard Reference Data System and a system of information analysis centers dealing with the broader aspects of the National Measurement System; provides appropriate services to ensure that the NBS staff has optimum accessibility to the scientific information of the world, and directs the public information activities of the Bureau. The Office consists of the following organizational units:

Office of Standard Reference Data-Office of Technical Information and Publications-Library-Office of International Relations.

\footnotetext{
1 Headquarters and Laboratories at Gaithersburg, Maryland, unless otherwise noted; mailing address Washington, D.C. 20234.

2 Part of the Center for Radiation Research.

3 Located at Boulder, Colorado 80302.
} 


\section{Na. 22 Thermophysical Properties}

\section{of Helium-4 from 4 to $3000 \mathrm{R}$ with Pressures to 15000 PSIA}

Robert D. McCarty

Cryogenics Division

Institute for Basic Standards

U.S. National Bureau of Standards

Boulder, Colorado 80302
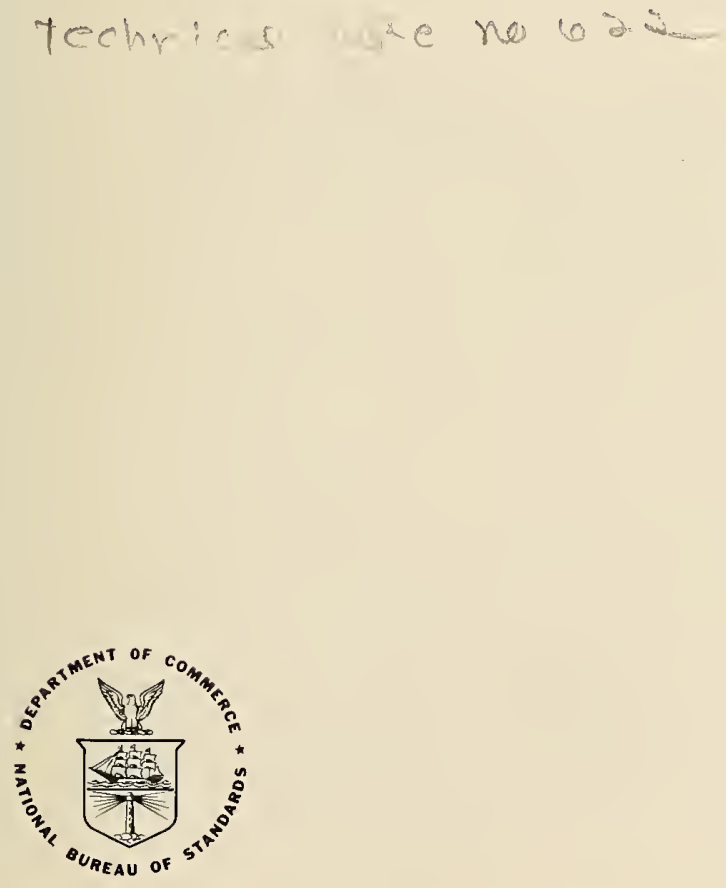

U.S. DePARTMENT OF COMMERCE. Peter G. Peterson, Secretary NATIONAL BUREAU OF STANDARDS, Lawrence M. Kushner, Acting Director 
National Bureau of Standards Technical Note 622

Nat. Bur. Stand. (U.S.), Tech. Note 622, 146 pages (September 1972) CODEN: NBTNAE

Issued September 1972 
1. Introduction . . . . . . . . . . . . . . . . . 1

2. Thermodynamic and Related Properties . . . . . . . . 1

2.1 PVT Surface . . . . . . . . . . . . . . 1

2.2 Derived Thermodynamic Properties . . . . . . 3

2.3 Related Properties . . . . . . . . . . . 3

2.4 Heat Capacities . . . . . . . . . . . . 3

3. Transport Properties . . . . . . . . . . . . . . 4

3.1 Thermal Conductivity, $300 \mathrm{~K}$ and Below . . . . . 4

3.2 Thermal Conductivity, $300 \mathrm{~K}$ and Above . . . . . 5

3.3 Viscosity Below $100 \mathrm{~K}$. . . . . . . . . . . 9

3.4 Viscosity Between 100 and $300 \mathrm{~K}$. . . . . . . 10

3.5 Viscosity for Temperatures Above $300 \mathrm{~K}$. . . . 10

4. Surface Tension . . . . . . . . . . . . . . . . 11

5. Dielectric Constant . . . . . . . . . . . . . . 11

6. Index of Refraction . . . . . . . . . . . . . . . 13

7. Thermal Diffusivity . . . . . . . . . . . . 16

8. Prandtl Number . . . . . . . . . . . . . . 16

9. Joule-Thomson Inversion Curve . . . . . . . . . . 16

10. The Melting Line . . . . . . . . . . . . . . 19

11. The Lambda Line . . . . . . . . . . . . . . . 20

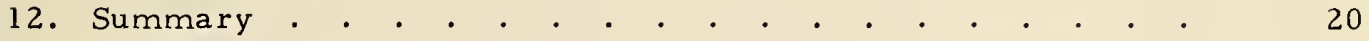

13. Bibliography . • . . . . . . . . . . . . . . . 21

Appendix A, List of Symbols and Units . . . . . . . . . . 23

Appendix B, Fixed Points . . . . . . . . . . . . . . 24

Appendix C, Conversion Factors . . . . . . . . . . . . 24

Appendix D, Saturation Properties . . . . . . . . . . 26

Appendix E, Isobaric Properties . . . . . . . . . . . 30 

Table

1. Uncertainties in the PVT Data . . . . . . . . . . 1

2. Index of Refraction of Saturated Liquid Helium at Three Wavelengths . . . . . . . . . 14

3. Joule-Thomson Inversion Curve . . . . . . . . . 17

4. Melting Line for Helium-4 . . . . . . . . . . 19

5. $\mathrm{P} \rho \mathrm{T}$ of the Lambda Line . . . . . . . . . . . . 20

List of Figures

$\underline{\text { Figure }}$

1. Range of PT Covered . . . . . . . . . . . . . 2

2. PVT Range of Experimental Data for Thermal Conductivity $\quad . \quad$. . . . . . . . 6

3. Deviations between Calculated Thermal Conductivity and Data by Kerrisk (1968) . . . . . . . 7

4. Deviations between Calculated Thermal Conductivities and Data by Golubev and Shpagina (1966).

5. Deviations between Calculated and Experimental

Surface Tension Data . . . . . . . . . . . . 12

6. Index of Refraction for Saturated Liquid and Gaseous

Helium. Points are from Edwards $(1957,1958)$

7. Joule-Thomson Inversion Curve . . . . . . . . 
Robert D. McCarty

Tables of thermophysical properties of helium 4 are presented for temperatures from 4 to 3000 Rankine for pressures to 15000 psia. The tables include, entropy, enthalpy, internal energy, density, volume, speed of sound, specific heat, thermal conductivity, viscosity, thermal diffusivity, Prandtl number and the dielectric constant for 74 isobars. Also included in the isobaric tables are quantities of special utility in heat transfer calculations: $(\partial \mathrm{P} / \partial \mathrm{V})_{\mathrm{T}},(\partial \mathrm{P} / \partial \mathrm{T})_{\rho}, \mathrm{V}(\partial \mathrm{H} / \partial \mathrm{V})_{\mathrm{P}}, \mathrm{V}(\partial \mathrm{P} / \partial \mathrm{U})_{\mathrm{V}},-\mathrm{V}(\partial \mathrm{P} / \partial \mathrm{V})_{\mathrm{T}}{ }$ $1 / \mathrm{V}(\partial \mathrm{V} / \partial \mathrm{T})_{\mathrm{P}^{\cdot}}$

In addition to the isobaric tables, tables for the saturated vapor and liquid are given which include all of the above properties, plus the surface tension. Tables for the $P \rho T$ of the freezing liquid, $P \rho T$ of the lambda line, index of refraction and the derived Joule-Thomson inversion curve are also presented.

Key Words: Density; dielectric constant; enthalpy; entropy; equation of state; fixed points; heat transfer coefficients; helium 4; index of refraction; Joule-Thomson coefficient; lambda line; latent heat; melting point; Prandtl number; specific heats; speed of sound; surface tension; thermal conductivity; thermal diffusivity; vapor pressure; viscosity; volume.

* This work carried out at the National Bureau of Standards, supported by NASA-MSC Contract T-1813A. 


\section{Introduction}

The purpose of this document is to assemble data on many of the properties of helium commonly used in engineering calculations over as wide a temperature and pressure range as is practical, and present these properties in a form which is convenient to the engineer. All of these properties have been critically evaluated and represent the "best values" for that property at this time.

The properties of helium 4 have been of great interest to the scientific and engineering community for many years. Much of the interest, and consequently the work, has been in the more spectacular properties of helium II, the superfluid phase. The properties of the superfluid are not included here except for the PPT of the boundary where the transition to the superfluid begins.

\section{Thermodynamic and Related Properties}

\subsection{PVT Surface}

The PVT surface described by McCarty (1972) was used to calculate all of the thermodynamic and related properties. The tables given here are essentially the same as those found in the referenced document. Figure 1 shows the range of PT covered by these tables, and table 1 gives estimates of uncertainties in density.

Table 1. Uncertainties in the PVT Data

Temperature Range

Pressure Range

$$
2 \text { - } 20 \mathrm{~K}
$$

2 - $20 \mathrm{~K}$

Critical region

$20-70 \mathrm{~K}$

$20-70 \mathrm{~K}$

$70-150 \mathrm{~K}$

$70-150 \mathrm{~K}$

$150-400 \mathrm{~K}$

$150-400 \mathrm{~K}$

$400-1500 \mathrm{~K}$

$400-1500 \mathrm{~K}$

$$
\begin{aligned}
& 0-2 \text { atm } \\
& 2-1000 \text { atm }
\end{aligned}
$$

$$
\mathrm{T}_{\mathrm{c}} \pm 5 \%, \rho_{\mathrm{c}} \pm 20 \%
$$

0 - 20 atm

$20-1000$ atm

0 - $100 \mathrm{~atm}$

0 - 100 atm

$100-1000 \mathrm{~atm}$

0 - 100 atm

$100-1000 \mathrm{~atm}$
$100-1000$ atm
Uncertainty in Density

Average Maximum

$0.1 \%$

$0.5 \%$

$3 \%$

$0.5 \%$

$1 \%$

$0.1 \%$

$0.5 \%$

$0.05 \%$

$0.1 \%$

$0.1 \%$

$.2 \%$ $\left.\begin{array}{l}0.5 \% \\ 1.5 \%\end{array}\right\} \begin{gathered}\text { (except in } \\ \text { critical region) }\end{gathered}$

\section{$8 \%$}

$1 \%$

$2 \%$ (no reliable experimental data)

$0.5 \%$

$2 \%$

$0.2 \%$

$1 \%$

$0.5 \%$

$2 \%$ 


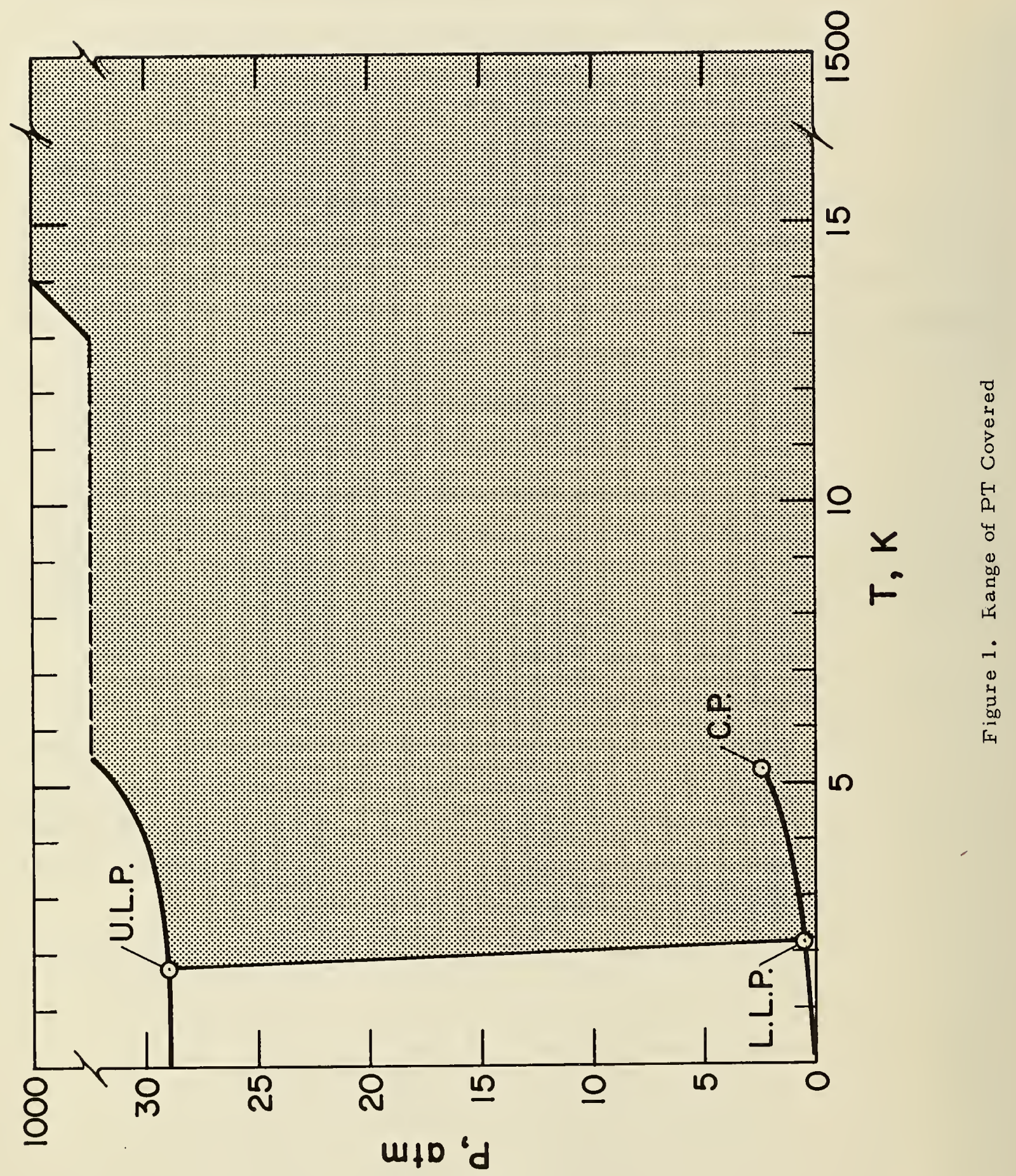




\section{2 Derived Thermodynamic Properties}

The enthalpy, $H$, speed of sound, $W$, entropy, $S$, and the internal energy, $U$, were calculated directly from the equations taken from McCarty (1972) and should be identical to those of McCarty when the proper unit conversions are applied.

\section{3 Related Properties}

A number of parameters such as the specific heat input $\left[\mathrm{V}(\partial \mathrm{H} / \partial \mathrm{V})_{\mathrm{P}}\right]$ are of use to the engineer. Several of the more us eful quantities of this kind have been tabulated here for the convenience of the user. These quantities have been derived from the equation of state in the following manner.

Specific heat input

$$
\theta=V\left(\frac{\partial H}{\partial V}\right)_{P}=\rho C_{p}\left[\left(\frac{\partial P}{\partial \rho}\right)_{T} /\left(\frac{\partial P}{\partial T}\right)_{V}\right]
$$

Energy derivative

$$
\Phi=V\left(\frac{\partial P}{\partial U}\right)_{V}=\frac{V}{C_{V}}\left(\frac{\partial P}{\partial T}\right)_{V}
$$

Is othermal bulk modulus

$$
\alpha=V\left(\frac{\partial P}{\partial V}\right)_{T}=-\rho\left(\frac{\partial P}{\partial \rho}\right)_{T}
$$

Volume expansivity

$$
\beta=\frac{1}{V}\left(\frac{\partial V}{\partial T}\right)_{P}=-\frac{1}{\rho}\left(\frac{\partial P}{\partial T}\right)_{\rho} /\left(\frac{\partial P}{\partial \rho}\right)_{T}
$$

\section{4 Heat Capacities}

The heat capacities, $\mathrm{C}_{\mathrm{V}}, \mathrm{C}_{\mathrm{P}}$ which appear in this document are taken from McCarty (1972). Except for the critical region and near the boundaries of phase changes, the uncertainty of the tabulated specific heats is estimated to be no greater than $5 \%$.

At the critical point, and along the lambda line, the specific heats become anomalous and no realistic estimates of accuracy may be made. 


\section{Transport Properties}

\subsection{Thermal Conductivity, $300 \mathrm{~K}$ and Below}

For temperatures below $300 \mathrm{~K}$, the thermal conductivity for helium- 4 has been calculated using the following equations.

$$
\lambda=\lambda_{o}(T) \lambda_{y}(\rho, T)+\lambda_{c}(\rho, T)
$$

where $\lambda_{0}(T)$ is the dilute gas contribution, $\lambda_{y}(\rho, T)$ corrects the dilute gas value for increasing densities and $\lambda_{c}(\rho, T)$ predicts the enhancement in thermal conductivity in the region near the critical point. The dilute gas contribution, $\lambda_{0}(T)$ for this temperature region has been calculated from

$$
\lambda_{0}(T)=e^{Z(\ln T)}
$$

If $x=\ln T$, then

$$
\begin{aligned}
Z(T)= & -4.3611622157+1.9250159286 x-0.52544120165 x^{2}+0.090045763885 x^{3} \\
& -0.0054773874708 x^{4}
\end{aligned}
$$

where $\mathrm{T}$ is in Kelvin and $\lambda_{0}$ is in $\mathrm{mW} / \mathrm{cm}-\mathrm{K}$.

The $\lambda_{y}(\rho, T)$ of equation $(5)$ is given by

$$
\lambda_{y}(\rho, T)=e^{\left(B(T) \rho+C(T) \rho^{2}\right)},
$$

if $\mathrm{x}=\ln \mathrm{T}$, then

$$
\begin{aligned}
B(T) & =\operatorname{EXP}\left(4.7470660612-5.3641468153 x+3.4639703698 x^{2}-1.0702455443 x^{3}\right. \\
& +0.1571349306 x^{4}-0.00892140047 x^{5}
\end{aligned}
$$

and

$$
\begin{aligned}
C(T) & =2.2109006708+.187 .74174808 / T-1281.0947055 / T^{2}+3645.2393216 / T^{3} \\
& -3986.6937948 / T^{4}
\end{aligned}
$$

where $\mathrm{T}$ is in Kelvin and $\rho$ is in $\mathrm{g} / \mathrm{cm}^{3}$. The $\lambda_{c}(\rho, \mathrm{T})$ of equation $(5)$ is given by

$$
\lambda_{c}(\rho, T)=0.000649578 \Delta C_{p}(\rho, T)
$$

where the $\Delta \mathrm{C}_{\mathrm{p}}$ is the $\mathrm{C}_{\mathrm{p}}$ at $\rho$ and $\mathrm{T}$ minus the $\mathrm{C}_{\mathrm{p}}$ at $\rho$ and $\mathrm{T}=11.83 \mathrm{~K}$. For $\mathrm{T}>11.83 \mathrm{~K}$, $\rho>.12 \mathrm{~g} / \mathrm{cm}^{3}$ or $\Delta \mathrm{C}_{\mathrm{p}}$ negative, $\lambda_{c}(\rho, \mathrm{T})$ is taken to be zero. The units of $\mathrm{C}_{\mathrm{p}}$ must be $\mathrm{J} / \mathrm{mol}-\mathrm{K}$ and the resulting units of thermal conductivity are $\mathrm{mW} / \mathrm{cm}-\mathrm{K}$. There are no known thermal conductivity measurements for helium-4 in the region of the critical 
point, and equation (11) is based on values scaled from hydrogen. The other term in equation (5), the $\lambda_{0}(\mathrm{~T}) \lambda_{y}(\rho, T)$ term relies heavily on two sources of experimental data, Golubev and Shpagina (1966) and Kerrisk (1968). These data were used by Roder (1971) and Arp (1971) to determine equations (7, 9, 10). Figure 2 shows the pressure and temperature regions covered by these two sources and figures 3 and 4 show typical differences, including the maximum differences, between the calculated and experimental conductivities.

\section{2 Thermal Conductivity, $300 \mathrm{~K}$ and Above}

When correlating thermal conductivity data it is common procedure to separate the equation into additive parts, with the $\lambda_{0}(T)$, or dilute gas contribution being a function of temperature only and $\lambda_{E}(\rho, T)$, called the excess or dense gas contribution being a function of density and temperature. For the heavier fluids, the temperature dependence of $\lambda_{E}(\rho, T)$ is so slight that it is usually neglected; however for helium this is not the case, and although equation (5) is multiplicative rather than additive, it does take into account the temperature dependence of the excess function. Since no experimental data above $300 \mathrm{~K}$ were used in obtaining the various parts of equation (5) it was not used for temperatures above $300 \mathrm{~K}$. For temperatures greater than $300 \mathrm{~K}$, the thermal conductivity has been calculated using the following equations

$$
\lambda=\lambda_{0}^{\prime}(T)+\lambda_{E}(\rho, T)
$$

$$
\lambda_{0}^{\prime}(\mathrm{T})=\left(1.53220256 \mathrm{~T}^{0.71938}\right)\left(\mathrm{e}^{\left(12.451 / \mathrm{T}-295.67 / \mathrm{T}^{2}-4.1249\right)}\right)+\mathrm{C}
$$

where $T$ is in Kelvin, $\lambda_{0}^{\prime}(T)$ is in $\mathrm{mW} / \mathrm{cm}-\mathrm{K}$ and $\mathrm{C}$ is a constant such that $\lambda_{0}^{\prime}(T)$ from equation $(13)=\lambda_{0}(T)$ from equation $(6)$ at $T=300 \mathrm{~K}$. 


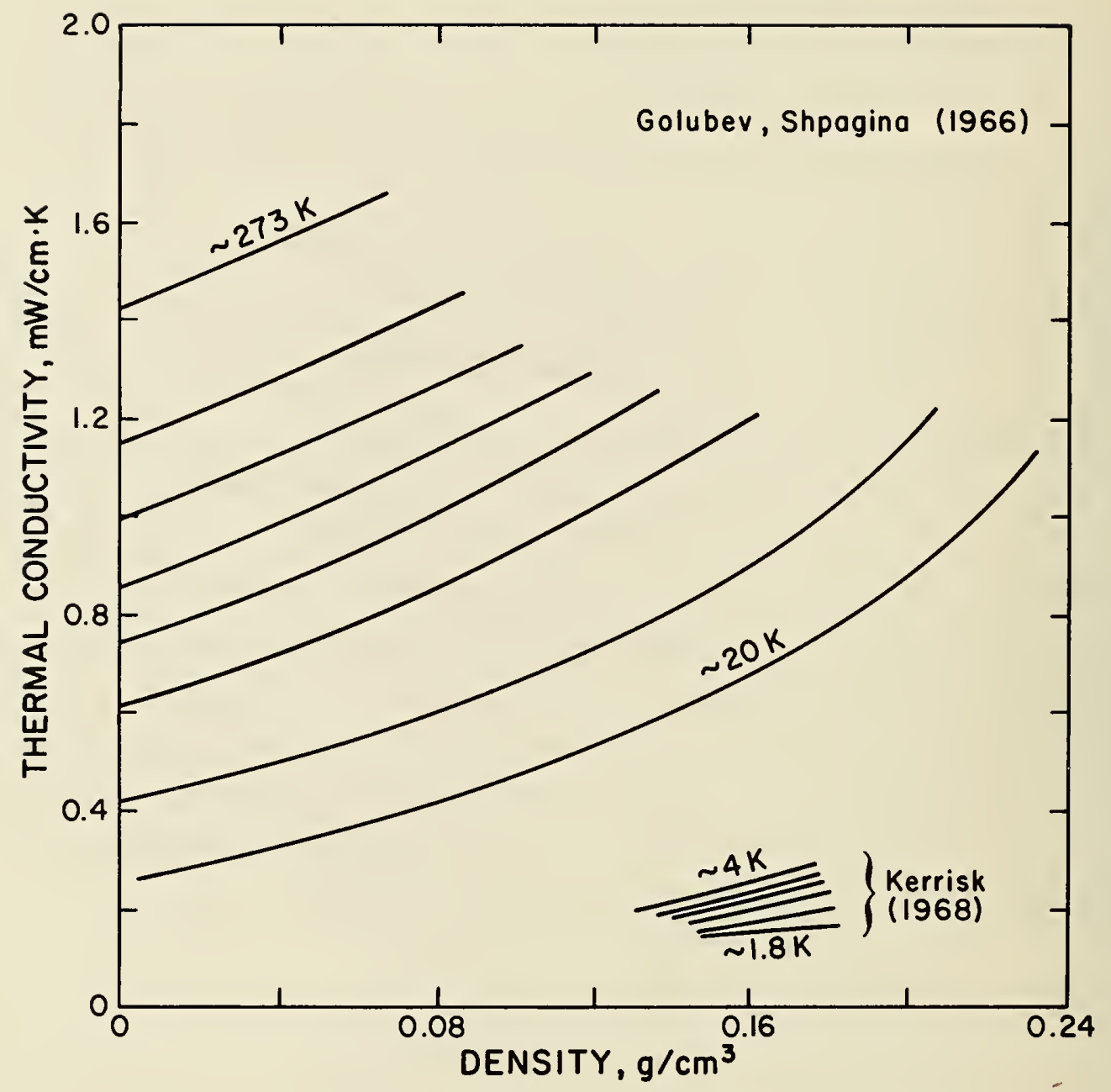

Figure 2. PVT Range of Experimental Data for Thermal Conductivity. 


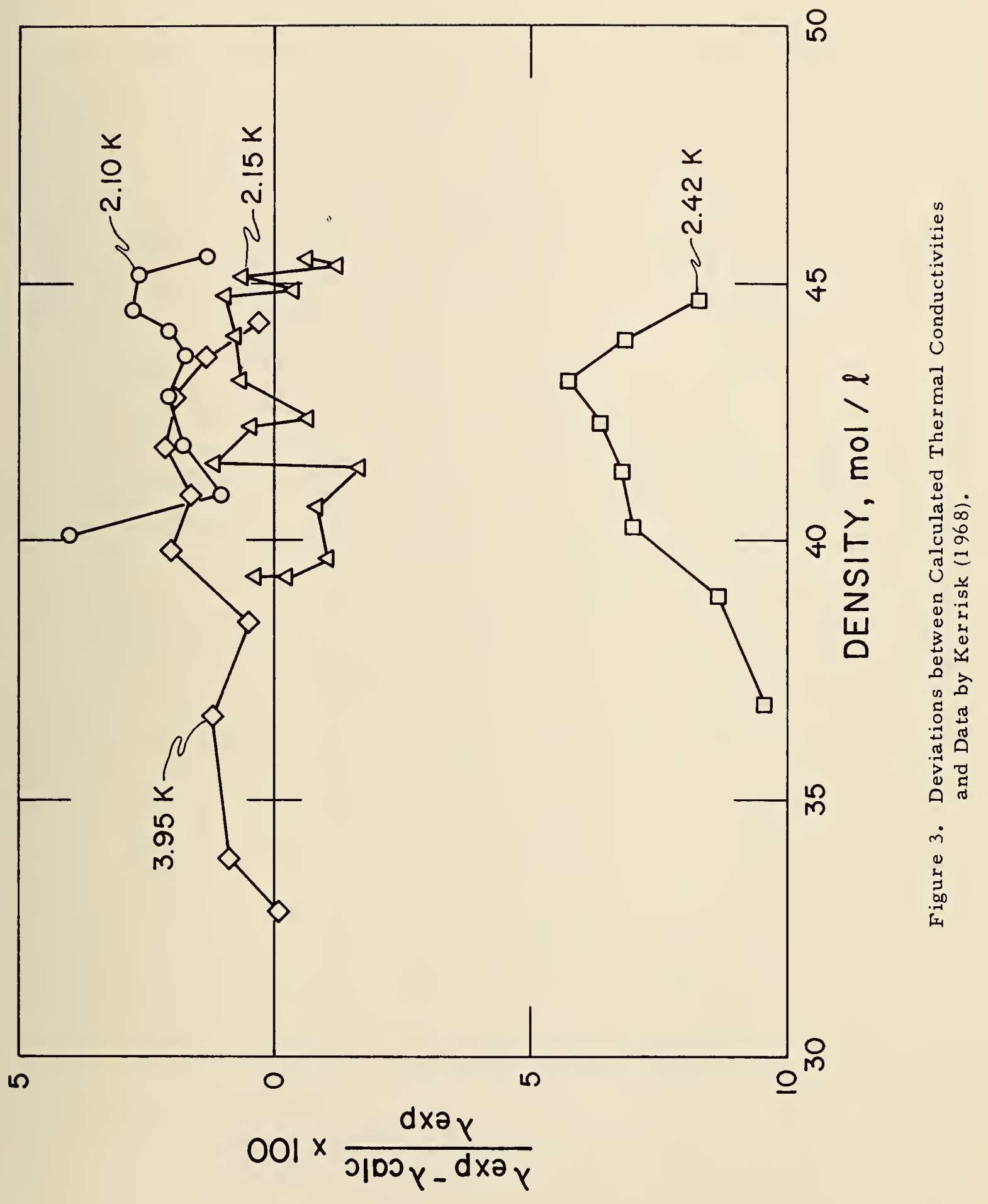




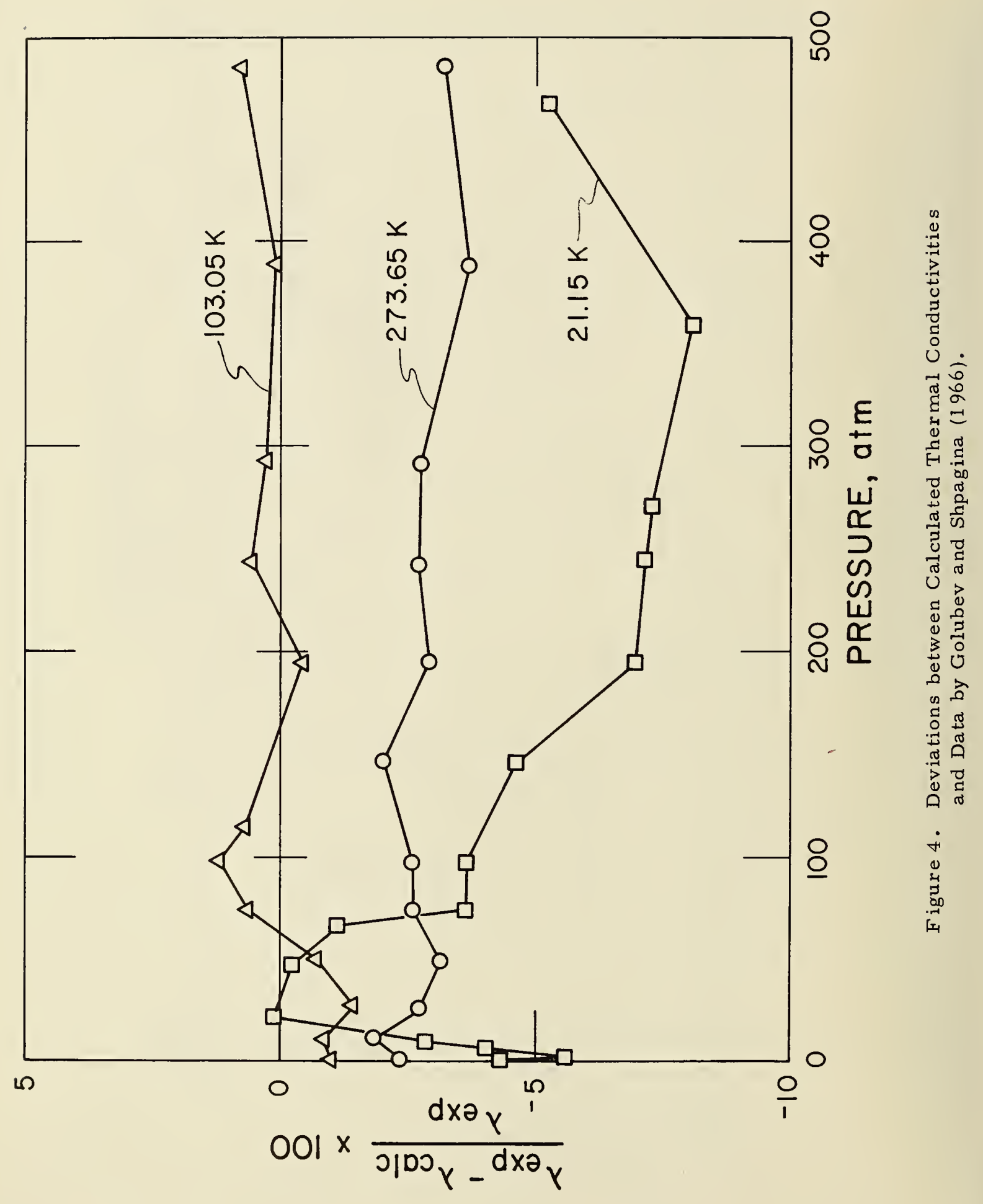


In selecting a suitable representation of the dilute gas contribution for temperatures above $300 \mathrm{~K}$, the correlation by Tsederberg, et al., (1969) was considered but was not used for two reasons. First, their value at $300 \mathrm{~K}$ was in disagreement with our best estimates by an excessive amount and, second, the $\lambda_{0}$ at higher temperatures, $\left(2000^{\circ} \mathrm{C}\right)$, were not theor etically consistent with the recently mea sured dilute gas viscosities, Guevara, et al., (1969). The representation which was finally selected (equation 13) is from Maitland and Smith (1971), and was selected because the above two objections to the equation proposed by Tsederberg, et al., (1969) were not present. The original equation from Maitland and Smith (1971) is for the dilute gas viscosity and has been converted to thermal conductivity by a proportionality constant.

The $\lambda_{E}(\rho, T)$ of equation (12) is given by

$$
\lambda_{E}(\rho, T)=\lambda_{E}(\rho)=\lambda_{o}(300)\left[\lambda_{y}(\rho, 300)-1\right]
$$

evaluated at $\rho$ and $T=300 \mathrm{~K}$. Thus equation (5) and equation (12) will give identical results for all $\rho$ at $300 \mathrm{~K}$.

\subsection{Viscosity Below $100 \mathrm{~K}$}

For temperatures of $100 \mathrm{~K}$ and below the equation

$$
\ln \eta=\eta_{0}^{\prime}(\mathrm{T})+\eta_{\mathrm{E}}^{\prime}(\rho, \mathrm{T})
$$

was used to calculate the viscosity for helium. If $x=\ln (T)$, then

$$
\begin{aligned}
\eta_{0}^{\prime}(\mathrm{T})=-0.135311743 / x+1.00347841 & +1.20654649 x-0.149564551 x^{2} \\
& +0.0125208416 x^{3}
\end{aligned}
$$

and

$$
\eta_{E}^{\prime}(\rho, T)=\rho B(T)+\rho^{2} C(T)+\rho^{3} D(T)
$$

where $\rho$ is in $\mathrm{g} / \mathrm{cm}^{3}$, and

$B(T)=-47.5295259 / x+87.6799309-42.0741589 x+8.33128289 x^{2}-0.589252385 x^{3}$ 
$C(T)=547.309267 / x-904.870586+431.404928 x-81.4504854 x^{2}$

$D(T)=-1684.39324 / x+3331.08630-1632.19172 x+308.804413 x^{2}$

$$
-20.2936367 x^{3}
$$

The resulting viscosities are in $\mu \mathrm{g} / \mathrm{cm} \cdot \mathrm{s}$. Equations (15-20) are from Steward, et al. (1971). Steward's work included new measurements from 4 to $20 \mathrm{~K}$ at pressures from the dilute gas region to $10 \mathrm{MN} / \mathrm{m}^{2}$. Steward reports a standard deviation of .032 in the natural log of the viscosity in the units of $\mu \mathrm{g} / \mathrm{cm} \cdot \mathrm{s}$. In addition, Steward proposes the possibility of an uncertainty of $\pm 8 \%$.

\subsection{Viscosity Between 100 and $300 \mathrm{~K}$}

Steward included a few points calculated from the Enskog theory (Hanley, et al., 1971) when the equations (15-20) were derived. He found this necessary to enable the use of these equations up to $300 \mathrm{~K}$; however, from 100 to $300 \mathrm{~K}$ the dilute gas values of Steward differ by $2.5 \%$ from a recent correlation by Maitland and Smith (1971). Since Steward reports using calculated dilute gas values and the correlation of Maitland and Smith is based on experimental data, the dilute gas values of Maitland and Smith were used for all $\mathrm{T}>110$. Between 100 and $110 \mathrm{~K}$, a linear average of the dilute gas values of Steward and Maitland and Smith was used. In the 100 to $110 \mathrm{~K}$ temperature range the dense gas contribution for viscosity was calculated from Steward's equations. The equations for viscositv between 100 and $300 \mathrm{~K}$ are:

$$
\eta(\rho, T)=\eta_{0}(T)+\eta_{E}(\rho, T)
$$

where

$$
\eta_{0}(T)=196 T^{.71938} e^{\left(12.451 / T-295.67 / T^{2}-4.1249\right)}
$$

and

$$
\eta_{E}=e^{\left[\eta_{0}^{\prime}(T)+\eta_{E}^{\prime}(\rho, T)\right]}-e^{\left[\eta_{o}^{\prime}(T)+\eta_{E}^{\prime}(0, T)\right]}
$$

where $\rho$ is in $g / \mathrm{cm}^{3}, T$ in Kelvin, and $\eta$ is in $\mu \mathrm{g} / \mathrm{cm} \cdot \mathrm{s}$.

\section{5 Viscosities for Temperatures Above $300 \mathrm{~K}$}

Since Steward's analysis did not include any dense gas data for temperatures above $300 \mathrm{~K}$, either calculated or experimental, the temperature dependence of the excess function given by equation (23) was frozen at $300 \mathrm{~K}$. When equation (23) is fixed at $300 \mathrm{~K}$, the resulting equation is a function of density alone and gives results similar to the excess 
function of Tsederberg, et al., (1969). Therefore, for temperatures above $300 \mathrm{~K}$, the viscosities were calculated using equations (21-23) except that equation (23) was always evaluated at $\rho$ and $T=300 \mathrm{~K}$. The uncertainty of the viscosity for $\mathrm{T}>100 \mathrm{~K}$ is estimated to be maximum of $\pm 10 \%$.

\section{Surface Tension}

The surface tension for helium- 4 has been calculated using the equation

$$
\varphi=\gamma_{0}\left(1-T / T_{c}\right)
$$

where $\gamma_{0}=0.5308 \mathrm{dyn} / \mathrm{cm}$ and $\mathrm{T}_{\mathrm{c}}=5.2014 \mathrm{~K}$. The $\gamma_{0}$ is based on a least squares fit of equation (24) to the data of van Urk, et al., (1925). Since the least squares fit of equation (24) was performed two sources of experimental data have appeared. These are Dickson, et al., (1970), and Devaraj and Hollis-Hallett (1967). Figure 5 shows that $V_{0}$ would not change appreciably if the se new data were included in a refit.

\section{Dielectric Constant}

The dielectric constant of a fluid may be calculated from the Clausius-Mossotti equation:

$$
\frac{\epsilon-1}{\epsilon+2} \frac{1}{p}=p
$$

where $\epsilon$ is the dielectric constant, $\rho$ is the density, and $P$ is the specific polarizability, a property of the substance having dimensions of specific volume. Recent measurements of the dielectric constant by Kerr and Sherman (1970) indicate that for helium-4 the zpecific polarizability is a weak function of density and that the first density correction is negative. For the calculations here, the equation:

$$
p=0.123396-0.0014 \rho
$$

was used, where $p$ is the specific polarizability in $\mathrm{cm}^{3} / g$ and $\rho$ is the density in $\mathrm{g} / \mathrm{cm}^{3}$. The uncertainty of the tabulated values of dielectric constant is estimated to be $0.01 \%$. 


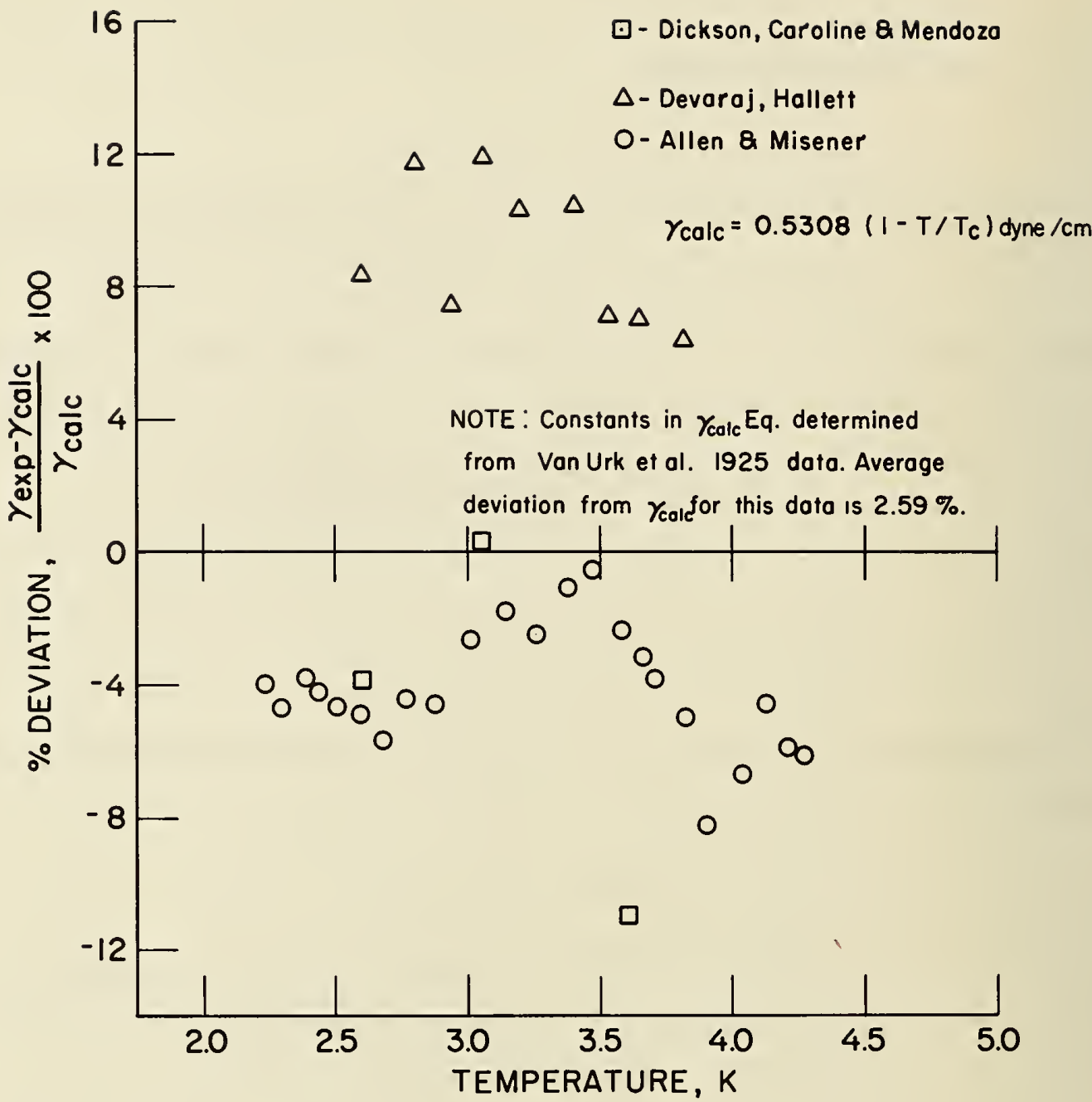

Figure 5. Deviations between Calculated and Experimental Surface Tension Data. 


\section{Index of Refraction}

The refractive index of a non-polar fluid depends on the wavelength of the incident radiation and on the density of the fluid. However, the dependence on wavelength can be treated independently of the dependence on density. The Cauchy dispersion formula:

$$
r_{\Lambda}=r(\rho, \Lambda)=r_{\infty}(\rho)+\theta_{1} / \Lambda^{2}+\theta_{2} / \Lambda^{4}
$$

assuming the equivalence of the Maxwell's relation

$$
\epsilon=\mathrm{n}_{\infty}^{2}
$$

to

$$
p=r_{\infty}
$$

allows the calculation of $r_{\infty}$ from the polarizability as a function of density and

$$
r_{\infty}(\rho)=0.123396-0.0014 \rho
$$

where $\rho$ is density in $\mathrm{g} / \mathrm{cm}^{3}$. Equation (30) was then substituted for the first term in equation (27) and $\theta_{1}$ and $\theta_{3}$ were determined by least squares estimation using dispersion data from Landolt-Börnstein (1962). The resulting values were $\theta_{1}=33701.617944$ and $\theta_{2}=-12325284955$. The specific refraction, $r_{\Lambda}$, is in $\mathrm{cm}^{3} / \mathrm{g}$, density, $\rho$, is in $\mathrm{g} / \mathrm{cm}^{3}$, and the wavelength, $\Lambda$, is in $\AA$. Values of the index of refraction $n$ in table 2 have been calculated from equations (27), (30), and (31).

$$
r_{\Lambda}=\frac{n^{2}-1}{n^{2}+2} \cdot \frac{1}{\rho}
$$

A comparison between experimental measurements of the index of refraction (Edwards, 1956, 1957, and 1958) and those calculated using equation (31) shows agreement to better than 0.1\%, except with those values from the 1956 reference. Edwards (1956) and (1958) papers both report values of the index of refraction for the saturated liquid of helium-4. However, the values in the 1956 paper are about $4 \%$ higher than those in the 1958 paper, and the 1956 values are presumed to be in error. Figure 6 shows $\mathrm{n}$ as a function of density for the saturated vapor and saturated liquid conditions. The points are from Edwards (1957 and 1958). 
Table 2. Index of Refraction of Saturated Liquid Helium at Three Wavelengths

$\begin{array}{cccc}\text { Temp } & 4358 \AA & 5462 \AA & 6939 \AA \\ \mathrm{K} & \mathrm{n} & \mathrm{n} & \mathrm{n} \\ 2.2 & 1.02881 & 1.02867 & 1.02857 \\ 2.4 & 1.02864 & 1.02849 & 1.02839 \\ 2.6 & 1.02841 & 1.02827 & 1.02817 \\ 2.8 & 1.02814 & 1.02800 & 1.02790 \\ 3.0 & 1.02782 & 1.02768 & 1.02759 \\ 3.2 & 1.02745 & 1.02731 & 1.02722 \\ 3.4 & 1.02703 & 1.02690 & 1.02681 \\ 3.6 & 1.02656 & 1.02643 & 1.02634 \\ 3.8 & 1.02602 & 1.02589 & 1.02580 \\ 4.0 & 1.02541 & 1.02528 & 1.02520 \\ 4.2 & 1.02471 & 1.02459 & 1.02450 \\ 4.4 & 1.02389 & 1.02377 & 1.02369 \\ 4.6 & 1.02290 & 1.02278 & 1.02270 \\ 4.8 & 1.02163 & 1.02152 & 1.02145 \\ 5.0 & 1.01986 & 1.01976 & 1.01969\end{array}$




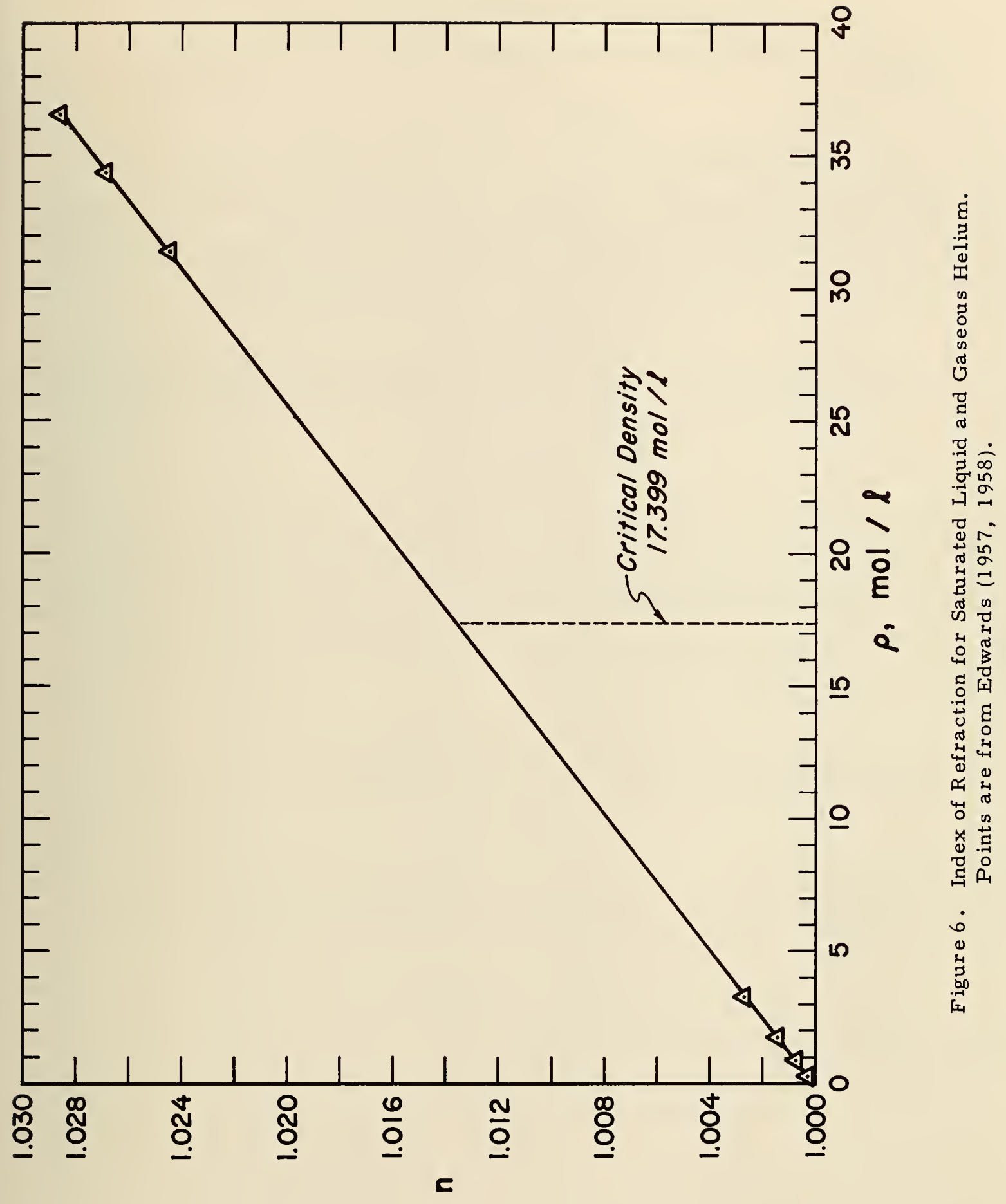


7. Thermal Diffusivity

The thermal diffusivity of a fluid is defined as

$$
\propto=\lambda /\left(\rho C_{p}\right)
$$

where $\propto$ is the thermal diffusivity, $\lambda$ is the thermal conductivity, and $C_{p}$ is the specific heat at constant pressure. The tabulations of thermal diffusivity in appendices $D$ and $E$ have been calculated using the above equation, and $\rho, \lambda$, and $C_{p}$ in the tables. The uncertainty of $\propto$ is estimated to be $20 \%$, except in the critical region.

\section{Prandtl Number}

The Prandtl number is frequently used in engineering calculations and is defined as:

$$
\operatorname{Pr}=C_{p} \eta / \lambda
$$

where $\mathrm{Pr}$ is the Prandtl number, $\mathrm{C}_{\mathrm{p}}$ is the specific heat at constant pressure, $\eta$ is the viscosity, and $\lambda$ is the thermal conductivity. The tabulations of the Prandtl number in appendices $D$ and $E$ have been calculated from equation (33) using values of $\eta, \lambda$, and $C_{p}$ from adjacent entries in the tables. Since $\operatorname{Pr}$ is a function of both $\eta$ and $\lambda$, the uncertainty in $\mathrm{Pr}$ could be as much as $25 \%$.

\section{Joule-Thomson Inversion Curve}

The Joule-Thomson coefficient for a fluid is defined as:

$$
\mathrm{J}=(\partial \mathrm{T} / \partial \mathrm{P})_{\mathrm{H}}
$$

The locus of points where $J=0$ is called the Joule-Thomson inversion curve: see figure 7. The inversion curve as given in table 3 has been calculated using the relationship:

$$
T(\partial P / \partial T)_{\rho}=\rho(\partial P / \partial \rho)_{T}
$$

and the equation of state from McCarty (1972). 
Table 3. Joule-Thomson Inversion Curve

Temperature

K

4.5

59.0

$6 \quad 10.8$

$7 \quad 12.6$

$8 \quad 12.4$

$9 \quad 16.2$

$10 \quad 18.0$

$12 \quad 21.6$

$14 \quad 25.2$

$16 \quad 28.8$

$18 \quad 32.4$

$20 \quad 36.0$

$22 \quad 39.6$

$24 \quad 43.2$

$26 \quad 46.8$

$28 \quad 50.4$

$30 \quad 54.0$

$32 \quad 57.6$

$34 \quad 61.2$

$36 \quad 64.8$

$38 \quad 68.4$

$40 \quad 72.0$

$42 \quad 75.6$

$43 \quad 77.4$
Pressure

atm

psia

1.821

3.768

26.76

55.37

7.266

106.8

10.74

14.10

17.31

20.36

25.57

29.29

32.07

34.44

36.18

37.33

37.93

37.98

37.48

36.40

34.71

32.32

29.13

24.89

19.11

9.80

.03
157.8

207.2

254.4

299.2

375.8

430.5

471.3

506.2

531.7

548.6

557.4

558.2

550.8

535.0

510.1

475.0

428.0

365.8

280.8

144.0

.5
Density

$\mathrm{mol} / \mathrm{e}$

$\mathrm{lb} / \mathrm{ft}^{3}$

30.83

7. 703

30.68

7.667

30.03

7.504

29.53

7.378

28. 99

7.245

28.43

7.106

27.86

6.962

26.42

6.602

24.72

6. 177

23.07

5. 764

21.61

5.400

20.20

5.046

18.82

4. 703

17.48

4.367

16.15

4.035

14.83

3. 705

13.49

3. 372

12.13

3.030

10.71

2.675

9.194

2.297

7.527

1.881

5.567

1.391

2. 780

.695

.009 


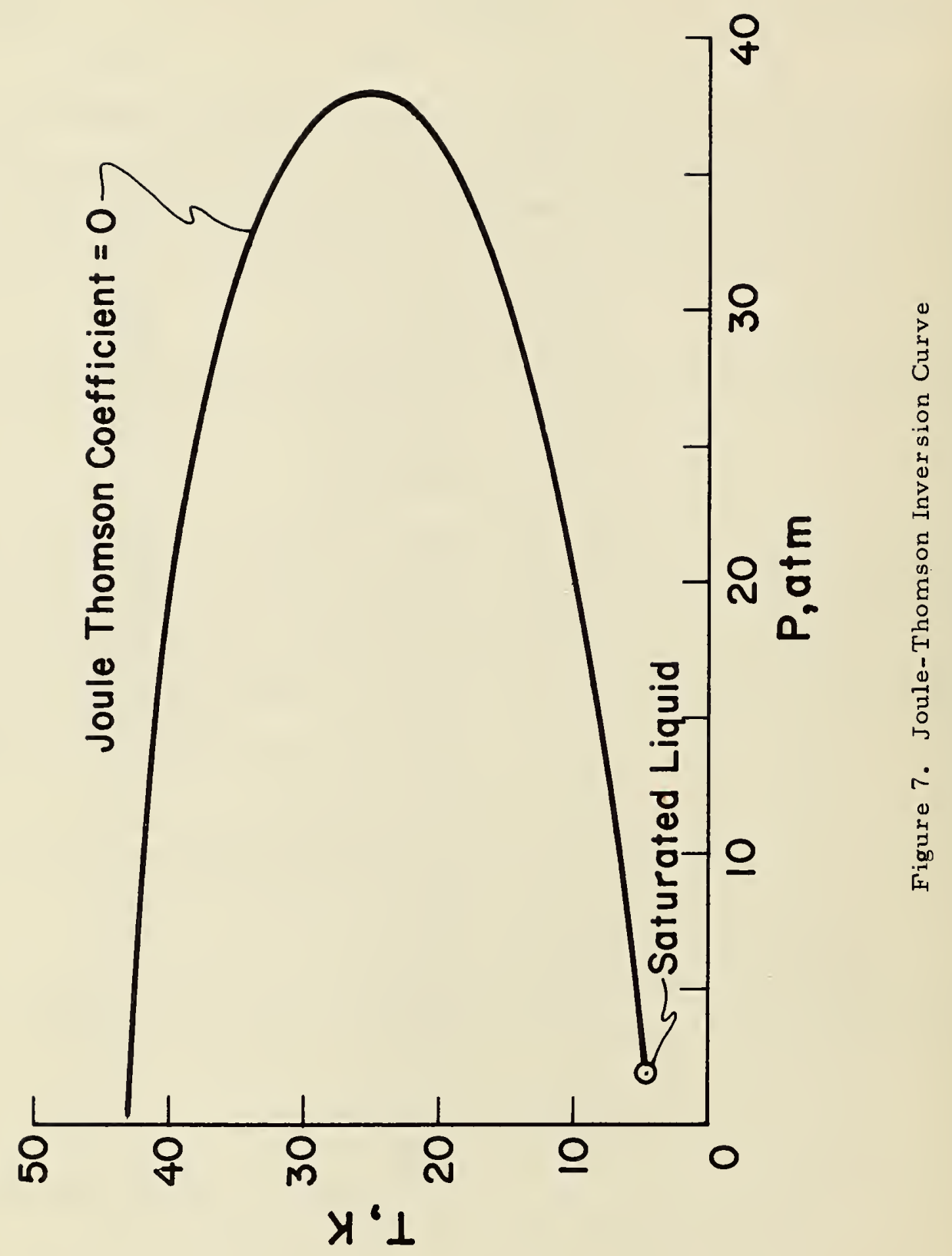


10. The Melting Line

The melting curve for helium may be calculated using the Simon melting equation.

$$
P=-17.80+17.31457 \mathrm{~T}^{1.555414}
$$

where $P$ is in $\mathrm{kg} / \mathrm{cm}^{2}$ and $T$ is in Kelvin. The constants in equation (36) were reported by Mills and Grilly (1955), and were based on their experimental data which extended from the upper lambda point to $30.77 \mathrm{~K}$ where they found the melting pressure to be $3555.6 \mathrm{~kg} / \mathrm{cm}^{2}$. In a later paper (Grilly and Mills, 1959) a separate equation is reported for temperatures between 1.8 and $5.2 \mathrm{~K}$. This equation is:

$$
P=33.28-44.156 \mathrm{~T}+31.799 \mathrm{~T}^{2}-4.8159 \mathrm{~T}^{3}+0.30313 \mathrm{~T}^{4}
$$

where $P$ is in $\mathrm{kg} / \mathrm{cm}^{2}$ and $T$ is in Kelvin. The values in table 4 have been calculated using equation (37) for $T \leq 5.2$ and equation (36) for $T>5.2$

\begin{tabular}{|c|c|}
\hline $\mathrm{K}$ & $\mathrm{R}$ \\
\hline 2.0 & 3.60 \\
\hline 2.5 & 4.50 \\
\hline 3.0 & 5.40 \\
\hline 3.5 & 6.30 \\
\hline 4.0 & 7.20 \\
\hline 5.0 & 9.00 \\
\hline 6.0 & 10.80 \\
\hline 7.0 & 12.60 \\
\hline 8.0 & 14.40 \\
\hline 9.0 & 16.20 \\
\hline 10.0 & 18.00 \\
\hline 12.0 & 21.60 \\
\hline 14.0 & 25.20 \\
\hline 16.0 & 28.80 \\
\hline 18.0 & 32.40 \\
\hline 20.0 & 36.00 \\
\hline 22.0 & 39.60 \\
\hline 24.0 & 43.20 \\
\hline 26.0 & 46.80 \\
\hline 28.0 & 50.40 \\
\hline 30.0 & 54.00 \\
\hline
\end{tabular}

Table 4. Melting Line for Helium-4

\begin{tabular}{rrrc}
\multicolumn{2}{c}{ Pressure } & Liquid & Density \\
atm & psia & mol/ $\ell$ & lb/cu-ft \\
37.25 & 547.4 & 46.18 & 11.540 \\
56.35 & 828.2 & 48.67 & 12.162 \\
78.91 & 1159.6 & 50.97 & 12.737 \\
103.83 & 1525.9 & 52.94 & 13.229 \\
130.49 & 1917.7 & 54.63 & 13.650 \\
188.67 & 2772.8 & 57.54 & 14.378 \\
254.77 & 3744.1 & 60.25 & 15.055 \\
328.47 & 4827.1 & 62.85 & 15.704 \\
408.27 & 5999.9 & 65.30 & 16.317 \\
493.81 & 7257.1 & 67.63 & 16.900 \\
584.82 & 8594.4 & 69.85 & 17.455 \\
782.21 & 11495.4 & 73.91 & 18.468 \\
998.83 & 14678.8 & 77.37 & 19.332 \\
1233.37 & 18125.7 & 80.43 & 20.096 \\
1484.81 & 21820.8 & 83.34 & 20.824 \\
1752.29 & 25751.6 & 86.07 & 21.507 \\
2035.05 & 29907.1 & 88.67 & 22.156 \\
2332.48 & 34278.1 & 91.17 & 22.780 \\
2644.01 & 38856.3 & 93.60 & 23.387 \\
2969.14 & 43634.5 & 95.98 & 23.983 \\
3307.44 & 48606.2 & 98.34 & 24.573
\end{tabular}


Table 5. PpT of the Lambda Line

\begin{tabular}{cccccc} 
Temperature & \multicolumn{2}{c}{ Pressure } & \multicolumn{2}{c}{ Density } \\
$\mathrm{K}$ & $\mathrm{R}$ & $\mathrm{atm}$ & $\mathrm{psia}$ & $\mathrm{g} / \mathrm{cm}^{3}$ & $\mathrm{lb} / \mathrm{ft}^{3}$ \\
2.172 & 3.910 & 0.0497 & .730 & 0.1462 & 9.127 \\
2.15 & 3.87 & 2.33 & 34.24 & 0.1506 & 9.402 \\
2.10 & 3.78 & 6.84 & 100.5 & 0.1576 & 9.839 \\
2.05 & 3.69 & 10.91 & 160.3 & 0.1627 & 10.16 \\
2.00 & 3.6 & 14.68 & 215.7 & 0.1669 & 10.42 \\
1.95 & 3.51 & 18.22 & 267.8 & 0.1705 & 10.64 \\
1.90 & 3.42 & 21.55 & 316.7 & 0.1736 & 10.84 \\
1.85 & 3.33 & 24.70 & 363.0 & 0.1763 & 11.01 \\
1.80 & 3.24 & 27.67 & 406.6 & 0.1788 & 11.16 \\
1.763 & 3.174 & 29.74 & 437.1 & 0.1804 & 11.26
\end{tabular}

\section{The Lambda Line}

The boundary of the superfluid phase (helium II) of liquid helium-4 is known as the lambda line. The lambda line begins at $2.172 \mathrm{~K}$ on the saturated liquid line and continues through the liquid phase to intersect the melting curve at $1.763 \mathrm{~K}$. Table 5 gives the $\mathrm{P} \rho \mathrm{T}$ of the lambda line as reported by Kierstead (1967).

\section{Summary}

The lack of sufficiently accurate experimental data for helium between the temperatures of 2 and $70 \mathrm{~K}$ has hampered the efforts of correlating the PVT surface by McCarty (1972). This is especially important for the temperature range of 2 - $20 \mathrm{~K}$ where small amounts of accurate data do exist but these data cover very restricted ranges of temperature and pressure. A single set of self consistent data covering the entire pressure and temperature range is needed.

The purpose of the previous sections has been to describe where or how the values were obtained in assembling the various tables and graphs presented here. Most of the material has been taken from McCarty (1972) and formulas and descriptions given there have not been repeated here.

In addition, an effort has been made to assign realistic uncertainties in the data wherever possible. Uncertainty is defined here to be an estimate of accuracy at a $95 \%$ confidence level. These assignments are made in the text of the section or subsection concerned with that property. Finally, the number of digits in the tables of appendices $D$ and $E$ should not be taken as an indication of accuracy of the number. The tabulations are a direct copy of computer printouts where it is of ten necessary to present more digits for a property than its accuracy justifies. 


\section{Bibliography}

Allen, J. F. and Miesner, A. D. The Surface Tension of Liquid Helium, Camb. Phil. Soc. $34^{\circ}, 299$ (1938).

Arp, V., Ballinger, E. R., Giarratano, P. J., Roder, H. M., Smith, R. V. , Snyder, N. S. , Steward, W. G., and Wallace, G. H., Helium Heat Transfer, Unpublished Data (1971).

Devaraj, N. and Hollis-Hallet, A. G., The Surface Tension of Liquid Helium I and the Law of Corresponding States, Can. J. Phys. $\underline{45}, 2113$ (1967).

Dickson, D. P. E., Caroline, D., and Mendoza, E., Surface Tension of Liquid ${ }^{4}$ Helium, Phys. Lett. 33A, No. 3, 139 (1970).

Edwards, M. H. , The Index of Refraction of Liquid Helium, Can. J. Phys. 34 , $898(1956)$.

Edwards, M. H. , Refractive Index of $\mathrm{He}^{4}$ : Saturated Vapor, Phys. Rev. 108, No. 5, 1243 (Dec 1957).

Edwards, M. H., Refractive Index of $\mathrm{He}^{4}$ : Liquid, Can. J. Phys. 36,884 (1958).

Grilly, E. R. and Mills, R. L. , Melting Properties of $\mathrm{He}^{3}$ and $\mathrm{He}^{4}$ up to $3500 \mathrm{~kg} / \mathrm{cm}^{2}$, Ann. Phys. 8,1 (1959).

Golubev, I. F. and Shpagina, I. B., Thermal Conductivity of Helium at Temperatures from 273.65 to 21.15 Degrees $\mathrm{K}$ and Pressures From 1 to 500 Atmospheres, Gaz. Prom. 11, No. 8, 40 (Aug 1966).

Guevara, F. A., McInteer, B. B., and Wageman, W. E., High Temperature Viscosity Ratios for Hydrogen, Helium, Argon and Nitrogen, Phys. Fluids 12, No. 12, 2493 (Dec 1969).

Hanley, H. J. M. McCarty, R. D., and Cohen, E. G. D., Analysis of Transport Coefficients for Simple Dense Fluids: Application of the Modified Enskog Theory, Physica (1972).

Kerr, E. C. and Sherman, R. H. , The Molar Polarizability of ${ }^{3} \mathrm{He}$ at Low

Temperatures and its Density Dependence, J. Low Temp. Phys. $\underline{3}$, No. 5, 451 (1970). Kerrisk, J. F., The Thermal Conductivity of Liquid Helium-4 and Liquid Helium-3, Doctoral Dissertation, University of New Mexico, Albuquerque, N.M. (Jun 1968).

Kierstead, H. A., Lambda Curve of Liquid He ${ }^{4}$, Phys. Rev. 162, No. 1, (Oct 1967). Landolt-Börnste in Tables, 6th Edition II, Part 8, 872 (1962).

Maitland, G. C. and Smith, E. B., The Viscosities of Eleven Common Gases: A Critical Compilation, Physical Chemistry Laboratory, South Parks Road, Oxford, England (Sep 1971).

McCarty, R. D., The Thermodynamic Functions for Helium-4 for Temperatures from 2 to $1500 \mathrm{~K}$ with Pressures to $100 \mathrm{MN} / \mathrm{m}^{2}$, NSRD Monograph (to be published) (1972). 
Mechtly, E. A., The International System of Units, Physical Constants and Conversion Factors, NASA-SP-7012 (1964).

Mills, R. L. and Grilly, E. R., Melting Curves of $\mathrm{He}^{3}, \mathrm{He}^{4}, \mathrm{H}_{2}, \mathrm{D}_{2}, \mathrm{Ne}, \mathrm{N}_{2}$ and $\mathrm{O}_{2}$ up to $3500 \mathrm{~kg} / \mathrm{cm}^{2}$, Phys. Rev. 99, No. 2, 480 (Jul 1955).

Roder, H. M., Private Communication (1971).

Steward, W. G. and Wallace, G. H., Helium ${ }^{4}$ Viscosity Measuremen ts 4 to $20 \mathrm{~K}, 0$ to $10 \mathrm{MN} / \mathrm{m}^{2}$, Unpublished Data (Sep 1971).

Tsederberg, N. V., Popov, V. N., and Morozova, N. A., Thermodynamic and

Thermophysical Properties of Helium, Moscow (1969), Translated by the Israel

Program for Scientific Translations, Jerusalem (1971), Available from the U. S.

Department of Commerce National Technical Information Service, Springfield, Va. van Urk, A. Th., Keesom, W. H., and Onnes, H. K., Measurements of the Surface

Tension of Liquid Helium, Communs. Phys. Lab. Univ. Leiden, No. 179a (1925). 


\section{Appendix A. List of Symbols and Units}

The calculation of the tables and properties presented here was performed in many different systems of units, and converted to engineering units at the very end of the calculations; therefore the reader is cautioned to pay particular attention to the units when consulting individual sections of this document. All conversion factors have been taken from the National Aeronautics and Space Administration Report Number SP-7012 (Mechtly 1964).

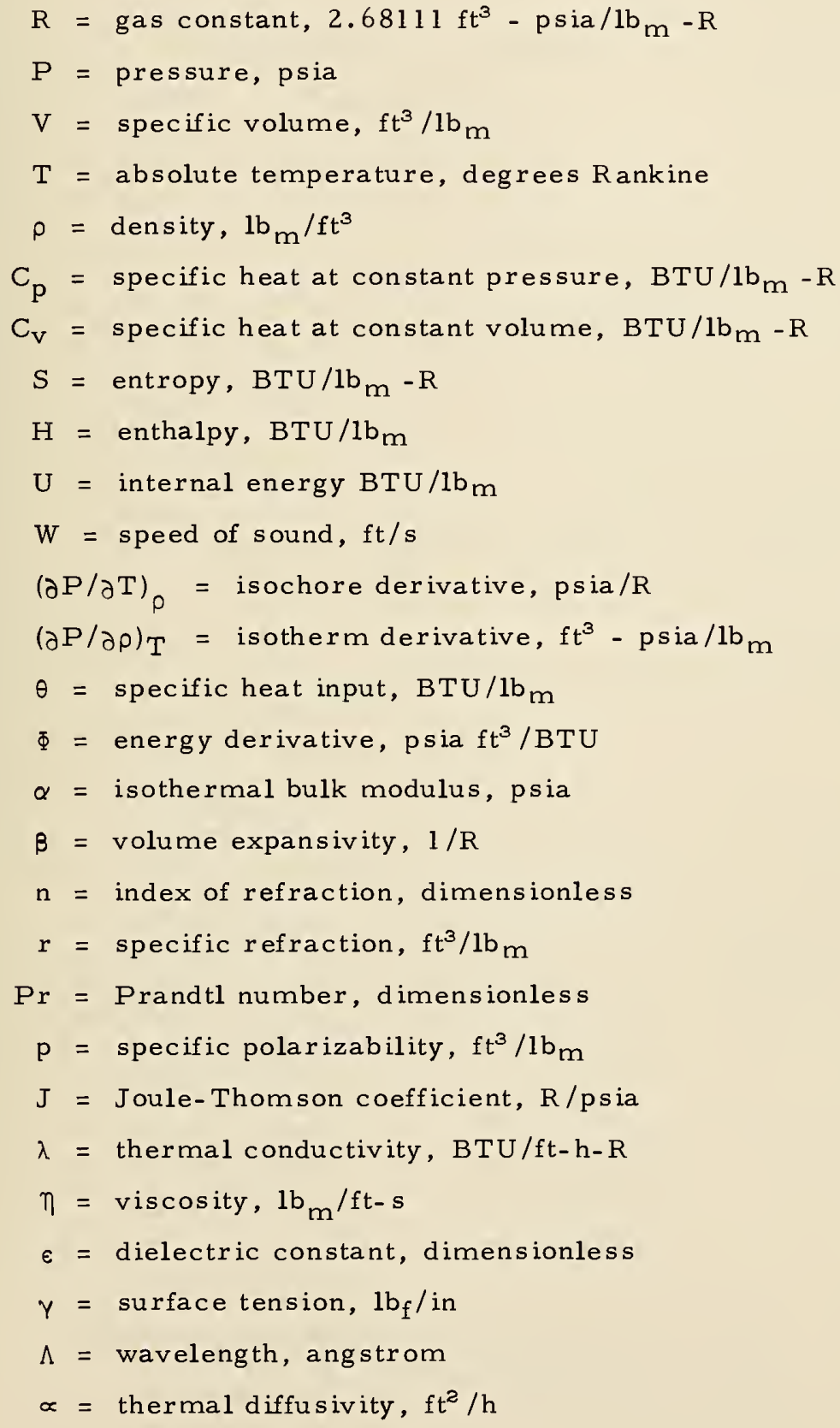


Appendix B. Fixed Points *

Critical Point

$$
\begin{aligned}
& \mathrm{P}_{\mathrm{c}}=32.99 \mathrm{psia}(2.245 \mathrm{~atm}) \\
& \mathrm{T}_{\mathrm{c}}=9.3625 \mathrm{R}(5.2014 \mathrm{~K}) \\
& \rho_{\mathrm{c}}=4.348 \mathrm{lb} / \mathrm{ft}^{3}\left(0.017399 \mathrm{~mol} / \mathrm{cm}^{3}\right)
\end{aligned}
$$

Normal Boiling Point

$$
\begin{array}{ll}
\mathrm{P} & =14.696 \text { psia }(1 \mathrm{~atm}) \\
\mathrm{T} & =7.604 \mathrm{R}(4.224 \mathrm{~K}) \\
\rho_{\text {gas }} & =1.054 \mathrm{lb} / \mathrm{ft}^{3}\left(0.004220 \mathrm{~mol} / \mathrm{cm}^{3}\right) \\
\rho_{\text {liquid }} & =7.802 \mathrm{lb} / \mathrm{ft}^{3}\left(0.03122 \mathrm{~mol} / \mathrm{cm}^{3}\right)
\end{array}
$$

Lower Lambda Point

$$
\begin{array}{ll}
\mathrm{P} & =0.730 \mathrm{psia}(0.0497 \mathrm{~atm}) \\
\mathrm{T} & =3.919 \mathrm{R}(2.177 \mathrm{~K}) \\
\rho_{\text {liquid }} & =9.127 \mathrm{lb} \mathrm{m} / \mathrm{ft}^{3}\left(0.03653 \mathrm{~mol} / \mathrm{cm}^{3}\right)
\end{array}
$$

Upper Lambda Point

$$
\begin{array}{ll}
\mathrm{P} & =437.1 \mathrm{psia}(29.74 \mathrm{~atm}) \\
\mathrm{T} & =3.174 \mathrm{R}(1.763 \mathrm{~K}) \\
\rho_{\text {liquid }} & =11.26 \mathrm{lb} \mathrm{m} / \mathrm{ft}^{3}\left(0.04507 \mathrm{~mol} / \mathrm{cm}^{3}\right)
\end{array}
$$

\section{Appendix C. Conversion Factors}

$\begin{array}{ll}\text { Temperature } & 1.8 \mathrm{R}=1 \mathrm{~K} \\ \text { Pressure } & 14.695949 \mathrm{psia}=1 \mathrm{~atm}=1.01325 \times 10^{5} \mathrm{~N} / \mathrm{m}^{2} \\ \text { Specific Volume } & 0.004002013 \mathrm{ft}^{3} / \mathrm{lb}=1 \mathrm{~cm} / \mathrm{mol} \\ \text { Internal Energy } & 0.107483 \mathrm{BTU} / 1 \mathrm{~b}_{\mathrm{m}}=1 \mathrm{~J} / \mathrm{mol} \\ \text { Entropy, Specific Heat } & 0.0597126 \mathrm{BTU} / \mathrm{lb} \mathrm{m}=1 \mathrm{~J} / \mathrm{mol}-\mathrm{K} \\ \text { Thermal Conductivity } & 0.0578176 \mathrm{BTU} / \mathrm{ft}-\mathrm{hr}-\mathrm{R}=1 \mathrm{~mW} / \mathrm{cm}-\mathrm{K} \\ \text { Viscosity } & 0.0671968971 \mathrm{bm} / \mathrm{ft}-\mathrm{s}=1 \mathrm{~g} / \mathrm{cm}-\mathrm{s} \\ \text { Speed of Sound } & 3.2808 \mathrm{ft} / \mathrm{s}=1 \mathrm{~m} / \mathrm{s}=\mathrm{m} / \mathrm{s} \\ \text { Molecular Weight } & 4.0026 * * \\ \text { Surface Tension } & 0.5710147 \times 10^{-5} \mathrm{lb} / \mathrm{in}=1 \mathrm{dyn} / \mathrm{cm} \\ & \left(1 \mathrm{dyn}=10^{-5} \mathrm{~N}\right) \\ & * \text { Fixed Points from McCarty (1972) }\end{array}$


Appendix D, Saturation Properties

THERMOOYNAMIC PROPERTIES OF COEXISTING GASEOUS ANO LIOUIO HELIUM

\begin{tabular}{|c|c|c|c|c|c|c|c|c|c|c|c|}
\hline TEMP & PRESS & VOLUMF & $\begin{array}{l}\text { ISOTHERM } \\
\text { ERIVAT I : }\end{array}$ & $\begin{array}{l}\text { ISOCHORE } \\
\text { OERIVATIVE }\end{array}$ & $\begin{array}{l}\text { INTERNAL } \\
\text { ENERGY }\end{array}$ & ENTHALPY & ENTPOPY & $\mathrm{CV}$ & $C P$ & $\begin{array}{l}\text { VELOCITY } \\
\text { OF SOUND }\end{array}$ & $\begin{array}{l}\text { SURF ACE } \\
\text { TENSION }\end{array}$ \\
\hline OEG. R & PSIA & CU FT/LB CU & FT-PSIA / L $B$ & PSIA/R & BTU/LP B & BTU/LB & ETU/LE-R & BTU / L8 & $-R$ & FT/SEC & LB/IN $\times 10$ \\
\hline $\begin{array}{r}3.919 \\
* 3.919\end{array}$ & $\begin{array}{l}0.7303 \\
0.7303\end{array}$ & $\begin{array}{l}0.1096 \\
13.61\end{array}$ & 9.36 & 0.201 & 9.093 & 10.93 & 2.848 & 0.764 & $1 \cdot 34$ & 275.9 & \\
\hline $\begin{array}{l}4.0 \\
4.0\end{array}$ & $\begin{array}{l}0.8146 \\
0.8146\end{array}$ & $\begin{array}{l}0.1097 \\
12.40\end{array}$ & $\begin{array}{r}106.8 \\
9.47\end{array}$ & $\begin{array}{l}13.7 \\
0.220\end{array}$ & $\begin{array}{l}1.423 \\
9.141\end{array}$ & $\begin{array}{l}1.440 \\
11.01\end{array}$ & $\begin{array}{l}0.4068 \\
2.817\end{array}$ & $\begin{array}{l}0.699 \\
0.765\end{array}$ & $\begin{array}{l}0.715 \\
1.35\end{array}$ & $\begin{array}{l}709.4 \\
278.2\end{array}$ & $17 \cdot 36$ \\
\hline $\begin{array}{l}4.1 \\
4.1\end{array}$ & $\begin{array}{l}0.9266 \\
0.9266\end{array}$ & $\begin{array}{l}0.1099 \\
11.12\end{array}$ & $\begin{array}{r}105.0 \\
9.61\end{array}$ & $\begin{array}{l}15.7 \\
0.246\end{array}$ & $\begin{array}{l}1.490 \\
9.200\end{array}$ & $\begin{array}{l}1.509 \\
11.11\end{array}$ & $\begin{array}{l}0.4233 \\
2.780\end{array}$ & $\begin{array}{l}0.614 \\
0.766\end{array}$ & $\begin{array}{l}0.635 \\
1.36\end{array}$ & $\begin{array}{l}710.5 \\
280.8\end{array}$ & 17.04 \\
\hline $\begin{array}{l}4.2 \\
4.2\end{array}$ & $\begin{array}{l}1.049 \\
1.049\end{array}$ & $\begin{array}{l}0.1100 \\
10.01\end{array}$ & $\begin{array}{r}104.0 \\
9.73\end{array}$ & $\begin{array}{l}17.5 \\
0.274\end{array}$ & $\begin{array}{l}1.550 \\
9.258\end{array}$ & $\begin{array}{l}1.572 \\
11.20\end{array}$ & $\begin{array}{l}0.4379 \\
2.746\end{array}$ & $\begin{array}{l}0.553 \\
0.767\end{array}$ & $\begin{array}{l}0.580 \\
1.37\end{array}$ & $\begin{array}{l}711.9 \\
283.4\end{array}$ & 16.71 \\
\hline $\begin{array}{l}4.3 \\
4.3\end{array}$ & $\begin{array}{l}1.181 \\
1.181\end{array}$ & $\begin{array}{l}0.1102 \\
9.052\end{array}$ & $\begin{array}{r}103.0 \\
9.85\end{array}$ & $\begin{array}{l}19.0 \\
0.303\end{array}$ & $\begin{array}{l}1.606 \\
9.316\end{array}$ & $\begin{array}{r}1.630 \\
11.30\end{array}$ & $\begin{array}{l}0.4510 \\
2.713\end{array}$ & $\begin{array}{l}0.510 \\
0.768\end{array}$ & $\begin{array}{l}0.544 \\
1.38\end{array}$ & $\begin{array}{l}713.2 \\
285.9\end{array}$ & 16.39 \\
\hline $\begin{array}{l}4.4 \\
4.4\end{array}$ & $\begin{array}{l}1.325 \\
1.325\end{array}$ & $\begin{array}{l}0.1104 \\
8.211\end{array}$ & $\begin{array}{r}102.0 \\
10.0\end{array}$ & $\begin{array}{l}20.4 \\
0.334\end{array}$ & $\begin{array}{l}1.659 \\
9.372\end{array}$ & $\begin{array}{l}1.686 \\
11.39\end{array}$ & $\begin{array}{l}0.4632 \\
2.681\end{array}$ & $\begin{array}{l}0.480 \\
0.770\end{array}$ & $\begin{array}{l}0.521 \\
1.39\end{array}$ & $\begin{array}{l}714.2 \\
288.3\end{array}$ & 16.07 \\
\hline $\begin{array}{l}4.5 \\
4.5\end{array}$ & $\begin{array}{l}1.481 \\
1.431\end{array}$ & $\begin{array}{l}0.1106 \\
7.473\end{array}$ & $\begin{array}{r}100.0 \\
10.1\end{array}$ & $\begin{array}{l}21.6 \\
0.368\end{array}$ & $\begin{array}{l}1.710 \\
9.427\end{array}$ & $\begin{array}{l}1.740 \\
11.48\end{array}$ & $\begin{array}{l}0.4746 \\
2.650\end{array}$ & $\begin{array}{l}0.461 \\
0.771\end{array}$ & $\begin{array}{l}0.508 \\
1.40\end{array}$ & $\begin{array}{l}714.8 \\
290.7\end{array}$ & 15.74 \\
\hline $\begin{array}{l}4.6 \\
4.6\end{array}$ & $\begin{array}{l}1.649 \\
1.649\end{array}$ & $\begin{array}{l}0.1109 \\
6.821\end{array}$ & $\begin{array}{l}98.3 \\
10.1\end{array}$ & $\begin{array}{l}22.6 \\
0.404\end{array}$ & $\begin{array}{l}1.760 \\
9.481\end{array}$ & $\begin{array}{l}1.794 \\
11.56\end{array}$ & $\begin{array}{l}0.4857 \\
2.620\end{array}$ & $\begin{array}{l}0.448 \\
0.772\end{array}$ & $\begin{array}{l}0.503 \\
1.41\end{array}$ & $\begin{array}{l}714.7 \\
292.9\end{array}$ & 15.42 \\
\hline $\begin{array}{l}4.7 \\
4.7\end{array}$ & $\begin{array}{l}1.829^{\circ} \\
1.829\end{array}$ & $\begin{array}{l}0.1112 \\
6.244\end{array}$ & $\begin{array}{l}96.6 \\
10.2\end{array}$ & $\begin{array}{l}23.5 \\
0.442\end{array}$ & $\begin{array}{l}1.810 \\
9.534\end{array}$ & $\begin{array}{l}1.847 \\
11.65\end{array}$ & $\begin{array}{l}0.4964 \\
2.592\end{array}$ & $\begin{array}{l}0.442 \\
0.773\end{array}$ & $\begin{array}{l}0.503 \\
1.42\end{array}$ & $\begin{array}{l}714.1 \\
295.1\end{array}$ & 15.09 \\
\hline $\begin{array}{l}4.8 \\
4.3\end{array}$ & $\begin{array}{l}2.022 \\
2.022\end{array}$ & $\begin{array}{l}0.1115 \\
5.731\end{array}$ & $\begin{array}{l}94.8 \\
10.3\end{array}$ & $\begin{array}{l}24.3 \\
0.482\end{array}$ & $\begin{array}{l}1.860 \\
9.58 E\end{array}$ & $\begin{array}{l}1.932 \\
11.73\end{array}$ & $\begin{array}{l}0.5069 \\
2.564\end{array}$ & $\begin{array}{l}0.439 \\
0.774\end{array}$ & $\begin{array}{l}0.508 \\
1.43\end{array}$ & $\begin{array}{l}712.7 \\
297.2\end{array}$ & 14.77 \\
\hline $\begin{array}{l}4.9 \\
4.9\end{array}$ & $\begin{array}{l}2.229 \\
2.229\end{array}$ & $\begin{array}{l}0.1118 \\
5.273\end{array}$ & $\begin{array}{l}92.8 \\
10.4\end{array}$ & $\begin{array}{l}25.1 \\
0.525\end{array}$ & $\begin{array}{l}1.910 \\
9.636\end{array}$ & $\begin{array}{c}1.956 \\
11.81\end{array}$ & $\begin{array}{l}0.5174 \\
2.538\end{array}$ & $\begin{array}{l}0.439 \\
0.775\end{array}$ & $\begin{array}{l}0.516 \\
1.45\end{array}$ & $\begin{array}{l}710.8 \\
299.2\end{array}$ & 14.45 \\
\hline $\begin{array}{l}5.0 \\
5.0\end{array}$ & $\begin{array}{l}2.450 \\
2.450\end{array}$ & $\begin{array}{l}0.1121 \\
4.862\end{array}$ & $\begin{array}{l}90.9 \\
10.4\end{array}$ & $\begin{array}{l}25.7 \\
0.570\end{array}$ & $\begin{array}{l}1.962 \\
9.68 f\end{array}$ & $\begin{array}{l}2.012 \\
11.89\end{array}$ & $\begin{array}{l}0.5278 \\
2.512\end{array}$ & $\begin{array}{l}0.441 \\
0.776\end{array}$ & $\begin{array}{l}0.526 \\
1.46\end{array}$ & $\begin{array}{l}708.2 \\
301.2\end{array}$ & 14.12 \\
\hline $\begin{array}{l}5.1 \\
5.1\end{array}$ & $\begin{array}{l}2.686 \\
2.686\end{array}$ & $\begin{array}{l}0.1124 \\
4.494\end{array}$ & $\begin{array}{l}88.8 \\
10.5\end{array}$ & $\begin{array}{r}26.3 \\
0.618\end{array}$ & $\begin{array}{l}2.014 \\
9.734\end{array}$ & $\begin{array}{c}2.070 \\
11.97\end{array}$ & $\begin{array}{l}0.5381 \\
2.487\end{array}$ & $\begin{array}{l}0.445 \\
0.777\end{array}$ & $\begin{array}{l}0.538 \\
1.47\end{array}$ & $\begin{array}{l}705.2 \\
303.1\end{array}$ & 13.80 \\
\hline $\begin{array}{l}5.2 \\
5.2\end{array}$ & $\begin{array}{l}2.936 \\
2.936\end{array}$ & $\begin{array}{l}0.1127 \\
4.162\end{array}$ & $\begin{array}{l}86.7 \\
10.5\end{array}$ & $\begin{array}{l}26.8 \\
0.668\end{array}$ & $\begin{array}{l}2.067 \\
9.781\end{array}$ & $\begin{array}{l}2.128 \\
12.04\end{array}$ & $\begin{array}{l}0.5485 \\
2.462\end{array}$ & $\begin{array}{l}0.449 \\
0.778\end{array}$ & $\begin{array}{l}0.551 \\
1.49\end{array}$ & $\begin{array}{l}701.8 \\
304.9\end{array}$ & 13.48 \\
\hline $\begin{array}{l}5.3 \\
5.3\end{array}$ & $\begin{array}{l}3.202 \\
3.202\end{array}$ & $\begin{array}{l}0.1131 \\
3.861\end{array}$ & $\begin{array}{l}84.5 \\
10.5\end{array}$ & $\begin{array}{l}27.3 \\
0.722\end{array}$ & $\begin{array}{l}2.122 \\
9.827\end{array}$ & $\begin{array}{c}2.189 \\
12.12\end{array}$ & $\begin{array}{l}0.5589 \\
2.439\end{array}$ & $\begin{array}{l}0.454 \\
0.779\end{array}$ & $\begin{array}{l}0.565 \\
1.50\end{array}$ & $\begin{array}{l}698.0 \\
306.7\end{array}$ & 13.15 \\
\hline $\begin{array}{l}5.4 \\
5.4\end{array}$ & $\begin{array}{l}3.484 \\
3.484\end{array}$ & $\begin{array}{l}0.1135 \\
3.589\end{array}$ & $\begin{array}{l}82.4 \\
10.5\end{array}$ & $\begin{array}{l}27.7 \\
0.778\end{array}$ & $\begin{array}{l}2.177 \\
9.871\end{array}$ & $\begin{array}{r}2.251 \\
12.19\end{array}$ & $\begin{array}{l}0.5694 \\
2.415\end{array}$ & $\begin{array}{l}0.459 \\
0.780\end{array}$ & $\begin{array}{l}0.573 \\
1.52\end{array}$ & $\begin{array}{l}694.0 \\
308.4\end{array}$ & 12.83 \\
\hline $\begin{array}{l}5.5 \\
5.5\end{array}$ & $\begin{array}{l}3.782 \\
3.732\end{array}$ & $\begin{array}{l}0.1139 \\
3.342\end{array}$ & $\begin{array}{l}80.2 \\
10.5\end{array}$ & $\begin{array}{l}28.1 \\
0.837\end{array}$ & $\begin{array}{l}2.234 \\
9.914\end{array}$ & $\begin{array}{r}2.314 \\
12.25\end{array}$ & $\begin{array}{l}0.5799 \\
2.393\end{array}$ & $\begin{array}{l}0.464 \\
0.781\end{array}$ & $\begin{array}{l}0.595 \\
1.54\end{array}$ & $\begin{array}{l}689.7 \\
310.0\end{array}$ & 12.50 \\
\hline $\begin{array}{l}5.6 \\
5.6\end{array}$ & $\begin{array}{l}4.097 \\
4.097\end{array}$ & $\begin{array}{l}0.1143 \\
3.116\end{array}$ & $\begin{array}{l}77.9 \\
10.5\end{array}$ & $\begin{array}{l}28.5 \\
n .899\end{array}$ & $\begin{array}{l}2.293 \\
9.96\end{array}$ & $\begin{array}{r}2.380 \\
12.32\end{array}$ & $\begin{array}{l}0.5905 \\
2.371\end{array}$ & $\begin{array}{l}0.470 \\
0.782\end{array}$ & $\begin{array}{l}0.611 \\
1.55\end{array}$ & $\begin{array}{l}685.2 \\
311.5\end{array}$ & 12.18 \\
\hline $\begin{array}{l}5.7 \\
5.7\end{array}$ & $\begin{array}{l}4.430 \\
4.430\end{array}$ & $\begin{array}{l}0.1147 \\
2.910\end{array}$ & $\begin{array}{l}75.7 \\
10.5\end{array}$ & $\begin{array}{l}28.8 \\
0.965\end{array}$ & $\begin{array}{c}2.353 \\
10.00\end{array}$ & $\begin{array}{r}2.447 \\
12.38\end{array}$ & $\begin{array}{l}0.6012 \\
2.349\end{array}$ & $\begin{array}{l}0.475 \\
0.782\end{array}$ & $\begin{array}{l}0.627 \\
1.57\end{array}$ & $\begin{array}{l}680.5 \\
313.0\end{array}$ & 11.86 \\
\hline $\begin{array}{l}5.8 \\
5.8\end{array}$ & $\begin{array}{l}4.780 \\
4.780\end{array}$ & $\begin{array}{l}0.1152 \\
2.721\end{array}$ & $\begin{array}{l}73.4 \\
10.5\end{array}$ & $\begin{array}{c}29.1 \\
1.03\end{array}$ & $\begin{array}{c}2.414 \\
10.03\end{array}$ & $\begin{aligned} 2.516 \\
12.44\end{aligned}$ & $\begin{array}{l}0.6119 \\
2.328\end{array}$ & $\begin{array}{l}0.480 \\
0.793\end{array}$ & $\begin{array}{l}0.645 \\
1.59\end{array}$ & $\begin{array}{l}675.6 \\
314.5\end{array}$ & 11.53 \\
\hline $\begin{array}{l}5.9 \\
5.9\end{array}$ & $\begin{array}{l}5.148 \\
5.148\end{array}$ & $\begin{array}{l}0.1157 \\
2.548\end{array}$ & $\begin{array}{l}71.1 \\
10.5\end{array}$ & $\begin{array}{l}29.4 \\
1.11\end{array}$ & $\begin{array}{r}2.477 \\
10.07\end{array}$ & $\begin{array}{r}2.587 \\
12.50\end{array}$ & $\begin{array}{l}0.6227 \\
2.307\end{array}$ & $\begin{array}{l}0.485 \\
0.784\end{array}$ & $\begin{array}{l}0.662 \\
1.62\end{array}$ & $\begin{array}{l}670.7 \\
315.8\end{array}$ & 11.21 \\
\hline $\begin{array}{l}6.0 \\
6.0\end{array}$ & $\begin{array}{l}5.536 \\
5.536\end{array}$ & $\begin{array}{l}0.1162 \\
2.389\end{array}$ & $\begin{array}{l}68.8 \\
10.4\end{array}$ & $\begin{array}{l}29.6 \\
1.19\end{array}$ & $\begin{array}{r}2.541 \\
10.11\end{array}$ & $\begin{array}{r}2.660 \\
12.55\end{array}$ & $\begin{array}{l}0.6336 \\
2.286\end{array}$ & $\begin{array}{l}0.490 \\
0.785\end{array}$ & $\begin{array}{l}0.681 \\
1.64\end{array}$ & $\begin{array}{l}665.6 \\
317.1\end{array}$ & 10.89 \\
\hline $\begin{array}{l}6.1 \\
6.1\end{array}$ & $\begin{array}{l}5.942 \\
5.942\end{array}$ & $\begin{array}{l}0.1167 \\
2.242\end{array}$ & $\begin{array}{l}66.5 \\
10.3\end{array}$ & $\begin{array}{l}29.8 \\
1.25\end{array}$ & $\begin{array}{l}2.607 \\
10.14\end{array}$ & $\begin{array}{l}2.735 \\
12.61\end{array}$ & $\begin{array}{l}0.6445 \\
2.266\end{array}$ & $\begin{array}{l}0.495 \\
0.786\end{array}$ & $\begin{array}{l}0.700 \\
1.66\end{array}$ & $\begin{array}{l}660.4 \\
318.3\end{array}$ & 10.56 \\
\hline $\begin{array}{l}6.2 \\
6.2\end{array}$ & $\begin{array}{l}6.368 \\
6.368\end{array}$ & $\begin{array}{l}0.1172 \\
2.107\end{array}$ & $\begin{array}{l}64.2 \\
10.3\end{array}$ & $\begin{array}{l}30.0 \\
1.35\end{array}$ & $\begin{array}{c}2.674 \\
10.17\end{array}$ & $\begin{array}{c}2.812 \\
12.65\end{array}$ & $\begin{array}{l}0.6556 \\
2.246\end{array}$ & $\begin{array}{l}0.500 \\
0.787\end{array}$ & $\begin{array}{l}0.721 \\
1.69\end{array}$ & $\begin{array}{l}655.0 \\
319.5\end{array}$ & 10.24 \\
\hline $\begin{array}{l}8.3 \\
6.3\end{array}$ & $\begin{array}{l}6.815 \\
6.815\end{array}$ & $\begin{array}{l}0.1173 \\
1.981\end{array}$ & $\begin{array}{l}61.9 \\
10.2\end{array}$ & $\begin{array}{c}30.2 \\
1.44\end{array}$ & $\begin{array}{c}2.74 ? \\
10.20\end{array}$ & $\begin{array}{l}2.892 \\
12.79\end{array}$ & $\begin{array}{l}0.6657 \\
2.226\end{array}$ & $\begin{array}{l}0.504 \\
0.788\end{array}$ & $\begin{array}{l}0.74 ? \\
1.72\end{array}$ & $\begin{array}{l}649.6 \\
320.6\end{array}$ & 9.914 \\
\hline $\begin{array}{l}6.4 \\
6.4\end{array}$ & $\begin{array}{l}7.282 \\
7.232\end{array}$ & $\begin{array}{l}0.1183 \\
1.865\end{array}$ & $\begin{array}{l}59.6 \\
10.1\end{array}$ & $\begin{array}{c}30.3 \\
1.53\end{array}$ & $\begin{array}{c}2.814 \\
19.23\end{array}$ & $\begin{array}{r}2.974 \\
12.74\end{array}$ & $\begin{array}{l}3.6780 \\
2.207\end{array}$ & $\begin{array}{l}0.509 \\
0.789\end{array}$ & $\begin{array}{l}0.764 \\
1.74\end{array}$ & $\begin{array}{l}644.1 \\
321.6\end{array}$ & 9.591 \\
\hline $\begin{array}{l}6.5 \\
6.5\end{array}$ & $\begin{array}{l}7.770 \\
7.770\end{array}$ & $\begin{array}{l}0.1190 \\
1.757\end{array}$ & $\begin{array}{l}57.3 \\
10.0\end{array}$ & $\begin{array}{l}30.4 \\
1.53\end{array}$ & $\begin{array}{c}2.886 \\
10.25\end{array}$ & $\begin{array}{l}2.058 \\
12.78\end{array}$ & $\begin{array}{l}0.6994 \\
2.187\end{array}$ & $\begin{array}{l}0.513 \\
0.790\end{array}$ & $\begin{array}{l}0.788 \\
1.78\end{array}$ & $\begin{array}{l}638.4 \\
322.6\end{array}$ & 9.267 \\
\hline $\begin{array}{l}6.6 \\
6.6\end{array}$ & $\begin{array}{l}8.280 \\
8.230\end{array}$ & $\begin{array}{l}0.1196 \\
1.657\end{array}$ & $\begin{array}{l}55.0 \\
9.88\end{array}$ & $\begin{array}{c}30.5 \\
1.73\end{array}$ & $\begin{array}{l}2.961 \\
10.28\end{array}$ & $\begin{array}{c}3.144 \\
12.82\end{array}$ & $\begin{array}{l}0.7009 \\
2.168\end{array}$ & $\begin{array}{l}0.518 \\
0.791\end{array}$ & $\begin{array}{l}0.813 \\
1.81\end{array}$ & $\begin{array}{l}632.6 \\
323.5\end{array}$ & 8.943 \\
\hline $\begin{array}{l}6.7 \\
6.7\end{array}$ & $\begin{array}{l}8.311 \\
8.811\end{array}$ & $\begin{array}{l}0.1203 \\
1.564\end{array}$ & $\begin{array}{l}52.7 \\
9.75\end{array}$ & $\begin{array}{c}30.5 \\
1.84\end{array}$ & $\begin{array}{l}3.037 \\
10.30\end{array}$ & $\begin{array}{l}3.23^{7} \\
12.85\end{array}$ & $\begin{array}{l}0.7125 \\
2.150\end{array}$ & $\begin{array}{l}0.522 \\
0.792\end{array}$ & $\begin{array}{l}0.840 \\
1.84\end{array}$ & $\begin{array}{l}626.7 \\
324.3\end{array}$ & 8.619 \\
\hline $\begin{array}{l}6.8 \\
6.8\end{array}$ & $\begin{array}{l}9.365 \\
9.365\end{array}$ & $\begin{array}{l}0.1210 \\
1.477\end{array}$ & $\begin{array}{l}50.5 \\
9 . \notin 1\end{array}$ & $\begin{array}{l}30.6 \\
1.95\end{array}$ & $\begin{array}{c}3.115 \\
10.32\end{array}$ & $\begin{array}{r}3.325 \\
12.89\end{array}$ & $\begin{array}{l}3.7242 \\
2.131\end{array}$ & $\begin{array}{l}0.527 \\
0.793\end{array}$ & $\begin{array}{l}0.868 \\
1.88\end{array}$ & $\begin{array}{l}620.5 \\
325.1\end{array}$ & 8.296 \\
\hline
\end{tabular}


THERMDOYNAHIC PRDPERTIES OF T.DEXISTING GASEDUS AND LIDUIO HELIUM

\begin{tabular}{|c|c|c|c|c|c|c|c|c|c|c|}
\hline $\begin{array}{c}\text { TENPERATURE } \\
\text { DEG. } R\end{array}$ & DENSITY & $\begin{array}{c}V(D H / D V)_{P} \\
\text { BTU/LA }\end{array}$ & $\begin{array}{c}V(D P / D U)_{V} \\
\text { PSIA-CU FT/BTU }\end{array}$ & $\begin{array}{l}-V(D P I D V) T \\
\text { PSIA }\end{array}$ & $\begin{array}{c}(O V / D T)_{p}^{\prime V} \\
\text { OEG. R }\end{array}$ & $\begin{array}{c}\text { THERMAL } \\
\text { CONDUCTIVITY } \\
\text { BTU/FT-HR-R }\end{array}$ & $\begin{array}{l}\text { VISCDSITY } \\
\text { LB/FT-SEC } \\
\times 10^{B}\end{array}$ & $\begin{array}{l}\text { THERMAL } \\
\text { DIFFUSIVITY } \\
\text { SD FT/HR }\end{array}$ & $\begin{array}{l}\text { DIELECTRIC } \\
\text { CDNSTANT }\end{array}$ & $\begin{array}{l}\text { PRANDTL } \\
\text { NUMBER }\end{array}$ \\
\hline $\begin{array}{r}3.919 \\
+3.919\end{array}$ & $\begin{array}{l}9.128 \\
0.07350\end{array}$ & 4.60 & 3.57 & 0.688 & 0.292 & 0.00258 & 0.361 & 0.0262 & $\begin{array}{l}1.02040 \\
1.00028\end{array}$ & 0.675 \\
\hline $\begin{array}{l}4.0 \\
4.0\end{array}$ & $\begin{array}{l}9.119 \\
0.08063\end{array}$ & $\begin{array}{c}50.4 \\
4.68\end{array}$ & $\begin{array}{l}2.16 \\
3.57\end{array}$ & $\begin{array}{r}969.0 \\
0.764\end{array}$ & $\begin{array}{l}0.0142 \\
0.288\end{array}$ & $\begin{array}{l}0.00866 \\
0.00265\end{array}$ & $\begin{array}{l}2.44 \\
0.371\end{array}$ & $\begin{array}{l}0.00133 \\
0.0244\end{array}$ & $\begin{array}{l}1.02039 \\
1.00030\end{array}$ & $\begin{array}{l}0.724 \\
0.678\end{array}$ \\
\hline $\begin{array}{l}4.1 \\
4.1\end{array}$ & $\begin{array}{l}9.136 \\
0.08993\end{array}$ & $\begin{array}{c}38.7 \\
4.77\end{array}$ & $\begin{array}{l}2.82 \\
3.57\end{array}$ & $\begin{array}{l}959.0 \\
0.864\end{array}$ & $\begin{array}{l}0.0164 \\
0.285\end{array}$ & $\begin{array}{l}0.00888 \\
0.00274\end{array}$ & $\begin{array}{l}2.46 \\
0.382\end{array}$ & $\begin{array}{l}0.00154 \\
0.0225\end{array}$ & $\begin{array}{l}1.02038 \\
1.00034\end{array}$ & $\begin{array}{l}0.633 \\
0.681\end{array}$ \\
\hline $\begin{array}{l}4.2 \\
4.2\end{array}$ & $\begin{array}{l}9.092 \\
0.0999\end{array}$ & $\begin{array}{c}31.4 \\
4.86\end{array}$ & $\begin{array}{l}3.48 \\
3.57\end{array}$ & $\begin{array}{r}947.0 \\
0.972\end{array}$ & $\begin{array}{l}0.0185 \\
0.281\end{array}$ & $\begin{array}{l}0.00907 \\
0.00283\end{array}$ & $\begin{array}{l}2.48 \\
0.394\end{array}$ & $\begin{array}{l}0.00172 \\
0.0207\end{array}$ & $\begin{array}{l}1.02037 \\
1.00038\end{array}$ & $\begin{array}{l}0.570 \\
0.685\end{array}$ \\
\hline $\begin{array}{l}4.3 \\
4.3\end{array}$ & $\begin{array}{l}9.075 \\
0.1105\end{array}$ & $\begin{array}{c}26.7 \\
4.94\end{array}$ & $\begin{array}{l}4.12 \\
3.57\end{array}$ & $\begin{array}{l}934.0 \\
1.09\end{array}$ & $\begin{array}{l}0.0204 \\
0.278\end{array}$ & $\begin{array}{l}0.00924 \\
0.00292\end{array}$ & $\begin{array}{l}2.49 \\
0.406\end{array}$ & $\begin{array}{l}0.00187 \\
0.0192\end{array}$ & $\begin{array}{l}1.02036 \\
1.00042\end{array}$ & $\begin{array}{l}0.527 \\
0.589\end{array}$ \\
\hline $\begin{array}{l}4.4 \\
4.4\end{array}$ & $\begin{array}{l}9.057 \\
0.1218\end{array}$ & $\begin{array}{l}23.5 \\
5.03\end{array}$ & $\begin{array}{l}4.69 \\
3.57\end{array}$ & $\begin{array}{r}919.0 \\
1.21\end{array}$ & $\begin{array}{l}0.0222 \\
0.276\end{array}$ & $\begin{array}{l}0.00938 \\
0.00300\end{array}$ & $\begin{array}{l}2.50 \\
0.417\end{array}$ & $\begin{array}{l}0.00199 \\
0.0178\end{array}$ & $\begin{array}{l}1.02035 \\
1.00046\end{array}$ & $\begin{array}{l}0.499 \\
0.693\end{array}$ \\
\hline $\begin{array}{l}4.5 \\
4.5\end{array}$ & $\begin{array}{l}9.038 \\
0.1338\end{array}$ & $\begin{array}{c}21.3 \\
5.11\end{array}$ & $\begin{array}{l}5.18 \\
3.57\end{array}$ & $\begin{array}{r}904.0 \\
1.35\end{array}$ & $\begin{array}{l}0.0239 \\
0.273\end{array}$ & $\begin{array}{l}0.00951 \\
0.00309\end{array}$ & $\begin{array}{l}2.50 \\
0.429\end{array}$ & $\begin{array}{l}0.00207 \\
0.0165\end{array}$ & $\begin{array}{l}1.02034 \\
1.00050\end{array}$ & $\begin{array}{l}0.482 \\
0.698\end{array}$ \\
\hline $\begin{array}{l}4.6 \\
4.6\end{array}$ & $\begin{array}{l}9.017 \\
0.1466\end{array}$ & $\begin{array}{c}19.7 \\
5.19\end{array}$ & $\begin{array}{l}5.59 \\
3.57\end{array}$ & $\begin{array}{c}887.0 \\
1.49\end{array}$ & $\begin{array}{l}0.0255 \\
0.271\end{array}$ & $\begin{array}{l}0.00963 \\
0.00318\end{array}$ & $\begin{array}{l}2.51 \\
0.441\end{array}$ & $\begin{array}{l}0.00212 \\
0.0154\end{array}$ & $\begin{array}{l}1.02032 \\
1.00055\end{array}$ & $\begin{array}{l}0.472 \\
0.703\end{array}$ \\
\hline $\begin{array}{l}4.7 \\
4.7\end{array}$ & $\begin{array}{l}8.996 \\
0.1602\end{array}$ & $\begin{array}{r}18.6 \\
5.27\end{array}$ & $\begin{array}{l}5.92 \\
3.57\end{array}$ & $\begin{array}{l}869.0 \\
1.64\end{array}$ & $\begin{array}{l}0.0271 \\
0.270\end{array}$ & $\begin{array}{l}0.00973 \\
0.00327\end{array}$ & $\begin{array}{l}2.51 \\
0.452\end{array}$ & $\begin{array}{l}0.00215 \\
0.0144\end{array}$ & $\begin{array}{l}1.02031 \\
1.00060\end{array}$ & $\begin{array}{l}0.467 \\
0.708\end{array}$ \\
\hline $\begin{array}{l}4.8 \\
4.8\end{array}$ & $\begin{array}{l}8.973 \\
0.1745\end{array}$ & $\begin{array}{c}17.7 \\
5.34\end{array}$ & $\begin{array}{l}6.18 \\
3.57\end{array}$ & $\begin{array}{r}850.0 \\
1.80\end{array}$ & $\begin{array}{l}0.0286 \\
0.268\end{array}$ & $\begin{array}{l}0.00983 \\
0.00335\end{array}$ & $\begin{array}{l}2.51 \\
0.464\end{array}$ & $\begin{array}{l}0.00216 \\
0.0134\end{array}$ & $\begin{array}{l}1.02029 \\
1.00065\end{array}$ & $\begin{array}{l}0.467 \\
0.713\end{array}$ \\
\hline $\begin{array}{l}4.9 \\
4.9\end{array}$ & $\begin{array}{l}8.948 \\
0.1897\end{array}$ & $\begin{array}{c}17.1 \\
5.41\end{array}$ & $\begin{array}{l}6.38 \\
3.57\end{array}$ & $\begin{array}{r}831.0 \\
1.97\end{array}$ & $\begin{array}{l}0.0302 \\
0.267\end{array}$ & $\begin{array}{l}0.00992 \\
0.00344\end{array}$ & $\begin{array}{l}2.51 \\
0.476\end{array}$ & $\begin{array}{l}0.00215 \\
0.0126\end{array}$ & $\begin{array}{l}1.02027 \\
1.00071\end{array}$ & $\begin{array}{l}0.469 \\
0.719\end{array}$ \\
\hline $\begin{array}{l}5.0 \\
5.0\end{array}$ & $\begin{array}{l}8.923 \\
0.2057\end{array}$ & $\begin{array}{c}16.6 \\
5.48\end{array}$ & $\begin{array}{l}6.53 \\
3.57\end{array}$ & $\begin{array}{r}811.0 \\
2.14\end{array}$ & $\begin{array}{l}0.0317 \\
0.266\end{array}$ & $\begin{array}{l}0.0100 \\
0.00353\end{array}$ & $\begin{array}{l}2.50 \\
0.487\end{array}$ & $\begin{array}{l}0.00213 \\
0.0118\end{array}$ & $\begin{array}{l}1.02025 \\
1.00077\end{array}$ & $\begin{array}{l}0.473 \\
0.725\end{array}$ \\
\hline $\begin{array}{l}5.1 \\
5.1\end{array}$ & $\begin{array}{l}8.897 \\
0.2225\end{array}$ & $\begin{array}{l}16.1 \\
5.55\end{array}$ & $\begin{array}{l}6.65 \\
3.57\end{array}$ & $\begin{array}{r}790.0 \\
2.33\end{array}$ & $\begin{array}{l}0.0333 \\
0.265\end{array}$ & $\begin{array}{l}0.0101 \\
0.00362\end{array}$ & $\begin{array}{l}2.50 \\
0.499\end{array}$ & $\begin{array}{l}0.00211 \\
0.0113\end{array}$ & $\begin{array}{l}1.02023 \\
1.00083\end{array}$ & $\begin{array}{l}0.479 \\
0.732\end{array}$ \\
\hline $\begin{array}{l}5.2 \\
5.2\end{array}$ & $\begin{array}{l}8.963 \\
0.2403\end{array}$ & $\begin{array}{r}15.8 \\
5.61\end{array}$ & $\begin{array}{l}6.74 \\
3.58\end{array}$ & $\begin{array}{r}769.0 \\
2.52\end{array}$ & $\begin{array}{l}0.0349 \\
0.265\end{array}$ & $\begin{array}{l}0.0102 \\
0.00370\end{array}$ & $\begin{array}{l}2.49 \\
0.511\end{array}$ & $\begin{array}{l}0.00208 \\
0.0104\end{array}$ & $\begin{array}{l}1.02021 \\
1.00990\end{array}$ & $\begin{array}{l}0.485 \\
0.739\end{array}$ \\
\hline $\begin{array}{l}5.3 \\
5.3\end{array}$ & $\begin{array}{l}8.841 \\
0.2590\end{array}$ & $\begin{array}{r}15.5 \\
5.67\end{array}$ & $\begin{array}{l}6.81 \\
3.58\end{array}$ & $\begin{array}{r}748.0 \\
2.72\end{array}$ & $\begin{array}{l}0.0365 \\
0.265\end{array}$ & $\begin{array}{l}0.0102 \\
0.00379\end{array}$ & $\begin{array}{l}2.48 \\
0.523\end{array}$ & $\begin{array}{l}0.00205 \\
0.00974\end{array}$ & $\begin{array}{l}1.02019 \\
1.00097\end{array}$ & $\begin{array}{l}0.493 \\
0.747\end{array}$ \\
\hline $\begin{array}{l}5.4 \\
5.4\end{array}$ & $\begin{array}{l}8.811 \\
0.2786\end{array}$ & $\begin{array}{c}15.2 \\
5.73\end{array}$ & $\begin{array}{l}6.86 \\
3.58\end{array}$ & $\begin{array}{r}726.0 \\
2.94\end{array}$ & $\begin{array}{l}0.0382 \\
0.265\end{array}$ & $\begin{array}{l}0.0103 \\
0.00388\end{array}$ & $\begin{array}{l}2.47 \\
0.535\end{array}$ & $\begin{array}{l}0.00202 \\
0.00916\end{array}$ & $\begin{array}{l}1.02017 \\
1.00104\end{array}$ & $\begin{array}{l}0.500 \\
0.755\end{array}$ \\
\hline $\begin{array}{l}5.5 \\
5.5\end{array}$ & $\begin{array}{l}8.780 \\
0.2992\end{array}$ & $\begin{array}{c}14.9 \\
5.79\end{array}$ & $\begin{array}{l}6.90 \\
3.58\end{array}$ & $\begin{array}{r}704.0 \\
3.15\end{array}$ & $\begin{array}{l}0.0400 \\
0.265\end{array}$ & $\begin{array}{l}0.0104 \\
0.00397\end{array}$ & $\begin{array}{l}2.46 \\
0.547\end{array}$ & $\begin{array}{l}0.00199 \\
0.00863\end{array}$ & $\begin{array}{l}1.02015 \\
1.00112\end{array}$ & $\begin{array}{l}0.508 \\
0.763\end{array}$ \\
\hline $\begin{array}{l}5.6 \\
5.6\end{array}$ & $\begin{array}{l}8.749 \\
0.3239\end{array}$ & $\begin{array}{l}14.6 \\
5.84\end{array}$ & $\begin{array}{l}6.93 \\
3.59\end{array}$ & $\begin{array}{c}692.0 \\
3.38\end{array}$ & $\begin{array}{l}0.0418 \\
0.266\end{array}$ & $\begin{array}{l}0.0104 \\
0.00406\end{array}$ & $\begin{array}{l}2.45 \\
0.559\end{array}$ & $\begin{array}{l}0.00195 \\
0.00813\end{array}$ & $\begin{array}{l}1.02012 \\
1.00120\end{array}$ & $\begin{array}{l}0.516 \\
0.772\end{array}$ \\
\hline $\begin{array}{l}5.7 \\
5.7\end{array}$ & $\begin{array}{l}8.716 \\
0.3436\end{array}$ & $\begin{array}{c}14.4 \\
5.89\end{array}$ & $\begin{array}{l}6.96 \\
3.59\end{array}$ & $\begin{array}{l}659.0 \\
3.61\end{array}$ & $\begin{array}{l}0.0437 \\
0.267\end{array}$ & $\begin{array}{l}0.0105 \\
0.00415\end{array}$ & $\begin{array}{l}2.44 \\
0.572\end{array}$ & $\begin{array}{l}0.00192 \\
0.00767\end{array}$ & $\begin{array}{l}1.02009 \\
1.00128\end{array}$ & $\begin{array}{l}0.524 \\
0.781\end{array}$ \\
\hline $\begin{array}{l}5.8 \\
5.8\end{array}$ & $\begin{array}{l}8.681 \\
0.3675\end{array}$ & $\begin{array}{c}14.1 \\
5.94\end{array}$ & $\begin{array}{l}6.98 \\
3.59\end{array}$ & $\begin{array}{r}637.0 \\
3.85\end{array}$ & $\begin{array}{l}0.0457 \\
0.268\end{array}$ & $\begin{array}{l}0.0106 \\
0.00424\end{array}$ & $\begin{array}{l}2.43 \\
0.584\end{array}$ & $\begin{array}{l}0.00189 \\
0.00723\end{array}$ & $\begin{array}{l}1.02007 \\
1.00137\end{array}$ & $\begin{array}{l}0.533 \\
0.791\end{array}$ \\
\hline $\begin{array}{l}5.9 \\
5.9\end{array}$ & $\begin{array}{l}8.646 \\
0.3924\end{array}$ & $\begin{array}{c}13.9 \\
5.99\end{array}$ & $\begin{array}{l}7.00 \\
3.60\end{array}$ & $\begin{array}{r}615.0 \\
4.10\end{array}$ & $\begin{array}{l}0.0478 \\
0.270\end{array}$ & $\begin{array}{l}0.0106 \\
0.00433\end{array}$ & $\begin{array}{l}2.41 \\
0.597\end{array}$ & $\begin{array}{l}0.00186 \\
0.00682\end{array}$ & $\begin{array}{l}1.02004 \\
1.00146\end{array}$ & $\begin{array}{l}0.542 \\
0.802\end{array}$ \\
\hline $\begin{array}{l}6.0 \\
6.0\end{array}$ & $\begin{array}{l}8.610 \\
0.4186\end{array}$ & $\begin{array}{c}13.6 \\
6.03\end{array}$ & $\begin{array}{l}7.02 \\
3.60\end{array}$ & $\begin{array}{r}592.0 \\
4.35\end{array}$ & $\begin{array}{l}0.0500 \\
0.272\end{array}$ & $\begin{array}{l}0.0107 \\
0.00442\end{array}$ & $\begin{array}{l}2.40 \\
0.609\end{array}$ & $\begin{array}{l}0.00182 \\
0.00644\end{array}$ & $\begin{array}{l}1.02001 \\
1.00155\end{array}$ & $\begin{array}{l}0.551 \\
0.813\end{array}$ \\
\hline $\begin{array}{l}6.1 \\
6.1\end{array}$ & $\begin{array}{l}8.572 \\
0.4460\end{array}$ & $\begin{array}{c}13.4 \\
6.07\end{array}$ & $\begin{array}{l}7.03 \\
3.60\end{array}$ & $\begin{array}{r}570.0 \\
4.61\end{array}$ & $\begin{array}{l}0.0523 \\
0.274\end{array}$ & $\begin{array}{l}0.0107 \\
0.00451\end{array}$ & $\begin{array}{l}2.39 \\
0.622\end{array}$ & $\begin{array}{l}0.00179 \\
0.00608\end{array}$ & $\begin{array}{l}1.01997 \\
1.00165\end{array}$ & $\begin{array}{l}0.560 \\
0.825\end{array}$ \\
\hline $\begin{array}{l}6.2 \\
6.2\end{array}$ & $\begin{array}{l}8.532 \\
0.4747\end{array}$ & $\begin{array}{c}13.2 \\
6.11\end{array}$ & $\begin{array}{l}7.04 \\
3.61\end{array}$ & $\begin{array}{r}548.0 \\
4.88\end{array}$ & $\begin{array}{l}0.0548 \\
0.276\end{array}$ & $\begin{array}{l}0.0108 \\
0.00460\end{array}$ & $\begin{array}{l}2.37 \\
0.635\end{array}$ & $\begin{array}{l}0.00176 \\
0.00575\end{array}$ & $\begin{array}{l}1.01994 \\
1.00176\end{array}$ & $\begin{array}{l}0.570 \\
0.838\end{array}$ \\
\hline $\begin{array}{l}6.3 \\
6.3\end{array}$ & $\begin{array}{l}8.492 \\
0.5047\end{array}$ & $\begin{array}{c}12.9 \\
6.14\end{array}$ & $\begin{array}{l}7.05 \\
3.61\end{array}$ & $\begin{array}{c}526.0 \\
5.14\end{array}$ & $\begin{array}{l}0.0574 \\
0.279\end{array}$ & $\begin{array}{l}0.0109 \\
0.00470\end{array}$ & $\begin{array}{l}2.36 \\
0.648\end{array}$ & $\begin{array}{l}0.00172 \\
0.00543\end{array}$ & $\begin{array}{l}1.01991 \\
1.00186\end{array}$ & $\begin{array}{l}0.580 \\
0.852\end{array}$ \\
\hline $\begin{array}{l}6.4 \\
6.4\end{array}$ & $\begin{array}{l}8.450 \\
0.5361\end{array}$ & $\begin{array}{r}12.7 \\
6.17\end{array}$ & $\begin{array}{l}7.05 \\
3.62\end{array}$ & $\begin{array}{c}504.0 \\
5.41\end{array}$ & $\begin{array}{l}0.0602 \\
0.283\end{array}$ & $\begin{array}{l}0.0109 \\
0.00480\end{array}$ & $\begin{array}{l}2.34 \\
0.661\end{array}$ & $\begin{array}{l}0.00169 \\
0.00513\end{array}$ & $\begin{array}{l}1.01987 \\
1.00198\end{array}$ & $\begin{array}{l}0.590 \\
0.866\end{array}$ \\
\hline $\begin{array}{l}6.5 \\
8.5\end{array}$ & $\begin{array}{l}8.436 \\
0.5630\end{array}$ & $\begin{array}{c}12.5 \\
6.23\end{array}$ & $\begin{array}{l}7.05 \\
3.62\end{array}$ & $\begin{array}{r}492.0 \\
5.69\end{array}$ & $\begin{array}{l}0.0631 \\
0.286\end{array}$ & $\begin{array}{l}0.0110 \\
0.00489\end{array}$ & $\begin{array}{l}2.33 \\
0.675\end{array}$ & $\begin{array}{l}0.00166 \\
0.00484\end{array}$ & $\begin{array}{l}1.01983 \\
1.00210\end{array}$ & $\begin{array}{l}0.602 \\
0.832\end{array}$ \\
\hline $\begin{array}{l}6 . E \\
6.6\end{array}$ & $\begin{array}{l}8.361 \\
0.6034\end{array}$ & $\begin{array}{r}12.3 \\
6.23\end{array}$ & $\begin{array}{l}7.04 \\
3.67\end{array}$ & $\begin{array}{c}450.9 \\
5.96\end{array}$ & $\begin{array}{l}0.0563 \\
0.290\end{array}$ & $\begin{array}{l}0.0110 \\
0.00499\end{array}$ & $\begin{array}{l}2.31 \\
0.688\end{array}$ & $\begin{array}{l}0.00162 \\
0.00457\end{array}$ & $\begin{array}{l}1.01979 \\
1.00222\end{array}$ & $\begin{array}{l}0.614 \\
0.898\end{array}$ \\
\hline $\begin{array}{l}8.7 \\
6.7\end{array}$ & $\begin{array}{l}8.314 \\
0.5394\end{array}$ & $\begin{array}{c}12.1 \\
6.25\end{array}$ & $\begin{array}{l}7.03 \\
3.63\end{array}$ & $\begin{array}{c}438.0 \\
6.23\end{array}$ & $\begin{array}{l}0.0696 \\
0.205\end{array}$ & $\begin{array}{l}9.0111 \\
0.00509\end{array}$ & $\begin{array}{l}2.29 \\
0.702\end{array}$ & $\begin{array}{l}0.00159 \\
0.00432\end{array}$ & $\begin{array}{l}1.01974 \\
1.00235\end{array}$ & $\begin{array}{l}0.627 \\
0.915\end{array}$ \\
\hline $\begin{array}{l}6.8 \\
6.8\end{array}$ & $\begin{array}{l}8.265 \\
0.6771\end{array}$ & $\begin{array}{c}11.8 \\
6.27\end{array}$ & $\begin{array}{l}7.02 \\
3.54\end{array}$ & $\begin{array}{r}417.0 \\
6.50\end{array}$ & $\begin{array}{l}0.0733 \\
0.300\end{array}$ & $\begin{array}{l}0.0111 \\
0.00520\end{array}$ & $\begin{array}{l}2.29 \\
0.716\end{array}$ & $\begin{array}{l}0.00155 \\
0.00408\end{array}$ & $\begin{array}{l}1.01970 \\
1.00248\end{array}$ & $\begin{array}{l}0.640 \\
0.934\end{array}$ \\
\hline
\end{tabular}


THEPMODYNAMIC PROPERTIES OF COEXISTING GASEOUS AND LIQUID HELIUM

\begin{tabular}{|c|c|c|c|c|c|c|c|c|c|c|c|c|}
\hline TEMP & PRESS & & LUME & $\begin{array}{l}\text { SOTHERM } \\
\text { RI VAT I VE }\end{array}$ & $\begin{array}{l}\text { I SOCHORE } \\
\text { OERI VATI IVE }\end{array}$ & $\begin{array}{l}\text { INTERNAL } \\
\text { ENERGY }\end{array}$ & ENTHAL PY & ENTROPY & cv & $C P$ & $\begin{array}{l}\text { VELOCITY } \\
\text { OF SOUND }\end{array}$ & $\begin{array}{l}\text { SURF ACE } \\
\text { TENSION }\end{array}$ \\
\hline OEG. $R$ & PSIA & cu & FT/LB CU & $F T-P S I A / L B$ & PSIA/R & BTU/LB B & BTU/LB & BTU/LB-R & BTU / LP & $-R$ & FT/SEC & LB/IN $\times 10^{7}$ \\
\hline $\begin{array}{l}6.9 \\
6.9\end{array}$ & $\begin{array}{l}9.942 \\
9.942\end{array}$ & & $\begin{array}{l}0.1217 \\
1.396\end{array}$ & $\begin{array}{r}48.2 \\
9.45\end{array}$ & $\begin{array}{c}30.6 \\
2.07\end{array}$ & $\begin{array}{l}3.195 \\
10.34\end{array}$ & $\begin{array}{l}3.419 \\
12.91\end{array}$ & $\begin{array}{l}0.7362 \\
2.112\end{array}$ & $\begin{array}{l}0.531 \\
0.794\end{array}$ & $\begin{array}{l}0.898 \\
1.92\end{array}$ & $\begin{array}{l}614.3 \\
325.8\end{array}$ & 7.972 \\
\hline $\begin{array}{l}7.0 \\
7.0\end{array}$ & $\begin{array}{l}10.54 \\
10.54\end{array}$ & & $\begin{array}{l}0.1225 \\
1.320\end{array}$ & $\begin{array}{r}46.8 \\
9.29\end{array}$ & $\begin{array}{r}30.5 \\
2.20\end{array}$ & $\begin{array}{l}3.278 \\
10.35\end{array}$ & $\begin{array}{l}3.517 \\
12.93\end{array}$ & $\begin{array}{l}0.7482 \\
2.093\end{array}$ & $\begin{array}{l}0.536 \\
0.795\end{array}$ & $\begin{array}{l}0.930 \\
1.97\end{array}$ & $\begin{array}{l}607.9 \\
326.4\end{array}$ & 7.648 \\
\hline $\begin{array}{l}7.1 \\
7.1\end{array}$ & $\begin{array}{l}11.17 \\
11.17\end{array}$ & & $\begin{array}{l}0.1233 \\
1.248\end{array}$ & $\begin{array}{r}43.7 \\
9.11\end{array}$ & $\begin{array}{c}30.5 \\
2.33\end{array}$ & $\begin{array}{l}3.363 \\
10.36\end{array}$ & $\begin{array}{l}3.618 \\
12.94\end{array}$ & $\begin{array}{l}0.7605 \\
2.075\end{array}$ & $\begin{array}{l}0.541 \\
0.795\end{array}$ & $\begin{array}{l}0.965 \\
2.02\end{array}$ & $\begin{array}{l}601.2 \\
327.0\end{array}$ & 7.324 \\
\hline $\begin{array}{l}7.2 \\
7.2\end{array}$ & $\begin{array}{l}11.82 \\
11.82\end{array}$ & & $\begin{array}{l}0.1242 \\
1.181\end{array}$ & $\begin{array}{r}41.5 \\
8.91\end{array}$ & $\begin{array}{c}30.4 \\
2.47\end{array}$ & $\begin{array}{l}3.450 \\
10.37\end{array}$ & $\begin{array}{l}3.721 \\
12.96\end{array}$ & $\begin{array}{l}0.7729 \\
2.056\end{array}$ & $\begin{array}{l}0.545 \\
0.796\end{array}$ & $\begin{array}{l}1.00 \\
2.07\end{array}$ & $\begin{array}{l}594.4 \\
327.5\end{array}$ & 7.001 \\
\hline $\begin{array}{l}7.3 \\
7.3\end{array}$ & $\begin{array}{l}12.49 \\
12.49\end{array}$ & & $\begin{array}{l}0.1251 \\
1.118\end{array}$ & $\begin{array}{r}39.3 \\
8.71\end{array}$ & $\begin{array}{r}30.3 \\
2.61\end{array}$ & $\begin{array}{c}3.539 \\
10.38\end{array}$ & $\begin{array}{l}3.829 \\
12.97\end{array}$ & $\begin{array}{l}0.7856 \\
2.038\end{array}$ & $\begin{array}{l}0.550 \\
0.797\end{array}$ & $\begin{array}{l}1.04 \\
2.13\end{array}$ & $\begin{array}{l}587.3 \\
327.9\end{array}$ & 6.677 \\
\hline $\begin{array}{l}7.4 \\
7.4\end{array}$ & $\begin{array}{l}13.19 \\
13.19\end{array}$ & & $\begin{array}{l}0.1260 \\
1.059\end{array}$ & $\begin{array}{c}37.1 \\
8.48\end{array}$ & $\begin{array}{c}30.1 \\
2.77\end{array}$ & $\begin{array}{l}3.632 \\
10.38\end{array}$ & $\begin{array}{l}3.939 \\
12.97\end{array}$ & $\begin{array}{l}0.7984 \\
2.019\end{array}$ & $\begin{array}{l}0.555 \\
0.798\end{array}$ & $\begin{array}{l}1.09 \\
2.19\end{array}$ & $\begin{array}{l}580.0 \\
328.2\end{array}$ & 6.353 \\
\hline $\begin{array}{l}7.5 \\
7.5\end{array}$ & $\begin{array}{l}13.91 \\
13.91\end{array}$ & & $\begin{array}{l}0.1271 \\
1.003\end{array}$ & $\begin{array}{r}34.9 \\
8.25\end{array}$ & $\begin{array}{l}29.9 \\
2.93\end{array}$ & $\begin{array}{l}3.727 \\
10.38\end{array}$ & $\begin{array}{l}4.054 \\
12.97\end{array}$ & $\begin{array}{l}0.8115 \\
2.000\end{array}$ & $\begin{array}{l}0.550 \\
0.799\end{array}$ & $\begin{array}{l}1.14 \\
2.26\end{array}$ & $\begin{array}{l}572.4 \\
328.4\end{array}$ & 6.030 \\
\hline $\begin{array}{l}7.6 \\
7.6\end{array}$ & $\begin{array}{l}14.67 \\
14.67\end{array}$ & & $\begin{array}{l}0.1281 \\
0.9505\end{array}$ & $\begin{array}{r}32.8 \\
7.99\end{array}$ & $\begin{array}{l}29.7 \\
3.11\end{array}$ & $\begin{array}{l}3.825 \\
10.38\end{array}$ & $\begin{array}{l}4.173 \\
12.96\end{array}$ & $\begin{array}{l}0.8249 \\
1.981\end{array}$ & $\begin{array}{l}0.566 \\
0.800\end{array}$ & $\begin{array}{l}1.19 \\
2.33\end{array}$ & $\begin{array}{l}564.6 \\
328.6\end{array}$ & 5.706 \\
\hline $\begin{array}{l}7.604 \\
7.504\end{array}$ & $\begin{array}{l}14.70 \\
14.70\end{array}$ & & $\begin{array}{l}0.1282 \\
0.9484\end{array}$ & $\begin{array}{l}32.7 \\
7.98\end{array}$ & $\begin{array}{l}29.7 \\
3.11\end{array}$ & $\begin{array}{l}3.829 \\
10.38\end{array}$ & $\begin{array}{l}4.178 \\
12.96\end{array}$ & $\begin{array}{l}0.8255 \\
1.961\end{array}$ & $\begin{array}{l}0.566 \\
0.600\end{array}$ & $\begin{array}{l}1.19 \\
2.34\end{array}$ & $\begin{array}{l}564.2 \\
328.6\end{array}$ & 5.693 \\
\hline $\begin{array}{l}7.7 \\
7.7\end{array}$ & $\begin{array}{l}15.44 \\
15.44\end{array}$ & & $\begin{array}{l}0.1293 \\
0.9004\end{array}$ & $\begin{array}{c}30.6 \\
7.72\end{array}$ & $\begin{array}{l}29.5 \\
3.29\end{array}$ & $\begin{array}{l}3.926 \\
10.37\end{array}$ & $\begin{array}{l}4.296 \\
12.95\end{array}$ & $\begin{array}{l}0.8386 \\
1.962\end{array}$ & $\begin{array}{l}0.571 \\
0.801\end{array}$ & $\begin{array}{l}1.25 \\
2.42\end{array}$ & $\begin{array}{l}556.4 \\
328.7\end{array}$ & 5.382 \\
\hline $\begin{array}{l}7.8 \\
7.8\end{array}$ & $\begin{array}{l}16 \cdot 25 \\
16 \cdot 25\end{array}$ & & $\begin{array}{l}0.1305 \\
0.8530\end{array}$ & $\begin{array}{r}28.5 \\
7.43\end{array}$ & $\begin{array}{r}29.2 \\
3.48\end{array}$ & $\begin{array}{l}4.031 \\
10.36\end{array}$ & $\begin{array}{l}4.424 \\
12.93\end{array}$ & $\begin{array}{l}0.8526 \\
1.943\end{array}$ & $\begin{array}{l}0.577 \\
0.802\end{array}$ & $\begin{array}{l}1.31 \\
2.52\end{array}$ & $\begin{array}{l}547.9 \\
328.7\end{array}$ & 5.058 \\
\hline $\begin{array}{l}7.9 \\
7.9\end{array}$ & $\begin{array}{l}17.09 \\
17.09\end{array}$ & & $\begin{array}{l}0.1318 \\
0.8078\end{array}$ & $\begin{array}{r}26.4 \\
7.13\end{array}$ & $\begin{array}{l}28.9 \\
3.69\end{array}$ & $\begin{array}{l}4.140 \\
10.35\end{array}$ & $\begin{array}{l}4.557 \\
12.90\end{array}$ & $\begin{array}{l}0.8670 \\
1.923\end{array}$ & $\begin{array}{l}0.583 \\
0.803\end{array}$ & $\begin{array}{l}1.39 \\
2.63\end{array}$ & $\begin{array}{l}539.1 \\
328.6\end{array}$ & 4.735 \\
\hline $\begin{array}{l}8.0 \\
8.0\end{array}$ & $\begin{array}{l}17.95 \\
17.95\end{array}$ & & $\begin{array}{l}0.1332 \\
0.7649\end{array}$ & $\begin{array}{l}24.3 \\
6.80\end{array}$ & $\begin{array}{c}28.5 \\
3.91\end{array}$ & $\begin{array}{c}4.253 \\
10.33\end{array}$ & $\begin{array}{l}4.696 \\
12.37\end{array}$ & $\begin{array}{l}0.8817 \\
1.903\end{array}$ & $\begin{array}{l}0.589 \\
0.804\end{array}$ & $\begin{array}{l}1.47 \\
2.75\end{array}$ & $\begin{array}{l}530.0 \\
328.4\end{array}$ & 4.411 \\
\hline $\begin{array}{l}8.1 \\
8.1\end{array}$ & $\begin{array}{l}18.84 \\
18.84\end{array}$ & & $\begin{array}{l}0.1347 \\
0.7238\end{array}$ & $\begin{array}{r}22.2 \\
6.45\end{array}$ & $\begin{array}{r}28.2 \\
4.15\end{array}$ & $\begin{array}{r}4.370 \\
10.30\end{array}$ & $\begin{array}{r}4.840 \\
12.83\end{array}$ & $\begin{array}{l}0.8969 \\
1.882\end{array}$ & $\begin{array}{l}0.595 \\
0.805\end{array}$ & $\begin{array}{l}1.56 \\
2.90\end{array}$ & $\begin{array}{l}520.5 \\
328.0\end{array}$ & 4.087 \\
\hline $\begin{array}{l}8.2 \\
8.2\end{array}$ & $\begin{array}{l}19.77 \\
19.77\end{array}$ & & $\begin{array}{l}0.1363 \\
0.6845\end{array}$ & $\begin{array}{l}20.2 \\
5.08\end{array}$ & $\begin{array}{l}27.7 \\
4.40\end{array}$ & $\begin{array}{c}4.493 \\
10.27\end{array}$ & $\begin{array}{c}4.992 \\
12.77\end{array}$ & $\begin{array}{l}0.9127 \\
1.861\end{array}$ & $\begin{array}{l}0.602 \\
0.806\end{array}$ & $\begin{array}{l}1.68 \\
3.07\end{array}$ & $\begin{array}{l}510.5 \\
327.6\end{array}$ & 3.763 \\
\hline $\begin{array}{l}8.3 \\
8.3\end{array}$ & $\begin{array}{l}20.72 \\
20.72\end{array}$ & & $\begin{array}{l}0.1381 \\
0.6467\end{array}$ & $\begin{array}{c}18.2 \\
5.69\end{array}$ & $\begin{array}{c}27.3 \\
4.67\end{array}$ & $\begin{array}{l}4.621 \\
10.23\end{array}$ & $\begin{array}{l}5.150 \\
12.71\end{array}$ & $\begin{array}{l}0.9290 \\
1.839\end{array}$ & $\begin{array}{l}0.608 \\
0.307\end{array}$ & $\begin{array}{l}1.81 \\
3.27\end{array}$ & $\begin{array}{l}500.2 \\
327.1\end{array}$ & 3.440 \\
\hline $\begin{array}{l}8.4 \\
8.4\end{array}$ & $\begin{array}{l}21.71 \\
21.71\end{array}$ & & $\begin{array}{l}0.1400 \\
0.6103\end{array}$ & $\begin{array}{c}16.2 \\
5.28\end{array}$ & $\begin{array}{l}26.8 \\
4.97\end{array}$ & $\begin{array}{l}4.755 \\
10.18\end{array}$ & $\begin{array}{l}5.318 \\
12.54\end{array}$ & $\begin{array}{l}3.9450 \\
1.816\end{array}$ & $\begin{array}{l}0.616 \\
0.807\end{array}$ & $\begin{array}{l}1.96 \\
3.52\end{array}$ & $\begin{array}{l}489.4 \\
326.5\end{array}$ & 3.116 \\
\hline $\begin{array}{l}8.5 \\
8.5\end{array}$ & $\begin{array}{l}22.73 \\
22.73\end{array}$ & & $\begin{array}{l}0.1421 \\
0.5751\end{array}$ & $\begin{array}{c}14.3 \\
4.83\end{array}$ & $\begin{array}{l}26.2 \\
5.30\end{array}$ & $\begin{array}{l}4.897 \\
10.12\end{array}$ & $\begin{array}{r}5.495 \\
12.55\end{array}$ & $\begin{array}{l}0.9638 \\
1.793\end{array}$ & $\begin{array}{l}0.623 \\
0.808\end{array}$ & $\begin{array}{l}2.16 \\
3.83\end{array}$ & $\begin{array}{l}478.2 \\
325.7\end{array}$ & 2.792 \\
\hline $\begin{array}{l}8.6 \\
8.6\end{array}$ & $\begin{array}{l}23.79 \\
23.79\end{array}$ & & $\begin{array}{l}0.1445 \\
0.5409\end{array}$ & $\begin{array}{r}12.3 \\
4.36\end{array}$ & $\begin{array}{r}25.6 \\
5.66\end{array}$ & $\begin{array}{l}5.047 \\
10.06\end{array}$ & $\begin{array}{l}5.584 \\
12.44\end{array}$ & $\begin{array}{l}0.9826 \\
1.767\end{array}$ & $\begin{array}{l}0.631 \\
0.809\end{array}$ & $\begin{array}{l}2.40 \\
4.22\end{array}$ & $\begin{array}{l}466.4 \\
324.9\end{array}$ & 2.469 \\
\hline $\begin{array}{l}8.7 \\
8.7\end{array}$ & $\begin{array}{l}24.87 \\
24.87\end{array}$ & & $\begin{array}{l}0.1472 \\
0.5073\end{array}$ & $\begin{array}{c}10.5 \\
3.86\end{array}$ & $\begin{array}{c}25.0 \\
6.06\end{array}$ & $\begin{array}{l}5.208 \\
9.97\end{array}$ & $\begin{array}{l}5.886 \\
12.31\end{array}$ & $\begin{array}{l}1.003 \\
1.740\end{array}$ & $\begin{array}{l}0.640 \\
0.809\end{array}$ & $\begin{array}{l}2.72 \\
4.75\end{array}$ & $\begin{array}{l}454.1 \\
323.9\end{array}$ & 2.145 \\
\hline $\begin{array}{l}8.8 \\
8.8\end{array}$ & $\begin{array}{l}26.00 \\
26.00\end{array}$ & & $\begin{array}{l}0.1503 \\
0.4742\end{array}$ & $\begin{array}{l}8.86 \\
3.33\end{array}$ & $\begin{array}{c}24.3 \\
6.51\end{array}$ & $\begin{array}{l}5.382 \\
9.873\end{array}$ & $\begin{array}{l}6.106 \\
12.16\end{array}$ & $\begin{array}{l}1.0224 \\
1.711\end{array}$ & $\begin{array}{l}0.649 \\
0.810\end{array}$ & $\begin{array}{l}3.15 \\
5.47\end{array}$ & $\begin{array}{l}441.2 \\
322.9\end{array}$ & 1.821 \\
\hline $\begin{array}{l}8.9 \\
8.9\end{array}$ & $\begin{array}{l}27 \cdot 15 \\
27.15\end{array}$ & & $\begin{array}{l}0.1540 \\
0.4412\end{array}$ & $\begin{array}{l}6.90 \\
2.77\end{array}$ & $\begin{array}{l}23.5 \\
7.03\end{array}$ & $\begin{array}{l}5.573 \\
9.751\end{array}$ & $\begin{array}{l}6.348 \\
11.97\end{array}$ & $\begin{array}{l}1.048 \\
1.679\end{array}$ & $\begin{array}{l}0.560 \\
0.810\end{array}$ & $\begin{array}{l}3.78 \\
6.54\end{array}$ & $\begin{array}{l}427.7 \\
321.9\end{array}$ & 1.497 \\
\hline $\begin{array}{l}9.0 \\
9.0\end{array}$ & $\begin{array}{l}28.35 \\
28.35\end{array}$ & & $\begin{array}{l}0.1585 \\
0.4076\end{array}$ & $\begin{array}{l}5.21 \\
2.18\end{array}$ & $\begin{array}{c}22.5 \\
7.66\end{array}$ & $\begin{array}{l}5.787 \\
9.596\end{array}$ & $\begin{array}{l}5.519 \\
11.74\end{array}$ & $\begin{array}{l}1.074 \\
1.642\end{array}$ & $\begin{array}{l}0.671 \\
0.809\end{array}$ & $\begin{array}{l}4.75 \\
8.26\end{array}$ & $\begin{array}{l}413.3 \\
321.0\end{array}$ & 1.174 \\
\hline $\begin{array}{l}9.1 \\
9.1\end{array}$ & $\begin{array}{l}29.58 \\
29.58\end{array}$ & & $\begin{array}{l}0.1642 \\
0.3726\end{array}$ & $\begin{array}{l}3.61 \\
1.56\end{array}$ & $\begin{array}{l}21.5 \\
8.43\end{array}$ & $\begin{array}{l}5.035 \\
9.393\end{array}$ & $\begin{array}{l}6.934 \\
11.43\end{array}$ & $\begin{array}{l}1.105 \\
1.599\end{array}$ & $\begin{array}{l}0.68,4 \\
0.808\end{array}$ & $\begin{array}{l}6.48 \\
11.5\end{array}$ & $\begin{array}{l}398.1 \\
320.3\end{array}$ & 0.8499 \\
\hline $\begin{array}{l}9.2 \\
9.2\end{array}$ & $\begin{array}{l}30.85 \\
30.85\end{array}$ & & $\begin{array}{l}0.1721 \\
0.3342\end{array}$ & $\begin{array}{l}2.13 \\
0.915\end{array}$ & $\begin{array}{l}20.2 \\
9.48\end{array}$ & $\begin{array}{l}6.339 \\
9.107\end{array}$ & $\begin{array}{l}7.322 \\
11.02\end{array}$ & $\begin{array}{l}1.143 \\
1.544\end{array}$ & $\begin{array}{l}0.700 \\
0.805\end{array}$ & $\begin{array}{l}10.3 \\
19.5\end{array}$ & $\begin{array}{l}391.4 \\
320.4\end{array}$ & 0.5261 \\
\hline $\begin{array}{l}9.3 \\
9.3\end{array}$ & $\begin{array}{l}32 \cdot 15 \\
32 \cdot 15\end{array}$ & & $\begin{array}{l}0.1857 \\
0.2867\end{array}$ & $\begin{array}{l}0.793 \\
0.293\end{array}$ & $\begin{array}{l}18.3 \\
11.2\end{array}$ & $\begin{array}{l}6.778 \\
8.627\end{array}$ & $\begin{array}{c}7.833 \\
10.33\end{array}$ & $\begin{array}{l}1.199 \\
1.453\end{array}$ & $\begin{array}{l}0.724 \\
0.797\end{array}$ & $\begin{array}{l}25.8 \\
61.2\end{array}$ & $\begin{array}{l}361.6 \\
322.9\end{array}$ & 0.2024 \\
\hline $\begin{array}{r}9.363 \\
.9 .363\end{array}$ & $\begin{array}{l}32.99 \\
32.99\end{array}$ & & $\begin{array}{l}0.2300 \\
0.2300\end{array}$ & & & & & & & & & 0.000 \\
\hline
\end{tabular}


THERMODYNAMIC PRDPERTIES OF COEXISTING GASEOUS AND LIQUIR HELIUM

\begin{tabular}{|c|c|c|c|c|c|c|c|c|c|c|}
\hline $\begin{array}{c}\text { TEMPERA TURE } \\
\text { OEG. R }\end{array}$ & $\begin{array}{l}\text { DENSITY } \\
\text { LB/CU FT }\end{array}$ & $\begin{array}{l}V(D H / O V){ }_{p} \\
\text { BTU/LA }\end{array}$ & $\begin{array}{c}V(D P / D U)_{V} \\
\text { PSIA-CU FT/9TU }\end{array}$ & $\begin{array}{c}-V(D P / D V)_{T} \\
\text { PSIA }\end{array}$ & $\begin{array}{c}(O V / D T)_{\mathrm{P}}^{\prime} \mathrm{V} \\
\text { DEG. R }\end{array}$ & $\begin{array}{c}\text { THERMAL } \\
\text { CDNDUCTIVITY } \\
\text { BTU/FT-HQ-R }\end{array}$ & $\begin{array}{l}\text { VISCDSITY } \\
\begin{array}{r}\text { LA/FT-SEC } \\
\times 10^{8}\end{array}\end{array}$ & $\begin{array}{l}\text { IHERMAL } \\
\text { DIFFUSIVITY } \\
\text { SO FT/HR }\end{array}$ & $\begin{array}{l}\text { OIELECTRIC } \\
\text { CDNSTANT }\end{array}$ & $\begin{array}{r}\text { PRAND TL } \\
\text { NUM9ER }\end{array}$ \\
\hline $\begin{array}{l}6.9 \\
6.9\end{array}$ & $\begin{array}{l}8.215 \\
0.7165\end{array}$ & $\begin{array}{c}11.6 \\
6.29\end{array}$ & $\begin{array}{l}7.00 \\
3.64\end{array}$ & $\begin{array}{c}396.0 \\
6.77\end{array}$ & $\begin{array}{l}0.0771 \\
0.306\end{array}$ & $\begin{array}{l}0.0112 \\
0.00530\end{array}$ & $\begin{array}{l}2.26 \\
0.730\end{array}$ & $\begin{array}{l}0.00151 \\
0.00385\end{array}$ & $\begin{array}{l}1.01965 \\
1.00262\end{array}$ & $\begin{array}{l}0.655 \\
0.954\end{array}$ \\
\hline $\begin{array}{l}7.0 \\
7.0\end{array}$ & $\begin{array}{l}8.163 \\
0.7578\end{array}$ & $\begin{array}{r}11.4 \\
6.30\end{array}$ & $\begin{array}{l}6.98 \\
3.65\end{array}$ & $\begin{array}{r}375.0 \\
7.04\end{array}$ & $\begin{array}{l}0.0814 \\
0.312\end{array}$ & $\begin{array}{l}0.0112 \\
0.00541\end{array}$ & $\begin{array}{l}2.24 \\
0.745\end{array}$ & $\begin{array}{l}0.00147 \\
0.00363\end{array}$ & $\begin{array}{l}1.01960 \\
1.00277\end{array}$ & $\begin{array}{l}0.671 \\
0.975\end{array}$ \\
\hline $\begin{array}{l}7.1 \\
7.1\end{array}$ & $\begin{array}{l}8.109 \\
0.8011\end{array}$ & $\begin{array}{c}11.2 \\
6.31\end{array}$ & $\begin{array}{l}6.95 \\
3.66\end{array}$ & $\begin{array}{r}355.0 \\
7.30\end{array}$ & $\begin{array}{l}0.0859 \\
0.319\end{array}$ & $\begin{array}{l}0.0112 \\
0.00552\end{array}$ & $\begin{array}{l}2.22 \\
0.759\end{array}$ & $\begin{array}{l}0.00143 \\
0.00342\end{array}$ & $\begin{array}{l}1.01954 \\
1.00292\end{array}$ & $\begin{array}{l}0.688 \\
1.00\end{array}$ \\
\hline $\begin{array}{l}7.2 \\
7.2\end{array}$ & $\begin{array}{l}8.053 \\
0.8465\end{array}$ & $\begin{array}{c}11.0 \\
6.32\end{array}$ & $\begin{array}{l}6.92 \\
3.66\end{array}$ & $\begin{array}{r}334.0 \\
7.55\end{array}$ & $\begin{array}{l}0.0909 \\
0.327\end{array}$ & $\begin{array}{l}\text { C.0113 } \\
0.00563\end{array}$ & $\begin{array}{l}2.21 \\
0.774\end{array}$ & $\begin{array}{l}0.00139 \\
0.00322\end{array}$ & $\begin{array}{l}1.01949 \\
1.00308\end{array}$ & $\begin{array}{l}0.707 \\
1.02\end{array}$ \\
\hline $\begin{array}{l}7.3 \\
7.3\end{array}$ & $\begin{array}{l}7.995 \\
0.8941\end{array}$ & $\begin{array}{c}10.8 \\
6.33\end{array}$ & $\begin{array}{l}6.88 \\
3.67\end{array}$ & $\begin{array}{r}314.0 \\
7.78\end{array}$ & $\begin{array}{l}0.0963 \\
0.336\end{array}$ & $\begin{array}{l}0.0113 \\
0.00575\end{array}$ & $\begin{array}{l}2.19 \\
0.790\end{array}$ & $\begin{array}{l}0.00135 \\
0.00303\end{array}$ & $\begin{array}{l}1.01942 \\
1.00325\end{array}$ & $\begin{array}{l}0.726 \\
1.05\end{array}$ \\
\hline $\begin{array}{l}7.4 \\
7.4\end{array}$ & $\begin{array}{l}7.934 \\
0.9441\end{array}$ & $\begin{array}{c}10.6 \\
6.33\end{array}$ & $\begin{array}{l}6.83 \\
3.67\end{array}$ & $\begin{array}{r}294.0 \\
8.01\end{array}$ & $\begin{array}{l}0.102 \\
0.346\end{array}$ & $\begin{array}{l}0.0113 \\
0.00587\end{array}$ & $\begin{array}{l}2.17 \\
0.805\end{array}$ & $\begin{array}{l}0.00131 \\
0.00284\end{array}$ & $\begin{array}{l}1.01936 \\
1.00342\end{array}$ & $\begin{array}{l}0.750 \\
1.08\end{array}$ \\
\hline $\begin{array}{l}7.5 \\
7.5\end{array}$ & $\begin{array}{l}7.871 \\
0.997\end{array}$ & $\begin{array}{c}10.4 \\
6.33\end{array}$ & $\begin{array}{l}6.78 \\
3.68\end{array}$ & $\begin{array}{r}275.0 \\
8.22\end{array}$ & $\begin{array}{l}0.109 \\
0.357\end{array}$ & $\begin{array}{l}0.0113 \\
0.00600\end{array}$ & $\begin{array}{l}2.15 \\
0.821\end{array}$ & $\begin{array}{l}0.00127 \\
0.00267\end{array}$ & $\begin{array}{l}1.01929 \\
1.00360\end{array}$ & $\begin{array}{l}0.775 \\
1.11\end{array}$ \\
\hline $\begin{array}{l}7.6 \\
7.6\end{array}$ & $\begin{array}{l}7.805 \\
1.052\end{array}$ & $\begin{array}{c}10.2 \\
6.32\end{array}$ & $\begin{array}{l}6.73 \\
3.69\end{array}$ & $\begin{array}{c}256.0 \\
8.41\end{array}$ & $\begin{array}{l}0.116 \\
0.369\end{array}$ & $\begin{array}{l}0.0114 \\
0.00614\end{array}$ & $\begin{array}{l}2.13 \\
0.837\end{array}$ & $\begin{array}{l}0.00122 \\
0.00250\end{array}$ & $\begin{array}{l}1.01922 \\
1.00379\end{array}$ & $\begin{array}{l}0.803 \\
1.15\end{array}$ \\
\hline $\begin{array}{l}7.604 \\
7.604\end{array}$ & $\begin{array}{l}7.802 \\
1.054\end{array}$ & $\begin{array}{c}10.2 \\
6.32\end{array}$ & $\begin{array}{l}6.73 \\
3.69\end{array}$ & $\begin{array}{r}255.0 \\
8.42\end{array}$ & $\begin{array}{l}0.117 \\
0.370\end{array}$ & $\begin{array}{l}0.0114 \\
0.00614\end{array}$ & $\begin{array}{l}2.13 \\
0.836\end{array}$ & $\begin{array}{l}0.00122 \\
0.00249\end{array}$ & $\begin{array}{l}1.01921 \\
1.00380\end{array}$ & $\begin{array}{l}0.804 \\
1.15\end{array}$ \\
\hline $\begin{array}{l}7.7 \\
7.7\end{array}$ & $\begin{array}{l}7.736 \\
1.111\end{array}$ & $\begin{array}{c}10.0 \\
6.31\end{array}$ & $\begin{array}{l}6.67 \\
3.70\end{array}$ & $\begin{array}{r}237.0 \\
8.58\end{array}$ & $\begin{array}{l}0.124 \\
0.383\end{array}$ & $\begin{array}{l}0.0114 \\
0.00628\end{array}$ & $\begin{array}{l}2.11 \\
0.854\end{array}$ & $\begin{array}{l}0.00118 \\
0.00234\end{array}$ & $\begin{array}{l}1.01914 \\
1.00399\end{array}$ & $\begin{array}{l}0.833 \\
1.18\end{array}$ \\
\hline $\begin{array}{l}7.8 \\
7.8\end{array}$ & $\begin{array}{l}7.664 \\
1.172\end{array}$ & $\begin{array}{l}9.82 \\
6.30\end{array}$ & $\begin{array}{l}6.60 \\
3.70\end{array}$ & $\begin{array}{r}218.0 \\
8.72\end{array}$ & $\begin{array}{l}0.134 \\
0.399\end{array}$ & $\begin{array}{l}0.0114 \\
0.00643\end{array}$ & $\begin{array}{l}2.09 \\
0.871\end{array}$ & $\begin{array}{l}0.00113 \\
0.00218\end{array}$ & $\begin{array}{l}1.01906 \\
1.00420\end{array}$ & $\begin{array}{l}0.868 \\
1.23\end{array}$ \\
\hline $\begin{array}{l}7.9 \\
7.9\end{array}$ & $\begin{array}{l}7.588 \\
1.238\end{array}$ & $\begin{array}{l}9.61 \\
6.28\end{array}$ & $\begin{array}{l}6.53 \\
3.71\end{array}$ & $\begin{array}{r}200.0 \\
\text { A. } 92\end{array}$ & $\begin{array}{l}0.144 \\
0.418\end{array}$ & $\begin{array}{l}0.0114 \\
0.00659\end{array}$ & $\begin{array}{l}2.07 \\
0.888\end{array}$ & $\begin{array}{l}0.00108 \\
0.00203\end{array}$ & $\begin{array}{l}1.01897 \\
1.00442\end{array}$ & $\begin{array}{l}0.907 \\
1.27\end{array}$ \\
\hline $\begin{array}{l}8.0 \\
8.0\end{array}$ & $\begin{array}{l}7.509 \\
1.307\end{array}$ & $\begin{array}{l}9.39 \\
6.26\end{array}$ & $\begin{array}{l}6.45 \\
3.72\end{array}$ & $\begin{array}{r}182.0 \\
8.89\end{array}$ & $\begin{array}{l}0.156 \\
0.440\end{array}$ & $\begin{array}{l}0.0114 \\
0.00676\end{array}$ & $\begin{array}{l}2.05 \\
0.906\end{array}$ & $\begin{array}{l}0.00103 \\
0.00188\end{array}$ & $\begin{array}{l}1.01887 \\
1.00466\end{array}$ & $\begin{array}{l}0.952 \\
1.33\end{array}$ \\
\hline $\begin{array}{l}8.1 \\
8.1\end{array}$ & $\begin{array}{l}7.426 \\
1.382\end{array}$ & $\begin{array}{l}9.18 \\
6.23\end{array}$ & $\begin{array}{l}6.37 \\
3.73\end{array}$ & $\begin{array}{r}165.0 \\
8.92\end{array}$ & $\begin{array}{l}0.171 \\
0.465\end{array}$ & $\begin{array}{l}0.0115 \\
0.00694\end{array}$ & $\begin{array}{l}2.03 \\
0.924\end{array}$ & $\begin{array}{l}0.000988 \\
0.00173\end{array}$ & $\begin{array}{l}1.01877 \\
1.00490\end{array}$ & $\begin{array}{l}0.994 \\
1.39\end{array}$ \\
\hline $\begin{array}{l}8.2 \\
8.2\end{array}$ & $\begin{array}{l}7.337 \\
1.461\end{array}$ & $\begin{array}{l}8.95 \\
6.20\end{array}$ & $\begin{array}{l}6.28 \\
3.74\end{array}$ & $\begin{array}{r}148.0 \\
8.89\end{array}$ & $\begin{array}{l}0.187 \\
0.495\end{array}$ & $\begin{array}{l}0.0115 \\
0.00715\end{array}$ & $\begin{array}{l}2.00 \\
0.944\end{array}$ & $\begin{array}{l}0.000937 \\
0.00160\end{array}$ & $\begin{array}{l}1.01866 \\
1.00517\end{array}$ & $\begin{array}{l}1.05 \\
1.46\end{array}$ \\
\hline $\begin{array}{l}8.3 \\
8.3\end{array}$ & $\begin{array}{l}7.244 \\
1.546\end{array}$ & $\begin{array}{l}8.73 \\
6.16\end{array}$ & $\begin{array}{l}6.19 \\
3.75\end{array}$ & $\begin{array}{r}132.0 \\
8.80\end{array}$ & $\begin{array}{l}0.257 \\
0.531\end{array}$ & $\begin{array}{l}0.0116 \\
0.00738\end{array}$ & $\begin{array}{l}1.98 \\
0.964\end{array}$ & $\begin{array}{l}0.000884 \\
0.00146\end{array}$ & $\begin{array}{l}1.01853 \\
1.00545\end{array}$ & $\begin{array}{l}1.11 \\
1.54\end{array}$ \\
\hline $\begin{array}{l}8.4 \\
8.4\end{array}$ & $\begin{array}{l}7.144 \\
1.638\end{array}$ & $\begin{array}{l}8.49 \\
6.12\end{array}$ & $\begin{array}{l}6.09 \\
3.76\end{array}$ & $\begin{array}{r}11 F .0 \\
8.64\end{array}$ & $\begin{array}{l}0.231 \\
0.575\end{array}$ & $\begin{array}{l}0.0116 \\
0.00764\end{array}$ & $\begin{array}{l}1.96 \\
0.984\end{array}$ & $\begin{array}{l}0.000829 \\
0.00132\end{array}$ & $\begin{array}{l}1.01840 \\
1.00575\end{array}$ & $\begin{array}{l}1.19 \\
1.63\end{array}$ \\
\hline $\begin{array}{l}8.5 \\
8.5\end{array}$ & $\begin{array}{l}7.036 \\
1.739\end{array}$ & $\begin{array}{l}8.25 \\
6.06\end{array}$ & $\begin{array}{l}5.98 \\
3.77\end{array}$ & $\begin{array}{r}100.0 \\
8.40\end{array}$ & $\begin{array}{l}0.261 \\
0.630\end{array}$ & $\begin{array}{l}0.0117 \\
0.00795\end{array}$ & $\begin{array}{l}1.93 \\
1.01\end{array}$ & $\begin{array}{l}0.000771 \\
0.00119\end{array}$ & $\begin{array}{l}1.01825 \\
1.00607\end{array}$ & $\begin{array}{l}1.28 \\
1.75\end{array}$ \\
\hline $\begin{array}{l}8.6 \\
8.6\end{array}$ & $\begin{array}{l}6.920 \\
1.849\end{array}$ & $\begin{array}{l}8.00 \\
6.02\end{array}$ & $\begin{array}{l}5.87 \\
3.78\end{array}$ & $\begin{array}{c}85.4 \\
8.06\end{array}$ & $\begin{array}{l}0.300 \\
0.701\end{array}$ & $\begin{array}{l}0.0118 \\
0.00832\end{array}$ & $\begin{array}{l}1.90 \\
1.03\end{array}$ & $\begin{array}{l}0.000709 \\
0.00136\end{array}$ & $\begin{array}{l}1.01809 \\
1.00642\end{array}$ & $\begin{array}{l}1.40 \\
1.88\end{array}$ \\
\hline $\begin{array}{l}8.7 \\
8.7\end{array}$ & $\begin{array}{l}6.793 \\
1.971\end{array}$ & $\begin{array}{l}7.75 \\
5.97\end{array}$ & $\begin{array}{l}5.75 \\
3.80\end{array}$ & $\begin{array}{l}71.2 \\
7.61\end{array}$ & $\begin{array}{l}0.351 \\
0.796\end{array}$ & $\begin{array}{l}0.0119 \\
0.00877\end{array}$ & $\begin{array}{l}1.88 \\
1.05\end{array}$ & $\begin{array}{l}0.000645 \\
0.000937\end{array}$ & $\begin{array}{l}1.01790 \\
1.00680\end{array}$ & $\begin{array}{l}1.54 \\
2.05\end{array}$ \\
\hline $\begin{array}{l}8.8 \\
8.8\end{array}$ & $\begin{array}{l}6.652 \\
2.109\end{array}$ & $\begin{array}{l}7.48 \\
5.90\end{array}$ & $\begin{array}{l}5.62 \\
3.81\end{array}$ & $\begin{array}{l}57.6 \\
7.03\end{array}$ & $\begin{array}{l}0.421 \\
0.927\end{array}$ & $\begin{array}{l}0.0121 \\
0.00936\end{array}$ & $\begin{array}{l}1.84 \\
1.03\end{array}$ & $\begin{array}{l}0.000576 \\
0.000812\end{array}$ & $\begin{array}{l}1.01769 \\
1.00723\end{array}$ & $\begin{array}{l}1.73 \\
2.27\end{array}$ \\
\hline $\begin{array}{l}8.9 \\
8.9\end{array}$ & $\begin{array}{l}6.494 \\
2.267\end{array}$ & $\begin{array}{l}7.21 \\
5.84\end{array}$ & $\begin{array}{l}5.48 \\
3.83\end{array}$ & $\begin{array}{l}44.8 \\
6.28\end{array}$ & $\begin{array}{l}0.524 \\
1.12\end{array}$ & $\begin{array}{l}0.0124 \\
0.0102\end{array}$ & $\begin{array}{l}1.81 \\
1.11\end{array}$ & $\begin{array}{l}0.000504 \\
0.000687\end{array}$ & $\begin{array}{l}1.01745 \\
1.00771\end{array}$ & $\begin{array}{l}1.99 \\
2.56\end{array}$ \\
\hline $\begin{array}{l}9.0 \\
9.0\end{array}$ & $\begin{array}{l}6.310 \\
2.453\end{array}$ & $\begin{array}{l}6.93 \\
5.77\end{array}$ & $\begin{array}{l}5.32 \\
3.86\end{array}$ & $\begin{array}{c}32.9 \\
5.34\end{array}$ & $\begin{array}{l}0.685 \\
1.43\end{array}$ & $\begin{array}{l}0.0128 \\
0.0114\end{array}$ & $\begin{array}{l}1.77 \\
1.14\end{array}$ & $\begin{array}{l}0.000428 \\
0.000565\end{array}$ & $\begin{array}{l}1.01715 \\
1.00827\end{array}$ & $\begin{array}{l}2.37 \\
2.97\end{array}$ \\
\hline $\begin{array}{l}9.1 \\
9.1\end{array}$ & $\begin{array}{l}6.091 \\
2.684\end{array}$ & $\begin{array}{l}6.64 \\
5.67\end{array}$ & $\begin{array}{l}5.15 \\
3.89\end{array}$ & $\begin{array}{l}22.0 \\
4.18\end{array}$ & $\begin{array}{l}0.975 \\
2.0 ?\end{array}$ & $\begin{array}{l}0.0137 \\
0.0137\end{array}$ & $\begin{array}{l}1.73 \\
1.18\end{array}$ & $\begin{array}{l}0.000348 \\
0.000444\end{array}$ & $\begin{array}{l}1.01679 \\
1.00894\end{array}$ & $\begin{array}{l}2.94 \\
3.57\end{array}$ \\
\hline $\begin{array}{l}9.2 \\
9.2\end{array}$ & $\begin{array}{l}5.810 \\
2.993\end{array}$ & $\begin{array}{l}6.34 \\
5.63\end{array}$ & $\begin{array}{l}4.95 \\
3.94\end{array}$ & $\begin{array}{r}12.4 \\
2.74\end{array}$ & $\begin{array}{l}1.63 \\
3.46\end{array}$ & $\begin{array}{l}0.0159 \\
0.0190\end{array}$ & $\begin{array}{l}1.68 \\
1.23\end{array}$ & $\begin{array}{l}0.000265 \\
0.000326\end{array}$ & $\begin{array}{l}1.01629 \\
1.00982\end{array}$ & $\begin{array}{l}3.93 \\
4.54\end{array}$ \\
\hline $\begin{array}{l}9.3 \\
9.3\end{array}$ & $\begin{array}{l}5.385 \\
3.488\end{array}$ & $\begin{array}{l}6.02 \\
5.59\end{array}$ & $\begin{array}{l}4.69 \\
4.02\end{array}$ & $\begin{array}{l}4.27 \\
1.02\end{array}$ & $\begin{array}{l}4.28 \\
10.9\end{array}$ & $\begin{array}{l}0.0252 \\
0.0457\end{array}$ & $\begin{array}{l}1.60 \\
1.30\end{array}$ & $\begin{array}{l}0.000181 \\
0.000214\end{array}$ & $\begin{array}{l}1.01549 \\
1.01115\end{array}$ & $\begin{array}{l}5.90 \\
6.29\end{array}$ \\
\hline
\end{tabular}

$* .363 \quad 4.349$
-9.363 
1 PSIA ISOGAR

Appendix E, Isobaric Propertie

THERMOOYNAMIC PROPERTIES OF HELIUM 4

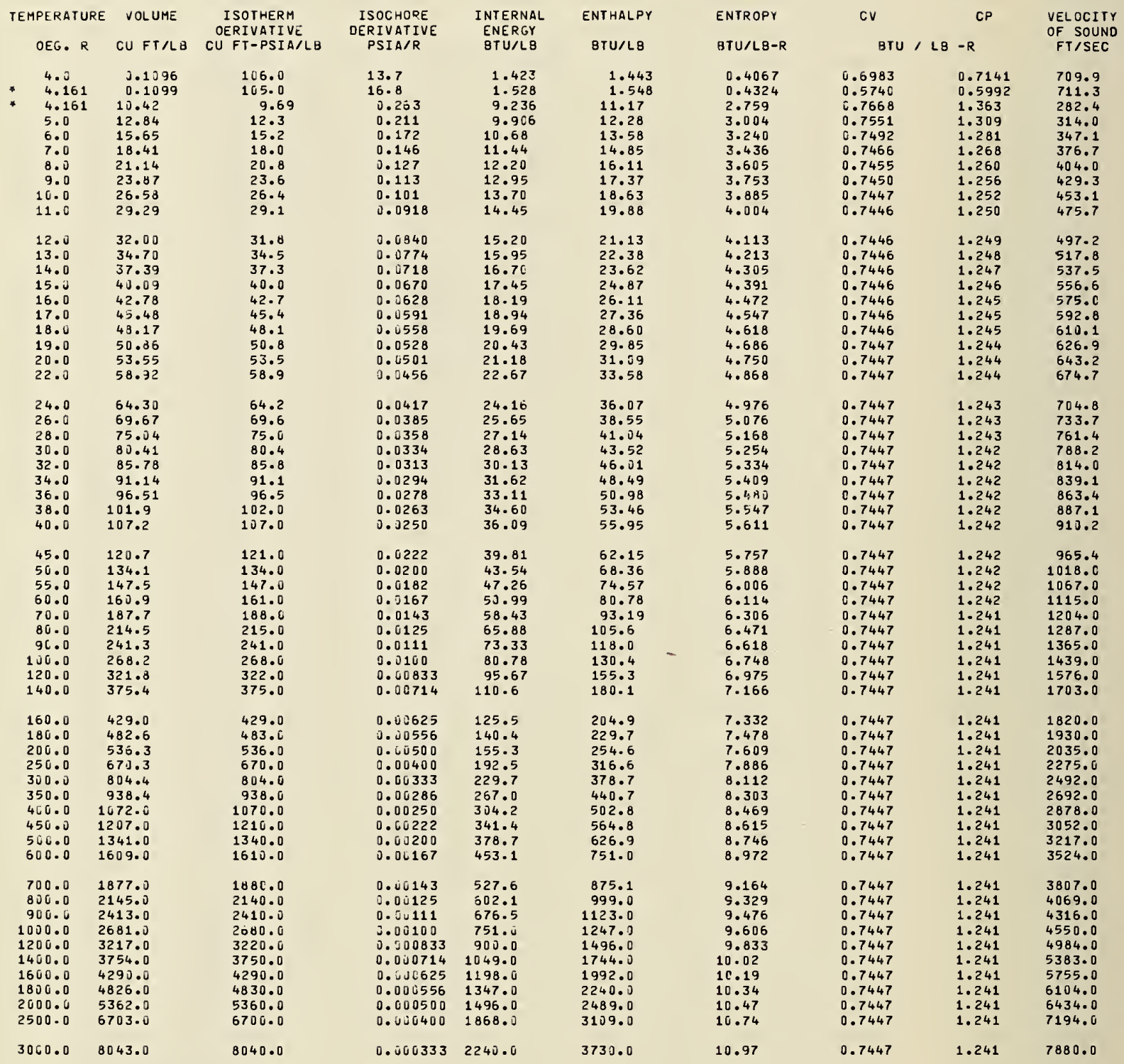

* two-phase gounoary 
1 PSIA ISDBAR

\begin{tabular}{|c|c|c|c|c|c|c|c|c|c|c|}
\hline TEMPERATURE & DENSI TY & $V(O H / O V)_{P}$ & $V(D P / O U)_{v}-V$ & $(0 P / O V)_{T}$ & $(O V / D T) / V$ & $\begin{array}{l}\text { THERMAL } \\
\text { CONOUC I I ITIY }\end{array}$ & VISCOSITY & $\begin{array}{l}\text { THERMAL } \\
\text { OIFFUSIVITY }\end{array}$ & $\begin{array}{l}\text { OIELECTRIC } \\
\text { CONSTANT }\end{array}$ & $\begin{array}{l}\text { PRANDTL } \\
\text { NUMBER }\end{array}$ \\
\hline OEG. $R$ & $L B / C \cup F T$ & BTU/LB & PSIA-CU FT/BTU & PSIA & 1/OEG. R & STU/FT-HR-R & $\begin{array}{r}\text { LB/FT-SEC } \\
\times 10 \bar{E}+6\end{array}$ & SQ FT/HR & & \\
\hline $\begin{array}{l}4.0 \\
+4.161 \\
+\quad 4.161 \\
5.0 \\
6.0 \\
7.0 \\
8.0 \\
9.0 \\
10.0 \\
11.0\end{array}$ & $\begin{array}{l}9.121 \\
9.097 \\
0.09592 \\
0.07786 \\
0.06392 \\
0.05433 \\
0.04729 \\
0.04190 \\
0.03762 \\
0.03414\end{array}$ & $\begin{array}{l}50.4 \\
33.9 \\
4.82 \\
5.93 \\
7.22 \\
8.49 \\
9.76 \\
11.0 \\
12.3 \\
13.5\end{array}$ & $\begin{array}{l}2.16 \\
3.23 \\
3.57 \\
3.59 \\
3.60 \\
3.61 \\
3.61 \\
3.61 \\
3.61 \\
3.61\end{array}$ & $\begin{array}{l}970.0 \\
952.0 \\
0.929 \\
0.956 \\
0.972 \\
0.980 \\
0.986 \\
0.989 \\
0.991 \\
0.993\end{array}$ & $\begin{array}{l}0.0142 \\
0.0177 \\
0.283 \\
0.221 \\
0.177 \\
0.149 \\
0.129 \\
0.114 \\
0.102 \\
0.0924\end{array}$ & $\begin{array}{l}0.00866 \\
0.00900 \\
0.00283 \\
0.00340 \\
0.00409 \\
0.00474 \\
0.00534 \\
0.00591 \\
0.00644 \\
0.00694\end{array}$ & $\begin{array}{l}2.44 \\
2.47 \\
0.390 \\
0.481 \\
0.581 \\
0.674 \\
0.762 \\
0.846 \\
0.925 \\
1.00\end{array}$ & $\begin{array}{l}0.00133 \\
0.00165 \\
0.0214 \\
0.0333 \\
0.0499 \\
0.0688 \\
0.0896 \\
0.112 \\
0.137 \\
0.163\end{array}$ & $\begin{array}{l}1.02039 \\
1.02038 \\
1.00036 \\
1.00029 \\
1.00024 \\
1.00020 \\
1.00018 \\
1.00016 \\
1.00014 \\
1.00013\end{array}$ & $\begin{array}{l}0.724 \\
0.592 \\
0.684 \\
0.667 \\
0.656 \\
0.650 \\
0.647 \\
0.647 \\
0.648 \\
0.650\end{array}$ \\
\hline $\begin{array}{l}12.0 \\
13.0 \\
14.0 \\
15.0 \\
16.0 \\
17.0 \\
18.0 \\
19.0 \\
20.0 \\
22.0\end{array}$ & $\begin{array}{l}0.03125 \\
0.02882 \\
0.02674 \\
0.02494 \\
0.02337 \\
0.02199 \\
0.02076 \\
0.01966 \\
0.01868 \\
0.01697\end{array}$ & $\begin{array}{l}14.8 \\
16.0 \\
17.3 \\
18.5 \\
19.8 \\
21.0 \\
22.3 \\
23.5 \\
24.8 \\
27.3\end{array}$ & $\begin{array}{l}3.61 \\
3.61 \\
3.61 \\
3.61 \\
3.61 \\
3.61 \\
3.61 \\
3.61 \\
3.61 \\
3.60\end{array}$ & $\begin{array}{l}0.994 \\
1.00 \\
1.00 \\
1.00 \\
1.00 \\
1.00 \\
1.00 \\
1.00 \\
1.00 \\
1.00\end{array}$ & $\begin{array}{l}0.0845 \\
0.0778 \\
0.0721 \\
0.0672 \\
0.0629 \\
0.0592 \\
0.0559 \\
0.0529 \\
0.0502 \\
0.0456\end{array}$ & $\begin{array}{l}0.00742 \\
0.00787 \\
0.00830 \\
0.00872 \\
0.00911 \\
0.00950 \\
0.00987 \\
0.0102 \\
0.0106 \\
0.0113\end{array}$ & $\begin{array}{l}1.08 \\
1.15 \\
1.21 \\
1.28 \\
1.35 \\
1.41 \\
1.47 \\
1.53 \\
1.59 \\
1.70\end{array}$ & $\begin{array}{l}0.190 \\
0.219 \\
0.249 \\
0.280 \\
0.313 \\
0.347 \\
0.382 \\
0.418 \\
0.456 \\
0.533\end{array}$ & $\begin{array}{l}1.00012 \\
1.00011 \\
1.00010 \\
1.00009 \\
1.00009 \\
1.00008 \\
1.00008 \\
1.00007 \\
1.00007 \\
1.00006\end{array}$ & $\begin{array}{l}0.652 \\
0.654 \\
0.657 \\
0.659 \\
0.662 \\
0.665 \\
0.667 \\
0.669 \\
0.672 \\
0.676\end{array}$ \\
\hline $\begin{array}{l}24.0 \\
26.0 \\
28.0 \\
30.0 \\
32.0 \\
34.0 \\
36.0 \\
38.0 \\
40.0\end{array}$ & $\begin{array}{l}0.01555 \\
0.01435 \\
0.01333 \\
0.01244 \\
0.01166 \\
0.01097 \\
0.01036 \\
0.009816 \\
0.009325\end{array}$ & $\begin{array}{l}29.8 \\
32.2 \\
34.7 \\
37.2 \\
39.7 \\
42.2 \\
44.7 \\
47.2 \\
49.6\end{array}$ & $\begin{array}{l}3.60 \\
3.60 \\
3.60 \\
3.60 \\
3.60 \\
3.60 \\
3.60 \\
3.60 \\
3.60\end{array}$ & $\begin{array}{l}1.00 \\
1.00 \\
1.00 \\
1.00 \\
1.30 \\
1.00 \\
1.00 \\
1.00 \\
1.00\end{array}$ & $\begin{array}{l}0.0418 \\
0.0385 \\
0.0358 \\
0.0334 \\
0.0313 \\
0.0294 \\
0.0278 \\
0.0263 \\
0.0250\end{array}$ & $\begin{array}{l}0.0119 \\
0.0125 \\
0.0131 \\
0.0137 \\
0.0142 \\
0.0148 \\
0.0153 \\
0.0158 \\
0.0163\end{array}$ & $\begin{array}{l}1.81 \\
1.91 \\
2.01 \\
2.11 \\
2.20 \\
2.29 \\
2.38 \\
2.47 \\
2.55\end{array}$ & $\begin{array}{l}0.615 \\
0.702 \\
0.792 \\
0.885 \\
0.983 \\
1.08 \\
1.19 \\
1.30 \\
1.41\end{array}$ & $\begin{array}{l}1.00006 \\
1.000005 \\
1.00005 \\
1.00005 \\
1.00004 \\
1.00004 \\
1.00004 \\
1.00004 \\
1.00004\end{array}$ & $\begin{array}{l}0.680 \\
0.683 \\
0.686 \\
0.689 \\
0.692 \\
0.694 \\
0.696 \\
0.698 \\
0.699\end{array}$ \\
\hline $\begin{array}{r}45.0 \\
50.0 \\
55.0 \\
60.0 \\
70.0 \\
80.0 \\
90.0 \\
100.0 \\
120.0 \\
140.0\end{array}$ & $\begin{array}{l}0.008288 \\
0.007459 \\
0.006781 \\
0.005215 \\
0.005327 \\
0.004662 \\
0.004144 \\
0.003729 \\
0.003108 \\
0.002664\end{array}$ & $\begin{array}{r}55.9 \\
62.1 \\
68.3 \\
74.5 \\
86.9 \\
99.3 \\
112.0 \\
124.0 \\
149.0 \\
174.0\end{array}$ & $\begin{array}{l}3.60 \\
3.60 \\
3.60 \\
3.60 \\
3.60 \\
3.60 \\
3.60 \\
3.60 \\
3.60 \\
3.60\end{array}$ & $\begin{array}{l}1.00 \\
1.00 \\
1.00 \\
1.00 \\
1.00 \\
1.00 \\
1.00 \\
1.00 \\
1.00 \\
1.00\end{array}$ & $\begin{array}{l}0.0222 \\
0.0200 \\
0.0182 \\
0.0167 \\
0.0143 \\
0.0125 \\
0.0111 \\
0.0100 \\
0.00833 \\
0.00714\end{array}$ & $\begin{array}{l}0.0175 \\
0.0187 \\
0.0198 \\
0.0209 \\
0.0230 \\
0.0250 \\
0.0269 \\
0.0288 \\
0.0324 \\
0.0359\end{array}$ & $\begin{array}{l}2.76 \\
2.95 \\
3.13 \\
3.31 \\
3.65 \\
3.96 \\
4.26 \\
4.55 \\
5.09 \\
5.61\end{array}$ & $\begin{array}{l}1.70 \\
2.02 \\
2.35 \\
2.71 \\
3.48 \\
4.32 \\
5.24 \\
6.23 \\
8.41 \\
10.9\end{array}$ & $\begin{array}{l}1.00003 \\
1.00003 \\
1.00003 \\
1.00002 \\
1.00002 \\
1.00002 \\
1.00002 \\
1.00001 \\
1.00001 \\
1.00001\end{array}$ & $\begin{array}{l}0.703 \\
0.705 \\
0.706 \\
0.707 \\
0.708 \\
0.708 \\
0.707 \\
0.705 \\
0.702 \\
0.698\end{array}$ \\
\hline $\begin{array}{l}160.0 \\
180.0 \\
200.0 \\
250.0 \\
360.0 \\
350.0 \\
400.0 \\
450.0 \\
500.0 \\
600.0\end{array}$ & $\begin{array}{l}0.002331 \\
0.002072 \\
0.001865 \\
0.001492 \\
0.001243 \\
0.001066 \\
0.0009324 \\
0.0008288 \\
0.0007459 \\
0.0006216\end{array}$ & $\begin{array}{l}199.0 \\
223.0 \\
248.0 \\
310.0 \\
372.0 \\
434.0 \\
496.0 \\
559.0 \\
621.0 \\
745.0\end{array}$ & $\begin{array}{l}3.60 \\
3.60 \\
3.60 \\
3.60 \\
3.60 \\
3.60 \\
3.60 \\
3.60 \\
3.60 \\
3.60\end{array}$ & $\begin{array}{l}1.00 \\
1.00 \\
1.00 \\
1.00 \\
1.00 \\
1.00 \\
1.00 \\
1.00 \\
1.00 \\
1.00\end{array}$ & $\begin{array}{l}0.00625 \\
0.00556 \\
0.00500 \\
0.00400 \\
0.00333 \\
0.00286 \\
0.00250 \\
0.00222 \\
0.00200 \\
0.00167\end{array}$ & $\begin{array}{l}0.0392 \\
0.0425 \\
0.0456 \\
0.0531 \\
0.0602 \\
0.0669 \\
0.0732 \\
0.0793 \\
0.0850 \\
0.0962\end{array}$ & $\begin{array}{l}6.09 \\
6.56 \\
6.89 \\
7.98 \\
9.00 \\
10.0 \\
10.9 \\
11.8 \\
12.7 \\
14.4\end{array}$ & $\begin{array}{r}13.6 \\
16.5 \\
19.7 \\
28.7 \\
39.0 \\
50.6 \\
63.3 \\
77.1 \\
91.8 \\
125.0\end{array}$ & $\begin{array}{l}1.00001 \\
1.00001 \\
1.00001 \\
1.00001 \\
1.00000 \\
1.00000 \\
1.00000 \\
1.00000 \\
1.00000 \\
1.00000\end{array}$ & $\begin{array}{l}0.694 \\
0.691 \\
0.675 \\
0.671 \\
0.669 \\
0.667 \\
0.666 \\
0.667 \\
0.668 \\
0.669\end{array}$ \\
\hline $\begin{array}{r}700.0 \\
800.0 \\
900.0 \\
1000.0 \\
1200.0 \\
1400.0 \\
1600.0 \\
1800.0 \\
2000.0 \\
2500.0\end{array}$ & $\begin{array}{l}0.0005328 \\
0.0004662 \\
0.0004144 \\
0.0003730 \\
0.0003108 \\
0.0002664 \\
0.0002331 \\
0.0002072 \\
0.0001865 \\
0.0001492\end{array}$ & $\begin{array}{r}869.0 \\
993.0 \\
1120.0 \\
1240.0 \\
1490.0 \\
1740.0 \\
1990.0 \\
2230.0 \\
2480.0 \\
3100.0\end{array}$ & $\begin{array}{l}3.60 \\
3.60 \\
3.60 \\
3.60 \\
3.60 \\
3.60 \\
3.60 \\
3.60 \\
3.60 \\
3.60\end{array}$ & $\begin{array}{l}1.00 \\
1.00 \\
1.00 \\
1.00 \\
1.00 \\
1.00 \\
1.00 \\
1.00 \\
1.00 \\
1.00\end{array}$ & $\begin{array}{l}0.00143 \\
0.00125 \\
0.00111 \\
0.00100 \\
0.000833 \\
0.000714 \\
0.000625 \\
0.000556 \\
0.000500 \\
0.000400\end{array}$ & $\begin{array}{l}0.107 \\
0.117 \\
0.128 \\
0.137 \\
0.156 \\
0.174 \\
0.191 \\
0.208 \\
0.224 \\
0.263\end{array}$ & $\begin{array}{l}16.0 \\
17.6 \\
19.1 \\
20.5 \\
23.3 \\
26.0 \\
28.5 \\
31.0 \\
33.4 \\
39.2\end{array}$ & $\begin{array}{r}162.0 \\
203.0 \\
248.0 \\
297.0 \\
405.0 \\
526.0 \\
661.0 \\
909.0 \\
968.0 \\
1420.0\end{array}$ & $\begin{array}{l}1.00000 \\
1.00000 \\
1.00000 \\
1.00000 \\
1.00000 \\
1.00000 \\
1.00000 \\
1.00000 \\
1.00000 \\
1.00000\end{array}$ & $\begin{array}{l}0.668 \\
0.668 \\
0.668 \\
0.667 \\
0.667 \\
0.667 \\
0.666 \\
0.666 \\
0.666 \\
0.666\end{array}$ \\
\hline 3000.0 & 0.0001243 & 3720.0 & 3.60 & 1.00 & 0.000333 & 0.299 & 44.6 & 1940.0 & 1.00000 & 0.666 \\
\hline
\end{tabular}




\begin{tabular}{|c|c|c|c|c|c|c|c|c|c|}
\hline $\begin{array}{l}\text { TEMPERATUR } \\
\text { DEG. R }\end{array}$ & $\begin{array}{l}\text { VOLUAE } \\
\text { CU FT/LO }\end{array}$ & $\begin{array}{c}\text { ISOTHERM } \\
\text { OERIVATIVE } \\
\text { CU FT-PSIA/LB }\end{array}$ & $\begin{array}{l}\text { ISOCHORE } \\
\text { DERIVATIVE } \\
\text { PSIA/R }\end{array}$ & $\begin{array}{l}\text { INTERNAL } \\
\text { ENERGY } \\
\text { BTU/LB }\end{array}$ & $\begin{array}{l}\text { ENTHALPY } \\
\text { BTU/LO }\end{array}$ & $\begin{array}{l}\text { ENTROPY } \\
\text { OTU/LB-R }\end{array}$ & $\begin{array}{l}\text { CV } \\
\text { BTU / L8 }\end{array}$ & $\begin{array}{r}C P \\
-R\end{array}$ & $\begin{array}{l}\text { VELOCITY } \\
\text { OF SOUND } \\
\text { FT/SEC }\end{array}$ \\
\hline $\begin{array}{l}4.0 \\
4.789 \\
4.789 \\
5.0 \\
6.0 \\
7.0 \\
8.0 \\
9.0 \\
10.0 \\
11.0\end{array}$ & $\begin{array}{l}0.1095 \\
0.1114 \\
5.786 \\
6.110 \\
7.589 \\
9.016 \\
10.42 \\
11.80 \\
13.18 \\
14.54\end{array}$ & $\begin{array}{r}107.0 \\
95.0 \\
10.3 \\
11.0 \\
14.3 \\
17.3 \\
20.2 \\
23.1 \\
25.9 \\
28.7\end{array}$ & $\begin{array}{l}13.7 \\
24.3 \\
0.477 \\
0.450 \\
0.358 \\
0.300 \\
0.259 \\
0.229 \\
0.205 \\
0.185\end{array}$ & $\begin{array}{l}1.421 \\
1.854 \\
9.580 \\
9.759 \\
10.57 \\
11.36 \\
12.13 \\
12.89 \\
13.65 \\
14.40\end{array}$ & $\begin{array}{l}1.462 \\
1.895 \\
11.72 \\
12.02 \\
13.38 \\
14.70 \\
15.98 \\
17.26 \\
18.53 \\
19.79\end{array}$ & $\begin{array}{l}0.4063 \\
0.5058 \\
2.567 \\
2.629 \\
2.877 \\
3.079 \\
3.251 \\
3.402 \\
3.535 \\
3.656\end{array}$ & $\begin{array}{l}0.6951 \\
0.4392 \\
0.7737 \\
0.7686 \\
0.7547 \\
0.7490 \\
0.7465 \\
0.7453 \\
0.7448 \\
0.7446\end{array}$ & $\begin{array}{l}0.7107 \\
0.5074 \\
1.431 \\
1.403 \\
1.330 \\
1.298 \\
1.281 \\
1.271 \\
1.264 \\
1.260\end{array}$ & $\begin{array}{l}713.0 \\
712.9 \\
297.0 \\
305.5 \\
341.4 \\
372.7 \\
400.9 \\
426.9 \\
451.3 \\
474.2\end{array}$ \\
\hline $\begin{array}{l}12.0 \\
13.0 \\
14.0 \\
15.0 \\
16.0 \\
17.0 \\
18.0 \\
19.0 \\
20.0 \\
22.0\end{array}$ & $\begin{array}{l}15.91 \\
17.27 \\
18.63 \\
19.98 \\
21.33 \\
22.69 \\
24.04 \\
25.39 \\
26.73 \\
29.43\end{array}$ & $\begin{array}{l}31.5 \\
34.2 \\
37.0 \\
39.7 \\
42.4 \\
45.2 \\
47.9 \\
50.6 \\
53.3 \\
58.7\end{array}$ & $\begin{array}{l}0.169 \\
0.156 \\
0.145 \\
0.135 \\
0.126 \\
0.119 \\
0.112 \\
0.106 \\
0.101 \\
0.0913\end{array}$ & $\begin{array}{l}15.16 \\
15.91 \\
16.66 \\
17.41 \\
18.16 \\
18.91 \\
19.65 \\
20.40 \\
21.15 \\
22.64\end{array}$ & $\begin{array}{l}21.05 \\
22.30 \\
23.56 \\
24.81 \\
26.06 \\
27.31 \\
28.56 \\
29.80 \\
31.05 \\
33.54\end{array}$ & $\begin{array}{l}3.765 \\
3.866 \\
3.958 \\
4.045 \\
4.125 \\
4.201 \\
4.273 \\
4.340 \\
4.404 \\
4.523\end{array}$ & $\begin{array}{l}0.7445 \\
0.7444 \\
0.7445 \\
0.7445 \\
0.7445 \\
0.7446 \\
0.7446 \\
0.7446 \\
0.7446 \\
0.7447\end{array}$ & $\begin{array}{l}1.257 \\
1.254 \\
1.252 \\
1.251 \\
1.250 \\
1.249 \\
1.248 \\
1.247 \\
1.247 \\
1.246\end{array}$ & $\begin{array}{l}496.0 \\
516.8 \\
536.8 \\
556.0 \\
574.5 \\
592.4 \\
609.8 \\
626.6 \\
643.1 \\
674.7\end{array}$ \\
\hline $\begin{array}{l}24.0 \\
26.0 \\
28.0 \\
30.0 \\
32.0 \\
34.0 \\
36.0 \\
38.0 \\
40.0\end{array}$ & $\begin{array}{l}32.12 \\
34.81 \\
37.50 \\
40.19 \\
42.88 \\
45.56 \\
48.25 \\
50.94 \\
53.62\end{array}$ & $\begin{array}{r}64.1 \\
69.5 \\
74.9 \\
80.3 \\
85.7 \\
91.1 \\
96.5 \\
102.0 \\
107.0\end{array}$ & $\begin{array}{l}0.0837 \\
0.0772 \\
0.0716 \\
0.0668 \\
0.0626 \\
0.0589 \\
0.0557 \\
0.0527 \\
0.0501\end{array}$ & $\begin{array}{l}24.14 \\
25.63 \\
27.12 \\
28.62 \\
30.11 \\
31.60 \\
33.09 \\
34.58 \\
36.07\end{array}$ & $\begin{array}{l}36.03 \\
38.52 \\
41.01 \\
43.50 \\
45.99 \\
48.47 \\
50.96 \\
53.45 \\
55.93\end{array}$ & $\begin{array}{l}4.631 \\
4.731 \\
4.823 \\
4.909 \\
4.989 \\
5.064 \\
5.135 \\
5.203 \\
5.266\end{array}$ & $\begin{array}{l}0.7447 \\
0.7447 \\
0.7448 \\
0.7448 \\
0.7448 \\
0.7448 \\
0.7448 \\
0.7448 \\
0.7448\end{array}$ & $\begin{array}{l}1.245 \\
1.245 \\
1.244 \\
1.244 \\
1.243 \\
1.243 \\
1.243 \\
1.243 \\
1.243\end{array}$ & $\begin{array}{l}704.8 \\
733.7 \\
761.5 \\
788.3 \\
814.2 \\
839.3 \\
863.7 \\
887.3 \\
910.4\end{array}$ \\
\hline $\begin{array}{r}45.0 \\
50.0 \\
55.0 \\
60.0 \\
70.0 \\
80.0 \\
90.0 \\
100.0 \\
120.0 \\
140.0\end{array}$ & $\begin{array}{r}60.33 \\
67.04 \\
73.75 \\
80.46 \\
93.87 \\
107.3 \\
120.7 \\
134.1 \\
160.9 \\
187.7\end{array}$ & $\begin{array}{l}121.0 \\
134.0 \\
148.0 \\
161.0 \\
188.0 \\
215.0 \\
241.0 \\
268.0 \\
322.0 \\
376.0\end{array}$ & $\begin{array}{l}0.0445 \\
0.0400 \\
0.0364 \\
0.0334 \\
0.0286 \\
0.0250 \\
0.0222 \\
0.0200 \\
0.0167 \\
0.0143\end{array}$ & $\begin{array}{c}39.80 \\
43.53 \\
47.25 \\
50.98 \\
58.43 \\
65.88 \\
73.33 \\
80.77 \\
95.67 \\
110.6\end{array}$ & $\begin{array}{r}62.14 \\
68.35 \\
74.56 \\
80.77 \\
93.19 \\
105.6 \\
118.0 \\
130.4 \\
155.3 \\
180.1\end{array}$ & $\begin{array}{l}5.413 \\
5.544 \\
5.662 \\
5.770 \\
5.961 \\
6.127 \\
6.274 \\
6.404 \\
6.631 \\
6.822\end{array}$ & $\begin{array}{l}0.7448 \\
0.7448 \\
0.7448 \\
0.7448 \\
0.7448 \\
0.7448 \\
0.7448 \\
0.7447 \\
0.7447 \\
0.7447\end{array}$ & $\begin{array}{l}1.242 \\
1.242 \\
1.242 \\
1.242 \\
1.242 \\
1.242 \\
1.241 \\
1.241 \\
1.241 \\
1.241\end{array}$ & $\begin{array}{r}965.6 \\
1018.0 \\
1068.0 \\
1115.0 \\
1204.0 \\
1287.0 \\
1365.0 \\
1439.0 \\
1577.0 \\
1703.0\end{array}$ \\
\hline $\begin{array}{l}160.0 \\
180.0 \\
200.0 \\
250.0 \\
300.0 \\
350.0 \\
400.0 \\
450.0 \\
500.0 \\
600.0\end{array}$ & $\begin{array}{l}214.5 \\
241.3 \\
268.2 \\
335.2 \\
402.2 \\
469.2 \\
536.3 \\
603.3 \\
670.3 \\
804.4\end{array}$ & $\begin{array}{r}429.0 \\
483.0 \\
536.0 \\
670.0 \\
805.0 \\
939.0 \\
1070.0 \\
1210.0 \\
1340.0 \\
1610.0\end{array}$ & $\begin{array}{l}0.0125 \\
0.0111 \\
0.0100 \\
0.00800 \\
0.00667 \\
0.00571 \\
0.00500 \\
0.00444 \\
0.00400 \\
0.00333\end{array}$ & $\begin{array}{l}125.5 \\
140.4 \\
155.3 \\
192.5 \\
229.7 \\
267.0 \\
304.2 \\
341.4 \\
378.7 \\
453.1\end{array}$ & $\begin{array}{l}204.9 \\
229.7 \\
254.6 \\
316.6 \\
378.7 \\
440.7 \\
502.8 \\
564.9 \\
626.9 \\
751.0\end{array}$ & $\begin{array}{l}6.988 \\
7.134 \\
7.265 \\
7.542 \\
7.768 \\
7.959 \\
8.125 \\
8.271 \\
8.402 \\
8.628\end{array}$ & $\begin{array}{l}0.7447 \\
0.7447 \\
0.7447 \\
0.7447 \\
0.7447 \\
0.7447 \\
0.7447 \\
0.7447 \\
0.7447 \\
0.7447\end{array}$ & $\begin{array}{l}1.241 \\
1.241 \\
1.241 \\
1.241 \\
1.241 \\
1.241 \\
1.241 \\
1.241 \\
1.241 \\
1.241\end{array}$ & $\begin{array}{l}1820.0 \\
1931.0 \\
2035.0 \\
2275.0 \\
2492.0 \\
2692.0 \\
2878.0 \\
3052.0 \\
3217.0 \\
3524.0\end{array}$ \\
\hline $\begin{array}{r}700.0 \\
800.0 \\
900.0 \\
1000.0 \\
1200.0 \\
1400.0 \\
1600.0 \\
1800.0 \\
2000.0 \\
2500.0\end{array}$ & $\begin{array}{r}938.4 \\
1072.0 \\
1207.0 \\
1341.0 \\
1609.0 \\
1877.0 \\
2145.0 \\
2413.0 \\
2681.0 \\
3351.0\end{array}$ & $\begin{array}{l}1860.0 \\
2150.0 \\
2410.0 \\
2680.0 \\
3220.0 \\
3750.0 \\
4290.0 \\
4830.0 \\
5360.0 \\
6700.0\end{array}$ & $\begin{array}{l}0.00286 \\
0.00250 \\
0.00222 \\
0.00200 \\
0.00167 \\
0.00143 \\
0.00125 \\
0.00111 \\
0.00100 \\
0.000800\end{array}$ & $\begin{array}{r}527.6 \\
602.1 \\
676.5 \\
751.0 \\
900.0 \\
1049.0 \\
1198.0 \\
1347.0 \\
1496.0 \\
1868.0\end{array}$ & $\begin{array}{r}875.2 \\
999.0 \\
1123.0 \\
1248.0 \\
1496.0 \\
1744.0 \\
1992.0 \\
2240.0 \\
2489.0 \\
3109.0\end{array}$ & $\begin{array}{l}8.820 \\
8.985 \\
9.132 \\
9.262 \\
9.489 \\
9.680 \\
9.846 \\
9.99 \\
10.12 \\
10.40\end{array}$ & $\begin{array}{l}0.7447 \\
0.7447 \\
0.7447 \\
0.7447 \\
0.7447 \\
0.7447 \\
0.7447 \\
0.7447 \\
0.7447 \\
0.7447\end{array}$ & $\begin{array}{l}1.241 \\
1.241 \\
1.241 \\
1.241 \\
1.241 \\
1.241 \\
1.241 \\
1.241 \\
1.241 \\
1.241\end{array}$ & $\begin{array}{l}3807.0 \\
4070.0 \\
4316.0 \\
4550.0 \\
4984.0 \\
5383.0 \\
5755.0 \\
6104.0 \\
6434.0 \\
7194.0\end{array}$ \\
\hline 3000.0 & 4022.0 & 8040.0 & 0.000667 & 2240.0 & 3730.0 & 10.63 & 0.7447 & 1.241 & 7880.0 \\
\hline
\end{tabular}


2 PSIA ISOBAR

\begin{tabular}{|c|c|c|c|c|c|c|c|c|c|c|}
\hline TEMPERATURE & OENSI TY & $V(\mathrm{OH} / \mathrm{OV})_{\mathrm{P}}$ & $V(D P / D U)_{v}-$ & $\left.{ }^{(D P / O V}\right)_{T}$ & (OV/OT) ${ }_{\mathrm{P}}{ }_{\mathrm{C}}$ & $\begin{array}{c}\text { THERMAL } \\
\text { CDNDUCTIVITY }\end{array}$ & VISCDSITY & $\begin{array}{l}\text { THERMAL } \\
\text { OIFFUSIVITY }\end{array}$ & $\begin{array}{l}\text { DIELECTRIC } \\
\text { CDNSTANT }\end{array}$ & $\begin{array}{l}\text { PRANDTL } \\
\text { NUMBER }\end{array}$ \\
\hline DEG. $R$ & LB/CU FT & BTU/LB & PSIA-CU FT/BTU & PSIA & 1/OEG. R & BTU/FT-HR-R & $\begin{array}{r}\text { LB/FT }-S E C \\
\times 10 E+6\end{array}$ & SQ FT/HR & & \\
\hline $\begin{array}{l}4.0 \\
4.789 \\
4.789 \\
5.0 \\
6.0 \\
7.0 \\
8.0 \\
9.0 \\
10.0 \\
11.0\end{array}$ & $\begin{array}{l}9.131 \\
8.975 \\
0.1728 \\
0.1637 \\
0.1318 \\
0.1109 \\
0.09601 \\
0.08474 \\
0.07589 \\
0.06875\end{array}$ & $\begin{array}{c}50.7 \\
17.8 \\
5.33 \\
5.63 \\
6.98 \\
8.30 \\
9.59 \\
10.9 \\
12.1 \\
13.4\end{array}$ & $\begin{array}{l}2.17 \\
6.15 \\
3.57 \\
3.58 \\
3.60 \\
3.61 \\
3.62 \\
3.62 \\
3.62 \\
3.62\end{array}$ & $\begin{array}{c}980.0 \\
852.0 \\
1.78 \\
1.81 \\
1.88 \\
1.92 \\
1.94 \\
1.96 \\
1.97 \\
1.97\end{array}$ & $\begin{array}{l}0.0140 \\
0.0285 \\
0.268 \\
0.249 \\
0.191 \\
0.156 \\
0.134 \\
0.117 \\
0.104 \\
0.0939\end{array}$ & $\begin{array}{l}0.00868 \\
0.00982 \\
0.00334 \\
0.00348 \\
0.00414 \\
0.00477 \\
0.00537 \\
0.00593 \\
0.00646 \\
0.00696\end{array}$ & $\begin{array}{l}2.45 \\
2.51 \\
0.463 \\
0.485 \\
0.587 \\
0.680 \\
0.768 \\
0.851 \\
0.931 \\
1.01\end{array}$ & $\begin{array}{l}0.00134 \\
0.00216 \\
0.0135 \\
0.0152 \\
0.0236 \\
0.0332 \\
0.0437 \\
0.0551 \\
0.0673 \\
0.0804\end{array}$ & $\begin{array}{l}1.02040 \\
1.02029 \\
1.00065 \\
1.00061 \\
1.00049 \\
1.00042 \\
1.00036 \\
1.00032 \\
1.00029 \\
1.00026\end{array}$ & $\begin{array}{l}0.723 \\
0.467 \\
0.713 \\
0.704 \\
0.679 \\
0.666 \\
0.659 \\
0.656 \\
0.656 \\
0.656\end{array}$ \\
\hline $\begin{array}{l}12.0 \\
13.0 \\
14.0 \\
15.0 \\
16.0 \\
17.0 \\
18.0 \\
19.0 \\
20.0 \\
22.0\end{array}$ & $\begin{array}{l}0.06286 \\
0.05791 \\
0.05369 \\
0.05005 \\
0.04687 \\
0.04408 \\
0.04160 \\
0.03939 \\
0.03740 \\
0.03398\end{array}$ & $\begin{array}{l}14.7 \\
15.9 \\
17.2 \\
18.5 \\
19.7 \\
21.0 \\
22.2 \\
23.5 \\
24.7 \\
27.2\end{array}$ & $\begin{array}{l}3.62 \\
3.62 \\
3.62 \\
3.61 \\
3.61 \\
3.61 \\
3.61 \\
3.61 \\
3.61 \\
3.61\end{array}$ & $\begin{array}{l}1.98 \\
1.98 \\
1.98 \\
1.99 \\
1.99 \\
1.99 \\
1.99 \\
1.99 \\
1.99 \\
2.00\end{array}$ & $\begin{array}{l}0.0856 \\
0.0787 \\
0.0728 \\
0.0678 \\
0.0634 \\
0.0596 \\
0.0562 \\
0.0531 \\
0.0504 \\
0.0458\end{array}$ & $\begin{array}{l}0.00743 \\
0.00789 \\
0.00832 \\
0.00873 \\
0.00913 \\
0.00952 \\
0.00989 \\
0.0102 \\
0.0106 \\
0.0113\end{array}$ & $\begin{array}{l}1.08 \\
1.15 \\
1.22 \\
1.29 \\
1.35 \\
1.41 \\
1.47 \\
1.53 \\
1.59 \\
1.70\end{array}$ & $\begin{array}{l}0.0941 \\
0.109 \\
0.124 \\
0.139 \\
0.156 \\
0.173 \\
0.190 \\
0.209 \\
0.227 \\
0.266\end{array}$ & $\begin{array}{l}1.00024 \\
1.00022 \\
1.00020 \\
1.00019 \\
1.00018 \\
1.00017 \\
1.00016 \\
1.00015 \\
1.00014 \\
1.00013\end{array}$ & $\begin{array}{l}0.657 \\
0.659 \\
0.661 \\
0.663 \\
0.665 \\
0.667 \\
0.669 \\
0.672 \\
0.674 \\
0.678\end{array}$ \\
\hline $\begin{array}{l}24.0 \\
26.0 \\
28.0 \\
30.0 \\
32.0 \\
34.0 \\
36.0 \\
38.0 \\
40.0\end{array}$ & $\begin{array}{l}0.03113 \\
0.02872 \\
0.02666 \\
0.02488 \\
0.02332 \\
0.02195 \\
0.02072 \\
0.01963 \\
0.01865\end{array}$ & $\begin{array}{l}29.7 \\
32.2 \\
34.7 \\
37.2 \\
39.7 \\
42.2 \\
44.7 \\
47.1 \\
49.6\end{array}$ & $\begin{array}{l}3.61 \\
3.61 \\
3.61 \\
3.61 \\
3.61 \\
3.61 \\
3.61 \\
3.61 \\
3.60\end{array}$ & $\begin{array}{l}2.00 \\
2.00 \\
2.00 \\
2.00 \\
2.00 \\
2.00 \\
2.00 \\
2.00 \\
2.00\end{array}$ & $\begin{array}{l}0.0419 \\
0.0386 \\
0.0358 \\
0.0334 \\
0.0313 \\
0.0295 \\
0.0278 \\
0.0264 \\
0.0250\end{array}$ & $\begin{array}{l}0.0119 \\
0.0125 \\
0.0131 \\
0.0137 \\
0.0142 \\
0.0148 \\
0.0153 \\
0.0158 \\
0.0163\end{array}$ & $\begin{array}{l}1.81 \\
1.91 \\
2.01 \\
2.11 \\
2.20 \\
2.29 \\
2.38 \\
2.47 \\
2.55\end{array}$ & $\begin{array}{l}0.307 \\
0.350 \\
0.395 \\
0.442 \\
0.491 \\
0.542 \\
0.594 \\
0.649 \\
0.705\end{array}$ & $\begin{array}{l}1.00012 \\
1.00011 \\
1.00010 \\
1.00009 \\
1.00009 \\
1.00008 \\
1.00008 \\
1.00007 \\
1.00007\end{array}$ & $\begin{array}{l}0.681 \\
0.684 \\
0.687 \\
0.690 \\
0.692 \\
0.695 \\
0.697 \\
0.698 \\
0.700\end{array}$ \\
\hline $\begin{array}{r}45.0 \\
50.0 \\
55.0 \\
60.0 \\
70.0 \\
80.0 \\
90.0 \\
100.0 \\
120.0 \\
140.0\end{array}$ & $\begin{array}{l}0.01657 \\
0.01492 \\
0.01356 \\
0.01243 \\
0.01065 \\
0.009322 \\
0.008286 \\
0.007457 \\
0.006215 \\
0.005327\end{array}$ & $\begin{array}{r}55.9 \\
62.1 \\
68.3 \\
74.5 \\
86.9 \\
99.3 \\
112.0 \\
124.0 \\
149.0 \\
174.0\end{array}$ & $\begin{array}{l}3.60 \\
3.60 \\
3.60 \\
3.60 \\
3.60 \\
3.60 \\
3.60 \\
3.60 \\
3.60 \\
3.60\end{array}$ & $\begin{array}{l}2.00 \\
2.00 \\
2.00 \\
2.00 \\
2.00 \\
2.00 \\
2.00 \\
2.00 \\
2.00 \\
2.00\end{array}$ & $\begin{array}{l}0.0222 \\
0.0200 \\
0.0182 \\
0.0167 \\
0.0143 \\
0.0125 \\
0.0111 \\
0.0100 \\
0.00833 \\
0.00714\end{array}$ & $\begin{array}{l}0.0175 \\
0.0187 \\
0.0198 \\
0.0209 \\
0.0230 \\
0.0250 \\
0.0270 \\
0.0288 \\
0.0324 \\
0.0359\end{array}$ & $\begin{array}{l}2.76 \\
2.95 \\
3.13 \\
3.31 \\
3.65 \\
3.96 \\
4.26 \\
4.55 \\
5.09 \\
5.61\end{array}$ & $\begin{array}{l}0.852 \\
1.01 \\
1.18 \\
1.36 \\
1.74 \\
2.16 \\
2.62 \\
3.11 \\
4.21 \\
5.43\end{array}$ & $\begin{array}{l}1.00006 \\
1.00006 \\
1.00005 \\
1.00005 \\
1.00004 \\
1.00004 \\
1.00003 \\
1.00003 \\
1.00002 \\
1.00002\end{array}$ & $\begin{array}{l}0.703 \\
0.705 \\
0.707 \\
0.707 \\
0.708 \\
0.708 \\
0.707 \\
0.705 \\
0.702 \\
0.698\end{array}$ \\
\hline $\begin{array}{l}160.0 \\
180.0 \\
200.0 \\
250.0 \\
300.0 \\
350.0 \\
400.0 \\
450.0 \\
500.0 \\
600.0\end{array}$ & $\begin{array}{l}0.004661 \\
0.004143 \\
0.003729 \\
0.002983 \\
0.002486 \\
0.002131 \\
0.001865 \\
0.001658 \\
0.001492 \\
0.001243\end{array}$ & $\begin{array}{l}199.0 \\
223.0 \\
248.0 \\
310.0 \\
372.0 \\
434.0 \\
497.0 \\
559.0 \\
621.0 \\
745.0\end{array}$ & $\begin{array}{l}3.60 \\
3.60 \\
3.60 \\
3.60 \\
3.60 \\
3.60 \\
3.60 \\
3.60 \\
3.60 \\
3.60\end{array}$ & $\begin{array}{l}2.00 \\
2.00 \\
2.00 \\
2.00 \\
2.000 \\
2.00 \\
2.00 \\
2.00 \\
2.00 \\
2.00\end{array}$ & $\begin{array}{l}0.00625 \\
0.00555 \\
0.00500 \\
0.00400 \\
0.00333 \\
0.00286 \\
0.00250 \\
0.00222 \\
0.00200 \\
0.00167\end{array}$ & $\begin{array}{l}0.0392 \\
0.0425 \\
0.0456 \\
0.0531 \\
0.0602 \\
0.0669 \\
0.0732 \\
0.0793 \\
0.0850 \\
0.0962\end{array}$ & $\begin{array}{c}6.09 \\
6.56 \\
6.89 \\
7.98 \\
9.00 \\
10.0 \\
10.9 \\
11.8 \\
12.7 \\
14.4\end{array}$ & $\begin{array}{l}6.78 \\
8.26 \\
9.85 \\
14.3 \\
19.5 \\
25.3 \\
31.6 \\
38.5 \\
45.9 \\
62.3\end{array}$ & $\begin{array}{l}1.00002 \\
1.00002 \\
1.00001 \\
1.00001 \\
1.00001 \\
1.00001 \\
1.00001 \\
1.00001 \\
1.00001 \\
1.00000\end{array}$ & $\begin{array}{l}0.694 \\
0.691 \\
0.675 \\
0.671 \\
0.669 \\
0.667 \\
0.666 \\
0.667 \\
0.668 \\
0.669\end{array}$ \\
\hline $\begin{array}{r}700.0 \\
800.0 \\
900.0 \\
1000.0 \\
1200.0 \\
1400.0 \\
1600.0 \\
1800.0 \\
2000.0 \\
2500.0\end{array}$ & $\begin{array}{l}0.001066 \\
0.0009324 \\
0.0008288 \\
0.0007459 \\
0.0006216 \\
0.0005328 \\
0.0004662 \\
0.0004144 \\
0.0003730 \\
0.0002984\end{array}$ & $\begin{array}{r}869.0 \\
993.0 \\
1120.0 \\
1240.0 \\
1490.0 \\
1740.0 \\
1990.0 \\
2230.0 \\
2480.0 \\
3100.0\end{array}$ & $\begin{array}{l}3.60 \\
3.60 \\
3.60 \\
3.60 \\
3.60 \\
3.60 \\
3.60 \\
3.60 \\
3.60 \\
3.60\end{array}$ & $\begin{array}{l}2.00 \\
2.00 \\
2.00 \\
2.00 \\
2.00 \\
2.00 \\
2.00 \\
2.00 \\
2.00 \\
2.00\end{array}$ & $\begin{array}{l}0.00143 \\
0.00125 \\
0.00111 \\
0.00100 \\
0.000833 \\
0.000714 \\
0.000625 \\
0.000556 \\
0.000500 \\
0.000400\end{array}$ & $\begin{array}{l}0.107 \\
0.117 \\
0.128 \\
0.137 \\
0.156 \\
0.174 \\
0.191 \\
0.208 \\
0.224 \\
0.263\end{array}$ & $\begin{array}{l}16.0 \\
17.6 \\
19.1 \\
20.5 \\
23.3 \\
26.0 \\
28.5 \\
31.0 \\
33.4 \\
39.2\end{array}$ & $\begin{array}{r}80.9 \\
102.0 \\
124.0 \\
148.0 \\
202.0 \\
263.0 \\
331.0 \\
404.0 \\
484.0 \\
709.0\end{array}$ & $\begin{array}{l}1.00000 \\
1.00000 \\
1.00000 \\
1.00000 \\
1.00000 \\
1.00000 \\
1.00000 \\
1.00000 \\
1.00000 \\
1.00000\end{array}$ & $\begin{array}{l}0.668 \\
0.668 \\
0.668 \\
0.667 \\
0.667 \\
0.667 \\
0.666 \\
0.666 \\
0.666 \\
0.666\end{array}$ \\
\hline 3000.0 & 0.0032487 & 3720.0 & 3.60 & 2.00 & 0.000333 & 0.299 & 44.6 & 970.0 & 1.00000 & 0.666 \\
\hline
\end{tabular}

- tho-phase boundary 


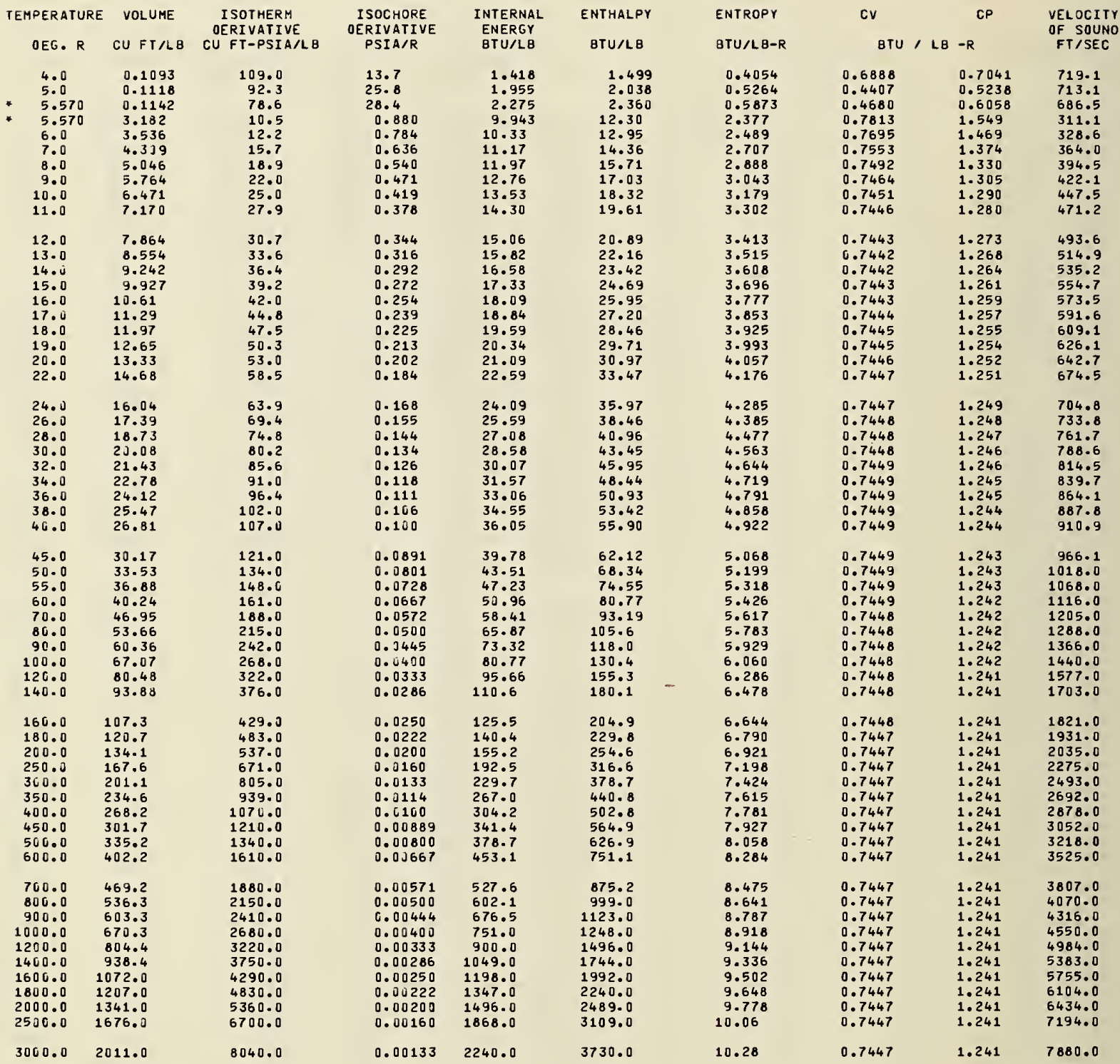


4 PSIA ISDBAR

\begin{tabular}{|c|c|c|c|c|c|c|c|c|c|c|}
\hline $\begin{array}{c}\text { TEMPERATURE } \\
\text { DEG. } R\end{array}$ & $\begin{array}{l}\text { DENSITY } \\
\text { LB/CU FT }\end{array}$ & $\begin{array}{c}\text { V(DH/DV) } \\
\text { BTU/LB }\end{array}$ & $\begin{array}{c}V(D P / D U)^{V}-V \\
P S I A-C U \text { FT/BTU }\end{array}$ & $\begin{array}{l}-V(D P / D V) T \\
\text { PSIA }\end{array}$ & $\begin{array}{l}(D V / D T) / V \\
1 / D E G . R\end{array}$ & $\begin{array}{c}\text { THERMAL } \\
\text { CDNDUCTIVITY } \\
\text { BTU/FT-HR-R }\end{array}$ & $\begin{array}{l}\text { VISCOSITY } \\
\text { LB/FT-SEC } \\
\times 10 E+6\end{array}$ & $\begin{array}{l}\text { THERMAL } \\
\text { DIFFUSIVITY } \\
\text { SQ FT/HR }\end{array}$ & $\begin{array}{l}\text { OIELECTRIC } \\
\text { CONSTANT }\end{array}$ & $\begin{array}{l}\text { PRANDTL } \\
\text { NUMBER }\end{array}$ \\
\hline $\begin{array}{l}4.0 \\
5.0 \\
+5.570 \\
+5.570 \\
6.0 \\
7.0 \\
8.0 \\
9.0 \\
10.0 \\
11.0\end{array}$ & $\begin{array}{l}9.150 \\
8.941 \\
8.758 \\
0.3142 \\
0.2828 \\
0.2321 \\
0.1932 \\
0.1735 \\
0.1545 \\
0.1395\end{array}$ & $\begin{array}{c}51.2 \\
16.8 \\
14.7 \\
5.83 \\
6.47 \\
7.89 \\
9.24 \\
10.6 \\
11.9 \\
13.2\end{array}$ & $\begin{array}{l}2.18 \\
6.54 \\
6.93 \\
3.58 \\
3.60 \\
3.63 \\
3.64 \\
3.64 \\
3.64 \\
3.64\end{array}$ & $\begin{array}{r}1000.0 \\
826.0 \\
638.0 \\
3.31 \\
3.45 \\
3.65 \\
3.75 \\
3.82 \\
3.86 \\
3.89\end{array}$ & $\begin{array}{l}0.0137 \\
0.0312 \\
0.0412 \\
0.266 \\
0.227 \\
0.174 \\
0.144 \\
0.123 \\
0.109 \\
0.0971\end{array}$ & $\begin{array}{l}0.00870 \\
0.0100 \\
0.0104 \\
0.00403 \\
0.00427 \\
0.00486 \\
0.00544 \\
0.00599 \\
0.00651 \\
0.00700\end{array}$ & $\begin{array}{l}2.47 \\
2.52 \\
2.45 \\
0.556 \\
0.599 \\
0.692 \\
0.780 \\
0.862 \\
0.941 \\
1.02\end{array}$ & $\begin{array}{l}0.00135 \\
0.00214 \\
0.00196 \\
0.00828 \\
0.0103 \\
0.0152 \\
0.0206 \\
0.0264 \\
0.0326 \\
0.0392\end{array}$ & $\begin{array}{l}1.02041 \\
1.02027 \\
1.02013 \\
1.00117 \\
1.00106 \\
1.00087 \\
1.00074 \\
1.00065 \\
1.00058 \\
1.00052\end{array}$ & $\begin{array}{l}0.720 \\
0.474 \\
0.514 \\
0.769 \\
0.741 \\
0.704 \\
0.686 \\
0.677 \\
0.672 \\
0.669\end{array}$ \\
\hline $\begin{array}{l}12.0 \\
13.0 \\
14.0 \\
15.0 \\
16.0 \\
17.0 \\
18.0 \\
19.0 \\
20.0 \\
22.0\end{array}$ & $\begin{array}{l}0.1272 \\
0.1159 \\
0.1082 \\
0.1007 \\
0.09425 \\
0.08857 \\
0.08353 \\
0.07905 \\
0.07503 \\
0.06810\end{array}$ & $\begin{array}{l}14.5 \\
15.8 \\
17.0 \\
18.3 \\
19.6 \\
20.8 \\
22.1 \\
23.4 \\
24.6 \\
27.1\end{array}$ & $\begin{array}{l}3.63 \\
3.63 \\
3.63 \\
3.63 \\
3.63 \\
3.63 \\
3.62 \\
3.62 \\
3.62 \\
3.62\end{array}$ & $\begin{array}{l}3.91 \\
3.93 \\
3.94 \\
3.95 \\
3.96 \\
3.96 \\
3.97 \\
3.97 \\
3.98 \\
3.98\end{array}$ & $\begin{array}{l}0.0880 \\
0.0805 \\
0.0742 \\
0.0689 \\
0.0643 \\
0.0603 \\
0.0568 \\
0.0537 \\
0.0509 \\
0.0461\end{array}$ & $\begin{array}{l}0.00748 \\
0.00793 \\
0.00836 \\
0.00877 \\
0.00916 \\
0.00955 \\
0.00992 \\
0.0103 \\
0.0106 \\
0.0113\end{array}$ & $\begin{array}{l}1.09 \\
1.16 \\
1.23 \\
1.29 \\
1.36 \\
1.42 \\
1.48 \\
1.54 \\
1.60 \\
1.71\end{array}$ & $\begin{array}{l}0.0462 \\
0.0535 \\
0.0611 \\
0.0690 \\
0.0773 \\
0.0858 \\
0.0946 \\
0.104 \\
0.113 \\
0.133\end{array}$ & $\begin{array}{l}1.00048 \\
1.00044 \\
1.00041 \\
1.00038 \\
1.00035 \\
1.00033 \\
1.00031 \\
1.00030 \\
1.00028 \\
1.00026\end{array}$ & $\begin{array}{l}0.668 \\
0.668 \\
0.669 \\
0.670 \\
0.671 \\
0.673 \\
0.674 \\
0.676 \\
0.677 \\
0.681\end{array}$ \\
\hline $\begin{array}{l}24.0 \\
26.0 \\
28.0 \\
30.0 \\
32.0 \\
34.0 \\
36.0 \\
38.0 \\
40.0\end{array}$ & $\begin{array}{l}0.06236 \\
0.05752 \\
0.05338 \\
0.04979 \\
0.04666 \\
0.04391 \\
0.04146 \\
0.03927 \\
0.03730\end{array}$ & $\begin{array}{l}29.6 \\
32.2 \\
34.7 \\
37.2 \\
39.7 \\
42.1 \\
44.6 \\
47.1 \\
49.6\end{array}$ & $\begin{array}{l}3.62 \\
3.62 \\
3.61 \\
3.61 \\
3.61 \\
3.61 \\
3.61 \\
3.61 \\
3.61\end{array}$ & $\begin{array}{l}3.99 \\
3.99 \\
3.99 \\
3.99 \\
4.00 \\
4.00 \\
4.00 \\
4.00 \\
4.00\end{array}$ & $\begin{array}{l}0.0421 \\
0.0388 \\
0.0360 \\
0.0335 \\
0.0314 \\
0.0295 \\
0.0279 \\
0.0264 \\
0.0251\end{array}$ & $\begin{array}{l}0.0119 \\
0.0126 \\
0.0131 \\
0.0137 \\
0.0143 \\
0.0148 \\
0.0153 \\
0.0158 \\
0.0163\end{array}$ & $\begin{array}{l}1.82 \\
1.92 \\
2.02 \\
2.11 \\
2.21 \\
2.30 \\
2.39 \\
2.47 \\
2.56\end{array}$ & $\begin{array}{l}0.153 \\
0.175 \\
0.197 \\
0.221 \\
0.245 \\
0.271 \\
0.297 \\
0.324 \\
0.352\end{array}$ & $\begin{array}{l}1.00023 \\
1.00022 \\
1.00020 \\
1.00019 \\
1.00018 \\
1.00017 \\
1.00016 \\
1.00015 \\
1.00014\end{array}$ & $\begin{array}{l}0.684 \\
0.687 \\
0.689 \\
0.692 \\
0.694 \\
0.696 \\
0.698 \\
0.699 \\
0.701\end{array}$ \\
\hline $\begin{array}{r}45.0 \\
50.0 \\
55.0 \\
60.0 \\
70.0 \\
80.0 \\
90.0 \\
100.0 \\
120.0 \\
140.0\end{array}$ & $\begin{array}{l}0.03315 \\
0.02983 \\
0.02711 \\
0.02485 \\
0.02130 \\
0.01864 \\
0.01657 \\
0.01491 \\
0.01243 \\
0.01065\end{array}$ & $\begin{array}{r}55.9 \\
62.1 \\
68.3 \\
74.5 \\
86.9 \\
99.4 \\
112.0 \\
124.0 \\
149.0 \\
174.0\end{array}$ & $\begin{array}{l}3.61 \\
3.61 \\
3.61 \\
3.61 \\
3.60 \\
3.60 \\
3.60 \\
3.60 \\
3.60 \\
3.60\end{array}$ & $\begin{array}{l}4.00 \\
4.00 \\
4.00 \\
4.00 \\
4.00 \\
4.00 \\
4.00 \\
4.00 \\
4.00 \\
4.00\end{array}$ & $\begin{array}{l}0.0223 \\
0.0200 \\
0.0182 \\
0.0 .267 \\
0.0143 \\
0.0125 \\
0.0111 \\
0.0100 \\
0.00833 \\
0.00714\end{array}$ & $\begin{array}{l}0.0176 \\
0.0187 \\
0.0198 \\
0.0209 \\
0.0230 \\
0.0250 \\
0.0270 \\
0.0288 \\
0.0325 \\
0.0359\end{array}$ & $\begin{array}{l}2.76 \\
2.95 \\
3.14 \\
3.31 \\
3.65 \\
3.96 \\
4.26 \\
4.55 \\
5.10 \\
5.61\end{array}$ & $\begin{array}{l}0.426 \\
0.505 \\
0.589 \\
0.678 \\
0.870 \\
1.08 \\
1.31 \\
1.56 \\
2.10 \\
2.72\end{array}$ & $\begin{array}{l}1.00013 \\
1.00011 \\
1.00010 \\
1.00009 \\
1.00008 \\
1.00007 \\
1.00006 \\
1.00006 \\
1.00005 \\
1.00004\end{array}$ & $\begin{array}{l}0.703 \\
0.706 \\
0.707 \\
0.708 \\
0.708 \\
0.708 \\
0.707 \\
0.705 \\
0.702 \\
0.698\end{array}$ \\
\hline $\begin{array}{l}160.0 \\
180.0 \\
200.0 \\
250.0 \\
360.0 \\
350.0 \\
400.0 \\
450.0 \\
500.0 \\
600.0\end{array}$ & $\begin{array}{l}0.009320 \\
0.008285 \\
0.007457 \\
0.005966 \\
0.004972 \\
0.004262 \\
0.003729 \\
0.003315 \\
0.002983 \\
0.002486\end{array}$ & $\begin{array}{l}199.0 \\
224.0 \\
248.0 \\
310.0 \\
372.0 \\
435.0 \\
497.0 \\
559.0 \\
621.0 \\
745.0\end{array}$ & $\begin{array}{l}3.60 \\
3.60 \\
3.60 \\
3.60 \\
3.60 \\
3.60 \\
3.60 \\
3.60 \\
3.60 \\
3.60\end{array}$ & $\begin{array}{l}4.00 \\
4.00 \\
4.00 \\
4.00 \\
4.00 \\
4.00 \\
4.00 \\
4.00 \\
4.00 \\
4.00\end{array}$ & $\begin{array}{l}0.00625 \\
0.00555 \\
0.00500 \\
0.0040 C \\
0.06333 \\
0.00286 \\
0.00250 \\
0.00222 \\
0.00200 \\
0.00167\end{array}$ & $\begin{array}{l}0.0392 \\
0.0425 \\
0.0456 \\
0.0531 \\
0.0602 \\
0.0669 \\
0.1732 \\
0.0793 \\
0.0850 \\
0.0962\end{array}$ & $\begin{array}{c}6.10 \\
6.56 \\
6.89 \\
7.98 \\
9.00 \\
10.0 \\
10.9 \\
11.8 \\
12.7 \\
14.4\end{array}$ & $\begin{array}{l}3.39 \\
4.13 \\
4.93 \\
7.17 \\
9.75 \\
12.6 \\
15.8 \\
19.3 \\
23.0 \\
31.2\end{array}$ & $\begin{array}{l}1.00004 \\
1.00003 \\
1.00003 \\
1.00002 \\
1.00002 \\
1.00002 \\
1.00001 \\
1.00001 \\
1.00001 \\
1.00001\end{array}$ & $\begin{array}{l}0.694 \\
0.691 \\
0.675 \\
0.671 \\
0.669 \\
0.657 \\
0.666 \\
0.667 \\
0.668 \\
0.669\end{array}$ \\
\hline $\begin{array}{r}700.0 \\
800.0 \\
900.0 \\
1060.0 \\
1200.0 \\
1400.0 \\
1600.0 \\
1800.0 \\
2000.0 \\
2500.0\end{array}$ & $\begin{array}{l}0.002131 \\
0.001865 \\
0.001658 \\
0.001492 \\
0.001243 \\
0.001066 \\
0.0009324 \\
0.0008288 \\
0.0007459 \\
0.0005968\end{array}$ & $\begin{array}{r}869.0 \\
993.0 \\
1120.0 \\
1240.0 \\
1490.0 \\
1740.0 \\
1990.0 \\
2230.0 \\
2480.0 \\
3100.0\end{array}$ & $\begin{array}{l}3.60 \\
3.60 \\
3.60 \\
3.60 \\
3.60 \\
3.60 \\
3.60 \\
3.60 \\
3.60 \\
3.60\end{array}$ & $\begin{array}{l}4.00 \\
4.00 \\
4.00 \\
4.00 \\
4.00 \\
4.00 \\
4.00 \\
4.00 \\
4.00 \\
4.00\end{array}$ & $\begin{array}{l}0.00143 \\
0.00125 \\
0.00111 \\
0.00100 \\
0.000833 \\
0.000714 \\
0.000625 \\
0.000556 \\
0.000500 \\
0.000400\end{array}$ & $\begin{array}{l}0.107 \\
0.117 \\
0.128 \\
0.137 \\
0.156 \\
0.174 \\
0.191 \\
0.208 \\
0.224 \\
0.263\end{array}$ & $\begin{array}{l}16.0 \\
17.6 \\
19.1 \\
20.5 \\
23.3 \\
26.0 \\
28.5 \\
31.0 \\
33.4 \\
39.2\end{array}$ & $\begin{array}{r}40.5 \\
50.8 \\
62.0 \\
74.2 \\
101.0 \\
132.0 \\
165.0 \\
202.0 \\
242.0 \\
355.0\end{array}$ & $\begin{array}{l}1.00001 \\
1.00001 \\
1.00001 \\
1.00001 \\
1.00000 \\
1.00000 \\
1.00000 \\
1.00000 \\
1.00000 \\
1.00000\end{array}$ & $\begin{array}{l}0.668 \\
0.668 \\
0.668 \\
0.667 \\
0.667 \\
0.667 \\
0.666 \\
0.666 \\
0.666 \\
0.666\end{array}$ \\
\hline 3000.0 & 0.0004973 & 3720.0 & 3.60 & 4.00 & 0.000333 & 0.299 & 44.6 & 485.0 & 1.00000 & 0.666 \\
\hline
\end{tabular}

* Tho-PHASE BoUndarY 


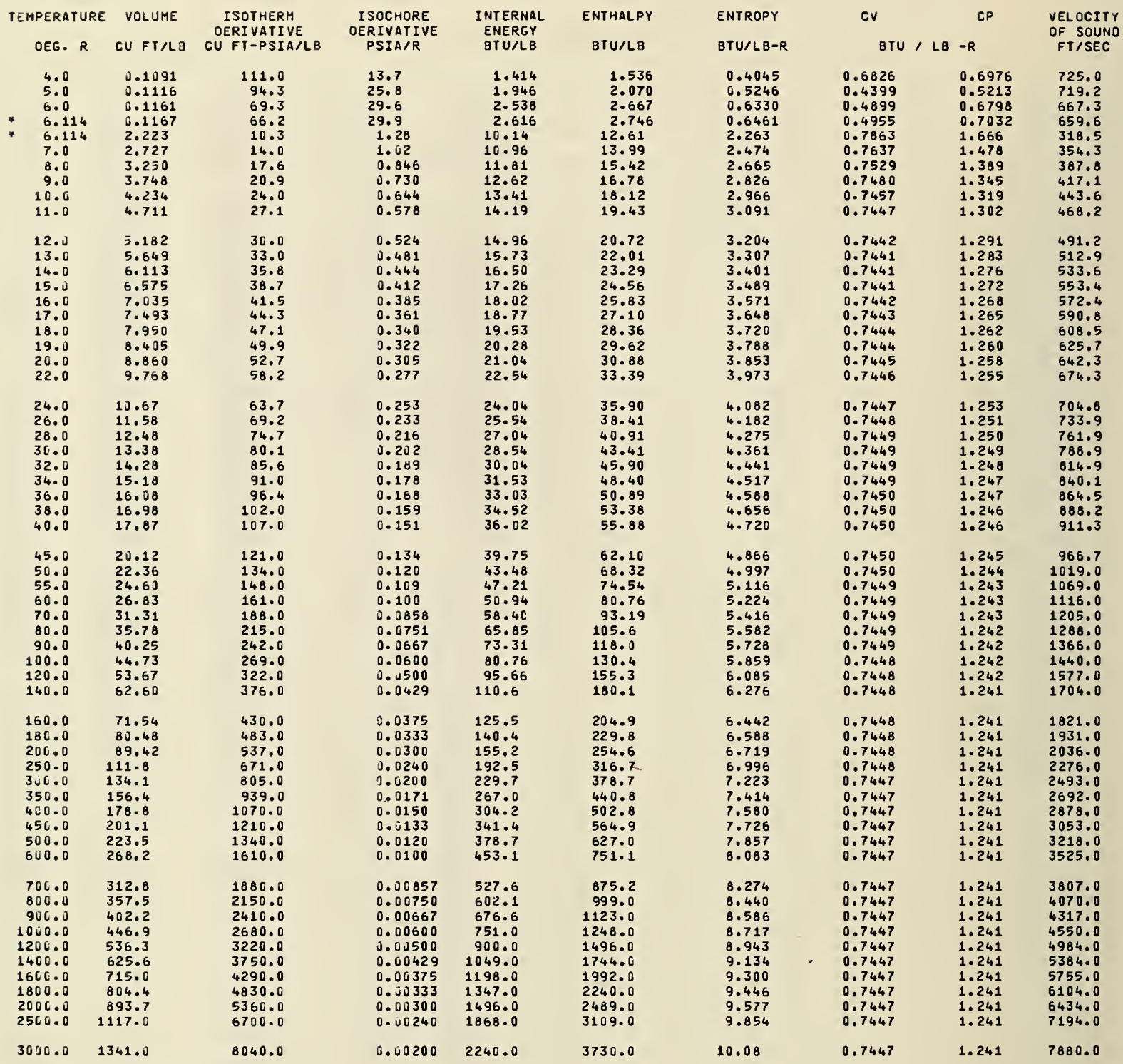

- tho-PHASE BOUNOARY 
THERMOPHYSICAL PROPERTIES OF HELIUM 4

6 PSIA ISOBAR

TEMPERATURE DENSITY VIDH/OV) $\mathrm{P}$ V(DP/OU) $-V(O P / O V)_{T}$ DEG. R LB/CUFT BTU/LB PSIA-CU FT/BTU PSIA

$\begin{array}{r}4.0 \\ +5 \\ +\quad 6.0 \\ \hline\end{array}$

$*$

\begin{tabular}{|c|c|}
\hline $\begin{array}{l}4.0 \\
5.0 \\
6.0 \\
6.114 \\
6.114 \\
7.0 \\
8.0 \\
9.0 \\
10.0 \\
11.0\end{array}$ & $\begin{array}{l}9.168 \\
8.964 \\
8.617 \\
8.566 \\
0.4499 \\
0.3666 \\
0.3377 \\
0.2668 \\
0.2362 \\
0.2123\end{array}$ \\
\hline $\begin{array}{l}12.0 \\
13.0 \\
14.0 \\
15.0 \\
16.0 \\
17.0 \\
18.0 \\
19.0 \\
20.0 \\
22.0\end{array}$ & $\begin{array}{l}0.1930 \\
0.1770 \\
0.1636 \\
0.1521 \\
0.1422 \\
0.1335 \\
0.1258 \\
0.1190 \\
0.1129 \\
0.1024\end{array}$ \\
\hline $\begin{array}{l}24.0 \\
26.0 \\
28.0 \\
30.0 \\
32.0 \\
34.0 \\
36.0 \\
38.0 \\
40.0\end{array}$ & $\begin{array}{l}0.09369 \\
0.08638 \\
0.08314 \\
0.07474 \\
0.07003 \\
0.06588 \\
0.06220 \\
0.05891 \\
0.05595\end{array}$ \\
\hline $\begin{array}{l}45.0 \\
50.0 \\
55.0 \\
60.0 \\
70.0 \\
80.0 \\
90.0 \\
100.0 \\
120.0 \\
140.0\end{array}$ & $\begin{array}{l}0.04971 \\
0.04473 \\
0.04066 \\
0.03727 \\
0.03194 \\
0.02795 \\
0.02484 \\
0.02236 \\
0.01863 \\
0.01597\end{array}$ \\
\hline $\begin{array}{l}160.0 \\
180.0 \\
200.0 \\
250.0 \\
300.0 \\
350.0 \\
400.0 \\
450.0 \\
500.0 \\
600.0\end{array}$ & $\begin{array}{l}0.01398 \\
0.01243 \\
0.01118 \\
0.008948 \\
0.007457 \\
0.006392 \\
0.005593 \\
0.004972 \\
0.004475 \\
0.003729\end{array}$ \\
\hline $\begin{array}{l}700.0 \\
800.0 \\
900.0 \\
000.0 \\
200.0 \\
400.0 \\
600.0 \\
800.0 \\
000.0 \\
500.0\end{array}$ & $\begin{array}{l}0.003196 \\
0.002797 \\
0.002486 \\
0.002238 \\
0.001865 \\
0.001598 \\
0.001399 \\
0.001243 \\
0.001119 \\
0.0008951\end{array}$ \\
\hline 000. & 0.0007459 \\
\hline
\end{tabular}

$\begin{array}{ll}2000.0 & 0.001119 \\ 2500.0 & 0.0008951\end{array}$

3000.0

51.8
17.1
13.7
13.4
6.08
7.45
8.89
10.3
11.6

14.3

15.6

16.9

18.2

19.4
20.7

22.0
23.3

23.3

27.1

29.6
32.1

34.6

37.1

42.1
44.6

44.6
47.1

49.6

55.9
62.1

62.1

74.5

87.0

99.4

112.0

124.0

149.0
174.0

199.0

224.0

248.0
310.0

373.0

435.0

597.0

621.0

745.0

869.0

993.0
1120.0

1120.0

1240.0
1490.0

1740.0

1990.0

2480.0

3100.0

3720.0
2.19
6.54
7.02
7.03
3.60
3.64
3.65
3.66
3.66
3.65

1020.0

845.0
597.0

567.0

4.65

5.14
5.41

5.57

5.67
5.74

3.65

3.65

3.65

3.64

3.64
3.64
3.64
3.63

3.63

3.63
3.63

3.63

3.62

3.62
3.62

3.62

3.62

3.62

3.61
3.61

3.61

3.61
3.61
3.61

3.61

3.61

3.61

3.61

3.60
3.60

3.60
3.60

3.60

3.60
3.60

3.60
3.60

3.60

3.60

3.60
3.60

3.60

3.60

3.60

3.60
3.60

3.60
3.60

3.60

3.60

3.60

3.60

3.60

5.80

5.83

5.86

5.90
5.92

5.92
5.93

5.94
5.95

5.95
5.96

5.97
5.98

5.98

5.99

5.99

5.99
6.00

6.00
6.00

6.00

6.00
6.00

6.00

6.01

6.01

6.01

6.01

6.01
6.00

6.00
6.00

6.00

6.00
6.00

6.00
6.00

6.00

6.00

6.00

6.00

6.00

6.00

6.00

6.00
6.00

6.00

6.00

6.00

6.00

6.00

6.00

(OV/OT) PV THERMAL VIS 1/DEG. $R$ QTU/FT-HR-R LB/FT-SEC

$\begin{array}{ll}0.0135 & 0.00872 \\ 0.0305 & 0.0101 \\ 0.0497 & 0.0107 \\ 0.0526 & 0.0108 \\ 0.274 & 0.00452 \\ 0.198 & 0.00497 \\ 0.156 & 0.00551 \\ 0.131 & 0.00605 \\ 0.114 & 0.00656 \\ 0.101 & 0.00705\end{array}$

2.49
2.54
2.41
2.38
0.624
0.706
0.792
0.874
0.952
1.03

0.0905

0.0824

0.0757

0.0701

0.0652

0.0611

0.0542

0.0513
0.0464

0.00752

0.00796

0.00839

0.00880

0.00920

0.00958

0.0100

0.0103

0.0107
0.0113

0.0424

0.0390

0.0361

0.0337

0.0315

0.0296
0.0279

0.0279
0.0264

0.0264
0.0251

0.0223

0.0200

0.0182

0.0167

0.0143

0.0111

0.0100

0.00833

0.00625

0.00555

0.00500

0.00400

0.00286

0.00250

0.00222

0.00200

0.0120

0.0126

0.0132
0.0137

0.0143

0.0143

0.0148

0.0154

0.0159
0.0164

0.0176

0.0187

0.0199

0.0210

0.0230

0.0250

0.0270

0.0289
0.0325

0.0325
0.0359

0.0393

0.0425

0.0456

0.0531

0.0602

0.0669

0.0732

0.0793

0.0650

0.00143

0.00125

0.107
0.117

0.00100

0.000833

0.000714

0.000625

0.000556

0.000500

0.128
0.137

0.156

0.174

0.191

0.208
0.224

0.224
0.263

0.0003330 .299

1.10
1.17

1.24

1. 30

1.37
1.43

1.43
1.49

1.49
1.55

1.60
1.71

1.92

2.02
2.12

2.12

2.21

2.30

2.39

2.56

2.76

2.95

3.32

3.65

3.97

4.27

4.55
5.10

THERMAL OIELECTRIC DIFFUSIVITY CONSTANT SQ FT/HR

0.00136
0.00215
0.00183
0.00179
0.00604
0.00917
0.0129
0.0169
0.0211
0.0255

$1.02042 \quad 0.718$

$1.01997 \quad 0.561$

$1.00167 \quad 0.827$

$\begin{array}{ll}1.00136 & 0.756 \\ 1.00115 & 0.719\end{array}$

$\begin{array}{ll}1.00115 & 0.719 \\ 1.00100 & 0.700\end{array}$

$1.00088 \quad 0.689$

$1.10 \quad 0.0302 \quad 1.00072 \quad 0.680$

0.0351

0.0402

0.0455

0.0510

0.0568
0.0627

0.0688

0.0751

.00066

1.00061

1.00057

1.00053

1.00050

1.00047
1.00045

1.00045
1.00042

1.00039

$1.82 \quad 0.102$

0.116

0.131

0.147

0.164

0.181
0.198

0.216

0.235

1.00035

1.00033

1.00030

1.00028

1.00026

1.00025

1.00023
1.00022

1.00022
1.00021

0.284 


\begin{tabular}{|c|c|c|c|c|c|c|c|c|c|}
\hline $\begin{array}{c}\text { TEMPERATUR } \\
\text { OEG. R }\end{array}$ & $\begin{array}{l}\text { VOLUME } \\
\text { CU FT/LB }\end{array}$ & $\begin{array}{c}\text { ISOTHERM } \\
\text { OERI VATIVE } \\
\text { CU FT-PSIA/LB }\end{array}$ & $\begin{array}{l}\text { ISOCHORE } \\
\text { OERIVAT IVE } \\
\text { PSIA/R }\end{array}$ & $\begin{array}{l}\text { INTERNAL } \\
\text { ENERGY } \\
\text { BTU/LB }\end{array}$ & $\begin{array}{l}\text { ENTHALPY } \\
\text { BTU/LB }\end{array}$ & $\begin{array}{l}\text { ENTROPY } \\
\text { BTU/LB-R }\end{array}$ & $\begin{array}{l}\text { CV } \\
\text { BTU / LB }\end{array}$ & $-R$ & $\begin{array}{l}\text { VELOCITY } \\
\text { OF SOUNO } \\
\text { FT/SEC }\end{array}$ \\
\hline $\begin{array}{l}4.0 \\
5.0 \\
6.0 \\
6.546 \\
6 \quad 6.546 \\
7.0 \\
8.0 \\
9.0 \\
10.0 \\
11.0\end{array}$ & $\begin{array}{l}0.1189 \\
j .1113 \\
j .1157 \\
j .1193 \\
1.711 \\
1.924 \\
2.346 \\
2.738 \\
3.114 \\
3.480\end{array}$ & $\begin{array}{c}113.0 \\
96.1 \\
71.3 \\
56.3 \\
9.94 \\
12.1 \\
16.2 \\
19.7 \\
23.1 \\
26.2\end{array}$ & $\begin{array}{l}13.7 \\
25.8 \\
29.8 \\
30.4 \\
1.67 \\
1.47 \\
1.18 \\
1.01 \\
0.881 \\
0.786\end{array}$ & $\begin{array}{l}1.411 \\
1.938 \\
2.523 \\
2.920 \\
10.27 \\
10.72 \\
11.63 \\
12.48 \\
13.29 \\
14.08\end{array}$ & $\begin{array}{l}1.573 \\
2.103 \\
2.694 \\
3.097 \\
12.80 \\
13.57 \\
15.11 \\
16.53 \\
17.90 \\
19.24\end{array}$ & $\begin{array}{l}0.4037 \\
0.5229 \\
0.6305 \\
0.6946 \\
2.179 \\
2.293 \\
2.498 \\
2.666 \\
2.810 \\
2.938\end{array}$ & $\begin{array}{l}0.6766 \\
0.4392 \\
0.4894 \\
0.5153 \\
0.7903 \\
0.7749 \\
0.7576 \\
0.7501 \\
0.7466 \\
0.7450\end{array}$ & $\begin{array}{l}0.6912 \\
0.5188 \\
0.6745 \\
0.7993 \\
1.790 \\
1.629 \\
1.465 \\
1.392 \\
1.351 \\
1.326\end{array}$ & $\begin{array}{l}730.8 \\
725.2 \\
674.7 \\
635.8 \\
323.0 \\
343.4 \\
380.5 \\
411.8 \\
439.7 \\
465.1\end{array}$ \\
\hline $\begin{array}{l}12.0 \\
13.0 \\
14.0 \\
15.0 \\
16.0 \\
17.0 \\
18.0 \\
19.0 \\
26.0 \\
22.0\end{array}$ & $\begin{array}{l}3.840 \\
4.196 \\
4.549 \\
4.899 \\
5.247 \\
5.594 \\
5.939 \\
6.233 \\
6.626 \\
7.310\end{array}$ & $\begin{array}{l}29.3 \\
32.3 \\
35.3 \\
38.2 \\
41.1 \\
43.9 \\
46.8 \\
49.6 \\
52.4 \\
58.0\end{array}$ & $\begin{array}{l}0.711 \\
0.650 \\
0.599 \\
0.555 \\
0.518 \\
0.486 \\
0.457 \\
0.432 \\
0.409 \\
0.371\end{array}$ & $\begin{array}{l}14.87 \\
15.64 \\
16.41 \\
17.18 \\
17.94 \\
18.71 \\
19.47 \\
20.22 \\
20.98 \\
22.49\end{array}$ & $\begin{array}{l}20.56 \\
21.86 \\
23.15 \\
24.44 \\
25.72 \\
26.99 \\
28.26 \\
29.53 \\
30.79 \\
33.32\end{array}$ & $\begin{array}{l}3.052 \\
3.157 \\
3.253 \\
3.341 \\
3.424 \\
3.501 \\
3.574 \\
3.542 \\
3.707 \\
3.827\end{array}$ & $\begin{array}{l}0.7443 \\
0.7440 \\
0.7439 \\
0.7439 \\
0.7440 \\
0.7441 \\
0.7443 \\
0.7444 \\
0.7445 \\
0.7446\end{array}$ & $\begin{array}{l}1.310 \\
1.298 \\
1.289 \\
1.282 \\
1.277 \\
1.273 \\
1.269 \\
1.266 \\
1.264 \\
1.260\end{array}$ & $\begin{array}{l}488.8 \\
511.0 \\
532.1 \\
552.2 \\
571.4 \\
590.0 \\
607.9 \\
625.2 \\
642.0 \\
674.2\end{array}$ \\
\hline $\begin{array}{l}24.0 \\
26.0 \\
28.0 \\
31.0 \\
32.0 \\
34.0 \\
36.0 \\
38.0 \\
40.0\end{array}$ & $\begin{array}{r}7.992 \\
8.672 \\
9.351 \\
10.03 \\
10.73 \\
11.38 \\
12.00 \\
12.73 \\
13.40\end{array}$ & $\begin{array}{r}63.5 \\
69.0 \\
74.5 \\
80.0 \\
85.5 \\
90.9 \\
96.4 \\
102.0 \\
107.0\end{array}$ & $\begin{array}{l}0.339 \\
0.312 \\
0.289 \\
0.269 \\
0.252 \\
0.237 \\
0.224 \\
0.212 \\
0.211\end{array}$ & $\begin{array}{l}24.00 \\
25.50 \\
27.00 \\
28.50 \\
30.00 \\
31.50 \\
33.00 \\
34.49 \\
35.99\end{array}$ & $\begin{array}{l}35.83 \\
38.35 \\
40.85 \\
43.36 \\
45.86 \\
48.36 \\
50.86 \\
53.35 \\
55.85\end{array}$ & $\begin{array}{l}3.937 \\
4.037 \\
4.130 \\
4.217 \\
4.298 \\
4.373 \\
4.445 \\
4.512 \\
4.576\end{array}$ & $\begin{array}{l}0.7448 \\
0.7449 \\
0.7449 \\
0.7450 \\
0.7450 \\
0.7450 \\
0.7450 \\
0.7451 \\
0.7451\end{array}$ & $\begin{array}{l}1.257 \\
1.255 \\
1.253 \\
1.252 \\
1.250 \\
1.249 \\
1.248 \\
1.248 \\
1.247\end{array}$ & $\begin{array}{l}704.8 \\
734.1 \\
762.1 \\
789.1 \\
815.2 \\
840.4 \\
864.9 \\
888.7 \\
911.8\end{array}$ \\
\hline $\begin{array}{r}45.0 \\
50.0 \\
55.0 \\
60.0 \\
70.0 \\
80.0 \\
90.0 \\
100.0 \\
120.0 \\
140.0\end{array}$ & $\begin{array}{l}15.09 \\
15.77 \\
13.45 \\
23.13 \\
23.49 \\
26.85 \\
30.20 \\
33.55 \\
40.26 \\
46.97\end{array}$ & $\begin{array}{l}121.0 \\
134.0 \\
148.0 \\
161.0 \\
188.0 \\
215.0 \\
242.0 \\
269.0 \\
322.0 \\
376.0\end{array}$ & $\begin{array}{l}0.179 \\
0.161 \\
0.146 \\
0.134 \\
0.114 \\
0.16 C \\
0.0890 \\
0.0501 \\
0.0667 \\
0.0572\end{array}$ & $\begin{array}{l}39.73 \\
43.46 \\
47.20 \\
50.93 \\
58.39 \\
65.84 \\
73.30 \\
80.75 \\
95.65 \\
110.6\end{array}$ & $\begin{array}{c}62.03 \\
68.31 \\
74.53 \\
80.75 \\
93.18 \\
105.6 \\
118.0 \\
130.5 \\
155.3 \\
180.1\end{array}$ & $\begin{array}{l}4.723 \\
4.854 \\
4.973 \\
5.081 \\
5.273 \\
5.439 \\
5.585 \\
5.716 \\
5.942 \\
6.134\end{array}$ & $\begin{array}{l}0.7451 \\
0.7450 \\
0.7450 \\
0.7450 \\
0.7450 \\
0.7449 \\
0.7449 \\
0.7449 \\
0.7449 \\
0.7448\end{array}$ & $\begin{array}{l}1.246 \\
1.245 \\
1.244 \\
1.244 \\
1.243 \\
1.243 \\
1.242 \\
1.242 \\
1.242 \\
1.242\end{array}$ & $\begin{array}{r}967.2 \\
1019.0 \\
1069.0 \\
1117.0 \\
1206.0 \\
1289.0 \\
1367.0 \\
1441.0 \\
1578.0 \\
1704.0\end{array}$ \\
\hline $\begin{array}{l}160.0 \\
180.0 \\
200.0 \\
250.0 \\
300.0 \\
350.0 \\
400.0 \\
450.0 \\
500.0 \\
600.0\end{array}$ & $\begin{array}{l}53.67 \\
60.37 \\
67.08 \\
83.83 \\
100.6 \\
117.3 \\
134.1 \\
150.9 \\
167.6 \\
201.1\end{array}$ & $\begin{array}{r}430.0 \\
483.0 \\
537.0 \\
671.0 \\
805.0 \\
939.0 \\
1070.0 \\
1210.0 \\
1340.0 \\
1610.0\end{array}$ & $\begin{array}{l}0.0500 \\
0.0444 \\
0.0400 \\
0.0320 \\
0.0267 \\
0.0229 \\
0.0200 \\
0.0178 \\
0.0160 \\
0.0133\end{array}$ & $\begin{array}{l}125.5 \\
140.3 \\
155.2 \\
192.5 \\
229.7 \\
267.0 \\
304.2 \\
341.4 \\
378.7 \\
453.1\end{array}$ & $\begin{array}{l}205.0 \\
229.8 \\
254.6 \\
316.7 \\
378.7 \\
440.8 \\
502.9 \\
564.9 \\
627.0 \\
751.1\end{array}$ & $\begin{array}{l}6.299 \\
6.446 \\
6.576 \\
6.853 \\
7.080 \\
7.271 \\
7.437 \\
7.583 \\
7.714 \\
7.940\end{array}$ & $\begin{array}{l}0.7448 \\
0.7448 \\
0.7448 \\
0.7448 \\
0.7448 \\
0.7447 \\
0.7447 \\
0.7447 \\
0.7447 \\
0.7447\end{array}$ & $\begin{array}{l}1.241 \\
1.241 \\
1.241 \\
1.241 \\
1.241 \\
1.241 \\
1.241 \\
1.241 \\
1.241 \\
1.241\end{array}$ & $\begin{array}{l}1822.0 \\
1932.0 \\
2036.0 \\
2276.0 \\
2493.0 \\
2693.0 \\
2878.0 \\
3053.0 \\
3218.0 \\
3525.0\end{array}$ \\
\hline $\begin{array}{r}700.0 \\
800.0 \\
960.0 \\
1000.0 \\
1200.0 \\
1400.0 \\
1600.0 \\
1800.0 \\
2006.0 \\
2500.0\end{array}$ & $\begin{array}{l}234.6 \\
268.2 \\
301.7 \\
335.2 \\
402.2 \\
469.2 \\
536.3 \\
603.3 \\
670.3 \\
837.9\end{array}$ & $\begin{array}{l}1880.0 \\
2150.0 \\
2410.0 \\
2680.0 \\
3220.0 \\
3750.0 \\
4290.0 \\
4830.0 \\
5360.0 \\
6700.0\end{array}$ & $\begin{array}{l}0.0114 \\
0.0160 \\
0.00889 \\
0.00800 \\
0.00667 \\
0.00571 \\
0.00500 \\
0.00444 \\
0.00400 \\
0.00320\end{array}$ & $\begin{array}{r}527.6 \\
602.1 \\
576.6 \\
751.0 \\
900.0 \\
1049.0 \\
1198.0 \\
1347.0 \\
1496.0 \\
1868.0\end{array}$ & $\begin{array}{r}875.2 \\
999.0 \\
1123.0 \\
1248.0 \\
1496.0 \\
1744.0 \\
1992.0 \\
2240.0 \\
2489.0 \\
3109.0\end{array}$ & $\begin{array}{l}8.131 \\
8.297 \\
8.443 \\
8.574 \\
8.800 \\
8.992 \\
9.157 \\
9.304 \\
9.434 \\
9.711\end{array}$ & $\begin{array}{l}0.7447 \\
0.7447 \\
0.7447 \\
0.7447 \\
0.7447 \\
0.7447 \\
0.7447 \\
0.7447 \\
0.7447 \\
0.7447\end{array}$ & $\begin{array}{l}1.241 \\
1.241 \\
1.241 \\
1.241 \\
1.241 \\
1.241 \\
1.241 \\
1.241 \\
1.241 \\
1.241\end{array}$ & $\begin{array}{l}3807.0 \\
4070.0 \\
4317.0 \\
4550.0 \\
4984.0 \\
5384.0 \\
5755.0 \\
6104.0 \\
6434.0 \\
7194.0\end{array}$ \\
\hline 3000.0 & 1405.0 & 8040.0 & 0.00267 & $2240 . \mathrm{C}$ & 3730.0 & 9.938 & 0.7447 & 1.241 & 7880.0 \\
\hline
\end{tabular}


o PSIA ISOBAR

\begin{tabular}{|c|c|c|c|c|c|c|c|c|c|c|}
\hline TEMPERATURE & OENSITY & $V(\mathrm{OH} / \mathrm{OV})_{\mathrm{P}}$ & $V(O P / O U)-$ & $-V(O P / O V)_{T}$ & $(0 \mathrm{~V} / 0 \mathrm{~T})_{\mathrm{p}}^{\mathrm{V}}$ & $\begin{array}{l}\text { THERMAL } \\
\text { CONDUCTIVITY }\end{array}$ & VISCOSITY & $\begin{array}{c}\text { THERMAL } \\
\text { OIFFUSIVITY }\end{array}$ & $\begin{array}{l}\text { OIELECTRIC } \\
\text { CONSTANT }\end{array}$ & $\begin{array}{l}\text { PRANOTL } \\
\text { NUMBER }\end{array}$ \\
\hline OEG. $R$ & LB/CU FT & BTU/L3 & PSIA-CU FT/UTU & PSIA & 1/OEG. R & $B T U / F T-H R-R$ & $\begin{array}{r}\text { LB/FT }- \text { SEC } \\
\times 10 E+6\end{array}$ & SQ FT/HR & & \\
\hline $\begin{array}{l}4.0 \\
5.0 \\
6.0 \\
6.546 \\
6.546 \\
7.0 \\
8.0 \\
9.0 \\
10.0 \\
11.0\end{array}$ & $\begin{array}{l}9.187 \\
8.980 \\
8.646 \\
8.380 \\
0.5845 \\
0.5199 \\
0.4262 \\
0.3653 \\
0.3212 \\
0.2873\end{array}$ & $\begin{array}{c}52.3 \\
17.4 \\
14.0 \\
12.4 \\
6.21 \\
6.98 \\
8.52 \\
10.0 \\
11.4 \\
12.7\end{array}$ & $\begin{array}{l}2.20 \\
6.54 \\
7.04 \\
7.04 \\
3.62 \\
3.64 \\
3.67 \\
3.67 \\
3.67 \\
3.67\end{array}$ & $\begin{array}{c}1040.0 \\
864.0 \\
616.0 \\
472.0 \\
5.81 \\
6.30 \\
6.89 \\
7.21 \\
7.41 \\
7.54\end{array}$ & $\begin{array}{l}0.0132 \\
0.0299 \\
0.0483 \\
0.0645 \\
0.288 \\
0.233 \\
0.172 \\
0.140 \\
0.119 \\
0.104\end{array}$ & $\begin{array}{l}0.00874 \\
0.0101 \\
0.0107 \\
0.0110 \\
0.00494 \\
0.00511 \\
0.00560 \\
0.00612 \\
0.00662 \\
0.00710\end{array}$ & $\begin{array}{l}2.51 \\
2.56 \\
2.43 \\
2.32 \\
0.681 \\
0.721 \\
0.806 \\
0.887 \\
0.964 \\
1.04\end{array}$ & $\begin{array}{l}0.00138 \\
0.00216 \\
0.00184 \\
0.00164 \\
0.00472 \\
0.00604 \\
0.00897 \\
0.0120 \\
0.0152 \\
0.0186\end{array}$ & $\begin{array}{l}1.02043 \\
1.02030 \\
1.02004 \\
1.01981 \\
1.00215 \\
1.00192 \\
1.00158 \\
1.00136 \\
1.00120 \\
1.00107\end{array}$ & $\begin{array}{l}0.716 \\
0.474 \\
0.549 \\
0.607 \\
0.889 \\
0.827 \\
0.759 \\
0.726 \\
0.709 \\
0.698\end{array}$ \\
\hline $\begin{array}{l}12.0 \\
13.0 \\
14.0 \\
15.0 \\
16.0 \\
17.0 \\
18.0 \\
19.0 \\
20.0 \\
22.0\end{array}$ & $\begin{array}{l}0.2634 \\
0.2383 \\
0.2138 \\
0.2041 \\
0.1906 \\
0.1788 \\
0.1684 \\
0.1592 \\
0.1539 \\
0.1368\end{array}$ & $\begin{array}{l}14.1 \\
15.4 \\
16.7 \\
18.0 \\
19.3 \\
20.6 \\
21.9 \\
23.2 \\
24.4 \\
27.0\end{array}$ & $\begin{array}{l}3.67 \\
3.66 \\
3.66 \\
3.66 \\
3.65 \\
3.65 \\
3.65 \\
3.64 \\
3.64 \\
3.64\end{array}$ & $\begin{array}{l}7.63 \\
7.70 \\
7.75 \\
7.79 \\
7.83 \\
7.85 \\
7.88 \\
7.89 \\
7.91 \\
7.93\end{array}$ & $\begin{array}{l}0.0932 \\
0.0844 \\
0.0772 \\
0.0712 \\
0.0662 \\
0.0618 \\
0.0580 \\
0.0547 \\
0.0517 \\
0.0467\end{array}$ & $\begin{array}{l}0.00756 \\
0.00801 \\
0.00843 \\
0.00884 \\
0.00923 \\
0.00962 \\
0.0100 \\
0.0103 \\
0.0107 \\
0.0114\end{array}$ & $\begin{array}{l}1.11 \\
1.18 \\
1.25 \\
1.31 \\
1.37 \\
1.43 \\
1.49 \\
1.55 \\
1.61 \\
1.72\end{array}$ & $\begin{array}{l}0.0222 \\
0.0259 \\
0.0298 \\
0.0338 \\
0.0379 \\
0.0423 \\
0.0467 \\
0.0513 \\
0.0560 \\
0.0659\end{array}$ & $\begin{array}{l}1.00097 \\
1.00089 \\
1.00082 \\
1.00076 \\
1.00071 \\
1.00067 \\
1.00063 \\
1.00060 \\
1.00057 \\
1.00051\end{array}$ & $\begin{array}{l}0.692 \\
0.688 \\
0.685 \\
0.684 \\
0.684 \\
0.684 \\
0.684 \\
0.684 \\
0.685 \\
0.687\end{array}$ \\
\hline $\begin{array}{l}24.0 \\
26.0 \\
28.0 \\
30.0 \\
32.0 \\
34.0 \\
36.0 \\
38.0 \\
46.0\end{array}$ & $\begin{array}{l}0.1251 \\
0.1153 \\
0.1069 \\
0.0997 \\
0.09341 \\
0.08787 \\
0.08295 \\
0.07855 \\
0.07460\end{array}$ & $\begin{array}{l}29.5 \\
32.0 \\
34.6 \\
37.1 \\
39.6 \\
42.1 \\
44.6 \\
47.1 \\
49.6\end{array}$ & $\begin{array}{l}3.63 \\
3.63 \\
3.63 \\
3.63 \\
3.62 \\
3.62 \\
3.62 \\
3.62 \\
3.62\end{array}$ & $\begin{array}{l}7.95 \\
7.96 \\
7.97 \\
7.98 \\
7.99 \\
7.99 \\
7.99 \\
8.00 \\
8.00\end{array}$ & $\begin{array}{l}0.0426 \\
0.0332 \\
0.0363 \\
0.0338 \\
0.0316 \\
0.0297 \\
0.0280 \\
0.0265 \\
0.0251\end{array}$ & $\begin{array}{l}0.0120 \\
0.0126 \\
0.0132 \\
0.0138 \\
0.0143 \\
0.0149 \\
0.0154 \\
0.01159 \\
0.0164\end{array}$ & $\begin{array}{l}1.83 \\
1.93 \\
2.03 \\
2.12 \\
2.22 \\
2.31 \\
2.39 \\
2.48 \\
2.56\end{array}$ & $\begin{array}{l}0.0763 \\
0.0871 \\
0.0985 \\
0.110 \\
0.123 \\
0.135 \\
0.149 \\
0.162 \\
0.176\end{array}$ & $\begin{array}{l}1.00047 \\
1.00043 \\
1.00040 \\
1.00038 \\
1.00035 \\
1.00033 \\
1.00031 \\
1.00030 \\
1.00028\end{array}$ & $\begin{array}{l}0.689 \\
0.691 \\
0.693 \\
0.695 \\
0.697 \\
0.698 \\
0.700 \\
0.701 \\
0.702\end{array}$ \\
\hline $\begin{array}{r}45.0 \\
50.0 \\
55.0 \\
60.0 \\
70.0 \\
80.0 \\
90.0 \\
100.0 \\
120.0 \\
140.0\end{array}$ & $\begin{array}{l}0.00028 \\
0.05963 \\
0.05420 \\
0.04967 \\
0.04257 \\
0.03725 \\
0.03311 \\
0.02980 \\
0.02484 \\
0.02129\end{array}$ & $\begin{array}{r}55.8 \\
62.1 \\
68.3 \\
74.5 \\
87.0 \\
99.4 \\
112.0 \\
124.0 \\
149.0 \\
174.0\end{array}$ & $\begin{array}{l}3.62 \\
3.61 \\
3.61 \\
3.61 \\
3.61 \\
3.61 \\
3.61 \\
3.61 \\
3.60 \\
3.60\end{array}$ & $\begin{array}{l}8.00 \\
8.01 \\
8.01 \\
8.01 \\
8.01 \\
8.01 \\
8.01 \\
8.01 \\
8.01 \\
8.01\end{array}$ & $\begin{array}{l}0.0223 \\
0.0201 \\
0.0182 \\
0.0167 \\
0.0143 \\
0.0125 \\
0.0111 \\
0.0100 \\
0.00833 \\
0.00714\end{array}$ & $\begin{array}{l}0.0176 \\
0.0188 \\
0.0199 \\
0.0210 \\
0.0231 \\
0.0251 \\
0.0270 \\
0.0289 \\
0.0325 \\
0.0359\end{array}$ & $\begin{array}{l}2.77 \\
2.96 \\
3.14 \\
3.32 \\
3.65 \\
3.97 \\
4.27 \\
4.56 \\
5.10 \\
5.61\end{array}$ & $\begin{array}{l}0.213 \\
0.253 \\
0.295 \\
0.339 \\
0.436 \\
0.541 \\
0.656 \\
0.780 \\
1.05 \\
1.36\end{array}$ & $\begin{array}{l}1.00025 \\
1.00022 \\
1.00020 \\
1.00019 \\
1.00016 \\
1.000114 \\
1.00012 \\
1.00011 \\
1.00009 \\
1.00008\end{array}$ & $\begin{array}{l}0.705 \\
0.706 \\
0.708 \\
0.708 \\
0.709 \\
0.708 \\
0.707 \\
0.705 \\
0.702 \\
0.698\end{array}$ \\
\hline $\begin{array}{l}160.0 \\
100.0 \\
200.0 \\
250.0 \\
300.0 \\
350.0 \\
400.0 \\
450.0 \\
500.0 \\
600.0\end{array}$ & $\begin{array}{l}0.01863 \\
0.01656 \\
0.01491 \\
0.01193 \\
0.009941 \\
0.008522 \\
0.007457 \\
0.006629 \\
0.005966 \\
0.004972\end{array}$ & $\begin{array}{l}199.0 \\
224.0 \\
248.0 \\
310.0 \\
373.0 \\
435.0 \\
497.0 \\
559.0 \\
621.0 \\
745.0\end{array}$ & $\begin{array}{l}3.60 \\
3.60 \\
3.60 \\
3.60 \\
3.60 \\
3.60 \\
3.60 \\
3.60 \\
3.60 \\
3.60\end{array}$ & $\begin{array}{l}8.01 \\
8.01 \\
8.01 \\
8.00 \\
8.00 \\
8.00 \\
8.00 \\
8.00 \\
8.00 \\
8.00\end{array}$ & $\begin{array}{l}0.00625 \\
0.00555 \\
0.00500 \\
0.00400 \\
0.00333 \\
0.00286 \\
0.00250 \\
0.00222 \\
0.00200 \\
0.00167\end{array}$ & $\begin{array}{l}0.0393 \\
0.0425 \\
0.0456 \\
0.0531 \\
0.0602 \\
0.0669 \\
0.0732 \\
0.0793 \\
0.0850 \\
0.0962\end{array}$ & $\begin{array}{l}6.10 \\
6.57 \\
6.89 \\
7.98 \\
9.01 \\
10.0 \\
10.9 \\
11.8 \\
12.7 \\
14.4\end{array}$ & $\begin{array}{l}1.70 \\
2.07 \\
2.47 \\
3.59 \\
4.88 \\
6.32 \\
7.91 \\
9.64 \\
11.5 \\
15.6\end{array}$ & $\begin{array}{l}1.00007 \\
1.00006 \\
1.00006 \\
1.00005 \\
1.00004 \\
1.00003 \\
1.00003 \\
1.00003 \\
1.00002 \\
1.00002\end{array}$ & $\begin{array}{l}0.694 \\
0.691 \\
0.675 \\
0.671 \\
0.669 \\
0.667 \\
0.666 \\
0.667 \\
0.668 \\
0.669\end{array}$ \\
\hline $\begin{array}{r}700.0 \\
800.0 \\
900.0 \\
1000.0 \\
1200.0 \\
1406.0 \\
1600.0 \\
1860.0 \\
2006.0 \\
2500.0\end{array}$ & $\begin{array}{l}0.004262 \\
0.003729 \\
0.003315 \\
0.002983 \\
0.002486 \\
0.002131 \\
0.001865 \\
0.001658 \\
0.001492 \\
0.001193\end{array}$ & $\begin{array}{r}869.0 \\
993.0 \\
1120.0 \\
1240.0 \\
1490.0 \\
1740.0 \\
1990.0 \\
2230.0 \\
2480.0 \\
3100.0\end{array}$ & $\begin{array}{l}3.60 \\
3.60 \\
3.60 \\
3.60 \\
3.63 \\
3.60 \\
3.60 \\
3.60 \\
3.60 \\
3.60\end{array}$ & $\begin{array}{l}8.00 \\
8.00 \\
8.00 \\
8.00 \\
8.00 \\
8.00 \\
8.00 \\
8.00 \\
8.00 \\
8.00\end{array}$ & $\begin{array}{l}0.00143 \\
0.00125 \\
0.00111 \\
0.00100 \\
0.000833 \\
0.000714 \\
0.000625 \\
0.000556 \\
0.000500 \\
0.000400\end{array}$ & $\begin{array}{l}0.107 \\
0.117 \\
0.128 \\
0.137 \\
0.156 \\
0.174 \\
0.191 \\
0.208 \\
0.224 \\
0.263\end{array}$ & $\begin{array}{l}16.0 \\
17.6 \\
19.1 \\
20.5 \\
23.3 \\
26.0 \\
28.5 \\
31.0 \\
33.4 \\
39.2\end{array}$ & $\begin{array}{r}20.2 \\
25.4 \\
31.0 \\
37.1 \\
50.6 \\
65.8 \\
82.7 \\
101.0 \\
121.0 \\
177.0\end{array}$ & $\begin{array}{l}1.000002 \\
1.000001 \\
1.00001 \\
1.00001 \\
1.00001 \\
1.00001 \\
1.000001 \\
1.00001 \\
1.00001 \\
1.000000\end{array}$ & $\begin{array}{l}0.668 \\
0.668 \\
0.668 \\
0.667 \\
0.667 \\
0.667 \\
0.666 \\
0.666 \\
0.666 \\
0.666\end{array}$ \\
\hline 3000.0 & $0.0 ง 09946$ & 3725.0 & 3.60 & 8.00 & 0.000333 & 0.299 & 44.6 & 242.0 & 1.00000 & 0.666 \\
\hline
\end{tabular}

- two-phase goundary 
10 PSIA ISOBAR

THERMOOYNAMIC PROPERTIES OF HELIUM 4

\begin{tabular}{|c|c|c|c|c|c|c|c|c|c|}
\hline $\begin{array}{c}\text { TEMPERATURE } \\
\text { OEG. R }\end{array}$ & $\begin{array}{l}\text { E VOLUME } \\
\text { CU FT/LB }\end{array}$ & $\begin{array}{c}\text { ISOTHERM } \\
\text { OERIVATIVE } \\
\text { CU FT-PSIA/LB }\end{array}$ & $\begin{array}{l}\text { ISOCHORE } \\
\text { OERIVATIVE } \\
\text { PSIA/R }\end{array}$ & $\begin{array}{l}\text { INTERNAL } \\
\text { ENERGY } \\
\text { BTU/LB }\end{array}$ & $\begin{array}{l}\text { ENTHALPY } \\
\text { BTU/LB }\end{array}$ & $\begin{array}{l}\text { ENTROPY } \\
\text { BTU/LB-R }\end{array}$ & $\begin{array}{l}\text { CV } \\
\text { BTU / LB }\end{array}$ & $\begin{array}{l}C P \\
-R\end{array}$ & $\begin{array}{l}\text { VEL OCITY } \\
\text { OF SOUND } \\
\text { FT/SEC }\end{array}$ \\
\hline $\begin{array}{l}4.0 \\
5.0 \\
6.0 \\
6.910 \\
6.910 \\
7.0 \\
8.0 \\
9.0 \\
10.0 \\
11.0\end{array}$ & $\begin{array}{l}0.1086 \\
0.1110 \\
0.1153 \\
0.1218 \\
1.388 \\
1.426 \\
1.798 \\
2.129 \\
2.440 \\
2.741\end{array}$ & $\begin{array}{c}115.0 \\
98.0 \\
73.3 \\
48.0 \\
9.44 \\
9.94 \\
14.6 \\
18.5 \\
22.1 \\
25.4\end{array}$ & $\begin{array}{l}13.7 \\
25.9 \\
29.9 \\
30.6 \\
2.08 \\
2.02 \\
1.56 \\
1.31 \\
1.13 \\
1.00\end{array}$ & $\begin{array}{l}1.408 \\
1.930 \\
2.509 \\
3.203 \\
10.34 \\
10.44 \\
11.44 \\
12.32 \\
13.16 \\
13.97\end{array}$ & $\begin{array}{l}1.610 \\
2.135 \\
2.723 \\
3.429 \\
12.91 \\
13.08 \\
14.77 \\
16.26 \\
17.58 \\
19.05\end{array}$ & $\begin{array}{l}0.4029 \\
0.5212 \\
0.6280 \\
0.7373 \\
2.110 \\
2.135 \\
2.361 \\
2.537 \\
2.686 \\
2.817\end{array}$ & $\begin{array}{l}0.6707 \\
0.4385 \\
0.4889 \\
0.5318 \\
0.7937 \\
0.7897 \\
0.7634 \\
0.7526 \\
0.7477 \\
0.7454\end{array}$ & $\begin{array}{l}0.6850 \\
0.5164 \\
0.6695 \\
0.9013 \\
1.927 \\
1.872 \\
1.563 \\
1.447 \\
1.387 \\
1.352\end{array}$ & $\begin{array}{l}736.5 \\
731.1 \\
681.8 \\
613.7 \\
325.8 \\
330.4 \\
372.7 \\
406.3 \\
435.6 \\
462.0\end{array}$ \\
\hline $\begin{array}{l}12.0 \\
13.0 \\
14.0 \\
15.0 \\
16.0 \\
17.0 \\
18.0 \\
19.0 \\
20.0 \\
22.0\end{array}$ & $\begin{array}{l}3.035 \\
3.324 \\
3.610 \\
3.893 \\
4.174 \\
+.454 \\
4.732 \\
5.009 \\
5.285 \\
5.835\end{array}$ & $\begin{array}{l}28.6 \\
31.7 \\
34.7 \\
37.7 \\
40.6 \\
43.5 \\
46.4 \\
49.3 \\
52.1 \\
57.7\end{array}$ & $\begin{array}{l}0.904 \\
0.824 \\
0.757 \\
0.701 \\
0.653 \\
0.612 \\
0.575 \\
0.543 \\
0.514 \\
0.465\end{array}$ & $\begin{array}{l}14.77 \\
15.55 \\
16.33 \\
17.10 \\
17.87 \\
18.64 \\
19.40 \\
20.16 \\
20.92 \\
22.44\end{array}$ & $\begin{array}{l}20.39 \\
21.71 \\
23.02 \\
24.31 \\
25.60 \\
26.89 \\
28.16 \\
29.44 \\
30.71 \\
33.24\end{array}$ & $\begin{array}{l}2.933 \\
3.039 \\
3.136 \\
3.225 \\
3.309 \\
3.386 \\
3.459 \\
3.528 \\
3.594 \\
3.714\end{array}$ & $\begin{array}{l}0.7444 \\
0.7439 \\
0.7438 \\
0.7438 \\
0.7439 \\
0.7440 \\
0.7442 \\
0.7443 \\
0.7444 \\
0.7446\end{array}$ & $\begin{array}{l}1.330 \\
1.314 \\
1.302 \\
1.293 \\
1.287 \\
1.281 \\
1.277 \\
1.273 \\
1.270 \\
1.265\end{array}$ & $\begin{array}{l}486.3 \\
509.0 \\
530.5 \\
550.9 \\
570.4 \\
589.2 \\
607.2 \\
624.7 \\
641.6 \\
674.0\end{array}$ \\
\hline $\begin{array}{l}24.0 \\
26.0 \\
28.0 \\
30.0 \\
32.0 \\
34.0 \\
36.0 \\
38.0 \\
40.0\end{array}$ & $\begin{array}{c}6.383 \\
6.929 \\
7.474 \\
8.017 \\
8.560 \\
9.102 \\
9.643 \\
10.18 \\
10.72\end{array}$ & $\begin{array}{r}63.3 \\
68.9 \\
74.4 \\
79.9 \\
85.4 \\
90.9 \\
96.3 \\
102.0 \\
107.0\end{array}$ & $\begin{array}{l}0.425 \\
0.391 \\
0.362 \\
0.338 \\
0.316 \\
0.297 \\
0.280 \\
0.265 \\
0.252\end{array}$ & $\begin{array}{l}23.95 \\
25.46 \\
26.96 \\
28.47 \\
29.97 \\
31.47 \\
32.97 \\
34.47 \\
35.96\end{array}$ & $\begin{array}{l}35.77 \\
38.29 \\
40.80 \\
43.31 \\
45.82 \\
48.32 \\
50.82 \\
53.32 \\
55.82\end{array}$ & $\begin{array}{l}3.824 \\
3.925 \\
4.018 \\
4.105 \\
4.186 \\
4.262 \\
4.333 \\
4.401 \\
4.465\end{array}$ & $\begin{array}{l}0.7448 \\
0.7449 \\
0.7450 \\
0.7450 \\
0.7451 \\
0.7451 \\
0.7451 \\
0.7451 \\
0.7451\end{array}$ & $\begin{array}{l}1.261 \\
1.258 \\
1.256 \\
1.254 \\
1.253 \\
1.251 \\
1.250 \\
1.249 \\
1.248\end{array}$ & $\begin{array}{l}704.8 \\
734.2 \\
762.3 \\
789.4 \\
815.5 \\
840.8 \\
865.3 \\
889.1 \\
912.3\end{array}$ \\
\hline $\begin{array}{r}45.0 \\
50.0 \\
55.0 \\
60.0 \\
70.0 \\
80.0 \\
90.0 \\
100.0 \\
120.0 \\
140.0\end{array}$ & $\begin{array}{l}12.07 \\
13.42 \\
14.77 \\
16.11 \\
18.80 \\
21.48 \\
24.17 \\
26.85 \\
32.22 \\
37.53\end{array}$ & $\begin{array}{l}121.0 \\
134.0 \\
148.0 \\
161.0 \\
188.0 \\
215.0 \\
242.0 \\
269.0 \\
323.0 \\
376.0\end{array}$ & $\begin{array}{l}0.223 \\
0.201 \\
0.182 \\
0.167 \\
0.143 \\
0.125 \\
0.111 \\
0.100 \\
0.0834 \\
0.0715\end{array}$ & $\begin{array}{l}39.70 \\
43.44 \\
47.18 \\
50.91 \\
58.37 \\
65.83 \\
73.29 \\
80.74 \\
95.65 \\
110.5\end{array}$ & $\begin{array}{c}62.06 \\
68.29 \\
74.52 \\
80.74 \\
93.18 \\
105.6 \\
118.0 \\
130.5 \\
155.3 \\
180.1\end{array}$ & $\begin{array}{l}4.612 \\
4.743 \\
4.862 \\
4.970 \\
5.162 \\
5.328 \\
5.474 \\
5.605 \\
5.831 \\
6.023\end{array}$ & $\begin{array}{l}0.7451 \\
0.7451 \\
0.7451 \\
0.7451 \\
0.7450 \\
0.7450 \\
0.7450 \\
0.7449 \\
0.7449 \\
0.7449\end{array}$ & $\begin{array}{l}1.247 \\
1.246 \\
1.245 \\
1.244 \\
1.243 \\
1.243 \\
1.243 \\
1.242 \\
1.242 \\
1.242\end{array}$ & $\begin{array}{r}967.7 \\
1020.0 \\
1070.0 \\
1117.0 \\
1206.0 \\
1290.0 \\
1368.0 \\
1441.0 \\
1578.0 \\
1705.0\end{array}$ \\
\hline $\begin{array}{l}160.0 \\
180.0 \\
200.0 \\
250.0 \\
300.0 \\
350.0 \\
400.0 \\
450.0 \\
500.0 \\
600.0\end{array}$ & $\begin{array}{c}42.94 \\
48.31 \\
53.67 \\
67.08 \\
80.48 \\
93.89 \\
107.3 \\
120.7 \\
134.1 \\
160.9\end{array}$ & $\begin{array}{r}430.0 \\
484.0 \\
537.0 \\
671.0 \\
805.0 \\
939.0 \\
1070.0 \\
1210.0 \\
1340.0 \\
1610.0\end{array}$ & $\begin{array}{l}0.0625 \\
0.0556 \\
0.0560 \\
0.0400 \\
0.0333 \\
0.0286 \\
0.0250 \\
0.0222 \\
0.0200 \\
0.0167\end{array}$ & $\begin{array}{l}125.4 \\
140.3 \\
155.2 \\
192.5 \\
229.7 \\
267.0 \\
304.2 \\
341.4 \\
378.7 \\
453.1\end{array}$ & $\begin{array}{l}205.0 \\
229.8 \\
254.6 \\
316.7 \\
378.8 \\
440.8 \\
502.9 \\
564.9 \\
627.0 \\
751.1\end{array}$ & $\begin{array}{l}6.189 \\
6.335 \\
6.466 \\
6.743 \\
6.969 \\
7.160 \\
7.326 \\
7.472 \\
7.603 \\
7.829\end{array}$ & $\begin{array}{l}0.7448 \\
0.7448 \\
0.7448 \\
0.7448 \\
0.7448 \\
0.7448 \\
0.7448 \\
0.7447 \\
0.7447 \\
0.7447\end{array}$ & $\begin{array}{l}1.242 \\
1.241 \\
1.241 \\
1.241 \\
1.241 \\
1.241 \\
1.241 \\
1.241 \\
1.241 \\
1.241\end{array}$ & $\begin{array}{l}1822.0 \\
1932.0 \\
2037.0 \\
2276.0 \\
2493.0 \\
2693.0 \\
2879.0 \\
3053.0 \\
3218.0 \\
3525.0\end{array}$ \\
\hline $\begin{array}{r}700.0 \\
800.0 \\
900.0 \\
1000.0 \\
1200.0 \\
1400.0 \\
1600.0 \\
1800.0 \\
2000.0 \\
2500.0\end{array}$ & $\begin{array}{l}187.7 \\
214.5 \\
241.3 \\
268.2 \\
321.8 \\
375.4 \\
429.0 \\
482.6 \\
536.3 \\
670.3\end{array}$ & $\begin{array}{l}1880.0 \\
2150.0 \\
2410.0 \\
2680.0 \\
3220.0 \\
3750.0 \\
4290.0 \\
4830.0 \\
5360.0 \\
6700.0\end{array}$ & $\begin{array}{l}0.0143 \\
0.0125 \\
0.0111 \\
0.0100 \\
0.00833 \\
0.00714 \\
0.00625 \\
0.00556 \\
0.00500 \\
0.00400\end{array}$ & $\begin{array}{r}527.6 \\
602.1 \\
676.6 \\
751.0 \\
900.0 \\
1049.0 \\
1198.0 \\
1347.0 \\
1496.0 \\
1868.0\end{array}$ & $\begin{array}{r}875.2 \\
999.0 \\
1123.0 \\
1248.0 \\
1496.0 \\
1744.0 \\
1992.0 \\
2241.0 \\
2489.0 \\
3109.0\end{array}$ & $\begin{array}{l}8.021 \\
8.186 \\
8.332 \\
8.463 \\
8.690 \\
8.881 \\
9.047 \\
9.193 \\
9.324 \\
9.601\end{array}$ & $\begin{array}{l}0.7447 \\
0.7447 \\
0.7447 \\
0.7447 \\
0.7447 \\
0.7447 \\
0.7447 \\
0.7447 \\
0.7447 \\
0.7447\end{array}$ & $\begin{array}{l}1.241 \\
1.241 \\
1.241 \\
1.241 \\
1.241 \\
1.241 \\
1.241 \\
1.241 \\
1.241 \\
1.241\end{array}$ & $\begin{array}{l}3807.0 \\
4070.0 \\
4317.0 \\
4550.0 \\
4984.0 \\
5384.0 \\
5755.0 \\
6104.0 \\
6435.0 \\
7194.0\end{array}$ \\
\hline 3000.0 & 804.4 & 8040.0 & 0.00333 & 2240.0 & 3730.0 & 9.827 & 0.7447 & 1.241 & 7880.0 \\
\hline
\end{tabular}

- TWO-PHASE BOUNDARY 
THERMOPHYSICAL PRDPERTIES OF HELIUM 4

1) PSIA I SOBAR

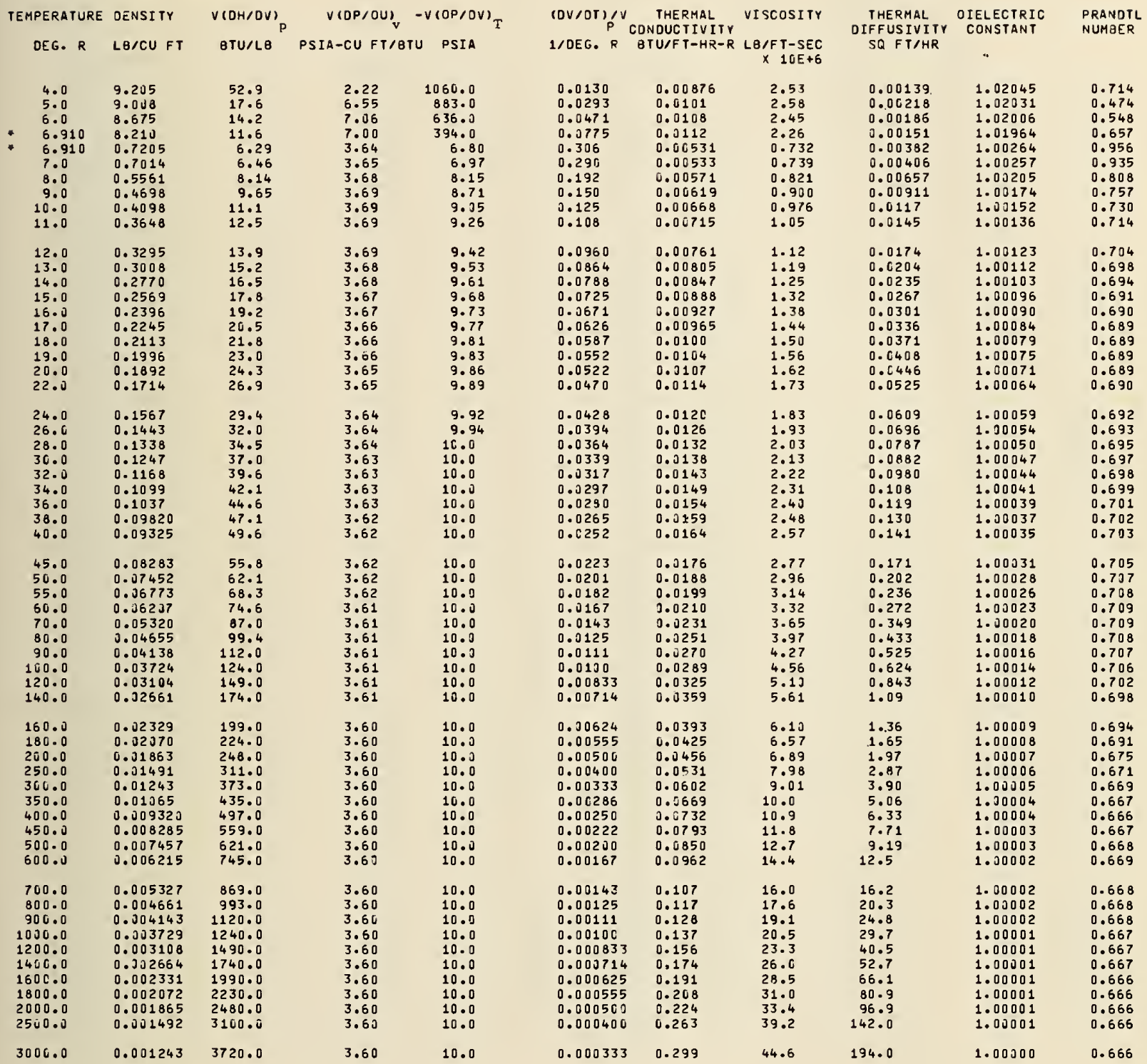

- TWD-PHASE gDUNDARY 
12 PSIA ISOBAR

THERMOOYNAMIC PROPERTIES OF HELIUM 4

\begin{tabular}{|c|c|c|c|c|c|c|c|c|c|}
\hline $\begin{array}{c}\text { TEMPERATURE } \\
\text { OEG. R }\end{array}$ & $\begin{array}{l}\text { VOLUME } \\
\text { CUFTILB }\end{array}$ & $\begin{array}{c}\text { I SOTHERM } \\
\text { OERIVATIVE } \\
\text { SU FT-PSIA L B }\end{array}$ & $\begin{array}{l}\text { ISOCHORE } \\
\text { OERIVAIIVE } \\
\text { PSIA/R }\end{array}$ & $\begin{array}{l}\text { INTERNAL } \\
\text { ENERGY } \\
\text { BTU/LB }\end{array}$ & $\begin{array}{l}\text { ENTHALPY } \\
\text { BTU/LB }\end{array}$ & $\begin{array}{l}\text { ENTROPY } \\
\text { ATU/LB-R }\end{array}$ & $\begin{array}{l}\text { CV } \\
\text { BTU, LB }\end{array}$ & $\begin{array}{l}C P \\
-R\end{array}$ & $\begin{array}{l}\text { VELOCITY } \\
\text { OF SOUNO } \\
\text { FT/SEC }\end{array}$ \\
\hline $\begin{array}{l}4.0 \\
5.0 \\
6.0 \\
7.0 \\
7.228 \\
7.228 \\
8.0 \\
9.0 \\
10.0 \\
11.0\end{array}$ & $\begin{array}{l}j .1384 \\
j .1108 \\
0.1149 \\
0.1220 \\
j .1244 \\
1.134 \\
1.427 \\
1.720 \\
1.934 \\
2.248\end{array}$ & $\begin{array}{c}116.0 \\
100.0 \\
75.3 \\
47.6 \\
40.9 \\
8.85 \\
13.0 \\
17.3 \\
21.1 \\
24.5\end{array}$ & $\begin{array}{l}13.7 \\
25.9 \\
30.1 \\
33.7 \\
30.3 \\
2.51 \\
2.00 \\
1.63 \\
1.40 \\
1.23\end{array}$ & $\begin{array}{l}1.406 \\
1.922 \\
2.496 \\
3.259 \\
3.474 \\
10.38 \\
11.22 \\
12.16 \\
13.02 \\
13.85\end{array}$ & $\begin{array}{l}1.646 \\
2.168 \\
2.751 \\
3.533 \\
3.751 \\
12.96 \\
14.39 \\
15.98 \\
17.45 \\
18.85\end{array}$ & $\begin{array}{l}0.4029 \\
0.5195 \\
0.6257 \\
0.7454 \\
0.7764 \\
2.051 \\
2.239 \\
2.427 \\
2.581 \\
2.715\end{array}$ & $\begin{array}{l}0.6650 \\
0.4378 \\
0.4884 \\
0.5352 \\
0.5467 \\
0.7967 \\
0.7707 \\
0.7558 \\
0.7491 \\
0.7460\end{array}$ & $\begin{array}{l}0.6789 \\
0.5140 \\
0.6647 \\
0.9186 \\
1.013 \\
2.083 \\
1.694 \\
1.513 \\
1.428 \\
1.381\end{array}$ & $\begin{array}{l}742.1 \\
736.9 \\
688.8 \\
614.9 \\
592.4 \\
327.6 \\
363.9 \\
400.5 \\
431.3 \\
458.7\end{array}$ \\
\hline $\begin{array}{l}12.0 \\
13.0 \\
14.0 \\
15.0 \\
16.0 \\
17.0 \\
18.0 \\
19.0 \\
20.0 \\
22.0\end{array}$ & $\begin{array}{l}2.438 \\
2.743 \\
2.984 \\
3.223 \\
3.459 \\
3.674 \\
3.928 \\
4.100 \\
4.392 \\
4.852\end{array}$ & $\begin{array}{l}27.8 \\
31.0 \\
34.1 \\
37.2 \\
40.2 \\
43.1 \\
46.5 \\
48.9 \\
51.8 \\
57.5\end{array}$ & $\begin{array}{l}1.10 \\
1.00 \\
3.920 \\
0.850 \\
0.791 \\
0.740 \\
0.695 \\
0.656 \\
0.621 \\
0.561\end{array}$ & $\begin{array}{l}14.66 \\
15.46 \\
16.25 \\
17.03 \\
17.80 \\
10.57 \\
19.34 \\
20.10 \\
20.87 \\
22.39\end{array}$ & $\begin{array}{l}20.21 \\
21.55 \\
22.88 \\
24.19 \\
25.49 \\
26.78 \\
28.07 \\
29.35 \\
30.62 \\
33.17\end{array}$ & $\begin{array}{l}2.834 \\
2.941 \\
3.039 \\
3.130 \\
3.214 \\
3.292 \\
3.365 \\
3.435 \\
3.500 \\
3.621\end{array}$ & $\begin{array}{l}0.7445 \\
0.7439 \\
0.7437 \\
0.7437 \\
0.7438 \\
0.7439 \\
0.7441 \\
0.7442 \\
0.7443 \\
0.7446\end{array}$ & $\begin{array}{l}1.351 \\
1.330 \\
1.316 \\
1.305 \\
1.296 \\
1.289 \\
1.284 \\
1.280 \\
1.276 \\
1.270\end{array}$ & $\begin{array}{l}483.8 \\
507.0 \\
528.9 \\
549.6 \\
569.4 \\
588.4 \\
606.6 \\
624.2 \\
641.2 \\
673.9\end{array}$ \\
\hline $\begin{array}{l}24.0 \\
26.0 \\
28.0 \\
31.0 \\
32.0 \\
34.0 \\
36.0 \\
38.0 \\
40.0\end{array}$ & $\begin{array}{l}5.311 \\
5.767 \\
6.223 \\
6.677 \\
7.130 \\
7.583 \\
0.034 \\
8.486 \\
8.936\end{array}$ & $\begin{array}{r}63.1 \\
68.7 \\
74.3 \\
79.8 \\
85.3 \\
90.8 \\
96.3 \\
102.0 \\
107.0\end{array}$ & $\begin{array}{l}0.512 \\
0.471 \\
0.436 \\
0.406 \\
0.380 \\
3.357 \\
0.337 \\
0.319 \\
0.302\end{array}$ & $\begin{array}{l}23.90 \\
25.41 \\
26.92 \\
28.43 \\
29.93 \\
31.44 \\
32.94 \\
34.44 \\
35.94\end{array}$ & $\begin{array}{l}35.70 \\
38.23 \\
40.75 \\
43.26 \\
45.78 \\
48.28 \\
50.79 \\
53.29 \\
55.79\end{array}$ & $\begin{array}{l}3.732 \\
3.833 \\
3.926 \\
4.013 \\
4.094 \\
4.170 \\
4.242 \\
4.309 \\
4.373\end{array}$ & $\begin{array}{l}0.7448 \\
0.7449 \\
0.7450 \\
0.7451 \\
0.7452 \\
0.7452 \\
0.7452 \\
0.7452 \\
0.7452\end{array}$ & $\begin{array}{l}1.265 \\
1.262 \\
1.259 \\
1.257 \\
1.255 \\
1.253 \\
1.252 \\
1.251 \\
1.250\end{array}$ & $\begin{array}{l}704.8 \\
734.3 \\
762.5 \\
789.7 \\
815.9 \\
841.2 \\
865.7 \\
889.6 \\
912.7\end{array}$ \\
\hline $\begin{array}{r}45.0 \\
50.0 \\
55.0 \\
60.0 \\
70.0 \\
00.0 \\
90.0 \\
160.0 \\
120.0 \\
146.0\end{array}$ & $\begin{array}{l}13.06 \\
11.19 \\
12.31 \\
13.43 \\
15.57 \\
17.31 \\
20.15 \\
22.33 \\
26.85 \\
31.33\end{array}$ & $\begin{array}{l}121.0 \\
134.0 \\
148.0 \\
161.0 \\
188.0 \\
215.0 \\
242.0 \\
269.0 \\
323.0 \\
376.0\end{array}$ & $\begin{array}{l}0.208 \\
0.241 \\
0.219 \\
0.201 \\
0.172 \\
0.150 \\
0.134 \\
0.120 \\
0.100 \\
0.0857\end{array}$ & $\begin{array}{r}39.68 \\
43.42 \\
47.16 \\
50.89 \\
58.36 \\
65.82 \\
73.28 \\
80.73 \\
95.64 \\
110.5\end{array}$ & $\begin{array}{c}62.34 \\
68.27 \\
74.51 \\
80.73 \\
93.18 \\
105.6 \\
118.0 \\
130.5 \\
155.3 \\
180.1\end{array}$ & $\begin{array}{l}4.521 \\
4.652 \\
4.771 \\
4.879 \\
5.071 \\
5.237 \\
5.383 \\
5.514 \\
5.741 \\
5.932\end{array}$ & $\begin{array}{l}0.7452 \\
0.7452 \\
0.7452 \\
0.7451 \\
0.7451 \\
0.7450 \\
0.7450 \\
0.7450 \\
0.7449 \\
0.7449\end{array}$ & $\begin{array}{l}1.248 \\
1.247 \\
1.246 \\
1.245 \\
1.244 \\
1.243 \\
1.243 \\
1.242 \\
1.242 \\
1.242\end{array}$ & $\begin{array}{r}968.2 \\
1021.0 \\
1070.0 \\
1118.0 \\
1207.0 \\
1290.0 \\
1368.0 \\
1442.0 \\
1579.0 \\
1705.0\end{array}$ \\
\hline $\begin{array}{l}160.0 \\
180.0 \\
200.0 \\
250.0 \\
300.0 \\
350.0 \\
400.0 \\
450.0 \\
500.0 \\
600.0\end{array}$ & $\begin{array}{l}35.80 \\
40.26 \\
44.73 \\
55.91 \\
67.08 \\
78.25 \\
89.42 \\
100.6 \\
111.8 \\
134.1\end{array}$ & $\begin{array}{r}430.0 \\
484.0 \\
537.0 \\
671.0 \\
806.0 \\
940.0 \\
1070.0 \\
1210.0 \\
1340.0 \\
1610.0\end{array}$ & $\begin{array}{l}0.0750 \\
0.0667 \\
3.0600 \\
0.0480 \\
0.0400 \\
0.0343 \\
0.0300 \\
0.0267 \\
0.0240 \\
0.0200\end{array}$ & $\begin{array}{l}125.4 \\
140.3 \\
155.2 \\
192.5 \\
229.7 \\
267.0 \\
304.2 \\
341.4 \\
378.7 \\
453.1\end{array}$ & $\begin{array}{l}205.0 \\
229.8 \\
254.6 \\
316.7 \\
378.8 \\
440.8 \\
502.9 \\
565.0 \\
627.0 \\
751.1\end{array}$ & $\begin{array}{l}6.098 \\
6.244 \\
6.375 \\
6.652 \\
6.878 \\
7.070 \\
7.235 \\
7.382 \\
7.512 \\
7.739\end{array}$ & $\begin{array}{l}0.7449 \\
0.7448 \\
0.7448 \\
0.7448 \\
0.7448 \\
0.7448 \\
0.7448 \\
0.7448 \\
0.7448 \\
0.7447\end{array}$ & $\begin{array}{l}1.242 \\
1.241 \\
1.241 \\
1.241 \\
1.241 \\
1.241 \\
1.241 \\
1.241 \\
1.241 \\
1.241\end{array}$ & $\begin{array}{l}1822.0 \\
1933.0 \\
2037.0 \\
2277.0 \\
2494.0 \\
2693.0 \\
2879.0 \\
3053.0 \\
3218.0 \\
3525.0\end{array}$ \\
\hline $\begin{array}{r}700.0 \\
800.0 \\
900.0 \\
1000.0 \\
1200.0 \\
1400.0 \\
1600.0 \\
1800.0 \\
2000.0 \\
2500.0\end{array}$ & $\begin{array}{l}156.4 \\
170.8 \\
201.1 \\
223.5 \\
268.2 \\
312.8 \\
357.5 \\
402.2 \\
446.9 \\
558.6\end{array}$ & $\begin{array}{l}1880.0 \\
2150.0 \\
2410.0 \\
2680.0 \\
3220.0 \\
3750.0 \\
4290.0 \\
4830.0 \\
5360.0 \\
5700.0\end{array}$ & $\begin{array}{l}0.0171 \\
0.0150 \\
0.0133 \\
0.0120 \\
0.0100 \\
0.00857 \\
0.00750 \\
0.00667 \\
0.00600 \\
0.00480\end{array}$ & $\begin{array}{r}527.6 \\
602.1 \\
676.6 \\
751.0 \\
900.0 \\
1049.0 \\
1198.0 \\
1347.0 \\
1496.0 \\
1868.0\end{array}$ & $\begin{array}{r}875.2 \\
999.0 \\
1123.0 \\
1248.0 \\
1496.0 \\
1744.0 \\
1992.0 \\
2241.0 \\
2489.0 \\
3109.0\end{array}$ & $\begin{array}{l}7.930 \\
8.096 \\
8.242 \\
8.373 \\
8.599 \\
8.790 \\
8.956 \\
9.102 \\
9.233 \\
9.510\end{array}$ & $\begin{array}{l}0.7447 \\
0.7447 \\
0.7447 \\
0.7447 \\
0.7447 \\
0.7447 \\
0.7447 \\
0.7447 \\
0.7447 \\
0.7447\end{array}$ & $\begin{array}{l}1.241 \\
1.241 \\
1.241 \\
1.241 \\
1.241 \\
1.241 \\
1.241 \\
1.241 \\
1.241 \\
1.241\end{array}$ & $\begin{array}{l}3808.0 \\
4070.0 \\
4317.0 \\
4550.0 \\
4985.0 \\
5384.0 \\
5755.0 \\
6104.0 \\
6435.0 \\
7194.0\end{array}$ \\
\hline $30 \cup[.0$ & 670.3 & 8040.0 & 0.00400 & 2240.0 & 3730.0 & 9.736 & 0.7447 & 1.241 & 7881.0 \\
\hline
\end{tabular}

- two-PHASE gOUNOARY 
12 PSIA ISOBAR

\begin{tabular}{|c|c|c|c|c|c|c|c|c|c|c|}
\hline $\begin{array}{c}\text { TEMPERATURE } \\
\text { OEG. R }\end{array}$ & $\begin{array}{l}\text { OENSITY } \\
\text { LЗ/CU FT }\end{array}$ & $\begin{array}{c}\text { V(OH/OV) } \\
\text { BTU/LB }\end{array}$ & $\begin{array}{l}V(O P / O U)^{V}- \\
\text { PSIA-CU FT/RTU }\end{array}$ & $\begin{array}{l}-V(O P / D V) T \\
\text { JSIA }\end{array}$ & $\begin{array}{l}(O V / O T) / V \\
1 / O E G . R\end{array}$ & $\begin{array}{c}\text { THERMAL } \\
\text { CONOUCTIVITY } \\
\text { BTU/FT-HR-R }\end{array}$ & $\begin{array}{l}\text { VISCOSITY } \\
\text { LB/FT-SEC } \\
\times 10 E+6\end{array}$ & $\begin{array}{l}\text { THERMAL } \\
\text { OIFFUSIVIIY } \\
\text { SQ FI/HR }\end{array}$ & $\begin{array}{l}\text { OIELECTRIC } \\
\text { CONSTANT }\end{array}$ & $\begin{array}{l}\text { PRANOTL } \\
\text { NUMBER }\end{array}$ \\
\hline $\begin{array}{l}4.0 \\
5.0 \\
6.0 \\
7.0 \\
7.228 \\
7.228 \\
8.0 \\
9.0 \\
11.0 \\
11.0\end{array}$ & $\begin{array}{l}9.223 \\
9.029 \\
8.703 \\
8.195 \\
8.037 \\
0.8594 \\
0.7008 \\
0.5814 \\
0.5025 \\
0.4449\end{array}$ & $\begin{array}{l}53.4 \\
17.9 \\
14.5 \\
11.6 \\
11.0 \\
6.32 \\
7.74 \\
9.34 \\
10.8 \\
12.3\end{array}$ & $\begin{array}{l}2.23 \\
6.55 \\
7.08 \\
7.01 \\
6.91 \\
3.66 \\
3.69 \\
3.71 \\
3.71 \\
3.71\end{array}$ & $\begin{array}{c}1070.0 \\
991.0 \\
655.0 \\
390.0 \\
329.0 \\
7.61 \\
9.12 \\
10.1 \\
10.6 \\
10.9\end{array}$ & $\begin{array}{l}0.0127 \\
0.0287 \\
0.0459 \\
0.0789 \\
0.0923 \\
0.329 \\
0.219 \\
0.162 \\
0.132 \\
0.113\end{array}$ & $\begin{array}{l}0.00877 \\
0.0102 \\
0.0108 \\
0.0112 \\
0.0113 \\
0.00567 \\
0.00585 \\
0.00628 \\
0.00674 \\
0.00721\end{array}$ & $\begin{array}{l}2.56 \\
2.60 \\
2.47 \\
2.26 \\
2.20 \\
0.779 \\
0.837 \\
0.914 \\
0.988 \\
1.06\end{array}$ & $\begin{array}{l}0.00140 \\
0.00219 \\
0.00187 \\
0.00149 \\
0.00138 \\
0.00316 \\
0.00493 \\
0.00714 \\
0.00939 \\
0.0117\end{array}$ & $\begin{array}{l}1.02046 \\
1.02033 \\
1.02008 \\
1.01963 \\
1.01947 \\
1.00312 \\
1.00257 \\
1.00214 \\
1.00186 \\
1.00165\end{array}$ & $\begin{array}{l}0.712 \\
0.475 \\
0.546 \\
0.665 \\
0.713 \\
1.03 \\
0.873 \\
0.793 \\
0.754 \\
0.731\end{array}$ \\
\hline $\begin{array}{l}12.0 \\
13.0 \\
14.0 \\
15.0 \\
16.0 \\
17.0 \\
18.0 \\
19.0 \\
20.0 \\
22.0\end{array}$ & $\begin{array}{l}0.4004 \\
0.3546 \\
0.3351 \\
0.3103 \\
0.2891 \\
0.2737 \\
0.2546 \\
0.2434 \\
0.2277 \\
0.2061\end{array}$ & $\begin{array}{l}13.6 \\
15.0 \\
16.4 \\
17.7 \\
19.0 \\
20.3 \\
21.6 \\
22.9 \\
24.2 \\
26.8\end{array}$ & $\begin{array}{l}3.70 \\
3.70 \\
3.69 \\
3.68 \\
3.68 \\
3.67 \\
3.67 \\
3.67 \\
3.66 \\
3.66\end{array}$ & $\begin{array}{l}11.1 \\
11.3 \\
11.4 \\
11.5 \\
11.6 \\
11.7 \\
11.7 \\
11.8 \\
11.8 \\
11.8\end{array}$ & $\begin{array}{l}0.0990 \\
0.0886 \\
0.0834 \\
0.0737 \\
0.0681 \\
0.0634 \\
0.0593 \\
0.0558 \\
0.0526 \\
0.0474\end{array}$ & $\begin{array}{l}0.00766 \\
0.00809 \\
0.00851 \\
0.00892 \\
0.00931 \\
0.00968 \\
0.0101 \\
0.0104 \\
0.0108 \\
0.0114\end{array}$ & $\begin{array}{l}1.13 \\
1.20 \\
1.26 \\
1.33 \\
1.39 \\
1.45 \\
1.51 \\
1.57 \\
1.62 \\
1.73\end{array}$ & $\begin{array}{l}0.0142 \\
0.0167 \\
0.0193 \\
0.0220 \\
0.0248 \\
0.0277 \\
0.0307 \\
0.0338 \\
0.0370 \\
0.0436\end{array}$ & $\begin{array}{l}1.00149 \\
1.00136 \\
1.00125 \\
1.00116 \\
1.00108 \\
1.00101 \\
1.00095 \\
1.00090 \\
1.00085 \\
1.00077\end{array}$ & $\begin{array}{l}0.718 \\
0.709 \\
0.703 \\
0.699 \\
0.696 \\
0.695 \\
0.694 \\
0.693 \\
0.693 \\
0.694\end{array}$ \\
\hline $\begin{array}{l}24.0 \\
26.0 \\
28.0 \\
30.0 \\
32.0 \\
34.0 \\
36.0 \\
38.0 \\
40.0\end{array}$ & $\begin{array}{l}0.1883 \\
0.1734 \\
0.1637 \\
0.1498 \\
0.1403 \\
0.1319 \\
0.1245 \\
0.1178 \\
0.1119\end{array}$ & $\begin{array}{l}29.4 \\
31.9 \\
34.5 \\
37.0 \\
39.5 \\
42.0 \\
44.6 \\
47.1 \\
49.6\end{array}$ & $\begin{array}{l}3.65 \\
3.65 \\
3.64 \\
3.64 \\
3.64 \\
3.63 \\
3.63 \\
3.63 \\
3.63\end{array}$ & $\begin{array}{l}11.9 \\
11.9 \\
11.9 \\
12.0 \\
12.0 \\
12.0 \\
12.0 \\
12.0 \\
12.0\end{array}$ & $\begin{array}{l}0.0431 \\
0.0395 \\
0.0365 \\
0.0340 \\
0.0318 \\
0.0298 \\
0.0281 \\
0.0266 \\
0.0252\end{array}$ & $\begin{array}{l}0.0121 \\
0.0127 \\
0.0133 \\
0.0138 \\
0.01144 \\
0.01149 \\
0.0154 \\
0.0159 \\
0.0164\end{array}$ & $\begin{array}{l}1.84 \\
1.94 \\
2.04 \\
2.13 \\
2.22 \\
2.31 \\
2.45 \\
2.49 \\
2.57\end{array}$ & $\begin{array}{l}0.0506 \\
0.0579 \\
0.0655 \\
0.0734 \\
0.0816 \\
0.0902 \\
0.0990 \\
0.108 \\
0.118\end{array}$ & $\begin{array}{l}1.00071 \\
1.00065 \\
1.00060 \\
1.00056 \\
1.00053 \\
1.00050 \\
1.00047 \\
1.00044 \\
1.00042\end{array}$ & $\begin{array}{l}0.694 \\
0.696 \\
0.697 \\
0.698 \\
0.699 \\
0.701 \\
0.702 \\
0.703 \\
0.704\end{array}$ \\
\hline $\begin{array}{r}45.0 \\
50.0 \\
55.0 \\
60.0 \\
76.0 \\
80.0 \\
90.0 \\
100.0 \\
120.0 \\
140.0\end{array}$ & $\begin{array}{l}0.09339 \\
0.08940 \\
0.08125 \\
0.07447 \\
0.06382 \\
0.05584 \\
0.04964 \\
0.04468 \\
0.03724 \\
0.03192\end{array}$ & $\begin{array}{r}55.8 \\
62.1 \\
68.3 \\
74.0 \\
87.0 \\
99.5 \\
112.0 \\
124.0 \\
149.0 \\
174.0\end{array}$ & $\begin{array}{l}3.62 \\
3.62 \\
3.62 \\
3.62 \\
3.61 \\
3.61 \\
3.61 \\
3.61 \\
3.61 \\
3.61\end{array}$ & $\begin{array}{l}12.0 \\
12.0 \\
12.0 \\
12.0 \\
12.0 \\
12.0 \\
12.0 \\
12.0 \\
12.0 \\
12.0\end{array}$ & $\begin{array}{l}0.0223 \\
0.0201 \\
0.0182 \\
0.0157 \\
0.0143 \\
0.0125 \\
0.0111 \\
0.0100 \\
0.00832 \\
0.00714\end{array}$ & $\begin{array}{l}4.0176 \\
0.0188 \\
0.0199 \\
0.0210 \\
0.0231 \\
0.0251 \\
0.0270 \\
0.3289 \\
0.3325 \\
0.0360\end{array}$ & $\begin{array}{l}2.77 \\
2.96 \\
3.15 \\
3.32 \\
3.66 \\
3.97 \\
4.27 \\
4.56 \\
5.10 \\
5.61\end{array}$ & $\begin{array}{l}0.142 \\
0.169 \\
0.197 \\
0.227 \\
0.291 \\
0.361 \\
0.438 \\
0.521 \\
0.703 \\
0.907\end{array}$ & $\begin{array}{l}1.00037 \\
1.00034 \\
1.00031 \\
1.00028 \\
1.00024 \\
1.00021 \\
1.00019 \\
1.00017 \\
1.00014 \\
1.00012\end{array}$ & $\begin{array}{l}0.706 \\
0.707 \\
0.708 \\
0.709 \\
0.709 \\
0.709 \\
0.707 \\
0.706 \\
0.702 \\
0.698\end{array}$ \\
\hline $\begin{array}{l}160.0 \\
180.0 \\
200.0 \\
250.0 \\
306.0 \\
350.0 \\
400.0 \\
450.0 \\
500.0 \\
600.0\end{array}$ & $\begin{array}{l}0.02794 \\
0.02484 \\
0.02235 \\
0.01789 \\
0.01491 \\
0.01278 \\
0.01118 \\
0.009941 \\
0.008948 \\
0.007457\end{array}$ & $\begin{array}{l}199.0 \\
224.0 \\
249.0 \\
311.0 \\
373.0 \\
435.0 \\
497.0 \\
559.0 \\
621.0 \\
745.0\end{array}$ & $\begin{array}{l}3.61 \\
3.60 \\
3.60 \\
3.60 \\
3.60 \\
3.60 \\
3.60 \\
3.60 \\
3.60 \\
3.63\end{array}$ & $\begin{array}{l}12.0 \\
12.0 \\
12.0 \\
12.0 \\
12.0 \\
12.0 \\
12.0 \\
12.0 \\
12.0 \\
12.0\end{array}$ & $\begin{array}{l}0.00624 \\
0.00555 \\
0.00500 \\
0.06400 \\
0.00333 \\
0.00286 \\
0.00250 \\
0.00222 \\
0.00200 \\
0.00167\end{array}$ & $\begin{array}{l}0.0393 \\
0.0425 \\
0.0457 \\
0.0531 \\
0.0602 \\
0.0669 \\
0.3732 \\
0.0793 \\
0.0850 \\
0.0962\end{array}$ & $\begin{array}{c}6.13 \\
6.57 \\
6.90 \\
7.98 \\
9.01 \\
10.0 \\
10.9 \\
11.8 \\
12.7 \\
14.4\end{array}$ & $\begin{array}{c}1.13 \\
1.38 \\
1.64 \\
2.39 \\
3.25 \\
4.22 \\
5.28 \\
6.43 \\
7.66 \\
10.4\end{array}$ & $\begin{array}{l}1.00011 \\
1.00009 \\
1.00008 \\
1.00007 \\
1.00006 \\
1.00005 \\
1.00004 \\
1.00004 \\
1.00003 \\
1.00003\end{array}$ & $\begin{array}{l}0.694 \\
0.691 \\
0.675 \\
0.671 \\
0.669 \\
0.667 \\
0.666 \\
0.667 \\
0.668 \\
0.669\end{array}$ \\
\hline $\begin{array}{r}700.0 \\
800.0 \\
900.0 \\
1000.0 \\
1200.0 \\
1400.0 \\
1600.0 \\
1800.0 \\
2000.0 \\
2500.0\end{array}$ & $\begin{array}{l}0.006392 \\
0.005593 \\
0.004972 \\
0.004475 \\
0.003729 \\
0.003197 \\
0.002797 \\
0.302486 \\
0.002238 \\
0.001790\end{array}$ & $\begin{array}{r}869.0 \\
993.0 \\
1120.0 \\
1240.0 \\
1490.0 \\
1740.0 \\
1990.0 \\
2230.0 \\
2480.0 \\
3100.0\end{array}$ & $\begin{array}{l}3.60 \\
3.60 \\
3.60 \\
3.60 \\
3.60 \\
3.60 \\
3.60 \\
3.60 \\
3.60 \\
3.60\end{array}$ & $\begin{array}{l}12.0 \\
12.0 \\
12.0 \\
12.0 \\
12.0 \\
12.0 \\
12.0 \\
12.0 \\
12.0 \\
12.0\end{array}$ & $\begin{array}{l}0.00143 \\
0.00125 \\
0.00111 \\
0.00100 \\
0.000833 \\
0.000714 \\
0.000625 \\
0.000555 \\
0.000500 \\
0.000400\end{array}$ & $\begin{array}{l}0.107 \\
0.117 \\
0.128 \\
0.137 \\
0.156 \\
0.174 \\
0.191 \\
0.208 \\
0.224 \\
0.263\end{array}$ & $\begin{array}{l}16.0 \\
17.6 \\
19.1 \\
20.5 \\
23.3 \\
26.0 \\
28.5 \\
31.0 \\
33.4 \\
39.2\end{array}$ & $\begin{array}{r}13.5 \\
16.9 \\
20.7 \\
24.7 \\
33.7 \\
43.9 \\
55.1 \\
67.4 \\
80.7 \\
118.0\end{array}$ & $\begin{array}{l}1.00002 \\
1.00002 \\
1.00002 \\
1.00002 \\
1.00001 \\
1.00001 \\
1.00001 \\
1.00001 \\
1.00001 \\
1.00001\end{array}$ & $\begin{array}{l}0.668 \\
0.668 \\
0.668 \\
0.667 \\
0.667 \\
0.667 \\
0.666 \\
0.666 \\
0.666 \\
0.666\end{array}$ \\
\hline 3000.0 & 0.031492 & 3720.0 & 3.63 & 12.0 & 0.000333 & 0.299 & 44.6 & 162.0 & 1.00001 & 0.666 \\
\hline
\end{tabular}




\begin{tabular}{|c|c|c|c|c|c|c|c|c|c|}
\hline $\begin{array}{c}\text { TEMPERATURE } \\
\text { DEG. R }\end{array}$ & $\begin{array}{l}\text { E VOLUME } \\
\text { CU FT/LB }\end{array}$ & $\begin{array}{c}\text { ISOTHERM } \\
\text { OERIVATIVE } \\
\text { CU FT PSIA/LB }\end{array}$ & $\begin{array}{l}\text { IS OCHORE } \\
\text { DERIVATIVE } \\
\text { PSIA/R }\end{array}$ & $\begin{array}{l}\text { INTERNAL } \\
\text { ENERGY } \\
\text { BTU/LB }\end{array}$ & $\begin{array}{l}\text { ENT HALPY } \\
\text { BTU } / L B\end{array}$ & $\begin{array}{l}\text { ENTROPY } \\
\text { BTU/LB-R }\end{array}$ & $\begin{array}{l}\text { CV } \\
\text { BTU / LB }\end{array}$ & $\begin{array}{l}C P \\
-R\end{array}$ & $\begin{array}{l}\text { VELOCITY } \\
\text { OF SOUND } \\
\text { FT/SEC }\end{array}$ \\
\hline $\begin{array}{l}4.0 \\
5.0 \\
6.0 \\
7.0 \\
7.604 \\
7.604 \\
8.0 \\
9.0 \\
10.0 \\
11.0\end{array}$ & $\begin{array}{l}0.1081 \\
0.1104 \\
0.1144 \\
0.1212 \\
0.1282 \\
0.9484 \\
1.075 \\
1.341 \\
1.575 \\
1.794\end{array}$ & $\begin{array}{c}119.0 \\
102.0 \\
77.9 \\
50.4 \\
32.7 \\
7.98 \\
10.5 \\
15.5 \\
19.7 \\
23.4\end{array}$ & $\begin{array}{l}13.6 \\
25.9 \\
30.3 \\
31.1 \\
29.7 \\
3.11 \\
2.70 \\
2.12 \\
1.78 \\
1.55\end{array}$ & $\begin{array}{l}1.402 \\
1.912 \\
2.478 \\
3.227 \\
3.829 \\
10.38 \\
10.88 \\
11.92 \\
12.83 \\
13.69\end{array}$ & $\begin{array}{l}1.696 \\
2.213 \\
2.790 \\
3.556 \\
4.178 \\
12.96 \\
13.80 \\
15.57 \\
17.12 \\
18.58\end{array}$ & $\begin{array}{l}0.4010 \\
0.5174 \\
0.6226 \\
0.7405 \\
0.8255 \\
1.981 \\
2.089 \\
2.297 \\
2.460 \\
2.599\end{array}$ & $\begin{array}{l}0.6574 \\
0.4369 \\
0.4877 \\
0.5338 \\
0.5659 \\
0.8002 \\
0.7830 \\
0.7609 \\
0.7514 \\
0.7470\end{array}$ & $\begin{array}{l}0.6709 \\
0.5110 \\
0.6587 \\
0.8991 \\
1.191 \\
2.337 \\
1.970 \\
1.626 \\
1.493 \\
1.424\end{array}$ & $\begin{array}{l}749.5 \\
744.5 \\
697.9 \\
627.3 \\
564.2 \\
328.6 \\
350.3 \\
392.1 \\
425.4 \\
454.3\end{array}$ \\
\hline $\begin{array}{l}12.0 \\
13.0 \\
14.0 \\
15.0 \\
16.0 \\
17.0 \\
18.0 \\
19.0 \\
20.0 \\
22.0\end{array}$ & $\begin{array}{l}2.004 \\
2.209 \\
2.410 \\
2.608 \\
2.803 \\
2.997 \\
3.190 \\
3.381 \\
3.572 \\
3.951\end{array}$ & $\begin{array}{l}26.8 \\
30.2 \\
33.4 \\
36.5 \\
39.5 \\
42.6 \\
45.5 \\
48.5 \\
51.4 \\
57.1\end{array}$ & $\begin{array}{l}1.38 \\
1.25 \\
1.14 \\
1.06 \\
0.981 \\
0.916 \\
0.859 \\
0.810 \\
0.766 \\
0.691\end{array}$ & $\begin{array}{l}14.52 \\
15.33 \\
16.13 \\
16.92 \\
17.70 \\
18.48 \\
19.25 \\
20.02 \\
20.79 \\
22.32\end{array}$ & $\begin{array}{l}19.98 \\
21.34 \\
22.69 \\
24.02 \\
25.33 \\
26.64 \\
27.93 \\
29.22 \\
30.51 \\
33.07\end{array}$ & $\begin{array}{l}2.721 \\
2.831 \\
2.930 \\
3.022 \\
3.107 \\
3.186 \\
3.260 \\
3.330 \\
3.396 \\
3.518\end{array}$ & $\begin{array}{l}0.7449 \\
0.7440 \\
0.7436 \\
0.7435 \\
0.7436 \\
0.7438 \\
0.7439 \\
0.7441 \\
0.7443 \\
0.7446\end{array}$ & $\begin{array}{l}1.382 \\
1.355 \\
1.335 \\
1.321 \\
1.310 \\
1.301 \\
1.294 \\
1.289 \\
1.284 \\
1.277\end{array}$ & $\begin{array}{l}480.3 \\
504.3 \\
526.7 \\
547.9 \\
568.0 \\
587.3 \\
605.7 \\
623.5 \\
640.7 \\
673.6\end{array}$ \\
\hline $\begin{array}{l}24.0 \\
26.0 \\
28.0 \\
30.0 \\
32.0 \\
34.0 \\
36.0 \\
38.0 \\
40.0\end{array}$ & $\begin{array}{l}4.327 \\
4.702 \\
5.075 \\
5.447 \\
5.818 \\
6.189 \\
6.559 \\
6.928 \\
7.297\end{array}$ & $\begin{array}{r}62.8 \\
68.5 \\
74.1 \\
79.7 \\
85.2 \\
90.7 \\
96.3 \\
102.0 \\
107.0\end{array}$ & $\begin{array}{l}0.630 \\
0.579 \\
0.536 \\
0.499 \\
0.467 \\
0.438 \\
0.413 \\
0.391 \\
0.371\end{array}$ & $\begin{array}{l}23.84 \\
25.35 \\
26.87 \\
28.38 \\
29.88 \\
31.39 \\
32.89 \\
34.40 \\
35.90\end{array}$ & $\begin{array}{l}35.61 \\
38.15 \\
40.60 \\
43.20 \\
45.72 \\
48.23 \\
50.74 \\
53.25 \\
55.76\end{array}$ & $\begin{array}{l}3.628 \\
3.730 \\
3.824 \\
3.911 \\
3.992 \\
4.068 \\
4.140 \\
4.208 \\
4.272\end{array}$ & $\begin{array}{l}0.7448 \\
0.7450 \\
0.7451 \\
0.7452 \\
0.7453 \\
0.7453 \\
0.7453 \\
0.7453 \\
0.7453\end{array}$ & $\begin{array}{l}1.271 \\
1.267 \\
1.263 \\
1.260 \\
1.258 \\
1.256 \\
1.254 \\
1.253 \\
1.252\end{array}$ & $\begin{array}{l}704.8 \\
734.5 \\
762.8 \\
790.1 \\
816.3 \\
841.7 \\
866.3 \\
890.2 \\
913.4\end{array}$ \\
\hline $\begin{array}{r}45.0 \\
50.0 \\
55.0 \\
60.0 \\
70.0 \\
80.0 \\
90.0 \\
100.0 \\
120.0 \\
140.0\end{array}$ & $\begin{array}{l}8.217 \\
9.136 \\
10.05 \\
10.97 \\
12.80 \\
14.63 \\
16.46 \\
18.28 \\
21.94 \\
25.59\end{array}$ & $\begin{array}{l}121.0 \\
134.0 \\
148.0 \\
162.0 \\
189.0 \\
215.0 \\
242.0 \\
269.0 \\
323.0 \\
377.0\end{array}$ & $\begin{array}{l}0.329 \\
0.296 \\
0.269 \\
0.246 \\
0.211 \\
0.184 \\
0.164 \\
0.147 \\
0.123 \\
0.105\end{array}$ & $\begin{array}{r}39.65 \\
43.39 \\
47.13 \\
50.87 \\
58.34 \\
65.80 \\
73.26 \\
80.72 \\
95.63 \\
110.5\end{array}$ & $\begin{array}{c}62.01 \\
68.25 \\
74.49 \\
80.72 \\
93.17 \\
105.6 \\
118.0 \\
130.5 \\
155.3 \\
180.2\end{array}$ & $\begin{array}{l}4.419 \\
4.551 \\
4.670 \\
4.778 \\
4.970 \\
5.136 \\
5.283 \\
5.414 \\
5.640 \\
5.832\end{array}$ & $\begin{array}{l}0.7453 \\
0.7453 \\
0.7453 \\
0.7452 \\
0.7452 \\
0.7451 \\
0.7451 \\
0.7450 \\
0.7450 \\
0.7449\end{array}$ & $\begin{array}{l}1.250 \\
1.248 \\
1.247 \\
1.246 \\
1.245 \\
1.244 \\
1.243 \\
1.243 \\
1.242 \\
1.242\end{array}$ & $\begin{array}{r}968.9 \\
1021.0 \\
1071.0 \\
1118.0 \\
1208.0 \\
1291.0 \\
1369.0 \\
1442.0 \\
1580.0 \\
1706.0\end{array}$ \\
\hline $\begin{array}{l}160.0 \\
180.0 \\
200.0 \\
250.0 \\
300.0 \\
350.0 \\
400.0 \\
450.0 \\
500.0 \\
600.0\end{array}$ & $\begin{array}{c}29.24 \\
32.89 \\
36.54 \\
45.66 \\
54.78 \\
63.90 \\
73.02 \\
82.15 \\
91.27 \\
109.5\end{array}$ & $\begin{array}{r}430.0 \\
484.0 \\
538.0 \\
672.0 \\
806.0 \\
940.0 \\
1070.0 \\
1210.0 \\
1340.0 \\
1610.0\end{array}$ & $\begin{array}{l}0.0919 \\
0.0817 \\
0.0735 \\
0.0588 \\
0.0490 \\
0.0420 \\
0.0367 \\
0.0327 \\
0.0294 \\
0.0245\end{array}$ & $\begin{array}{l}125.4 \\
140.3 \\
155.2 \\
192.5 \\
229.7 \\
267.0 \\
304.2 \\
341.4 \\
378.7 \\
453.1\end{array}$ & $\begin{array}{l}205.0 \\
229.8 \\
254.7 \\
316.7 \\
378.8 \\
440.9 \\
502.9 \\
565.0 \\
627.0 \\
751.2\end{array}$ & $\begin{array}{l}5.997 \\
6.144 \\
6.274 \\
6.551 \\
6.778 \\
6.969 \\
7.135 \\
7.281 \\
7.412 \\
7.638\end{array}$ & $\begin{array}{l}0.7449 \\
0.7449 \\
0.7449 \\
0.7448 \\
0.7448 \\
0.7448 \\
0.7448 \\
0.7448 \\
0.7448 \\
0.7448\end{array}$ & $\begin{array}{l}1.242 \\
1.242 \\
1.241 \\
1.241 \\
1.241 \\
1.241 \\
1.241 \\
1.241 \\
1.241 \\
1.241\end{array}$ & $\begin{array}{l}1823.0 \\
1933.0 \\
2037.0 \\
2277.0 \\
2494.0 \\
2694.0 \\
2879.0 \\
3054.0 \\
3219.0 \\
3526.0\end{array}$ \\
\hline $\begin{array}{r}700.0 \\
800.0 \\
900.0 \\
1000.0 \\
1200.0 \\
1400.0 \\
1600.0 \\
1800.0 \\
2000.0 \\
2500.0\end{array}$ & $\begin{array}{l}127.8 \\
146.0 \\
164.2 \\
182.5 \\
219.0 \\
255.5 \\
291.9 \\
328.4 \\
364.9 \\
456.1\end{array}$ & $\begin{array}{l}1880.0 \\
2150.0 \\
2410.0 \\
2680.0 \\
3220.0 \\
3750.0 \\
4290.0 \\
4830.0 \\
5360.0 \\
6700.0\end{array}$ & $\begin{array}{l}0.0210 \\
0.0184 \\
0.0163 \\
0.0147 \\
0.0122 \\
0.0105 \\
0.00918 \\
0.00816 \\
0.00735 \\
0.00588\end{array}$ & $\begin{array}{r}527.6 \\
602.1 \\
676.6 \\
751.0 \\
900.0 \\
1049.0 \\
1198.0 \\
1347.0 \\
1496.0 \\
1868.0\end{array}$ & $\begin{array}{r}875.3 \\
999.0 \\
1124.0 \\
1248.0 \\
1496.0 \\
1744.0 \\
1992.0 \\
2241.0 \\
2489.0 \\
3109.0\end{array}$ & $\begin{array}{l}7.829 \\
7.995 \\
8.141 \\
8.272 \\
8.498 \\
8.690 \\
8.855 \\
9.002 \\
9.132 \\
9.409\end{array}$ & $\begin{array}{l}0.7447 \\
0.7447 \\
0.7447 \\
0.7447 \\
0.7447 \\
0.7447 \\
0.7447 \\
0.7447 \\
0.7447 \\
0.7447\end{array}$ & $\begin{array}{l}1.241 \\
1.241 \\
1.241 \\
1.241 \\
1.241 \\
1.241 \\
1.241 \\
1.241 \\
1.241 \\
1.241\end{array}$ & $\begin{array}{l}3808.0 \\
4070.0 \\
4317.0 \\
4551.0 \\
4985.0 \\
5384.0 \\
5756.0 \\
6105.0 \\
6435.0 \\
7194.0\end{array}$ \\
\hline 3000.0 & 547.3 & 8040.0 & 0.00490 & 2240.0 & 3730.0 & 9.636 & 0.7447 & 1.241 & 7881.0 \\
\hline
\end{tabular}


14.096 PSIA ISOBAR

\begin{tabular}{|c|c|c|c|c|c|c|c|c|c|c|}
\hline $\begin{array}{c}\text { TEMPERATURE } \\
\text { OEG. R }\end{array}$ & DENSITY & $\begin{array}{c}V(\mathrm{OH} / \mathrm{OV})_{\mathrm{P}} \\
\text { BTU/LB }\end{array}$ & $\begin{array}{l}V(O P / O U)_{V}-V \\
P S I A-C U F T / B T U\end{array}$ & $\begin{array}{l}V(O P / O V)_{T} \\
\text { PSIA }\end{array}$ & $\begin{array}{l}\text { COV/OT)/V } \\
1 / O E G . R\end{array}$ & $\begin{array}{c}\text { THERMAL } \\
\text { CONDUCTIVITY } \\
\text { BTU/FT-HR-R }\end{array}$ & $\begin{array}{l}\text { VISCOSITY } \\
\text { LB/FT-SEC } \\
\times \quad 10 E+6\end{array}$ & $\begin{array}{l}\text { THERMAL } \\
\text { OIFFUSIVITY } \\
\text { SQ FT/HR }\end{array}$ & $\begin{array}{l}\text { OIELECTRIC } \\
\text { CONST ANT }\end{array}$ & $\begin{array}{l}\text { PRANOTL } \\
\text { NUMBER }\end{array}$ \\
\hline $\begin{array}{l}4.0 \\
5.0 \\
6.0 \\
7.0 \\
7.604 \\
7.604 \\
8.0 \\
9.0 \\
10.0 \\
11.0\end{array}$ & $\begin{array}{l}9.240 \\
9.057 \\
8.740 \\
8.250 \\
7.832 \\
1.354 \\
0.9331 \\
0.7456 \\
0.6350 \\
0.5575\end{array}$ & $\begin{array}{c}54.1 \\
18.3 \\
14.8 \\
12.0 \\
10.2 \\
6.32 \\
7.14 \\
8.89 \\
10.5 \\
11.9\end{array}$ & $\begin{array}{l}2.24 \\
6.55 \\
7.10 \\
7.06 \\
6.73 \\
3.69 \\
3.71 \\
3.73 \\
3.73 \\
3.73\end{array}$ & $\begin{array}{c}1100.0 \\
927.0 \\
680.0 \\
416.0 \\
255.0 \\
8.42 \\
9.79 \\
11.6 \\
12.5 \\
13.0\end{array}$ & $\begin{array}{l}0.0124 \\
0.0280 \\
0.0445 \\
0.0747 \\
0.117 \\
0.370 \\
0.276 \\
0.183 \\
0.143 \\
0.119\end{array}$ & $\begin{array}{l}0.06880 \\
0.0102 \\
0.0109 \\
0.0113 \\
0.0114 \\
0.00614 \\
0.00611 \\
0.00642 \\
0.00684 \\
0.00729\end{array}$ & $\begin{array}{l}2.58 \\
2.63 \\
2.50 \\
2.29 \\
2.13 \\
0.838 \\
0.863 \\
0.935 \\
1.01 \\
1.08\end{array}$ & $\begin{array}{l}0.00142 \\
0.06220 \\
0.00189 \\
0.00153 \\
0.00122 \\
0.00249 \\
0.00334 \\
0.00529 \\
0.00722 \\
0.00918\end{array}$ & $\begin{array}{l}1.02047 \\
1.02035 \\
1.02011 \\
1.01968 \\
1.01921 \\
1.00380 \\
1.00337 \\
1.00273 \\
1.00233 \\
1.00205\end{array}$ & $\begin{array}{l}0.709 \\
0.475 \\
0.545 \\
0.656 \\
0.804 \\
1.15 \\
1.00 \\
0.852 \\
0.790 \\
0.757\end{array}$ \\
\hline $\begin{array}{l}12.0 \\
13.0 \\
14.0 \\
15.0 \\
16.0 \\
17.0 \\
18.0 \\
19.0 \\
20.0 \\
22.0\end{array}$ & $\begin{array}{l}0.4990 \\
0.4527 \\
0.4150 \\
0.3835 \\
0.3507 \\
0.3336 \\
0.3135 \\
0.2957 \\
0.2800 \\
0.2531\end{array}$ & $\begin{array}{l}13.4 \\
14.8 \\
16.1 \\
17.5 \\
18.8 \\
20.2 \\
21.5 \\
22.8 \\
24.1 \\
26.7\end{array}$ & $\begin{array}{l}3.72 \\
3.72 \\
3.71 \\
3.70 \\
3.70 \\
3.69 \\
3.69 \\
3.68 \\
3.68 \\
3.67\end{array}$ & $\begin{array}{l}13.4 \\
13.7 \\
13.8 \\
14.3 \\
14.1 \\
14.2 \\
14.3 \\
14.3 \\
14.4 \\
14.5\end{array}$ & $\begin{array}{l}0.103 \\
0.0917 \\
0.0827 \\
0.0755 \\
0.0695 \\
0.0645 \\
0.0602 \\
0.0565 \\
0.0533 \\
0.0478\end{array}$ & $\begin{array}{l}0.00773 \\
0.00815 \\
0.00857 \\
0.00897 \\
0.00936 \\
0.00973 \\
0.0101 \\
0.0105 \\
0.0108 \\
0.0115\end{array}$ & $\begin{array}{l}1.14 \\
1.21 \\
1.28 \\
1.34 \\
1.40 \\
1.46 \\
1.52 \\
1.58 \\
1.63 \\
1.74\end{array}$ & $\begin{array}{l}0.0112 \\
0.0133 \\
0.0155 \\
0.0177 \\
0.0200 \\
0.0224 \\
0.0249 \\
0.0274 \\
0.0300 \\
0.0355\end{array}$ & $\begin{array}{l}1.00184 \\
1.00168 \\
1.00154 \\
1.00142 \\
1.00133 \\
1.00124 \\
1.00117 \\
1.00110 \\
1.00104 \\
1.00095\end{array}$ & $\begin{array}{l}0.737 \\
0.724 \\
0.715 \\
0.709 \\
0.705 \\
0.702 \\
0.700 \\
0.699 \\
0.699 \\
0.698\end{array}$ \\
\hline $\begin{array}{l}24.0 \\
26.0 \\
28.0 \\
30.0 \\
32.0 \\
34.0 \\
36.0 \\
38.0 \\
40.0\end{array}$ & $\begin{array}{l}0.2311 \\
0.2127 \\
0.1970 \\
0.1836 \\
0.1719 \\
0.1616 \\
0.1525 \\
0.1443 \\
0.1370\end{array}$ & $\begin{array}{l}29.3 \\
31.8 \\
34.4 \\
36.9 \\
39.5 \\
42.0 \\
44.5 \\
47.1 \\
49.6\end{array}$ & $\begin{array}{l}3.66 \\
3.66 \\
3.65 \\
3.65 \\
3.64 \\
3.64 \\
3.64 \\
3.64 \\
3.63\end{array}$ & $\begin{array}{l}14.5 \\
14.6 \\
14.6 \\
14.6 \\
14.6 \\
14.7 \\
14.7 \\
14.7 \\
14.7\end{array}$ & $\begin{array}{l}0.0434 \\
0.0398 \\
0.0367 \\
0.0341 \\
0.0319 \\
0.0299 \\
0.0282 \\
0.0266 \\
0.0253\end{array}$ & $\begin{array}{l}0.0121 \\
0.0127 \\
0.0133 \\
0.0139 \\
0.0144 \\
0.0149 \\
0.0155 \\
0.0160 \\
0.0165\end{array}$ & $\begin{array}{l}1.84 \\
1.95 \\
2.04 \\
2.14 \\
2.23 \\
2.32 \\
2.41 \\
2.49 \\
2.58\end{array}$ & $\begin{array}{l}0.0412 \\
0.0471 \\
0.0534 \\
0.0599 \\
0.0666 \\
0.0736 \\
0.0808 \\
0.0883 \\
0.0960\end{array}$ & $\begin{array}{l}1.00086 \\
1.00080 \\
1.00074 \\
1.00069 \\
1.00064 \\
1.00061 \\
1.00057 \\
1.00054 \\
1.00051\end{array}$ & $\begin{array}{l}0.698 \\
0.699 \\
0.699 \\
0.700 \\
0.701 \\
0.702 \\
0.703 \\
0.704 \\
0.705\end{array}$ \\
\hline $\begin{array}{r}45.0 \\
50.0 \\
55.0 \\
60.0 \\
70.0 \\
80.0 \\
90.0 \\
100.0 \\
120.0 \\
140.0\end{array}$ & $\begin{array}{l}0.1217 \\
0.1095 \\
0.09947 \\
0.09116 \\
0.07812 \\
0.06836 \\
0.06077 \\
0.05469 \\
0.04559 \\
0.03908\end{array}$ & $\begin{array}{r}55.8 \\
62.1 \\
68.4 \\
74.6 \\
87.1 \\
100.0 \\
112.0 \\
124.0 \\
149.0 \\
174.0\end{array}$ & $\begin{array}{l}3.63 \\
3.63 \\
3.62 \\
3.62 \\
3.62 \\
3.61 \\
3.61 \\
3.61 \\
3.61 \\
3.61\end{array}$ & $\begin{array}{l}14.7 \\
14.7 \\
14.7 \\
14.7 \\
14.7 \\
14.7 \\
14.7 \\
14.7 \\
14.7 \\
14.7\end{array}$ & $\begin{array}{l}0.0224 \\
0.02 J 1 \\
0.0182 \\
0.0167 \\
0.0143 \\
0.0125 \\
0.0111 \\
0.0100 \\
0.00832 \\
0.00713\end{array}$ & $\begin{array}{l}0.0177 \\
0.0188 \\
0.0199 \\
0.0216 \\
0.0231 \\
0.0251 \\
0.0270 \\
0.0289 \\
0.0325 \\
0.0360\end{array}$ & $\begin{array}{l}2.78 \\
2.97 \\
3.15 \\
3.33 \\
3.66 \\
3.97 \\
4.27 \\
4.56 \\
5.10 \\
5.61\end{array}$ & $\begin{array}{l}0.116 \\
0.138 \\
0.161 \\
0.185 \\
0.238 \\
0.295 \\
0.358 \\
0.425 \\
0.574 \\
0.741\end{array}$ & $\begin{array}{l}1.00046 \\
1.00041 \\
1.00037 \\
1.00034 \\
1.00029 \\
1.00026 \\
1.00023 \\
1.00021 \\
1.00017 \\
1.00015\end{array}$ & $\begin{array}{l}0.707 \\
0.708 \\
0.709 \\
0.709 \\
0.709 \\
0.709 \\
0.707 \\
0.706 \\
0.702 \\
0.698\end{array}$ \\
\hline $\begin{array}{l}160.0 \\
180.0 \\
200.0 \\
250.0 \\
360.0 \\
350.0 \\
400.0 \\
450.0 \\
500.0 \\
600.0\end{array}$ & $\begin{array}{l}0.03420 \\
0.03341 \\
0.02737 \\
0.02190 \\
0.01825 \\
0.01565 \\
0.01369 \\
0.01217 \\
0.31096 \\
0.009132\end{array}$ & $\begin{array}{l}199.0 \\
224.0 \\
249.0 \\
311.0 \\
373.0 \\
435.0 \\
497.0 \\
559.0 \\
621.0 \\
745.0\end{array}$ & $\begin{array}{l}3.61 \\
3.61 \\
3.60 \\
3.60 \\
3.60 \\
3.60 \\
3.60 \\
3.60 \\
3.60 \\
3.60\end{array}$ & $\begin{array}{l}14.7 \\
14.7 \\
14.7 \\
14.7 \\
14.7 \\
14.7 \\
14.7 \\
14.7 \\
14.7 \\
14.7\end{array}$ & $\begin{array}{l}0.00624 \\
0.00555 \\
0.00499 \\
0.00400 \\
0.00333 \\
0.00285 \\
0.00250 \\
0.00222 \\
0.00200 \\
0.00167\end{array}$ & $\begin{array}{l}0.0393 \\
0.0425 \\
0.0457 \\
0.0532 \\
0.0602 \\
0.0669 \\
0.0733 \\
0.0793 \\
0.0851 \\
0.0962\end{array}$ & $\begin{array}{c}6.10 \\
6.57 \\
6.90 \\
7.98 \\
9.01 \\
10.0 \\
10.9 \\
11.8 \\
12.7 \\
14.4\end{array}$ & $\begin{array}{l}0.925 \\
1.13 \\
1.34 \\
1.96 \\
2.66 \\
3.44 \\
4.31 \\
5.25 \\
6.25 \\
8.49\end{array}$ & $\begin{array}{l}1.00013 \\
1.00011 \\
1.00010 \\
1.00008 \\
1.00007 \\
1.00006 \\
1.00005 \\
1.00005 \\
1.00004 \\
1.00003\end{array}$ & $\begin{array}{l}0.694 \\
0.691 \\
0.675 \\
0.671 \\
0.669 \\
0.667 \\
0.666 \\
0.667 \\
0.668 \\
0.669\end{array}$ \\
\hline $\begin{array}{r}700.0 \\
800.0 \\
900.0 \\
1000.0 \\
1200.0 \\
1400.0 \\
1600.0 \\
1800.0 \\
2000.0 \\
2500.0\end{array}$ & $\begin{array}{l}0.007828 \\
0.00685 J \\
0.006089 \\
0.035483 \\
0.004567 \\
0.003915 \\
0.003425 \\
0.003045 \\
0.002740 \\
0.302192\end{array}$ & $\begin{array}{r}869.0 \\
993.0 \\
1120.0 \\
1240.0 \\
1490.0 \\
1740.0 \\
1990.0 \\
2230.0 \\
2480.0 \\
3100.0\end{array}$ & $\begin{array}{l}3.60 \\
3.60 \\
3.60 \\
3.60 \\
3.60 \\
3.60 \\
3.60 \\
3.60 \\
3.60 \\
3.60\end{array}$ & $\begin{array}{l}14.7 \\
14.7 \\
14.7 \\
14.7 \\
14.7 \\
14.7 \\
14.7 \\
14.7 \\
14.7 \\
14.7\end{array}$ & $\begin{array}{l}0.00143 \\
0.00125 \\
0.00111 \\
0.00100 \\
0.060833 \\
0.000714 \\
0.000625 \\
0.000555 \\
0.000500 \\
0.000400\end{array}$ & $\begin{array}{l}0.107 \\
0.117 \\
0.128 \\
0.137 \\
0.156 \\
0.174 \\
0.191 \\
0.208 \\
0.224 \\
0.263\end{array}$ & $\begin{array}{l}16.0 \\
17.6 \\
19.1 \\
20.5 \\
23.3 \\
26.0 \\
28.5 \\
31.0 \\
33.4 \\
39.2\end{array}$ & $\begin{array}{l}11.0 \\
13.8 \\
16.9 \\
20.2 \\
27.5 \\
35.8 \\
45.0 \\
55.0 \\
65.9 \\
96.6\end{array}$ & $\begin{array}{l}1.00003 \\
1.00003 \\
1.00002 \\
1.00002 \\
1.00002 \\
1.00001 \\
1.00001 \\
1.00001 \\
1.00001 \\
1.00001\end{array}$ & $\begin{array}{l}0.668 \\
0.668 \\
0.668 \\
0.667 \\
0.667 \\
0.667 \\
0.666 \\
0.666 \\
0.6666 \\
0.6666\end{array}$ \\
\hline 3000.0 & [. 311827 & 3720.0 & 3.60 & 14.7 & $0.0 \cos 33$ & 0.299 & 44.6 & 132.0 & 1.00001 & 0.666 \\
\hline
\end{tabular}


16 PSIA ISOBAR

THERMOOYNAMIC PROPERTIES OF HELIUM 4

\begin{tabular}{|c|c|c|c|c|c|c|c|c|c|c|}
\hline $\begin{array}{c}\text { TEMPERATURE } \\
\text { OEG. } R\end{array}$ & $\begin{array}{l}\text { VOLUME } \\
\text { CUFT/LB }\end{array}$ & $\begin{array}{c}\text { ISOTHERM } \\
\text { OERIVATIVE } \\
\text { CU FT-PSIA/LB }\end{array}$ & $\begin{array}{l}\text { ISOCHORE } \\
\text { OERIVATIVE } \\
\text { PSIA/R }\end{array}$ & $\begin{array}{l}\text { INTERNAL } \\
\text { ENERGY } \\
\text { BTU/LB }\end{array}$ & $\begin{array}{l}\text { ENTHALPY } \\
\text { BTU/LB }\end{array}$ & $\begin{array}{l}\text { ENTROPY } \\
\text { BTU/LB-R }\end{array}$ & $\begin{array}{l}\text { CV } \\
\text { BTU }\end{array}$ & , L8 & $\begin{array}{l}C P \\
-R\end{array}$ & $\begin{array}{l}\text { VELOCI IY } \\
\text { OF SOUND } \\
\text { FT/SEC }\end{array}$ \\
\hline $\begin{array}{l}4.0 \\
5.0 \\
6.0 \\
7.0 \\
7.769 \\
+7.769 \\
8.0 \\
9.0 \\
10.0 \\
11.0\end{array}$ & $\begin{array}{l}0.1080 \\
0.1102 \\
j .1142 \\
0.1208 \\
0.1301 \\
0.8673 \\
0.9411 \\
1.232 \\
1.423 \\
1.629\end{array}$ & $\begin{array}{c}120.0 \\
103.0 \\
79.1 \\
51.8 \\
29.1 \\
7.53 \\
9.17 \\
14.6 \\
19.0 \\
22.8\end{array}$ & $\begin{array}{l}13.6 \\
25.9 \\
30.3 \\
31.3 \\
29.3 \\
3.42 \\
3.12 \\
2.38 \\
1.98 \\
1.72\end{array}$ & $\begin{array}{c}1.400 \\
1.908 \\
2.470 \\
3.212 \\
3.999 \\
10.37 \\
10.68 \\
11.79 \\
12.74 \\
13.61\end{array}$ & $\begin{array}{l}1.720 \\
2.234 \\
2.809 \\
3.570 \\
4.384 \\
12.94 \\
13.47 \\
15.35 \\
16.95 \\
18.44\end{array}$ & $\begin{array}{l}0.4005 \\
0.5164 \\
0.6212 \\
0.7382 \\
0.8483 \\
1.949 \\
2.017 \\
2.239 \\
2.408 \\
2.549\end{array}$ & $\begin{array}{l}0.6538 \\
0.4364 \\
0.4874 \\
0.5332 \\
0.5750 \\
0.8018 \\
0.7904 \\
0.7637 \\
0.7526 \\
0.7475\end{array}$ & & $\begin{array}{l}0.6672 \\
0.5096 \\
0.6559 \\
0.8905 \\
1.291 \\
2.485 \\
2.183 \\
1.694 \\
1.529 \\
1.447\end{array}$ & $\begin{array}{l}753.0 \\
748.1 \\
702.2 \\
633.2 \\
550.6 \\
328.7 \\
342.5 \\
387.7 \\
422.4 \\
452.1\end{array}$ \\
\hline $\begin{array}{l}12.0 \\
13.0 \\
14.0 \\
15.0 \\
16.0 \\
17.0 \\
18.0 \\
19.0 \\
20.0 \\
22.0\end{array}$ & $\begin{array}{l}1.825 \\
2.015 \\
2.231 \\
2.384 \\
2.565 \\
2.745 \\
2.922 \\
3.099 \\
3.274 \\
3.623\end{array}$ & $\begin{array}{l}26.4 \\
29.7 \\
33.0 \\
36.2 \\
39.3 \\
42.3 \\
45.3 \\
48.3 \\
51.2 \\
57.0\end{array}$ & $\begin{array}{l}1.53 \\
1.38 \\
1.26 \\
1.16 \\
1.07 \\
1.00 \\
0.940 \\
0.885 \\
0.837 \\
0.755\end{array}$ & $\begin{array}{l}14.45 \\
15.27 \\
16.07 \\
16.87 \\
17.65 \\
18.43 \\
19.21 \\
19.98 \\
20.75 \\
22.28\end{array}$ & $\begin{array}{l}19.86 \\
21.24 \\
22.60 \\
23.93 \\
25.25 \\
26.57 \\
27.87 \\
29.26 \\
30.45 \\
33.02\end{array}$ & $\begin{array}{l}2.673 \\
2.784 \\
2.884 \\
2.976 \\
3.062 \\
3.141 \\
3.216 \\
3.285 \\
3.352 \\
3.474\end{array}$ & $\begin{array}{l}0.7451 \\
0.7440 \\
0.7436 \\
0.7435 \\
0.7435 \\
0.7437 \\
0.7439 \\
0.7440 \\
0.7442 \\
0.7445\end{array}$ & & $\begin{array}{l}1.398 \\
1.367 \\
1.345 \\
1.329 \\
1.316 \\
1.307 \\
1.299 \\
1.293 \\
1.288 \\
1.280\end{array}$ & $\begin{array}{l}478.6 \\
503.0 \\
525.7 \\
547.1 \\
567.4 \\
586.7 \\
605.3 \\
623.2 \\
640.5 \\
673.5\end{array}$ \\
\hline $\begin{array}{l}24.0 \\
26.0 \\
28.0 \\
30.0 \\
32.0 \\
34.0 \\
36.0 \\
38.0 \\
40.0\end{array}$ & $\begin{array}{l}3.970 \\
4.315 \\
4.659 \\
5.001 \\
5.343 \\
5.683 \\
0.023 \\
6.363 \\
6.7132\end{array}$ & $\begin{array}{r}62.7 \\
68.4 \\
74.0 \\
79.6 \\
85.2 \\
90.7 \\
96.2 \\
102.0 \\
107.0\end{array}$ & $\begin{array}{l}0.688 \\
0.632 \\
0.585 \\
0.544 \\
0.509 \\
0.478 \\
0.451 \\
0.426 \\
0.404\end{array}$ & $\begin{array}{l}23.81 \\
25.32 \\
26.84 \\
28.35 \\
29.86 \\
31.37 \\
32.87 \\
34.38 \\
35.88\end{array}$ & $\begin{array}{l}35.57 \\
38.11 \\
40.64 \\
43.17 \\
45.69 \\
48.21 \\
50.72 \\
53.23 \\
55.74\end{array}$ & $\begin{array}{l}3.585 \\
3.687 \\
3.780 \\
3.868 \\
3.949 \\
4.025 \\
4.097 \\
4.165 \\
4.229\end{array}$ & $\begin{array}{l}0.7448 \\
0.7450 \\
0.7451 \\
0.7452 \\
0.7453 \\
0.7454 \\
0.7454 \\
0.7454 \\
0.7454\end{array}$ & & $\begin{array}{l}1.274 \\
1.269 \\
1.265 \\
1.262 \\
1.260 \\
1.257 \\
1.256 \\
1.254 \\
1.253\end{array}$ & $\begin{array}{l}704.8 \\
734.5 \\
763.0 \\
790.3 \\
816.6 \\
842.0 \\
866.6 \\
890.5 \\
913.7\end{array}$ \\
\hline $\begin{array}{r}45.0 \\
50.0 \\
55.0 \\
60.0 \\
70.0 \\
80.0 \\
90.0 \\
100.0 \\
120.0 \\
140.0\end{array}$ & $\begin{array}{c}7.548 \\
8.392 \\
9.235 \\
10.08 \\
11.76 \\
13.44 \\
15.12 \\
16.83 \\
20.15 \\
23.51\end{array}$ & $\begin{array}{l}121.0 \\
134.0 \\
148.0 \\
162.0 \\
189.0 \\
216.0 \\
242.0 \\
269.0 \\
323.0 \\
377.0\end{array}$ & $\begin{array}{l}0.359 \\
0.322 \\
0.293 \\
0.268 \\
0.229 \\
0.240 \\
0.178 \\
0.160 \\
0.133 \\
0.114\end{array}$ & $\begin{array}{c}39.63 \\
43.38 \\
47.12 \\
50.86 \\
58.33 \\
65.80 \\
73.26 \\
80.72 \\
95.63 \\
110.5\end{array}$ & $\begin{array}{c}62.00 \\
68.24 \\
74.48 \\
80.72 \\
93.17 \\
105.6 \\
118.1 \\
130.5 \\
155.3 \\
180.2\end{array}$ & $\begin{array}{l}4.377 \\
4.508 \\
4.627 \\
4.736 \\
4.928 \\
5.094 \\
5.240 \\
5.371 \\
5.598 \\
5.789\end{array}$ & $\begin{array}{l}0.7454 \\
0.7454 \\
0.7453 \\
0.7453 \\
0.7452 \\
0.7452 \\
0.7451 \\
0.7451 \\
0.7450 \\
0.7450\end{array}$ & & $\begin{array}{l}1.250 \\
1.249 \\
1.247 \\
1.246 \\
1.245 \\
1.244 \\
1.243 \\
1.243 \\
1.242 \\
1.242\end{array}$ & $\begin{array}{r}969.2 \\
1022.0 \\
1071.0 \\
1119.0 \\
1208.0 \\
1291.0 \\
1369.0 \\
1443.0 \\
1580.0 \\
1706.0\end{array}$ \\
\hline $\begin{array}{l}160.0 \\
180.0 \\
200.0 \\
250.0 \\
300.0 \\
350.0 \\
400.0 \\
450.0 \\
500.0 \\
600.0\end{array}$ & $\begin{array}{c}26.86 \\
30.21 \\
33.56 \\
41.94 \\
50.32 \\
58.70 \\
67.08 \\
75.45 \\
83.83 \\
100.6\end{array}$ & $\begin{array}{r}430.0 \\
484.0 \\
538.0 \\
672.0 \\
806.0 \\
940.0 \\
1070.0 \\
1210.0 \\
1340.0 \\
1610.0\end{array}$ & $\begin{array}{l}3.100 \\
0.0889 \\
0.0800 \\
0.0640 \\
0.0533 \\
0.0457 \\
0.0400 \\
0.0356 \\
0.0320 \\
0.0267\end{array}$ & $\begin{array}{l}125.4 \\
140.3 \\
155.2 \\
192.5 \\
229.7 \\
267.0 \\
304.2 \\
341.4 \\
378.7 \\
453.1\end{array}$ & $\begin{array}{l}205.0 \\
229.8 \\
254.7 \\
316.7 \\
378.8 \\
440.9 \\
502.9 \\
565.0 \\
627.1 \\
751.2\end{array}$ & $\begin{array}{l}5.955 \\
6.101 \\
6.232 \\
6.509 \\
6.736 \\
6.927 \\
7.093 \\
7.239 \\
7.370 \\
7.596\end{array}$ & $\begin{array}{l}0.7449 \\
0.7449 \\
0.7449 \\
0.7448 \\
0.7448 \\
0.7448 \\
0.7448 \\
0.7448 \\
0.7448 \\
0.7448\end{array}$ & & $\begin{array}{l}1.242 \\
1.242 \\
1.242 \\
1.241 \\
1.241 \\
1.241 \\
1.241 \\
1.241 \\
1.241 \\
1.241\end{array}$ & $\begin{array}{l}1823.0 \\
1933.0 \\
2038.0 \\
2277.0 \\
2494.0 \\
2694.0 \\
2879.0 \\
3054.0 \\
3219.0 \\
3526.0\end{array}$ \\
\hline $\begin{array}{r}740.0 \\
800.0 \\
900.0 \\
1000.0 \\
1200.0 \\
1400.0 \\
1600.0 \\
1800.0 \\
2000.0 \\
2500.0\end{array}$ & $\begin{array}{l}117.3 \\
134.1 \\
150.9 \\
167.6 \\
201.1 \\
234.6 \\
268.1 \\
331.7 \\
335.2 \\
419.0\end{array}$ & $\begin{array}{l}1880.0 \\
2150.0 \\
2410.0 \\
2680.0 \\
3220.0 \\
3750.0 \\
4290.0 \\
4830.0 \\
5360.0 \\
6700.0\end{array}$ & $\begin{array}{l}0.0229 \\
0.0200 \\
0.0178 \\
0.0160 \\
0.0133 \\
0.0114 \\
0.0100 \\
0.00889 \\
0.00800 \\
0.00640\end{array}$ & $\begin{array}{r}527.6 \\
602.1 \\
676.6 \\
751.0 \\
900.0 \\
1049.0 \\
1198.0 \\
1347.0 \\
1496.0 \\
1868.0\end{array}$ & $\begin{array}{r}875.3 \\
999.0 \\
1124.0 \\
1248.0 \\
1496.0 \\
1744.0 \\
1992.0 \\
2241.0 \\
2489.0 \\
3109.0\end{array}$ & $\begin{array}{l}7.787 \\
7.953 \\
8.099 \\
8.230 \\
8.456 \\
8.648 \\
8.813 \\
8.959 \\
9.090 \\
9.367\end{array}$ & $\begin{array}{l}0.7448 \\
0.7447 \\
0.7447 \\
0.7447 \\
0.7447 \\
0.7447 \\
0.7447 \\
0.7447 \\
0.7447 \\
0.7447\end{array}$ & & $\begin{array}{l}1.241 \\
1.241 \\
1.241 \\
1.241 \\
1.241 \\
1.241 \\
1.241 \\
1.241 \\
1.241 \\
1.241\end{array}$ & $\begin{array}{l}3808.0 \\
4071.0 \\
4317.0 \\
4551.0 \\
4985.0 \\
5384.0 \\
5756.0 \\
6105.0 \\
6435.0 \\
7194.0\end{array}$ \\
\hline 3000.0 & 502.7 & 8040.0 & 0.00533 & 2240.0 & 3730.0 & 9.593 & 0.7447 & & 1.241 & 7881.0 \\
\hline
\end{tabular}

* tho-phase boundary 
16 PSIA ISDBAR

TEMPERATURE DENSITY VIDH/OVI $V(D P / D U)_{V}-V\left(D P / O V I_{T}\right.$ DEG. R LB/CUFT BTULLA PSIA-CU FT/BTU PSIA

\begin{tabular}{|c|c|}
\hline $\begin{array}{l}4.0 \\
5.0 \\
6.0 \\
7.0 \\
7.769 \\
7.769 \\
8.0 \\
9.0 \\
10.0 \\
11.0\end{array}$ & $\begin{array}{l}9.258 \\
9.071 \\
8.757 \\
8.275 \\
7.686 \\
1.153 \\
1.063 \\
0.8321 \\
0.7025 \\
0.6140\end{array}$ \\
\hline $\begin{array}{l}12.0 \\
13.0 \\
14.0 \\
15.0 \\
16.0 \\
17.0 \\
18.0 \\
19.0 \\
20.0 \\
22.0\end{array}$ & $\begin{array}{l}0.5480 \\
0.4963 \\
0.4543 \\
0.4194 \\
0.3898 \\
0.3644 \\
0.3422 \\
0.3227 \\
0.3054 \\
0.2760\end{array}$ \\
\hline $\begin{array}{l}24.0 \\
26.0 \\
28.0 \\
30.0 \\
32.0 \\
34.0 \\
36.0 \\
38.0 \\
40.0\end{array}$ & $\begin{array}{l}0.2519 \\
0.2317 \\
0.2147 \\
0.2000 \\
0.1872 \\
0.1760 \\
0.1660 \\
0.1572 \\
0.1492\end{array}$ \\
\hline $\begin{array}{r}45.0 \\
50.0 \\
55.0 \\
60.0 \\
70.0 \\
80.0 \\
90.0 \\
100.0 \\
120.0 \\
140.0\end{array}$ & $\begin{array}{l}0.1325 \\
0.1192 \\
0.1083 \\
0.09923 \\
0.08504 \\
0.07441 \\
0.06614 \\
0.05954 \\
0.04962 \\
0.04254\end{array}$ \\
\hline $\begin{array}{l}160.0 \\
180.0 \\
200.0 \\
250.0 \\
300.0 \\
350.0 \\
400.0 \\
450.0 \\
500.0 \\
600.0\end{array}$ & $\begin{array}{l}0.03723 \\
0.03310 \\
0.02980 \\
0.02384 \\
0.01987 \\
0.01704 \\
0.01491 \\
0.01325 \\
0.01193 \\
0.009941\end{array}$ \\
\hline $\begin{array}{r}700 \cdot 0 \\
800 \cdot 0 \\
900.0 \\
1000 \cdot 0 \\
1200 \cdot 0 \\
1400.0 \\
1600.0 \\
1800.0 \\
2000.0 \\
2500.0\end{array}$ & $\begin{array}{l}0.008522 \\
0.007457 \\
0.006629 \\
0.005966 \\
0.004972 \\
0.004262 \\
0.003729 \\
0.003315 \\
0.002984 \\
0.002387\end{array}$ \\
\hline
\end{tabular}

3000.0

\begin{tabular}{|c|c|c|}
\hline $\begin{array}{c}54.4 \\
18.4 \\
15.0 \\
12.2 \\
9.88 \\
6.30 \\
6.82 \\
8.67 \\
10.3 \\
11.8\end{array}$ & $\begin{array}{l}2.25 \\
6.55 \\
7.11 \\
7.09 \\
6.62 \\
3.70 \\
3.71 \\
3.74 \\
3.75 \\
3.74\end{array}$ & $\begin{array}{c}1110.0 \\
939.0 \\
693.0 \\
429.0 \\
224.0 \\
8.68 \\
9.74 \\
12.2 \\
13.3 \\
14.0\end{array}$ \\
\hline $\begin{array}{l}13.2 \\
14.7 \\
16.0 \\
17.4 \\
18.8 \\
20.1 \\
21.4 \\
22.7 \\
24.0 \\
26.7\end{array}$ & $\begin{array}{l}3.74 \\
3.73 \\
3.72 \\
3.71 \\
3.70 \\
3.70 \\
3.69 \\
3.69 \\
3.68 \\
3.67\end{array}$ & $\begin{array}{l}14.4 \\
14.8 \\
15.0 \\
15.2 \\
15.3 \\
15.4 \\
15.5 \\
15.6 \\
15.6 \\
15.7\end{array}$ \\
\hline $\begin{array}{l}29.2 \\
31.8 \\
34.4 \\
36.9 \\
39.5 \\
42.0 \\
44.5 \\
47.0 \\
49.6\end{array}$ & $\begin{array}{l}3.67 \\
3.66 \\
3.66 \\
3.65 \\
3.65 \\
3.64 \\
3.64 \\
3.64 \\
3.64\end{array}$ & $\begin{array}{l}15.8 \\
15.8 \\
15.9 \\
15.9 \\
15.9 \\
16.0 \\
16.0 \\
16.0 \\
16.0\end{array}$ \\
\hline $\begin{array}{r}55.8 \\
62.1 \\
68.4 \\
74.6 \\
87.1 \\
100.0 \\
112.0 \\
124.0 \\
149.0 \\
174.0\end{array}$ & $\begin{array}{l}3.63 \\
3.63 \\
3.62 \\
3.62 \\
3.62 \\
3.62 \\
3.61 \\
3.61 \\
3.61 \\
3.61\end{array}$ & $\begin{array}{l}16.0 \\
16.0 \\
16.0 \\
16.0 \\
16.0 \\
16.0 \\
16.0 \\
16.0 \\
16.0 \\
16.0\end{array}$ \\
\hline $\begin{array}{l}199.0 \\
224.0 \\
249.0 \\
311.0 \\
373.0 \\
435.0 \\
497.0 \\
559.0 \\
621.0 \\
745.0\end{array}$ & $\begin{array}{l}3.61 \\
3.61 \\
3.61 \\
3.60 \\
3.60 \\
3.60 \\
3.60 \\
3.60 \\
3.60 \\
3.60\end{array}$ & $\begin{array}{l}16.0 \\
16.0 \\
16.0 \\
16.0 \\
16.0 \\
16.0 \\
16.0 \\
16.0 \\
16.0 \\
16.0\end{array}$ \\
\hline $\begin{array}{r}869.0 \\
993.0 \\
1120.0 \\
1240.0 \\
1490.0 \\
1740.0 \\
1990.0 \\
2230.0 \\
2480.0 \\
3100.0\end{array}$ & $\begin{array}{l}3.60 \\
3.60 \\
3.60 \\
3.60 \\
3.60 \\
3.60 \\
3.60 \\
3.60 \\
3.60 \\
3.60\end{array}$ & $\begin{array}{l}16.0 \\
16.0 \\
16.0 \\
16.0 \\
16.0 \\
16.0 \\
16.0 \\
16.0 \\
16.0 \\
16.0\end{array}$ \\
\hline 3720.0 & 3.60 & 16.0 \\
\hline
\end{tabular}

0.001989

3720.0
(DV/OT) $/ V$ THERMAL $V$ 1/DEG. R BTU/FT-HR-R LA/FT-SEC FT $-S E C$
$\times \quad 10 E+6$

$\begin{array}{ll}0.0123 & 0 \\ 0.0276 & 0 \\ 0.0438 & 0 \\ 0.0729 & 0 \\ 0.131 & 0 \\ 0.394 & 0 \\ 0.320 & 0 \\ 0.195 & 0 \\ 0.149 & 0 \\ 0.123 & 0 \\ 0.106 & 0 \\ 0.0933 & 0.0 \\ 0.0838 & 0.0\end{array}$

0.0838

0.0702

0.0702

0.0606

0.0569

0.0536

0.0480

0.0436

0.0399

0.0368

0.0342

0.3299

0.0282

0.0282
0.0267

0.0253

0.0224

0.0201

0.0182

0.0167

0.0125

0.0111

0.0100

0.00832

0.00624

0.00555

0.00499

0.00400

0.00333

0.00285

0.00250

0.00200

0.00167

0.00143

0.00125

0.00111

0.000833

0.000714

0.000625

0.000555

0.000500

0.00881

0.0102

0.0109

0.0114

0.0114

0.00630

0.00650

0.00690
0.00733

0.00776

0.00819

0.00860

0.00938

0.00938

0.00976

0.0101

0.0105
0.0108

0.0115

0.0121

0.0127

0.0133

0.0139

0.0150

0.0155

0.0160

0.0160
0.0165

0.0177

0.018

0.0200

0.0210

0.0231

0.0251

0.0270

0.0289

0.0325
0.0360

0.0393

0.0425

0.0457

0.0532

0.0669

0.0733

0.0793

0.0851

0.107

0.117

0.128
0.137

0.156

0.174

0.191
0.208

0.208
0.224

0.224
0.263

0.000333

0.299
2.60
2.65
2.51
2.31
2.10
0.865
0.878
0.945
1.02
1.08

\subsection{5}

1.22

1. 28

1.41

1.41
1.46

1.46
1.52

1.58

1.64
1.74

1.85

1.95

2.14

2.23

2.32

2.41

2.49
2.58

2.78

2.97
3.15

3.15
3.33

3.66

3.98

4.28

4.56

5.10
5.62

6.10

6.57
6.90

7.98

9.01

10.0

10.9

11.8
12.7

12.7

16.0

16.0
17.6
19.1

19.1
20.5

20.5

26.0

28.5

31.0

33.4

44.6

44.6
THERMAL DIELECTRIC DIFFUSIVITY CDNSTANT SQ FT/HR

0.00143
0.00221
0.00190
0.00154
0.00115
0.00223
0.00271
0.00461
0.00642
0.00825
0.0101

1.02048
1.02036
1.02013
1.01971
1.01908
1.00414
1.00383
1.00303
1.00257
1.00226

0.798

0.545

0.652

0.857

1.21

1.10
0.887

0.810

0.771

0.747

$0.0121 \quad 1.00183 \quad 0.732$

$0.0141 \quad 1.00168$

$0.0161 \quad 1.00156$

$0.0183 \quad 1.00145$

0.0205

$0.0228 \quad 1.00127 \quad 0.704$

$\begin{array}{lll}0.0251 & 1.00120 & 0.702\end{array}$

$\begin{array}{lll}0.0275 & 1.00114 & 0.701\end{array}$

$0.0377 \quad 1.00094 \quad 0.700$

$0.0432 \quad 1.00087 \quad 0.700$

$\begin{array}{lll}0.0490 & 1.00080 & 0.701\end{array}$

$0.0612 \quad 1.00070 \quad 0.702$

$0.0676 \quad 1.00066$

$0.0742 \quad 1.00062 \quad 0.704$

$0.0811 \quad 1.00059 \quad 0.705$

1.00050

1.00045

1.00041

1.00037

1.00032

1.00028

. 00025

1.00019
1.00016

1. 00014

1.00012

1.00011

1. 00008

1.00006

1.00006

1.00005

1. 00005

1.00004

1.00003

1.00003

1. 00003

1.00002
1.00002

1.00002

1. 00001

1.00001

1. 00001

50.6

50.5
88.7

1.00001

0.708

0.709

0.710

0.709

0.707

0.706

0.702

0.694

0.691

0.675

0.671

0.669

0.667

0.666

0.66

0.669

0.668

0.668

0.668

0.667

0.667

0.667

0.666

0.666

0.666

0.666

0.666

- tho-phase bounoarY 
THERMOOYNAMIC PROPERTIES OF HELIUM 4

\begin{tabular}{|c|c|c|c|c|c|c|c|c|c|}
\hline $\begin{array}{c}\text { TEMPERATURE } \\
\text { OEG. R }\end{array}$ & $\begin{array}{l}\text { E VOLUME } \\
\text { CU FT/LB }\end{array}$ & $\begin{array}{c}\text { ISOTHERM } \\
\text { OERIVATIVE } \\
\text { CU FT-PSIA/LB }\end{array}$ & $\begin{array}{l}\text { ISOCHORE } \\
\text { OERIVATIVE } \\
\text { PSIA/R }\end{array}$ & $\begin{array}{l}\text { INTERNAL } \\
\text { ENERGY } \\
\text { BTU/LB }\end{array}$ & $\begin{array}{l}\text { ENT HALPY } \\
\text { BTU/LB }\end{array}$ & $\begin{array}{l}\text { ENTROPY } \\
\text { BTU/LB-R }\end{array}$ & $\begin{array}{l}\text { CV } \\
\text { BTU / LB }\end{array}$ & $\begin{array}{l}C P \\
-R\end{array}$ & $\begin{array}{l}\text { VEL OCITY } \\
\text { OF SOUND } \\
\text { FT/SEG }\end{array}$ \\
\hline $\begin{array}{r}4.0 \\
5.0 \\
6.0 \\
7.0 \\
8.0 \\
8.0 \\
8.0 \\
9.0 \\
10.0 \\
11.0\end{array}$ & $\begin{array}{l}0.1078 \\
0.1100 \\
0.1139 \\
0.1203 \\
0.1331 \\
0.7025 \\
0.1333 \\
1.024 \\
1.233 \\
1.421\end{array}$ & $\begin{array}{c}122.0 \\
105.0 \\
81.0 \\
53.9 \\
24.4 \\
6.78 \\
24.2 \\
13.2 \\
17.9 \\
21.9\end{array}$ & $\begin{array}{c}13.6 \\
26.0 \\
30.5 \\
31.5 \\
28.5 \\
3.92 \\
28.5 \\
2.82 \\
2.30 \\
1.98\end{array}$ & $\begin{array}{c}1.398 \\
1.901 \\
2.459 \\
3.190 \\
4.252 \\
10.33 \\
4.259 \\
11.59 \\
12.58 \\
13.49\end{array}$ & $\begin{array}{c}1.757 \\
2.267 \\
2.838 \\
3.591 \\
4.695 \\
12.87 \\
4.704 \\
15.00 \\
16.69 \\
18.22\end{array}$ & $\begin{array}{l}0.3997 \\
0.5148 \\
0.6190 \\
0.7348 \\
0.8815 \\
1.902 \\
0.8826 \\
2.154 \\
2.332 \\
2.479\end{array}$ & $\begin{array}{l}0.6484 \\
0.4357 \\
0.4869 \\
0.5322 \\
0.5886 \\
0.8040 \\
0.5890 \\
0.7687 \\
0.7548 \\
0.7485\end{array}$ & $\begin{array}{l}0.6614 \\
0.5074 \\
0.6518 \\
0.8782 \\
1.467 \\
2.759 \\
1.474 \\
1.824 \\
1.592 \\
1.485\end{array}$ & $\begin{array}{l}758.3 \\
753.5 \\
708.7 \\
641.8 \\
530.3 \\
328.3 \\
529.5 \\
380.4 \\
417.6 \\
448.6\end{array}$ \\
\hline $\begin{array}{l}12.0 \\
13.0 \\
14.0 \\
15.0 \\
16.0 \\
17.0 \\
18.0 \\
19.0 \\
20.0 \\
22.0\end{array}$ & $\begin{array}{l}1.600 \\
1.772 \\
1.940 \\
2.105 \\
2.267 \\
2.428 \\
2.587 \\
2.745 \\
2.902 \\
3.214\end{array}$ & $\begin{array}{l}25.6 \\
29.1 \\
32.4 \\
35.6 \\
38.8 \\
41.9 \\
44.9 \\
47.9 \\
50.9 \\
56.7\end{array}$ & $\begin{array}{l}1.75 \\
1.57 \\
1.43 \\
1.32 \\
1.22 \\
1.14 \\
1.06 \\
1.00 \\
0.947 \\
0.853\end{array}$ & $\begin{array}{l}14.34 \\
15.17 \\
15.99 \\
16.79 \\
17.58 \\
18.37 \\
19.15 \\
19.92 \\
20.69 \\
22.23\end{array}$ & $\begin{array}{l}19.68 \\
21.08 \\
22.45 \\
23.80 \\
25.14 \\
26.46 \\
27.77 \\
29.07 \\
30.37 \\
32.94\end{array}$ & $\begin{array}{l}2.605 \\
2.717 \\
2.819 \\
2.912 \\
2.998 \\
3.079 \\
3.153 \\
3.224 \\
3.290 \\
3.413\end{array}$ & $\begin{array}{l}0.7455 \\
0.7441 \\
0.7436 \\
0.7434 \\
0.7434 \\
0.7436 \\
0.7438 \\
0.7440 \\
0.7442 \\
0.7445\end{array}$ & $\begin{array}{l}1.425 \\
1.387 \\
1.360 \\
1.341 \\
1.327 \\
1.316 \\
1.307 \\
1.300 \\
1.294 \\
1.285\end{array}$ & $\begin{array}{l}476.0 \\
501.0 \\
524.1 \\
545.8 \\
566.4 \\
585.9 \\
604.7 \\
622.7 \\
640.2 \\
673.4\end{array}$ \\
\hline $\begin{array}{l}24.0 \\
26.0 \\
28.0 \\
30.0 \\
32.0 \\
34.0 \\
36.0 \\
38.0 \\
40.0\end{array}$ & $\begin{array}{l}3.523 \\
3.831 \\
4.137 \\
4.443 \\
4.747 \\
5.050 \\
5.353 \\
5.655 \\
5.957\end{array}$ & $\begin{array}{r}62.5 \\
68.2 \\
73.9 \\
79.5 \\
85.1 \\
90.7 \\
96.2 \\
102.0 \\
107.0\end{array}$ & $\begin{array}{l}0.777 \\
0.714 \\
0.660 \\
0.614 \\
0.574 \\
0.539 \\
0.508 \\
0.480 \\
0.456\end{array}$ & $\begin{array}{l}23.76 \\
25.28 \\
26.80 \\
28.31 \\
29.83 \\
31.34 \\
32.84 \\
34.35 \\
35.85\end{array}$ & $\begin{array}{l}35.50 \\
38.05 \\
40.59 \\
43.12 \\
45.65 \\
48.17 \\
50.69 \\
53.20 \\
55.71\end{array}$ & $\begin{array}{l}3.524 \\
3.626 \\
3.721 \\
3.808 \\
3.889 \\
3.966 \\
4.038 \\
4.106 \\
4.170\end{array}$ & $\begin{array}{l}0.7448 \\
0.7450 \\
0.7452 \\
0.7453 \\
0.7454 \\
0.7454 \\
0.7455 \\
0.7455 \\
0.7455\end{array}$ & $\begin{array}{l}1.278 \\
1.273 \\
1.268 \\
1.265 \\
1.262 \\
1.260 \\
1.258 \\
1.256 \\
1.254\end{array}$ & $\begin{array}{l}704.8 \\
734.7 \\
763.2 \\
790.6 \\
816.9 \\
842.4 \\
867.0 \\
890.9 \\
914.2\end{array}$ \\
\hline $\begin{array}{r}45.0 \\
50.0 \\
55.0 \\
60.0 \\
70.0 \\
80.0 \\
90.0 \\
100.0 \\
120.0 \\
140.0\end{array}$ & $\begin{array}{c}6.710 \\
7.461 \\
8.211 \\
8.960 \\
10.46 \\
11.95 \\
13.44 \\
14.93 \\
17.92 \\
20.93\end{array}$ & $\begin{array}{l}121.0 \\
135.0 \\
148.0 \\
162.0 \\
189.0 \\
216.0 \\
243.0 \\
270.0 \\
323.0 \\
377.0\end{array}$ & $\begin{array}{l}0.444 \\
0.363 \\
0.329 \\
0.302 \\
0.258 \\
0.226 \\
0.200 \\
0.180 \\
0.150 \\
0.129\end{array}$ & $\begin{array}{c}39.61 \\
43.36 \\
47.10 \\
50.84 \\
58.32 \\
65.79 \\
73.25 \\
80.71 \\
95.62 \\
110.5\end{array}$ & $\begin{array}{c}61.97 \\
68.23 \\
74.47 \\
80.71 \\
93.17 \\
105.6 \\
118.1 \\
139.5 \\
155.3 \\
180.2\end{array}$ & $\begin{array}{l}4.318 \\
4.449 \\
4.568 \\
4.677 \\
4.869 \\
5.035 \\
5.182 \\
5.313 \\
5.539 \\
5.731\end{array}$ & $\begin{array}{l}0.7455 \\
0.7455 \\
0.7454 \\
0.7454 \\
0.7453 \\
0.7452 \\
0.7452 \\
0.7451 \\
0.7450 \\
0.7450\end{array}$ & $\begin{array}{l}1.252 \\
1.250 \\
1.248 \\
1.247 \\
1.245 \\
1.244 \\
1.244 \\
1.243 \\
1.242 \\
1.242\end{array}$ & $\begin{array}{r}969.7 \\
1022.0 \\
1072.0 \\
1119.0 \\
1209.0 \\
1292.0 \\
1370.0 \\
1443.0 \\
1580.0 \\
1706.0\end{array}$ \\
\hline $\begin{array}{l}160.0 \\
180.0 \\
200.0 \\
250.0 \\
300.0 \\
350.0 \\
400.0 \\
450.0 \\
500.0 \\
600.0\end{array}$ & $\begin{array}{l}23.88 \\
26.86 \\
29.84 \\
37.29 \\
44.73 \\
52.18 \\
59.63 \\
67.08 \\
74.52 \\
89.42\end{array}$ & $\begin{array}{r}431.0 \\
484.0 \\
538.0 \\
672.0 \\
806.0 \\
940.0 \\
1070.0 \\
1210.0 \\
1340.0 \\
1610.0\end{array}$ & $\begin{array}{l}0.113 \\
0.100 \\
0.0900 \\
0.0720 \\
0.0600 \\
0.0514 \\
0.0450 \\
0.0400 \\
0.0360 \\
0.0300\end{array}$ & $\begin{array}{l}125.4 \\
140.3 \\
155.2 \\
192.5 \\
229.7 \\
267.0 \\
304.2 \\
341.4 \\
378.7 \\
453.2\end{array}$ & $\begin{array}{l}205.0 \\
229.9 \\
254.7 \\
316.8 \\
378.8 \\
440.9 \\
503.0 \\
565.0 \\
627.1 \\
751.2\end{array}$ & $\begin{array}{l}5.897 \\
6.043 \\
6.174 \\
6.451 \\
6.677 \\
6.868 \\
7.034 \\
7.180 \\
7.311 \\
7.537\end{array}$ & $\begin{array}{l}0.7449 \\
0.7449 \\
0.7449 \\
0.7449 \\
0.7448 \\
0.7448 \\
0.7448 \\
0.7448 \\
0.7448 \\
0.7448\end{array}$ & $\begin{array}{l}1.242 \\
1.242 \\
1.242 \\
1.241 \\
1.241 \\
1.241 \\
1.241 \\
1.241 \\
1.241 \\
1.241\end{array}$ & $\begin{array}{l}1824.0 \\
1934.0 \\
2038.0 \\
2278.0 \\
2495.0 \\
2694.0 \\
2880.0 \\
3054.0 \\
3219.0 \\
3526.0\end{array}$ \\
\hline $\begin{array}{r}700.0 \\
800.0 \\
900.0 \\
1000.0 \\
1200.0 \\
1400.0 \\
1600.0 \\
1800.0 \\
2000.0 \\
2500.0\end{array}$ & $\begin{array}{l}104.3 \\
119.2 \\
134.1 \\
149.0 \\
178.8 \\
208.6 \\
238.4 \\
268.1 \\
297.9 \\
372.4\end{array}$ & $\begin{array}{l}1880.0 \\
2150.0 \\
2410.0 \\
2680.0 \\
3220.0 \\
3760.0 \\
4290.0 \\
4830.0 \\
5360.0 \\
6700.0\end{array}$ & $\begin{array}{l}0.0257 \\
0.0225 \\
0.0200 \\
0.0180 \\
0.0150 \\
0.0129 \\
0.0112 \\
0.0100 \\
0.00900 \\
0.00720\end{array}$ & $\begin{array}{r}527.6 \\
602.1 \\
676.6 \\
751.0 \\
900.0 \\
1049.0 \\
1198.0 \\
1347.0 \\
1496.0 \\
1868.0\end{array}$ & $\begin{array}{r}875.3 \\
999.0 \\
1124.0 \\
1248.0 \\
1496.0 \\
1744.0 \\
1992.0 \\
2241.0 \\
2489.0 \\
3109.0\end{array}$ & $\begin{array}{l}7.729 \\
7.895 \\
8.041 \\
8.171 \\
8.398 \\
8.589 \\
8.755 \\
8.901 \\
9.032 \\
9.309\end{array}$ & $\begin{array}{l}0.7448 \\
0.7448 \\
0.7447 \\
0.7447 \\
0.7447 \\
0.7447 \\
0.7447 \\
0.7447 \\
0.7447 \\
0.7447\end{array}$ & $\begin{array}{l}1.241 \\
1.241 \\
1.241 \\
1.241 \\
1.241 \\
1.241 \\
1.241 \\
1.241 \\
1.241 \\
1.241\end{array}$ & $\begin{array}{l}3808.0 \\
4071.0 \\
4317.0 \\
4551.0 \\
4985.0 \\
5384.0 \\
5756.0 \\
6105.0 \\
6435.0 \\
7194.0\end{array}$ \\
\hline 3000.0 & 446.9 & 8040.0 & 0.00600 & 2240.0 & 3730.0 & 9.535 & 0.7447 & 1.241 & 7881.0 \\
\hline
\end{tabular}


18 PSIA ISOBAR

TEMPERATURE OENSITY V(OH/OV) V V OP/OU) ${ }_{\mathrm{P}}-V(O P / O V)_{T}$ OEG. R LB/CUFT BTU/LB PSIA-CU FT/BTU PSIA

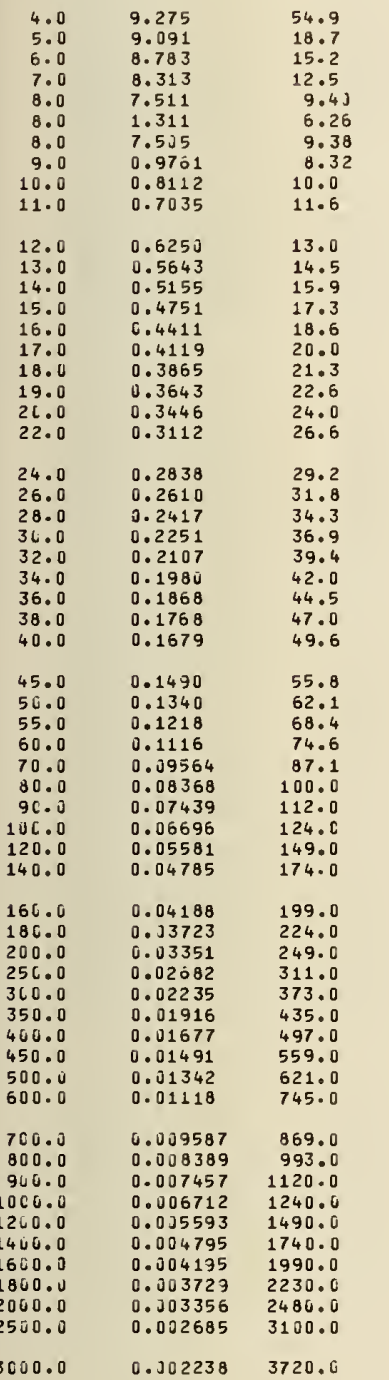

\begin{tabular}{|c|c|}
\hline $\begin{array}{l}2.26 \\
6.55 \\
7.12 \\
7.13 \\
6.46 \\
3.72 \\
6.45 \\
3.76 \\
3.76 \\
3.76\end{array}$ & $\begin{array}{c}1130.0 \\
957.0 \\
711.0 \\
448.0 \\
183.0 \\
8.89 \\
181.0 \\
12.9 \\
14.5 \\
15.4\end{array}$ \\
\hline $\begin{array}{l}3.75 \\
3.74 \\
3.73 \\
3.73 \\
3.72 \\
3.71 \\
3.70 \\
3.70 \\
3.69 \\
3.68\end{array}$ & $\begin{array}{l}16.0 \\
16.4 \\
16.7 \\
16.9 \\
17.1 \\
17.3 \\
17.4 \\
17.5 \\
17.5 \\
17.7\end{array}$ \\
\hline $\begin{array}{l}3.68 \\
3.67 \\
3.66 \\
3.66 \\
3.65 \\
3.65 \\
3.65 \\
3.64 \\
3.64\end{array}$ & $\begin{array}{l}17.7 \\
17.8 \\
17.9 \\
17.9 \\
17.9 \\
18.0 \\
18.0 \\
18.0 \\
18.0\end{array}$ \\
\hline $\begin{array}{l}3.64 \\
3.63 \\
3.63 \\
3.62 \\
3.62 \\
3.62 \\
3.62 \\
3.61 \\
3.61 \\
3.61\end{array}$ & $\begin{array}{l}18.0 \\
18.0 \\
18.0 \\
18.0 \\
18.1 \\
18.1 \\
18.1 \\
18.0 \\
18.0 \\
18.0\end{array}$ \\
\hline $\begin{array}{l}3.61 \\
3.61 \\
3.61 \\
3.60 \\
3.60 \\
3.60 \\
3.60 \\
3.60 \\
3.60 \\
3.60\end{array}$ & $\begin{array}{l}18.0 \\
18.0 \\
18.0 \\
18.0 \\
18.0 \\
18.0 \\
18.0 \\
18.0 \\
18.0 \\
18.0\end{array}$ \\
\hline $\begin{array}{l}3.60 \\
3.60 \\
3.60 \\
3.60 \\
3.60 \\
3.60 \\
3.60 \\
3.60 \\
3.60 \\
3.60\end{array}$ & $\begin{array}{l}18.0 \\
18.0 \\
18.0 \\
18.0 \\
18.0 \\
18.0 \\
18.0 \\
18.0 \\
18.0 \\
18.0\end{array}$ \\
\hline
\end{tabular}

(OV/OT) $N$ THERMAL VISCOSITY 1/OEG. R GTU/FT-HR-R LB/FT-SEC $\times 10 E+6$

0.0120
0.0271
0.0428
0.0704
0.156
0.441
0.157
0.219
0.159
0.129
0.109
0.0958
0.0857
0.0777
0.0712
0.0659
0.0613
0.0574
0.0540
0.0484

0.00883
0.0102
0.0109
0.0114
0.0114
0.00677
0.0114
0.00664
0.00699
0.00740
0.00782
0.00824
0.00864
0.00904
0.00942
0.00979
0.0102
0.0105
0.0108
0.0115

2.62

2.67

2.54
2.33

2.05

2.05
0.907

2. 05

0.963

1.03
1.10

\section{16}

1.23

1.29

1.35

1.41
1.47

1.47

1.53

1.59
1.64

1.64
1.75

0.0438

0.0401
0.0370

0.0343

0.0320

0.0300

0.0267

0.0224

0.0201

0.0183

0.0167

0.0143

0.0125

0.0111

0.0100

0.00713

0.00624

0.00555

0.00499

0.00399

0.00333

0.00285

0.00250

0.00222

0.00200

0.00143

0.00125

0.00125

0.00100

0.000833

0.000714

0.000625

0.000555

0.000500

0.0121

0.0127

0.0133

0.0139

0.0144

0.0150

0.0155

0.0160

0.0165

0.0177

0.0189
0.0200

0.0200
0.0211

0.0211

0.0251

0.0271

0.0289

0.0325

0.0360

0.0393

0.0425

0.0457

0.0532
0.0602

0.0669

0.0733

0.0793

0.0851
0.0962

1.85
1.95

1.95
2.05

2.15

2.24

2.33

2.41

2.50
2.58

2.78

2.97
3.15
3.33

3.15
3.33

3.66

3.98

4.28

4.56
5.11

5.11
5.62

6.10

6.57

6.90
7.99

7.99
9.01

10.0

10.9

11.8

12.7

0.107

0.107
0.118

0.128

0.137
0.156

0.174

0.191

0.208

0.224
0.263

0.000333

0.299

16.0

17.6

19.1

20.5
23.3

23.3
26.0

28.5

31.0

33.4
39.2

44.6
THERMAL OIELECTRIC OIFFUSIVITY CONSTANT SQ FT/HR

$\begin{array}{lll}0.00144 & 1.02049 & 0.706 \\ 0.00222 & 1.02037 & 0.476 \\ 0.00191 & 1.02015 & 0.544 \\ 0.00156 & 1.01974 & 0.646 \\ 0.00103 & 1.01887 & 0.951 \\ 0.00187 & 1.00467 & 1.33 \\ 0.00103 & 1.01887 & 0.955 \\ 0.00373 & 1.00353 & 0.952 \\ 0.00541 & 1.00296 & 0.845 \\ 0.00708 & 1.00258 & 0.793 \\ 0.00878 & 1.00230 & 0.763 \\ 0.0105 & 1.00208 & 0.744 \\ 0.0123 & 1.00190 & 0.731 \\ 0.0142 & 1.00176 & 0.723 \\ 0.0161 & 1.00163 & 0.717 \\ 0.0181 & 1.00153 & 0.712 \\ 0.0201 & 1.00144 & 0.709 \\ 0.0222 & 1.00135 & 0.707 \\ 0.0243 & 1.00128 & 0.705 \\ 0.0288 & 1.00116 & 0.703\end{array}$

0.0335

0.0384

0.0435

0.0488

0.0543

0.0601

0.0660

0.0721
0.0784

0.0949

0.113
0.131

0.131

0.151

0.241

0.293

0.348

0.469

3.52
4.29

5.11

9.00

9.00
11.3
13.8

16.5

16.5
22.5
29.3

36.7

44.9

53.8
78.8

108.0
0.705
0.703

1. 00116

0.702
0.702

1. 00106

1.00097

1.00084

1. 00079

1.00074

1.00070

1. 00066

1.00056

1.00056
1.00050

1.00050
1.00046

1.00042

1.00036

1.00031

1.00028

1.00025

1. 00021

1.00018

1.00016

1.00014

1.00013
1.00010

1. 00010

1. 00008

1. 00007

1.00006

1.00005

PRANOTL

NUMBER

.706

.544

.646

1.33

.955

845

0.763

.731

(7)

. 712

.709

0.703

0.703
0.704

0.704

0.705

0.706

0.708

0.709
0.709

0.710

0.710

0.709

0.738

0.706

0.698

0.694

0.691

0.675
0.671

0.669

0.667

0.666

0.667

0.668

1. 00004

1.00003

1.00003

1. 00003

1. 00002

1.00002

1. 00002

1.00001

0.668

0.668

0.668

0.667

0.667
0.667

0.666

0.666

0.666

1.00001

0.666

* two-phase bounoary 


\begin{tabular}{|c|c|c|c|c|c|c|c|c|c|}
\hline $\begin{array}{c}\text { TEMPERATURE } \\
\text { DEG. R }\end{array}$ & $\begin{array}{l}\text { VOLUME } \\
\text { CU FT/LB }\end{array}$ & $\begin{array}{c}\text { ISOTHERM } \\
\text { OERI VATIVE } \\
\text { CU FT - PSIA/LB }\end{array}$ & $\begin{array}{l}\text { ISOCHORE } \\
\text { DERIVATIVE } \\
\text { PSIA/R }\end{array}$ & $\begin{array}{l}\text { INTERNAL } \\
\text { ENERGY } \\
\text { BTU/LB }\end{array}$ & $\begin{array}{l}\text { ENTHALPY } \\
\text { BTU/LB }\end{array}$ & $\begin{array}{l}\text { ENTROPY } \\
\text { BTU/LB-R }\end{array}$ & $\begin{array}{l}\text { CV } \\
\text { BTU / LB }\end{array}$ & $\begin{array}{l}C P \\
-R\end{array}$ & $\begin{array}{l}\text { VEL OCITY } \\
\text { OF SOUND } \\
\text { FT/SEC }\end{array}$ \\
\hline $\begin{array}{c}4.0 \\
5.0 \\
6.0 \\
7.0 \\
8.0 \\
8.224 \\
+\quad 8.224 \\
9.0 \\
10.0 \\
11.0\end{array}$ & $\begin{array}{l}0.1076 \\
0.1098 \\
0.1135 \\
0.1198 \\
0.1318 \\
0.1367 \\
0.6751 \\
0.8789 \\
1.079 \\
1.255\end{array}$ & $\begin{array}{c}123.0 \\
107.0 \\
82.9 \\
55.9 \\
26.8 \\
19.7 \\
5.99 \\
11.6 \\
16.7 \\
21.0\end{array}$ & $\begin{array}{l}13.6 \\
26.0 \\
30.6 \\
31.8 \\
29.1 \\
27.6 \\
4.46 \\
3.32 \\
2.65 \\
2.26\end{array}$ & $\begin{array}{c}1.396 \\
1.894 \\
2.447 \\
3.169 \\
4.200 \\
4.523 \\
10.26 \\
11.36 \\
12.42 \\
13.36\end{array}$ & $\begin{array}{l}1.794 \\
2.301 \\
2.867 \\
3.612 \\
4.689 \\
5.030 \\
12.76 \\
14.61 \\
16.41 \\
18.00\end{array}$ & $\begin{array}{l}0.3990 \\
0.5133 \\
0.6169 \\
0.7315 \\
0.8746 \\
0.9166 \\
1.856 \\
2.072 \\
2.262 \\
2.414\end{array}$ & $\begin{array}{l}0.6431 \\
0.4350 \\
0.4864 \\
0.5313 \\
0.5862 \\
0.6032 \\
0.8059 \\
0.7743 \\
0.7572 \\
0.7496\end{array}$ & $\begin{array}{l}0.6558 \\
0.5053 \\
0.6479 \\
0.8668 \\
1.397 \\
1.706 \\
3.115 \\
2.000 \\
1.665 \\
1.528\end{array}$ & $\begin{array}{l}763.5 \\
758.9 \\
715.0 \\
650.2 \\
544.1 \\
508.0 \\
327.5 \\
372.5 \\
412.6 \\
445.1\end{array}$ \\
\hline $\begin{array}{l}12.0 \\
13.0 \\
14.0 \\
15.0 \\
16.0 \\
17.0 \\
18.0 \\
19.0 \\
20.0 \\
22.0\end{array}$ & $\begin{array}{l}1.420 \\
1.578 \\
1.731 \\
1.881 \\
2.029 \\
2.175 \\
2.319 \\
2.462 \\
2.604 \\
2.886\end{array}$ & $\begin{array}{l}24.8 \\
28.4 \\
31.8 \\
35.1 \\
38.3 \\
41.5 \\
44.6 \\
47.6 \\
50.6 \\
56.5\end{array}$ & $\begin{array}{l}1.98 \\
1.77 \\
1.61 \\
1.48 \\
1.37 \\
1.27 \\
1.19 \\
1.12 \\
1.06 \\
0.953\end{array}$ & $\begin{array}{l}14.23 \\
15.08 \\
15.90 \\
16.71 \\
17.51 \\
18.30 \\
19.08 \\
19.86 \\
20.64 \\
22.18\end{array}$ & $\begin{array}{l}19.49 \\
20.92 \\
22.31 \\
23.67 \\
25.02 \\
26.35 \\
27.67 \\
28.98 \\
30.28 \\
32.86\end{array}$ & $\begin{array}{l}2.543 \\
2.657 \\
2.760 \\
2.855 \\
2.941 \\
3.022 \\
3.098 \\
3.168 \\
3.235 \\
3.358\end{array}$ & $\begin{array}{l}0.7460 \\
0.7443 \\
0.7436 \\
0.7433 \\
0.7434 \\
0.7435 \\
0.7437 \\
0.7439 \\
0.7441 \\
0.7445\end{array}$ & $\begin{array}{l}1.453 \\
1.407 \\
1.376 \\
1.354 \\
1.338 \\
1.325 \\
1.315 \\
1.307 \\
1.300 \\
1.290\end{array}$ & $\begin{array}{l}473.4 \\
498.9 \\
522.5 \\
544.5 \\
565.3 \\
585.1 \\
604.1 \\
622.3 \\
639.8 \\
673.2\end{array}$ \\
\hline $\begin{array}{l}24.0 \\
26.0 \\
28.0 \\
30.0 \\
32.0 \\
34.0 \\
36.0 \\
38.0 \\
40.0\end{array}$ & $\begin{array}{l}3.166 \\
3.444 \\
3.720 \\
3.996 \\
4.270 \\
4.544 \\
4.817 \\
5.089 \\
5.361\end{array}$ & $\begin{array}{r}62.3 \\
68.0 \\
73.7 \\
79.4 \\
85.0 \\
90.6 \\
96.2 \\
102.0 \\
107.0\end{array}$ & $\begin{array}{l}0.867 \\
0.796 \\
0.735 \\
0.684 \\
0.639 \\
0.600 \\
0.565 \\
0.534 \\
0.507\end{array}$ & $\begin{array}{l}23.71 \\
25.24 \\
26.76 \\
28.28 \\
29.79 \\
31.30 \\
32.81 \\
34.32 \\
35.83\end{array}$ & $\begin{array}{l}35.43 \\
37.99 \\
40.54 \\
43.07 \\
45.61 \\
48.13 \\
50.65 \\
53.17 \\
55.68\end{array}$ & $\begin{array}{l}3.470 \\
3.572 \\
3.667 \\
3.754 \\
3.836 \\
3.913 \\
3.985 \\
4.053 \\
4.117\end{array}$ & $\begin{array}{l}0.7448 \\
0.7451 \\
0.7452 \\
0.7454 \\
0.7455 \\
0.7455 \\
0.7455 \\
0.7456 \\
0.7456\end{array}$ & $\begin{array}{l}1.282 \\
1.276 \\
1.271 \\
1.267 \\
1.264 \\
1.262 \\
1.259 \\
1.257 \\
1.256\end{array}$ & $\begin{array}{l}704.8 \\
734.8 \\
763.4 \\
790.8 \\
817.2 \\
842.7 \\
867.4 \\
891.4 \\
914.6\end{array}$ \\
\hline $\begin{array}{r}45.0 \\
50.0 \\
55.0 \\
60.0 \\
70.0 \\
80.0 \\
90.0 \\
100.0 \\
120.0 \\
140.0\end{array}$ & $\begin{array}{c}6.040 \\
6.717 \\
7.392 \\
8.066 \\
9.413 \\
10.76 \\
12.10 \\
13.45 \\
16.13 \\
18.81\end{array}$ & $\begin{array}{l}121.0 \\
135.0 \\
148.0 \\
162.0 \\
189.0 \\
216.0 \\
243.0 \\
270.0 \\
323.0 \\
377.0\end{array}$ & $\begin{array}{l}0.449 \\
0.403 \\
0.366 \\
0.335 \\
0.287 \\
0.251 \\
0.223 \\
0.200 \\
0.167 \\
0.143\end{array}$ & $\begin{array}{r}39.58 \\
43.34 \\
47.08 \\
50.83 \\
58.30 \\
65.77 \\
73.24 \\
80.70 \\
95.62 \\
110.5\end{array}$ & $\begin{array}{c}61.95 \\
68.21 \\
74.46 \\
80.70 \\
93.17 \\
105.6 \\
118.1 \\
138.5 \\
155.4 \\
180.2\end{array}$ & $\begin{array}{l}4.265 \\
4.397 \\
4.516 \\
4.624 \\
4.817 \\
4.983 \\
5.129 \\
5.260 \\
5.487 \\
5.679\end{array}$ & $\begin{array}{l}0.7456 \\
0.7455 \\
0.7455 \\
0.7454 \\
0.7454 \\
0.7453 \\
0.7452 \\
0.7452 \\
0.7451 \\
0.7450\end{array}$ & $\begin{array}{l}1.253 \\
1.250 \\
1.249 \\
1.248 \\
1.246 \\
1.245 \\
1.244 \\
1.243 \\
1.243 \\
1.242\end{array}$ & $\begin{array}{r}970.2 \\
1023.0 \\
1072.0 \\
1120.0 \\
1209.0 \\
1292.0 \\
1370.0 \\
1444.0 \\
1581.0 \\
1707.0\end{array}$ \\
\hline $\begin{array}{l}160.0 \\
180.0 \\
200.0 \\
250.0 \\
300.0 \\
350.0 \\
400.0 \\
450.0 \\
500.0 \\
600.0\end{array}$ & $\begin{array}{l}21.50 \\
24.18 \\
26.86 \\
33.56 \\
40.27 \\
46.97 \\
53.67 \\
60.37 \\
67.08 \\
80.48\end{array}$ & $\begin{array}{r}431.0 \\
485.0 \\
538.0 \\
672.0 \\
806.0 \\
940.0 \\
1070.0 \\
1210.0 \\
1340.0 \\
1610.0\end{array}$ & $\begin{array}{l}0.125 \\
0.111 \\
0.100 \\
0.0800 \\
0.0667 \\
0.0571 \\
0.0500 \\
0.0444 \\
0.0400 \\
0.0333\end{array}$ & $\begin{array}{l}125.4 \\
140.3 \\
155.2 \\
192.5 \\
229.7 \\
267.0 \\
304.2 \\
341.4 \\
378.7 \\
453.2\end{array}$ & $\begin{array}{l}205.0 \\
229.9 \\
254.7 \\
316.8 \\
378.8 \\
440.9 \\
503.0 \\
565.0 \\
627.1 \\
751.2\end{array}$ & $\begin{array}{l}5.844 \\
5.991 \\
6.121 \\
6.398 \\
6.625 \\
6.816 \\
6.982 \\
7.128 \\
7.259 \\
7.485\end{array}$ & $\begin{array}{l}0.7450 \\
0.7449 \\
0.7449 \\
0.7449 \\
0.7448 \\
0.7448 \\
0.7448 \\
0.7448 \\
0.7448 \\
0.7448\end{array}$ & $\begin{array}{l}1.242 \\
1.242 \\
1.242 \\
1.241 \\
1.241 \\
1.241 \\
1.241 \\
1.241 \\
1.241 \\
1.241\end{array}$ & $\begin{array}{l}1824.0 \\
1934.0 \\
2038.0 \\
2278.0 \\
2495.0 \\
2694.0 \\
2880.0 \\
3054.0 \\
3219.0 \\
3526.0\end{array}$ \\
\hline $\begin{array}{r}700.0 \\
800.0 \\
900.0 \\
1000.0 \\
1200.0 \\
1400.0 \\
1600.0 \\
1860.0 \\
2000.0 \\
2500.0\end{array}$ & $\begin{array}{l}93.89 \\
107.3 \\
120.7 \\
134.1 \\
160.9 \\
187.7 \\
214.5 \\
241.3 \\
268.1 \\
335.2\end{array}$ & $\begin{array}{l}1880.0 \\
2150.0 \\
2410.0 \\
2680.0 \\
3220.0 \\
3760.0 \\
4290.0 \\
4830.0 \\
5360.0 \\
6700.0\end{array}$ & $\begin{array}{l}0.0286 \\
0.0250 \\
0.0222 \\
0.0200 \\
0.0167 \\
0.0143 \\
0.0125 \\
0.0111 \\
0.0100 \\
0.00800\end{array}$ & $\begin{array}{r}527.6 \\
602.1 \\
676.6 \\
751.0 \\
900.0 \\
1049.0 \\
1198.0 \\
1347.0 \\
1496.0 \\
1868.0\end{array}$ & $\begin{array}{r}875.3 \\
999.0 \\
1124.0 \\
1248.0 \\
1496.0 \\
1744.0 \\
1992.0 \\
2241.0 \\
2489.0 \\
3109.0\end{array}$ & $\begin{array}{l}7.676 \\
7.842 \\
7.988 \\
8.119 \\
8.345 \\
8.537 \\
8.703 \\
8.849 \\
8.979 \\
9.256\end{array}$ & $\begin{array}{l}0.7448 \\
0.7448 \\
0.7448 \\
0.7447 \\
0.7447 \\
0.7447 \\
0.7447 \\
0.7447 \\
0.7447 \\
0.7447\end{array}$ & $\begin{array}{l}1.241 \\
1.241 \\
1.241 \\
1.241 \\
1.241 \\
1.241 \\
1.241 \\
1.241 \\
1.241 \\
1.241\end{array}$ & $\begin{array}{l}3808.0 \\
4071.0 \\
4318.0 \\
4551.0 \\
4985.0 \\
5384.0 \\
5756.0 \\
6105.0 \\
6435.0 \\
7194.0\end{array}$ \\
\hline 3000.0 & 402.2 & 8040.0 & 0.00667 & 2240.0 & 3730.0 & 9.483 & 0.7447 & 1.241 & 7881.0 \\
\hline
\end{tabular}

* two-phase gounoary 
THERMOPHYSICAL PRDPERTIES OF HELIUM 4

20 PSIA ISOBAR

TERPERATURE OENSITY

DEG. R LB/CUFT ETU/LB PSIA-CU FT/BTU PSIA

\begin{tabular}{|c|c|c|c|c|}
\hline $\begin{array}{l}4.0 \\
5.0 \\
6.0 \\
7.0 \\
8.0 \\
8.224 \\
8.224 \\
9.0 \\
10.0 \\
11.0\end{array}$ & $\begin{array}{l}9.292 \\
9.111 \\
8.808 \\
8.350 \\
7.585 \\
7.315 \\
1.481 \\
1.138 \\
0.9269 \\
0.7968\end{array}$ & $\begin{array}{c}55.5 \\
19.0 \\
15.5 \\
12.7 \\
9.78 \\
8.90 \\
6.19 \\
7.94 \\
9.72 \\
11.3\end{array}$ & $\begin{array}{l}2.27 \\
6.55 \\
7.13 \\
7.16 \\
6.54 \\
6.26 \\
3.74 \\
3.77 \\
3.78 \\
3.78\end{array}$ & $\begin{array}{c}1150.0 \\
975.0 \\
730.0 \\
467.0 \\
203.0 \\
144.0 \\
8.87 \\
13.2 \\
15.5 \\
16.7\end{array}$ \\
\hline $\begin{array}{l}12.0 \\
13.0 \\
14.0 \\
15.0 \\
16.0 \\
17.0 \\
18.0 \\
19.0 \\
20.0 \\
22.0\end{array}$ & $\begin{array}{l}0.7044 \\
0.6339 \\
0.5777 \\
0.5316 \\
0.4929 \\
0.4599 \\
0.4312 \\
0.4062 \\
0.3840 \\
0.3465\end{array}$ & $\begin{array}{l}12.8 \\
14.3 \\
15.7 \\
17.1 \\
18.5 \\
19.9 \\
21.2 \\
22.5 \\
23.9 \\
26.5\end{array}$ & $\begin{array}{l}3.77 \\
3.76 \\
3.75 \\
3.74 \\
3.73 \\
3.72 \\
3.71 \\
3.71 \\
3.70 \\
3.69\end{array}$ & $\begin{array}{l}17.5 \\
18.0 \\
18.4 \\
18.7 \\
18.9 \\
19.1 \\
19.2 \\
19.3 \\
19.4 \\
19.6\end{array}$ \\
\hline $\begin{array}{l}24.0 \\
26.0 \\
28.0 \\
36.0 \\
32.0 \\
34.0 \\
36.0 \\
38.0 \\
40.0\end{array}$ & $\begin{array}{l}0.3159 \\
0.2904 \\
0.2688 \\
0.2503 \\
0.2342 \\
0.2201 \\
0.2076 \\
0.1965 \\
0.1865\end{array}$ & $\begin{array}{l}29.1 \\
31.7 \\
34.3 \\
36.8 \\
39.4 \\
41.9 \\
44.5 \\
47.0 \\
49.5\end{array}$ & $\begin{array}{l}3.68 \\
3.68 \\
3.67 \\
3.66 \\
3.66 \\
3.66 \\
3.65 \\
3.65 \\
3.65\end{array}$ & $\begin{array}{l}19.7 \\
19.8 \\
19.8 \\
19.9 \\
19.9 \\
19.9 \\
20.0 \\
20.0 \\
20.0\end{array}$ \\
\hline $\begin{array}{r}45.0 \\
50.0 \\
55.0 \\
60.0 \\
70.0 \\
80.0 \\
90.0 \\
160.0 \\
120.0 \\
140.0\end{array}$ & $\begin{array}{l}0.1656 \\
0.1489 \\
0.1353 \\
0.1240 \\
0.1062 \\
0.09295 \\
0.08263 \\
0.07437 \\
0.06200 \\
0.05315\end{array}$ & $\begin{array}{r}55.8 \\
62.1 \\
68.4 \\
74.6 \\
87.1 \\
100.0 \\
112.0 \\
124.0 \\
149.0 \\
174.0\end{array}$ & $\begin{array}{l}3.64 \\
3.63 \\
3.63 \\
3.63 \\
3.62 \\
3.62 \\
3.62 \\
3.61 \\
3.61 \\
3.61\end{array}$ & $\begin{array}{l}20.0 \\
20.0 \\
20.1 \\
20.1 \\
20.1 \\
20.1 \\
20.1 \\
20.1 \\
20.1 \\
20.0\end{array}$ \\
\hline $\begin{array}{l}160.0 \\
180.0 \\
200.0 \\
250.0 \\
300.0 \\
350.0 \\
400.0 \\
450.0 \\
500.0 \\
600.0\end{array}$ & $\begin{array}{l}0.04652 \\
0.04136 \\
0.03723 \\
0.02979 \\
0.02483 \\
0.02129 \\
0.01863 \\
0.01656 \\
0.01491 \\
0.01243\end{array}$ & $\begin{array}{l}199.0 \\
224.0 \\
249.0 \\
311.0 \\
373.0 \\
435.0 \\
497.0 \\
559.0 \\
621.0 \\
745.0\end{array}$ & $\begin{array}{l}3.61 \\
3.61 \\
3.61 \\
3.60 \\
3.60 \\
3.60 \\
3.60 \\
3.60 \\
3.60 \\
3.60\end{array}$ & $\begin{array}{l}20.0 \\
20.0 \\
20.0 \\
20.0 \\
20.0 \\
20.0 \\
20.0 \\
20.0 \\
20.0 \\
20.0\end{array}$ \\
\hline $\begin{array}{r}700.0 \\
800.0 \\
900.0 \\
1000.0 \\
1200.0 \\
1400.0 \\
1600.0 \\
1800.0 \\
2000.0 \\
2500.0\end{array}$ & $\begin{array}{l}0.01065 \\
0.009321 \\
0.008285 \\
0.007457 \\
0.006215 \\
0.005327 \\
0.004661 \\
0.004144 \\
0.003729 \\
0.002984\end{array}$ & $\begin{array}{r}869.0 \\
993.0 \\
1120.0 \\
1240.0 \\
1490.0 \\
1740.0 \\
1990.0 \\
2230.0 \\
2480.0 \\
3100.0\end{array}$ & $\begin{array}{l}3.60 \\
3.60 \\
3.60 \\
3.60 \\
3.60 \\
3.60 \\
3.60 \\
3.60 \\
3.60 \\
3.60\end{array}$ & $\begin{array}{l}20.0 \\
20.0 \\
20.0 \\
20.0 \\
20.0 \\
20.0 \\
20.0 \\
20.0 \\
20.0 \\
20.0\end{array}$ \\
\hline 3000.0 & 0.002486 & 3720.0 & 3.60 & 20.0 \\
\hline
\end{tabular}

COV/DT Y $/$ THERMAL TONDUCTIVITY $V I S C$ 1/OEG. R BTU/FT-HR-R LB/FT-SEC $X 10 E+6$

0.0118
0.0266
0.0419
0.0680
0.143
0.192
0.503
0.252
0.171
0.135
0.113
0.0984
0.0876
0.0791
0.0723
0.0667
0.0620
0.0580
0.0545
0.0487
0.0441
0.0403
0.0371
0.0344
0.0321
0.0301
0.0283
0.0267
0.0253
0.0224
0.0201
0.0183
0.0167
0.0143
0.0125
0.0111
0.0100
0.00832
0.00713

0.00885
0.0103
0.0110
0.0115
0.0115
0.0115
0.00720
0.00682
0.00709
0.00747
0.00788
0.00829
0.00869
0.00908
0.00946
0.00983
0.0102
0.0105
0.0109
0.0115

2.64
2.69
2.56
2.35
2.08
2.00
0.948
0.983
1.04

0.0122

0.0122 0.0128 0.0134
0.0139 0.0145 0.0150 0.0155 0.0160

0.0177

0.0189 0.0189 0.0200 0.0211
0.0232 0.0232 0.0251
0.0271 0.0271
0.0289 0.0325 0.0360

\subsection{4} 0.00555 0.00499 0.00399 0.00333 0.00285 0.00250
0.00222 0.00222
0.00200 0.00167

\subsection{3}

0.0426

0.0426
0.0457

0.0457
0.0532

0.0532
0.0602 0.0602
0.0669 0.0669
0.0733 0.0733
0.0793 0.0793
0.0851 0.0962

1.17
1.24

1.24
1.30

1.36

1.42

1.48

1.54

1.59

1.65
1.76

1.86
1.96

1.96
2.06

2.15

2.24

2.33
2.42

2.42
2.50
2.58

\subsection{8}

2. 97

3.16

3.33
3.67

3.67
3.98

4.28

4.57

5.11
5.62

6.11

6.57

6.90

7.99
9.01 9.01
10.0 10.0
10.9

10.9
11.8

11.8
12.7

0.00143

0.00125

0.00111

$0.0010 \mathrm{C}$

0.00083 0.000714
0.000625 0.000625 0.000500 0.000400

\subsection{7}

0.118

0.128
0.137

0.137
0.156

0.156
0.174

0.174
0.191

0.191

0.208
0.224

0.224
0.263

0.000333

0.299
16.0

17.6

19.1

20.5

23.3
26.0
28.5

28.5
31.0

33.4
39.2

44.6
THERMAL DIELECTRIC OIFFUSIVITY CDNSTANT SQ FT/HR

0.00145
0.00223
0.00192
0.00158
0.00108
0.000924
0.00156
0.00300
0.00459
0.00614
0.00770
0.00929
0.0109
0.0126
0.0143
0.0161
0.0180
0.0199
0.0218
0.00258

$\begin{array}{ll}1.02050 & 0.704 \\ 1.02038 & 0.476 \\ 1.02017 & 0.543 \\ 1.01978 & 0.641 \\ 1.01896 & 0.911 \\ 1.01863 & 1.06 \\ 1.00523 & 1.48 \\ 1.00408 & 1.04 \\ 1.00336 & 0.884 \\ 1.00291 & 0.817 \\ 1.00258 & 0.780 \\ 1.00233 & 0.757 \\ 1.00213 & 0.742 \\ 1.00196 & 0.731 \\ 1.00182 & 0.723 \\ 1.00170 & 0.718 \\ 1.00160 & 0.714 \\ 1.00151 & 0.711 \\ 1.00143 & 0.709 \\ 1.00129 & 0.707\end{array}$

0.0300

0.0345

0.0391

0.0439

0.0540

0.0594

0.0649

0.0855

0.101

D. 118

0.136

0.175
0.217

0.263

0.313

0.422

1.00118
1.00108

1.00108
1.00100

1.00094

1.00088

1. 00082

1. 00078

1.00074
1.00070

1.00062

1.00056

1. 00051

1.00051
1.00047

1.00040

1.00040
1.00035

1. 00031

1. 00028

1.00023

1.00020

0.681

0.829
0.988

0.988
1.44

1.44
1.95

1.95
2.53

3.17

3.86

4.60
6.24

1. 00018

1. 00016

1.00014

1. 00011

1.00008

1.00007

1. 00006

1. 00006

1.00005

8.10

10.2

12.4

14.8

20.2

26.3
33.1

33.1
40.4

40.4
48.4

71.0

1.00004

1.00004

1.00003

1.00003

1.00002

1.00002
1.00002

1.00002
1.00002

1. 00001

1.00001

1.00001
PRANOTL

NUMBER

704

48

.04

0.817

731

.718

0.711

0.705

0.705

0.705

0.705

0.706

0.706

0.707

0.708

0.709

0.710

0.710

0.710

0.708

0.706

0.702

.698

0.694

0.675

0.675

0.671

0.669

0.667

0.666

0.666

0.668
0.669

0.668

0.668

0.668
0.667

0.667

0.667

0.667
0.666

0.666

0.666

0.666

0.666

- two-phase gDUNDary 
22 PSIA ISOBAR

THERMOOYNAMIC PROPERTIES OF HELIUM 4

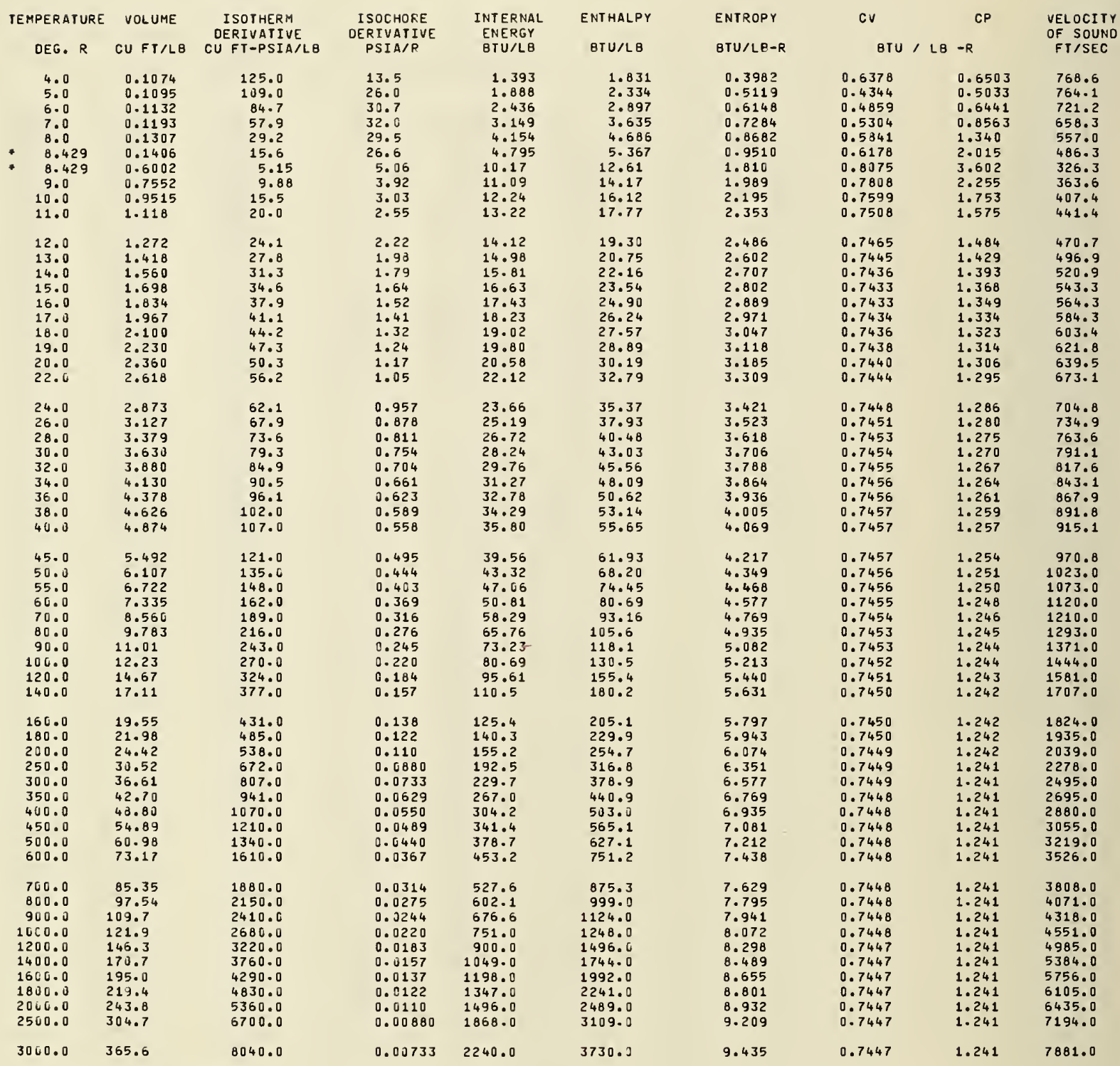

* tho-phase bounoary 
22 PSIA ISOBAR

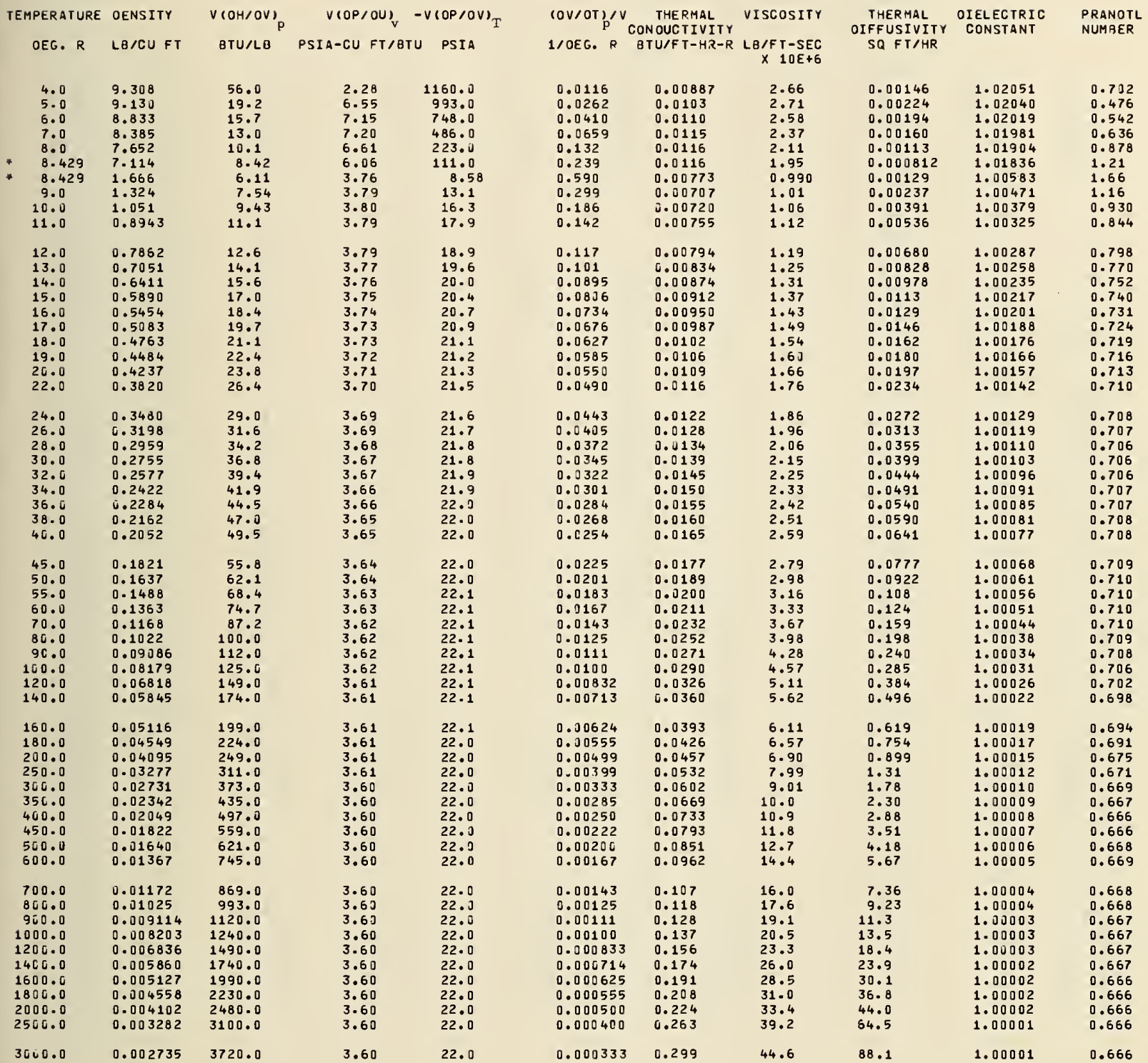

- tho-phase bounoart 


\begin{tabular}{|c|c|c|c|c|c|c|c|c|c|}
\hline $\begin{array}{c}\text { TEHPERATURE } \\
\text { OEG. R }\end{array}$ & $\begin{array}{l}\text { E VOLUHE } \\
\text { CUFT/LB }\end{array}$ & $\begin{array}{c}\text { I SOTHERH } \\
\text { OERIVATIVE } \\
\text { CU FT-PSIA/LA }\end{array}$ & $\begin{array}{l}\text { ISOCHORE } \\
\text { OERIVATIVE } \\
\text { PSIA/R }\end{array}$ & $\begin{array}{l}\text { INTERNAL } \\
\text { ENERGY } \\
\text { BTU/LB }\end{array}$ & $\begin{array}{l}\text { ENTHALPY } \\
\text { BTU/LB }\end{array}$ & $\begin{array}{l}\text { ENTROPY } \\
\text { BTU/LB-R }\end{array}$ & $\begin{array}{l}\text { CV } \\
\text { BTU / LB }\end{array}$ & $\begin{array}{l}C P \\
-R\end{array}$ & $\begin{array}{l}\text { VELOCI TY } \\
\text { OF SOUNO } \\
\text { FT/SEC }\end{array}$ \\
\hline $\begin{array}{l}4.0 \\
5.0 \\
6.0 \\
7.0 \\
8.0 \\
8.620 \\
8.620 \\
9.0 \\
10.0 \\
11.0\end{array}$ & $\begin{array}{l}0.1072 \\
0.1093 \\
0.1129 \\
0.1168 \\
0.1296 \\
0.1450 \\
0.5341 \\
0.6457 \\
0.8437 \\
1.004\end{array}$ & $\begin{array}{c}127.0 \\
111.0 \\
86.5 \\
59.9 \\
31.5 \\
12.0 \\
4.26 \\
7.97 \\
14.3 \\
19.1\end{array}$ & $\begin{array}{l}13.5 \\
26.0 \\
30.8 \\
32.2 \\
30.0 \\
25.5 \\
5.73 \\
4.64 \\
3.45 \\
2.86\end{array}$ & $\begin{array}{l}1.391 \\
1.881 \\
2.425 \\
3.130 \\
4.111 \\
5.579 \\
10.04 \\
10.78 \\
12.06 \\
13.08\end{array}$ & $\begin{array}{l}1.868 \\
2.367 \\
2.927 \\
3.657 \\
4.687 \\
5.723 \\
12.41 \\
13.65 \\
15.81 \\
17.54\end{array}$ & $\begin{array}{l}0.3975 \\
0.5104 \\
0.6123 \\
0.7254 \\
0.8622 \\
0.9865 \\
1.762 \\
1.903 \\
2.131 \\
2.296\end{array}$ & $\begin{array}{l}0.6327 \\
0.4337 \\
0.4854 \\
0.5295 \\
0.5821 \\
0.6331 \\
0.8088 \\
0.7883 \\
0.7629 \\
0.7521\end{array}$ & $\begin{array}{l}0.6450 \\
0.5013 \\
0.6405 \\
0.8465 \\
1.292 \\
2.458 \\
4.317 \\
2.667 \\
1.861 \\
1.629\end{array}$ & $\begin{array}{l}773.7 \\
769.3 \\
727.3 \\
666.1 \\
569.1 \\
464.0 \\
324.7 \\
353.4 \\
401.9 \\
437.7\end{array}$ \\
\hline $\begin{array}{l}12.0 \\
13.0 \\
14.0 \\
15.0 \\
16.0 \\
17.0 \\
18.0 \\
19.0 \\
20.0 \\
22.0\end{array}$ & $\begin{array}{l}1.148 \\
1.285 \\
1.417 \\
1.545 \\
1.671 \\
1.795 \\
1.917 \\
2.037 \\
2.157 \\
2.394\end{array}$ & $\begin{array}{l}23.3 \\
27.1 \\
30.7 \\
34.1 \\
37.4 \\
40.7 \\
43.8 \\
46.9 \\
50.0 \\
56.0\end{array}$ & $\begin{array}{l}2.47 \\
2.20 \\
1.98 \\
1.81 \\
1.67 \\
1.55 \\
1.45 \\
1.36 \\
1.28 \\
1.15\end{array}$ & $\begin{array}{l}14.00 \\
14.87 \\
15.72 \\
16.55 \\
17.36 \\
18.16 \\
18.95 \\
19.74 \\
20.52 \\
22.07\end{array}$ & $\begin{array}{l}19.10 \\
20.59 \\
22.02 \\
23.41 \\
24.78 \\
26.13 \\
27.47 \\
28.79 \\
30.11 \\
32.71\end{array}$ & $\begin{array}{l}2.432 \\
2.551 \\
2.657 \\
2.753 \\
2.841 \\
2.923 \\
3.000 \\
3.071 \\
3.139 \\
3.263\end{array}$ & $\begin{array}{l}0.7471 \\
0.7447 \\
0.7436 \\
0.7432 \\
0.7432 \\
0.7433 \\
0.7435 \\
0.7437 \\
0.7440 \\
0.7444\end{array}$ & $\begin{array}{l}1.518 \\
1.453 \\
1.411 \\
1.381 \\
1.360 \\
1.344 \\
1.331 \\
1.321 \\
1.313 \\
1.300\end{array}$ & $\begin{array}{l}467.9 \\
494.8 \\
519.3 \\
542.0 \\
563.3 \\
583.5 \\
602.8 \\
621.3 \\
639.1 \\
673.0\end{array}$ \\
\hline $\begin{array}{l}24.0 \\
26.0 \\
28.0 \\
30.0 \\
32.0 \\
34.0 \\
36.0 \\
38.0 \\
40.0\end{array}$ & $\begin{array}{l}2.629 \\
2.863 \\
3.095 \\
3.325 \\
3.555 \\
3.784 \\
4.313 \\
4.240 \\
4.468\end{array}$ & $\begin{array}{r}61.9 \\
67.7 \\
73.5 \\
79.2 \\
84.9 \\
90.5 \\
96.1 \\
102.0 \\
107.0\end{array}$ & $\begin{array}{l}1.05 \\
0.961 \\
0.888 \\
0.825 \\
0.770 \\
0.722 \\
0.681 \\
0.643 \\
0.610\end{array}$ & $\begin{array}{l}23.61 \\
25.15 \\
26.68 \\
28.20 \\
29.72 \\
31.24 \\
32.75 \\
34.26 \\
35.77\end{array}$ & $\begin{array}{l}35.30 \\
37.87 \\
40.43 \\
42.98 \\
45.52 \\
48.06 \\
50.58 \\
53.11 \\
55.63\end{array}$ & $\begin{array}{l}3.376 \\
3.478 \\
3.573 \\
3.661 \\
3.743 \\
3.820 \\
3.892 \\
3.961 \\
4.025\end{array}$ & $\begin{array}{l}0.7448 \\
0.7451 \\
0.7453 \\
0.7455 \\
0.7456 \\
0.7457 \\
0.7457 \\
0.7457 \\
0.7457\end{array}$ & $\begin{array}{l}1.291 \\
1.284 \\
1.278 \\
1.273 \\
1.269 \\
1.266 \\
1.263 \\
1.261 \\
1.259\end{array}$ & $\begin{array}{l}704.8 \\
735.1 \\
763.9 \\
791.4 \\
818.0 \\
843.5 \\
868.3 \\
892.3 \\
915.6\end{array}$ \\
\hline $\begin{array}{r}45.0 \\
50.0 \\
55.0 \\
60.0 \\
70.0 \\
80.0 \\
90.0 \\
100.0 \\
120.0 \\
140.0\end{array}$ & $\begin{array}{c}5.035 \\
5.599 \\
6.163 \\
6.726 \\
7.849 \\
8.971 \\
10.09 \\
11.21 \\
13.45 \\
15.69\end{array}$ & $\begin{array}{l}121.0 \\
135.0 \\
148.0 \\
162.0 \\
189.0 \\
216.0 \\
243.0 \\
270.0 \\
324.0 \\
378.0\end{array}$ & $\begin{array}{l}0.540 \\
0.485 \\
0.440 \\
0.403 \\
0.344 \\
0.301 \\
0.267 \\
0.240 \\
0.200 \\
0.172\end{array}$ & $\begin{array}{l}39.54 \\
43.29 \\
47.05 \\
50.79 \\
58.28 \\
65.75 \\
73.22 \\
80.68 \\
95.60 \\
110.5\end{array}$ & $\begin{array}{c}61.91 \\
68.18 \\
74.44 \\
80.68 \\
93.16 \\
105.6 \\
118.1 \\
130.5 \\
155.4 \\
180.2\end{array}$ & $\begin{array}{l}4.173 \\
4.305 \\
4.425 \\
4.533 \\
4.726 \\
4.892 \\
5.039 \\
5.170 \\
5.396 \\
5.588\end{array}$ & $\begin{array}{l}0.7457 \\
0.7457 \\
0.7456 \\
0.7456 \\
0.7455 \\
0.7454 \\
0.7453 \\
0.7453 \\
0.7452 \\
0.7451\end{array}$ & $\begin{array}{l}1.255 \\
1.252 \\
1.250 \\
1.249 \\
1.247 \\
1.245 \\
1.244 \\
1.244 \\
1.243 \\
1.242\end{array}$ & $\begin{array}{r}971.3 \\
1024.0 \\
1074.0 \\
1121.0 \\
1210.0 \\
1293.0 \\
1371.0 \\
1445.0 \\
1582.0 \\
1708.0\end{array}$ \\
\hline $\begin{array}{l}160.0 \\
180.0 \\
200.0 \\
250.0 \\
300.0 \\
350.0 \\
400.0 \\
450.0 \\
500.0 \\
660.0\end{array}$ & $\begin{array}{l}17.92 \\
20.16 \\
22.39 \\
27.98 \\
33.56 \\
39.15 \\
44.73 \\
50.32 \\
55.90 \\
67.07\end{array}$ & $\begin{array}{r}431.0 \\
485.0 \\
539.0 \\
673.0 \\
807.0 \\
941.0 \\
1070.0 \\
1210.0 \\
1340.0 \\
1610.0\end{array}$ & $\begin{array}{l}0.150 \\
0.133 \\
0.120 \\
0.0960 \\
0.0800 \\
0.0686 \\
0.0600 \\
0.0533 \\
0.0480 \\
0.0400\end{array}$ & $\begin{array}{l}125.4 \\
140.3 \\
155.2 \\
192.5 \\
229.7 \\
267.0 \\
304.2 \\
341.4 \\
378.7 \\
453.2\end{array}$ & $\begin{array}{l}205.1 \\
229.9 \\
254.7 \\
316.8 \\
378.9 \\
440.9 \\
503.0 \\
565.1 \\
627.1 \\
751.2\end{array}$ & $\begin{array}{l}5.754 \\
5.900 \\
6.031 \\
6.308 \\
6.534 \\
6.726 \\
6.891 \\
7.038 \\
7.168 \\
7.395\end{array}$ & $\begin{array}{l}0.7450 \\
0.7450 \\
0.7450 \\
0.7449 \\
0.7449 \\
0.7448 \\
0.7448 \\
0.7448 \\
0.7448 \\
0.7448\end{array}$ & $\begin{array}{l}1.242 \\
1.242 \\
1.242 \\
1.241 \\
1.241 \\
1.241 \\
1.241 \\
1.241 \\
1.241 \\
1.241\end{array}$ & $\begin{array}{l}1825.0 \\
1935.0 \\
2039.0 \\
2279.0 \\
2496.0 \\
2695.0 \\
2800.0 \\
3055.0 \\
3220.0 \\
3526.0\end{array}$ \\
\hline $\begin{array}{r}700.0 \\
800.0 \\
900.0 \\
1000.0 \\
1200.0 \\
1400.0 \\
1600.0 \\
1800.0 \\
2000.0 \\
2500.0\end{array}$ & $\begin{array}{l}78.25 \\
89.42 \\
100.6 \\
111.8 \\
134.1 \\
156.4 \\
178.8 \\
201.1 \\
223.5 \\
279.3\end{array}$ & $\begin{array}{l}1880.0 \\
2150.0 \\
2420.0 \\
2680.0 \\
3220.0 \\
3760.0 \\
4290.0 \\
4830.0 \\
5360.0 \\
6700.0\end{array}$ & $\begin{array}{l}0.0343 \\
0.0300 \\
0.0267 \\
0.0240 \\
0.0200 \\
0.0171 \\
0.0150 \\
0.0133 \\
0.0120 \\
0.00960\end{array}$ & $\begin{array}{r}527.6 \\
602.1 \\
676.6 \\
751.0 \\
900.0 \\
1049.0 \\
1198.0 \\
1347.0 \\
1496.0 \\
1868.0\end{array}$ & $\begin{array}{r}875.4 \\
999.0 \\
1124.0 \\
1248.0 \\
1496.0 \\
1744.0 \\
1992.0 \\
2241.0 \\
2489.0 \\
3109.0\end{array}$ & $\begin{array}{l}7.586 \\
7.752 \\
7.898 \\
8.029 \\
8.255 \\
8.446 \\
8.612 \\
8.758 \\
8.889 \\
9.166\end{array}$ & $\begin{array}{l}0.7448 \\
0.7448 \\
0.7448 \\
0.7448 \\
0.7448 \\
0.7447 \\
0.7447 \\
0.7447 \\
0.7447 \\
0.7447\end{array}$ & $\begin{array}{l}1.241 \\
1.241 \\
1.241 \\
1.241 \\
1.241 \\
1.241 \\
1.241 \\
1.241 \\
1.241 \\
1.241\end{array}$ & $\begin{array}{l}3809.0 \\
4071.0 \\
4318.0 \\
4551.0 \\
4985.0 \\
5384.0 \\
5756.0 \\
6105.0 \\
6435.0 \\
7194.0\end{array}$ \\
\hline 3000.0 & 335.2 & 8040.0 & 0.46800 & 2240.0 & 3730.0 & 9.392 & 0.7447 & 1.241 & 7881.0 \\
\hline
\end{tabular}

\footnotetext{
- two-phase boUnoARY
} 
24 PSIA ISOBAR

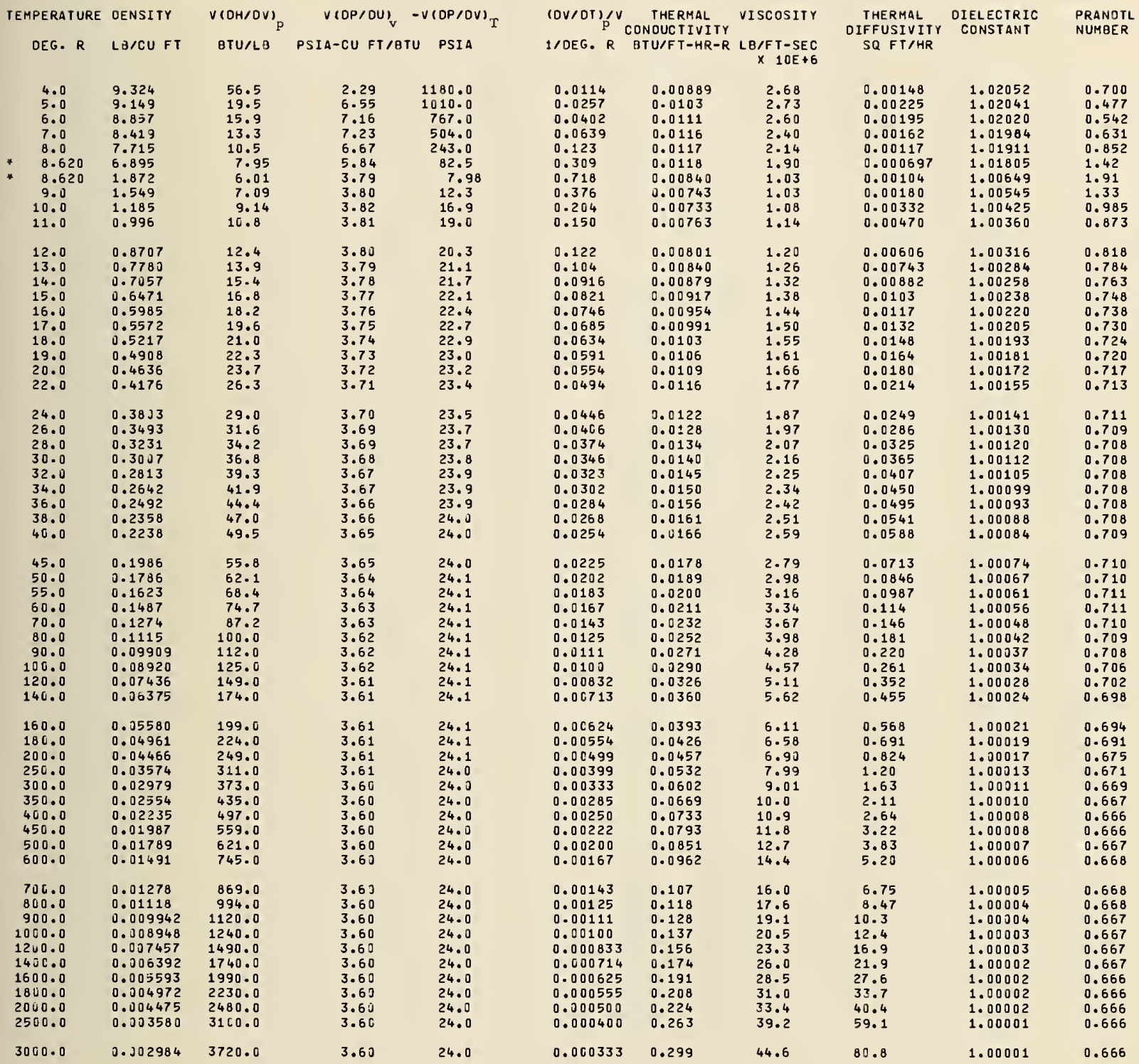

- THO-PHASE gOUNOARY 


\begin{tabular}{|c|c|c|c|c|c|c|c|c|c|}
\hline $\begin{array}{c}\text { TEMPERATURE } \\
\text { OEG. } R\end{array}$ & $\begin{array}{l}\text { VOLUME } \\
\text { CU FT/LB }\end{array}$ & $\begin{array}{c}\text { ISOTHERM } \\
\text { OERIVATIVE } \\
\text { CU FT-PSIA/LB }\end{array}$ & $\begin{array}{l}\text { ISOCHORE } \\
\text { OERIVATIVE } \\
\text { PSIA/R }\end{array}$ & $\begin{array}{l}\text { INTERNAL } \\
\text { ENERGY } \\
\text { BTU/LB }\end{array}$ & $\begin{array}{l}\text { ENTHALPY } \\
\text { BTU/LE }\end{array}$ & $\begin{array}{l}\text { ENTROPY } \\
\text { BTU/LB-R }\end{array}$ & $\begin{array}{l}\text { CV } \\
\text { BTU / LB }\end{array}$ & $\begin{array}{l}C P \\
-R\end{array}$ & $\begin{array}{l}\text { VELOCITY } \\
\text { OF SOUNO } \\
\text { FT/SEC }\end{array}$ \\
\hline $\begin{array}{l}4.0 \\
5.0 \\
6.0 \\
7.0 \\
8.0 \\
8.800 \\
8.800 \\
9.0 \\
10.0 \\
11.0\end{array}$ & $\begin{array}{l}0.1071 \\
0.1091 \\
0.1126 \\
0.1183 \\
0.1287 \\
0.1503 \\
0.4741 \\
0.5431 \\
0.7509 \\
0.9058\end{array}$ & $\begin{array}{c}128.0 \\
112.0 \\
88.4 \\
61.9 \\
33.7 \\
8.65 \\
3.33 \\
5.74 \\
13.0 \\
18.1\end{array}$ & $\begin{array}{c}13.5 \\
26.0 \\
30.9 \\
32.4 \\
30.4 \\
24.3 \\
6.51 \\
5.61 \\
3.91 \\
3.19\end{array}$ & $\begin{array}{c}1.389 \\
1.875 \\
2.415 \\
3.111 \\
4.071 \\
5.383 \\
9.873 \\
10.39 \\
11.85 \\
12.93\end{array}$ & $\begin{array}{l}1.905 \\
2.401 \\
2.957 \\
3.681 \\
4.690 \\
5.107 \\
12.16 \\
13.00 \\
15.47 \\
17.29\end{array}$ & $\begin{array}{l}0.3968 \\
0.5090 \\
0.6109 \\
0.7225 \\
0.8567 \\
1.024 \\
1.711 \\
1.806 \\
2.068 \\
2.241\end{array}$ & $\begin{array}{l}0.6277 \\
0.4330 \\
0.4849 \\
0.5286 \\
0.5802 \\
0.6494 \\
0.8095 \\
0.7969 \\
0.7660 \\
0.7535\end{array}$ & $\begin{array}{l}0.6397 \\
0.4994 \\
0.6370 \\
0.8373 \\
1.251 \\
3.154 \\
5.474 \\
3.486 \\
1.995 \\
1.690\end{array}$ & $\begin{array}{l}778.6 \\
774.3 \\
733.3 \\
673.8 \\
580.5 \\
441.2 \\
322.9 \\
341.1 \\
396.0 \\
433.9\end{array}$ \\
\hline $\begin{array}{l}12.0 \\
13.0 \\
14.0 \\
15.0 \\
16.0 \\
17.0 \\
18.0 \\
19.0 \\
20.0 \\
22.0\end{array}$ & $\begin{array}{l}1.044 \\
1.173 \\
1.296 \\
1.416 \\
1.533 \\
1.648 \\
1.752 \\
1.874 \\
1.985 \\
2.205\end{array}$ & $\begin{array}{l}22.5 \\
26.4 \\
30.1 \\
33.6 \\
37.0 \\
40.3 \\
43.5 \\
46.6 \\
49.7 \\
55.7\end{array}$ & $\begin{array}{l}2.74 \\
2.42 \\
2.18 \\
1.98 \\
1.83 \\
1.69 \\
1.58 \\
1.48 \\
1.40 \\
1.26\end{array}$ & $\begin{array}{l}13.88 \\
14.77 \\
15.63 \\
16.46 \\
17.28 \\
18.09 \\
18.89 \\
19.68 \\
20.46 \\
22.02\end{array}$ & $\begin{array}{l}18.90 \\
20.42 \\
21.87 \\
23.28 \\
24.66 \\
26.02 \\
27.37 \\
28.70 \\
30.02 \\
32.64\end{array}$ & $\begin{array}{l}2.382 \\
2.503 \\
2.610 \\
2.708 \\
2.797 \\
2.880 \\
2.956 \\
3.028 \\
3.096 \\
3.221\end{array}$ & $\begin{array}{l}0.7477 \\
0.7450 \\
0.7437 \\
0.7432 \\
0.7431 \\
0.7432 \\
0.7434 \\
0.7437 \\
0.7439 \\
0.7444\end{array}$ & $\begin{array}{l}1.554 \\
1.478 \\
1.429 \\
1.396 \\
1.372 \\
1.353 \\
1.339 \\
1.328 \\
1.319 \\
1.305\end{array}$ & $\begin{array}{l}465.2 \\
492.7 \\
517.6 \\
540.7 \\
562.3 \\
582.8 \\
602.2 \\
620.8 \\
638.8 \\
672.8\end{array}$ \\
\hline $\begin{array}{l}24.0 \\
26.0 \\
28.0 \\
30.0 \\
32.0 \\
34.0 \\
36.0 \\
38.0 \\
40.0\end{array}$ & $\begin{array}{l}2.423 \\
2.639 \\
2.854 \\
3.068 \\
3.280 \\
3.492 \\
3.703 \\
3.914 \\
4.124\end{array}$ & $\begin{array}{r}61.7 \\
67.6 \\
73.4 \\
79.1 \\
84.8 \\
90.4 \\
96.1 \\
102.0 \\
107.0\end{array}$ & $\begin{array}{l}1.14 \\
1.04 \\
0.964 \\
0.895 \\
0.836 \\
0.784 \\
0.738 \\
0.698 \\
0.662\end{array}$ & $\begin{array}{l}23.56 \\
25.10 \\
26.63 \\
28.16 \\
29.69 \\
31.20 \\
32.72 \\
34.23 \\
35.74\end{array}$ & $\begin{array}{l}35.23 \\
37.81 \\
40.38 \\
42.93 \\
45.48 \\
48.02 \\
50.55 \\
53.08 \\
55.60\end{array}$ & $\begin{array}{l}3.334 \\
3.437 \\
3.532 \\
3.620 \\
3.702 \\
3.779 \\
3.852 \\
3.920 \\
3.985\end{array}$ & $\begin{array}{l}0.7448 \\
0.7451 \\
0.7454 \\
0.7456 \\
0.7457 \\
0.7457 \\
0.7458 \\
0.7458 \\
0.7458\end{array}$ & $\begin{array}{l}1.295 \\
1.287 \\
1.281 \\
1.276 \\
1.271 \\
1.268 \\
1.265 \\
1.262 \\
1.260\end{array}$ & $\begin{array}{l}704.8 \\
735.2 \\
764.1 \\
791.7 \\
818.3 \\
843.9 \\
868.7 \\
892.7 \\
916.1\end{array}$ \\
\hline $\begin{array}{r}45.0 \\
50.0 \\
55.0 \\
60.0 \\
70.0 \\
80.0 \\
90.0 \\
100.0 \\
120.0 \\
140.0\end{array}$ & $\begin{array}{c}4.648 \\
5.170 \\
5.690 \\
6.210 \\
7.248 \\
8.284 \\
9.318 \\
10.35 \\
12.42 \\
14.48\end{array}$ & $\begin{array}{l}121.0 \\
135.0 \\
148.0 \\
162.0 \\
189.0 \\
216.0 \\
243.0 \\
270.0 \\
324.0 \\
378.0\end{array}$ & $\begin{array}{l}0.586 \\
0.526 \\
0.477 \\
0.437 \\
0.373 \\
0.326 \\
0.290 \\
0.261 \\
0.217 \\
0.186\end{array}$ & $\begin{array}{c}39.51 \\
43.27 \\
47.03 \\
50.78 \\
58.26 \\
65.74 \\
73.21 \\
80.68 \\
95.60 \\
110.5\end{array}$ & $\begin{array}{c}51.89 \\
68.16 \\
74.42 \\
80.67 \\
93.16 \\
105.6 \\
118.1 \\
130.5 \\
155.4 \\
180.2\end{array}$ & $\begin{array}{l}4.133 \\
4.255 \\
4.385 \\
4.493 \\
4.686 \\
4.852 \\
4.999 \\
5.130 \\
5.357 \\
5.548\end{array}$ & $\begin{array}{l}0.7458 \\
0.7458 \\
0.7457 \\
0.7457 \\
0.7456 \\
0.7455 \\
0.7454 \\
0.7453 \\
0.7452 \\
0.7451\end{array}$ & $\begin{array}{l}1.256 \\
1.253 \\
1.251 \\
1.249 \\
1.247 \\
1.246 \\
1.245 \\
1.244 \\
1.243 \\
1.242\end{array}$ & $\begin{array}{r}971.0 \\
1024.0 \\
1074.0 \\
1122.0 \\
1211.0 \\
1294.0 \\
1372.0 \\
1445.0 \\
1582.0 \\
1708.0\end{array}$ \\
\hline $\begin{array}{l}160.0 \\
180.0 \\
200.0 \\
250.0 \\
300.0 \\
350.0 \\
400.0 \\
450.0 \\
500.0 \\
600.0\end{array}$ & $\begin{array}{l}16.55 \\
18.01 \\
20.67 \\
25.83 \\
30.99 \\
36.14 \\
41.30 \\
46.45 \\
51.61 \\
61.92\end{array}$ & $\begin{array}{r}431.0 \\
485.0 \\
539.0 \\
673.0 \\
807.0 \\
941.0 \\
1070.0 \\
1210.0 \\
1340.0 \\
1610.0\end{array}$ & $\begin{array}{l}0.163 \\
0.144 \\
0.130 \\
0.104 \\
0.0867 \\
0.0743 \\
0.0650 \\
0.0578 \\
0.0520 \\
0.0433\end{array}$ & $\begin{array}{l}125.4 \\
140.3 \\
155.2 \\
192.5 \\
229.7 \\
257.0 \\
304.2 \\
341.4 \\
378.7 \\
453.2\end{array}$ & $\begin{array}{l}205.1 \\
229.9 \\
254.8 \\
316.8 \\
378.9 \\
441.9 \\
503.0 \\
565.1 \\
627.2 \\
751.3\end{array}$ & $\begin{array}{l}5.714 \\
5.860 \\
5.991 \\
6.268 \\
6.495 \\
6.686 \\
6.852 \\
6.998 \\
7.129 \\
7.355\end{array}$ & $\begin{array}{l}0.7451 \\
0.7450 \\
0.7450 \\
0.7449 \\
0.7449 \\
0.7449 \\
0.7448 \\
0.7448 \\
0.7448 \\
0.7448\end{array}$ & $\begin{array}{l}1.242 \\
1.242 \\
1.242 \\
1.241 \\
1.241 \\
1.241 \\
1.241 \\
1.241 \\
1.241 \\
1.241\end{array}$ & $\begin{array}{l}1825.0 \\
1935.0 \\
2040.0 \\
2279.0 \\
2496.0 \\
2695.0 \\
2881.0 \\
3055.0 \\
3220.0 \\
3527.0\end{array}$ \\
\hline $\begin{array}{r}700.0 \\
800.0 \\
900.0 \\
1000.0 \\
1200.0 \\
1400.0 \\
1600.0 \\
1800.0 \\
2000.0 \\
2500.0\end{array}$ & $\begin{array}{c}72.23 \\
82.54 \\
92.85 \\
103.2 \\
123.8 \\
144.4 \\
165.0 \\
185.7 \\
200.3 \\
257.8\end{array}$ & $\begin{array}{l}1880.0 \\
2150.0 \\
2420.0 \\
2680.0 \\
3220.0 \\
3760.0 \\
4290.0 \\
4830.0 \\
5360.0 \\
6700.0\end{array}$ & $\begin{array}{l}0.0371 \\
0.0325 \\
0.0289 \\
0.0260 \\
0.0217 \\
0.0186 \\
0.0162 \\
0.0144 \\
0.0130 \\
0.0104\end{array}$ & $\begin{array}{r}527.6 \\
602.1 \\
676.6 \\
751.1 \\
900.0 \\
1049.0 \\
1198.0 \\
1347.0 \\
1496.0 \\
1868.0\end{array}$ & $\begin{array}{r}875.4 \\
1000.0 \\
1124.0 \\
1248.0 \\
1496.0 \\
1744.0 \\
1992.0 \\
2241.0 \\
2489.0 \\
3109.0\end{array}$ & $\begin{array}{l}7.546 \\
7.712 \\
7.858 \\
7.989 \\
8.215 \\
8.407 \\
8.572 \\
8.718 \\
8.849 \\
9.126\end{array}$ & $\begin{array}{l}0.7448 \\
0.7448 \\
0.7448 \\
0.7448 \\
0.7448 \\
0.7448 \\
0.7447 \\
0.7447 \\
0.7447 \\
0.7447\end{array}$ & $\begin{array}{l}1.241 \\
1.241 \\
1.241 \\
1.241 \\
1.241 \\
1.241 \\
1.241 \\
1.241 \\
1.241 \\
1.241\end{array}$ & $\begin{array}{l}3809.0 \\
4071.0 \\
4318.0 \\
4551.0 \\
4985.0 \\
5385.0 \\
5756.0 \\
5105.0 \\
5435.0 \\
7194.0\end{array}$ \\
\hline 3000.0 & 309.4 & 8040.0 & 0.00867 & 2240.0 & 3730.0 & 9.352 & 0.7447 & 1.241 & 7881.0 \\
\hline
\end{tabular}


THERMOPHYSICAL PROPERTIES OF HELIUM 4

26 PSIA ISDBAR

\begin{tabular}{|c|c|c|c|c|c|c|c|c|c|c|}
\hline $\begin{array}{c}\text { TEMPERATURE } \\
\text { DEG. R }\end{array}$ & OENSITY & $\begin{array}{l}V(\mathrm{OH} / \mathrm{OV})_{P} \\
\text { BrU/LB }\end{array}$ & $\begin{array}{l}V(O P / O U)_{V}-V \\
\text { PSIA-CU FT/BTU }\end{array}$ & $\begin{array}{l}-V(O P / O V) T \\
\text { PSIA }\end{array}$ & $\begin{array}{l}\text { COV/OT })_{\mathrm{P}} / \mathrm{V} \\
\text { 1/OEG. } \mathrm{R}\end{array}$ & $\begin{array}{c}\text { THERMAL } \\
\text { CONDUCTIVITY } \\
\text { BTU/FT }=H R=R\end{array}$ & $\begin{array}{l}\text { VISCOSITY } \\
\text { LB/FT-SEC } \\
\times 10 E+6\end{array}$ & $\begin{array}{c}\text { THERMAL } \\
\text { DIFFUSIVITY } \\
\text { SQ FT/HR }\end{array}$ & $\begin{array}{l}\text { OIELECTRIC } \\
\text { CONSTANT }\end{array}$ & $\begin{array}{l}\text { PRANDTL } \\
\text { NUMBER }\end{array}$ \\
\hline $\begin{array}{l}4.0 \\
5.0 \\
6.0 \\
7.0 \\
8.0 \\
8.800 \\
8.850 \\
9.0 \\
10.0 \\
11.0\end{array}$ & $\begin{array}{l}9.341 \\
9.168 \\
8.881 \\
8.452 \\
7.773 \\
6.651 \\
2.109 \\
1.841 \\
1.332 \\
1.104\end{array}$ & $\begin{array}{c}57.0 \\
19.8 \\
16.2 \\
13.5 \\
10.8 \\
7.48 \\
5.90 \\
6.58 \\
8.83 \\
10.6\end{array}$ & $\begin{array}{l}2.30 \\
6.55 \\
7.17 \\
7.26 \\
6.74 \\
5.62 \\
3.81 \\
3.82 \\
3.83 \\
3.83\end{array}$ & $\begin{array}{c}1200.0 \\
1030.0 \\
785.0 \\
523.0 \\
262.0 \\
57.5 \\
7.02 \\
10.6 \\
17.3 \\
20.0\end{array}$ & $\begin{array}{l}0.0112 \\
0.0253 \\
0.0394 \\
0.0620 \\
0.116 \\
0.422 \\
0.927 \\
0.530 \\
0.226 \\
0.159\end{array}$ & $\begin{array}{l}0.00890 \\
0.0104 \\
0.0111 \\
0.0116 \\
0.0118 \\
0.0121 \\
0.00937 \\
0.00810 \\
0.00749 \\
0.00773\end{array}$ & $\begin{array}{l}2.70 \\
2.75 \\
2.62 \\
2.42 \\
2.16 \\
1.84 \\
1.08 \\
1.07 \\
1.10 \\
1.15\end{array}$ & $\begin{array}{l}0.00149 \\
0.00226 \\
0.00196 \\
0.00164 \\
0.00121 \\
0.000576 \\
0.000811 \\
0.00126 \\
0.00282 \\
0.00414\end{array}$ & $\begin{array}{l}1.02053 \\
1.02042 \\
1.02022 \\
1.01987 \\
1.01918 \\
1.01769 \\
1.00723 \\
1.00639 \\
1.00474 \\
1.00397\end{array}$ & $\begin{array}{l}0.698 \\
0.477 \\
0.541 \\
0.627 \\
0.829 \\
1.73 \\
2.27 \\
1.66 \\
1.05 \\
0.907\end{array}$ \\
\hline $\begin{array}{l}12.0 \\
13.0 \\
14.0 \\
15.0 \\
16.0 \\
17.0 \\
18.0 \\
19.0 \\
20.0 \\
22.0\end{array}$ & $\begin{array}{l}0.9582 \\
0.8528 \\
0.7715 \\
0.7062 \\
0.6522 \\
0.6067 \\
0.5675 \\
0.5336 \\
0.5337 \\
0.4534\end{array}$ & $\begin{array}{l}12.2 \\
13.8 \\
15.2 \\
16.7 \\
18.1 \\
19.5 \\
20.9 \\
22.2 \\
23.6 \\
26.3\end{array}$ & $\begin{array}{l}3.82 \\
3.81 \\
3.79 \\
3.78 \\
3.77 \\
3.76 \\
3.75 \\
3.74 \\
3.73 \\
3.72\end{array}$ & $\begin{array}{l}21.5 \\
22.5 \\
23.2 \\
23.7 \\
24.1 \\
24.4 \\
24.7 \\
24.9 \\
25.0 \\
25.3\end{array}$ & $\begin{array}{l}0.127 \\
0.107 \\
0.0937 \\
0.0836 \\
0.0757 \\
0.0694 \\
0.0641 \\
0.0597 \\
0.0559 \\
0.0497\end{array}$ & $\begin{array}{l}0.00808 \\
0.00845 \\
0.00884 \\
0.00921 \\
0.00958 \\
0.00995 \\
0.0103 \\
0.0106 \\
0.0110 \\
0.0116\end{array}$ & $\begin{array}{l}1.21 \\
1.27 \\
1.33 \\
1.39 \\
1.45 \\
1.50 \\
1.56 \\
1.61 \\
1.67 \\
1.77\end{array}$ & $\begin{array}{l}0.00542 \\
0.00671 \\
0.00801 \\
0.00935 \\
0.0107 \\
0.0121 \\
0.0136 \\
0.0150 \\
0.0165 \\
0.0197\end{array}$ & $\begin{array}{l}1.00347 \\
1.00310 \\
1.00282 \\
1.00259 \\
1.00239 \\
1.00223 \\
1.00209 \\
1.00197 \\
1.00186 \\
1.00168\end{array}$ & $\begin{array}{l}0.838 \\
0.799 \\
0.774 \\
0.757 \\
0.745 \\
0.736 \\
0.730 \\
0.725 \\
0.721 \\
0.716\end{array}$ \\
\hline $\begin{array}{l}24.0 \\
26.0 \\
28.0 \\
36.0 \\
32.0 \\
34.0 \\
36.0 \\
38.0 \\
40.0\end{array}$ & $\begin{array}{l}0.4127 \\
0.3789 \\
0.3504 \\
0.3260 \\
0.3048 \\
0.2864 \\
0.2700 \\
0.2555 \\
0.2425\end{array}$ & $\begin{array}{l}28.9 \\
31.5 \\
34.1 \\
36.7 \\
39.3 \\
41.9 \\
44.4 \\
47.0 \\
49.5\end{array}$ & $\begin{array}{l}3.71 \\
3.70 \\
3.69 \\
3.68 \\
3.68 \\
3.67 \\
3.67 \\
3.66 \\
3.66\end{array}$ & $\begin{array}{l}25.5 \\
25.6 \\
25.7 \\
25.8 \\
25.8 \\
25.9 \\
25.9 \\
26.0 \\
26.0\end{array}$ & $\begin{array}{l}0.0448 \\
0.0408 \\
0.0375 \\
0.0347 \\
0.0323 \\
0.0303 \\
0.0285 \\
0.0269 \\
0.0255\end{array}$ & $\begin{array}{l}0.0123 \\
0.0129 \\
0.0134 \\
0.0140 \\
0.0145 \\
0.0151 \\
0.0156 \\
0.0161 \\
0.0166\end{array}$ & $\begin{array}{l}1.88 \\
1.97 \\
2.07 \\
2.16 \\
2.25 \\
2.34 \\
2.43 \\
2.51 \\
2.59\end{array}$ & $\begin{array}{l}0.0229 \\
0.0264 \\
0.0299 \\
0.0337 \\
0.0375 \\
0.0415 \\
0.0456 \\
0.0499 \\
0.0543\end{array}$ & $\begin{array}{l}1.00153 \\
1.00141 \\
1.00130 \\
1.00121 \\
1.00114 \\
1.00107 \\
1.00101 \\
1.00095 \\
1.00091\end{array}$ & $\begin{array}{l}0.713 \\
0.711 \\
0.710 \\
0.710 \\
0.709 \\
0.709 \\
0.709 \\
0.709 \\
0.710\end{array}$ \\
\hline $\begin{array}{r}45.0 \\
50.0 \\
55.0 \\
60.0 \\
70.0 \\
80.0 \\
90.0 \\
100.0 \\
120.0 \\
140.0\end{array}$ & $\begin{array}{l}0.2152 \\
0.1934 \\
0.1757 \\
0.1610 \\
0.1380 \\
0.1237 \\
0.1373 \\
0.09660 \\
0.08353 \\
0.06905\end{array}$ & $\begin{array}{r}55.8 \\
62.1 \\
68.4 \\
74.7 \\
87.2 \\
140.0 \\
112.0 \\
125.0 \\
149.0 \\
174.0\end{array}$ & $\begin{array}{l}3.65 \\
3.64 \\
3.64 \\
3.64 \\
3.63 \\
3.62 \\
3.62 \\
3.62 \\
3.62 \\
3.61\end{array}$ & $\begin{array}{l}26.0 \\
26.1 \\
26.1 \\
26.1 \\
26.1 \\
26.1 \\
26.1 \\
26.1 \\
26.1 \\
26.1\end{array}$ & $\begin{array}{l}0.0225 \\
0.0232 \\
0.0183 \\
0.0167 \\
0.0143 \\
0.0125 \\
0.0111 \\
0.0100 \\
0.00832 \\
0.00713\end{array}$ & $\begin{array}{l}0.0178 \\
0.0189 \\
0.0200 \\
0.0211 \\
0.0232 \\
0.0252 \\
0.0271 \\
0.0290 \\
0.0326 \\
0.0360\end{array}$ & $\begin{array}{l}2.79 \\
2.98 \\
3.16 \\
3.34 \\
3.67 \\
3.99 \\
4.28 \\
4.57 \\
5.11 \\
5.62\end{array}$ & $\begin{array}{l}0.0658 \\
0.0781 \\
0.0912 \\
0.105 \\
0.135 \\
0.168 \\
0.203 \\
0.241 \\
0.325 \\
0.420\end{array}$ & $\begin{array}{l}1.00081 \\
1.00072 \\
1.00066 \\
1.00060 \\
1.00052 \\
1.00045 \\
1.00040 \\
1.00036 \\
1.00030 \\
1.00026\end{array}$ & $\begin{array}{l}0.710 \\
0.711 \\
0.711 \\
0.711 \\
0.711 \\
0.709 \\
0.708 \\
0.706 \\
0.702 \\
0.698\end{array}$ \\
\hline $\begin{array}{l}160.0 \\
180.0 \\
200.0 \\
250.0 \\
300.0 \\
350.0 \\
400.0 \\
456.0 \\
500.0 \\
600.0\end{array}$ & $\begin{array}{l}0.06044 \\
\text { C.05374 } \\
0.04837 \\
0.03872 \\
0 . J 3227 \\
0.02767 \\
0 . J 2421 \\
0.02153 \\
0.01938 \\
0.01615\end{array}$ & $\begin{array}{l}199.0 \\
224.0 \\
249.0 \\
311.0 \\
373.0 \\
435.0 \\
497.0 \\
559.0 \\
621.0 \\
745.0\end{array}$ & $\begin{array}{l}3.61 \\
3.61 \\
3.61 \\
3.61 \\
3.61 \\
3.60 \\
3.60 \\
3.60 \\
3.60 \\
3.60\end{array}$ & $\begin{array}{l}26.1 \\
26.1 \\
26.1 \\
26.0 \\
26.0 \\
26.0 \\
26.0 \\
26.0 \\
26.0 \\
26.0\end{array}$ & $\begin{array}{l}0.00624 \\
0.00554 \\
0.00499 \\
0.00399 \\
0.06333 \\
0.00285 \\
0.00250 \\
0.00222 \\
0.06200 \\
0.00167\end{array}$ & $\begin{array}{l}0.0394 \\
c .0426 \\
0.0457 \\
0.0532 \\
0.0603 \\
0.0669 \\
0.0733 \\
0.0793 \\
0.0851 \\
0.0962\end{array}$ & $\begin{array}{c}6.11 \\
6.58 \\
6.90 \\
7.99 \\
9.01 \\
10.0 \\
10.9 \\
11.8 \\
12.7 \\
14.4\end{array}$ & $\begin{array}{l}0.524 \\
0.638 \\
0.761 \\
1.11 \\
1.50 \\
1.95 \\
2.44 \\
2.97 \\
3.54 \\
4.80\end{array}$ & $\begin{array}{l}1.00023 \\
1.00020 \\
1.00018 \\
1.00015 \\
1.00012 \\
1.00010 \\
1.00009 \\
1.00008 \\
1.00007 \\
1.00006\end{array}$ & $\begin{array}{l}0.694 \\
0.691 \\
0.675 \\
0.671 \\
0.669 \\
0.667 \\
0.666 \\
0.666 \\
0.667 \\
0.668\end{array}$ \\
\hline $\begin{array}{r}70 C .0 \\
86 C .0 \\
9 C C .0 \\
100 C .0 \\
12 J C .0 \\
140 C .0 \\
1600.0 \\
180 C .0 \\
200 C .0 \\
2500.0\end{array}$ & $\begin{array}{l}0.01384 \\
0.01212 \\
0.01077 \\
0.003693 \\
0.008078 \\
0.036925 \\
0.006059 \\
0.005386 \\
0.004848 \\
0.003878\end{array}$ & $\begin{array}{r}869.0 \\
994.0 \\
1120.0 \\
1240.0 \\
1490.0 \\
1740.0 \\
1990.0 \\
2230.0 \\
2480.0 \\
3100.0\end{array}$ & $\begin{array}{l}3.60 \\
3.60 \\
3.60 \\
3.60 \\
3.60 \\
3.60 \\
3.60 \\
3.60 \\
3.60 \\
3.60\end{array}$ & $\begin{array}{l}26.0 \\
26.0 \\
26.0 \\
26.0 \\
26.0 \\
26.0 \\
26.0 \\
26.0 \\
26.0 \\
26.0\end{array}$ & $\begin{array}{l}0.00143 \\
0.00125 \\
0.00111 \\
0.00100 \\
0.000833 \\
0.000714 \\
0.000625 \\
0.000555 \\
0.00050 c \\
0.000400\end{array}$ & $\begin{array}{l}0.107 \\
0.118 \\
0.128 \\
0.137 \\
0.156 \\
0.174 \\
0.191 \\
0.208 \\
0.224 \\
0.263\end{array}$ & $\begin{array}{l}16.0 \\
17.6 \\
19.1 \\
20.5 \\
23.3 \\
26.0 \\
28.5 \\
31.0 \\
33.4 \\
39.2\end{array}$ & $\begin{array}{l}6.23 \\
7.81 \\
9.55 \\
11.4 \\
15.6 \\
20.3 \\
25.4 \\
31.1 \\
37.3 \\
54.6\end{array}$ & $\begin{array}{l}1.00005 \\
1.00005 \\
1.00004 \\
1.00004 \\
1.00003 \\
1.00003 \\
1.00002 \\
1.00002 \\
1.00002 \\
1.00001\end{array}$ & $\begin{array}{l}0.668 \\
0.668 \\
0.667 \\
0.667 \\
0.667 \\
0.667 \\
0.6666 \\
0.666 \\
0.666 \\
0.6666\end{array}$ \\
\hline 3600.0 & 0.003232 & $372 u ̈ .0$ & 3.60 & 26.0 & 0.000333 & 0.299 & 44.6 & 74.6 & 1.00001 & 0.666 \\
\hline
\end{tabular}

* thd-phase boundary 
23 PSIA ISOBAR

THERMOOYNAMIC PROPERTIES OF HELIUM 4

\begin{tabular}{|c|c|c|c|c|c|c|c|c|c|}
\hline $\begin{array}{c}\text { TEMPERATURE } \\
\text { DEG. R }\end{array}$ & $\begin{array}{l}\text { E VOLUME } \\
\text { CU FT/La }\end{array}$ & $\begin{array}{c}\text { ISOTHERM } \\
\text { OERIVATIVE } \\
\text { CU FT-PSIA/LB }\end{array}$ & $\begin{array}{l}\text { ISOCHORE } \\
\text { OERIVATIVE } \\
\text { PSIA/R }\end{array}$ & $\begin{array}{l}\text { INTERNAL } \\
\text { ENERGY } \\
\text { BTU/LB }\end{array}$ & $\begin{array}{l}\text { ENTHALPY } \\
\text { BTU/LB }\end{array}$ & $\begin{array}{l}\text { ENTROPY } \\
\text { BTU/LB-R }\end{array}$ & $\begin{array}{l}\text { CV } \\
\text { BTU / LB }\end{array}$ & $\begin{array}{l}C P \\
-R\end{array}$ & $\begin{array}{l}\text { VELOCITY } \\
\text { OF SOUND } \\
\text { FT/SEC }\end{array}$ \\
\hline $\begin{array}{l}4.0 \\
5.0 \\
6.0 \\
7.0 \\
8.0 \\
8.971 \\
8.971 \\
9.0 \\
10.0 \\
11.0\end{array}$ & $\begin{array}{l}0.1069 \\
0.1089 \\
0.1123 \\
0.1179 \\
0.1278 \\
0.1571 \\
0.4174 \\
0.4320 \\
0.6693 \\
0.8214\end{array}$ & $\begin{array}{c}130.0 \\
114.0 \\
90.1 \\
63.8 \\
35.9 \\
5.69 \\
2.35 \\
2.85 \\
11.6 \\
17.1\end{array}$ & $\begin{array}{l}13.4 \\
26.0 \\
31.0 \\
32.6 \\
30.8 \\
22.8 \\
7.47 \\
7.19 \\
4.43 \\
3.54\end{array}$ & $\begin{array}{l}1.388 \\
1.870 \\
2.405 \\
3.094 \\
4.034 \\
5.723 \\
9.644 \\
9.770 \\
11.64 \\
12.77\end{array}$ & $\begin{array}{l}1.942 \\
2.434 \\
2.987 \\
3.705 \\
4.696 \\
6.537 \\
11.81 \\
12.01 \\
15.11 \\
17.03\end{array}$ & $\begin{array}{l}0.3961 \\
0.5076 \\
0.6090 \\
0.7196 \\
0.8515 \\
1.066 \\
1.653 \\
1.676 \\
2.005 \\
2.189\end{array}$ & $\begin{array}{l}0.6227 \\
0.4323 \\
0.4844 \\
0.5278 \\
0.5785 \\
0.6676 \\
0.8093 \\
0.8072 \\
0.7694 \\
0.7551\end{array}$ & $\begin{array}{l}0.6345 \\
0.4975 \\
0.6336 \\
0.8287 \\
1.216 \\
4.418 \\
7.668 \\
6.443 \\
2.167 \\
1.759\end{array}$ & $\begin{array}{l}783.5 \\
779.3 \\
739.1 \\
681.2 \\
591.4 \\
417.6 \\
321.2 \\
324.8 \\
389.9 \\
430.0\end{array}$ \\
\hline $\begin{array}{l}12.0 \\
13.0 \\
14.0 \\
15.0 \\
16.0 \\
17.0 \\
18.0 \\
19.0 \\
20.0 \\
22.0\end{array}$ & $\begin{array}{l}0.9535 \\
1.076 \\
1.192 \\
1.3 J 5 \\
1.415 \\
1.523 \\
1.629 \\
1.734 \\
1.838 \\
2.043\end{array}$ & $\begin{array}{l}21.7 \\
25.7 \\
29.5 \\
33.1 \\
36.5 \\
39.9 \\
43.1 \\
46.3 \\
49.4 \\
55.5\end{array}$ & $\begin{array}{l}3.01 \\
2.65 \\
2.38 \\
2.16 \\
1.99 \\
1.84 \\
1.72 \\
1.61 \\
1.52 \\
1.36\end{array}$ & $\begin{array}{l}13.75 \\
14.67 \\
15.53 \\
16.38 \\
17.20 \\
18.02 \\
18.82 \\
19.61 \\
20.40 \\
21.97\end{array}$ & $\begin{array}{l}18.70 \\
20.24 \\
21.72 \\
23.15 \\
24.54 \\
25.91 \\
27.27 \\
28.61 \\
29.93 \\
32.56\end{array}$ & $\begin{array}{l}2.334 \\
2.457 \\
2.567 \\
2.665 \\
2.755 \\
2.839 \\
2.916 \\
2.988 \\
3.056 \\
3.182\end{array}$ & $\begin{array}{l}0.7484 \\
0.7452 \\
0.7438 \\
0.7432 \\
0.7431 \\
0.7432 \\
0.7434 \\
0.7436 \\
0.7438 \\
0.7443\end{array}$ & $\begin{array}{l}1.594 \\
1.504 \\
1.448 \\
1.411 \\
1.383 \\
1.363 \\
1.347 \\
1.335 \\
1.326 \\
1.310\end{array}$ & $\begin{array}{l}462.4 \\
490.6 \\
516.0 \\
539.4 \\
561.3 \\
582.0 \\
601.6 \\
620.4 \\
636.4 \\
672.7\end{array}$ \\
\hline $\begin{array}{l}24.0 \\
26.0 \\
28.0 \\
30.0 \\
32.0 \\
34.0 \\
36.0 \\
38.0 \\
40.0\end{array}$ & $\begin{array}{l}2.246 \\
2.448 \\
2.648 \\
2.847 \\
3.045 \\
3.242 \\
3.438 \\
3.634 \\
3.829\end{array}$ & $\begin{array}{r}61.5 \\
67.4 \\
73.2 \\
79.0 \\
84.7 \\
90.4 \\
96.0 \\
102.0 \\
107.0\end{array}$ & $\begin{array}{l}1.23 \\
1.13 \\
1.04 \\
0.967 \\
0.902 \\
0.846 \\
0.797 \\
0.753 \\
3.714\end{array}$ & $\begin{array}{l}23.52 \\
25.06 \\
26.59 \\
28.12 \\
29.65 \\
31.17 \\
32.69 \\
34.20 \\
35.72\end{array}$ & $\begin{array}{l}35.16 \\
37.75 \\
40.32 \\
42.88 \\
45.44 \\
47.98 \\
50.52 \\
53.05 \\
55.57\end{array}$ & $\begin{array}{l}3.295 \\
3.398 \\
3.494 \\
3.582 \\
3.665 \\
3.742 \\
3.814 \\
3.883 \\
3.947\end{array}$ & $\begin{array}{l}0.7448 \\
0.7452 \\
0.7454 \\
0.7456 \\
0.7457 \\
0.7458 \\
0.7459 \\
0.7459 \\
0.7459\end{array}$ & $\begin{array}{l}1.299 \\
1.291 \\
1.284 \\
1.278 \\
1.274 \\
1.270 \\
1.267 \\
1.264 \\
1.262\end{array}$ & $\begin{array}{l}704.9 \\
735.4 \\
764.3 \\
792.0 \\
818.7 \\
844.3 \\
869.1 \\
893.2 \\
916.6\end{array}$ \\
\hline $\begin{array}{r}45.0 \\
50.0 \\
55.0 \\
60.0 \\
70.0 \\
80.0 \\
90.0 \\
100.0 \\
120.0 \\
140.0\end{array}$ & $\begin{array}{l}4.316 \\
4.802 \\
5.285 \\
5.768 \\
6.732 \\
7.694 \\
8.655 \\
9.615 \\
11.53 \\
13.45\end{array}$ & $\begin{array}{l}121.0 \\
135.0 \\
149.0 \\
162.0 \\
189.0 \\
216.0 \\
243.0 \\
270.0 \\
324.0 \\
378.0\end{array}$ & $\begin{array}{l}0.632 \\
0.567 \\
0.514 \\
0.470 \\
0.402 \\
0.351 \\
0.312 \\
0.281 \\
0.234 \\
0.200\end{array}$ & $\begin{array}{l}39.49 \\
43.25 \\
47.01 \\
50.76 \\
58.25 \\
65.73 \\
73.20 \\
80.67 \\
95.59 \\
110.5\end{array}$ & $\begin{array}{l}61.87 \\
68.15 \\
74.41 \\
80.67 \\
93.15 \\
105.6 \\
118.1 \\
130.5 \\
155.4 \\
180.2\end{array}$ & $\begin{array}{l}4.096 \\
4.228 \\
4.347 \\
4.456 \\
4.649 \\
4.815 \\
4.962 \\
5.093 \\
5.320 \\
5.511\end{array}$ & $\begin{array}{l}0.7459 \\
0.7459 \\
0.7458 \\
0.7457 \\
0.7456 \\
0.7455 \\
0.7454 \\
0.7453 \\
0.7452 \\
0.7451\end{array}$ & $\begin{array}{l}1.257 \\
1.254 \\
1.252 \\
1.250 \\
1.248 \\
1.246 \\
1.245 \\
1.244 \\
1.243 \\
1.243\end{array}$ & $\begin{array}{r}972.3 \\
1025.0 \\
1075.0 \\
1122.0 \\
1211.0 \\
1294.0 \\
1372.0 \\
1446.0 \\
1583.0 \\
1709.0\end{array}$ \\
\hline $\begin{array}{l}160.0 \\
180.0 \\
200.0 \\
250.0 \\
300.0 \\
350.0 \\
400.0 \\
450.0 \\
500.0 \\
600.0\end{array}$ & $\begin{array}{l}15.37 \\
17.28 \\
19.20 \\
23.99 \\
28.78 \\
33.56 \\
38.35 \\
43.14 \\
47.93 \\
57.50\end{array}$ & $\begin{array}{r}432.0 \\
485.0 \\
539.0 \\
673.0 \\
807.0 \\
941.0 \\
1080.0 \\
1210.0 \\
1340.0 \\
1610.0\end{array}$ & $\begin{array}{l}0.175 \\
0.156 \\
0.140 \\
0.112 \\
0.0933 \\
0.0800 \\
0.0700 \\
0.0622 \\
0.0560 \\
0.0467\end{array}$ & $\begin{array}{l}125.4 \\
140.3 \\
155.2 \\
192.5 \\
229.7 \\
267.0 \\
304.2 \\
341.4 \\
378.7 \\
453.2\end{array}$ & $\begin{array}{l}205.1 \\
229.9 \\
254.8 \\
316.9 \\
378.9 \\
441.0 \\
503.1 \\
565.1 \\
627.2 \\
751.3\end{array}$ & $\begin{array}{l}5.677 \\
5.824 \\
5.954 \\
6.231 \\
6.458 \\
6.549 \\
6.815 \\
5.961 \\
7.092 \\
7.318\end{array}$ & $\begin{array}{l}0.7451 \\
0.7450 \\
0.7450 \\
0.7449 \\
0.7449 \\
0.7449 \\
0.7448 \\
0.7448 \\
0.7448 \\
0.7448\end{array}$ & $\begin{array}{l}1.242 \\
1.242 \\
1.242 \\
1.241 \\
1.241 \\
1.241 \\
1.241 \\
1.241 \\
1.241 \\
1.241\end{array}$ & $\begin{array}{l}1826.0 \\
1936.0 \\
2040.0 \\
2279.0 \\
2496.0 \\
2695.0 \\
2881.0 \\
3055.0 \\
3220.0 \\
3527.0\end{array}$ \\
\hline $\begin{array}{r}700.0 \\
800.0 \\
900.0 \\
1000.0 \\
1200.0 \\
1400.0 \\
1600.0 \\
1800.0 \\
2000.0 \\
2500.0\end{array}$ & $\begin{array}{r}67.07 \\
76.05 \\
86.22 \\
95.83 \\
114.9 \\
134.1 \\
153.2 \\
172.4 \\
191.5 \\
239.4\end{array}$ & $\begin{array}{l}1880.0 \\
2150.0 \\
2420.0 \\
2680.0 \\
3220.0 \\
3760.0 \\
4290.0 \\
4830.0 \\
5360.0 \\
6700.0\end{array}$ & $\begin{array}{l}0.0400 \\
0.0350 \\
0.0311 \\
0.0280 \\
0.0233 \\
0.0200 \\
0.0175 \\
0.0156 \\
0.0140 \\
0.0112\end{array}$ & $\begin{array}{r}527.6 \\
602.1 \\
676.6 \\
751.1 \\
900.0 \\
1049.0 \\
1198.0 \\
1347.0 \\
1496.0 \\
1868.0\end{array}$ & $\begin{array}{r}875.4 \\
1000.0 \\
1124.0 \\
1248.0 \\
1496.0 \\
1744.0 \\
1992.0 \\
2241.0 \\
2489.0 \\
3109.0\end{array}$ & $\begin{array}{l}7.509 \\
7.675 \\
7.821 \\
7.952 \\
8.178 \\
8.370 \\
8.535 \\
8.682 \\
8.812 \\
9.089\end{array}$ & $\begin{array}{l}0.7448 \\
0.7448 \\
0.7448 \\
0.7448 \\
0.7448 \\
0.7448 \\
0.7448 \\
0.7447 \\
0.7447 \\
0.7447\end{array}$ & $\begin{array}{l}1.241 \\
1.241 \\
1.241 \\
1.241 \\
1.241 \\
1.241 \\
1.241 \\
1.241 \\
1.241 \\
1.241\end{array}$ & $\begin{array}{l}3809.0 \\
4072.0 \\
4318.0 \\
4551.0 \\
4985.0 \\
5385.0 \\
5756.0 \\
6105.0 \\
6435.0 \\
7194.0\end{array}$ \\
\hline 3000.0 & 287.3 & 8050.0 & 0.00933 & 2240.0 & 3730.0 & 9.316 & 0.7447 & 1.241 & 7881.0 \\
\hline
\end{tabular}

- two-phase boundarr 
THERMOPHYSICAL PROPERTIES OF HELIUM 4

28 PSIA ISOBAR

\begin{tabular}{|c|c|c|c|c|c|c|c|c|c|c|}
\hline $\begin{array}{c}\text { TEMPERA TURE } \\
\text { OEG. R }\end{array}$ & DENSI TY & $\begin{array}{c}V(D H / O V)_{p} \\
\text { BTU/LB }\end{array}$ & $\begin{array}{c}V(O P / O U)_{V}-1 \\
P S I A-C U F T / B T U\end{array}$ & $\begin{array}{l}-V(O P / O V)_{T} T \\
\text { PSIA }\end{array}$ & $\begin{array}{l}(O V / O T)_{P} / V \\
1 / O E G . R\end{array}$ & $\begin{array}{c}\text { THERMAL } \\
\text { CONDUCTIVITY } \\
\text { BTU/FT-HR-R }\end{array}$ & $\begin{array}{l}\text { VISCOSITY } \\
\text { LB/FT-SEC } \\
\times 10 E+6\end{array}$ & $\begin{array}{c}\text { THERMAL } \\
\text { OIFFUSIVITY } \\
\text { SQ FT/HR }\end{array}$ & $\begin{array}{l}\text { OIELECTRIC } \\
\text { CONSTANT }\end{array}$ & $\begin{array}{l}\text { PRANOTL } \\
\text { NUMBER }\end{array}$ \\
\hline $\begin{array}{l}4.0 \\
5.0 \\
6.0 \\
7.0 \\
8.0 \\
8.971 \\
8.971 \\
9.0 \\
10.0 \\
11.0\end{array}$ & $\begin{array}{l}9.355 \\
9.187 \\
8.904 \\
8.484 \\
7.828 \\
6.366 \\
2.396 \\
2.315 \\
1.494 \\
1.217\end{array}$ & $\begin{array}{c}57.5 \\
20.0 \\
16.4 \\
13.7 \\
11.1 \\
7.01 \\
5.79 \\
5.92 \\
8.51 \\
10.4\end{array}$ & $\begin{array}{l}2.31 \\
6.55 \\
7.18 \\
7.29 \\
6.80 \\
5.37 \\
3.85 \\
3.85 \\
3.85 \\
3.85\end{array}$ & $\begin{array}{c}1220.0 \\
1050.0 \\
803.0 \\
541.0 \\
281.0 \\
36.2 \\
5.63 \\
6.60 \\
17.4 \\
20.9\end{array}$ & $\begin{array}{l}0.0110 \\
0.0249 \\
0.0386 \\
0.0603 \\
0.109 \\
0.630 \\
1.33 \\
1.09 \\
0.255 \\
0.170\end{array}$ & $\begin{array}{l}0.00892 \\
0.0104 \\
0.0111 \\
0.0117 \\
0.0118 \\
0.0127 \\
0.0110 \\
0.0102 \\
0.00768 \\
0.00783\end{array}$ & $\begin{array}{l}2.72 \\
2.77 \\
2.64 \\
2.44 \\
2.19 \\
1.79 \\
1.13 \\
1.13 \\
1.12 \\
1.17\end{array}$ & $\begin{array}{l}0.00150 \\
0.00227 \\
0.00197 \\
0.00166 \\
0.00124 \\
0.000450 \\
0.000600 \\
0.000685 \\
0.00237 \\
0.00366\end{array}$ & $\begin{array}{l}1.02054 \\
1.02043 \\
1.02024 \\
1.01990 \\
1.01924 \\
1.01724 \\
1.00810 \\
1.00786 \\
1.00528 \\
1.00435\end{array}$ & $\begin{array}{l}0.696 \\
0.478 \\
0.541 \\
0.624 \\
0.809 \\
2.24 \\
2.84 \\
2.55 \\
1.14 \\
0.944\end{array}$ \\
\hline $\begin{array}{l}12.0 \\
13.0 \\
14.0 \\
15.0 \\
16.0 \\
17.0 \\
18.0 \\
19.0 \\
20.0 \\
22.0\end{array}$ & $\begin{array}{l}1.049 \\
0.9295 \\
0.8386 \\
0.7662 \\
0.7066 \\
0.6566 \\
0.5137 \\
0.5767 \\
0.5441 \\
0.4894\end{array}$ & $\begin{array}{l}12.0 \\
13.6 \\
15.1 \\
16.6 \\
18.0 \\
19.4 \\
20.8 \\
22.1 \\
23.5 \\
26.2\end{array}$ & $\begin{array}{l}3.84 \\
3.82 \\
3.81 \\
3.79 \\
3.78 \\
3.77 \\
3.76 \\
3.75 \\
3.74 \\
3.73\end{array}$ & $\begin{array}{l}22.7 \\
23.9 \\
24.8 \\
25.4 \\
25.8 \\
26.2 \\
26.5 \\
26.7 \\
26.9 \\
27.2\end{array}$ & $\begin{array}{l}0.132 \\
0.111 \\
0.0959 \\
0.0852 \\
0.0769 \\
0.0703 \\
0.0648 \\
0.0603 \\
0.0564 \\
0.0500\end{array}$ & $\begin{array}{l}0.00815 \\
0.00852 \\
0.00889 \\
0.00926 \\
0.00963 \\
0.0100 \\
0.0103 \\
0.0107 \\
0.0110 \\
0.0117 .\end{array}$ & $\begin{array}{l}1.22 \\
1.28 \\
1.34 \\
1.40 \\
1.46 \\
1.51 \\
1.57 \\
1.62 \\
1.68 \\
1.78\end{array}$ & $\begin{array}{l}0.00488 \\
0.00609 \\
0.00732 \\
0.00857 \\
0.00985 \\
0.0112 \\
0.0125 \\
0.0139 \\
0.0153 \\
0.0182\end{array}$ & $\begin{array}{l}1.00378 \\
1.00337 \\
1.00305 \\
1.00280 \\
1.00259 \\
1.00241 \\
1.00226 \\
1.00212 \\
1.00201 \\
1.00181\end{array}$ & $\begin{array}{l}0.861 \\
0.815 \\
0.786 \\
0.766 \\
0.753 \\
0.743 \\
0.735 \\
0.730 \\
0.726 \\
0.720\end{array}$ \\
\hline $\begin{array}{l}24.0 \\
26.0 \\
28.0 \\
30.0 \\
32.0 \\
34.0 \\
36.0 \\
38.0 \\
40.0\end{array}$ & $\begin{array}{l}0.4452 \\
0.4085 \\
0.3777 \\
0.3513 \\
0.3284 \\
0.3085 \\
0.2909 \\
0.2752 \\
0.2611\end{array}$ & $\begin{array}{l}28.8 \\
31.5 \\
34.1 \\
36.7 \\
39.3 \\
41.8 \\
44.4 \\
47.0 \\
49.5\end{array}$ & $\begin{array}{l}3.72 \\
3.71 \\
3.70 \\
3.69 \\
3.68 \\
3.68 \\
3.67 \\
3.67 \\
3.66\end{array}$ & $\begin{array}{l}27.4 \\
27.5 \\
27.7 \\
27.7 \\
27.8 \\
27.9 \\
27.9 \\
28.0 \\
28.0\end{array}$ & $\begin{array}{l}0.0451 \\
0.0410 \\
0.0377 \\
0.0348 \\
0.0324 \\
0.0303 \\
0.0285 \\
0.0269 \\
0.0255\end{array}$ & $\begin{array}{l}0.0123 \\
0.0129 \\
0.0135 \\
0.0140 \\
0.0146 \\
0.0151 \\
0.0156 \\
0.0161 \\
0.0166\end{array}$ & $\begin{array}{l}1.88 \\
1.98 \\
2.07 \\
2.17 \\
2.26 \\
2.35 \\
2.43 \\
2.52 \\
2.60\end{array}$ & $\begin{array}{l}0.0212 \\
0.0244 \\
0.0278 \\
0.0312 \\
0.0348 \\
0.0385 \\
0.0424 \\
0.0463 \\
0.0504\end{array}$ & $\begin{array}{l}1.00165 \\
1.00152 \\
1.00140 \\
1.00131 \\
1.00122 \\
1.00115 \\
1.00108 \\
1.00103 \\
1.00098\end{array}$ & $\begin{array}{l}0.716 \\
0.714 \\
0.712 \\
0.711 \\
0.711 \\
0.710 \\
0.710 \\
0.710 \\
0.710\end{array}$ \\
\hline $\begin{array}{r}45.0 \\
50.0 \\
55.0 \\
60.0 \\
70.0 \\
80.0 \\
90.0 \\
100.0 \\
120.0 \\
140.0\end{array}$ & $\begin{array}{l}0.2317 \\
0.2083 \\
0.1892 \\
0.1734 \\
0.1485 \\
0.1300 \\
0.1155 \\
0.1040 \\
0.08670 \\
0.07434\end{array}$ & $\begin{array}{r}55.8 \\
62.2 \\
68.4 \\
74.7 \\
87.2 \\
100.0 \\
112.0 \\
125.0 \\
150.0 \\
174.0\end{array}$ & $\begin{array}{l}3.65 \\
3.65 \\
3.64 \\
3.64 \\
3.63 \\
3.63 \\
3.62 \\
3.62 \\
3.62 \\
3.61\end{array}$ & $\begin{array}{l}28.0 \\
28.1 \\
28.1 \\
28.1 \\
28.1 \\
28.1 \\
28.1 \\
28.1 \\
28.1 \\
28.1\end{array}$ & $\begin{array}{l}0.0225 \\
0.0202 \\
0.0183 \\
0.0167 \\
0.0143 \\
0.0125 \\
0.0111 \\
0.0100 \\
0.00831 \\
0.00713\end{array}$ & $\begin{array}{l}0.0178 \\
0.0190 \\
0.0201 \\
0.0211 \\
0.0232 \\
0.0252 \\
0.0271 \\
0.0290 \\
0.0326 \\
0.0360\end{array}$ & $\begin{array}{l}2.80 \\
2.99 \\
3.17 \\
3.34 \\
3.67 \\
3.99 \\
4.29 \\
4.57 \\
5.11 \\
5.62\end{array}$ & $\begin{array}{l}0.0611 \\
0.0726 \\
0.0847 \\
0.0976 \\
0.125 \\
0.156 \\
0.189 \\
0.224 \\
0.302 \\
0.390\end{array}$ & $\begin{array}{l}1.00087 \\
1.00078 \\
1.00071 \\
1.00065 \\
1.00056 \\
1.00049 \\
1.00043 \\
1.00039 \\
1.00033 \\
1.00028\end{array}$ & $\begin{array}{l}0.711 \\
0.711 \\
0.711 \\
0.711 \\
0.711 \\
0.710 \\
0.708 \\
0.706 \\
0.702 \\
0.698\end{array}$ \\
\hline $\begin{array}{l}160.0 \\
180.0 \\
200.0 \\
250.0 \\
300.0 \\
350.0 \\
400.0 \\
450.0 \\
500.0 \\
600.0\end{array}$ & $\begin{array}{l}0.06507 \\
0.05786 \\
0.05208 \\
0.04169 \\
0.03475 \\
0.02979 \\
0.02608 \\
0.02318 \\
0.02087 \\
0.01739\end{array}$ & $\begin{array}{l}199.0 \\
224.0 \\
249.0 \\
311.0 \\
373.0 \\
435.0 \\
497.0 \\
559.0 \\
621.0 \\
745.0\end{array}$ & $\begin{array}{l}3.61 \\
3.61 \\
3.61 \\
3.61 \\
3.61 \\
3.60 \\
3.60 \\
3.60 \\
3.60 \\
3.60\end{array}$ & $\begin{array}{l}28.1 \\
28.1 \\
28.1 \\
28.1 \\
28.0 \\
28.0 \\
28.0 \\
28.0 \\
28.0 \\
28.0\end{array}$ & $\begin{array}{l}0.00623 \\
0.00554 \\
0.00499 \\
0.00399 \\
0.00333 \\
0.00285 \\
0.00250 \\
0.00222 \\
0.00200 \\
0.00167\end{array}$ & $\begin{array}{l}0.0394 \\
0.0426 \\
0.0457 \\
0.0532 \\
0.0603 \\
0.0669 \\
0.0733 \\
0.0793 \\
0.0851 \\
0.0962\end{array}$ & $\begin{array}{c}6.11 \\
6.58 \\
6.90 \\
7.99 \\
9.01 \\
10.0 \\
10.9 \\
11.8 \\
12.7 \\
14.4\end{array}$ & $\begin{array}{l}0.487 \\
0.593 \\
0.707 \\
1.03 \\
1.40 \\
1.81 \\
2.26 \\
2.76 \\
3.29 \\
4.46\end{array}$ & $\begin{array}{l}1.00025 \\
1.00022 \\
1.00020 \\
1.00016 \\
1.00013 \\
1.00011 \\
1.00010 \\
1.00009 \\
1.00008 \\
1.00007\end{array}$ & $\begin{array}{l}0.694 \\
0.691 \\
0.675 \\
0.671 \\
0.669 \\
0.667 \\
0.666 \\
0.666 \\
0.667 \\
0.668\end{array}$ \\
\hline $\begin{array}{r}700.0 \\
800.0 \\
900.0 \\
1000.0 \\
1200.0 \\
1400.0 \\
1600.0 \\
1800.0 \\
2000.0 \\
2500.0\end{array}$ & $\begin{array}{l}0.01491 \\
0.01305 \\
0.01160 \\
0.01044 \\
0.038700 \\
0.007457 \\
0.006525 \\
0.005801 \\
0.005221 \\
0.024177\end{array}$ & $\begin{array}{r}870.0 \\
994.0 \\
1120.0 \\
1240.0 \\
1490.0 \\
1740.0 \\
1996.0 \\
2230.0 \\
2480.0 \\
3100.0\end{array}$ & $\begin{array}{l}3.60 \\
3.60 \\
3.60 \\
3.60 \\
3.60 \\
3.60 \\
3.60 \\
3.60 \\
3.60 \\
3.60\end{array}$ & $\begin{array}{l}28.0 \\
28.0 \\
28.0 \\
28.0 \\
28.0 \\
28.0 \\
28.0 \\
28.0 \\
28.0 \\
28.0\end{array}$ & $\begin{array}{l}0.00143 \\
0.00125 \\
0.00111 \\
0.00100 \\
0.000833 \\
0.000714 \\
0.000625 \\
0.000555 \\
0.000500 \\
0.000400\end{array}$ & $\begin{array}{l}0.107 \\
0.118 \\
0.128 \\
0.137 \\
0.156 \\
0.174 \\
0.191 \\
0.208 \\
0.224 \\
0.263\end{array}$ & $\begin{array}{l}16.0 \\
17.6 \\
19.1 \\
20.5 \\
23.3 \\
26.0 \\
28.5 \\
31.0 \\
33.4 \\
39.2\end{array}$ & $\begin{array}{l}5.79 \\
7.26 \\
8.86 \\
10.6 \\
14.5 \\
18.8 \\
23.6 \\
28.9 \\
34.6 \\
50.7\end{array}$ & $\begin{array}{l}1.000006 \\
1.00005 \\
1.00004 \\
1.00004 \\
1.000003 \\
1.000003 \\
1.000002 \\
1.000002 \\
1.00002 \\
1.000002\end{array}$ & $\begin{array}{l}0.668 \\
0.668 \\
0.667 \\
0.667 \\
0.667 \\
0.667 \\
0.666 \\
0.666 \\
0.666 \\
0.666\end{array}$ \\
\hline 3060.0 & 0.003481 & 3720.0 & 3.60 & 28.0 & 0.000333 & 0.299 & 44.6 & 69.3 & 1.00001 & 0.666 \\
\hline
\end{tabular}

* two-phase boUnoarY 
3J PSIA ISOBAR

THERMOOYNAMIC PROPERTIES OF HELIUM 4

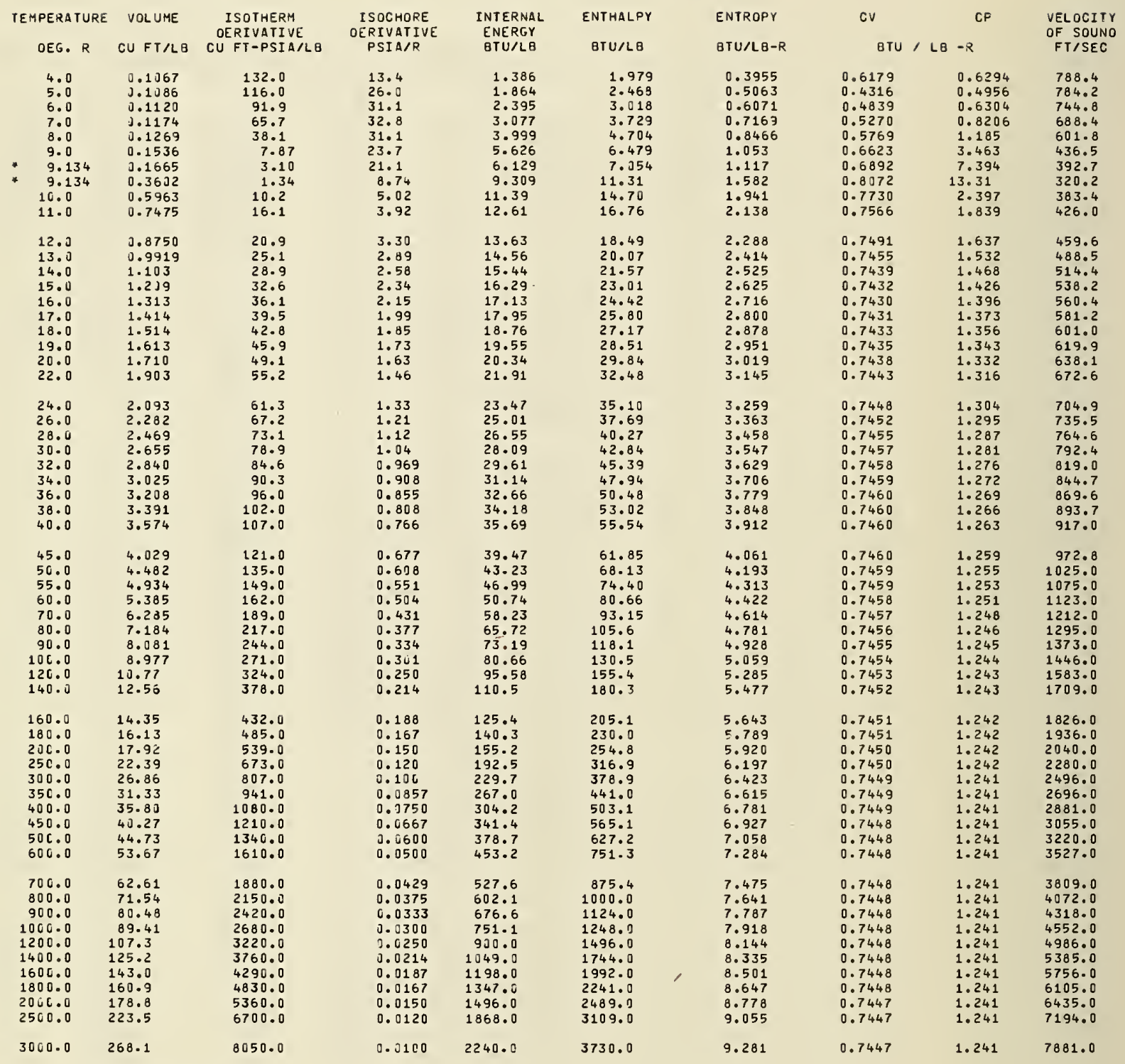

- two-phase bounoary 
30 PSIA ISOBAR

\begin{tabular}{|c|c|c|c|c|c|c|c|c|c|c|}
\hline $\begin{array}{c}\text { TEMPERATURE } \\
\text { DEG. R }\end{array}$ & $\begin{array}{l}\text { DENSITY } \\
\text { LB/CU FT }\end{array}$ & $\begin{array}{c}\text { V(DH/DV) } \\
\text { BTU/LB }\end{array}$ & $\begin{array}{l}V(D P / D U)_{v}^{-} \\
P S I A-C U F T / B T U\end{array}$ & $\begin{array}{l}-V(D P / D V)_{T} \\
\text { PSIA }\end{array}$ & $\begin{array}{l}\text { (DV/DT })_{\mathrm{P}} / \mathrm{V} \\
1 / D E G . R\end{array}$ & $\begin{array}{c}\text { THE RMAL } \\
\text { CONOUCTIVITY } \\
\text { BTU/FT T HR }-R\end{array}$ & $\begin{array}{l}\text { VISCOSITY } \\
\text { LB/FT-SEC } \\
\times 10 E+6\end{array}$ & $\begin{array}{l}\text { THERMAL } \\
\text { DIFFUSIVITY } \\
\text { SQ FI/HR }\end{array}$ & $\begin{array}{l}\text { DIELECTRIC } \\
\text { CONSTANT }\end{array}$ & $\begin{array}{l}\text { PRANDTL } \\
\text { NUMBER }\end{array}$ \\
\hline $\begin{array}{l}4.0 \\
5.0 \\
6.0 \\
7.0 \\
8.0 \\
9.0 \\
9.134 \\
9.134 \\
10.0 \\
11.0\end{array}$ & $\begin{array}{l}9.372 \\
9.205 \\
8.927 \\
8.515 \\
7.879 \\
6.510 \\
6.006 \\
2.776 \\
1.677 \\
1.338\end{array}$ & $\begin{array}{c}58.0 \\
20.3 \\
16.6 \\
14.0 \\
11.4 \\
7.49 \\
6.54 \\
5.67 \\
8.19 \\
10.1\end{array}$ & $\begin{array}{l}2.31 \\
6.55 \\
7.20 \\
7.32 \\
6.85 \\
5.49 \\
5.09 \\
3.90 \\
3.88 \\
3.87\end{array}$ & $\begin{array}{c}1230.0 \\
1060.0 \\
821.0 \\
559.0 \\
300.0 \\
51.2 \\
18.6 \\
3.73 \\
17.2 \\
21.6\end{array}$ & $\begin{array}{l}0.0109 \\
0.0244 \\
0.0379 \\
0.0587 \\
0.104 \\
0.462 \\
1.13 \\
2.35 \\
0.293 \\
0.182\end{array}$ & $\begin{array}{l}0.00894 \\
0.0104 \\
0.0112 \\
0.0117 \\
0.0119 \\
0.0123 \\
0.0142 \\
0.0149 \\
0.00793 \\
0.00795\end{array}$ & $\begin{array}{l}2.74 \\
2.79 \\
2.66 \\
2.46 \\
2.21 \\
1.83 \\
1.71 \\
1.20 \\
1.14 \\
1.18\end{array}$ & $\begin{array}{l}0.00152 \\
0.00228 \\
0.00198 \\
0.00168 \\
0.00128 \\
0.000544 \\
0.000320 \\
0.000404 \\
0.00197 \\
0.00323\end{array}$ & $\begin{array}{l}1.02055 \\
1.02045 \\
1.02026 \\
1.01993 \\
1.01930 \\
1.01747 \\
1.01664 \\
1.00921 \\
1.00587 \\
1.00476\end{array}$ & $\begin{array}{l}0.695 \\
0.478 \\
0.540 \\
0.620 \\
0.793 \\
1.86 \\
3.21 \\
3.84 \\
1.24 \\
0.986\end{array}$ \\
\hline $\begin{array}{l}12.0 \\
13.0 \\
14.0 \\
15.0 \\
16.0 \\
17.0 \\
18.0 \\
19.0 \\
20.0 \\
22.0\end{array}$ & $\begin{array}{l}1.143 \\
1.008 \\
0.9075 \\
0.8271 \\
0.7617 \\
0.7070 \\
0.6633 \\
0.62 J 1 \\
0.5847 \\
0.5255\end{array}$ & $\begin{array}{l}11.8 \\
13.4 \\
14.9 \\
16.4 \\
17.9 \\
19.3 \\
20.7 \\
22.1 \\
23.4 \\
26.1\end{array}$ & $\begin{array}{l}3.85 \\
3.84 \\
3.82 \\
3.81 \\
3.79 \\
3.78 \\
3.77 \\
3.76 \\
3.75 \\
3.74\end{array}$ & $\begin{array}{l}23.8 \\
25.3 \\
26.2 \\
27.0 \\
27.5 \\
27.9 \\
28.2 \\
28.5 \\
28.7 \\
29.0\end{array}$ & $\begin{array}{l}0.138 \\
0.114 \\
0.0983 \\
0.0868 \\
0.0781 \\
0.0712 \\
0.0656 \\
0.0609 \\
0.0569 \\
0.0504\end{array}$ & $\begin{array}{l}0.00823 \\
0.00858 \\
0.00894 \\
0.00931 \\
0.00967 \\
0.0100 \\
0.0104 \\
0.0107 \\
0.0111 \\
0.0117\end{array}$ & $\begin{array}{l}1.24 \\
1.29 \\
1.35 \\
1.41 \\
1.46 \\
1.52 \\
1.57 \\
1.63 \\
1.68 \\
1.79\end{array}$ & $\begin{array}{l}0.00440 \\
0.00555 \\
0.00671 \\
0.00789 \\
0.00910 \\
0.0103 \\
0.0116 \\
0.0129 \\
0.0142 \\
0.0169\end{array}$ & $\begin{array}{l}1.00410 \\
1.00364 \\
1.00329 \\
1.00301 \\
1.00278 \\
1.00259 \\
1.00242 \\
1.00228 \\
1.00215 \\
1.00194\end{array}$ & $\begin{array}{l}0.885 \\
0.831 \\
0.798 \\
0.776 \\
0.760 \\
0.749 \\
0.741 \\
0.734 \\
0.730 \\
0.723\end{array}$ \\
\hline $\begin{array}{l}24.0 \\
26.0 \\
28.0 \\
30.0 \\
32.0 \\
34.0 \\
36.0 \\
38.0 \\
40.0\end{array}$ & $\begin{array}{l}0.4777 \\
0.4382 \\
0.4050 \\
0.3766 \\
0.3521 \\
0.3316 \\
0.3117 \\
0.2949 \\
0.2798\end{array}$ & $\begin{array}{l}28.8 \\
31.4 \\
34.0 \\
36.7 \\
39.2 \\
41.8 \\
44.4 \\
46.9 \\
49.5\end{array}$ & $\begin{array}{l}3.73 \\
3.72 \\
3.71 \\
3.70 \\
3.69 \\
3.68 \\
3.68 \\
3.67 \\
3.67\end{array}$ & $\begin{array}{l}29.3 \\
29.5 \\
29.6 \\
29.7 \\
29.8 \\
29.9 \\
29.9 \\
30.0 \\
30.0\end{array}$ & $\begin{array}{l}0.0453 \\
0.0412 \\
0.0378 \\
0.0349 \\
0.0325 \\
0.0304 \\
0.0286 \\
0.0270 \\
0.0255\end{array}$ & $\begin{array}{l}0.0123 \\
0.0129 \\
0.0135 \\
0.0141 \\
0.0146 \\
0.0151 \\
0.0156 \\
0.0161 \\
0.0166\end{array}$ & $\begin{array}{l}1.89 \\
1.98 \\
2.08 \\
2.17 \\
2.26 \\
2.35 \\
2.44 \\
2.52 \\
2.67\end{array}$ & $\begin{array}{l}0.0198 \\
0.0228 \\
0.0259 \\
0.0291 \\
0.0325 \\
0.0360 \\
0.0395 \\
0.0432 \\
0.0471\end{array}$ & $\begin{array}{l}1.00177 \\
1.00162 \\
1.00150 \\
1.00140 \\
1.00131 \\
1.00123 \\
1.00116 \\
1.00110 \\
1.00104\end{array}$ & $\begin{array}{l}0.719 \\
0.716 \\
0.714 \\
0.713 \\
0.712 \\
0.711 \\
0.711 \\
0.711 \\
0.711\end{array}$ \\
\hline $\begin{array}{r}45.0 \\
50.0 \\
55.0 \\
60.0 \\
70.0 \\
80.0 \\
90.0 \\
100.0 \\
120.0 \\
140.0\end{array}$ & $\begin{array}{l}0.2482 \\
0.2231 \\
0.2827 \\
0.1857 \\
0.1591 \\
0.1392 \\
0.1238 \\
0.1114 \\
0.09287 \\
0.07963\end{array}$ & $\begin{array}{r}55.8 \\
62.2 \\
68.5 \\
74.7 \\
87.3 \\
100.0 \\
112.0 \\
125.0 \\
150.0 \\
174.0\end{array}$ & $\begin{array}{l}3.66 \\
3.65 \\
3.65 \\
3.64 \\
3.63 \\
3.63 \\
3.62 \\
3.62 \\
3.62 \\
3.61\end{array}$ & $\begin{array}{l}30 \cdot 1 \\
30 \cdot 1 \\
30 \cdot 1 \\
30 \cdot 1 \\
30.1 \\
30 \cdot 1 \\
30 \cdot 1 \\
30.1 \\
30.1 \\
30.1\end{array}$ & $\begin{array}{l}0.0225 \\
0.0202 \\
0.0183 \\
0.0167 \\
0.0143 \\
0.0125 \\
0.0111 \\
0.0100 \\
0.06831 \\
0.00712\end{array}$ & $\begin{array}{l}0.0178 \\
0.0190 \\
0.0201 \\
0.0212 \\
0.0232 \\
0.0252 \\
0.0271 \\
0.0290 \\
0.0326 \\
0.0361\end{array}$ & $\begin{array}{l}2.80 \\
2.99 \\
3.17 \\
3.34 \\
3.68 \\
3.99 \\
4.29 \\
4.57 \\
5.11 \\
5.63\end{array}$ & $\begin{array}{l}0.0571 \\
0.0678 \\
0.0791 \\
0.0911 \\
0.117 \\
0.145 \\
0.176 \\
0.209 \\
0.282 \\
0.364\end{array}$ & $\begin{array}{l}1.00093 \\
1.00083 \\
1.00076 \\
1.00070 \\
1.00060 \\
1.00052 \\
1.00046 \\
1.00042 \\
1.00035 \\
1.00030\end{array}$ & $\begin{array}{l}0.711 \\
0.712 \\
0.712 \\
0.712 \\
0.711 \\
0.710 \\
0.708 \\
0.706 \\
0.702 \\
0.698\end{array}$ \\
\hline $\begin{array}{l}160.0 \\
180.0 \\
200.0 \\
250.0 \\
300.0 \\
350.0 \\
400.0 \\
450.0 \\
500.0 \\
600.0\end{array}$ & $\begin{array}{l}0.06970 \\
0.06198 \\
0.05580 \\
0.04466 \\
0.03723 \\
0.03192 \\
0.02794 \\
0.02484 \\
0.02235 \\
0.01863\end{array}$ & $\begin{array}{l}199.0 \\
224.0 \\
249.0 \\
311.0 \\
373.0 \\
435.0 \\
497.0 \\
559.0 \\
621.0 \\
745.0\end{array}$ & $\begin{array}{l}3.61 \\
3.61 \\
3.61 \\
3.61 \\
3.61 \\
3.60 \\
3.60 \\
3.60 \\
3.60 \\
3.60\end{array}$ & $\begin{array}{l}30.1 \\
30.1 \\
30.1 \\
30.1 \\
30.1 \\
30.0 \\
30.0 \\
30.0 \\
30.0 \\
30.0\end{array}$ & $\begin{array}{l}0.00623 \\
0.00554 \\
0.00499 \\
0.00399 \\
0.00333 \\
0.00285 \\
0.00250 \\
0.00222 \\
0.00200 \\
0.00167\end{array}$ & $\begin{array}{l}0.0394 \\
0.0426 \\
0.0457 \\
0.0532 \\
0.0603 \\
0.0669 \\
0.0733 \\
0.0793 \\
0.0851 \\
0.0962\end{array}$ & $\begin{array}{c}6.11 \\
6.58 \\
6.91 \\
7.97 \\
9.01 \\
10.0 \\
10.9 \\
11.8 \\
12.7 \\
14.4\end{array}$ & $\begin{array}{l}0.455 \\
0.553 \\
0.660 \\
0.960 \\
1.30 \\
1.69 \\
2.11 \\
2.57 \\
3.07 \\
4.16\end{array}$ & $\begin{array}{l}1.00026 \\
1.00023 \\
1.00021 \\
1.00017 \\
1.00014 \\
1.00012 \\
1.00011 \\
1.00009 \\
1.00008 \\
1.00007\end{array}$ & $\begin{array}{l}0.694 \\
0.691 \\
0.675 \\
0.671 \\
0.669 \\
0.667 \\
0.666 \\
0.666 \\
0.667 \\
0.668\end{array}$ \\
\hline $\begin{array}{r}700.0 \\
800.0 \\
900.0 \\
1000.0 \\
1200.0 \\
1400.0 \\
1600.0 \\
1800.0 \\
2000.0 \\
2500.0\end{array}$ & $\begin{array}{l}0.01597 \\
0.01398 \\
0.01243 \\
0.01118 \\
0.009321 \\
0.007990 \\
0.006991 \\
0.006215 \\
0.005594 \\
0.004475\end{array}$ & $\begin{array}{r}870.0 \\
994.0 \\
1120.0 \\
1240.0 \\
1490.0 \\
1740.0 \\
1990.0 \\
2230.0 \\
2480.0 \\
3100.0\end{array}$ & $\begin{array}{l}3.60 \\
3.60 \\
3.60 \\
3.60 \\
3.60 \\
3.60 \\
3.60 \\
3.60 \\
3.60 \\
3.60\end{array}$ & $\begin{array}{l}30.0 \\
30.0 \\
30.0 \\
30.0 \\
30.0 \\
30.0 \\
30.0 \\
30.0 \\
30.0 \\
30.0\end{array}$ & $\begin{array}{l}0.00143 \\
0.00125 \\
0.00111 \\
0.00100 \\
0.000833 \\
0.000714 \\
0.000625 \\
0.000555 \\
0.000500 \\
0.000400\end{array}$ & $\begin{array}{l}0.107 \\
0.118 \\
0.128 \\
0.137 \\
0.156 \\
0.174 \\
0.191 \\
0.208 \\
0.224 \\
0.263\end{array}$ & $\begin{array}{l}16.0 \\
17.6 \\
19.1 \\
20.5 \\
23.3 \\
26.0 \\
28.5 \\
31.0 \\
33.4 \\
39.2\end{array}$ & $\begin{array}{c}5.40 \\
6.77 \\
8.27 \\
9.90 \\
13.5 \\
17.6 \\
22.1 \\
27.0 \\
32.3 \\
47.3\end{array}$ & $\begin{array}{l}1.00006 \\
1.00005 \\
1.00005 \\
1.00004 \\
1.00004 \\
1.00003 \\
1.00003 \\
1.00002 \\
1.00002 \\
1.00002\end{array}$ & $\begin{array}{l}0.668 \\
0.668 \\
0.667 \\
0.667 \\
0.667 \\
0.667 \\
0.666 \\
0.666 \\
0.666 \\
0.666\end{array}$ \\
\hline $300 \mathrm{C} .0$ & 0.003729 & 3720.0 & 3.60 & 30,0 & 0.000333 & 0.299 & 44.6 & 64.6 & 1.00001 & 0.666 \\
\hline
\end{tabular}

* tho-phase boundary 
32 PSIA ISOBAR

THERMOOYNAMIC PROPERTIES OF HELIUM 4

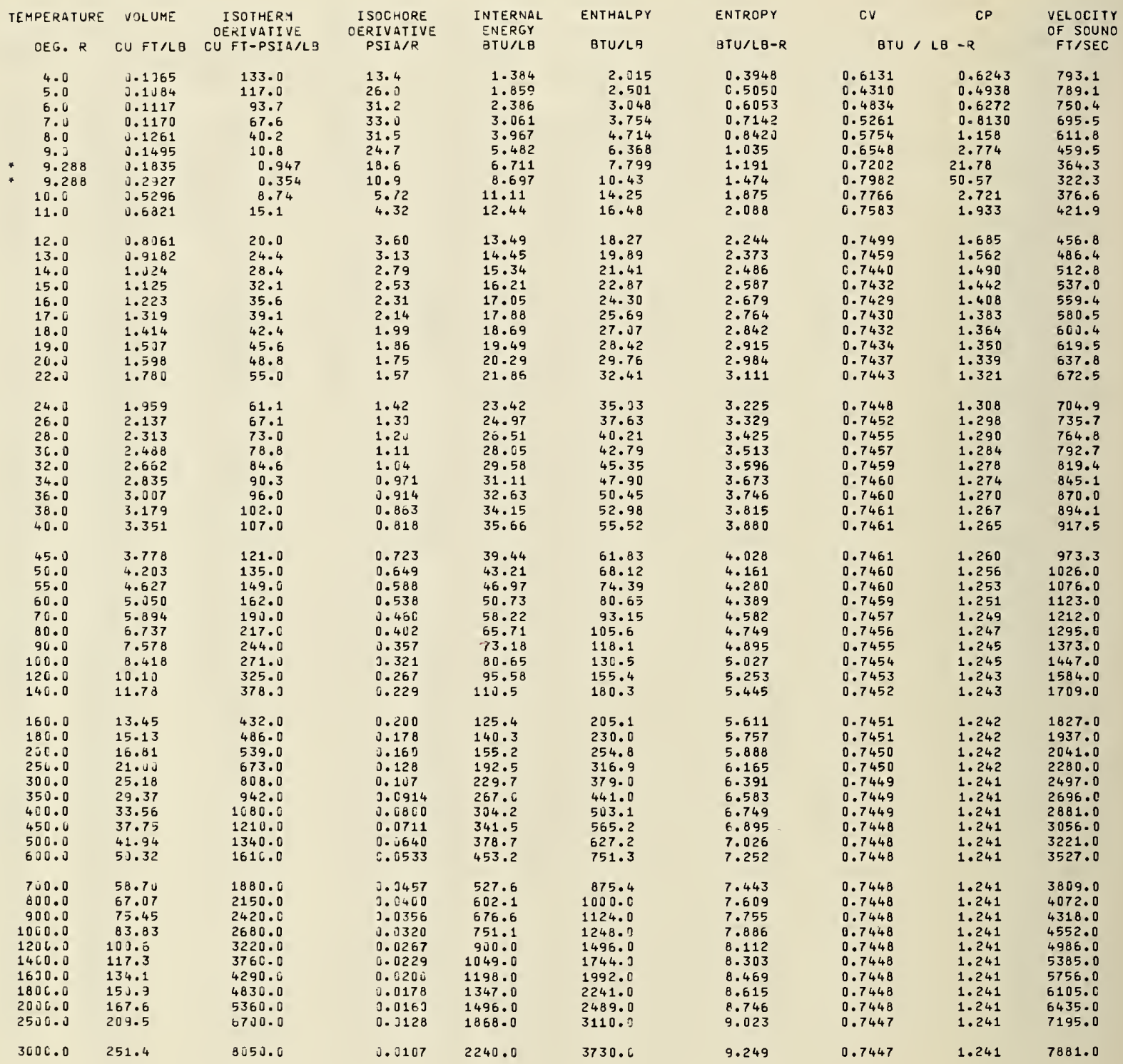


32 PSIA ISDBAR

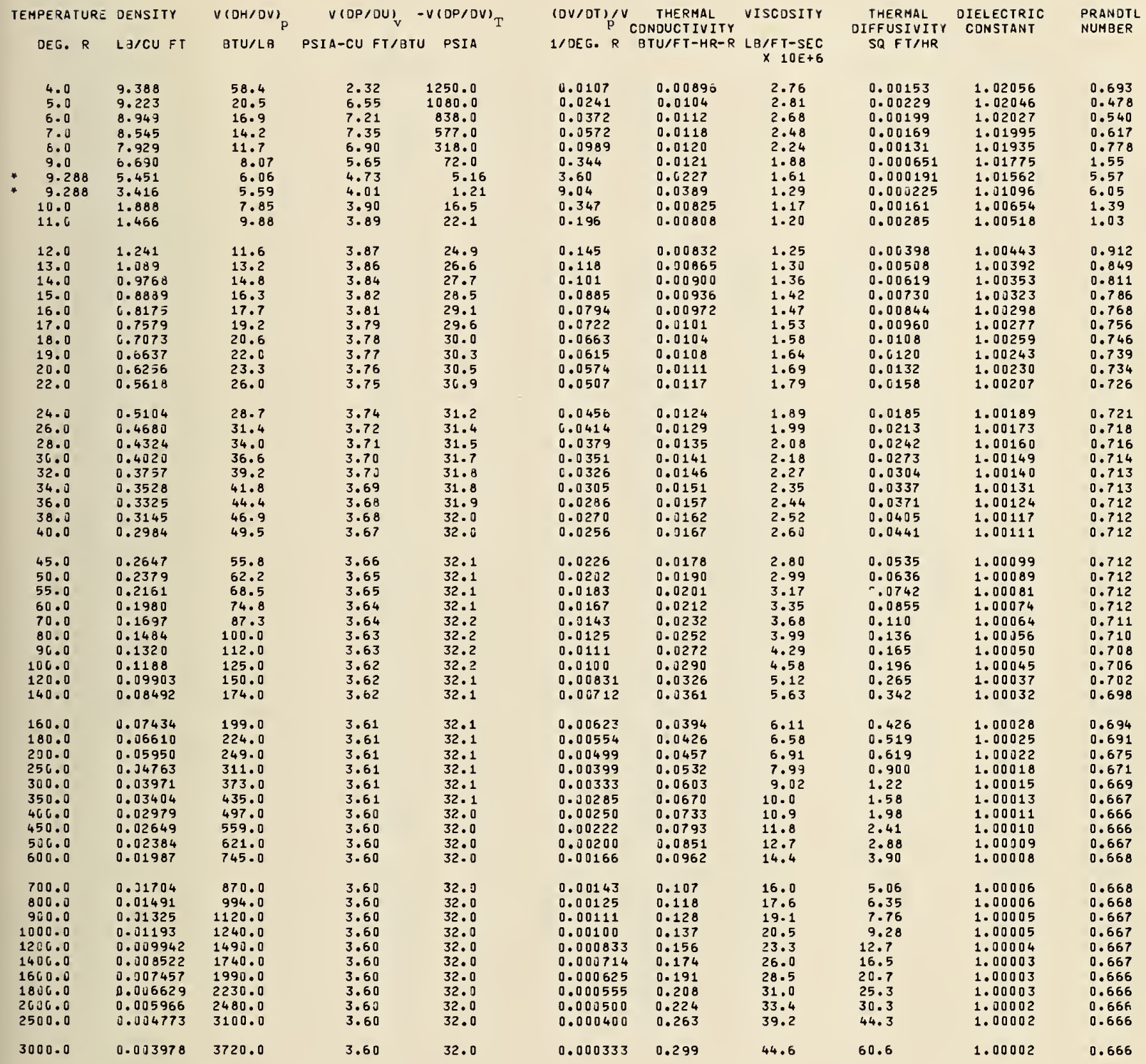


33 PSIA ISOBAR

THERMOOYNAMIC PROPERTIES OF HELIUM 4

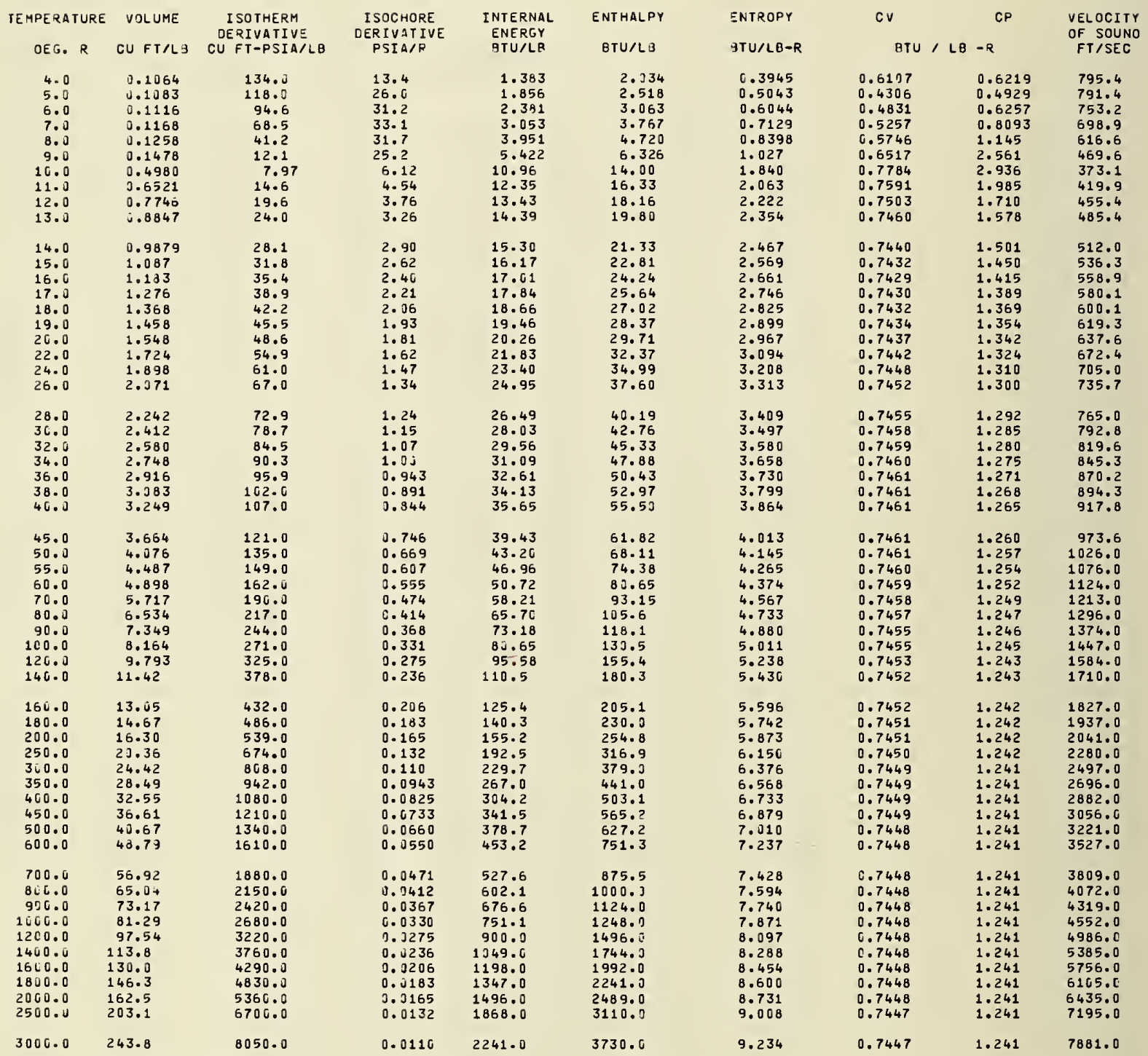


33 PSIA ISOBAR

\begin{tabular}{|c|c|c|c|c|c|c|c|c|c|c|}
\hline TEMPERATURE & OENSITY & $V(D H / O V)_{P}$ & $V(D P / D U)_{v}-v$ & $V(D P / O V)_{T}$ & (OV/OT) ${ }_{\mathrm{P}} \mathrm{V}$ & $\begin{array}{l}\text { THERMAL } \\
\text { CDNDUCTIVITY }\end{array}$ & VISCDSITY & $\begin{array}{l}\text { THERMAL } \\
\text { OIFFUSIVITY }\end{array}$ & $\begin{array}{l}\text { DIELECTRIC } \\
\text { CDNSTANT }\end{array}$ & $\begin{array}{l}\text { PRANOTL } \\
\text { NUMBER }\end{array}$ \\
\hline DEG. $R$ & LB/CU F T & $8 \mathrm{TU} / \mathrm{L}$ & PSIA-CU FT/BTU & PSIA & $1 / D E G \cdot R$ & ETU/FT-HR-R & $\begin{array}{r}L B / F T-S E C \\
\times 10 E+6\end{array}$ & SQ FT/HR & & \\
\hline $\begin{array}{r}4.0 \\
5.0 \\
6.0 \\
7.0 \\
8.0 \\
9.0 \\
10.0 \\
11.0 \\
12.0 \\
13.0\end{array}$ & $\begin{array}{l}9.395 \\
9.232 \\
8.963 \\
8.563 \\
7.952 \\
6.765 \\
2.308 \\
1.534 \\
1.291 \\
1.133\end{array}$ & $\begin{array}{c}58.7 \\
20.7 \\
17.0 \\
14.3 \\
11.8 \\
8.33 \\
7.68 \\
9.76 \\
11.5 \\
13.2\end{array}$ & $\begin{array}{l}2.33 \\
6.55 \\
7.21 \\
7.30 \\
6.93 \\
5.72 \\
3.91 \\
3.90 \\
3.88 \\
3.86\end{array}$ & $\begin{array}{r}1260.0 \\
1090.0 \\
847.0 \\
536.0 \\
328.0 \\
82.0 \\
16.0 \\
22.3 \\
25.4 \\
27.2\end{array}$ & $\begin{array}{l}0.0106 \\
0.0239 \\
0.0368 \\
0.0565 \\
0.0967 \\
0.307 \\
0.382 \\
0.203 \\
0.148 \\
0.120\end{array}$ & $\begin{array}{l}0.00896 \\
0.0104 \\
0.0112 \\
0.0118 \\
0.0120 \\
0.0121 \\
0.00845 \\
0.00815 \\
0.00836 \\
6.00868\end{array}$ & $\begin{array}{l}2.77 \\
2.82 \\
2.69 \\
2.49 \\
2.25 \\
1.93 \\
1.18 \\
1.21 \\
1.26 \\
1.31\end{array}$ & $\begin{array}{l}0.00153 \\
0.00229 \\
0.00200 \\
0.00170 \\
0.00132 \\
0.000696 \\
0.00143 \\
0.00268 \\
0.00379 \\
0.00487\end{array}$ & $\begin{array}{l}1.02056 \\
1.02046 \\
1.02028 \\
1.01996 \\
1.01938 \\
1.01786 \\
1.00692 \\
1.00540 \\
1.00460 \\
1.00406\end{array}$ & $\begin{array}{l}0.692 \\
0.479 \\
0.540 \\
0.616 \\
0.771 \\
1.45 \\
1.48 \\
1.06 \\
0.926 \\
0.858\end{array}$ \\
\hline $\begin{array}{l}14.0 \\
15.0 \\
16.0 \\
17.0 \\
18.0 \\
19.0 \\
26.0 \\
22.0 \\
24.0 \\
26.0\end{array}$ & $\begin{array}{l}1.012 \\
0.9232 \\
0.8456 \\
0.7836 \\
0.73 J 9 \\
0.6857 \\
0.6461 \\
0.5830 \\
0.5268 \\
0.4830\end{array}$ & $\begin{array}{l}14.7 \\
16.2 \\
17.7 \\
19.1 \\
20.5 \\
21.9 \\
23.3 \\
26.0 \\
28.7 \\
31.3\end{array}$ & $\begin{array}{l}3.85 \\
3.83 \\
3.81 \\
3.85 \\
3.79 \\
3.79 \\
3.77 \\
3.75 \\
3.74 \\
3.73\end{array}$ & $\begin{array}{l}28.4 \\
29.3 \\
29.9 \\
30.5 \\
30.9 \\
31.2 \\
31.4 \\
31.8 \\
32.1 \\
32.3\end{array}$ & $\begin{array}{l}0.102 \\
0.0894 \\
0.0800 \\
0.0727 \\
0.0667 \\
0.0618 \\
0.0576 \\
0.0509 \\
0.0457 \\
0.0415\end{array}$ & $\begin{array}{l}0.00903 \\
0.00939 \\
0.00974 \\
0.0101 \\
0.0104 \\
0.0108 \\
0.0111 \\
0.0117 \\
0.0124 \\
0.0130\end{array}$ & $\begin{array}{l}1.37 \\
1.42 \\
1.48 \\
1.53 \\
1.59 \\
1.64 \\
1.69 \\
1.79 \\
1.89 \\
1.99\end{array}$ & $\begin{array}{l}0.00594 \\
0.00703 \\
0.00814 \\
0.00927 \\
0.0104 \\
0.0116 \\
0.0128 \\
0.0153 \\
0.0179 \\
0.0206\end{array}$ & $\begin{array}{l}1.00365 \\
1.00334 \\
1.00308 \\
1.00286 \\
1.00267 \\
1.00251 \\
1.00237 \\
1.00214 \\
1.00194 \\
1.00179\end{array}$ & $\begin{array}{l}0.817 \\
0.791 \\
0.772 \\
0.759 \\
0.749 \\
0.742 \\
0.736 \\
0.728 \\
0.723 \\
0.719\end{array}$ \\
\hline $\begin{array}{l}28.0 \\
30.0 \\
32.0 \\
34.0 \\
36.0 \\
38.0 \\
40.0\end{array}$ & $\begin{array}{l}0.4461 \\
0.4147 \\
0.3875 \\
0.3638 \\
0.3429 \\
0.3244 \\
0.3078\end{array}$ & $\begin{array}{l}34.0 \\
36.6 \\
39.2 \\
41.8 \\
44.4 \\
46.9 \\
49.5\end{array}$ & $\begin{array}{l}3.72 \\
3.71 \\
3.70 \\
3.69 \\
3.69 \\
3.68 \\
3.67\end{array}$ & $\begin{array}{l}32.5 \\
32.7 \\
32.8 \\
32.8 \\
32.9 \\
33.0 \\
33.0\end{array}$ & $\begin{array}{l}0.0380 \\
0.0351 \\
0.0326 \\
0.0305 \\
0.0287 \\
0.0270 \\
0.0256\end{array}$ & $\begin{array}{l}0.0135 \\
0.0141 \\
0.0146 \\
0.0152 \\
0.0157 \\
0.0162 \\
0.0167\end{array}$ & $\begin{array}{l}2.09 \\
2.18 \\
2.27 \\
2.36 \\
2.44 \\
2.52 \\
2.61\end{array}$ & $\begin{array}{l}0.0235 \\
0.0264 \\
0.0295 \\
0.0327 \\
0.0359 \\
0.0393 \\
0.0428\end{array}$ & $\begin{array}{l}1.00165 \\
1.00154 \\
1.00144 \\
1.00135 \\
1.00128 \\
1.00121 \\
1.00115\end{array}$ & $\begin{array}{l}0.717 \\
0.715 \\
0.714 \\
0.713 \\
0.713 \\
0.713 \\
0.712\end{array}$ \\
\hline $\begin{array}{r}45.0 \\
50.0 \\
55.0 \\
6 C .0 \\
70.0 \\
80.0 \\
90.0 \\
100.0 \\
120.0 \\
140.0\end{array}$ & $\begin{array}{l}0.2730 \\
0.2453 \\
0.2228 \\
0.2342 \\
0.1749 \\
0.1531 \\
0.1361 \\
0.1225 \\
0.1021 \\
0.08757\end{array}$ & $\begin{array}{r}55.8 \\
62.2 \\
68.5 \\
74.8 \\
87.3 \\
100.0 \\
112.0 \\
125.0 \\
150.0 \\
174.0\end{array}$ & $\begin{array}{l}3.66 \\
3.66 \\
3.65 \\
3.64 \\
3.64 \\
3.63 \\
3.63 \\
3.62 \\
3.62 \\
3.62\end{array}$ & $\begin{array}{l}33.1 \\
33.1 \\
33.1 \\
33.2 \\
33.2 \\
33.2 \\
33.2 \\
33.2 \\
33.1 \\
33.1\end{array}$ & $\begin{array}{l}0.0226 \\
0.0202 \\
0.0183 \\
0.0167 \\
0.0143 \\
0.0125 \\
0.0111 \\
0.0100 \\
0.00831 \\
0.00712\end{array}$ & $\begin{array}{l}0.0179 \\
0.0190 \\
0.0201 \\
0.0212 \\
0.0233 \\
0.0252 \\
0.0272 \\
0.0290 \\
0.0326 \\
0.0361\end{array}$ & $\begin{array}{l}2.80 \\
2.99 \\
3.17 \\
3.35 \\
3.68 \\
3.99 \\
4.29 \\
4.58 \\
5.12 \\
5.63\end{array}$ & $\begin{array}{l}0.0519 \\
0.0616 \\
0.0720 \\
0.0829 \\
0.106 \\
0.132 \\
0.160 \\
0.190 \\
0.257 \\
0.331\end{array}$ & $\begin{array}{l}1.00102 \\
1.00092 \\
1.00083 \\
1.00076 \\
1.00066 \\
1.00057 \\
1.00051 \\
1.00046 \\
1.00038 \\
1.00033\end{array}$ & $\begin{array}{l}0.712 \\
0.712 \\
0.712 \\
0.712 \\
0.711 \\
0.710 \\
0.708 \\
0.706 \\
0.702 \\
0.698\end{array}$ \\
\hline $\begin{array}{l}160.0 \\
180.0 \\
200.0 \\
250.0 \\
300.0 \\
350.0 \\
400.0 \\
450.0 \\
500.0 \\
600.0\end{array}$ & $\begin{array}{l}0.07665 \\
0.06816 \\
0.06136 \\
0.04911 \\
0.04094 \\
0.03511 \\
0.03072 \\
0.02732 \\
0.02459 \\
0.02049\end{array}$ & $\begin{array}{l}199.0 \\
224.0 \\
249.0 \\
311.0 \\
373.0 \\
435.0 \\
497.0 \\
559.0 \\
621.0 \\
746.0\end{array}$ & $\begin{array}{l}3.61 \\
3.61 \\
3.61 \\
3.61 \\
3.61 \\
3.61 \\
3.60 \\
3.60 \\
3.60 \\
3.60\end{array}$ & $\begin{array}{l}33.1 \\
33.1 \\
33.1 \\
33.1 \\
33.1 \\
33.1 \\
33.0 \\
33.0 \\
33.0 \\
33.0\end{array}$ & $\begin{array}{l}0.00623 \\
0.00554 \\
0.00490 \\
0.00399 \\
0.00333 \\
0.00285 \\
0.00254 \\
0.00222 \\
0.00200 \\
0.00166\end{array}$ & $\begin{array}{l}0.0394 \\
0.0426 \\
0.0457 \\
0.0532 \\
0.0603 \\
0.0670 \\
0.0733 \\
0.0793 \\
0.0851 \\
0.0962\end{array}$ & $\begin{array}{l}6.11 \\
6.58 \\
6.91 \\
7.99 \\
9.02 \\
10.0 \\
10.9 \\
11.8 \\
12.7 \\
14.4\end{array}$ & $\begin{array}{l}0.414 \\
0.503 \\
0.600 \\
0.873 \\
1.19 \\
1.54 \\
1.92 \\
2.34 \\
2.79 \\
3.78\end{array}$ & $\begin{array}{l}1.00029 \\
1.00026 \\
1.00023 \\
1.00019 \\
1.00015 \\
1.00013 \\
1.00012 \\
1.00010 \\
1.00009 \\
1.00008\end{array}$ & $\begin{array}{l}0.694 \\
0.691 \\
0.675 \\
0.671 \\
0.669 \\
0.667 \\
0.666 \\
0.666 \\
0.667 \\
0.668\end{array}$ \\
\hline $\begin{array}{r}700.0 \\
800.0 \\
900.0 \\
1000.0 \\
1200.0 \\
1400.0 \\
1600.0 \\
1800.0 \\
2000.0 \\
2500.0\end{array}$ & $\begin{array}{l}0.01757 \\
0.01537 \\
0.01367 \\
0.01230 \\
0.01025 \\
0.008789 \\
0.007690 \\
0.006836 \\
0.036153 \\
0.004923\end{array}$ & $\begin{array}{r}870.0 \\
994.0 \\
1120.0 \\
1240.0 \\
1490.0 \\
1740.0 \\
1990.0 \\
2230.0 \\
2480.0 \\
3100.0\end{array}$ & $\begin{array}{l}3.60 \\
3.60 \\
3.60 \\
3.60 \\
3.60 \\
3.60 \\
3.60 \\
3.60 \\
3.60 \\
3.60\end{array}$ & $\begin{array}{l}33.0 \\
33.0 \\
33.0 \\
33.0 \\
33.0 \\
33.0 \\
33.0 \\
33.0 \\
33.0 \\
33.0\end{array}$ & $\begin{array}{l}0.00143 \\
0.00125 \\
0.00111 \\
0.00100 \\
0.000833 \\
0.000714 \\
0.000625 \\
0.000555 \\
0.000500 \\
0.000400\end{array}$ & $\begin{array}{l}0.107 \\
0.118 \\
0.128 \\
0.137 \\
0.156 \\
0.174 \\
0.191 \\
0.208 \\
0.224 \\
0.263\end{array}$ & $\begin{array}{l}16.0 \\
17.6 \\
19.1 \\
20.5 \\
23.3 \\
26.0 \\
28.5 \\
31.0 \\
33.4 \\
39.2\end{array}$ & $\begin{array}{l}4.91 \\
6.16 \\
7.52 \\
9.00 \\
12.3 \\
16.0 \\
20.0 \\
24.5 \\
29.4 \\
43.0\end{array}$ & $\begin{array}{l}1.00007 \\
1.00006 \\
1.00005 \\
1.00005 \\
1.00004 \\
1.00003 \\
1.00003 \\
1.00003 \\
1.00002 \\
1.00002\end{array}$ & $\begin{array}{l}0.668 \\
0.668 \\
0.667 \\
0.667 \\
0.667 \\
0.667 \\
0.666 \\
0.666 \\
0.666 \\
0.666\end{array}$ \\
\hline 3000.0 & 0.034102 & 3720.0 & 3.60 & 33.0 & 0.000333 & 0.299 & 44.6 & 58.8 & 1.00002 & 0.666 \\
\hline
\end{tabular}

* two-phase boundary 
34 PSIA ISOBAR

THERMDOYNAMIC PROPERTIES OF HELIUM 4

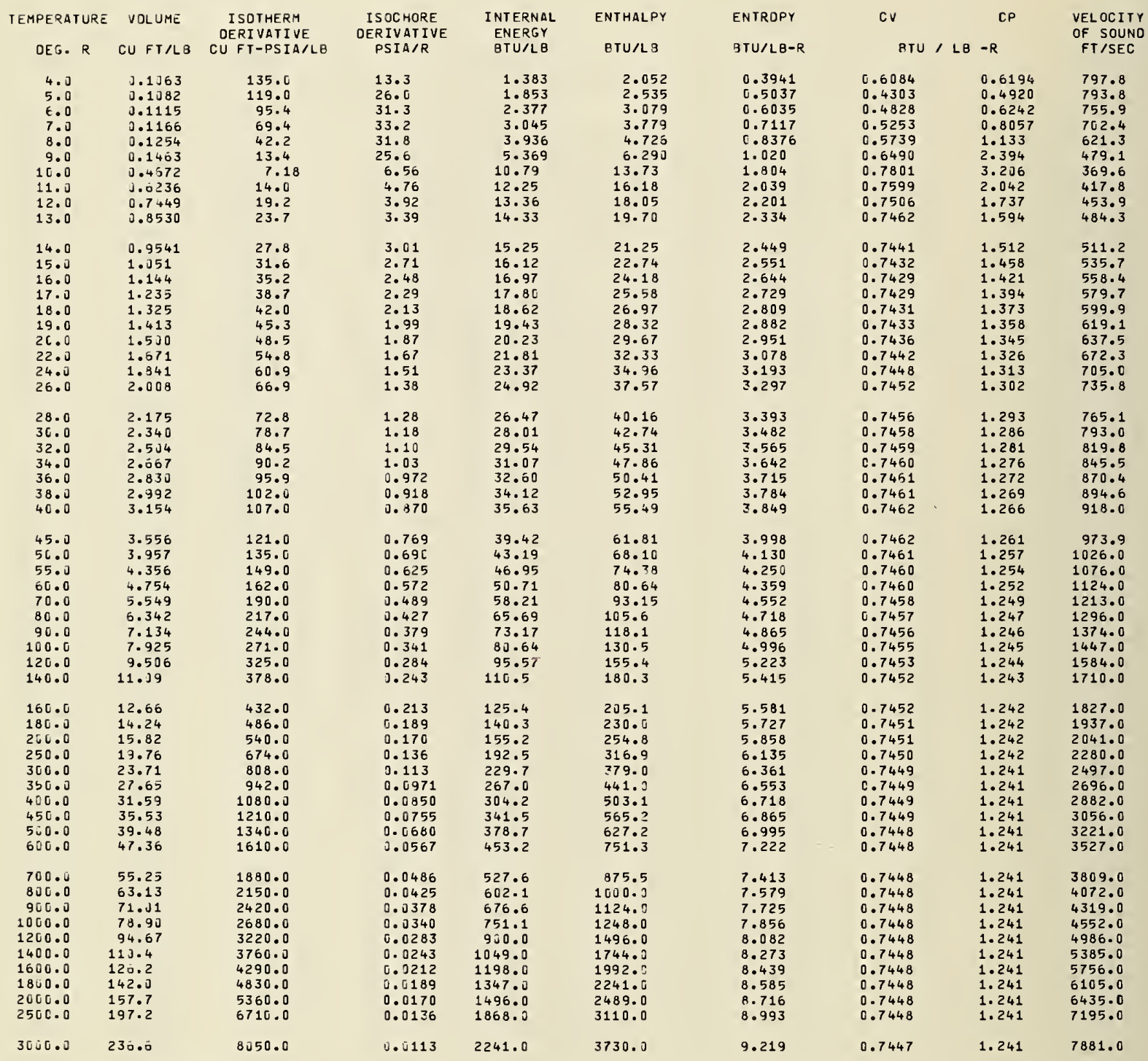

* two-phase goundarY 
34 PSIA ISOBAR

TEMPERATURE DENSITY V(OH/OV) V(DP/OU) $-V(O P / O V)_{T}$ DEG. R LB/CU FT

$\begin{array}{lcrr}9.403 & 58.9 & 2.33 & 1270.0 \\ 9.241 & 20.8 & 6.55 & 1100.0 \\ 8.971 & 17.1 & 7.22 & 856.0 \\ 8.574 & 14.4 & 7.38 & 595.0 \\ 7.975 & 12.0 & 6.95 & 337.0 \\ 6.834 & 8.58 & 5.78 & 91.8 \\ 2.140 & 7.50 & 3.93 & 15.4 \\ 1.604 & 9.64 & 3.91 & 22.5 \\ 1.342 & 11.4 & 3.89 & 25.8 \\ 1.172 & 13.1 & 3.87 & 27.8 \\ & & & \\ 1.048 & 14.6 & 3.85 & 29.1 \\ 0.9518 & 16.1 & 3.84 & 30.1 \\ 0.8740 & 17.6 & 3.82 & 30.8 \\ 0.8034 & 19.1 & 3.81 & 31.3 \\ 0.7547 & 20.5 & 3.79 & 31.7 \\ 0.7077 & 21.9 & 3.78 & 32.1 \\ 0.6567 & 23.2 & 3.77 & 32.3 \\ 0.5933 & 26.0 & 3.76 & 32.8 \\ 0.5432 & 28.7 & 3.74 & 33.1 \\ 0.4979 & 31.3 & 3.73 & 33.3 \\ 0.4598 & 34.0 & 3.72 & 33.5 \\ 0.4274 & 36.6 & 3.71 & 33.6 \\ 0.3994 & 39.2 & 3.75 & 33.7 \\ 0.3749 & 41.8 & 3.69 & 33.8 \\ 0.3534 & 44.4 & 3.69 & 33.9 \\ 0.3342 & 46.9 & 3.68 & 34.9 \\ 0.3171 & 49.5 & 3.68 & 34.0\end{array}$

0.2812

0.2527

0.2296

U. 2103

0.1832

0.1402

0.1262

0.1052

0.07896

0.07021

0.06321

0.05060
0.04218

0.04218
0.03617

0.03165

0.02814

0.02533
0.02111

0.01810

0.01584

0.01408
0.01267

0.01156

0. 009055

0.007923

0.007043
0.005339

0.005339
0.005072

$0.0 J+227$

49.5

55.8

62.2

68.5

87.3

100.0

112.0

125.0
150.0

175.0

199.0

224.0

349.0

311.0

373.0

435.0
497.0

497.0

621.0

746.0

870.0

994.0

1120.0

1240.0

1740.0

1990.0

2230.0

2480.0

3100.0

3720.0
3.68

3.67
3.66

3.66

3.65

3.65
3.64

3.64
3.63

3.63
3.62

3.62
3.62

3.61

3. 61

3.61

3.61

3.61
3.61

3.61
3.60

3.60
3.60

3.60
3.63

3.60

3.60

3.60

3.60

3.60

3.60

3.60

3.60
3.60

3.60

3.60
(DVIOT) P THERMAL VISCOSITY 1/OEG. $R$ BTU/FT-HR-R LB/FT-SEC
$\times 1 C E+6$

0.0105
0.0237
0.0365
0.0558
0.0945
0.279
0.427
0.212
0.152
0.122
0.103
0.0903
0.0807
0.0731
0.0671
0.0621
0.0579
0.0511
0.0458
0.0416
0.0381
0.0352
0.0327
0.0335
0.0287
0.0273
0.0256
0.0105

0.0118

0.0121

0.0121

0.00870

0.00823

0.00841
0.00872

0.00906

0.00941

0.00976

0.0101

0.0165

0.0111

0.3118

0.0124

0.0135

0. 0141

0.0146

0.0152

0.0157

0.0162

0.0226

0.0202

0.0183

0.0167

0.0143

0.0125

0.0111

0.00831

0.00712

0.00623

0.00554

0.00499

0.0139

0.00333

0.00285

0.00250

0.00222

0.00166

0.0179

0.0190

נ. 0201

0.0212

0.0233

0.0253

0.0272

0.0290

0.0326
0.0361

0.0394

0.01426

0.0457

0.0532

0.0603
0.0670

0.0733

0.0793

0.0851
0.0962

0.107

0.00143

C. 00111

0.000833

0.000714

0.000625

0.003555

0.000500

0.118

0.128

0.156
0.174

0.191

0.208

0.224
0.263

0.000333

0.299
2.78

2.78
2.83

2.70

2.50

2.26
1.92

1.92
1.29

1.23
1.22

1.22
1.26

1.32

1. 37

1.43
1.48

1.48
1.54

1.59

1.64

1.70

1.80

$1.9 \mathrm{~J}$

2.09

2.18

2.27

2. 36

2.44

2.54
2.51

2.81

2.99

3.17

3.35

3.68
3.99

4.29

4.58

5.12
5.63

6.11

6.91

7.93

9.02

10.0

10.9
11.8
12.7

14.4

16.0

19.1
20.5

23.3

26.0

28.5

31.0

33.4
39.2

44.6

THERMAL OIELECTRIC

IFFUSIVITY CONSTANT SQ FT/HR

$\begin{array}{lll}0.00154 & 1.02056 & 0.691 \\ 0.00230 & 1.02047 & 0.479 \\ 0.00200 & 1.02029 & 0.540 \\ 0.00171 & 1.01998 & 0.614 \\ 0.00133 & 1.01940 & 0.765 \\ 0.000737 & 1.01796 & 1.37 \\ 0.00127 & 1.00733 & 1.59 \\ 0.00251 & 1.00563 & 1.09 \\ 0.00361 & 1.00477 & 0.940 \\ 0.00466 & 1.00420 & 0.867 \\ 0.00572 & 1.00378 & 0.824 \\ 0.00678 & 1.00345 & 0.796 \\ 0.00786 & 1.00318 & 0.776 \\ 0.00896 & 1.00295 & 0.762 \\ 0.0101 & 1.00276 & 0.752 \\ 0.0112 & 1.00259 & 0.744 \\ 0.0124 & 1.00245 & 0.738 \\ 0.0148 & 1.03220 & 0.730 \\ 0.0174 & 1.00200 & 0.724 \\ 0.0230 & 1.00184 & 0.720 \\ 0.0228 & 1.00170 & 0.718 \\ 0.0228 & 1.00158 & 0.716 \\ 0.0257 & 1.00148 & 0.715 \\ 0.0286 & 1.00139 & 0.714 \\ 0.0317 & 1.00131 & 0.713 \\ 0.0349 & 1.00124 & 0.713 \\ 0.0382 & 1.00118 & 0.713 \\ 0.0415 & & \end{array}$

0.0504

0.0504

0.0699

0.0805

0.103

0.128

0.156

0.185

0.249
0.322

0.402

0.489

0.583

0.847

1.15

1.49

1.87
2.27

2.71
3.67

4.77

4.77
5.98

7.30

8.73

11.9

15.5

19.5

23.8
28.5

41.7

57.0

1.00105

1.00094

1.00086

1.00079

1.00059

1.00053

1.00047

1.03040

1.00034

1.00030

1.00026

1.00024

1. 00019

1.00016

1.00014
1.00012

1.00011

1.00010

1. 00008

1.00007

1.00006

1. 00005

1. 00005

1.00004

1.00003

1. 00003

1. 00003

1.00302

1.00002

1.03002

0.713

0.712

0.712

0.712

0.710

0.708

0.706

0.732
0.698

0.694

0.691

0.675

0.671
0.669

0.667

0.666

0.666 
35 PSIA ISOBAR

THERMOOYNAMIC PROPERTIES OF HELIUM 4

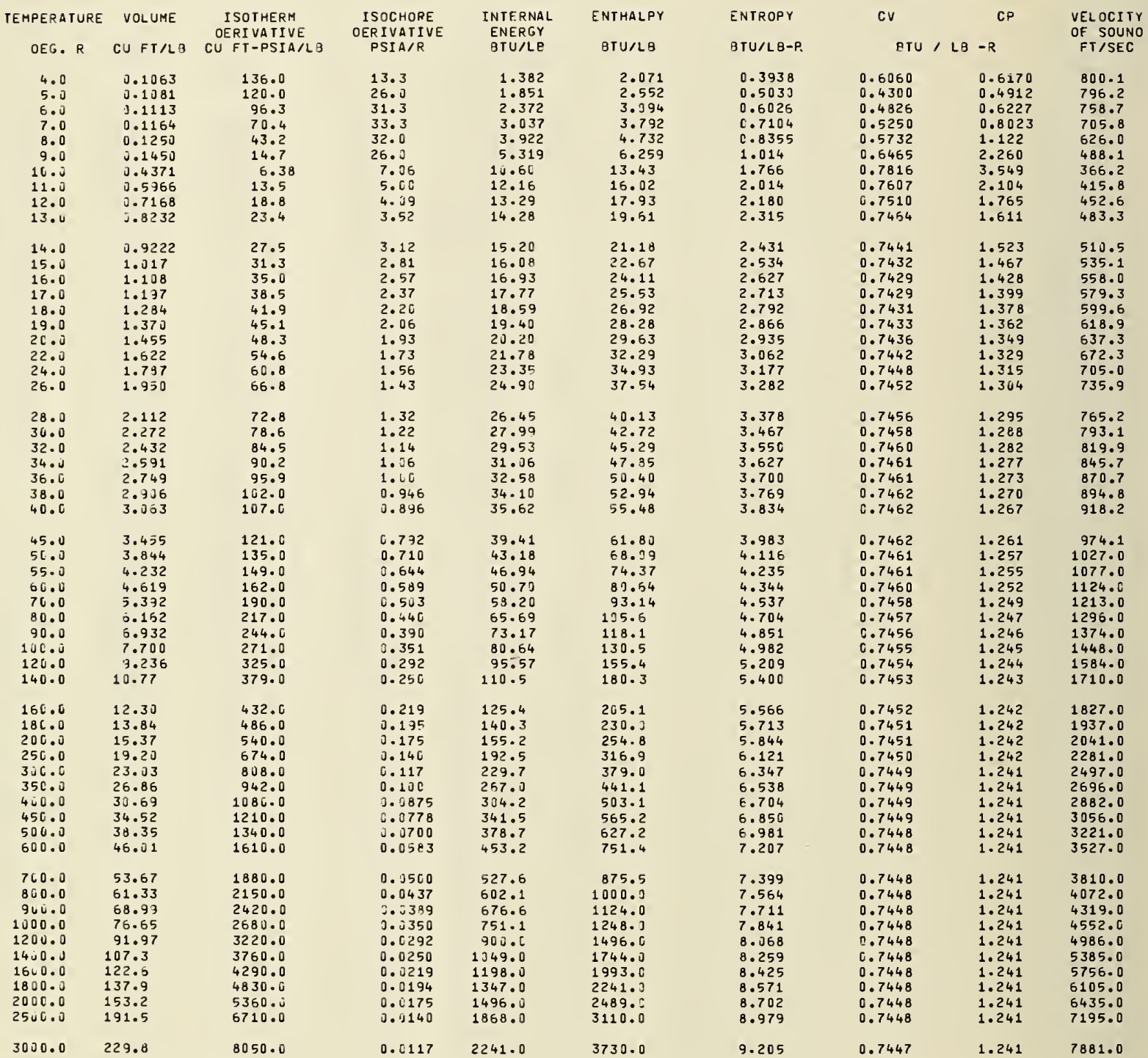

* two-phase gounoary 
35 PSIA ISOBAR

TEMPERATURE DENSITY V(DH/OV) ${ }_{\mathrm{P}} V(D P / O U)_{V}-V(D P / O V)_{T}$ DEG. R LB/CUFT BTU/LB PSIA-CU FT/BTU PSIA

\begin{tabular}{|c|c|c|c|c|}
\hline $\begin{array}{l}4.0 \\
5.0 \\
6.0 \\
7.0 \\
8.0 \\
9.0 \\
10.0 \\
11.0 \\
12.0 \\
13.0\end{array}$ & $\begin{array}{l}9.411 \\
9.249 \\
8.982 \\
8.589 \\
7.998 \\
6.897 \\
2.288 \\
1.676 \\
1.395 \\
1.215\end{array}$ & $\begin{array}{c}59.2 \\
20.9 \\
17.2 \\
14.5 \\
12.1 \\
8.81 \\
7.33 \\
9.52 \\
11.3 \\
13.0\end{array}$ & $\begin{array}{l}2.34 \\
6.54 \\
7.22 \\
7.39 \\
6.98 \\
5.84 \\
3.95 \\
3.92 \\
3.90 \\
3.88\end{array}$ & $\begin{array}{r}1280.0 \\
1110.0 \\
865.0 \\
604.0 \\
346.0 \\
101.0 \\
14.6 \\
22.6 \\
26.2 \\
28.4\end{array}$ \\
\hline $\begin{array}{l}14.0 \\
15.0 \\
16.0 \\
17.0 \\
18.0 \\
19.0 \\
20.0 \\
22.0 \\
24.0 \\
26.0\end{array}$ & $\begin{array}{l}1.084 \\
0.9836 \\
0.9025 \\
0.8353 \\
0.7785 \\
0.7299 \\
0.6874 \\
0.6165 \\
0.5597 \\
0.5128\end{array}$ & $\begin{array}{l}14.6 \\
16.1 \\
17.6 \\
19.0 \\
20.4 \\
21.8 \\
23.2 \\
25.9 \\
28.6 \\
31.3\end{array}$ & $\begin{array}{l}3.86 \\
3.84 \\
3.83 \\
3.81 \\
3.80 \\
3.79 \\
3.78 \\
3.76 \\
3.75 \\
3.74\end{array}$ & $\begin{array}{l}29.8 \\
30.8 \\
31.6 \\
32.1 \\
32.6 \\
32.9 \\
33.2 \\
33.7 \\
34.0 \\
34.3\end{array}$ \\
\hline $\begin{array}{l}28.0 \\
30.0 \\
32.0 \\
34.0 \\
36.0 \\
38.0 \\
40.0\end{array}$ & $\begin{array}{l}0.4736 \\
0.4401 \\
0.4112 \\
0.3860 \\
0.3638 \\
0.3441 \\
0.3264\end{array}$ & $\begin{array}{l}33.9 \\
36.6 \\
39.2 \\
41.8 \\
44.3 \\
46.9 \\
49.5\end{array}$ & $\begin{array}{l}3.72 \\
3.71 \\
3.71 \\
3.70 \\
3.69 \\
3.68 \\
3.68\end{array}$ & $\begin{array}{l}34.5 \\
34.6 \\
34.7 \\
34.8 \\
34.9 \\
34.9 \\
35.0\end{array}$ \\
\hline $\begin{array}{r}45.0 \\
50.0 \\
55.0 \\
60.0 \\
70.0 \\
80.0 \\
90.0 \\
100.0 \\
120.0 \\
140.0\end{array}$ & $\begin{array}{l}0.2895 \\
0.2601 \\
0.2363 \\
0.2165 \\
0.1855 \\
0.1623 \\
0.1443 \\
0.1299 \\
0.1083 \\
0.09285\end{array}$ & $\begin{array}{r}55.8 \\
62.2 \\
66.5 \\
74.8 \\
87.3 \\
100.0 \\
112.0 \\
125.0 \\
150.0 \\
175.0\end{array}$ & $\begin{array}{l}3.67 \\
3.66 \\
3.65 \\
3.65 \\
3.64 \\
3.63 \\
3.63 \\
3.63 \\
3.62 \\
3.62\end{array}$ & $\begin{array}{l}35.1 \\
35.1 \\
35.2 \\
35.2 \\
35.2 \\
35.2 \\
35.2 \\
35.2 \\
35.2 \\
35.1\end{array}$ \\
\hline $\begin{array}{l}160.0 \\
180.0 \\
200.0 \\
250.0 \\
300.0 \\
350.0 \\
400.0 \\
450.0 \\
560.0 \\
600.0\end{array}$ & $\begin{array}{l}0.08128 \\
0.07227 \\
0.06506 \\
0.05208 \\
0.04342 \\
0.03723 \\
0.03258 \\
0.02897 \\
0.02608 \\
0.02173\end{array}$ & $\begin{array}{l}199.0 \\
224.0 \\
249.0 \\
311.0 \\
373.0 \\
435.0 \\
497.0 \\
559.0 \\
621.0 \\
746.0\end{array}$ & $\begin{array}{l}3.61 \\
3.61 \\
3.61 \\
3.61 \\
3.61 \\
3.61 \\
3.60 \\
3.60 \\
3.60 \\
3.60\end{array}$ & $\begin{array}{l}35.1 \\
35.1 \\
35.1 \\
35.1 \\
35.1 \\
35.1 \\
35.1 \\
35.0 \\
35.0 \\
35.0\end{array}$ \\
\hline $\begin{array}{r}700.0 \\
860.0 \\
900.0 \\
1000.0 \\
1200.0 \\
1400.0 \\
1600.0 \\
1800.0 \\
2000.0 \\
2500.0\end{array}$ & $\begin{array}{l}0.01863 \\
0.01631 \\
0.01450 \\
0.01305 \\
0.01087 \\
0.009321 \\
0.008156 \\
0.007250 \\
0.006526 \\
0.005221\end{array}$ & $\begin{array}{r}870.0 \\
994.0 \\
1120.0 \\
1240.0 \\
1490.0 \\
1740.0 \\
1990.0 \\
2230.0 \\
2480.0 \\
3100.0\end{array}$ & $\begin{array}{l}3.60 \\
3.60 \\
3.60 \\
3.60 \\
3.60 \\
3.60 \\
3.60 \\
3.60 \\
3.60 \\
3.60\end{array}$ & $\begin{array}{l}35.0 \\
35.0 \\
35.0 \\
35.0 \\
35.0 \\
35.0 \\
35.0 \\
35.0 \\
35.0 \\
35.0\end{array}$ \\
\hline 3000.0 & 0.304351 & 3720.0 & 3.60 & 35.0 \\
\hline
\end{tabular}

(DV/DT) PV THERMAL VISCOSITY 1/OEG. R BTU/FT-HR-R LB/FT-SEC

0.0104
0.0235
0.0362
0.0551
0.0925
0.257
0.484
0.221
0.156
0.124

0.105$$
0.0912
$$

0.0813

0.0736

0.0674
0.0624

0.0624
0.0581

0.0581

0.0513

0.0417

0.0382

0.0352

0.0327

0.0306

0.0271

0.0256

0.0226

0.0202

0.0183

0.0167

0.0143

0.0125

0.0111

0.0100

0.00712

0.00623

0.00554
0.00499

0.00499
0.00399

0.00333

0.00285

0.00250

0.00222

0.00200

0.00143

0.00125

0.00125

0.00111

0.00100

0.000714

0.000625

0.000555

0.000500

0.000333

2.79
2.84
2.71
2.51
2.27
1.94
1.22
1.23
1.27
1.32

1.38

1.43

$\begin{array}{ll}0.00944 & 1.43 \\ 0.00979 & 1.49 \\ 0.0101 & 1.54\end{array}$

0.0101

0.0105

0.0108

0.0111
0.0118

0.0124

0.0130

0.0136

0.0141

0.0147

0.0152

0.0162

0.0162

0.0179

0.0179
0.0190

0.0190

0.0212

0.0233

0.0253

0.0272

0.0290

0.0326

0.0394

0.0394
0.0426

0.0426
0.0457

0.0532

0.0603

0.0670

0.0733

0.0793

0.0851

0.107
0.118

0.118
0.128

0.128
0.137

0.156

0.174

0.191
0.208

0.224

0.224
0.263

0.299

1.59
1.65

1.65
1.70

1.70
1.80

1.90
2.00

2.09

2.18
2.18
2.27

2.27
2.36

2.44

2.53
2.61

2.81
2.99

3.18
3.35

3.35
3.68

3.99

4.29

4.58

5.12

6.12

6.58

6.91
7.99

9.02

10.0
10.9
11.8

11.8

12.7
14.4

16.0
17.6

17.6

20.5

$23 \cdot 3$
26.0

28.5

31.0

33.4
39.2

44.6

THERMAL DIELECTRIC OIFFUSIVITY CDNSTANT

PRANOTL SQ FT/HR

$\begin{array}{lll}0.00155 & 1.02057 & 0.690 \\ 0.00230 & 1.02047 & 0.479 \\ 0.00201 & 1.02030 & 0.540 \\ 0.00172 & 1.01999 & 0.613 \\ 0.00135 & 1.01943 & 0.759 \\ 0.000774 & 1.01806 & 1.31 \\ 0.00111 & 1.00778 & 1.73 \\ 0.00236 & 1.00587 & 1.12 \\ 0.00344 & 1.00495 & 0.955 \\ 0.00447 & 1.00435 & 0.876\end{array}$

\subsection{0}

0.00654

0.00760

0.00867
0.00977

0.00977
0.0109

0.0120

0.0144

0.0168
0.0194

1. 00390

1.00356

1.00327

1.00304

1.00284
1.00267

$1.00267 \quad 0.754$

$\begin{array}{ll}1.00252 & 0.746\end{array}$

$\begin{array}{ll}1.00227 & 0.731\end{array}$

$\begin{array}{ll}1.00206 & 0.726 \\ 1.00189 & 0.722\end{array}$

0.0221

1.00175

$\begin{array}{ll}1.00175 & 0.719 \\ 1.00163 & 0.717\end{array}$

0.0249

0.0278

1. 00153

1.00143

1.00135

0.0339

1. 00128

0.715

0.0371

1.00122

0.714
0.714

0.0490

0.0582

1.00108

0.713

0.0679
0.0782

0.100

1. 00097

1. 00088

1. 00081

1.00069

$0.125 \quad 1.00061$

$0.151 \quad 1.00054$

0.180

1. 00049

0.313

1.00035

0.390

0.475

0.566
0.823

1.00031
1.00027

1.00025

1.12

1.45

1.81

2.21

2.63
3.57

1.00016

1.00014

1. 00012

1.00011

1.00010

0.713

0.713
0.713

0.712

0.711
0.710

0.708

0.706

0.702

4.63

5.81
7.09
8.48

11.6

15.1

18.9

23.1

27.7
40.5

1.00007

1.00006

1.00006

1.00005
1.00005

1.00005
1.00004

1. 00004

1.00003

1.00003

1.00002

0.694 0.691
0.675

0.671

0.668

0.667

0.666

0.666

0.668

0.668

0.668

0.667

0.667
0.667

0.667
0.667

0.666

0.666

0.666
0.666

55.4

1.00002

0.666 
36 PSIA ISOBAR

THERMOOYNAMIC PROPERTIES OF HELIUM 4

\begin{tabular}{|c|c|c|c|c|c|c|c|c|c|}
\hline $\begin{array}{c}\text { TEMPERATURE } \\
\text { OEG. } R\end{array}$ & $\begin{array}{l}\text { E VOLUME } \\
\text { CU FT/LB }\end{array}$ & $\begin{array}{c}\text { I SOTHERM } \\
\text { OERIVATI VE } \\
\text { CU FT-PSIA/LB }\end{array}$ & $\begin{array}{l}\text { ISOCHORE } \\
\text { OERIVATIVE } \\
\text { PSIA/R }\end{array}$ & $\begin{array}{l}\text { INTERNAL } \\
\text { ENERGY } \\
\text { BTU/LB }\end{array}$ & $\begin{array}{l}\text { ENTHALPY } \\
\text { BTU/LB }\end{array}$ & $\begin{array}{l}\text { ENTROPY } \\
\text { PTU/LB-R }\end{array}$ & $\begin{array}{l}\text { CV } \\
\text { BTU, LB }\end{array}$ & $\begin{array}{l}C P \\
-R\end{array}$ & $\begin{array}{l}\text { VELOCITY } \\
\text { OF SOUNO } \\
\text { FT/SEC }\end{array}$ \\
\hline $\begin{array}{r}4.0 \\
5.0 \\
6.0 \\
7.0 \\
8.0 \\
9.0 \\
10.0 \\
11.0 \\
12.0 \\
13.0\end{array}$ & $\begin{array}{l}0.1362 \\
0.1080 \\
0.1112 \\
0.1162 \\
0.1247 \\
0.1438 \\
0.4072 \\
1.5708 \\
0.6902 \\
0.7950\end{array}$ & $\begin{array}{c}137.0 \\
121.0 \\
97.2 \\
71.3 \\
44.2 \\
16.0 \\
5.57 \\
13.0 \\
18.4 \\
23.0\end{array}$ & $\begin{array}{c}13.3 \\
26.0 \\
31.3 \\
33.4 \\
32.2 \\
26.4 \\
7.63 \\
5.25 \\
4.26 \\
3.65\end{array}$ & $\begin{array}{c}1.381 \\
1.848 \\
2.368 \\
3.030 \\
3.937 \\
5.274 \\
10.39 \\
12.06 \\
13.21 \\
14.22\end{array}$ & $\begin{array}{c}2.089 \\
2.568 \\
3.109 \\
3.805 \\
4.739 \\
6.233 \\
13.10 \\
15.86 \\
17.82 \\
19.52\end{array}$ & $\begin{array}{l}0.3935 \\
0.5024 \\
0.6017 \\
0.7091 \\
0.8334 \\
1.008 \\
1.725 \\
1.990 \\
2.160 \\
2.296\end{array}$ & $\begin{array}{l}0.6037 \\
0.4296 \\
0.4823 \\
0.5246 \\
0.5725 \\
0.6443 \\
0.7829 \\
0.7615 \\
0.7514 \\
0.7465\end{array}$ & $\begin{array}{l}0.6146 \\
0.4903 \\
0.6213 \\
0.7989 \\
1.111 \\
2.149 \\
3.997 \\
2.171 \\
1.795 \\
1.628\end{array}$ & $\begin{array}{l}802.4 \\
798.5 \\
761.4 \\
709.1 \\
630.5 \\
496.6 \\
362.9 \\
413.8 \\
451.2 \\
482.3\end{array}$ \\
\hline $\begin{array}{l}14.0 \\
15.0 \\
16.0 \\
17.0 \\
18.0 \\
19.0 \\
20.0 \\
22.0 \\
24.0 \\
26.0\end{array}$ & $\begin{array}{l}3.8921 \\
3.9846 \\
1.074 \\
1.161 \\
1.246 \\
1.330 \\
1.412 \\
1.575 \\
1.736 \\
1.895\end{array}$ & $\begin{array}{l}27.2 \\
31.1 \\
34.7 \\
38.3 \\
41.7 \\
45.0 \\
48.2 \\
54.5 \\
60.7 \\
66.7\end{array}$ & $\begin{array}{l}3.23 \\
2.91 \\
2.65 \\
2.44 \\
2.27 \\
2.12 \\
1.99 \\
1.78 \\
1.61 \\
1.47\end{array}$ & $\begin{array}{l}15.15 \\
16.04 \\
16.89 \\
17.73 \\
18.56 \\
19.37 \\
20.17 \\
21.75 \\
23.32 \\
24.88\end{array}$ & $\begin{array}{l}21.10 \\
22.60 \\
24.05 \\
25.47 \\
26.86 \\
28.23 \\
29.58 \\
32.25 \\
34.89 \\
37.51\end{array}$ & $\begin{array}{l}2.413 \\
2.517 \\
2.611 \\
2.697 \\
2.776 \\
2.850 \\
2.920 \\
3.047 \\
3.162 \\
3.267\end{array}$ & $\begin{array}{l}0.7442 \\
0.7432 \\
0.7428 \\
0.7429 \\
0.7431 \\
0.7433 \\
0.7435 \\
0.7442 \\
0.7448 \\
0.7452\end{array}$ & $\begin{array}{l}1.535 \\
1.475 \\
1.435 \\
1.405 \\
1.382 \\
1.366 \\
1.352 \\
1.332 \\
1.317 \\
1.306\end{array}$ & $\begin{array}{l}509.7 \\
534.5 \\
557.5 \\
579.0 \\
599.3 \\
618.7 \\
637.2 \\
672.2 \\
705.0 \\
736.0\end{array}$ \\
\hline $\begin{array}{l}28.0 \\
30.0 \\
32.0 \\
34.0 \\
36.0 \\
38.0 \\
40.0\end{array}$ & $\begin{array}{l}2.052 \\
2.208 \\
2.364 \\
2.518 \\
2.672 \\
2.826 \\
2.978\end{array}$ & $\begin{array}{r}72.7 \\
78.6 \\
84.4 \\
90.2 \\
95.9 \\
102.0 \\
147.0\end{array}$ & $\begin{array}{l}1.35 \\
1.26 \\
1.17 \\
1.10 \\
1.03 \\
0.974 \\
0.922\end{array}$ & $\begin{array}{l}26.43 \\
27.97 \\
29.51 \\
31.04 \\
32.57 \\
34.09 \\
35.61\end{array}$ & $\begin{array}{l}40.11 \\
42.59 \\
45.27 \\
47.83 \\
50.38 \\
52.92 \\
55.46\end{array}$ & $\begin{array}{l}3.363 \\
3.452 \\
3.535 \\
3.613 \\
3.686 \\
3.755 \\
3.820\end{array}$ & $\begin{array}{l}0.7456 \\
0.7458 \\
0.7460 \\
0.7461 \\
0.7462 \\
0.7462 \\
0.7462\end{array}$ & $\begin{array}{l}1.297 \\
1.289 \\
1.283 \\
1.278 \\
1.274 \\
1.271 \\
1.268\end{array}$ & $\begin{array}{l}765.4 \\
793.3 \\
820.1 \\
845.9 \\
870.9 \\
895.0 \\
918.5\end{array}$ \\
\hline $\begin{array}{r}45.0 \\
50.0 \\
55.0 \\
60.0 \\
70.0 \\
80.0 \\
90.0 \\
100.0 \\
120.0 \\
140.0\end{array}$ & $\begin{array}{c}3.359 \\
3.738 \\
4.115 \\
4.492 \\
5.243 \\
5.992 \\
6.740 \\
7.487 \\
8.981 \\
10.47\end{array}$ & $\begin{array}{l}121.0 \\
135.0 \\
149.0 \\
163.0 \\
190.0 \\
217.0 \\
244.0 \\
271.0 \\
325.0 \\
379.0\end{array}$ & $\begin{array}{l}0.815 \\
0.731 \\
0.663 \\
0.606 \\
0.518 \\
0.452 \\
0.402 \\
0.361 \\
0.361 \\
0.257\end{array}$ & $\begin{array}{c}39.39 \\
43.17 \\
46.93 \\
50.69 \\
58.19 \\
65.68 \\
73.16 \\
80.63 \\
95.57 \\
110.5\end{array}$ & $\begin{array}{c}61.78 \\
68.38 \\
74.37 \\
80.63 \\
93.14 \\
105.6 \\
118.1 \\
130.5 \\
155.4 \\
180.3\end{array}$ & $\begin{array}{l}3.969 \\
4.102 \\
4.221 \\
4.330 \\
4.523 \\
4.690 \\
4.837 \\
4.958 \\
5.195 \\
5.386\end{array}$ & $\begin{array}{l}0.7462 \\
0.7462 \\
0.7461 \\
0.7460 \\
0.7459 \\
0.7457 \\
0.7456 \\
0.7455 \\
0.7454 \\
0.7453\end{array}$ & $\begin{array}{l}1.262 \\
1.258 \\
1.255 \\
1.253 \\
1.249 \\
1.247 \\
1.246 \\
1.245 \\
1.244 \\
1.243\end{array}$ & $\begin{array}{r}974.4 \\
1027.0 \\
1077.0 \\
1124.0 \\
1214.0 \\
1296.0 \\
1374.0 \\
1448.0 \\
1585.0 \\
1710.0\end{array}$ \\
\hline $\begin{array}{l}160.0 \\
180.0 \\
200.0 \\
250.0 \\
300.0 \\
350.0 \\
400.0 \\
450.0 \\
500.0 \\
600.0\end{array}$ & $\begin{array}{l}11.96 \\
13.45 \\
14.94 \\
18.67 \\
22.39 \\
26.12 \\
29.84 \\
33.56 \\
37.29 \\
44.73\end{array}$ & $\begin{array}{r}432.0 \\
486.0 \\
540.0 \\
674.0 \\
808.0 \\
942.0 \\
1080.0 \\
1210.0 \\
1340.0 \\
1610.0\end{array}$ & $\begin{array}{l}0.225 \\
0.200 \\
0.180 \\
0.144 \\
0.120 \\
0.163 \\
0.0900 \\
0.0800 \\
0.0720 \\
0.0600\end{array}$ & $\begin{array}{l}125.4 \\
140.3 \\
155.2 \\
192.5 \\
229.7 \\
267.0 \\
304.2 \\
341.5 \\
378.7 \\
453.2\end{array}$ & $\begin{array}{l}205.2 \\
230.0 \\
254.8 \\
316.9 \\
379.0 \\
441.1 \\
503.1 \\
565.2 \\
627.2 \\
751.4\end{array}$ & $\begin{array}{l}5.552 \\
5.699 \\
5.830 \\
6.107 \\
6.333 \\
6.524 \\
6.690 \\
6.836 \\
6.967 \\
7.193\end{array}$ & $\begin{array}{l}0.7452 \\
0.7451 \\
0.7451 \\
0.7450 \\
0.7449 \\
0.7449 \\
0.7449 \\
0.7449 \\
0.7449 \\
0.7448\end{array}$ & $\begin{array}{l}1.242 \\
1.242 \\
1.242 \\
1.242 \\
1.241 \\
1.241 \\
1.241 \\
1.241 \\
1.241 \\
1.241\end{array}$ & $\begin{array}{l}1827.0 \\
1937.0 \\
2041.0 \\
2281.0 \\
2497.0 \\
2696.0 \\
2882.0 \\
3056.0 \\
3221.0 \\
3528.0\end{array}$ \\
\hline $\begin{array}{r}700.0 \\
800.0 \\
900.0 \\
1000.0 \\
1200.0 \\
1400.0 \\
1600.0 \\
1800.0 \\
2000.0 \\
2500.0\end{array}$ & $\begin{array}{r}52.18 \\
59.63 \\
67.07 \\
74.52 \\
89.41 \\
104.3 \\
119.2 \\
134.1 \\
149.0 \\
186.2\end{array}$ & $\begin{array}{l}1880.0 \\
2150.0 \\
2420.0 \\
2680.0 \\
3220.0 \\
3760.0 \\
4290.0 \\
4830.0 \\
5360.0 \\
6710.0\end{array}$ & $\begin{array}{l}0.0514 \\
0.0450 \\
0.0400 \\
0.0360 \\
0.0300 \\
0.0257 \\
0.0225 \\
0.0200 \\
0.0180 \\
0.0144\end{array}$ & $\begin{array}{r}527.6 \\
602.1 \\
676.6 \\
751.1 \\
900.0 \\
1049.0 \\
1198.0 \\
1347.0 \\
1496.0 \\
1868.0\end{array}$ & $\begin{array}{r}875.5 \\
1000.0 \\
1124.0 \\
1248.0 \\
1496.0 \\
1744.0 \\
1993.0 \\
2241.0 \\
2489.0 \\
3110.0\end{array}$ & $\begin{array}{l}7.385 \\
7.550 \\
7.697 \\
7.827 \\
8.054 \\
8.245 \\
8.411 \\
8.557 \\
8.688 \\
8.965\end{array}$ & $\begin{array}{l}0.7448 \\
0.7448 \\
0.7448 \\
0.7448 \\
0.7448 \\
0.7448 \\
0.7448 \\
0.7448 \\
0.7448 \\
0.7448\end{array}$ & $\begin{array}{l}1.241 \\
1.241 \\
1.241 \\
1.241 \\
1.241 \\
1.241 \\
1.241 \\
1.241 \\
1.241 \\
1.241\end{array}$ & $\begin{array}{l}3810.0 \\
4072.0 \\
4319.0 \\
4552.0 \\
4986.0 \\
5385.0 \\
5756.0 \\
6105.0 \\
6435.0 \\
7195.0\end{array}$ \\
\hline 3000.0 & 223.5 & 8050.0 & 0.0120 & 2241.0 & 3730.0 & 9.191 & 0.7447 & 1.241 & 7881.0 \\
\hline
\end{tabular}


36 PSIA ISOGAR

\begin{tabular}{|c|c|c|c|c|c|c|c|c|c|c|}
\hline $\begin{array}{c}\text { TEMPERATURE } \\
\text { DEG. R }\end{array}$ & $\begin{array}{l}\text { DENSITY } \\
\text { LB/CU FT }\end{array}$ & $\begin{array}{c}\text { V(OH/OV) } \\
\text { BTU/LB }\end{array}$ & $\begin{array}{l}V(O P / O U)_{v}- \\
\text { PSIA-CU FT/BTU }\end{array}$ & $\begin{array}{l}-V(O P / O V) T \\
U \text { PSIA }\end{array}$ & $\begin{array}{l}\text { (DV/OT) } / V_{O} \\
\text { 1/DEG. R }\end{array}$ & $\begin{array}{c}\text { THERMAL } \\
\text { CONOUCTIVITY } \\
\text { B TU/FT-HR-R }\end{array}$ & $\begin{array}{l}\text { VISCOSITY } \\
\text { LB/FT-SEC } \\
\times \quad 10 E+6\end{array}$ & $\begin{array}{c}\text { THERMAL } \\
\text { OIFFUSIVITY } \\
\text { SQ FT/HR }\end{array}$ & $\begin{array}{l}\text { DIELECTRIC } \\
\text { CONSTANT }\end{array}$ & $\begin{array}{l}\text { PRANOTL } \\
\text { NUMBER }\end{array}$ \\
\hline $\begin{array}{r}4.0 \\
5.0 \\
6.0 \\
7.0 \\
8.0 \\
9.0 \\
16.0 \\
11.0 \\
12.0 \\
13.0\end{array}$ & $\begin{array}{l}9.418 \\
9.258 \\
8.992 \\
8.603 \\
8.020 \\
6.955 \\
2.456 \\
1.752 \\
1.449 \\
1.258\end{array}$ & $\begin{array}{c}59.4 \\
21.0 \\
17.3 \\
14.7 \\
12.3 \\
9.03 \\
7.16 \\
9.40 \\
11.2 \\
12.9\end{array}$ & $\begin{array}{l}2.34 \\
5.54 \\
7.23 \\
7.40 \\
7.00 \\
5.89 \\
3.97 \\
3.93 \\
3.91 \\
3.89\end{array}$ & $\begin{array}{r}1290.0 \\
1120.0 \\
874.0 \\
613.0 \\
355.0 \\
111.0 \\
13.7 \\
22.7 \\
26.7 \\
29.0\end{array}$ & $\begin{array}{l}0.0103 \\
0.0233 \\
0.0359 \\
0.0545 \\
0.0906 \\
0.238 \\
0.558 \\
0.231 \\
0.160 \\
0.126\end{array}$ & $\begin{array}{l}0.00899 \\
0.0105 \\
0.0113 \\
0.0118 \\
0.0121 \\
0.0121 \\
0.00940 \\
0.00839 \\
0.00851 \\
0.00879\end{array}$ & $\begin{array}{l}2.80 \\
2.85 \\
2.71 \\
2.52 \\
2.28 \\
1.96 \\
1.24 \\
1.24 \\
1.28 \\
1.33\end{array}$ & $\begin{array}{l}0.00155 \\
0.00231 \\
0.00201 \\
0.00172 \\
0.00136 \\
0.000808 \\
0.000958 \\
0.00221 \\
0.00327 \\
0.00429\end{array}$ & $\begin{array}{l}1.02057 \\
1.02048 \\
1.02030 \\
1.02000 \\
1.01945 \\
1.01814 \\
1.00828 \\
1.00611 \\
1.00513 \\
1.00449\end{array}$ & $\begin{array}{l}0.689 \\
0.479 \\
0.539 \\
0.512 \\
0.753 \\
1.26 \\
1.90 \\
1.15 \\
0.971 \\
0.886\end{array}$ \\
\hline $\begin{array}{l}14.0 \\
15.0 \\
16.0 \\
17.0 \\
18.0 \\
19.0 \\
20.0 \\
22.0 \\
24.0 \\
25.0\end{array}$ & $\begin{array}{l}1.121 \\
1.016 \\
0.9312 \\
0.8614 \\
0.8025 \\
0.7521 \\
0.7081 \\
0.5349 \\
0.5761 \\
0.5278\end{array}$ & $\begin{array}{l}14.5 \\
16.0 \\
17.5 \\
19.0 \\
20.4 \\
21.8 \\
23.2 \\
25.9 \\
28.5 \\
31.3\end{array}$ & $\begin{array}{l}3.87 \\
3.85 \\
3.83 \\
3.82 \\
3.80 \\
3.79 \\
3.78 \\
3.77 \\
3.75 \\
3.74\end{array}$ & $\begin{array}{l}30.5 \\
31.6 \\
32.4 \\
33.0 \\
33.4 \\
33.8 \\
34.1 \\
34.6 \\
35.0 \\
35.2\end{array}$ & $\begin{array}{l}0.106 \\
0.0921 \\
0.0820 \\
0.0741 \\
0.0678 \\
0.0627 \\
0.0584 \\
0.0514 \\
0.0461 \\
0.0418\end{array}$ & $\begin{array}{l}0.00912 \\
0.00946 \\
0.00981 \\
0.0102 \\
0.0105 \\
0.0108 \\
0.0112 \\
0.0118 \\
0.0124 \\
0.0130\end{array}$ & $\begin{array}{l}1.38 \\
1.44 \\
1.49 \\
1.54 \\
1.60 \\
1.65 \\
1.70 \\
1.80 \\
1.90 \\
2.00\end{array}$ & $\begin{array}{l}0.00530 \\
0.00632 \\
0.00735 \\
0.00839 \\
0.00946 \\
0.0105 \\
0.0117 \\
0.0140 \\
0.0164 \\
0.0189\end{array}$ & $\begin{array}{l}1.00403 \\
1.00367 \\
1.00337 \\
1.00313 \\
1.00293 \\
1.00275 \\
1.00259 \\
1.00233 \\
1.00212 \\
1.00195\end{array}$ & $\begin{array}{l}0.837 \\
0.806 \\
0.784 \\
0.759 \\
0.757 \\
0.749 \\
0.742 \\
0.733 \\
0.727 \\
0.723\end{array}$ \\
\hline $\begin{array}{l}28.0 \\
30.0 \\
32.0 \\
34.0 \\
36.0 \\
38.0 \\
40.0\end{array}$ & $\begin{array}{l}0.4873 \\
0.4528 \\
0.4230 \\
0.3971 \\
0.3742 \\
0.3539 \\
0.3358\end{array}$ & $\begin{array}{l}33.9 \\
36.5 \\
39.2 \\
41.8 \\
44.3 \\
46.9 \\
49.5\end{array}$ & $\begin{array}{l}3.73 \\
3.72 \\
3.71 \\
3.70 \\
3.69 \\
3.69 \\
3.68\end{array}$ & $\begin{array}{l}35.4 \\
35.6 \\
35.7 \\
35.8 \\
35.9 \\
35.9 \\
36.0\end{array}$ & $\begin{array}{l}0.0382 \\
0.0353 \\
0.0328 \\
0.0306 \\
0.0287 \\
0.0271 \\
0.0256\end{array}$ & $\begin{array}{l}0.0136 \\
0.0141 \\
0.0147 \\
0.0152 \\
0.0157 \\
0.0162 \\
0.0167\end{array}$ & $\begin{array}{l}2.09 \\
2.19 \\
2.27 \\
2.36 \\
2.45 \\
2.53 \\
2.61\end{array}$ & $\begin{array}{l}0.0215 \\
0.0242 \\
0.0270 \\
0.0299 \\
0.0329 \\
0.0360 \\
0.0392\end{array}$ & $\begin{array}{l}1.00180 \\
1.00168 \\
1.00157 \\
1.00147 \\
1.00139 \\
1.00132 \\
1.00125\end{array}$ & $\begin{array}{l}0.720 \\
0.718 \\
0.716 \\
0.715 \\
0.714 \\
0.714 \\
0.714\end{array}$ \\
\hline $\begin{array}{r}45.0 \\
50.0 \\
55.0 \\
60.0 \\
70.0 \\
80.0 \\
90.0 \\
100.0 \\
120.0 \\
140.0\end{array}$ & $\begin{array}{l}0.2977 \\
0.2676 \\
0.2430 \\
0.2226 \\
0.1907 \\
0.1669 \\
0.1484 \\
0.1336 \\
0.1114 \\
0.09549\end{array}$ & $\begin{array}{r}55.8 \\
62.2 \\
68.5 \\
74.8 \\
87.3 \\
100.0 \\
112.0 \\
125.0 \\
150.0 \\
175.0\end{array}$ & $\begin{array}{l}3.67 \\
3.66 \\
3.65 \\
3.65 \\
3.64 \\
3.63 \\
3.63 \\
3.63 \\
3.62 \\
3.62\end{array}$ & $\begin{array}{l}36.1 \\
36.1 \\
36.2 \\
36.2 \\
36.2 \\
36.2 \\
36.2 \\
36.2 \\
36.2 \\
36.2\end{array}$ & $\begin{array}{l}0.0226 \\
0.0202 \\
0.0183 \\
0.0167 \\
0.0143 \\
0.0125 \\
0.0111 \\
0.0100 \\
0.00831 \\
0.00712\end{array}$ & $\begin{array}{l}0.0179 \\
0.0190 \\
0.0201 \\
0.0212 \\
0.0233 \\
0.0253 \\
0.0272 \\
0.0290 \\
0.0326 \\
0.0361\end{array}$ & $\begin{array}{l}2.81 \\
3.00 \\
3.18 \\
3.35 \\
3.68 \\
4.00 \\
4.29 \\
4.58 \\
5.12 \\
5.63\end{array}$ & $\begin{array}{l}0.0476 \\
0.0566 \\
0.0650 \\
0.0761 \\
0.0977 \\
0.121 \\
0.147 \\
0.175 \\
0.236 \\
0.304\end{array}$ & $\begin{array}{l}1.00111 \\
1.00100 \\
1.00091 \\
1.00083 \\
1.00071 \\
1.00063 \\
1.00056 \\
1.00050 \\
1.00042 \\
1.00036\end{array}$ & $\begin{array}{l}0.713 \\
0.713 \\
0.713 \\
0.7112 \\
0.711 \\
0.710 \\
0.708 \\
0.706 \\
0.702 \\
0.698\end{array}$ \\
\hline $\begin{array}{l}160.0 \\
180.0 \\
200.0 \\
250.0 \\
300.0 \\
350.0 \\
400.0 \\
450.0 \\
500.0 \\
600.0\end{array}$ & $\begin{array}{l}0.08359 \\
0.07433 \\
0.06692 \\
0.05357 \\
0.04466 \\
0.03829 \\
0.03351 \\
0.02980 \\
0.02682 \\
0.02236\end{array}$ & $\begin{array}{l}199.0 \\
224.0 \\
249.0 \\
311.0 \\
373.0 \\
435.0 \\
497.0 \\
559.0 \\
521.0 \\
746.0\end{array}$ & $\begin{array}{l}3.61 \\
3.61 \\
3.61 \\
3.61 \\
3.61 \\
3.61 \\
3.60 \\
3.50 \\
3.50 \\
3.60\end{array}$ & $\begin{array}{l}36.1 \\
36.1 \\
36.1 \\
36.1 \\
36.1 \\
36.1 \\
36.1 \\
36.1 \\
36.0 \\
36.0\end{array}$ & $\begin{array}{l}0.00523 \\
0.06554 \\
0.00499 \\
0.00399 \\
0.00333 \\
0.00285 \\
0.00250 \\
0.00222 \\
0.00200 \\
0.00166\end{array}$ & $\begin{array}{l}0.0394 \\
0.0426 \\
0.0458 \\
0.0532 \\
0.0603 \\
0.0670 \\
0.0733 \\
0.0793 \\
0.0851 \\
0.0963\end{array}$ & $\begin{array}{c}6.12 \\
6.58 \\
5.91 \\
7.99 \\
9.02 \\
10.0 \\
10.9 \\
11.8 \\
12.7 \\
14.4\end{array}$ & $\begin{array}{l}0.379 \\
0.462 \\
0.551 \\
0.800 \\
1.09 \\
1.41 \\
1.76 \\
2.15 \\
2.56 \\
3.47\end{array}$ & $\begin{array}{l}1.00031 \\
1.00028 \\
1.00025 \\
1.00020 \\
1.00017 \\
1.00014 \\
1.00013 \\
1.00011 \\
1.00010 \\
1.00008\end{array}$ & $\begin{array}{l}0.694 \\
0.691 \\
0.675 \\
0.671 \\
0.668 \\
0.667 \\
0.656 \\
0.656 \\
0.657 \\
0.658\end{array}$ \\
\hline $\begin{array}{r}700.0 \\
800.0 \\
900.0 \\
1000.0 \\
1200.0 \\
1400.0 \\
1600.0 \\
1800.0 \\
2000.0 \\
2500.0\end{array}$ & $\begin{array}{l}0.01916 \\
0.01677 \\
0.01491 \\
0.01342 \\
0.01118 \\
0.009587 \\
0.008389 \\
0.007458 \\
0.006712 \\
0.035370\end{array}$ & $\begin{array}{r}870.0 \\
994.0 \\
1120.0 \\
1240.0 \\
1490.0 \\
1740.0 \\
1990.0 \\
2230.0 \\
2480.0 \\
31 \cup 0.0\end{array}$ & $\begin{array}{l}3.50 \\
3.60 \\
3.60 \\
3.50 \\
3.60 \\
3.60 \\
3.60 \\
3.60 \\
3.60 \\
3.60\end{array}$ & $\begin{array}{l}36.0 \\
36.0 \\
36.0 \\
36.0 \\
36.0 \\
36.0 \\
36.0 \\
36.0 \\
36.0 \\
36.0\end{array}$ & $\begin{array}{l}0.06143 \\
0.00125 \\
0.00111 \\
0.00100 \\
0.000833 \\
0.000714 \\
0.000625 \\
0.000555 \\
0.000500 \\
0.000403\end{array}$ & $\begin{array}{l}0.107 \\
0.118 \\
0.128 \\
0.137 \\
0.156 \\
0.174 \\
0.191 \\
0.208 \\
0.224 \\
0.263\end{array}$ & $\begin{array}{l}16.0 \\
17.6 \\
19.1 \\
20.5 \\
23.3 \\
26.0 \\
28.5 \\
31.0 \\
33.4 \\
39.2\end{array}$ & $\begin{array}{c}4.50 \\
5.65 \\
6.90 \\
8.25 \\
11.3 \\
14.6 \\
18.4 \\
22.5 \\
26.9 \\
39.4\end{array}$ & $\begin{array}{l}1.00007 \\
1.00006 \\
1.00006 \\
1.00005 \\
1.00004 \\
1.00004 \\
1.00003 \\
1.00003 \\
1.00003 \\
1.00002\end{array}$ & $\begin{array}{l}0.658 \\
0.658 \\
0.667 \\
0.667 \\
0.657 \\
0.667 \\
0.656 \\
0.656 \\
0.666 \\
0.666\end{array}$ \\
\hline 3000.0 & 0.004475 & 3720.0 & 3.60 & 36.0 & 0.000333 & 0.299 & 44.6 & 53.9 & 1.00002 & 0.666 \\
\hline
\end{tabular}

* tho-phase boundarY 


\begin{tabular}{|c|c|c|c|c|c|c|c|c|c|}
\hline $\begin{array}{c}\text { TEMPERATURE } \\
\text { OEG. R }\end{array}$ & $\begin{array}{l}\text { E VOLUME } \\
\text { CU FT/LB }\end{array}$ & $\begin{array}{c}\text { ISOTHERM } \\
\text { OERIVATIVE } \\
\text { CU FT-PSIA/LB }\end{array}$ & $\begin{array}{l}\text { I SOCHORE } \\
\text { OERIVATIVE } \\
\text { PSI A/R }\end{array}$ & $\begin{array}{l}\text { INTERNAL } \\
\text { ENERGY } \\
\text { BTU/LB }\end{array}$ & $\begin{array}{l}\text { ENTHALPY } \\
\text { BTU/LB }\end{array}$ & $\begin{array}{l}\text { ENTROPY } \\
\text { BTU/LB-R }\end{array}$ & $\begin{array}{l}\text { CV } \\
\text { BTU / LB }\end{array}$ & $\begin{array}{l}C P \\
-R\end{array}$ & $\begin{array}{l}\text { VELOCITY } \\
\text { OF SOUNO } \\
\text { FT/SEC }\end{array}$ \\
\hline $\begin{array}{r}4.0 \\
5.0 \\
6.0 \\
7.0 \\
8.0 \\
9.0 \\
10.0 \\
11.0 \\
12.0 \\
13.0\end{array}$ & $\begin{array}{l}0.1060 \\
0.1378 \\
0.1109 \\
0.1159 \\
0.1240 \\
0.1416 \\
0.3474 \\
0.5228 \\
0.6410 \\
0.7429\end{array}$ & $\begin{array}{c}138.0 \\
122.0 \\
98.9 \\
73.1 \\
46.2 \\
18.4 \\
4.00 \\
11.9 \\
17.6 \\
22.3\end{array}$ & $\begin{array}{c}13.3 \\
26.0 \\
31.4 \\
33.6 \\
32.5 \\
27.1 \\
9.10 \\
5.78 \\
4.61 \\
3.93\end{array}$ & $\begin{array}{r}1.380 \\
1.843 \\
2.360 \\
3.015 \\
3.880 \\
5.193 \\
9.876 \\
11.85 \\
13.07 \\
14.10\end{array}$ & $\begin{array}{c}2.126 \\
2.602 \\
3.140 \\
3.831 \\
4.753 \\
6.189 \\
12.32 \\
15.53 \\
17.58 \\
19.33\end{array}$ & $\begin{array}{l}0.3929 \\
0.5011 \\
0.6000 \\
0.7067 \\
0.8294 \\
0.997 \\
1.633 \\
1.941 \\
2.120 \\
2.260\end{array}$ & $\begin{array}{l}0.5992 \\
0.4290 \\
0.4818 \\
0.5238 \\
0.5712 \\
0.6403 \\
0.7835 \\
0.7631 \\
0.7522 \\
0.7469\end{array}$ & $\begin{array}{l}0.6098 \\
0.4886 \\
0.6184 \\
0.7924 \\
1.091 \\
1.975 \\
5.408 \\
2.325 \\
1.858 \\
1.665\end{array}$ & $\begin{array}{l}806.9 \\
803.1 \\
766.7 \\
715.7 \\
639.4 \\
512.4 \\
357.7 \\
409.8 \\
448.4 \\
480.3\end{array}$ \\
\hline $\begin{array}{l}14.0 \\
15.0 \\
16.0 \\
17.0 \\
18.0 \\
19.0 \\
20.0 \\
22.0 \\
24.0 \\
26.0\end{array}$ & $\begin{array}{l}0.8366 \\
0.9255 \\
1.011 \\
1.094 \\
1.176 \\
1.255 \\
1.334 \\
1.439 \\
1.642 \\
1.793\end{array}$ & $\begin{array}{l}26.6 \\
30.6 \\
34.3 \\
37.9 \\
41.3 \\
44.7 \\
47.9 \\
54.3 \\
60.5 \\
66.6\end{array}$ & $\begin{array}{l}3.46 \\
3.10 \\
2.83 \\
2.60 \\
2.41 \\
2.25 \\
2.12 \\
1.89 \\
1.71 \\
1.56\end{array}$ & $\begin{array}{l}15.05 \\
15.95 \\
16.81 \\
17.66 \\
18.49 \\
19.30 \\
20.11 \\
21.70 \\
23.27 \\
24.83\end{array}$ & $\begin{array}{l}20.94 \\
22.46 \\
23.93 \\
25.36 \\
26.76 \\
28.14 \\
29.49 \\
32.18 \\
34.82 \\
37.45\end{array}$ & $\begin{array}{l}2.379 \\
2.484 \\
2.579 \\
2.666 \\
2.746 \\
2.820 \\
2.890 \\
3.018 \\
3.133 \\
3.238\end{array}$ & $\begin{array}{l}0.7443 \\
0.7432 \\
0.7428 \\
0.7428 \\
0.7430 \\
0.7432 \\
0.7435 \\
0.7441 \\
0.7448 \\
0.7453\end{array}$ & $\begin{array}{l}1.559 \\
1.493 \\
1.448 \\
1.416 \\
1.391 \\
1.373 \\
1.359 \\
1.337 \\
1.321 \\
1.310\end{array}$ & $\begin{array}{l}508.2 \\
533.3 \\
556.6 \\
578.3 \\
598.7 \\
618.2 \\
636.9 \\
672.2 \\
705.1 \\
736.2\end{array}$ \\
\hline $\begin{array}{l}28.0 \\
30.0 \\
32.0 \\
34.0 \\
36.0 \\
38.0 \\
40.0\end{array}$ & $\begin{array}{l}1.942 \\
2.031 \\
2.238 \\
2.385 \\
2.531 \\
2.677 \\
2.822\end{array}$ & $\begin{array}{r}72.6 \\
78.5 \\
84.3 \\
90.1 \\
95.9 \\
102.0 \\
107.0\end{array}$ & $\begin{array}{l}1.43 \\
1.33 \\
1.24 \\
1.16 \\
1.09 \\
1.03 \\
0.975\end{array}$ & $\begin{array}{l}26.39 \\
27.93 \\
29.47 \\
31.01 \\
32.53 \\
34.06 \\
35.58\end{array}$ & $\begin{array}{l}40.05 \\
42.65 \\
45.22 \\
47.79 \\
50.34 \\
52.39 \\
55.43\end{array}$ & $\begin{array}{l}3.335 \\
3.424 \\
3.507 \\
3.585 \\
3.658 \\
3.727 \\
3.792\end{array}$ & $\begin{array}{l}0.7456 \\
0.7459 \\
0.7461 \\
0.7462 \\
0.7463 \\
0.7463 \\
0.7463\end{array}$ & $\begin{array}{l}1.300 \\
1.292 \\
1.286 \\
1.280 \\
1.276 \\
1.272 \\
1.269\end{array}$ & $\begin{array}{l}765.6 \\
793.6 \\
820.5 \\
846.4 \\
871.3 \\
895.5 \\
919.0\end{array}$ \\
\hline $\begin{array}{r}45.0 \\
50.0 \\
55.0 \\
60.0 \\
70.0 \\
80.0 \\
90.0 \\
100.0 \\
120.0 \\
140.0\end{array}$ & $\begin{array}{l}3.182 \\
3.542 \\
3.899 \\
4.256 \\
4.968 \\
5.678 \\
6.387 \\
7.075 \\
8.510 \\
9.923\end{array}$ & $\begin{array}{l}121.0 \\
135.0 \\
149.0 \\
163.0 \\
190.0 \\
217.0 \\
244.0 \\
271.0 \\
325.0 \\
379.0\end{array}$ & $\begin{array}{l}0.862 \\
0.772 \\
0.700 \\
0.640 \\
0.547 \\
0.478 \\
0.424 \\
0.381 \\
0.317 \\
0.272\end{array}$ & $\begin{array}{r}39.37 \\
43.15 \\
46.91 \\
50.67 \\
58.18 \\
65.67 \\
73.15 \\
80.63 \\
95.56 \\
110.5\end{array}$ & $\begin{array}{c}61.76 \\
68.07 \\
74.35 \\
80.63 \\
93.14 \\
105.6 \\
118.1 \\
130.6 \\
155.4 \\
180.3\end{array}$ & $\begin{array}{l}3.941 \\
4.074 \\
4.194 \\
4.303 \\
4.496 \\
4.663 \\
4.810 \\
4.941 \\
5.168 \\
5.360\end{array}$ & $\begin{array}{l}0.7463 \\
0.7463 \\
0.7462 \\
0.7461 \\
0.7459 \\
0.7458 \\
0.7457 \\
0.7456 \\
0.7454 \\
0.7453\end{array}$ & $\begin{array}{l}1.263 \\
1.259 \\
1.256 \\
1.253 \\
1.250 \\
1.248 \\
1.246 \\
1.245 \\
1.244 \\
1.243\end{array}$ & $\begin{array}{r}974.9 \\
1028.0 \\
1077.0 \\
1125.0 \\
1214.0 \\
1297.0 \\
1375.0 \\
1448.0 \\
1585.0 \\
1711.0\end{array}$ \\
\hline $\begin{array}{l}160.0 \\
180.0 \\
200.0 \\
250.0 \\
300.0 \\
350.0 \\
400.0 \\
450.0 \\
500.0 \\
600.0\end{array}$ & $\begin{array}{l}11.34 \\
12.75 \\
14.16 \\
17.69 \\
21.22 \\
24.74 \\
28.27 \\
31.80 \\
35.33 \\
42.38\end{array}$ & $\begin{array}{r}433.0 \\
486.0 \\
540.0 \\
674.0 \\
808.0 \\
942.0 \\
1080.0 \\
1210.0 \\
1340.0 \\
1610.0\end{array}$ & $\begin{array}{l}0.238 \\
0.211 \\
0.190 \\
0.152 \\
0.127 \\
0.109 \\
0.0950 \\
0.0844 \\
0.0760 \\
0.0633\end{array}$ & $\begin{array}{l}125.4 \\
140.3 \\
155.2 \\
192.5 \\
229.7 \\
267.0 \\
304.2 \\
341.5 \\
378.7 \\
453.2\end{array}$ & $\begin{array}{l}205.2 \\
230.0 \\
254.9 \\
316.9 \\
379.0 \\
441.1 \\
503.1 \\
565.2 \\
627.3 \\
751.4\end{array}$ & $\begin{array}{l}5.526 \\
5.672 \\
5.803 \\
6.080 \\
6.306 \\
6.497 \\
6.663 \\
6.809 \\
6.940 \\
7.167\end{array}$ & $\begin{array}{l}0.7452 \\
0.7452 \\
0.7451 \\
0.7450 \\
0.7450 \\
0.7449 \\
0.7449 \\
0.7449 \\
0.7449 \\
0.7448\end{array}$ & $\begin{array}{l}1.243 \\
1.242 \\
1.242 \\
1.242 \\
1.241 \\
1.241 \\
1.241 \\
1.241 \\
1.241 \\
1.241\end{array}$ & $\begin{array}{l}1828.0 \\
1938.0 \\
2042.0 \\
2281.0 \\
2498.0 \\
2697.0 \\
2882.0 \\
3056.0 \\
3221.0 \\
3528.0\end{array}$ \\
\hline $\begin{array}{r}700.0 \\
800.0 \\
900.0 \\
1000.0 \\
1200.0 \\
1400.0 \\
1600.0 \\
1800.0 \\
2000.0 \\
2500.0\end{array}$ & $\begin{array}{r}49.44 \\
50.49 \\
63.54 \\
70.60 \\
84.71 \\
98.82 \\
112.9 \\
127.0 \\
141.1 \\
176.4\end{array}$ & $\begin{array}{l}1880.0 \\
2150.0 \\
2420.0 \\
2680.0 \\
3220.0 \\
3760.0 \\
4290.0 \\
4830.0 \\
5360.0 \\
6710.0\end{array}$ & $\begin{array}{l}0.0543 \\
0.0475 \\
0.0422 \\
0.0380 \\
0.0317 \\
0.0271 \\
0.0237 \\
0.0211 \\
0.0190 \\
0.0152\end{array}$ & $\begin{array}{r}527.6 \\
602.1 \\
676.6 \\
751.1 \\
900.0 \\
1049.0 \\
1198.0 \\
1347.0 \\
1496.0 \\
1868.0\end{array}$ & $\begin{array}{r}875.5 \\
1000.0 \\
1124.0 \\
1248.0 \\
1496.0 \\
1744.0 \\
1993.0 \\
2241.0 \\
2489.0 \\
3110.0\end{array}$ & $\begin{array}{l}7.358 \\
7.524 \\
7.670 \\
7.801 \\
8.027 \\
8.218 \\
8.384 \\
8.530 \\
8.661 \\
8.938\end{array}$ & $\begin{array}{l}0.7448 \\
0.7448 \\
0.7448 \\
0.7448 \\
0.7448 \\
0.7448 \\
0.7448 \\
0.7448 \\
0.7448 \\
0.7448\end{array}$ & $\begin{array}{l}1.241 \\
1.241 \\
1.241 \\
1.241 \\
1.241 \\
1.241 \\
1.241 \\
1.241 \\
1.241 \\
1.241\end{array}$ & $\begin{array}{l}3810.0 \\
4072.0 \\
4319.0 \\
4552.0 \\
4986.0 \\
5385.0 \\
5757.0 \\
6106.0 \\
6436.0 \\
7195.0\end{array}$ \\
\hline 3000.0 & 211.7 & 8050.0 & 0.0127 & 2241.0 & 3730.0 & 9.164 & 0.7448 & 1.241 & 7881.0 \\
\hline
\end{tabular}


38 PSIA ISOBAR

\begin{tabular}{|c|c|c|c|c|c|c|c|c|c|c|}
\hline $\begin{array}{c}\text { TEMPERATURE } \\
\text { OEG, R }\end{array}$ & OENSITY & $\begin{array}{l}V(O H / O V)_{P} \\
\text { BTU/LB }\end{array}$ & $\begin{array}{l}V(O P / O U)_{V}-V \\
\text { PSIA-CU FT/BTU }\end{array}$ & $\begin{array}{l}-V(O P / O V) T \\
\text { PSIA }\end{array}$ & $\begin{array}{l}\text { COV/OT I / } / V_{C} \\
\text { 1/OEG. } R\end{array}$ & $\begin{array}{c}\text { THERMAL } \\
\text { CONOUCTIVITY } \\
\text { BTU/FT-HR-R }\end{array}$ & $\begin{array}{l}\text { VISCOSITY } \\
\text { LB/FT-SEC } \\
\times 10 E+6\end{array}$ & $\begin{array}{c}\text { THERMAL } \\
\text { OIFFUSIVITY } \\
\text { SQ FT/HR }\end{array}$ & $\begin{array}{l}\text { OIELECTRIC } \\
\text { CONSTANT }\end{array}$ & $\begin{array}{l}\text { PRANOTL } \\
\text { NUMBER }\end{array}$ \\
\hline $\begin{array}{r}4.0 \\
5.0 \\
6.0 \\
7.0 \\
8.0 \\
9.0 \\
10.0 \\
11.0 \\
12.0 \\
13.0\end{array}$ & $\begin{array}{l}9.433 \\
9.275 \\
9.014 \\
8.633 \\
8.062 \\
7.061 \\
2.378 \\
1.913 \\
1.560 \\
1.3+6\end{array}$ & $\begin{array}{c}59.9 \\
21.3 \\
17.5 \\
14.9 \\
12.5 \\
9.46 \\
6.85 \\
9.16 \\
11.0 \\
12.7\end{array}$ & $\begin{array}{l}2.35 \\
6.54 \\
7.24 \\
7.43 \\
7.05 \\
5.99 \\
4.03 \\
3.96 \\
3.93 \\
3.91\end{array}$ & $\begin{array}{r}1300.0 \\
1130.0 \\
891.0 \\
631.0 \\
373.0 \\
130.0 \\
11.5 \\
22.8 \\
27.4 \\
30.1\end{array}$ & $\begin{array}{l}0.0102 \\
0.0230 \\
0.0353 \\
0.0532 \\
0.0871 \\
0.209 \\
0.790 \\
0.254 \\
0.168 \\
0.131\end{array}$ & $\begin{array}{l}0.00901 \\
0.0105 \\
0.0113 \\
0.0119 \\
0.0122 \\
0.0121 \\
0.0106 \\
0.00859 \\
0.00862 \\
0.00887\end{array}$ & $\begin{array}{l}2.82 \\
2.87 \\
2.73 \\
2.54 \\
2.30 \\
1.99 \\
1.29 \\
1.26 \\
1.29 \\
1.34\end{array}$ & $\begin{array}{l}0.00157 \\
0.00232 \\
0.00202 \\
0.00174 \\
0.00139 \\
0.000869 \\
0.000680 \\
0.00193 \\
0.00297 \\
0.00396\end{array}$ & $\begin{array}{l}1.02058 \\
1.02049 \\
1.02032 \\
1.02002 \\
1.01949 \\
1.01829 \\
1.00950 \\
1.00662 \\
1.00549 \\
1.00479\end{array}$ & $\begin{array}{l}0.688 \\
0.490 \\
0.539 \\
0.609 \\
0.742 \\
1.17 \\
2.38 \\
1.23 \\
1.00 \\
0.907\end{array}$ \\
\hline $\begin{array}{l}14.0 \\
15.0 \\
16.0 \\
17.0 \\
18.0 \\
19.0 \\
24.0 \\
22.0 \\
24.0 \\
26.0\end{array}$ & $\begin{array}{l}1.195 \\
1.381 \\
0.9891 \\
0.9139 \\
0.8506 \\
0.7967 \\
0.7497 \\
0.6716 \\
0.6091 \\
0.5578\end{array}$ & $\begin{array}{l}14.3 \\
15.9 \\
17.4 \\
18.8 \\
20.3 \\
21.7 \\
23.1 \\
25.8 \\
28.5 \\
31.2\end{array}$ & $\begin{array}{l}3.87 \\
3.87 \\
3.85 \\
3.83 \\
3.82 \\
3.81 \\
3.80 \\
3.78 \\
3.76 \\
3.75\end{array}$ & $\begin{array}{l}31.8 \\
33.0 \\
33.9 \\
34.6 \\
35.2 \\
35.6 \\
35.9 \\
36.5 \\
36.8 \\
37.1\end{array}$ & $\begin{array}{l}0.109 \\
0.0940 \\
0.3833 \\
0.0751 \\
0.0686 \\
0.0633 \\
0.0589 \\
0.0518 \\
0.0463 \\
0.0420\end{array}$ & $\begin{array}{l}0.00918 \\
0.00952 \\
0.00986 \\
0.0102 \\
0.0105 \\
0.0109 \\
0.0112 \\
0.0118 \\
0.0124 \\
0.0130\end{array}$ & $\begin{array}{l}1.39 \\
1.45 \\
1.50 \\
1.55 \\
1.60 \\
1.66 \\
1.71 \\
1.81 \\
1.91 \\
2.00\end{array}$ & $\begin{array}{l}0.00493 \\
0.00590 \\
0.00688 \\
0.00788 \\
0.00890 \\
0.00993 \\
0.0110 \\
0.0132 \\
0.0155 \\
0.0178\end{array}$ & $\begin{array}{l}1.00428 \\
1.00389 \\
1.00357 \\
1.00331 \\
1.00309 \\
1.00290 \\
1.00274 \\
1.00246 \\
1.00224 \\
1.00206\end{array}$ & $\begin{array}{l}0.851 \\
0.816 \\
0.793 \\
0.775 \\
0.763 \\
0.754 \\
0.747 \\
0.736 \\
0.730 \\
0.725\end{array}$ \\
\hline $\begin{array}{l}26.0 \\
30.0 \\
32.0 \\
34.0 \\
36.0 \\
38.0 \\
46.0\end{array}$ & $\begin{array}{l}0.5148 \\
0.4783 \\
0.4468 \\
0.4193 \\
0.3951 \\
0.3736 \\
0.3544\end{array}$ & $\begin{array}{l}6.9 \\
36.5 \\
39.1 \\
41.7 \\
44.3 \\
46.9 \\
49.5\end{array}$ & $\begin{array}{l}3.74 \\
3.72 \\
3.71 \\
3.71 \\
3.70 \\
3.69 \\
3.69\end{array}$ & $\begin{array}{l}37.4 \\
37.5 \\
37.7 \\
37.8 \\
37.9 \\
37.9 \\
38.0\end{array}$ & $\begin{array}{l}0.0384 \\
0.0354 \\
0.0329 \\
0.0307 \\
0.0288 \\
0.0271 \\
0.0257\end{array}$ & $\begin{array}{l}0.0136 \\
0.0142 \\
0.0147 \\
0.0152 \\
0.0157 \\
0.0162 \\
0.0167\end{array}$ & $\begin{array}{l}2.10 \\
2.19 \\
2.28 \\
2.37 \\
2.45 \\
2.53 \\
2.61\end{array}$ & $\begin{array}{l}0.0203 \\
0.0229 \\
0.0256 \\
0.0284 \\
0.0312 \\
0.0342 \\
0.0372\end{array}$ & $\begin{array}{l}1.00190 \\
1.00177 \\
1.00165 \\
1.00155 \\
1.00147 \\
1.00139 \\
1.00132\end{array}$ & $\begin{array}{l}0.722 \\
0.719 \\
0.717 \\
0.716 \\
0.7115 \\
0.7115 \\
0.714\end{array}$ \\
\hline $\begin{array}{r}45.0 \\
50.0 \\
55.0 \\
60.0 \\
70.0 \\
80.0 \\
90.0 \\
100.0 \\
120.0 \\
140.0\end{array}$ & $\begin{array}{l}0.3142 \\
0.2824 \\
0.2504 \\
0.2349 \\
0.2313 \\
0.1761 \\
0.1566 \\
0.1409 \\
0.1175 \\
0.1008\end{array}$ & $\begin{array}{r}55.8 \\
62.2 \\
68.5 \\
74.8 \\
87.4 \\
100.0 \\
112.0 \\
125.0 \\
150.0 \\
175.0\end{array}$ & $\begin{array}{l}3.67 \\
3.66 \\
3.66 \\
3.65 \\
3.64 \\
3.64 \\
3.63 \\
3.63 \\
3.62 \\
3.62\end{array}$ & $\begin{array}{l}38 \cdot 1 \\
38 \cdot 2 \\
38 \cdot 2 \\
38 \cdot 2 \\
38 \cdot 2 \\
38 \cdot 2 \\
38.2 \\
38.2 \\
38 \cdot 2 \\
38.2\end{array}$ & $\begin{array}{l}0.0226 \\
0.0202 \\
0.0183 \\
0.0168 \\
0.0143 \\
0.0125 \\
0.0111 \\
0.0100 \\
0.00831 \\
0.00712\end{array}$ & $\begin{array}{l}0.0179 \\
0.0191 \\
0.0202 \\
0.0212 \\
0.0233 \\
0.0253 \\
0.0272 \\
0.0291 \\
0.0326 \\
0.0361\end{array}$ & $\begin{array}{l}2.81 \\
3.00 \\
3.18 \\
3.35 \\
3.68 \\
4.00 \\
4.29 \\
4.59 \\
5.12 \\
5.63\end{array}$ & $\begin{array}{l}0.0451 \\
0.0536 \\
0.0626 \\
0.0721 \\
0.0926 \\
0.115 \\
0.139 \\
0.166 \\
0.223 \\
0.288\end{array}$ & $\begin{array}{l}1.00117 \\
1.00105 \\
1.00096 \\
1.00088 \\
1.00075 \\
1.00066 \\
1.00059 \\
1.00053 \\
1.00044 \\
1.00038\end{array}$ & $\begin{array}{l}0.714 \\
0.713 \\
0.713 \\
0.713 \\
0.712 \\
0.710 \\
0.708 \\
0.706 \\
0.702 \\
0.698\end{array}$ \\
\hline $\begin{array}{l}160.0 \\
180.0 \\
200.0 \\
250.0 \\
300.0 \\
350.0 \\
400.0 \\
450.0 \\
500.0 \\
600.0\end{array}$ & $\begin{array}{l}0.08822 \\
0.07844 \\
0.07362 \\
0.05653 \\
0.04713 \\
0.034041 \\
0.03537 \\
0.03145 \\
0.02831 \\
0.02360\end{array}$ & $\begin{array}{l}199.0 \\
224.0 \\
249.0 \\
311.0 \\
373.0 \\
435.0 \\
497.0 \\
559.0 \\
622.0 \\
746.0\end{array}$ & $\begin{array}{l}3.62 \\
3.61 \\
3.61 \\
3.61 \\
3.61 \\
3.61 \\
3.61 \\
3.67 \\
3.60 \\
3.60\end{array}$ & $\begin{array}{l}38.2 \\
38.1 \\
38.1 \\
38.1 \\
38.1 \\
38.1 \\
38.1 \\
38.1 \\
38.1 \\
38.0\end{array}$ & $\begin{array}{l}0.00623 \\
0.00554 \\
0.00498 \\
0.00399 \\
0.00333 \\
0.00285 \\
0.00250 \\
0.00222 \\
0.00200 \\
0.00166\end{array}$ & $\begin{array}{l}0.0394 \\
0.0426 \\
0.0458 \\
0.0532 \\
0.0603 \\
0.0670 \\
0.0733 \\
0.0794 \\
0.0851 \\
0.0963\end{array}$ & $\begin{array}{l}6.12 \\
6.58 \\
6.91 \\
8.00 \\
9.02 \\
10.0 \\
10.9 \\
11.8 \\
12.7 \\
14.4\end{array}$ & $\begin{array}{l}0.360 \\
0.438 \\
0.522 \\
0.758 \\
1.03 \\
1.33 \\
1.67 \\
2.03 \\
2.42 \\
3.29\end{array}$ & $\begin{array}{l}1.00033 \\
1.00030 \\
1.00027 \\
1.00021 \\
1.00018 \\
1.00015 \\
1.00013 \\
1.00012 \\
1.00011 \\
1.00009\end{array}$ & $\begin{array}{l}0.694 \\
0.691 \\
0.675 \\
0.671 \\
0.668 \\
0.667 \\
0.666 \\
0.666 \\
0.667 \\
0.668\end{array}$ \\
\hline $\begin{array}{r}700.0 \\
800.0 \\
900.0 \\
1000.0 \\
126 L .0 \\
14 \mathrm{JC} .0 \\
16 i C .0 \\
180 C .0 \\
26 C L .0 \\
2500.0\end{array}$ & $\begin{array}{l}0 . J 2023 \\
0.01770 \\
0.01574 \\
0.011416 \\
0.01181 \\
0.01012 \\
0.008855 \\
0.007872 \\
0.037085 \\
u .005668\end{array}$ & $\begin{array}{r}870.0 \\
994.0 \\
1120.0 \\
1240.0 \\
1490.0 \\
1740.0 \\
1990.0 \\
2230.0 \\
2480.0 \\
3100.0\end{array}$ & $\begin{array}{l}3.60 \\
3.60 \\
3.60 \\
3.60 \\
3.60 \\
3.60 \\
3.60 \\
3.60 \\
3.60 \\
3.60\end{array}$ & $\begin{array}{l}38.0 \\
38.0 \\
38.0 \\
38.0 \\
38.0 \\
38.0 \\
38.0 \\
38.0 \\
38.0 \\
38.0\end{array}$ & $\begin{array}{l}0.00143 \\
0.00125 \\
0.00111 \\
0.00100 \\
0.000833 \\
0.000714 \\
0.000625 \\
0.000555 \\
0.000503 \\
0.000400\end{array}$ & $\begin{array}{l}0.107 \\
0.118 \\
0.128 \\
0.137 \\
0.156 \\
0.174 \\
0.191 \\
0.208 \\
0.224 \\
0.263\end{array}$ & $\begin{array}{l}16.0 \\
17.6 \\
19.1 \\
20.5 \\
23.3 \\
26.0 \\
28.5 \\
31.0 \\
33.4 \\
39.2\end{array}$ & $\begin{array}{l}4.27 \\
5.35 \\
6.53 \\
7.81 \\
10.7 \\
13.9 \\
17.4 \\
21.3 \\
25.5 \\
37.3\end{array}$ & $\begin{array}{l}1.00008 \\
1.00007 \\
1.00006 \\
1.00005 \\
1.00004 \\
1.00004 \\
1.00003 \\
1.00003 \\
1.00003 \\
1.00002\end{array}$ & $\begin{array}{l}0.668 \\
0.668 \\
0.667 \\
0.667 \\
0.667 \\
0.667 \\
0.666 \\
0.666 \\
0.656 \\
0.666\end{array}$ \\
\hline 3050.0 & 0.034724 & 3720.0 & 3.60 & 38.0 & 0.000333 & 0.299 & 44.6 & 51.0 & 1.00002 & 0.666 \\
\hline
\end{tabular}

* TWO-PHASE gOUNOARY 


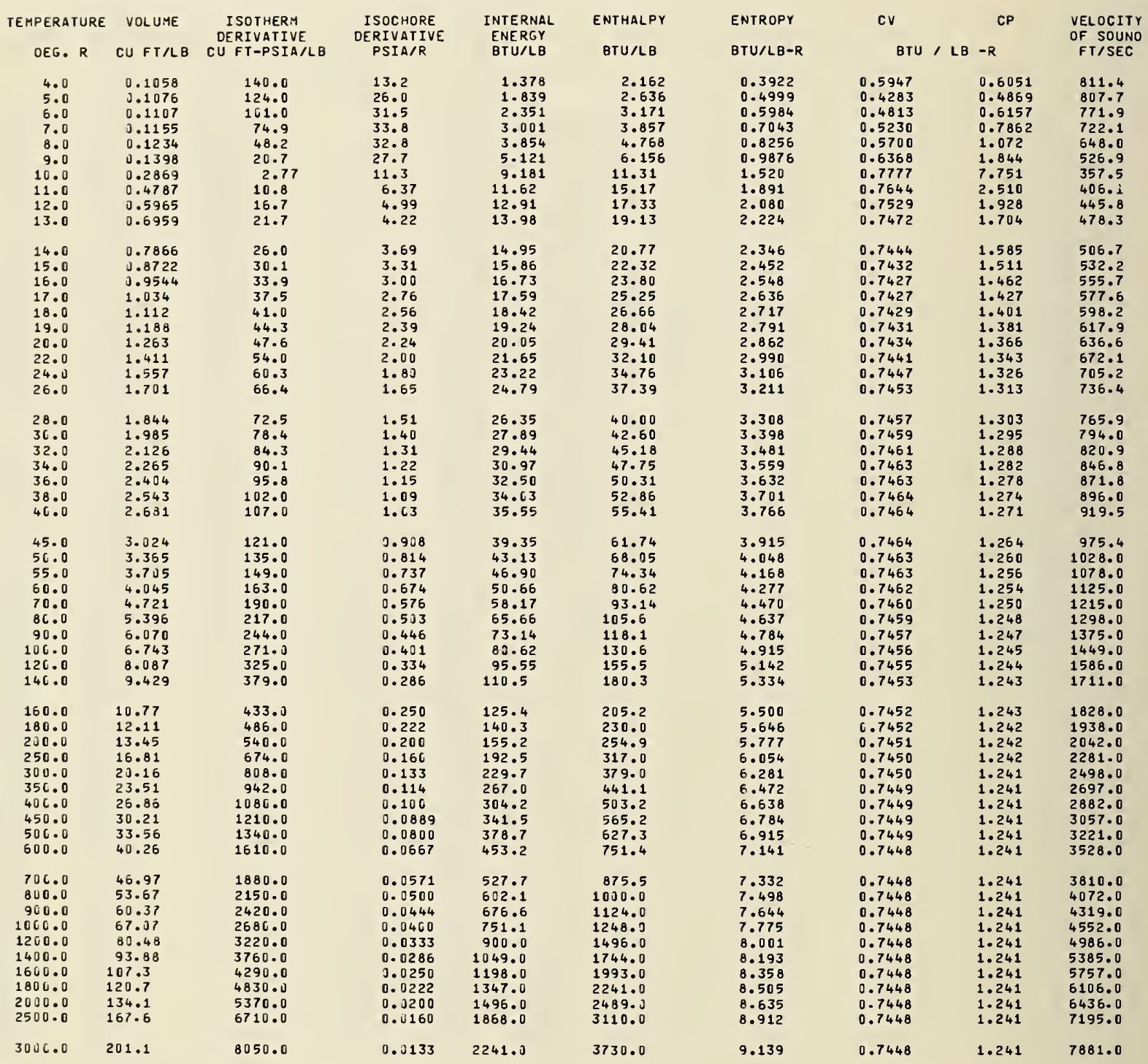


40 PSIA ISOBAR

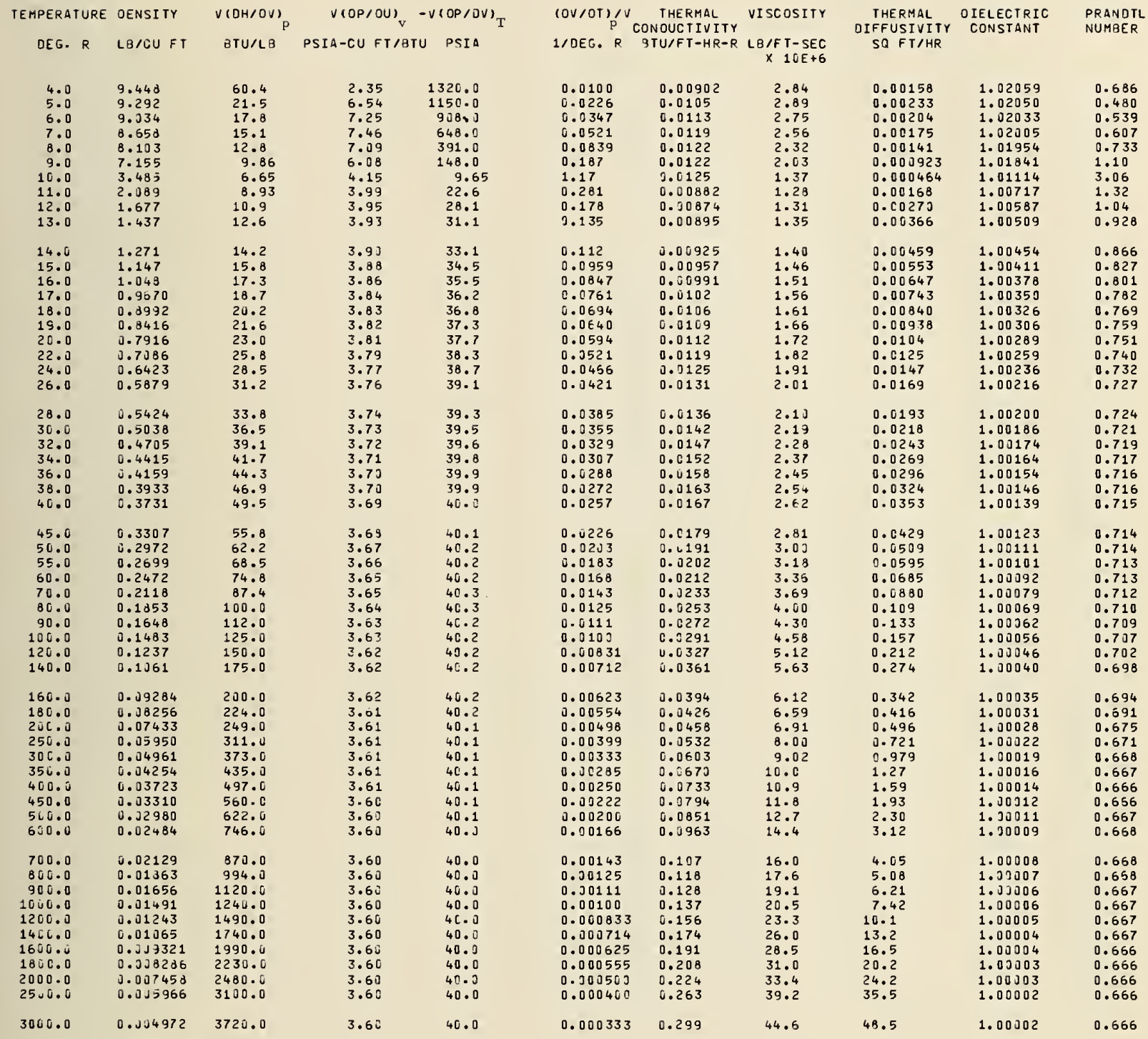


45 PSIA ISOBAR

THERMOOYNAMIC PROPERTIES OF HELIUM 4

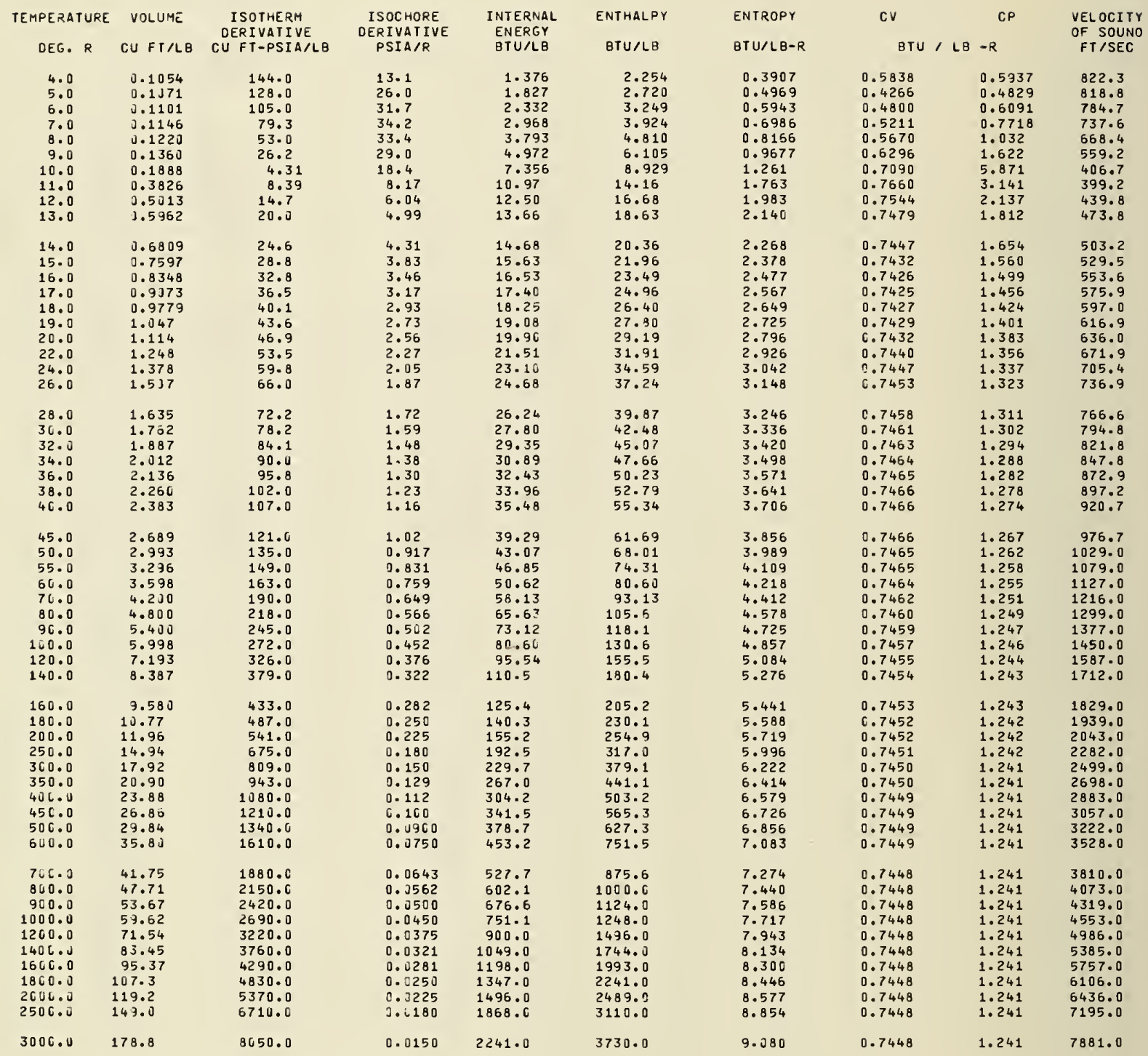


THERMOPHYSICAL PROPERTIES OF HELIUH 4

45 PSIA ISOBAR

\begin{tabular}{|c|c|c|c|c|c|c|c|c|c|c|}
\hline TEMPERATURE & DENSITY & $V(\mathrm{OH} / \mathrm{OV})_{P}$ & $V(D P / D U)_{v}-V$ & $V(O P / O V)_{T}$ & $(D V / D T)_{\mathrm{P}} / \mathrm{V}$ & $\begin{array}{c}\text { THERMAL } \\
\text { CONDUCTIVITY }\end{array}$ & VISCOSITY & $\begin{array}{c}\text { THERMAL } \\
\text { DIFFUSIVITY }\end{array}$ & $\begin{array}{l}\text { DIELECTRIC } \\
\text { CONSTANT }\end{array}$ & $\begin{array}{l}\text { PRANOTL } \\
\text { NUMBER }\end{array}$ \\
\hline OEG. $R$ & LE/CU FT & BTU/LB & PSIA-CU FT/BTU & PSIA & 1/OEG. R & BTU/FT-HR-R & $\begin{array}{r}\text { LB/FT }-S E C \\
\times 10 E+6\end{array}$ & $S Q F T / H R$ & & \\
\hline $\begin{array}{r}4.0 \\
5.0 \\
6.0 \\
7.0 \\
8.0 \\
9.0 \\
10.0 \\
11.0 \\
12.0 \\
13.0\end{array}$ & $\begin{array}{l}9.484 \\
9.334 \\
9.084 \\
8.723 \\
8.199 \\
7.353 \\
5.297 \\
2.613 \\
1.995 \\
1.677\end{array}$ & $\begin{array}{l}61.6 \\
22.2 \\
18.3 \\
15.6 \\
13.4 \\
10.8 \\
7.30 \\
8.43 \\
10.4 \\
12.2\end{array}$ & $\begin{array}{l}2.37 \\
6.53 \\
7.27 \\
7.52 \\
7.19 \\
6.27 \\
4.89 \\
4.08 \\
4.01 \\
3.98\end{array}$ & $\begin{array}{r}1360.0 \\
1190.0 \\
952.0 \\
692.0 \\
434.0 \\
193.0 \\
22.8 \\
21.9 \\
29.4 \\
33.5\end{array}$ & $\begin{array}{l}0.00964 \\
C .0218 \\
0.0333 \\
0.0494 \\
0.0770 \\
0.151 \\
0.804 \\
0.373 \\
0.205 \\
0.149\end{array}$ & $\begin{array}{l}0.00906 \\
0.0106 \\
0.0114 \\
0.0120 \\
0.0124 \\
0.0123 \\
0.0131 \\
0.00958 \\
0.00908 \\
0.00918\end{array}$ & $\begin{array}{l}2.89 \\
2.93 \\
2.80 \\
2.60 \\
2.38 \\
2.10 \\
1.66 \\
1.35 \\
1.35 \\
1.39\end{array}$ & $\begin{array}{l}0.00161 \\
0.00235 \\
0.00206 \\
0.00179 \\
0.00146 \\
0.00104 \\
0.000422 \\
0.00117 \\
0.00213 \\
0.00302\end{array}$ & $\begin{array}{l}1.02061 \\
1.02352 \\
1.02037 \\
1.02010 \\
1.01963 \\
1.01868 \\
1.01531 \\
1.00874 \\
1.00688 \\
1.00587\end{array}$ & $\begin{array}{l}0.682 \\
0.481 \\
0.539 \\
0.602 \\
0.713 \\
0.993 \\
2.67 \\
1.60 \\
1.15 \\
0.987\end{array}$ \\
\hline $\begin{array}{l}14.0 \\
15.0 \\
16.0 \\
17.0 \\
18.0 \\
19.0 \\
20.0 \\
22.0 \\
24.0 \\
26.0\end{array}$ & $\begin{array}{l}1.469 \\
1.310 \\
1.198 \\
1.102 \\
1.023 \\
0.9554 \\
0.8974 \\
0.8016 \\
0.7255 \\
0.6634\end{array}$ & $\begin{array}{l}13.9 \\
15.4 \\
17.0 \\
18.5 \\
19.9 \\
21.4 \\
22.8 \\
25.6 \\
28.3 \\
31.0\end{array}$ & $\begin{array}{l}3.94 \\
3.92 \\
3.90 \\
3.87 \\
3.86 \\
3.84 \\
3.83 \\
3.81 \\
3.79 \\
3.78\end{array}$ & $\begin{array}{l}36.2 \\
38.0 \\
39.3 \\
40.2 \\
41.0 \\
41.6 \\
42.1 \\
42.9 \\
43.4 \\
43.8\end{array}$ & $\begin{array}{l}0.119 \\
C .101 \\
0.0883 \\
0.0788 \\
0.0714 \\
0.0655 \\
0.0607 \\
0.0530 \\
0.0472 \\
0.0426\end{array}$ & $\begin{array}{l}0.00942 \\
0.00972 \\
0.0100 \\
0.0104 \\
0.0107 \\
0.0110 \\
0.0113 \\
0.0120 \\
0.0126 \\
0.0131\end{array}$ & $\begin{array}{l}1.43 \\
1.48 \\
1.53 \\
1.58 \\
1.63 \\
1.68 \\
1.73 \\
1.83 \\
1.93 \\
2.02\end{array}$ & $\begin{array}{l}0.00388 \\
0.00473 \\
0.00559 \\
0.00646 \\
0.00734 \\
0.00822 \\
0.00913 \\
0.0110 \\
0.0129 \\
0.0150\end{array}$ & $\begin{array}{l}1.00519 \\
1.00469 \\
1.00429 \\
1.00396 \\
1.00369 \\
1.00346 \\
1.00326 \\
1.00292 \\
1.00265 \\
1.00243\end{array}$ & $\begin{array}{l}0.905 \\
0.856 \\
0.823 \\
0.800 \\
0.783 \\
0.771 \\
0.762 \\
0.748 \\
0.739 \\
0.733\end{array}$ \\
\hline $\begin{array}{l}28.0 \\
30.0 \\
32.0 \\
34.0 \\
36.0 \\
38.0 \\
40.0\end{array}$ & $\begin{array}{l}0.6116 \\
0.5676 \\
0.5299 \\
0.4970 \\
0.4681 \\
0.4426 \\
0.4197\end{array}$ & $\begin{array}{l}33.7 \\
36.4 \\
39.0 \\
41.7 \\
44.3 \\
46.9 \\
49.4\end{array}$ & $\begin{array}{l}3.76 \\
3.75 \\
3.74 \\
3.73 \\
3.72 \\
3.71 \\
3.70\end{array}$ & $\begin{array}{l}44.1 \\
44.4 \\
44.6 \\
44.7 \\
44.8 \\
44.9 \\
45.9\end{array}$ & $\begin{array}{l}0.0389 \\
0.0353 \\
0.0331 \\
0.0309 \\
0.0290 \\
0.0273 \\
0.0258\end{array}$ & $\begin{array}{l}0.0137 \\
0.0143 \\
0.0148 \\
0.0153 \\
0.0158 \\
0.01163 \\
0.0168\end{array}$ & $\begin{array}{l}2.11 \\
2.23 \\
2.29 \\
2.38 \\
2.46 \\
2.55 \\
2.63\end{array}$ & $\begin{array}{l}0.0171 \\
0.0193 \\
0.0216 \\
0.0239 \\
0.0263 \\
0.0288 \\
0.0314\end{array}$ & $\begin{array}{l}1.00225 \\
1.00209 \\
1.00196 \\
1.00184 \\
1.00173 \\
1.00164 \\
1.00156\end{array}$ & $\begin{array}{l}0.728 \\
0.725 \\
0.722 \\
0.720 \\
0.719 \\
0.718 \\
0.717\end{array}$ \\
\hline $\begin{array}{r}45.0 \\
50.0 \\
55.0 \\
60.0 \\
70.0 \\
80.0 \\
90.0 \\
100.0 \\
120.0 \\
140.0\end{array}$ & $\begin{array}{l}0.3719 \\
0.3341 \\
0.3034 \\
0.2779 \\
0.2301 \\
0.2083 \\
0.1852 \\
0.1667 \\
0.1390 \\
0.1192\end{array}$ & $\begin{array}{r}55.8 \\
62.2 \\
68.6 \\
74.9 \\
87.4 \\
100.0 \\
112.0 \\
125.0 \\
150.0 \\
175.0\end{array}$ & $\begin{array}{l}3.69 \\
3.68 \\
3.67 \\
3.66 \\
3.65 \\
3.64 \\
3.64 \\
3.63 \\
3.63 \\
3.62\end{array}$ & $\begin{array}{l}45.1 \\
45.2 \\
45.3 \\
45.3 \\
45.3 \\
45.3 \\
45.3 \\
45.3 \\
45.3 \\
45.2\end{array}$ & $\begin{array}{l}C .0227 \\
0.0203 \\
0.0184 \\
0.0168 \\
c .0143 \\
0.0125 \\
0.0111 \\
0.0100 \\
C .00830 \\
0.00711\end{array}$ & $\begin{array}{l}0.0180 \\
0.0191 \\
0.0202 \\
0.0213 \\
0.0234 \\
0.0253 \\
0.0272 \\
0.0291 \\
0.0327 \\
0.0361\end{array}$ & $\begin{array}{l}2.82 \\
3.01 \\
3.19 \\
3.36 \\
3.69 \\
4.00 \\
4.30 \\
4.59 \\
5.13 \\
5.64\end{array}$ & $\begin{array}{l}0.0382 \\
0.0453 \\
0.0533 \\
0.0610 \\
0.0784 \\
0.0974 \\
0.118 \\
0.140 \\
0.189 \\
0.244\end{array}$ & $\begin{array}{l}1.00138 \\
1.00124 \\
1.00113 \\
1.00104 \\
1.00089 \\
1.00078 \\
1.00069 \\
1.00063 \\
1.00052 \\
1.00045\end{array}$ & $\begin{array}{l}0.716 \\
0.715 \\
0.714 \\
0.714 \\
0.712 \\
0.711 \\
0.709 \\
0.707 \\
0.702 \\
0.698\end{array}$ \\
\hline $\begin{array}{l}160.0 \\
180.0 \\
200.0 \\
250.0 \\
300.0 \\
350.0 \\
400.0 \\
450.0 \\
500.0 \\
600.0\end{array}$ & $\begin{array}{l}0.1344 \\
0.09283 \\
0.08358 \\
0.06691 \\
0.05579 \\
0.04784 \\
0.04187 \\
0.03723 \\
0.03351 \\
0.02794\end{array}$ & $\begin{array}{l}200.0 \\
224.0 \\
249.0 \\
311.0 \\
373.0 \\
436.0 \\
498.0 \\
560.0 \\
622.0 \\
746.0\end{array}$ & $\begin{array}{l}3.62 \\
3.62 \\
3.61 \\
3.61 \\
3.61 \\
3.61 \\
3.61 \\
3.61 \\
3.60 \\
3.60\end{array}$ & $\begin{array}{l}45.2 \\
45.2 \\
45.2 \\
45.1 \\
45.1 \\
45.1 \\
45.1 \\
45.1 \\
45.1 \\
45.1\end{array}$ & $\begin{array}{l}c .00623 \\
0.00553 \\
0.00498 \\
0.00399 \\
0.00332 \\
0.00285 \\
0.00249 \\
0.00222 \\
0.00200 \\
0.00166\end{array}$ & $\begin{array}{l}0.0394 \\
0.0427 \\
0.0458 \\
0.0533 \\
0.0603 \\
0.0670 \\
0.0733 \\
0.0794 \\
0.0851 \\
0.0963\end{array}$ & $\begin{array}{c}6.12 \\
6.59 \\
6.91 \\
8.00 \\
9.02 \\
10.0 \\
10.9 \\
11.8 \\
12.7 \\
14.4\end{array}$ & $\begin{array}{l}0.304 \\
0.370 \\
0.441 \\
0.641 \\
0.871 \\
1.13 \\
1.41 \\
1.72 \\
2.05 \\
2.78\end{array}$ & $\begin{array}{l}1.00039 \\
1.00035 \\
1.00031 \\
1.00025 \\
1.00021 \\
1.00018 \\
1.00016 \\
1.00014 \\
1.00013 \\
1.00011\end{array}$ & $\begin{array}{l}0.694 \\
0.691 \\
0.675 \\
0.671 \\
0.668 \\
0.667 \\
0.666 \\
0.666 \\
0.667 \\
0.668\end{array}$ \\
\hline $\begin{array}{r}700.0 \\
860.0 \\
900.0 \\
1000.0 \\
1200.0 \\
1400.0 \\
1600.0 \\
1800.0 \\
2000.0 \\
2500.0\end{array}$ & $\begin{array}{l}0.02395 \\
0.02096 \\
0.01863 \\
0.01677 \\
0.01398 \\
0.01198 \\
0.01049 \\
0.009321 \\
0.008389 \\
0.006712\end{array}$ & $\begin{array}{r}870.0 \\
994.0 \\
1120.0 \\
1240.0 \\
1490.0 \\
1740.0 \\
1990.0 \\
2240.0 \\
2480.0 \\
3160.0\end{array}$ & $\begin{array}{l}3.60 \\
3.60 \\
3.60 \\
3.60 \\
3.60 \\
3.60 \\
3.60 \\
3.60 \\
3.60 \\
3.60\end{array}$ & $\begin{array}{l}45.0 \\
45.0 \\
45.0 \\
45.0 \\
45.0 \\
45.0 \\
45.0 \\
45.0 \\
45.0 \\
45.0\end{array}$ & $\begin{array}{l}\text { c. } 00143 \\
0.00125 \\
0.00111 \\
0.00100 \\
0.000833 \\
0.000714 \\
0.000625 \\
0.000555 \\
c .000500 \\
0.000400\end{array}$ & $\begin{array}{l}0.107 \\
0.118 \\
0.128 \\
0.137 \\
0.156 \\
0.174 \\
0.191 \\
0.208 \\
0.224 \\
0.263\end{array}$ & $\begin{array}{l}16.0 \\
17.6 \\
19.1 \\
20.5 \\
23.3 \\
26.0 \\
28.5 \\
31.0 \\
33.4 \\
39.2\end{array}$ & $\begin{array}{c}3.60 \\
4.52 \\
5.52 \\
6.60 \\
9.00 \\
11.7 \\
14.7 \\
18.0 \\
21.5 \\
31.5\end{array}$ & $\begin{array}{l}1.00009 \\
1.00008 \\
1.00007 \\
1.00006 \\
1.00005 \\
1.00005 \\
1.00004 \\
1.00004 \\
1.00003 \\
1.00003\end{array}$ & $\begin{array}{l}0.668 \\
0.668 \\
0.667 \\
0.667 \\
0.667 \\
0.667 \\
0.666 \\
0.656 \\
0.666 \\
0.666\end{array}$ \\
\hline 3000.0 & ن. U05594 & 3720.0 & 3.60 & $45 . c$ & 0.000333 & 0.299 & 44.6 & 43.1 & 1. 00302 & 0.666 \\
\hline
\end{tabular}

- two-phase boUndarY 
5U PSIA ISOBAR

THERMOOYNAMIC PPOPERTIES OF HELIUM 4

\begin{tabular}{|c|c|c|c|c|c|c|c|c|c|}
\hline $\begin{array}{c}\text { TEMPERATURE } \\
\text { OEG. R }\end{array}$ & $\begin{array}{l}\text { VOLUME } \\
\text { CU FT/LB }\end{array}$ & $\begin{array}{l}\text { ISOTHERM } \\
\text { OERIVAIIVE } \\
\text { CU FT-PSIA/LB }\end{array}$ & $\begin{array}{l}\text { ISOCHORE } \\
\text { OERIVATIVE } \\
\text { PSIA/R }\end{array}$ & $\begin{array}{l}\text { INTERNAL } \\
\text { ENERGY } \\
\text { BTU/LB }\end{array}$ & BTU/LB & $\begin{array}{l}\text { ENTROPY } \\
\text { BTU/LB-R }\end{array}$ & $\begin{array}{l}\text { CV } \\
\text { BTU / LB }\end{array}$ & $-R$ & $\begin{array}{l}\text { VELOCITY } \\
\text { OF SOUNO } \\
\text { FT/SEC }\end{array}$ \\
\hline $\begin{array}{r}4.0 \\
5.0 \\
6.0 \\
7.0 \\
8.0 \\
9.0 \\
16.0 \\
11.0 \\
12.0 \\
13.0\end{array}$ & $\begin{array}{l}0.1051 \\
0.1067 \\
0.1095 \\
0.1138 \\
j .1207 \\
j .1330 \\
0.1651 \\
j .3042 \\
0.4242 \\
j .5161\end{array}$ & $\begin{array}{c}147.0 \\
132.0 \\
109.0 \\
83.6 \\
57.6 \\
31.4 \\
9.68 \\
5.70 \\
12.9 \\
18.4\end{array}$ & $\begin{array}{c}13.0 \\
26.0 \\
31.9 \\
34.5 \\
34.1 \\
39.2 \\
22.1 \\
10.6 \\
7.28 \\
5.85\end{array}$ & $\begin{array}{c}1.373 \\
1.817 \\
2.314 \\
2.938 \\
3.739 \\
4.851 \\
6.674 \\
10.17 \\
12.04 \\
13.33\end{array}$ & $\begin{array}{l}2.346 \\
2.805 \\
3.327 \\
3.992 \\
4.856 \\
6.083 \\
8.203 \\
12.99 \\
15.97 \\
18.13\end{array}$ & $\begin{array}{l}0.3893 \\
0.4940 \\
0.5904 \\
0.6933 \\
0.8084 \\
0.9515 \\
1.172 \\
1.628 \\
1.889 \\
2.650\end{array}$ & $\begin{array}{l}0.5733 \\
0.4250 \\
0.4786 \\
0.5193 \\
0.5643 \\
0.6240 \\
0.6738 \\
0.7618 \\
0.7551 \\
0.7484\end{array}$ & $\begin{array}{l}0.5827 \\
0.4790 \\
0.6029 \\
0.7590 \\
0.999 \\
1.480 \\
3.219 \\
3.927 \\
2.396 \\
1.939\end{array}$ & $\begin{array}{l}832.9 \\
829.5 \\
796.9 \\
752.4 \\
687.3 \\
587.3 \\
462.8 \\
400.0 \\
435.7 \\
470.1\end{array}$ \\
\hline $\begin{array}{l}14.0 \\
15.0 \\
16.0 \\
17.0 \\
18.0 \\
19.0 \\
26.0 \\
22.0 \\
24.0 \\
26.0\end{array}$ & $\begin{array}{l}0.5960 \\
0.6596 \\
0.7391 \\
0.8059 \\
3.8737 \\
0.3335 \\
0.975 \\
1.117 \\
1.235 \\
1.353\end{array}$ & $\begin{array}{l}23.2 \\
27.6 \\
31.7 \\
35.6 \\
39.3 \\
42.8 \\
46.2 \\
52.9 \\
59.4 \\
65.7\end{array}$ & $\begin{array}{l}4.99 \\
4.39 \\
3.95 \\
3.60 \\
3.31 \\
3.08 \\
2.88 \\
2.55 \\
2.30 \\
2.09\end{array}$ & $\begin{array}{l}14.41 \\
15.39 \\
16.32 \\
17.22 \\
18.08 \\
18.92 \\
19.75 \\
21.38 \\
22.98 \\
24.56\end{array}$ & $\begin{array}{l}19.93 \\
21.59 \\
23.17 \\
24.68 \\
26.14 \\
27.57 \\
28.97 \\
31.72 \\
34.42 \\
37.09\end{array}$ & $\begin{array}{l}2.195 \\
2.310 \\
2.41 . \\
2.503 \\
2.587 \\
2.664 \\
2.736 \\
2.867 \\
2.985 \\
3.092\end{array}$ & $\begin{array}{l}0.7449 \\
0.7431 \\
0.7424 \\
0.7423 \\
0.7425 \\
0.7426 \\
0.7430 \\
0.7438 \\
0.7446 \\
0.7453\end{array}$ & $\begin{array}{l}1.730 \\
1.613 \\
1.538 \\
1.486 \\
1.449 \\
1.422 \\
1.401 \\
1.370 \\
1.348 \\
1.332\end{array}$ & $\begin{array}{l}500.2 \\
527.1 \\
551.7 \\
574.5 \\
595.9 \\
616.2 \\
635.5 \\
671.8 \\
705.6 \\
737.4\end{array}$ \\
\hline $\begin{array}{l}28.0 \\
36.0 \\
32.0 \\
34.0 \\
36.0 \\
38.0 \\
40.0\end{array}$ & $\begin{array}{l}1.468 \\
1.533 \\
1.597 \\
1.310 \\
1.322 \\
2.033 \\
2.144\end{array}$ & $\begin{array}{r}71.9 \\
77.9 \\
83.9 \\
89.8 \\
95.7 \\
101.0 \\
107.0\end{array}$ & $\begin{array}{l}1.92 \\
1.77 \\
1.65 \\
1.54 \\
1.45 \\
1.37 \\
1.29\end{array}$ & $\begin{array}{l}26.14 \\
27.70 \\
29.26 \\
30.81 \\
32.35 \\
33.88 \\
35.42\end{array}$ & $\begin{array}{l}39.73 \\
42.36 \\
44.97 \\
47.56 \\
50.14 \\
52.71 \\
55.27\end{array}$ & $\begin{array}{l}3.190 \\
3.280 \\
3.365 \\
3.443 \\
3.517 \\
3.586 \\
3.652\end{array}$ & $\begin{array}{l}0.7458 \\
0.7462 \\
0.7464 \\
0.7466 \\
0.7467 \\
0.7468 \\
0.7468\end{array}$ & $\begin{array}{l}1.319 \\
1.308 \\
1.300 \\
1.293 \\
1.287 \\
1.282 \\
1.278\end{array}$ & $\begin{array}{l}767.3 \\
795.7 \\
822.8 \\
848.9 \\
874.0 \\
898.4 \\
921.9\end{array}$ \\
\hline $\begin{array}{r}45.0 \\
50.0 \\
55.0 \\
50.0 \\
70.0 \\
80.0 \\
96.0 \\
160.0 \\
120.0 \\
140.0\end{array}$ & $\begin{array}{l}2.421 \\
2.695 \\
2.908 \\
3.240 \\
3.783 \\
4.324 \\
4.903 \\
5.402 \\
6.478 \\
7.553\end{array}$ & $\begin{array}{l}121.0 \\
135.0 \\
149.0 \\
163.0 \\
191.0 \\
218.0 \\
245.0 \\
272.0 \\
326.0 \\
380.0\end{array}$ & $\begin{array}{l}1.14 \\
1.02 \\
0.925 \\
0.845 \\
0.721 \\
0.629 \\
0.559 \\
0.502 \\
0.418 \\
0.358\end{array}$ & $\begin{array}{c}39.23 \\
43.02 \\
46.80 \\
50.57 \\
58.10 \\
65.60 \\
73.09 \\
80.58 \\
95.52 \\
110.5\end{array}$ & $\begin{array}{r}61.54 \\
67.97 \\
74.23 \\
80.58 \\
93.12 \\
105.6 \\
118.1 \\
130.5 \\
155.5 \\
180.4\end{array}$ & $\begin{array}{l}3.802 \\
3.936 \\
4.056 \\
4.165 \\
4.059 \\
4.526 \\
4.573 \\
4.804 \\
5.031 \\
5.223\end{array}$ & $\begin{array}{l}0.7468 \\
0.7467 \\
0.7466 \\
0.7465 \\
0.7463 \\
0.7461 \\
0.7460 \\
0.7458 \\
0.7456 \\
0.7455\end{array}$ & $\begin{array}{l}1.270 \\
1.264 \\
1.260 \\
1.257 \\
1.253 \\
1.250 \\
1.248 \\
1.246 \\
1.245 \\
1.244\end{array}$ & $\begin{array}{r}978.0 \\
1031.0 \\
1081.0 \\
1128.0 \\
1217.0 \\
1300.0 \\
1378.0 \\
1451.0 \\
1588.0 \\
1713.0\end{array}$ \\
\hline $\begin{array}{l}160.0 \\
180.0 \\
200.0 \\
250.0 \\
300.0 \\
350.0 \\
400.0 \\
450.0 \\
500.0 \\
600.0\end{array}$ & $\begin{array}{r}8.627 \\
9.700 \\
10.77 \\
13.45 \\
16.14 \\
18.82 \\
21.53 \\
24.18 \\
26.80 \\
32.22\end{array}$ & $\begin{array}{r}434.0 \\
487.0 \\
541.0 \\
675.0 \\
809.0 \\
943.0 \\
1080.0 \\
1210.0 \\
1356.0 \\
1610.0\end{array}$ & $\begin{array}{l}0.313 \\
0.278 \\
j .250 \\
0.20 C \\
0.167 \\
0.143 \\
0.125 \\
0.111 \\
0.100 \\
0.0833\end{array}$ & $\begin{array}{l}125.4 \\
140.3 \\
155.2 \\
192.5 \\
229.7 \\
267.0 \\
304.2 \\
341.5 \\
378.7 \\
453.2\end{array}$ & $\begin{array}{l}205.2 \\
230.1 \\
254.9 \\
317.0 \\
379.1 \\
441.2 \\
593.3 \\
565.3 \\
627.4 \\
751.5\end{array}$ & $\begin{array}{l}5.389 \\
5.536 \\
5.666 \\
5.944 \\
5.170 \\
6.361 \\
6.527 \\
6.673 \\
6.804 \\
7.036\end{array}$ & $\begin{array}{l}0.7454 \\
0.7453 \\
0.7452 \\
0.7451 \\
0.7450 \\
0.7450 \\
0.7450 \\
0.7449 \\
0.7449 \\
0.7449\end{array}$ & $\begin{array}{l}1.243 \\
1.243 \\
1.242 \\
1.242 \\
1.242 \\
1.241 \\
1.241 \\
1.241 \\
1.241 \\
1.241\end{array}$ & $\begin{array}{l}1830.0 \\
1940.0 \\
2044.0 \\
2283.0 \\
2499.0 \\
2698.0 \\
2884.0 \\
3058.0 \\
3222.0 \\
3529.0\end{array}$ \\
\hline $\begin{array}{r}700.0 \\
800.0 \\
920.0 \\
1000.0 \\
1200.0 \\
1400.0 \\
1600.0 \\
1800.0 \\
2000.0 \\
2500.0\end{array}$ & $\begin{array}{l}37.53 \\
42.94 \\
49.33 \\
53.57 \\
64.39 \\
75.11 \\
85.83 \\
95.56 \\
137.3 \\
134.1\end{array}$ & $\begin{array}{l}1880.0 \\
2150.0 \\
2420.0 \\
2690.0 \\
3220.0 \\
3760.0 \\
4290.0 \\
4830.0 \\
5370.0 \\
6710.0\end{array}$ & $\begin{array}{l}0.0714 \\
0.0625 \\
0.0555 \\
0.0500 \\
0.0417 \\
\cup .0357 \\
0.0312 \\
0.0278 \\
0.3250 \\
0.02 C 0\end{array}$ & $\begin{array}{r}527.7 \\
602.1 \\
676.6 \\
751.1 \\
90 J .0 \\
1049.0 \\
1198.0 \\
1347.0 \\
1496.0 \\
1868.0\end{array}$ & $\begin{array}{r}875.0 \\
1003.0 \\
1124.0 \\
1248.0 \\
1496.0 \\
1744.0 \\
1993.0 \\
2241.0 \\
2489.3 \\
3110.0\end{array}$ & $\begin{array}{l}7.222 \\
7.387 \\
7.534 \\
7.664 \\
7.891 \\
8.082 \\
8.248 \\
8.394 \\
8.525 \\
8.802\end{array}$ & $\begin{array}{l}0.7449 \\
0.7448 \\
0.7448 \\
0.7448 \\
0.7448 \\
0.7448 \\
0.7448 \\
0.7448 \\
0.7448 \\
0.7448\end{array}$ & $\begin{array}{l}1.241 \\
1.241 \\
1.241 \\
1.241 \\
1.241 \\
1.241 \\
1.241 \\
1.241 \\
1.241 \\
1.241\end{array}$ & $\begin{array}{l}3811.0 \\
4073.0 \\
4320.0 \\
4553.0 \\
4987.0 \\
5386.0 \\
5757.0 \\
6106.0 \\
6436.0 \\
7195.0\end{array}$ \\
\hline $300 \mathrm{C} .0$ & 160.9 & 8050.0 & 0.3167 & $2241 \cdot 0$ & 3730.3 & 9.328 & 0.7448 & 1.241 & 7881.0 \\
\hline
\end{tabular}

* two-phase joundart 
5 J PSIA ISDBAR

\begin{tabular}{|c|c|c|c|c|c|c|c|c|c|c|}
\hline $\begin{array}{c}\text { TEMPERATURE } \\
\text { OEG. R }\end{array}$ & DENSI TY & $\begin{array}{c}V(D H / D V)_{p} \\
\text { BTU/LB }\end{array}$ & $\begin{array}{l}V(O P / D U)_{V}-V \\
P S I A-C U F T / B T U\end{array}$ & $\begin{array}{l}V(D P / D V)_{T} \\
\text { PSIA }\end{array}$ & $\begin{array}{l}\text { (OV/OT) }{ }_{\mathrm{P}} \mathrm{V} \\
1 / O E G . R\end{array}$ & $\begin{array}{c}\text { THERMAL } \\
\text { CONDUCTIVITY } \\
\text { BTU/FT-MR-R }\end{array}$ & $\begin{array}{l}\text { VI SCOSITY } \\
\text { LB/FT- JEC } \\
\times 10 E+6\end{array}$ & $\begin{array}{l}\text { TMERMAL } \\
\text { DIFFUSIVITY } \\
\text { SQ FT/MR }\end{array}$ & $\begin{array}{l}\text { DIELECIRIC } \\
\text { CONSTANT }\end{array}$ & $\begin{array}{l}\text { PRANOTL } \\
\text { NUMBER }\end{array}$ \\
\hline $\begin{array}{r}4.0 \\
5.0 \\
6.0 \\
7.0 \\
8.0 \\
9.0 \\
10.0 \\
11.0 \\
12.0 \\
13.0\end{array}$ & $\begin{array}{l}9.519 \\
9.374 \\
9.133 \\
8.784 \\
8.287 \\
7.517 \\
6.059 \\
3.287 \\
2.357 \\
1.938\end{array}$ & $\begin{array}{c}62.7 \\
22.8 \\
18.8 \\
16.1 \\
14.0 \\
11.6 \\
8.54 \\
8.15 \\
10.0 \\
11.8\end{array}$ & $\begin{array}{l}2.39 \\
6.52 \\
7.29 \\
7.57 \\
7.29 \\
6.44 \\
5.41 \\
4.24 \\
4.09 \\
4.03\end{array}$ & $\begin{array}{r}1400.0 \\
1240.0 \\
994.0 \\
735.0 \\
477.0 \\
236.0 \\
58.6 \\
22.0 \\
30.5 \\
35.7\end{array}$ & $\begin{array}{l}0.00929 \\
0.0210 \\
0.0321 \\
0.0470 \\
0.0714 \\
0.128 \\
0.377 \\
0.482 \\
0.239 \\
0.164\end{array}$ & $\begin{array}{l}0.00913 \\
0.0107 \\
0.0115 \\
0.0121 \\
0.0125 \\
0.0125 \\
0.0124 \\
0.0106 \\
0.00950 \\
0.20944\end{array}$ & $\begin{array}{l}2.94 \\
2.98 \\
2.85 \\
2.65 \\
2.43 \\
2.16 \\
1.82 \\
1.44 \\
1.40 \\
1.43\end{array}$ & $\begin{array}{l}3.00154 \\
0.00237 \\
0.00238 \\
0.00182 \\
0.00151 \\
0.00112 \\
0.000636 \\
0.000821 \\
0.00168 \\
0.00251\end{array}$ & $\begin{array}{l}1.02063 \\
1.02055 \\
1.02040 \\
1.02015 \\
1.01972 \\
1.01888 \\
1.01673 \\
1.01062 \\
1.00798 \\
1.00670\end{array}$ & $\begin{array}{l}0.677 \\
0.483 \\
0.539 \\
0.597 \\
0.696 \\
0.925 \\
1.70 \\
1.92 \\
1.27 \\
1.05\end{array}$ \\
\hline $\begin{array}{l}14.0 \\
15.0 \\
16.0 \\
17.0 \\
18.0 \\
19.0 \\
20.0 \\
22.0 \\
24.0 \\
26.0\end{array}$ & $\begin{array}{l}1.678 \\
1.493 \\
1.353 \\
1.241 \\
1.149 \\
1.371 \\
1.305 \\
0.8956 \\
0.3394 \\
0.7393\end{array}$ & $\begin{array}{l}13.5 \\
15.2 \\
16.7 \\
18.2 \\
19.7 \\
21.2 \\
22.6 \\
25.4 \\
28.2 \\
30.9\end{array}$ & $\begin{array}{l}3.99 \\
3.96 \\
3.93 \\
3.91 \\
3.89 \\
3.87 \\
3.86 \\
3.83 \\
3.81 \\
3.80\end{array}$ & $\begin{array}{l}39.0 \\
41.3 \\
42.9 \\
44.2 \\
45.1 \\
45.9 \\
46.5 \\
47.4 \\
48.0 \\
48.6\end{array}$ & $\begin{array}{l}0.128 \\
C .106 \\
0.0920 \\
0.0815 \\
c .0735 \\
0.0672 \\
0.0620 \\
0.0539 \\
c .0479 \\
0.0431\end{array}$ & $\begin{array}{l}0.00962 \\
0.00988 \\
0.0102 \\
0.0105 \\
0.0108 \\
0.0111 \\
0.0114 \\
0.0120 \\
0.0126 \\
0.0132\end{array}$ & $\begin{array}{l}1.45 \\
1.51 \\
1.55 \\
1.63 \\
1.65 \\
1.73 \\
1.75 \\
1.85 \\
1.94 \\
2.04\end{array}$ & $\begin{array}{l}0.00331 \\
0.00410 \\
0.00439 \\
0.00569 \\
0.00649 \\
0.00730 \\
0.00812 \\
0.00981 \\
0.0116 \\
0.0134\end{array}$ & $\begin{array}{l}1.00587 \\
1.00527 \\
1.00481 \\
1.00443 \\
1.00412 \\
1.00386 \\
1.00363 \\
1.00325 \\
1.00295 \\
1.00270\end{array}$ & $\begin{array}{l}0.947 \\
0.886 \\
0.846 \\
0.818 \\
0.798 \\
0.783 \\
0.772 \\
0.757 \\
0.746 \\
0.739\end{array}$ \\
\hline $\begin{array}{l}28.0 \\
30.0 \\
32.0 \\
34.0 \\
36.0 \\
38.0 \\
40.0\end{array}$ & $\begin{array}{l}0.6810 \\
0.6317 \\
0.5894 \\
0.5526 \\
0.5234 \\
0.4918 \\
0.4663\end{array}$ & $\begin{array}{l}33.6 \\
36.3 \\
39.0 \\
41.6 \\
44.2 \\
46.8 \\
49.4\end{array}$ & $\begin{array}{l}3.78 \\
3.76 \\
3.75 \\
3.74 \\
3.73 \\
3.72 \\
3.71\end{array}$ & $\begin{array}{l}48.9 \\
49.2 \\
49.5 \\
49.6 \\
49.8 \\
49.7 \\
50.0\end{array}$ & $\begin{array}{l}0.0392 \\
0.0360 \\
0.0334 \\
0.0311 \\
0.0291 \\
1.0274 \\
0.0259\end{array}$ & $\begin{array}{l}0.0138 \\
0.0143 \\
0.0149 \\
0.0154 \\
0.0159 \\
0.0164 \\
0.0169\end{array}$ & $\begin{array}{l}2.13 \\
2.22 \\
2.31 \\
2.39 \\
2.47 \\
2.55 \\
2.63\end{array}$ & $\begin{array}{l}0.0153 \\
0.0173 \\
0.0194 \\
0.0215 \\
0.0237 \\
0.0260 \\
0.0283\end{array}$ & $\begin{array}{l}1.00250 \\
1.00232 \\
1.00217 \\
1.00204 \\
1.00192 \\
1.00182 \\
1.00173\end{array}$ & $\begin{array}{l}0.733 \\
0.729 \\
0.726 \\
0.723 \\
0.721 \\
0.720 \\
0.719\end{array}$ \\
\hline $\begin{array}{r}45.0 \\
50.0 \\
55.0 \\
60.0 \\
70.0 \\
80.0 \\
90.0 \\
160.0 \\
120.0 \\
140.0\end{array}$ & $\begin{array}{l}0.4131 \\
0.3711 \\
0.3309 \\
0.3086 \\
0.2643 \\
0.2313 \\
0.2556 \\
0.1851 \\
0.1544 \\
0.1324\end{array}$ & $\begin{array}{r}55.9 \\
62.2 \\
68.6 \\
74.9 \\
87.5 \\
140.0 \\
113.0 \\
125.0 \\
150.0 \\
175.0\end{array}$ & $\begin{array}{l}3.70 \\
3.69 \\
3.68 \\
3.67 \\
3.66 \\
3.65 \\
3.64 \\
3.64 \\
3.63 \\
3.62\end{array}$ & $\begin{array}{l}50.2 \\
50.3 \\
50.3 \\
50.4 \\
50.4 \\
50.4 \\
50.4 \\
50.4 \\
50.3 \\
50.3\end{array}$ & $\begin{array}{l}0.0227 \\
0.0203 \\
0.0184 \\
0.0168 \\
0.0143 \\
{[.0125} \\
0.0111 \\
0.0100 \\
0.00830 \\
0.00711\end{array}$ & $\begin{array}{l}0.0180 \\
0.0192 \\
0.0203 \\
0.0213 \\
0.0234 \\
0.0254 \\
0.0273 \\
0.0291 \\
0.0327 \\
0.0362\end{array}$ & $\begin{array}{l}2.83 \\
3.02 \\
3.19 \\
3.37 \\
3.70 \\
4.01 \\
4.31 \\
4.59 \\
5.13 \\
5.64\end{array}$ & $\begin{array}{l}0.0344 \\
0.0409 \\
0.0477 \\
0.0550 \\
0.0706 \\
0.0878 \\
0.106 \\
0.126 \\
0.170 \\
0.220\end{array}$ & $\begin{array}{l}1.00153 \\
1.00138 \\
1.00125 \\
1.00115 \\
1.00099 \\
1.00086 \\
1.00077 \\
1.00069 \\
1.00058 \\
1.00050\end{array}$ & $\begin{array}{l}0.717 \\
0.716 \\
0.715 \\
0.714 \\
0.713 \\
0.711 \\
0.709 \\
0.707 \\
0.703 \\
0.698\end{array}$ \\
\hline $\begin{array}{l}160.0 \\
180.0 \\
200.0 \\
250.0 \\
300.0 \\
350.0 \\
406.0 \\
450.0 \\
500.0 \\
600.0\end{array}$ & $\begin{array}{l}0.1159 \\
0.1031 \\
0.09282 \\
0.07432 \\
0.06197 \\
0.05314 \\
0.04652 \\
0.04136 \\
0.03723 \\
0.03104\end{array}$ & $\begin{array}{l}200.0 \\
225.0 \\
249.0 \\
312.0 \\
374.0 \\
436.0 \\
498.0 \\
560.0 \\
622.0 \\
746.0\end{array}$ & $\begin{array}{l}3.62 \\
3.62 \\
3.62 \\
3.61 \\
3.61 \\
3.61 \\
3.61 \\
3.61 \\
3.61 \\
3.60\end{array}$ & $\begin{array}{l}50 \cdot 3 \\
50 \cdot 2 \\
50 \cdot 2 \\
50 \cdot 2 \\
50 \cdot 2 \\
50 \cdot 1 \\
50 \cdot 1 \\
50.1 \\
50.1 \\
50.1\end{array}$ & $\begin{array}{l}0.00622 \\
0.00553 \\
0.00498 \\
0.00399 \\
0.00332 \\
0.00285 \\
0.00249 \\
0.00222 \\
0.00200 \\
0.00166\end{array}$ & $\begin{array}{l}0.0395 \\
0.0427 \\
0.0458 \\
0.0533 \\
0.0603 \\
0.0670 \\
0.0733 \\
0.0794 \\
0.0851 \\
0.0963\end{array}$ & $\begin{array}{c}6.12 \\
6.59 \\
6.92 \\
8.00 \\
9.02 \\
10.0 \\
10.9 \\
11.8 \\
12.7 \\
14.4\end{array}$ & $\begin{array}{l}0.274 \\
0.333 \\
0.397 \\
0.577 \\
0.784 \\
1.02 \\
1.27 \\
1.55 \\
1.84 \\
2.50\end{array}$ & $\begin{array}{l}1.00044 \\
1.00039 \\
1.00335 \\
1.00028 \\
1.00023 \\
1.00020 \\
1.00018 \\
1.00016 \\
1.00014 \\
1.00012\end{array}$ & $\begin{array}{l}0.694 \\
0.691 \\
0.675 \\
0.671 \\
0.668 \\
0.667 \\
0.666 \\
0.666 \\
0.667 \\
0.668\end{array}$ \\
\hline $\begin{array}{r}700.0 \\
800.0 \\
900.0 \\
1000.0 \\
1200.0 \\
1400.0 \\
1600.0 \\
1800.0 \\
2060.0 \\
2500.0\end{array}$ & $\begin{array}{l}0.02661 \\
0.02329 \\
i .02070 \\
0.01863 \\
0.01553 \\
0.01331 \\
0.01165 \\
0.01336 \\
0.003321 \\
0.007458\end{array}$ & $\begin{array}{r}870.0 \\
994.0 \\
1120.0 \\
1240.0 \\
1490.0 \\
1740.0 \\
1990.0 \\
2240.0 \\
2480.0 \\
3100.0\end{array}$ & $\begin{array}{l}3.60 \\
3.60 \\
3.60 \\
3.60 \\
3.60 \\
3.60 \\
3.60 \\
3.60 \\
3.60 \\
3.60\end{array}$ & $\begin{array}{l}50.1 \\
50.1 \\
50.0 \\
50.0 \\
50.0 \\
50.0 \\
50.0 \\
50.0 \\
50.0 \\
50.0\end{array}$ & $\begin{array}{l}0.00143 \\
0.00125 \\
0.00111 \\
0.00100 \\
0.005833 \\
0.000714 \\
0.000625 \\
0.000555 \\
0.000500 \\
0.000400\end{array}$ & $\begin{array}{l}0.1 C 7 \\
0.118 \\
0.128 \\
0.137 \\
0.156 \\
0.174 \\
0.191 \\
0.208 \\
0.224 \\
0.263\end{array}$ & $\begin{array}{l}16.0 \\
17.6 \\
19.1 \\
20.5 \\
23.3 \\
26.0 \\
28.5 \\
31.0 \\
33.4 \\
39.2\end{array}$ & $\begin{array}{l}3.24 \\
4.07 \\
4.97 \\
5.94 \\
8.10 \\
10.5 \\
13.2 \\
16.2 \\
19.4 \\
28.4\end{array}$ & $\begin{array}{l}1.00010 \\
1.00009 \\
1.00008 \\
1.00007 \\
1.00006 \\
1.00005 \\
1.00004 \\
1.00004 \\
1.00004 \\
1.00003\end{array}$ & $\begin{array}{l}0.668 \\
0.668 \\
0.667 \\
0.667 \\
0.667 \\
0.667 \\
0.666 \\
0.666 \\
0.666 \\
0.666\end{array}$ \\
\hline 3000.0 & 0.005215 & 3720.0 & 3.60 & 50.0 & 0.000333 & 0.299 & 44.6 & 38.8 & 1.00002 & 0.656 \\
\hline
\end{tabular}

- tho-pHase gounoary 
IUJ PSIA ISOBAR

THERMOOYNAMIC PROPERTIES OF HELIUM 4

\begin{tabular}{|c|c|c|c|c|c|c|c|c|c|}
\hline $\begin{array}{c}\text { TEMPERATURE } \\
\text { OEG. R }\end{array}$ & $\begin{array}{l}\text { VOLUME } \\
\text { CU FTILB }\end{array}$ & $\begin{array}{c}\text { ISOTHERM } \\
\text { OERIVATIVE } \\
\text { CU FT - PSIA/LB }\end{array}$ & $\begin{array}{l}\text { ISOCHORE } \\
\text { OERIVATIVE } \\
\text { PSIA/R }\end{array}$ & $\begin{array}{l}\text { INTERNAL } \\
\text { ENERGY } \\
\text { BTU/LB }\end{array}$ & $\begin{array}{l}\text { ENTHALPY } \\
\text { BTU/LB }\end{array}$ & $\begin{array}{l}\text { ENTROPY } \\
\text { BTU/LB-R }\end{array}$ & $\begin{array}{l}\text { CV } \\
\text { BTU, LB }\end{array}$ & $\begin{array}{r}C P \\
-R\end{array}$ & $\begin{array}{l}\text { VELOCITY } \\
\text { OF SOUNO } \\
\text { FTISEC }\end{array}$ \\
\hline $\begin{array}{l}4.0 \\
5.0 \\
6.0 \\
7.0 \\
8.0 \\
9.0 \\
10.0 \\
11.0 \\
12.0 \\
13.0\end{array}$ & $\begin{array}{l}j .1017 \\
j .1029 \\
j .1049 \\
j .1078 \\
j .1121 \\
j .1182 \\
j .1267 \\
j .1391 \\
0.1596 \\
0.1931\end{array}$ & $\begin{array}{r}182.0 \\
168.0 \\
146.0 \\
123.0 \\
98.5 \\
74.8 \\
55.0 \\
37.1 \\
23.8 \\
18.3\end{array}$ & $\begin{array}{l}12.0 \\
25.5 \\
33.0 \\
37.3 \\
38.6 \\
37.2 \\
34.0 \\
29.7 \\
24.4 \\
19.0\end{array}$ & $\begin{array}{l}1.370 \\
1.750 \\
2.188 \\
2.725 \\
3.386 \\
4.214 \\
5.204 \\
6.361 \\
7.809 \\
9.568\end{array}$ & $\begin{array}{c}3.254 \\
3.655 \\
4.130 \\
4.722 \\
5.461 \\
6.402 \\
7.550 \\
8.936 \\
10.76 \\
13.14\end{array}$ & $\begin{array}{l}0.3772 \\
0.4703 \\
0.5590 \\
0.6513 \\
0.7499 \\
0.8590 \\
0.9785 \\
1.111 \\
1.270 \\
1.460\end{array}$ & $\begin{array}{l}0.4855 \\
0.4089 \\
0.4649 \\
0.5027 \\
0.5439 \\
0.5939 \\
0.6075 \\
0.6283 \\
0.6608 \\
0.6987\end{array}$ & $\begin{array}{l}0.4916 \\
0.4468 \\
0.5559 \\
0.6740 \\
0.8253 \\
1.023 \\
1.231 \\
1.564 \\
2.076 \\
2.470\end{array}$ & $\begin{array}{l}923.5 \\
921.8 \\
900.5 \\
873.0 \\
832.2 \\
772.7 \\
718.6 \\
653.9 \\
589.1 \\
547.8\end{array}$ \\
\hline $\begin{array}{l}14.0 \\
15.0 \\
16.0 \\
17.0 \\
18.0 \\
19.0 \\
26.0 \\
22.0 \\
24.0 \\
26.0\end{array}$ & $\begin{array}{l}0.2356 \\
1.2773 \\
0.3177 \\
0.3565 \\
0.3936 \\
0.4292 \\
0.4638 \\
0.5306 \\
0.5952 \\
3.6582\end{array}$ & $\begin{array}{l}19.6 \\
22.6 \\
26.2 \\
30.0 \\
33.9 \\
37.7 \\
41.5 \\
48.8 \\
56.0 \\
62.9\end{array}$ & $\begin{array}{c}14.9 \\
12.1 \\
10.3 \\
8.96 \\
7.98 \\
7.23 \\
6.63 \\
5.71 \\
5.05 \\
4.53\end{array}$ & $\begin{array}{l}11.32 \\
12.78 \\
14.05 \\
15.21 \\
16.27 \\
17.26 \\
18.20 \\
20.01 \\
21.74 \\
23.43\end{array}$ & $\begin{array}{l}15.68 \\
17.91 \\
19.94 \\
21.81 \\
23.56 \\
25.21 \\
26.79 \\
29.83 \\
32.76 \\
35.61\end{array}$ & $\begin{array}{l}1.649 \\
1.803 \\
1.933 \\
2.047 \\
2.147 \\
2.237 \\
2.318 \\
2.463 \\
2.591 \\
2.705\end{array}$ & $\begin{array}{l}0.7253 \\
0.7327 \\
0.7359 \\
0.7376 \\
0.7388 \\
0.7392 \\
0.7398 \\
0.7414 \\
0.7432 \\
0.7448\end{array}$ & $\begin{array}{l}2.347 \\
2.119 \\
1.940 \\
1.807 \\
1.709 \\
1.637 \\
1.583 \\
1.508 \\
1.460 \\
1.425\end{array}$ & $\begin{array}{l}541.8 \\
550.4 \\
565.7 \\
583.7 \\
602.6 \\
622.1 \\
641.3 \\
678.4 \\
713.5 \\
746.7\end{array}$ \\
\hline $\begin{array}{l}28.0 \\
36.0 \\
32.0 \\
34.0 \\
36.0 \\
38.0 \\
40.0\end{array}$ & $\begin{array}{l}0.7200 \\
0.7807 \\
0.8404 \\
0.8993 \\
0.3576 \\
1.015 \\
1.073\end{array}$ & $\begin{array}{r}69.7 \\
76.3 \\
82.7 \\
89.1 \\
95.3 \\
101.0 \\
108.0\end{array}$ & $\begin{array}{l}4.11 \\
3.76 \\
3.47 \\
3.23 \\
3.02 \\
2.83 \\
2.67\end{array}$ & $\begin{array}{l}25.09 \\
26.74 \\
28.37 \\
29.98 \\
31.58 \\
33.16 \\
34.73\end{array}$ & $\begin{array}{l}38.42 \\
41.20 \\
43.93 \\
46.63 \\
49.31 \\
51.96 \\
54.60\end{array}$ & $\begin{array}{l}2.809 \\
2.905 \\
2.993 \\
3.075 \\
3.152 \\
3.223 \\
3.291\end{array}$ & $\begin{array}{l}0.7461 \\
0.7470 \\
0.7476 \\
0.7481 \\
0.7483 \\
0.7485 \\
0.7486\end{array}$ & $\begin{array}{l}1.398 \\
1.376 \\
1.358 \\
1.344 \\
1.332 \\
1.322 \\
1.314\end{array}$ & $\begin{array}{l}777.7 \\
806.7 \\
834.5 \\
861.1 \\
886.6 \\
911.3 \\
935.1\end{array}$ \\
\hline $\begin{array}{r}45.0 \\
50.0 \\
55.0 \\
60.0 \\
70.0 \\
80.0 \\
90.0 \\
100.0 \\
120.0 \\
140.0\end{array}$ & $\begin{array}{l}1.215 \\
1.355 \\
1.494 \\
1.632 \\
1.906 \\
2.179 \\
2.450 \\
2.721 \\
3.261 \\
3.799\end{array}$ & $\begin{array}{l}122.0 \\
137.0 \\
151.0 \\
166.0 \\
194.0 \\
221.0 \\
249.0 \\
276.0 \\
330.0 \\
384.0\end{array}$ & $\begin{array}{l}2.34 \\
2.08 \\
1.88 \\
1.71 \\
1.46 \\
1.27 \\
1.12 \\
1.01 \\
0.838 \\
0.717\end{array}$ & $\begin{array}{l}38.63 \\
42.50 \\
46.34 \\
50.16 \\
57.76 \\
65.32 \\
72.85 \\
80.37 \\
95.37 \\
110.3\end{array}$ & $\begin{array}{c}61.13 \\
67.59 \\
74.00 \\
80.38 \\
93.06 \\
105.7 \\
118.2 \\
130.8 \\
155.7 \\
180.7\end{array}$ & $\begin{array}{l}3.445 \\
3.581 \\
3.703 \\
3.814 \\
4.010 \\
4.178 \\
4.326 \\
4.458 \\
4.686 \\
4.878\end{array}$ & $\begin{array}{l}0.7487 \\
0.7486 \\
0.7484 \\
0.7482 \\
0.7479 \\
0.7475 \\
0.7472 \\
0.7470 \\
0.7466 \\
0.7463\end{array}$ & $\begin{array}{l}1.298 \\
1.287 \\
1.279 \\
1.272 \\
1.264 \\
1.258 \\
1.254 \\
1.252 \\
1.248 \\
1.246\end{array}$ & $\begin{array}{r}991.7 \\
1045.0 \\
1095.0 \\
1142.0 \\
1231.0 \\
1314.0 \\
1391.0 \\
1464.0 \\
1600.0 \\
1724.0\end{array}$ \\
\hline $\begin{array}{l}160.0 \\
180.0 \\
200.0 \\
250.0 \\
300.0 \\
350.0 \\
400.0 \\
450.0 \\
500.0 \\
600.0\end{array}$ & $\begin{array}{c}4.337 \\
4.874 \\
5.411 \\
0.752 \\
8.093 \\
9.433 \\
10.77 \\
12.11 \\
13.45 \\
16.13\end{array}$ & $\begin{array}{r}438.0 \\
492.0 \\
546.0 \\
680.0 \\
814.0 \\
948.0 \\
1080.0 \\
1220.0 \\
1350.0 \\
1620.0\end{array}$ & $\begin{array}{l}0.626 \\
0.556 \\
0.500 \\
0.400 \\
0.333 \\
0.286 \\
0.250 \\
0.222 \\
0.206 \\
0.167\end{array}$ & $\begin{array}{l}125.3 \\
140.2 \\
155.2 \\
192.5 \\
229.7 \\
267.0 \\
304.2 \\
341.5 \\
378.7 \\
453.2\end{array}$ & $\begin{array}{l}205.6 \\
230.5 \\
255.4 \\
317.5 \\
379.6 \\
441.7 \\
503.7 \\
565.8 \\
627.9 \\
752.0\end{array}$ & $\begin{array}{l}5.044 \\
5.191 \\
5.322 \\
5.599 \\
5.826 \\
6.017 \\
6.183 \\
6.329 \\
6.460 \\
6.686\end{array}$ & $\begin{array}{l}0.7461 \\
0.7459 \\
0.7458 \\
0.7455 \\
0.7454 \\
0.7453 \\
0.7452 \\
0.7452 \\
0.7451 \\
0.7451\end{array}$ & $\begin{array}{l}1.245 \\
1.244 \\
1.243 \\
1.242 \\
1.242 \\
1.242 \\
1.241 \\
1.241 \\
1.241 \\
1.241\end{array}$ & $\begin{array}{l}1841.0 \\
1950.0 \\
2053.0 \\
2291.0 \\
2507.0 \\
2705.0 \\
2890.0 \\
3064.0 \\
3228.0 \\
3534.0\end{array}$ \\
\hline $\begin{array}{r}700.0 \\
800.0 \\
900.0 \\
1000.0 \\
1200.0 \\
1400.0 \\
1600.0 \\
1800.0 \\
2000.0 \\
2500.0\end{array}$ & $\begin{array}{l}18.81 \\
21.49 \\
24.17 \\
26.85 \\
32.22 \\
37.58 \\
42.94 \\
48.30 \\
53.66 \\
67.06\end{array}$ & $\begin{array}{l}1890.0 \\
2150.0 \\
2420.0 \\
2690.0 \\
3230.0 \\
3760.0 \\
4300.0 \\
4830.0 \\
5370.0 \\
6710.0\end{array}$ & $\begin{array}{l}0.143 \\
0.125 \\
0.111 \\
0.100 \\
0.0833 \\
0.0714 \\
0.0625 \\
0.0555 \\
0.0500 \\
0.0400\end{array}$ & $\begin{array}{r}527.7 \\
602.2 \\
676.7 \\
751.2 \\
900.1 \\
1049.0 \\
1198.0 \\
1347.0 \\
1496.0 \\
1868.0\end{array}$ & $\begin{array}{r}876.1 \\
1600.0 \\
1124.0 \\
1248.0 \\
1497.0 \\
1745.0 \\
1993.0 \\
2241.0 \\
2490.0 \\
3110.0\end{array}$ & $\begin{array}{l}6.878 \\
7.043 \\
7.189 \\
7.320 \\
7.547 \\
7.738 \\
7.904 \\
8.050 \\
8.181 \\
8.457\end{array}$ & $\begin{array}{l}0.7450 \\
0.7450 \\
0.7450 \\
0.7449 \\
0.7449 \\
0.7449 \\
0.7449 \\
0.7449 \\
0.7449 \\
0.7448\end{array}$ & $\begin{array}{l}1.241 \\
1.241 \\
1.241 \\
1.241 \\
1.241 \\
1.241 \\
1.241 \\
1.241 \\
1.241 \\
1.241\end{array}$ & $\begin{array}{l}3815.0 \\
4077.0 \\
4323.0 \\
4556.0 \\
4990.0 \\
5388.0 \\
5759.0 \\
6108.0 \\
6438.0 \\
7196.0\end{array}$ \\
\hline 3000.0 & 80.46 & 8050.0 & 0.0333 & 2241.0 & 3731.0 & 8.684 & 0.7448 & 1.241 & 7882.0 \\
\hline
\end{tabular}

* two-phase bounoary 


\begin{tabular}{|c|c|c|c|c|c|c|c|c|c|c|}
\hline $\begin{array}{c}\text { TEMPERATURE } \\
\text { DEG. } \cdot{ }^{\circ}\end{array}$ & $0 \equiv N S I T Y$ & $\begin{array}{c}V(O H / O V)_{P} \\
\text { BTU/LB }\end{array}$ & $\begin{array}{l}V(O P / O U)_{V}-V \\
\text { PSIA-CU FT/BTU }\end{array}$ & $\begin{array}{l}-V(O P / O V)_{T} \\
\text { PSIA }\end{array}$ & $\begin{array}{l}\text { IOV/OT } \mathrm{P}_{\mathrm{P}} / \mathrm{V} \\
\text { I/OEG. } \mathrm{R}\end{array}$ & $\begin{array}{l}\text { THERMAL } \\
\text { ONOUCTIVITY } \\
\text { 3TU/FT-HR-R }\end{array}$ & $\begin{array}{l}\text { VISCOSITY } \\
\text { LB/FT-SEC } \\
\times 10 E+6\end{array}$ & $\begin{array}{l}\text { THERMAL } \\
\text { OIFFUSIVITY } \\
\text { SQ FT/HR }\end{array}$ & $\begin{array}{l}\text { OIELECTRIC } \\
\text { CONSTANT }\end{array}$ & $\begin{array}{l}\text { PRANDTL } \\
\text { NUMBER }\end{array}$ \\
\hline $\begin{array}{r}4.0 \\
5.0 \\
6.0 \\
7.0 \\
8.0 \\
9.0 \\
10.0 \\
11.0 \\
12.0 \\
13.0\end{array}$ & $\begin{array}{l}9.830 \\
9.720 \\
9.535 \\
9.273 \\
8.323 \\
8.463 \\
7.894 \\
7.193 \\
6.267 \\
5.178\end{array}$ & $\begin{array}{l}73.0 \\
28.6 \\
23.5 \\
20.5 \\
18.8 \\
17.4 \\
15.7 \\
14.1 \\
12.7 \\
12.3\end{array}$ & $\begin{array}{l}2.52 \\
6.41 \\
7.45 \\
8.01 \\
7.95 \\
7.39 \\
7.08 \\
6.57 \\
5.90 \\
5.26\end{array}$ & $\begin{array}{r}1790.0 \\
1630.0 \\
1400.0 \\
1140.0 \\
879.0 \\
633.0 \\
434.0 \\
267.0 \\
149.0 \\
94.9\end{array}$ & $\begin{array}{l}0.00673 \\
0.0156 \\
0.0237 \\
0.0328 \\
0.0439 \\
0.0587 \\
0.0782 \\
0.111 \\
0.163 \\
0.200\end{array}$ & $\begin{array}{l}0.00946 \\
0.0112 \\
0.0121 \\
0.0129 \\
0.0136 \\
0.0140 \\
0.0141 \\
0.0140 \\
0.0137 \\
0.0131\end{array}$ & $\begin{array}{l}3.42 \\
3.44 \\
3.28 \\
3.07 \\
2.85 \\
2.62 \\
2.42 \\
2.24 \\
2.06 \\
1.92\end{array}$ & $\begin{array}{l}0.00196 \\
0.00258 \\
0.00229 \\
0.00207 \\
0.00185 \\
0.00162 \\
0.00145 \\
0.00125 \\
0.00105 \\
0.00102\end{array}$ & $\begin{array}{l}1.02077 \\
1.02073 \\
1.02064 \\
1.02049 \\
1.02025 \\
1.01988 \\
1.01932 \\
1.01846 \\
1.01708 \\
1.01507\end{array}$ & $\begin{array}{l}0.641 \\
0.495 \\
0.542 \\
0.576 \\
0.622 \\
0.690 \\
0.759 \\
0.898 \\
1.13 \\
1.30\end{array}$ \\
\hline $\begin{array}{l}14.0 \\
15.0 \\
16.0 \\
17.0 \\
18.0 \\
19.0 \\
20.0 \\
22.0 \\
24.0 \\
26.0\end{array}$ & $\begin{array}{l}4.245 \\
3.606 \\
3.147 \\
2.805 \\
2.540 \\
2.333 \\
2.150 \\
1.885 \\
1.680 \\
1.519\end{array}$ & $\begin{array}{l}13.1 \\
14.3 \\
15.6 \\
17.0 \\
18.4 \\
19.9 \\
21.4 \\
24.3 \\
27.2 \\
30.1\end{array}$ & $\begin{array}{l}4.83 \\
4.59 \\
4.43 \\
4.33 \\
4.25 \\
4.20 \\
4.15 \\
4.09 \\
4.04 \\
4.00\end{array}$ & $\begin{array}{l}83.1 \\
81.5 \\
82.5 \\
34.2 \\
86.1 \\
87.9 \\
89.5 \\
92.0 \\
94.0 \\
95.6\end{array}$ & $\begin{array}{l}0.179 \\
0.149 \\
0.125 \\
0.106 \\
0.0927 \\
0.0822 \\
0.0741 \\
0.0621 \\
0.0537 \\
0.0474\end{array}$ & $\begin{array}{l}0.0124 \\
0.0121 \\
0.0120 \\
0.0121 \\
0.0122 \\
0.0124 \\
0.01126 \\
0.0131 \\
0.0136 \\
0.0141\end{array}$ & $\begin{array}{l}1.84 \\
1.81 \\
1.82 \\
1.84 \\
1.86 \\
1.89 \\
1.93 \\
2.85 \\
2.88 \\
2.16\end{array}$ & $\begin{array}{l}0.00125 \\
0.00159 \\
0.00197 \\
0.00238 \\
0.00281 \\
0.00325 \\
0.00369 \\
0.00459 \\
0.00553 \\
0.00649\end{array}$ & $\begin{array}{l}1.01303 \\
1.01146 \\
1.01024 \\
1.00929 \\
1.00853 \\
1.00790 \\
1.00737 \\
1.00653 \\
1.00588 \\
1.00536\end{array}$ & $\begin{array}{l}1.25 \\
1.14 \\
1.05 \\
0.989 \\
0.939 \\
0.902 \\
0.873 \\
0.834 \\
0.808 \\
0.790\end{array}$ \\
\hline $\begin{array}{l}28.0 \\
30.0 \\
32.0 \\
34.0 \\
36.0 \\
38.4 \\
40.0\end{array}$ & $\begin{array}{l}1.389 \\
1.281 \\
1.190 \\
1.112 \\
1.044 \\
0.9848 \\
0.9321\end{array}$ & $\begin{array}{l}32.9 \\
35.7 \\
38.5 \\
41.2 \\
44.0 \\
46.6 \\
49.3\end{array}$ & $\begin{array}{l}3.97 \\
3.93 \\
3.91 \\
3.88 \\
3.86 \\
3.84 \\
3.83\end{array}$ & $\begin{array}{r}96.8 \\
97.7 \\
98.5 \\
99.1 \\
100.0 \\
100.0 \\
100.0\end{array}$ & $\begin{array}{l}0.0425 \\
0.0385 \\
0.0353 \\
0.0325 \\
0.0303 \\
C .0283 \\
0.0266\end{array}$ & $\begin{array}{l}0.0146 \\
0.0151 \\
0.0156 \\
0.0160 \\
0.0165 \\
0.01170 \\
0.0174\end{array}$ & $\begin{array}{l}2.25 \\
2.33 \\
2.41 \\
2.49 \\
2.56 \\
2.64 \\
2.72\end{array}$ & $\begin{array}{l}0.00750 \\
0.00855 \\
0.00962 \\
0.0107 \\
0.0119 \\
0.0130 \\
0.0142\end{array}$ & $\begin{array}{l}1.00493 \\
1.00457 \\
1.00426 \\
1.00400 \\
1.00376 \\
1.00356 \\
1.00338\end{array}$ & $\begin{array}{l}0.776 \\
0.765 \\
0.756 \\
0.750 \\
0.745 \\
0.740 \\
0.737\end{array}$ \\
\hline $\begin{array}{r}45.0 \\
50.0 \\
55.0 \\
60.0 \\
70.0 \\
80.0 \\
90.0 \\
100.0 \\
120.0 \\
140.0\end{array}$ & $\begin{array}{l}0.8233 \\
0.7381 \\
0.6694 \\
0.6128 \\
0.5246 \\
0.4590 \\
0.4081 \\
0.3675 \\
0.3507 \\
0.2632\end{array}$ & $\begin{array}{r}55.9 \\
62.5 \\
69.0 \\
75.4 \\
88.2 \\
101.0 \\
113.0 \\
126.0 \\
151.0 \\
176.0\end{array}$ & $\begin{array}{l}3.80 \\
3.77 \\
3.75 \\
3.74 \\
3.71 \\
3.70 \\
3.68 \\
3.67 \\
3.66 \\
3.65\end{array}$ & $\begin{array}{l}131.0 \\
101.0 \\
101.0 \\
101.0 \\
102.0 \\
142.0 \\
102.0 \\
101.0 \\
101.0 \\
101.0\end{array}$ & $\begin{array}{l}0.0232 \\
0.0206 \\
0.0185 \\
0.0169 \\
0.0143 \\
0.0125 \\
0.0111 \\
0.00993 \\
0.30826 \\
0.00708\end{array}$ & $\begin{array}{l}0.0186 \\
0.0197 \\
0.0207 \\
0.0218 \\
0.0238 \\
0.0257 \\
0.0276 \\
0.0295 \\
0.0330 \\
0.0364\end{array}$ & $\begin{array}{l}2.90 \\
3.08 \\
3.26 \\
3.43 \\
3.75 \\
4.06 \\
4.35 \\
4.63 \\
5.17 \\
5.67\end{array}$ & $\begin{array}{l}0.0174 \\
0.0207 \\
0.0242 \\
0.0279 \\
0.0359 \\
0.0446 \\
0.0540 \\
0.0640 \\
0.0862 \\
0.111\end{array}$ & $\begin{array}{l}1.00300 \\
1.00270 \\
1.00245 \\
1.00225 \\
1.00194 \\
1.00170 \\
1.00151 \\
1.00137 \\
1.00114 \\
1.00098\end{array}$ & $\begin{array}{l}0.731 \\
0.726 \\
0.723 \\
0.721 \\
0.717 \\
0.714 \\
0.711 \\
0.708 \\
0.703 \\
0.699\end{array}$ \\
\hline $\begin{array}{l}160.0 \\
180.0 \\
200.0 \\
250.0 \\
300.0 \\
350.0 \\
400.0 \\
450.0 \\
500.0 \\
600.0\end{array}$ & $\begin{array}{l}0.2306 \\
0.2052 \\
0.1348 \\
0.1481 \\
0.1236 \\
0.1060 \\
0.09282 \\
0.08255 \\
0.07433 \\
0.06198\end{array}$ & $\begin{array}{l}201.0 \\
226.0 \\
251.0 \\
313.0 \\
375.0 \\
437.0 \\
499.0 \\
561.0 \\
623.0 \\
747.0\end{array}$ & $\begin{array}{l}3.64 \\
3.64 \\
3.63 \\
3.62 \\
3.62 \\
3.62 \\
3.61 \\
3.61 \\
3.61 \\
3.61\end{array}$ & $\begin{array}{l}101.0 \\
101.0 \\
101.0 \\
101.0 \\
101.0 \\
101.0 \\
100.0 \\
100.0 \\
100.0 \\
100.0\end{array}$ & $\begin{array}{l}\text { C. } 00620 \\
0.00551 \\
0.00496 \\
0.00397 \\
C .00331 \\
0.00284 \\
C .00249 \\
C .00221 \\
0.00199 \\
0.00166\end{array}$ & $\begin{array}{l}0.0397 \\
0.0429 \\
0.0460 \\
0.0535 \\
0.0605 \\
0.0672 \\
0.0735 \\
0.0795 \\
0.0852 \\
0.0964\end{array}$ & $\begin{array}{c}6.16 \\
6.62 \\
6.94 \\
8.03 \\
9.04 \\
10.0 \\
10.9 \\
11.8 \\
12.7 \\
14.4\end{array}$ & $\begin{array}{l}0.138 \\
0.168 \\
0.200 \\
0.291 \\
0.394 \\
0.510 \\
0.638 \\
0.776 \\
0.924 \\
1.25\end{array}$ & $\begin{array}{l}1.00086 \\
1.00077 \\
1.00069 \\
1.00056 \\
1.00046 \\
1.00040 \\
1.00035 \\
1.00031 \\
1.00028 \\
1.00323\end{array}$ & $\begin{array}{l}0.695 \\
0.691 \\
0.675 \\
0.671 \\
0.668 \\
0.667 \\
0.666 \\
0.666 \\
0.667 \\
0.668\end{array}$ \\
\hline $\begin{array}{r}700.0 \\
800.0 \\
900.0 \\
1000.0 \\
1200.0 \\
1400.0 \\
1600.0 \\
1800.0 \\
2000.0 \\
2500.0\end{array}$ & $\begin{array}{l}0.05315 \\
0.04552 \\
0.04137 \\
0.03724 \\
0.03104 \\
0.02661 \\
0.02329 \\
0.02370 \\
0.01864 \\
0.01491\end{array}$ & $\begin{array}{r}971.0 \\
1060.0 \\
1120.0 \\
1240.0 \\
1490.0 \\
1740.0 \\
1990.0 \\
2240.0 \\
2480.0 \\
3110.0\end{array}$ & $\begin{array}{l}3.61 \\
3.61 \\
3.60 \\
3.60 \\
3.60 \\
3.60 \\
3.60 \\
3.60 \\
3.60 \\
3.60\end{array}$ & $\begin{array}{l}100.0 \\
100.0 \\
100.3 \\
100.0 \\
100.0 \\
100.0 \\
100.0 \\
100.0 \\
130.0 \\
100.0\end{array}$ & $\begin{array}{l}c .00142 \\
0.00125 \\
0.00111 \\
0.0010 C \\
C .000832 \\
0.000712 \\
C .000624 \\
c .000555 \\
0.000500 \\
0.00040 C\end{array}$ & $\begin{array}{l}0.107 \\
0.118 \\
0.128 \\
0.137 \\
0.156 \\
0.174 \\
0.191 \\
0.208 \\
0.224 \\
0.263\end{array}$ & $\begin{array}{l}16.0 \\
17.6 \\
19.1 \\
20.5 \\
23.3 \\
26.0 \\
28.5 \\
31.0 \\
33.4 \\
39.2\end{array}$ & $\begin{array}{c}1.62 \\
2.04 \\
2.49 \\
2.97 \\
4.06 \\
5.27 \\
6.62 \\
8.10 \\
9.69 \\
14.2\end{array}$ & $\begin{array}{l}1.00020 \\
1.00018 \\
1.00016 \\
1.00014 \\
1.00012 \\
1.00010 \\
1.00009 \\
1.00008 \\
1.00007 \\
1.00006\end{array}$ & $\begin{array}{l}0.668 \\
0.667 \\
0.667 \\
0.667 \\
0.667 \\
0.667 \\
0.666 \\
0.666 \\
0.666 \\
0.666\end{array}$ \\
\hline 3000.0 & 0.31243 & 3730.0 & 3.60 & 100.3 & 0.000333 & 0.299 & 44.6 & 19.4 & 1.00005 & 0.666 \\
\hline
\end{tabular}

* tho-phase goundar 
15. PSIA ISOBAR

THERMOOYNAMIC PROPERTIES OF MELIUM 4

\begin{tabular}{|c|c|c|c|c|c|c|c|c|c|}
\hline $\begin{array}{c}\text { TEMPERATURE } \\
\text { OEG. R }\end{array}$ & $\begin{array}{l}\text { VOLUME } \\
\text { CU FT/LB }\end{array}$ & $\begin{array}{c}\text { ISOTHERM } \\
\text { OERIVATIVE } \\
\text { CU FT-PSIA/LB }\end{array}$ & $\begin{array}{l}\text { ISOCHORE } \\
\text { OERIVATIVE } \\
\text { PSIA/R }\end{array}$ & $\begin{array}{l}\text { INTERNAL } \\
\text { ENERGY } \\
\text { BTU/LA }\end{array}$ & $\begin{array}{l}\text { ENTHALPY } \\
\text { BTU/LS }\end{array}$ & $\begin{array}{l}\text { ENTROPY } \\
\text { BTU/LB-R }\end{array}$ & $\begin{array}{l}\text { CV } \\
\text { פTU / LB }\end{array}$ & $\begin{array}{l}C P \\
-R\end{array}$ & $\begin{array}{l}\text { VEL OCITY } \\
\text { OF SOUNO } \\
\text { FT/SEC }\end{array}$ \\
\hline $\begin{array}{r}4.0 \\
5.0 \\
6.0 \\
7.0 \\
8.0 \\
9.0 \\
10.0 \\
11.0 \\
12.0 \\
13.0\end{array}$ & $\begin{array}{l}0.09912 \\
0.1000 \\
0.1015 \\
0.1038 \\
0.1370 \\
0.1112 \\
0.1165 \\
0.1231 \\
0.1318 \\
0.1435\end{array}$ & $\begin{array}{r}212.0 \\
200.0 \\
179.0 \\
157.0 \\
134.0 \\
111.0 \\
91.9 \\
73.6 \\
57.6 \\
44.8\end{array}$ & $\begin{array}{l}11.3 \\
24.8 \\
33.6 \\
39.2 \\
41.5 \\
41.2 \\
39.3 \\
36.5 \\
33.0 \\
29.2\end{array}$ & $\begin{array}{l}1.394 \\
1.728 \\
2.125 \\
2.609 \\
3.196 \\
3.916 \\
4.743 \\
5.640 \\
6.654 \\
7.812\end{array}$ & $\begin{array}{l}4.147 \\
4.506 \\
4.945 \\
5.493 \\
6.166 \\
7.004 \\
7.978 \\
9.358 \\
10.31 \\
11.80\end{array}$ & $\begin{array}{l}0.3682 \\
0.4526 \\
0.5358 \\
0.6215 \\
0.7115 \\
0.8083 \\
0.9093 \\
1.312 \\
1.122 \\
1.241\end{array}$ & $\begin{array}{l}0.4196 \\
0.3934 \\
0.4507 \\
0.4879 \\
0.5287 \\
0.5777 \\
0.5882 \\
0.6026 \\
0.5230 \\
0.6465\end{array}$ & $\begin{array}{l}0.4240 \\
0.4220 \\
0.5229 \\
0.6244 \\
0.7469 \\
0.8920 \\
1.009 \\
1.160 \\
1.355 \\
1.592\end{array}$ & $\begin{array}{l}995.0 \\
996.0 \\
982.1 \\
964.5 \\
935.7 \\
891.5 \\
854.8 \\
810 . C \\
7 E 1.6 \\
714.6\end{array}$ \\
\hline $\begin{array}{l}14.0 \\
15.0 \\
16.0 \\
17.0 \\
18.0 \\
19.0 \\
20.0 \\
22.0 \\
24.0 \\
2 E .0\end{array}$ & $\begin{array}{l}0.1593 \\
0.1796 \\
0.2030 \\
0.2276 \\
0.2514 \\
0.2748 \\
0.2930 \\
0.3433 \\
0.3877 \\
0.4313\end{array}$ & $\begin{array}{l}36.2 \\
32.2 \\
32.2 \\
34.5 \\
37.1 \\
39.9 \\
42.9 \\
49.3 \\
55.9 \\
62.7\end{array}$ & $\begin{array}{c}25.2 \\
21.5 \\
13.3 \\
15.3 \\
13.9 \\
12.4 \\
11.2 \\
9.43 \\
8.20 \\
7.27\end{array}$ & $\begin{array}{c}9.118 \\
10.53 \\
11.95 \\
13.32 \\
14.54 \\
15.65 \\
16.70 \\
18.68 \\
20.53 \\
22.32\end{array}$ & $\begin{array}{l}13.54 \\
15.52 \\
17.59 \\
19.64 \\
21.52 \\
23.28 \\
24.98 \\
28.21 \\
31.30 \\
34.30\end{array}$ & $\begin{array}{l}1.371 \\
1.508 \\
1.642 \\
1.766 \\
1.874 \\
1.970 \\
2.057 \\
2.212 \\
2.346 \\
2.466\end{array}$ & $\begin{array}{l}0.6712 \\
0.6941 \\
0.7123 \\
0.7250 \\
0.7306 \\
0.7328 \\
0.7346 \\
0.7376 \\
0.7404 \\
0.7430\end{array}$ & $\begin{array}{l}1.831 \\
1.978 \\
1.986 \\
1.909 \\
1.826 \\
1.752 \\
1.692 \\
1.604 \\
1.543 \\
1.499\end{array}$ & $\begin{array}{l}675.9 \\
652.3 \\
644.8 \\
648.4 \\
655.3 \\
665.0 \\
677.0 \\
754.7 \\
734.7 \\
765.2\end{array}$ \\
\hline $\begin{array}{l}28.0 \\
30.0 \\
32.0 \\
34.0 \\
36.0 \\
38.0 \\
40.0\end{array}$ & $\begin{array}{l}0.4740 \\
0.5160 \\
0.5572 \\
0.5378 \\
0.5379 \\
0.6775 \\
0.7167\end{array}$ & $\begin{array}{r}69.5 \\
76.2 \\
82.9 \\
89.4 \\
95.9 \\
102.0 \\
109.5\end{array}$ & $\begin{array}{l}5.54 \\
5.95 \\
5.46 \\
5.04 \\
4.69 \\
4.39 \\
4.13\end{array}$ & $\begin{array}{l}24.07 \\
25.80 \\
27.50 \\
29.17 \\
30.82 \\
32.45 \\
34.06\end{array}$ & $\begin{array}{l}37.24 \\
40.13 \\
42.98 \\
45.77 \\
48.53 \\
51.27 \\
53.97\end{array}$ & $\begin{array}{l}2.575 \\
2.675 \\
2.767 \\
2.852 \\
2.931 \\
3.365 \\
3.074\end{array}$ & $\begin{array}{l}0.7453 \\
0.7470 \\
0.7481 \\
0.7489 \\
0.7495 \\
0.7499 \\
0.7501\end{array}$ & $\begin{array}{l}1.463 \\
1.433 \\
1.409 \\
1.389 \\
1.373 \\
1.359 \\
1.347\end{array}$ & $\begin{array}{l}794.8 \\
823.0 \\
850.3 \\
876.5 \\
901.9 \\
926.4 \\
950.2\end{array}$ \\
\hline $\begin{array}{r}45.0 \\
50.0 \\
55.0 \\
60.0 \\
70.0 \\
80.0 \\
90.0 \\
100.0 \\
120.0 \\
140.0\end{array}$ & $\begin{array}{l}0.8135 \\
0.9087 \\
1.043 \\
1.096 \\
1.281 \\
1.404 \\
1.646 \\
1.827 \\
2.188 \\
2.548\end{array}$ & $\begin{array}{l}124.0 \\
139.0 \\
154.0 \\
168.0 \\
197.0 \\
225.0 \\
253.0 \\
284.0 \\
335.0 \\
389.0\end{array}$ & $\begin{array}{l}3.59 \\
3.18 \\
2.86 \\
2.60 \\
2.20 \\
1.91 \\
1.69 \\
1.52 \\
1.26 \\
1.00\end{array}$ & $\begin{array}{l}38.05 \\
41.99 \\
45.88 \\
49.75 \\
57.42 \\
65.04 \\
72.62 \\
80.17 \\
95.22 \\
110.2\end{array}$ & $\begin{array}{c}60.55 \\
67.23 \\
73.74 \\
80.19 \\
93.60 \\
105.7 \\
118.3 \\
130.9 \\
156.0 \\
181.0\end{array}$ & $\begin{array}{l}3.231 \\
3.370 \\
3.494 \\
3.606 \\
3.804 \\
3.973 \\
4.122 \\
4.255 \\
4.483 \\
4.676\end{array}$ & $\begin{array}{l}0.7503 \\
0.7503 \\
0.7501 \\
0.7498 \\
0.7493 \\
0.7488 \\
0.7484 \\
0.7480 \\
0.7475 \\
0.7471\end{array}$ & $\begin{array}{l}1.324 \\
1.308 \\
1.296 \\
1.287 \\
1.275 \\
1.266 \\
1.261 \\
1.257 \\
1.252 \\
1.248\end{array}$ & $\begin{array}{l}1007.0 \\
1059.0 \\
1109.0 \\
1157.0 \\
1245.0 \\
1327.0 \\
1404.0 \\
1477.0 \\
1611.0 \\
1736.0\end{array}$ \\
\hline $\begin{array}{l}160.0 \\
180.0 \\
200.0 \\
250.0 \\
300.0 \\
350.0 \\
400.0 \\
450.0 \\
500.0 \\
660.0\end{array}$ & $\begin{array}{c}2.907 \\
3.265 \\
3.623 \\
4.518 \\
5.412 \\
6.305 \\
7.197 \\
8.092 \\
8.985 \\
10.77\end{array}$ & $\begin{array}{r}443.0 \\
497.0 \\
551.0 \\
685.0 \\
819.0 \\
953.0 \\
1090.0 \\
1220.0 \\
1360.0 \\
1620.0\end{array}$ & $\begin{array}{l}0.941 \\
0.835 \\
0.751 \\
0.600 \\
0.500 \\
0.428 \\
0.375 \\
0.333 \\
0.360 \\
0.250\end{array}$ & $\begin{array}{l}125.2 \\
140.2 \\
155.1 \\
192.4 \\
229.7 \\
267.0 \\
304.3 \\
341.5 \\
378.8 \\
453.3\end{array}$ & $\begin{array}{l}205.9 \\
230.9 \\
255.8 \\
317.9 \\
380.9 \\
442.1 \\
514.2 \\
566.3 \\
628.4 \\
752.5\end{array}$ & $\begin{array}{l}4.843 \\
4.989 \\
5.120 \\
5.398 \\
5.624 \\
5.816 \\
5.982 \\
6.128 \\
6.259 \\
6.485\end{array}$ & $\begin{array}{l}0.7467 \\
0.7465 \\
0.7463 \\
0.7460 \\
0.7457 \\
0.7456 \\
0.7455 \\
0.7454 \\
0.7453 \\
0.7452\end{array}$ & $\begin{array}{l}1.246 \\
1.245 \\
1.244 \\
1.243 \\
1.242 \\
1.242 \\
1.242 \\
1.241 \\
1.241 \\
1.241\end{array}$ & $\begin{array}{l}1851.0 \\
1960.0 \\
2063.0 \\
2300.0 \\
2514.0 \\
2712.0 \\
2896.0 \\
3069.0 \\
3233.0 \\
3538.0\end{array}$ \\
\hline $\begin{array}{r}700.0 \\
800.0 \\
960.0 \\
1000.0 \\
1200.0 \\
1400.0 \\
1600.0 \\
1800.0 \\
2000.0 \\
2500.0\end{array}$ & $\begin{array}{l}12.50 \\
14.34 \\
16.13 \\
17.92 \\
21.49 \\
25.06 \\
28.64 \\
32.21 \\
35.78 \\
44.72\end{array}$ & $\begin{array}{l}1890.0 \\
2160.0 \\
2430.0 \\
2696.0 \\
3230.0 \\
3770.0 \\
4300.0 \\
4840.0 \\
5370.0 \\
6710.0\end{array}$ & $\begin{array}{l}0.214 \\
0.187 \\
0.107 \\
0.150 \\
0.125 \\
0.107 \\
0.4937 \\
0.0833 \\
0.0750 \\
0.0000\end{array}$ & $\begin{array}{r}527.8 \\
602.3 \\
676.8 \\
751.2 \\
900.2 \\
1049.0 \\
1198.0 \\
1347.0 \\
1496.0 \\
1368.0\end{array}$ & $\begin{array}{r}876.6 \\
1001.0 \\
1125.0 \\
1249.0 \\
1497.0 \\
1745.0 \\
1994.0 \\
2242.0 \\
2490.0 \\
3111.0\end{array}$ & $\begin{array}{l}6.676 \\
6.842 \\
6.988 \\
7.119 \\
7.345 \\
7.537 \\
7.702 \\
7.849 \\
7.979 \\
8.256\end{array}$ & $\begin{array}{l}0.7452 \\
0.7451 \\
0.7451 \\
0.7451 \\
0.7450 \\
0.7450 \\
0.7450 \\
0.7450 \\
0.7449 \\
0.7449\end{array}$ & $\begin{array}{l}1.241 \\
1.241 \\
1.241 \\
1.241 \\
1.241 \\
1.241 \\
1.241 \\
1.241 \\
1.241 \\
1.241\end{array}$ & $\begin{array}{l}3819.0 \\
4081.0 \\
4327.0 \\
4559.0 \\
4992.0 \\
5391.0 \\
5761.0 \\
6110.0 \\
6439.0 \\
7198.0\end{array}$ \\
\hline $3000 . \mathrm{C}$ & 53.55 & $805 c .0$ & 0.0500 & 2241.0 & 3731.3 & 8.482 & 0.7449 & 1.241 & 7883.0 \\
\hline
\end{tabular}


15) PSIA ISOBAR

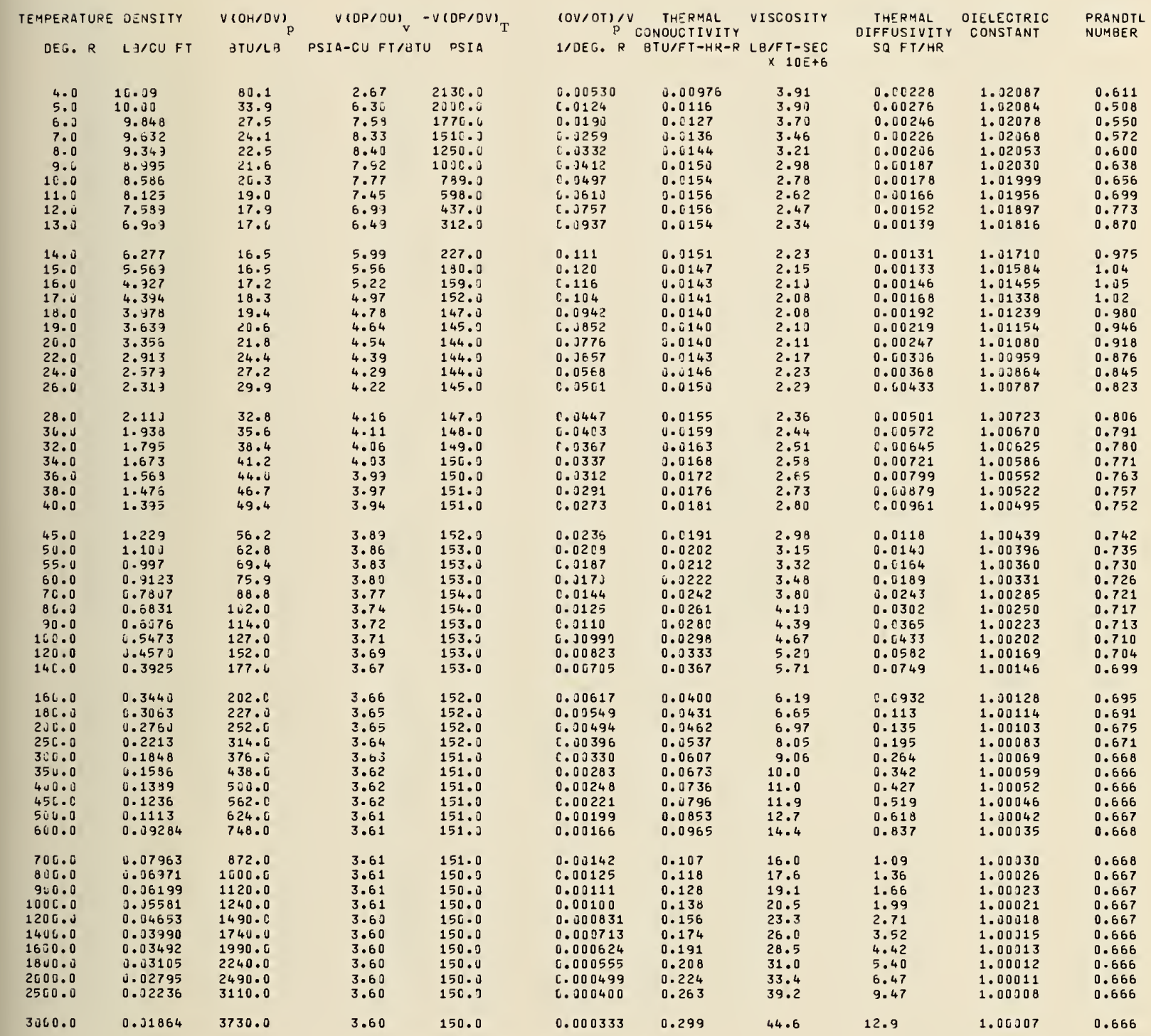

* tho-phase boundary 
200 PSIA ISOBAR

THERMOOYNAMIC PROPERTIES OF HELIUM 4

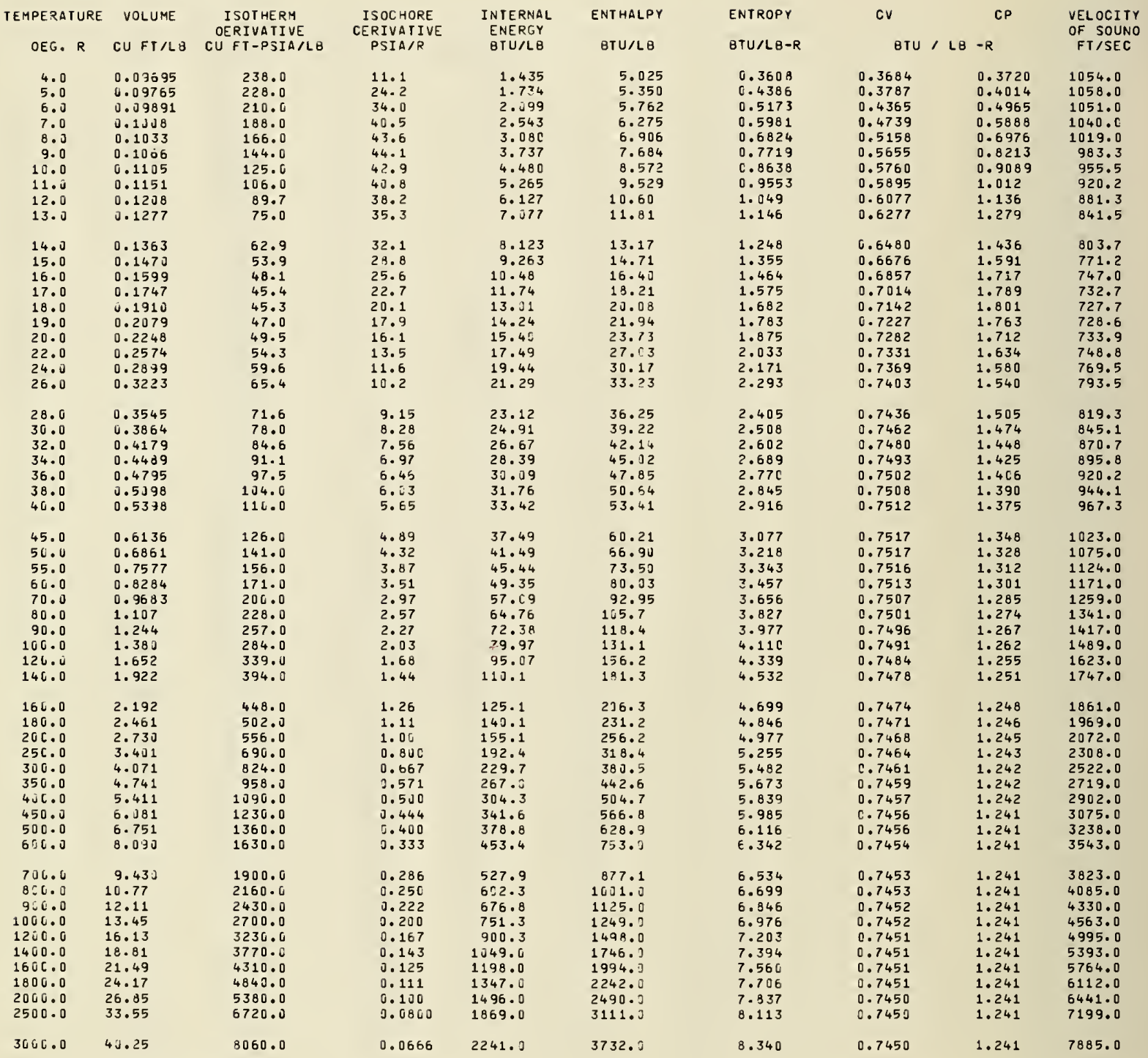

* tho-phase bounoary 
200 PSIA ISOBAR

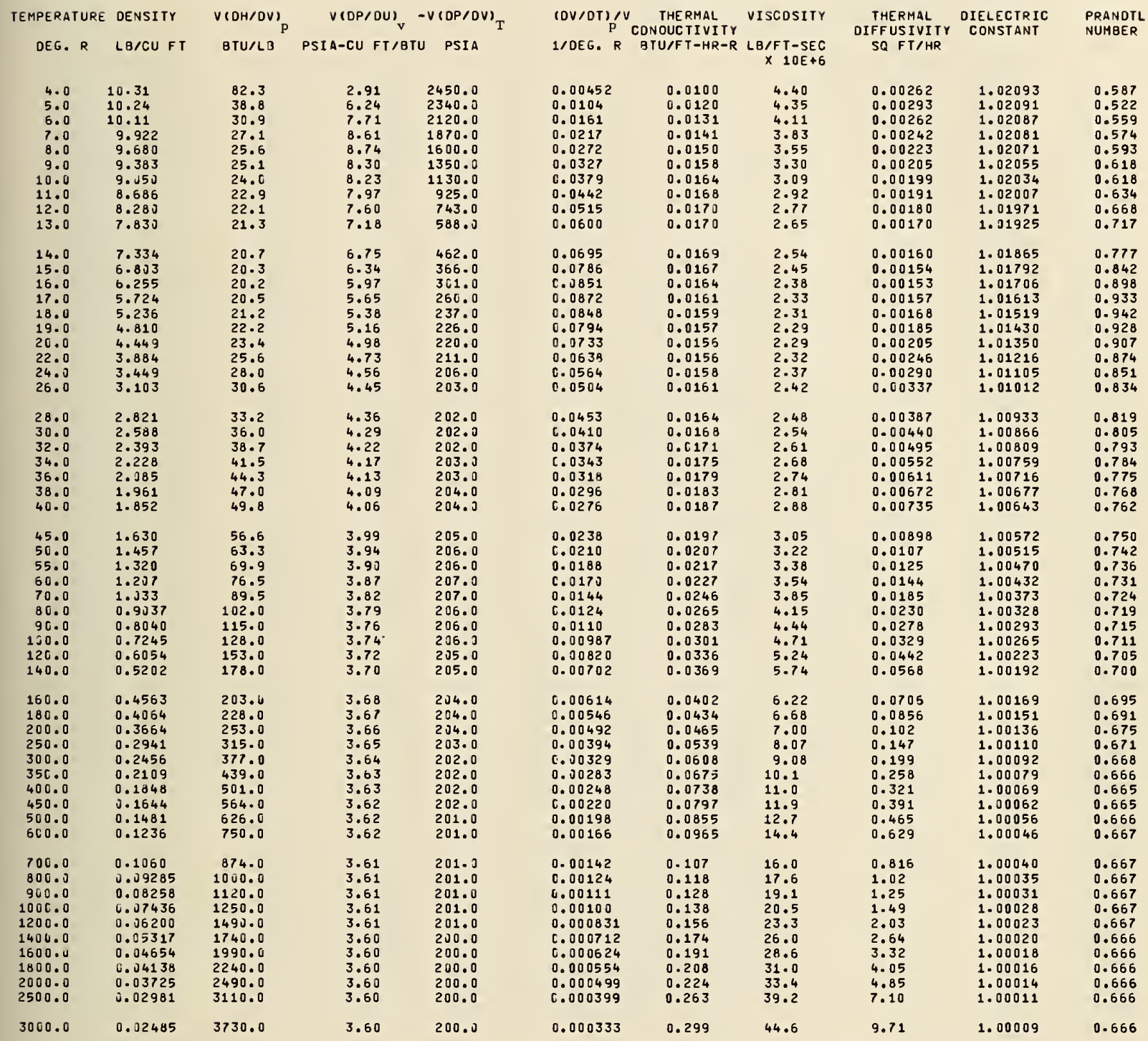

* two-phase boundary 


\begin{tabular}{|c|c|c|c|c|c|c|c|c|c|}
\hline $\begin{array}{c}\text { TEMPERATURE } \\
\text { OEG. R }\end{array}$ & $\begin{array}{l}\text { E VOLUME } \\
\text { CU FT/LB }\end{array}$ & $\begin{array}{c}\text { ISOTHERM } \\
\text { OERIVATIVE } \\
\text { CU FT-PSIA/LB }\end{array}$ & $\begin{array}{l}\text { ISOCHORE } \\
\text { OERIVATIVE } \\
\text { PSIA/R }\end{array}$ & $\begin{array}{l}\text { INTERNAL } \\
\text { ENERGY } \\
\text { BTU/LB }\end{array}$ & $\begin{array}{l}\text { ENTHALPY } \\
\text { BTU/LB }\end{array}$ & $\begin{array}{l}\text { ENTROPY } \\
\text { BTU/LB-R }\end{array}$ & $\begin{array}{l}\text { CV } \\
\text { BTU / LB }\end{array}$ & $-R$ & $\begin{array}{l}\text { VEL OCITY } \\
\text { OF SOUNO } \\
\text { FT/SEC }\end{array}$ \\
\hline $\begin{array}{l}4.0 \\
5.0 \\
6.0 \\
7.0 \\
8.0 \\
9.0 \\
10.0 \\
11.0 \\
12.0 \\
13.0\end{array}$ & $\begin{array}{l}0.09341 \\
0.09395 \\
0.09488 \\
0.09627 \\
0.09811 \\
0.1004 \\
0.1031 \\
0.1060 \\
0.1094 \\
0.1134\end{array}$ & $\begin{array}{l}281.0 \\
278.0 \\
263.0 \\
244.0 \\
223.0 \\
202.0 \\
184.0 \\
165.0 \\
148.0 \\
132.0\end{array}$ & $\begin{array}{l}12.7 \\
23.6 \\
34.7 \\
42.5 \\
46.8 \\
48.2 \\
47.9 \\
46.7 \\
45.0 \\
43.0\end{array}$ & $\begin{array}{l}1.548 \\
1.795 \\
2.111 \\
2.496 \\
2.966 \\
3.543 \\
4.192 \\
4.863 \\
5.585 \\
6.362\end{array}$ & $\begin{array}{r}6.733 \\
7.014 \\
7.382 \\
7.844 \\
8.416 \\
9.122 \\
9.917 \\
10.75 \\
11.66 \\
12.66\end{array}$ & $\begin{array}{l}0.3486 \\
0.4169 \\
0.4885 \\
0.5620 \\
0.6385 \\
0.7192 \\
0.8011 \\
0.8812 \\
0.9611 \\
1.041\end{array}$ & $\begin{array}{l}0.2974 \\
0.3522 \\
0.4092 \\
0.4483 \\
0.4938 \\
0.5466 \\
0.5592 \\
0.5738 \\
0.5921 \\
0.6111\end{array}$ & $\begin{array}{l}0.3011 \\
0.3685 \\
0.4549 \\
0.5375 \\
0.6339 \\
0.7398 \\
0.8049 \\
0.8762 \\
0.9563 \\
1.043\end{array}$ & $\begin{array}{l}1149.0 \\
1161.0 \\
1164.0 \\
1164.0 \\
1152.0 \\
1126.0 \\
1107.0 \\
1082.0 \\
1053.0 \\
1022.0\end{array}$ \\
\hline $\begin{array}{l}14.0 \\
15.0 \\
16.0 \\
17.0 \\
18.0 \\
19.0 \\
26.0 \\
22.0 \\
24.0 \\
26.0\end{array}$ & $\begin{array}{l}0.1179 \\
0.1230 \\
0.1290 \\
0.1358 \\
0.1434 \\
0.1521 \\
0.1613 \\
0.1811 \\
0.2012 \\
0.2214\end{array}$ & $\begin{array}{r}118.0 \\
105.0 \\
94.2 \\
85.5 \\
78.9 \\
74.3 \\
71.7 \\
71.2 \\
74.0 \\
77.7\end{array}$ & $\begin{array}{l}40.6 \\
38.1 \\
35.6 \\
33.0 \\
30.5 \\
27.9 \\
25.6 \\
21.6 \\
18.6 \\
16.3\end{array}$ & $\begin{array}{c}7.198 \\
8.092 \\
9.044 \\
10.05 \\
11.10 \\
12.22 \\
13.35 \\
15.55 \\
17.64 \\
19.60\end{array}$ & $\begin{array}{l}13.75 \\
14.93 \\
16.21 \\
17.59 \\
19.07 \\
20.67 \\
22.31 \\
25.61 \\
28.82 \\
31.90\end{array}$ & $\begin{array}{l}1.123 \\
1.205 \\
1.289 \\
1.373 \\
1.458 \\
1.546 \\
1.631 \\
1.789 \\
1.930 \\
2.054\end{array}$ & $\begin{array}{l}0.6296 \\
0.6469 \\
0.6628 \\
0.6772 \\
0.6901 \\
0.7019 \\
0.7108 \\
0.7226 \\
0.7297 \\
0.7346\end{array}$ & $\begin{array}{l}1.134 \\
1.229 \\
1.324 \\
1.415 \\
1.496 \\
1.556 \\
1.591 \\
1.600 \\
1.573 \\
1.544\end{array}$ & $\begin{array}{l}991.3 \\
961.5 \\
934.0 \\
909.9 \\
890.1 \\
873.3 \\
862.4 \\
854.8 \\
859.3 \\
869.9\end{array}$ \\
\hline $\begin{array}{l}28.0 \\
30.0 \\
32.0 \\
34.0 \\
36.0 \\
38.0 \\
40.0\end{array}$ & $\begin{array}{l}0.2418 \\
0.2626 \\
0.2834 \\
0.3141 \\
0.3247 \\
0.3452 \\
0.3655\end{array}$ & $\begin{array}{r}82.0 \\
87.2 \\
92.7 \\
98.5 \\
104.0 \\
111.0 \\
117.0\end{array}$ & $\begin{array}{c}14.6 \\
13.2 \\
12.5 \\
11.8 \\
10.2 \\
9.46 \\
8.84\end{array}$ & $\begin{array}{l}21.49 \\
23.36 \\
25.19 \\
26.99 \\
28.76 \\
30.50 \\
32.21\end{array}$ & $\begin{array}{l}34.93 \\
37.95 \\
40.94 \\
43.89 \\
46.80 \\
49.67 \\
52.52\end{array}$ & $\begin{array}{l}2.166 \\
2.271 \\
2.367 \\
2.456 \\
2.539 \\
2.617 \\
2.690\end{array}$ & $\begin{array}{l}0.7392 \\
0.7433 \\
0.7464 \\
0.7487 \\
0.7504 \\
0.7516 \\
0.7525\end{array}$ & $\begin{array}{l}1.524 \\
1.503 \\
1.483 \\
1.464 \\
1.446 \\
1.430 \\
1.415\end{array}$ & $\begin{array}{r}885.1 \\
903.8 \\
923.9 \\
944.7 \\
965.8 \\
987.0 \\
1008.0\end{array}$ \\
\hline $\begin{array}{r}45.0 \\
50.0 \\
55.0 \\
60.0 \\
70.0 \\
80.0 \\
90.0 \\
160.0 \\
120.0 \\
140.0\end{array}$ & $\begin{array}{l}0.4156 \\
0.4649 \\
0.5135 \\
0.5615 \\
0.6561 \\
0.7475 \\
0.8418 \\
0.9336 \\
1.116 \\
1.297\end{array}$ & $\begin{array}{l}132.0 \\
147.0 \\
163.0 \\
178.0 \\
207.0 \\
236.0 \\
265.0 \\
293.0 \\
348.0 \\
403.0\end{array}$ & $\begin{array}{l}7.60 \\
0.68 \\
5.96 \\
5.38 \\
4.52 \\
3.90 \\
3.44 \\
3.07 \\
2.54 \\
2.16\end{array}$ & $\begin{array}{c}36.42 \\
40.53 \\
44.58 \\
48.57 \\
56.45 \\
64.22 \\
71.92 \\
79.57 \\
94.77 \\
109.9\end{array}$ & $\begin{array}{c}59.51 \\
66.36 \\
73.10 \\
79.76 \\
92.90 \\
105.9 \\
118.7 \\
131.4 \\
156.7 \\
181.9\end{array}$ & $\begin{array}{l}2.855 \\
2.999 \\
3.128 \\
3.244 \\
3.446 \\
3.619 \\
3.770 \\
3.965 \\
4.136 \\
4.329\end{array}$ & $\begin{array}{l}0.7537 \\
0.7541 \\
0.7541 \\
0.7539 \\
0.7532 \\
0.7525 \\
0.7518 \\
0.7511 \\
0.7501 \\
0.7493\end{array}$ & $\begin{array}{l}1.383 \\
1.359 \\
1.340 \\
1.325 \\
1.303 \\
1.289 \\
1.279 \\
1.271 \\
1.261 \\
1.255\end{array}$ & $\begin{array}{l}1060.0 \\
1109.0 \\
1157.0 \\
1203.0 \\
1289.0 \\
1369.0 \\
1444.0 \\
1515.0 \\
1647.0 \\
1769.0\end{array}$ \\
\hline $\begin{array}{l}160.0 \\
180.0 \\
200.0 \\
250.0 \\
300.0 \\
350.0 \\
400.0 \\
450.0 \\
500.0 \\
600.0\end{array}$ & $\begin{array}{l}1.477 \\
1.650 \\
1.836 \\
2.283 \\
2.731 \\
3.177 \\
3.624 \\
4.070 \\
4.517 \\
5.409\end{array}$ & $\begin{array}{r}457.0 \\
512.0 \\
566.0 \\
700.0 \\
834.0 \\
968.0 \\
1100.0 \\
1240.0 \\
1370.0 \\
1640.0\end{array}$ & $\begin{array}{l}1.89 \\
1.67 \\
1.50 \\
1.20 \\
1.00 \\
0.857 \\
0.749 \\
0.666 \\
0.599 \\
0.499\end{array}$ & $\begin{array}{l}124.9 \\
140.0 \\
155.0 \\
192.4 \\
229.7 \\
267.1 \\
304.4 \\
341.7 \\
378.9 \\
453.5\end{array}$ & $\begin{array}{l}207.0 \\
232.0 \\
256.9 \\
319.2 \\
381.4 \\
443.6 \\
505.7 \\
567.8 \\
629.8 \\
754.0\end{array}$ & $\begin{array}{l}4.497 \\
4.644 \\
4.776 \\
5.054 \\
5.280 \\
5.472 \\
5.638 \\
5.784 \\
5.915 \\
6.141\end{array}$ & $\begin{array}{l}0.7487 \\
0.7483 \\
0.7479 \\
0.7472 \\
0.7458 \\
0.7465 \\
0.7463 \\
0.7461 \\
0.7460 \\
0.7458\end{array}$ & $\begin{array}{l}1.252 \\
1.249 \\
1.247 \\
1.245 \\
1.243 \\
1.242 \\
1.242 \\
1.242 \\
1.241 \\
1.241\end{array}$ & $\begin{array}{l}1882.0 \\
1989.0 \\
2090.0 \\
2324.0 \\
2536.0 \\
2732.0 \\
2914.0 \\
3086.0 \\
3249.0 \\
3552.0\end{array}$ \\
\hline $\begin{array}{r}706.0 \\
806.0 \\
960.0 \\
1000.0 \\
1200.0 \\
1400.0 \\
1660.0 \\
1800.0 \\
2000.0 \\
2500.0\end{array}$ & $\begin{array}{c}6.302 \\
7.195 \\
8.388 \\
8.980 \\
10.77 \\
12.55 \\
14.34 \\
16.12 \\
17.91 \\
22.38\end{array}$ & $\begin{array}{l}1900.0 \\
2170.0 \\
2440.0 \\
2710.0 \\
3240.0 \\
3780.0 \\
4310.0 \\
4850.0 \\
5380.0 \\
6720.0\end{array}$ & $\begin{array}{l}0.428 \\
0.375 \\
0.333 \\
0.360 \\
0.250 \\
0.214 \\
0.187 \\
0.167 \\
0.150 \\
0.120\end{array}$ & $\begin{array}{r}528.0 \\
602.5 \\
677.0 \\
751.5 \\
900.5 \\
1049.0 \\
1198.0 \\
1347.0 \\
1496.0 \\
1869.0\end{array}$ & $\begin{array}{r}878.1 \\
1002.0 \\
1126.0 \\
1250.0 \\
1499.0 \\
1747.0 \\
1995.0 \\
2243.0 \\
2491.0 \\
3112.0\end{array}$ & $\begin{array}{l}6.333 \\
6.498 \\
6.644 \\
6.775 \\
7.001 \\
7.193 \\
7.358 \\
7.505 \\
7.635 \\
7.912\end{array}$ & $\begin{array}{l}0.7457 \\
0.7456 \\
0.7455 \\
0.7455 \\
0.7454 \\
0.7453 \\
0.7453 \\
0.7452 \\
0.7452 \\
0.7451\end{array}$ & $\begin{array}{l}1.241 \\
1.241 \\
1.241 \\
1.241 \\
1.241 \\
1.241 \\
1.241 \\
1.241 \\
1.241 \\
1.241\end{array}$ & $\begin{array}{l}3832.0 \\
4092.0 \\
4337.0 \\
4569.0 \\
5001.0 \\
5398.0 \\
5768.0 \\
6116.0 \\
6445.0 \\
7202.0\end{array}$ \\
\hline 3000.0 & 26.34 & 8060.0 & 0.100 & 2241.0 & 3732.0 & 8.139 & 0.7451 & 1.241 & 7887.0 \\
\hline
\end{tabular}


THERMOPHYSICAL PROPERTIES OF HELIUM 4

$30 \circlearrowleft$ PSIA ISOBAR

\begin{tabular}{|c|c|c|c|c|c|c|c|c|c|c|}
\hline TEMPERATURE & E OENSITY & $V(D H / O V)_{P}$ & $V(D P / O U)_{v}-$ & $V(O P / O V)_{T}$ & (OV/OT) $/ \mathrm{V}$ & $\begin{array}{c}\text { THERMAL } \\
\text { CONOUCTIVITY }\end{array}$ & VISCOSITY & $\begin{array}{l}\text { THERMAL } \\
\text { DIFFUSIVITY }\end{array}$ & $\begin{array}{l}\text { DIELECTRIC } \\
\text { CONSTANT }\end{array}$ & $\begin{array}{l}\text { PRANOTL } \\
\text { NUMBER }\end{array}$ \\
\hline OEG. $R$ & $L B / C \cup F T$ & BTU/LA & PSIA-CU FT/BTU & PSIA & $1 / D E G \cdot R$ & $B T U / F T-H R-R$ & $\begin{array}{r}L B / F T-S E C \\
\times 10 E+6\end{array}$ & SQ FT/HR & & \\
\hline $\begin{array}{r}4.0 \\
5.0 \\
6.0 \\
7.0 \\
8.0 \\
9.0 \\
10.0 \\
11.0 \\
12.0 \\
13.0\end{array}$ & $\begin{array}{c}10.71 \\
10.04 \\
10.54 \\
10.39 \\
10.19 \\
9.96 \\
9.703 \\
9.431 \\
9.137 \\
8.822\end{array}$ & $\begin{array}{l}71.3 \\
46.3 \\
36.4 \\
32.0 \\
30.8 \\
30.9 \\
30.0 \\
29.3 \\
28.7 \\
28.3\end{array}$ & $\begin{array}{l}3.99 \\
6.29 \\
8.04 \\
9.13 \\
9.30 \\
8.86 \\
8.83 \\
8.64 \\
8.32 \\
7.97\end{array}$ & $\begin{array}{l}3010.0 \\
2960.0 \\
2770.0 \\
2530.0 \\
2270.0 \\
2310.0 \\
1780.0 \\
1560.0 \\
1350.0 \\
1170.0\end{array}$ & $\begin{array}{l}0.00422 \\
0.00796 \\
0.0125 \\
0.0168 \\
0.0206 \\
0.0239 \\
0.0269 \\
0.0300 \\
0.0333 \\
0.0368\end{array}$ & $\begin{array}{l}0.0105 \\
0.0128 \\
0.0143 \\
0.0151 \\
0.0161 \\
0.0170 \\
0.0173 \\
0.0185 \\
0.0189 \\
0.0193\end{array}$ & $\begin{array}{l}5.44 \\
5.29 \\
4.93 \\
4.56 \\
4.21 \\
3.90 \\
3.65 \\
3.45 \\
3.28 \\
3.14\end{array}$ & $\begin{array}{l}0.00326 \\
0.00326 \\
0.00291 \\
0.00270 \\
0.00250 \\
0.00231 \\
0.00228 \\
0.00224 \\
0.00217 \\
0.00209\end{array}$ & $\begin{array}{l}1.02100 \\
1.02099 \\
1.02098 \\
1.02095 \\
1.02090 \\
1.02082 \\
1.02072 \\
1.02058 \\
1.02040 \\
1.02018\end{array}$ & $\begin{array}{l}0.561 \\
0.549 \\
0.579 \\
0.585 \\
0.595 \\
0.509 \\
0.593 \\
0.589 \\
0.596 \\
0.612\end{array}$ \\
\hline $\begin{array}{l}14.0 \\
15.0 \\
16.0 \\
17.0 \\
18.0 \\
19.0 \\
26.0 \\
22.0 \\
24.0 \\
26.0\end{array}$ & $\begin{array}{l}8.485 \\
8.128 \\
7.753 \\
7.366 \\
6.972 \\
6.577 \\
6.193 \\
5.523 \\
4.969 \\
4.517\end{array}$ & $\begin{array}{l}27.9 \\
27.5 \\
27.2 \\
27.0 \\
27.0 \\
27.2 \\
27.5 \\
29.1 \\
31.1 \\
33.2\end{array}$ & $\begin{array}{l}7.61 \\
7.25 \\
6.92 \\
6.61 \\
6.33 \\
6.05 \\
5.81 \\
5.42 \\
5.13 \\
4.92\end{array}$ & $\begin{array}{r}1030.0 \\
854.0 \\
731.0 \\
630.0 \\
550.0 \\
438.0 \\
445.0 \\
393.0 \\
368.0 \\
351.0\end{array}$ & $\begin{array}{l}0.0407 \\
0.0447 \\
0.0487 \\
0.0524 \\
0.0554 \\
0.0572 \\
0.0575 \\
0.0550 \\
0.0506 \\
0.0465\end{array}$ & $\begin{array}{l}0.0194 \\
0.0195 \\
0.0194 \\
0.0193 \\
0.0191 \\
0.0189 \\
0.0187 \\
0.0184 \\
0.0183 \\
0.0184\end{array}$ & $\begin{array}{l}3.02 \\
2.92 \\
2.84 \\
2.77 \\
2.72 \\
2.68 \\
2.65 \\
2.63 \\
2.64 \\
2.67\end{array}$ & $\begin{array}{l}0.00202 \\
0.00195 \\
0.00189 \\
0.00185 \\
0.00183 \\
0.00185 \\
0.00190 \\
0.00208 \\
0.00235 \\
0.00264\end{array}$ & $\begin{array}{l}1.01990 \\
1.01956 \\
1.01916 \\
1.01869 \\
1.01816 \\
1.01758 \\
1.01697 \\
1.01575 \\
1.01464 \\
1.01366\end{array}$ & $\begin{array}{l}0.635 \\
0.665 \\
0.698 \\
0.733 \\
0.767 \\
0.794 \\
0.811 \\
0.822 \\
0.815 \\
0.807\end{array}$ \\
\hline $\begin{array}{l}28.0 \\
30.0 \\
32.0 \\
34.0 \\
36.0 \\
38.0 \\
40.0\end{array}$ & $\begin{array}{l}4.135 \\
3.807 \\
3.528 \\
3.288 \\
3.080 \\
2.897 \\
2.736\end{array}$ & $\begin{array}{l}35.5 \\
38.0 \\
40.5 \\
43.1 \\
45.8 \\
48.4 \\
51.1\end{array}$ & $\begin{array}{l}4.77 \\
4.65 \\
4.55 \\
4.47 \\
4.43 \\
4.34 \\
4.29\end{array}$ & $\begin{array}{l}339.0 \\
332.0 \\
327.0 \\
324.0 \\
322.0 \\
320.0 \\
319.0\end{array}$ & $\begin{array}{l}0.0429 \\
0.0396 \\
0.0366 \\
0.0340 \\
0.0316 \\
0.0295 \\
0.0277\end{array}$ & $\begin{array}{l}0.0185 \\
0.0187 \\
0.0189 \\
0.0192 \\
0.0195 \\
0.0198 \\
0.0201\end{array}$ & $\begin{array}{l}2.71 \\
2.75 \\
2.81 \\
2.86 \\
2.92 \\
2.98 \\
3.04\end{array}$ & $\begin{array}{l}0.00294 \\
0.00326 \\
0.00361 \\
0.00398 \\
0.00437 \\
0.00477 \\
0.00519\end{array}$ & $\begin{array}{l}1.01277 \\
1.01197 \\
1.01126 \\
1.01062 \\
1.01006 \\
1.00955 \\
1.00909\end{array}$ & $\begin{array}{l}0.803 \\
0.798 \\
0.792 \\
0.787 \\
0.781 \\
0.775 \\
0.770\end{array}$ \\
\hline $\begin{array}{r}45.0 \\
50.0 \\
55.0 \\
60.0 \\
70.0 \\
80.0 \\
90.0 \\
100.0 \\
120.0 \\
140.0\end{array}$ & $\begin{array}{l}2.405 \\
2.151 \\
1.947 \\
1.781 \\
1.524 \\
1.334 \\
1.188 \\
1.071 \\
0.8964 \\
0.7713\end{array}$ & $\begin{array}{r}57.8 \\
64.6 \\
71.3 \\
77.9 \\
91.1 \\
104.0 \\
117.0 \\
130.0 \\
155.0 \\
180.0\end{array}$ & $\begin{array}{l}4.19 \\
4.12 \\
4.65 \\
4.01 \\
3.94 \\
3.89 \\
3.85 \\
3.82 \\
3.77 \\
3.74\end{array}$ & $\begin{array}{l}318.0 \\
317.0 \\
317.0 \\
316.0 \\
316.0 \\
315.0 \\
314.0 \\
314.0 \\
312.0 \\
311.0\end{array}$ & $\begin{array}{l}0.0239 \\
C .0210 \\
0.0188 \\
0.0170 \\
0.0143 \\
C .0124 \\
0.0109 \\
0.00980 \\
0.00813 \\
0.00696\end{array}$ & $\begin{array}{l}0.0269 \\
0.0219 \\
0.0227 \\
0.0236 \\
0.0254 \\
0.0272 \\
0.0290 \\
0.0307 \\
0.0342 \\
0.0375\end{array}$ & $\begin{array}{l}3.19 \\
3.35 \\
3.50 \\
3.65 \\
3.95 \\
4.24 \\
4.52 \\
4.79 \\
5.31 \\
5.81\end{array}$ & $\begin{array}{l}0.00629 \\
0.00747 \\
0.00871 \\
0.0109 \\
0.0128 \\
0.0158 \\
0.0191 \\
0.0226 \\
0.0302 \\
0.0387\end{array}$ & $\begin{array}{l}1.00813 \\
1.00736 \\
1.00673 \\
1.00620 \\
1.00537 \\
1.00475 \\
1.00425 \\
1.00386 \\
1.00325 \\
1.00282\end{array}$ & $\begin{array}{l}0.759 \\
0.750 \\
0.743 \\
0.738 \\
0.729 \\
0.723 \\
0.718 \\
0.713 \\
0.706 \\
0.700\end{array}$ \\
\hline $\begin{array}{l}160.0 \\
18 C .00 \\
2 \dot{0} 0.0 \\
250.0 \\
360.0 \\
350.0 \\
400.0 \\
456.0 \\
500.0 \\
600.0\end{array}$ & $\begin{array}{l}0.6772 \\
0.6937 \\
0.5447 \\
0.4379 \\
0.3662 \\
0.3147 \\
0.2760 \\
0.2457 \\
0.2214 \\
0.1849\end{array}$ & $\begin{array}{l}225.0 \\
230.0 \\
255.0 \\
318.0 \\
380.0 \\
442.0 \\
504.0 \\
565.0 \\
628.0 \\
752.0\end{array}$ & $\begin{array}{l}3.72 \\
3.71 \\
3.69 \\
3.67 \\
3.66 \\
3.65 \\
3.64 \\
3.63 \\
3.63 \\
3.62\end{array}$ & $\begin{array}{l}310.0 \\
309.0 \\
308.0 \\
307.0 \\
305.0 \\
305.0 \\
304.0 \\
304.0 \\
303.0 \\
303.0\end{array}$ & $\begin{array}{l}0.00609 \\
0.00542 \\
0.00488 \\
C .00332 \\
C .00327 \\
c .00281 \\
c .00246 \\
{[.00219} \\
0.00198 \\
0.00165\end{array}$ & $\begin{array}{l}0.0407 \\
0.0438 \\
0.0469 \\
0.0542 \\
0.0612 \\
0.0673 \\
0.0743 \\
0.0803 \\
0.0857 \\
0.0967\end{array}$ & $\begin{array}{l}6.28 \\
6.74 \\
7.05 \\
8.12 \\
9.12 \\
10.1 \\
11.0 \\
11.9 \\
12.8 \\
14.4\end{array}$ & $\begin{array}{l}0.0480 \\
0.0581 \\
0.0690 \\
0.0995 \\
0.134 \\
0.173 \\
0.216 \\
0.262 \\
0.312 \\
0.422\end{array}$ & $\begin{array}{l}1.00248 \\
1.00222 \\
1.00201 \\
1.00162 \\
1.00136 \\
1.00117 \\
1.00103 \\
1.00092 \\
1.00083 \\
1.00069\end{array}$ & $\begin{array}{l}0.695 \\
0.691 \\
0.675 \\
0.671 \\
0.668 \\
0.666 \\
0.665 \\
0.665 \\
0.666 \\
0.667\end{array}$ \\
\hline $\begin{array}{r}700.0 \\
80 \mathrm{~L} .0 \\
90 \mathrm{C} .0 \\
100 \mathrm{C} 00 \\
1200.0 \\
140 \mathrm{C} .0 \\
160 \mathrm{C} .0 \\
1800.0 \\
200 \mathrm{~L} .0 \\
25 \cup C .0\end{array}$ & $\begin{array}{l}0.1587 \\
0.1370 \\
0.1236 \\
0.1114 \\
0.09288 \\
0.07967 \\
0.06974 \\
0.06202 \\
0.05583 \\
0.04469\end{array}$ & $\begin{array}{r}876.0 \\
1000.0 \\
1120.0 \\
1250.0 \\
1500.0 \\
1740.0 \\
1990.0 \\
2240.0 \\
2490.0 \\
3110.0\end{array}$ & $\begin{array}{l}3.62 \\
3.61 \\
3.61 \\
3.61 \\
3.61 \\
3.61 \\
3.60 \\
3.60 \\
3.60 \\
3.60\end{array}$ & $\begin{array}{l}302.0 \\
302.0 \\
302.0 \\
301.0 \\
301.0 \\
301.3 \\
301.0 \\
301.0 \\
301.0 \\
300.0\end{array}$ & $\begin{array}{l}0.00142 \\
0.00124 \\
0.00110 \\
0.000994 \\
C .000329 \\
C .000711 \\
C .000623 \\
0.000554 \\
C .000499 \\
c .000399\end{array}$ & $\begin{array}{l}0.108 \\
0.118 \\
0.128 \\
0.138 \\
0.156 \\
0.174 \\
0.192 \\
0.208 \\
0.224 \\
0.263\end{array}$ & $\begin{array}{l}16.0 \\
17.6 \\
19.1 \\
20.5 \\
23.3 \\
26.0 \\
28.6 \\
31.0 \\
33.4 \\
39.2\end{array}$ & $\begin{array}{l}0.546 \\
0.683 \\
0.834 \\
1.00 \\
1.36 \\
1.76 \\
2.21 \\
2.70 \\
3.24 \\
4.74\end{array}$ & $\begin{array}{l}1.00050 \\
1.00052 \\
1.00046 \\
1.00042 \\
1.00035 \\
1.00030 \\
1.00026 \\
1.00023 \\
1.00021 \\
1.00017\end{array}$ & $\begin{array}{l}0.667 \\
0.667 \\
0.667 \\
0.667 \\
0.666 \\
0.666 \\
0.666 \\
0.666 \\
0.666 \\
0.666\end{array}$ \\
\hline $30=0.0$ & 0.03726 & 3730.0 & 3.63 & 300.0 & 0.000333 & 0.299 & 44.6 & 6.47 & 1.00014 & 0.566 \\
\hline
\end{tabular}

* tho-phase boundary 


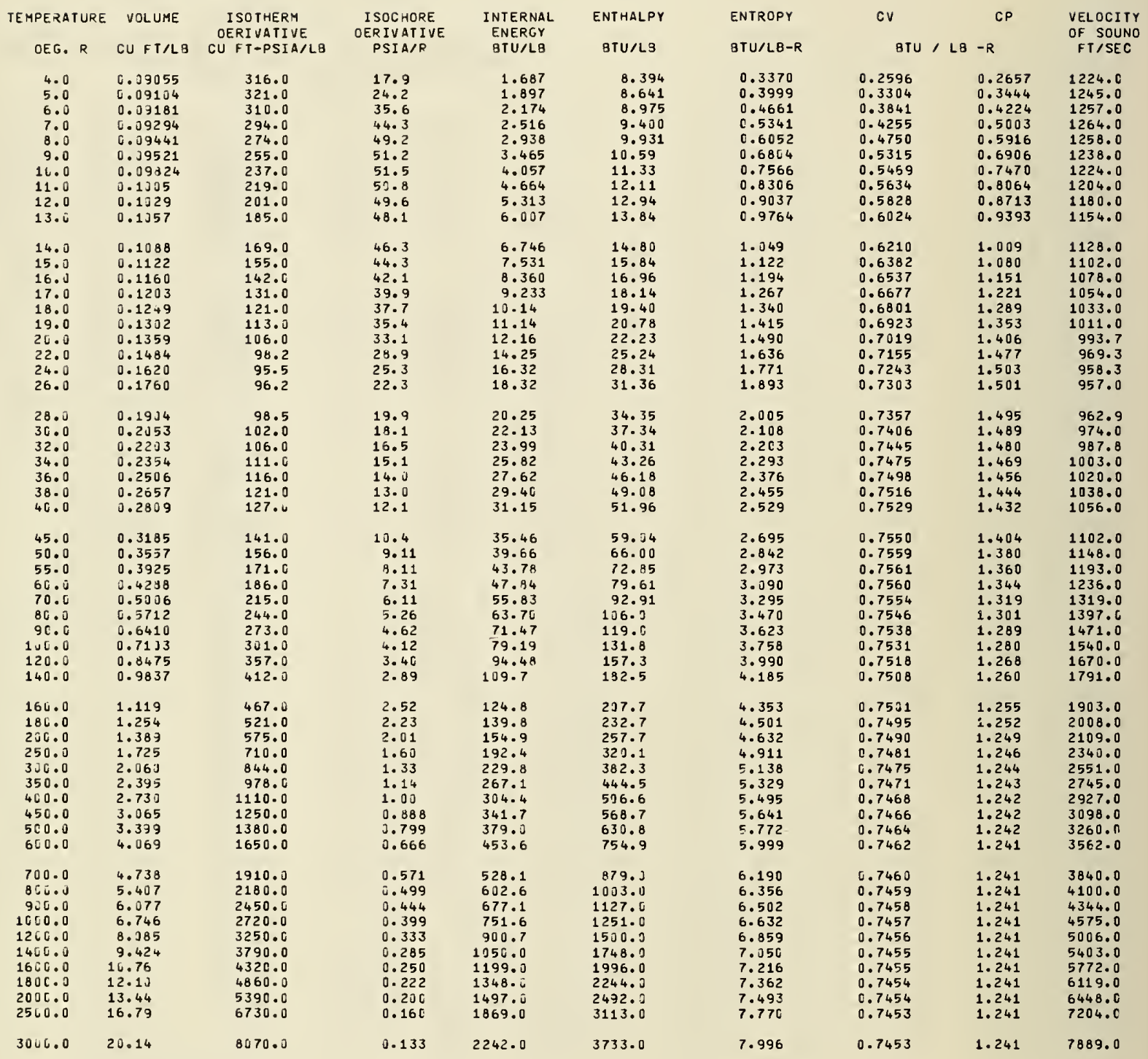


40 J PSIA ISOBAR

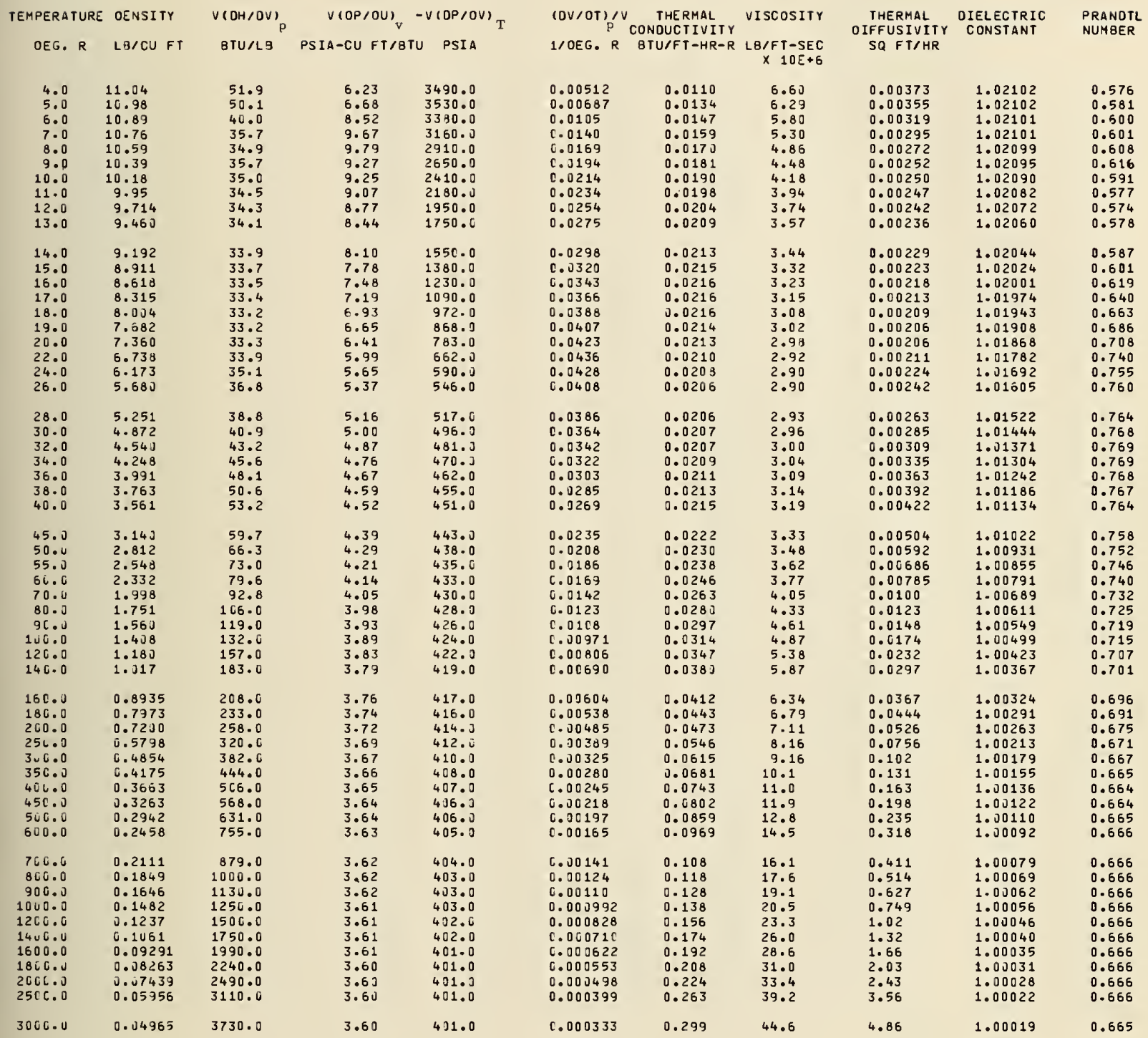

- TWO-PHASE bOUNDARY 


\begin{tabular}{|c|c|c|c|c|c|c|c|c|c|}
\hline $\begin{array}{c}\text { TEMPERATURE } \\
\text { OEG. R }\end{array}$ & $\begin{array}{l}\text { EOLUME } \\
\text { CU FT/LB }\end{array}$ & $\begin{array}{l}\text { ISOTHERM } \\
\text { OERIVATIVE } \\
\text { CU FT-PSIA/LB }\end{array}$ & $\begin{array}{l}\text { ISOCHORE } \\
\text { DERIVATIVE } \\
\text { PSIA/R }\end{array}$ & $\begin{array}{l}\text { INTERNAL } \\
\text { ENERGY } \\
\text { BTU/LB }\end{array}$ & $\begin{array}{l}\text { ENTHALPY } \\
\text { BTU/LB }\end{array}$ & $\begin{array}{l}\text { ENTROPY } \\
\text { BTU/LB-R }\end{array}$ & $\begin{array}{l}\text { CV } \\
\text { BTU / LB }\end{array}$ & $C P$ & $\begin{array}{l}\text { VEL OCITY } \\
\text { OF SOUND } \\
\text { FT/SEC }\end{array}$ \\
\hline $\begin{array}{l}4.0 \\
5.0 \\
6.0 \\
7.0 \\
8.0 \\
9.0 \\
10.0 \\
11.0 \\
12.0 \\
13.0\end{array}$ & $\begin{array}{l}0.38810 \\
0.08863 \\
0.08331 \\
0.09328 \\
0.09152 \\
0.09301 \\
0.09467 \\
0.09546 \\
0.09343 \\
0.1036\end{array}$ & $\begin{array}{l}342.0 \\
358.0 \\
353.0 \\
339.0 \\
321.0 \\
303.0 \\
286.0 \\
268.0 \\
250.0 \\
233.0\end{array}$ & $\begin{array}{l}27.3 \\
26.5 \\
37.2 \\
46.1 \\
51.4 \\
53.7 \\
54.3 \\
54.0 \\
53.1 \\
51.9\end{array}$ & $\begin{array}{l}1.837 \\
2.024 \\
2.269 \\
2.575 \\
2.961 \\
3.45 C \\
4.002 \\
4.567 \\
5.168 \\
5.809\end{array}$ & $\begin{array}{r}9.99 \\
10.23 \\
10.54 \\
10.93 \\
11.43 \\
12.06 \\
12.77 \\
13.50 \\
14.28 \\
15.12\end{array}$ & $\begin{array}{l}0.3238 \\
0.3851 \\
0.4473 \\
0.5110 \\
0.5781 \\
0.6493 \\
0.7216 \\
0.7916 \\
0.8505 \\
0.9286\end{array}$ & $\begin{array}{l}0.2511 \\
0.3144 \\
0.3619 \\
0.4053 \\
0.4587 \\
0.5188 \\
0.5369 \\
0.5553 \\
0.5759 \\
0.5964\end{array}$ & $\begin{array}{l}0.2636 \\
0.3287 \\
0.3967 \\
0.4714 \\
0.5605 \\
0.6561 \\
0.7082 \\
0.7616 \\
0.8190 \\
0.8779\end{array}$ & $\begin{array}{l}1290.0 \\
1317.0 \\
1338.0 \\
1351.0 \\
1349.0 \\
1332.0 \\
1321.0 \\
1304.0 \\
1283.0 \\
1261.0\end{array}$ \\
\hline $\begin{array}{l}14.0 \\
15.0 \\
16.0 \\
17.0 \\
18.0 \\
19.0 \\
20.0 \\
22.0 \\
24.0 \\
26.0\end{array}$ & $\begin{array}{l}0.1029 \\
0.1055 \\
0.1133 \\
0.1114 \\
0.1148 \\
0.1184 \\
0.1224 \\
0.1311 \\
0.14 j 8 \\
0.1512\end{array}$ & $\begin{array}{l}217.0 \\
202.0 \\
189.0 \\
176.0 \\
165.0 \\
155.0 \\
145.0 \\
132.0 \\
123.0 \\
120.0\end{array}$ & $\begin{array}{l}50.4 \\
48.8 \\
47.5 \\
45.1 \\
43.2 \\
41.4 \\
38.9 \\
34.9 \\
31.1 \\
27.8\end{array}$ & $\begin{array}{c}6.489 \\
7.209 \\
7.967 \\
8.761 \\
9.590 \\
10.51 \\
11.45 \\
13.41 \\
15.40 \\
17.38\end{array}$ & $\begin{array}{l}16.02 \\
16.98 \\
18.00 \\
19.08 \\
20.21 \\
21.47 \\
22.79 \\
25.55 \\
28.44 \\
31.38\end{array}$ & $\begin{array}{l}0.996 \\
1.063 \\
1.130 \\
1.196 \\
1.262 \\
1.331 \\
1.399 \\
1.533 \\
1.560 \\
1.778\end{array}$ & $\begin{array}{l}0.6155 \\
0.6330 \\
0.6487 \\
0.6527 \\
0.6751 \\
0.6876 \\
0.6975 \\
0.7120 \\
0.7216 \\
0.7283\end{array}$ & $\begin{array}{l}0.9373 \\
0.997 \\
1.056 \\
1.114 \\
1.171 \\
1.225 \\
1.276 \\
1.359 \\
1.413 \\
1.438\end{array}$ & $\begin{array}{l}1238.0 \\
1215.0 \\
1193.0 \\
1172.0 \\
1151.0 \\
1129.0 \\
1110.0 \\
1079.0 \\
1058.0 \\
1047.0\end{array}$ \\
\hline $\begin{array}{l}26.0 \\
30.0 \\
32.0 \\
34.0 \\
36.0 \\
38.0 \\
46.0\end{array}$ & $\begin{array}{l}0.1619 \\
0.1731 \\
0.1846 \\
0.1902 \\
0.2130 \\
0.2199 \\
0.2318\end{array}$ & $\begin{array}{l}119.0 \\
120.0 \\
123.0 \\
126.0 \\
130.0 \\
134.0 \\
139.0\end{array}$ & $\begin{array}{l}25.1 \\
22.8 \\
20.9 \\
19.2 \\
17.8 \\
16.5 \\
15.4\end{array}$ & $\begin{array}{l}19.30 \\
21.17 \\
23.03 \\
24.86 \\
26.68 \\
28.47 \\
30.25\end{array}$ & $\begin{array}{l}34.30 \\
37.20 \\
40.12 \\
43.03 \\
45.94 \\
48.83 \\
51.70\end{array}$ & $\begin{array}{l}1.887 \\
1.987 \\
2.081 \\
2.169 \\
2.252 \\
2.331 \\
2.404\end{array}$ & $\begin{array}{l}0.7339 \\
0.7390 \\
0.7431 \\
0.7465 \\
0.7492 \\
0.7513 \\
0.7533\end{array}$ & $\begin{array}{l}1.450 \\
1.457 \\
1.458 \\
1.455 \\
1.449 \\
1.442 \\
1.434\end{array}$ & $\begin{array}{l}1045.0 \\
1049.0 \\
1057.0 \\
1067.0 \\
1080.0 \\
1093.0 \\
1108.0\end{array}$ \\
\hline $\begin{array}{r}45.0 \\
56.0 \\
55.0 \\
60.0 \\
70.0 \\
86.0 \\
96.0 \\
100.0 \\
120.0 \\
140.0\end{array}$ & $\begin{array}{l}0.2615 \\
0.2912 \\
0.3207 \\
0.3499 \\
0.4976 \\
0.4645 \\
0.5237 \\
0.5764 \\
0.5867 \\
0.7960\end{array}$ & $\begin{array}{l}152.0 \\
166.0 \\
180.0 \\
195.0 \\
224.0 \\
253.0 \\
282.0 \\
311.0 \\
367.0 \\
422.0\end{array}$ & $\begin{array}{c}13.2 \\
11.6 \\
13.3 \\
9.27 \\
7.73 \\
6.64 \\
5.82 \\
5.19 \\
4.26 \\
3.63\end{array}$ & $\begin{array}{r}34.60 \\
38.87 \\
43.05 \\
47.17 \\
55.26 \\
63.20 \\
71.04 \\
78.81 \\
94.20 \\
109.5\end{array}$ & $\begin{array}{c}58.82 \\
65.83 \\
72.74 \\
79.56 \\
93.00 \\
106.2 \\
119.3 \\
132.2 \\
157.8 \\
183.2\end{array}$ & $\begin{array}{l}2.572 \\
2.720 \\
2.852 \\
2.970 \\
3.177 \\
3.354 \\
3.508 \\
3.644 \\
3.877 \\
4.073\end{array}$ & $\begin{array}{l}0.7557 \\
0.7571 \\
0.7577 \\
0.7578 \\
0.7574 \\
0.7566 \\
0.7557 \\
0.7549 \\
0.7535 \\
0.7523\end{array}$ & $\begin{array}{l}1.412 \\
1.392 \\
1.373 \\
1.357 \\
1.331 \\
1.312 \\
1.298 \\
1.288 \\
1.273 \\
1.264\end{array}$ & $\begin{array}{l}1148.0 \\
1189.0 \\
1231.0 \\
1272.0 \\
1351.0 \\
1427.0 \\
1498.0 \\
1566.0 \\
1694.0 \\
1813.0\end{array}$ \\
\hline $\begin{array}{l}160.0 \\
180.0 \\
200.0 \\
250.0 \\
360.0 \\
350.0 \\
400.0 \\
450.0 \\
500.0 \\
600.0\end{array}$ & $\begin{array}{l}0.9046 \\
1.313 \\
1.121 \\
1.393 \\
1.658 \\
1.926 \\
2.194 \\
2.461 \\
2.729 \\
3.264\end{array}$ & $\begin{array}{r}477.0 \\
531.0 \\
585.0 \\
720.0 \\
854.0 \\
988.0 \\
1120.0 \\
1260.0 \\
1390.0 \\
1660.0\end{array}$ & $\begin{array}{l}3.16 \\
2.80 \\
2.51 \\
2.03 \\
1.67 \\
1.43 \\
1.25 \\
1.11 \\
1.03 \\
0.832\end{array}$ & $\begin{array}{l}124.6 \\
139.7 \\
154.8 \\
192.3 \\
229.8 \\
267.1 \\
304.5 \\
341.8 \\
379.1 \\
453.7\end{array}$ & $\begin{array}{l}208.4 \\
233.5 \\
258.5 \\
321.7 \\
393.3 \\
445.4 \\
597.6 \\
569.7 \\
631.3 \\
755.9\end{array}$ & $\begin{array}{l}4.241 \\
4.389 \\
4.521 \\
4.806 \\
5.027 \\
5.219 \\
5.384 \\
5.531 \\
5.662 \\
5.888\end{array}$ & $\begin{array}{l}0.7514 \\
0.7506 \\
0.7500 \\
0.7489 \\
0.7482 \\
0.7477 \\
0.7474 \\
0.7471 \\
0.7469 \\
0.7465\end{array}$ & $\begin{array}{l}1.258 \\
1.254 \\
1.251 \\
1.247 \\
1.245 \\
1.243 \\
1.242 \\
1.242 \\
1.242 \\
1.241\end{array}$ & $\begin{array}{l}1923.0 \\
2028.0 \\
2127.0 \\
2356.0 \\
2565.0 \\
2758.0 \\
2939.0 \\
3109.0 \\
3270.0 \\
3571.0\end{array}$ \\
\hline $\begin{array}{r}700.0 \\
860.0 \\
900.0 \\
1000.0 \\
1260.0 \\
1400.0 \\
1600.0 \\
1800.0 \\
2000.0 \\
250 C .0\end{array}$ & $\begin{array}{r}3.800 \\
4.335 \\
4.873 \\
5.406 \\
6.475 \\
7.547 \\
8.618 \\
9.689 \\
10.76 \\
13.44\end{array}$ & $\begin{array}{l}1920.0 \\
2190.0 \\
2450.0 \\
2720.0 \\
3260.0 \\
3790.0 \\
4330.0 \\
4860.0 \\
5400.0 \\
6740.0\end{array}$ & $\begin{array}{l}0.713 \\
0.524 \\
0.555 \\
0.499 \\
0.416 \\
0.357 \\
0.312 \\
0.277 \\
0.250 \\
0.200\end{array}$ & $\begin{array}{r}528.2 \\
602.8 \\
677.3 \\
751.0 \\
900.0 \\
1050.0 \\
1199.0 \\
1348.0 \\
1497.0 \\
1869.0\end{array}$ & $\begin{array}{r}880.3 \\
1004.0 \\
1123.0 \\
1252.0 \\
1500.3 \\
1749.0 \\
1997.0 \\
2245.0 \\
2493.0 \\
3114.0\end{array}$ & $\begin{array}{l}6.079 \\
6.245 \\
6.391 \\
6.522 \\
6.748 \\
6.939 \\
7.105 \\
7.251 \\
7.382 \\
7.659\end{array}$ & $\begin{array}{l}0.7463 \\
0.7462 \\
0.7461 \\
0.7460 \\
0.7458 \\
0.7457 \\
0.7456 \\
0.7456 \\
0.7455 \\
0.7454\end{array}$ & $\begin{array}{l}1.241 \\
1.241 \\
1.241 \\
1.241 \\
1.241 \\
1.241 \\
1.241 \\
1.241 \\
1.241 \\
1.241\end{array}$ & $\begin{array}{l}3849.0 \\
4107.0 \\
4351.0 \\
4582.0 \\
5012.0 \\
5408.0 \\
5776.0 \\
6123.0 \\
6452.0 \\
7207.0\end{array}$ \\
\hline 3000.0 & 16.12 & 8070.0 & 0.167 & 2242 . C & 3734.9 & 7.885 & 0.7454 & 1.241 & 7891.0 \\
\hline
\end{tabular}


50J PSIA ISOBAR

\begin{tabular}{|c|c|c|c|c|c|c|c|c|c|c|}
\hline TEMPERATURE & E OENSITY & & $V(D P / D U)_{v}-$ & $-V(D P / D V)_{T}$ & $(O V / O T)_{\mathrm{p}} / \mathrm{V}$ & $\begin{array}{c}\text { THERMAL } \\
\text { CONDUCTIVITY }\end{array}$ & VISCOSITY & $\begin{array}{l}\text { THERMAL } \\
\text { OIFFUSIVITY }\end{array}$ & $\begin{array}{l}\text { DIELECTRIC } \\
\text { CONSTANT }\end{array}$ & $\begin{array}{l}\text { PRANDTL } \\
\text { NUMBER }\end{array}$ \\
\hline DEG. $R$ & LB/CU FT & BTU/LB & PSIA-CU FT/BTU & U PSIA & $1 / D E G . R$ & STU/FT $-M R-R$ & $\begin{array}{r}\text { LB/FT }- \text { SEC } \\
\times 10 E+6\end{array}$ & SQ FT/HR & & \\
\hline $\begin{array}{r}4.0 \\
5.0 \\
6.0 \\
7.0 \\
8.0 \\
9.0 \\
10.0 \\
11.0 \\
12.0 \\
13.0\end{array}$ & $\begin{array}{c}11.35 \\
11.28 \\
11.20 \\
11.08 \\
10.93 \\
10.75 \\
10.56 \\
10.37 \\
10.16 \\
9.942\end{array}$ & $\begin{array}{l}37.5 \\
50.2 \\
42.1 \\
38.4 \\
38.3 \\
39.8 \\
39.4 \\
39.1 \\
39.2 \\
39.2\end{array}$ & $\begin{array}{c}9.59 \\
7.47 \\
9.18 \\
10.3 \\
10.2 \\
9.63 \\
9.58 \\
9.38 \\
9.08 \\
8.76\end{array}$ & $\begin{array}{l}3880.0 \\
4040.0 \\
3950.0 \\
3750.0 \\
3510.0 \\
3260.0 \\
3020.0 \\
2770.0 \\
2540.0 \\
2320.0\end{array}$ & $\begin{array}{l}0.00704 \\
0.00655 \\
0.00942 \\
0.0123 \\
0.0146 \\
0.0165 \\
0.0180 \\
0.0195 \\
0.0209 \\
0.0224\end{array}$ & $\begin{array}{l}0.0114 \\
0.0140 \\
0.0153 \\
0.0166 \\
0.0179 \\
0.0190 \\
0.0201 \\
0.0210 \\
0.0217 \\
0.0223\end{array}$ & $\begin{array}{l}7.91 \\
7.39 \\
6.72 \\
6.08 \\
5.53 \\
5.07 \\
4.71 \\
4.42 \\
4.18 \\
3.99\end{array}$ & $\begin{array}{l}0.00380 \\
0.00378 \\
0.00345 \\
0.00318 \\
0.00292 \\
0.00270 \\
0.00268 \\
0.00266 \\
0.00261 \\
0.00256\end{array}$ & $\begin{array}{l}1.02100 \\
1.02100 \\
1.02101 \\
1.02102 \\
1.02102 \\
1.02101 \\
1.02098 \\
1.02094 \\
1.02089 \\
1.02082\end{array}$ & $\begin{array}{l}0.660 \\
0.624 \\
0.625 \\
0.621 \\
0.625 \\
0.630 \\
0.598 \\
0.578 \\
0.568 \\
0.565\end{array}$ \\
\hline $\begin{array}{l}14.0 \\
15.0 \\
16.0 \\
17.0 \\
18.0 \\
19.0 \\
20.0 \\
22.0 \\
24.0 \\
26.0\end{array}$ & $\begin{array}{l}9.714 \\
9.476 \\
9.230 \\
8.975 \\
8.714 \\
8.444 \\
0.171 \\
7.625 \\
7.100 \\
6.015\end{array}$ & $\begin{array}{l}39.2 \\
39.2 \\
39.1 \\
39.1 \\
39.0 \\
39.0 \\
38.9 \\
39.1 \\
39.8 \\
41.0\end{array}$ & $\begin{array}{l}8.44 \\
8.14 \\
7.85 \\
7.58 \\
7.34 \\
7.07 \\
6.83 \\
6.42 \\
6.07 \\
5.77\end{array}$ & $\begin{array}{r}2110.0 \\
1920.0 \\
1740.0 \\
1580.0 \\
1440.0 \\
1300.0 \\
1190.0 \\
1000.0 \\
877.0 \\
793.0\end{array}$ & $\begin{array}{l}0.0239 \\
0.0254 \\
0.0270 \\
0.0285 \\
0.0300 \\
0.0315 \\
0.0328 \\
0.0347 \\
0.0355 \\
0.0351\end{array}$ & $\begin{array}{l}0.0228 \\
0.0231 \\
0.0234 \\
0.0235 \\
0.0235 \\
0.0235 \\
0.0235 \\
0.0232 \\
0.0230 \\
0.0228\end{array}$ & $\begin{array}{l}3.83 \\
3.69 \\
3.58 \\
3.48 \\
3.40 \\
3.34 \\
3.28 \\
3.20 \\
3.15 \\
3.13\end{array}$ & $\begin{array}{l}0.00250 \\
0.00245 \\
0.00240 \\
0.00235 \\
0.00231 \\
0.00227 \\
0.00225 \\
0.00224 \\
0.00229 \\
0.00240\end{array}$ & $\begin{array}{l}1.02072 \\
1.02060 \\
1.02046 \\
1.02029 \\
1.02009 \\
1.01986 \\
1.01960 \\
1.01901 \\
1.01834 \\
1.01764\end{array}$ & $\begin{array}{l}0.567 \\
0.573 \\
0.582 \\
0.595 \\
0.609 \\
0.626 \\
0.642 \\
0.674 \\
0.697 \\
0.712\end{array}$ \\
\hline $\begin{array}{l}28.0 \\
36.0 \\
32.0 \\
34.0 \\
36.0 \\
38.0 \\
40.0\end{array}$ & $\begin{array}{l}6.175 \\
5.770 \\
5.417 \\
5.096 \\
4.807 \\
4.543 \\
4.315\end{array}$ & $\begin{array}{l}42.6 \\
44.5 \\
46.5 \\
48.7 \\
51.0 \\
53.4 \\
55.8\end{array}$ & $\begin{array}{l}5.53 \\
5.34 \\
5.18 \\
5.05 \\
4.93 \\
4.84 \\
4.75\end{array}$ & $\begin{array}{l}737.0 \\
696.0 \\
666.0 \\
643.0 \\
625.0 \\
612.0 \\
601.0\end{array}$ & $\begin{array}{l}0.0340 \\
0.0328 \\
0.0313 \\
0.0299 \\
0.0284 \\
0.0270 \\
0.0257\end{array}$ & $\begin{array}{l}0.0227 \\
0.0226 \\
0.0226 \\
0.0225 \\
0.0227 \\
0.0228 \\
0.0230\end{array}$ & $\begin{array}{l}3.14 \\
3.15 \\
3.18 \\
3.21 \\
3.25 \\
3.30 \\
3.34\end{array}$ & $\begin{array}{l}0.00253 \\
0.00268 \\
0.00286 \\
0.00305 \\
0.00326 \\
0.00348 \\
0.00372\end{array}$ & $\begin{array}{l}1.01693 \\
1.01623 \\
1.01555 \\
1.01491 \\
1.01430 \\
1.01373 \\
1.01319\end{array}$ & $\begin{array}{l}0.723 \\
0.732 \\
0.739 \\
0.744 \\
0.747 \\
0.749 \\
0.750\end{array}$ \\
\hline $\begin{array}{r}45.0 \\
50.0 \\
55.0 \\
60.0 \\
70.0 \\
80.0 \\
90.0 \\
100.0 \\
120.0 \\
140.0\end{array}$ & $\begin{array}{l}3.823 \\
3.434 \\
3.118 \\
2.858 \\
2.453 \\
2.153 \\
1.92 J \\
1.735 \\
1.456 \\
1.256\end{array}$ & $\begin{array}{r}62.1 \\
68.5 \\
75.0 \\
81.6 \\
94.7 \\
108.0 \\
121.0 \\
134.0 \\
159.0 \\
185.0\end{array}$ & $\begin{array}{l}4.58 \\
4.46 \\
4.36 \\
4.28 \\
4.16 \\
4.07 \\
4.01 \\
3.96 \\
3.89 \\
3.84\end{array}$ & $\begin{array}{l}582.0 \\
570.0 \\
562.0 \\
557.0 \\
550.0 \\
545.0 \\
542.0 \\
539.0 \\
534.0 \\
530.0\end{array}$ & $\begin{array}{l}0.0228 \\
0.0203 \\
0.0183 \\
c .0166 \\
c .0141 \\
0.0122 \\
c .0107 \\
c .00963 \\
0.00799 \\
c .00684\end{array}$ & $\begin{array}{l}0.0235 \\
0.0241 \\
0.0248 \\
0.0255 \\
0.0272 \\
0.0288 \\
0.0304 \\
0.0321 \\
0.0353 \\
0.0385\end{array}$ & $\begin{array}{l}3.47 \\
3.63 \\
3.74 \\
3.87 \\
4.15 \\
4.42 \\
4.69 \\
4.95 \\
5.45 \\
5.94\end{array}$ & $\begin{array}{l}0.00435 \\
0.00505 \\
0.00580 \\
0.00660 \\
0.00832 \\
0.0102 \\
0.0122 \\
0.0144 \\
0.0190 \\
0.0243\end{array}$ & $\begin{array}{l}1.01201 \\
1.01101 \\
1.01016 \\
1.00944 \\
1.00827 \\
1.00736 \\
1.00664 \\
1.00606 \\
1.00515 \\
1.00448\end{array}$ & $\begin{array}{l}0.750 \\
0.747 \\
0.744 \\
0.740 \\
0.732 \\
0.726 \\
0.721 \\
0.716 \\
0.708 \\
0.701\end{array}$ \\
\hline $\begin{array}{l}160.0 \\
180.0 \\
200.0 \\
250.0 \\
300.0 \\
350.0 \\
400.0 \\
450.0 \\
500.0 \\
600.0\end{array}$ & $\begin{array}{l}1.105 \\
0.9874 \\
0.8923 \\
0.7196 \\
0.6032 \\
0.5193 \\
0.4559 \\
0.4163 \\
0.3664 \\
0.3063\end{array}$ & $\begin{array}{l}210.0 \\
235.0 \\
260.0 \\
323.0 \\
385.0 \\
447.0 \\
509.0 \\
571.0 \\
633.0 \\
757.0\end{array}$ & $\begin{array}{l}3.89 \\
3.77 \\
3.75 \\
3.72 \\
3.69 \\
3.68 \\
3.66 \\
3.65 \\
3.65 \\
3.64\end{array}$ & $\begin{array}{l}527.0 \\
525.0 \\
522.0 \\
518.0 \\
515.0 \\
513.0 \\
511.0 \\
510.0 \\
509.0 \\
507.0\end{array}$ & $\begin{array}{l}c .00599 \\
c .00533 \\
c .00481 \\
c .00386 \\
0.00323 \\
0.00278 \\
0.00244 \\
c .00218 \\
0.00196 \\
c .00164\end{array}$ & $\begin{array}{l}0.0417 \\
0.0447 \\
0.0477 \\
0.0550 \\
0.0618 \\
0.0684 \\
0.0746 \\
0.0805 \\
0.0861 \\
0.0971\end{array}$ & $\begin{array}{c}6.40 \\
6.85 \\
7.16 \\
8.21 \\
9.20 \\
10.2 \\
11.1 \\
11.9 \\
12.8 \\
14.5\end{array}$ & $\begin{array}{l}0.0300 \\
0.0361 \\
0.0428 \\
0.0613 \\
0.0824 \\
0.106 \\
0.132 \\
0.159 \\
0.189 \\
0.255\end{array}$ & $\begin{array}{l}1.00397 \\
1.00357 \\
1.00324 \\
1.00263 \\
1.00222 \\
1.00192 \\
1.00169 \\
1.00151 \\
1.00136 \\
1.00114\end{array}$ & $\begin{array}{l}0.696 \\
0.691 \\
0.675 \\
0.670 \\
0.667 \\
0.665 \\
0.664 \\
0.664 \\
0.664 \\
0.666\end{array}$ \\
\hline $\begin{array}{r}700.0 \\
800.0 \\
900.0 \\
1000.0 \\
1200.0 \\
1400.0 \\
1600.0 \\
1800.0 \\
2000.0 \\
2500.0\end{array}$ & $\begin{array}{l}0.2632 \\
0.2337 \\
0.2053 \\
0.1850 \\
0.1544 \\
0.1325 \\
0.1160 \\
0.1032 \\
0.09293 \\
0.07441\end{array}$ & $\begin{array}{r}881.0 \\
1000.0 \\
1130.0 \\
1250.0 \\
1560.0 \\
1750.0 \\
2000.0 \\
2240.0 \\
2490.0 \\
3110.0\end{array}$ & $\begin{array}{l}3.63 \\
3.62 \\
3.62 \\
3.62 \\
3.61 \\
3.61 \\
3.61 \\
3.61 \\
3.60 \\
3.60\end{array}$ & $\begin{array}{l}536.0 \\
505.0 \\
505.0 \\
504.0 \\
503.0 \\
503.0 \\
502.0 \\
502.0 \\
502.0 \\
501.0\end{array}$ & $\begin{array}{l}c .00141 \\
0.00123 \\
0.00110 \\
0.000990 \\
c .000827 \\
c .000710 \\
0.000621 \\
c .000553 \\
0.000498 \\
c .000399\end{array}$ & $\begin{array}{l}0.108 \\
0.118 \\
0.128 \\
0.138 \\
0.157 \\
0.174 \\
0.192 \\
0.208 \\
0.224 \\
0.263\end{array}$ & $\begin{array}{l}16.1 \\
17.6 \\
19.1 \\
20.6 \\
23.3 \\
26.0 \\
28.6 \\
31.0 \\
33.4 \\
39.2\end{array}$ & $\begin{array}{l}0.330 \\
0.413 \\
0.503 \\
0.601 \\
0.817 \\
1.06 \\
1.33 \\
1.63 \\
1.95 \\
2.85\end{array}$ & $\begin{array}{l}1.00098 \\
1.00086 \\
1.00077 \\
1.00069 \\
1.00058 \\
1.00050 \\
1.00044 \\
1.00039 \\
1.00035 \\
1.00028\end{array}$ & $\begin{array}{l}0.666 \\
0.666 \\
0.666 \\
0.666 \\
0.666 \\
0.666 \\
0.666 \\
0.666 \\
0.656 \\
0.666\end{array}$ \\
\hline 3000.0 & 0.06204 & 3730.0 & 3.60 & 501.0 & c.000332 & 0.299 & 44.6 & 3.89 & 1.00023 & 0.665 \\
\hline
\end{tabular}


600 PSIA ISOBAR

THERMOOYNAMIC PROPERTIES OF HELIUM 4

\begin{tabular}{|c|c|c|c|c|c|c|c|c|c|}
\hline $\begin{array}{c}\text { TEMPERA TURE } \\
\text { DEG. R }\end{array}$ & $\begin{array}{l}\varepsilon \text { VOLUHE } \\
\text { CU FT/LB }\end{array}$ & $\begin{array}{c}\text { ISOTHERH } \\
\text { OERIVATIVE } \\
\text { CU FT-PSIA/LB }\end{array}$ & $\begin{array}{l}\text { ISOCHORE } \\
\text { OERIVATIVE } \\
\text { PSIA/R }\end{array}$ & $\begin{array}{l}\text { INTERNAL } \\
\text { ENERGY } \\
\text { BTU/LB }\end{array}$ & $\begin{array}{l}\text { ENTHALPY } \\
\text { BTU/LB }\end{array}$ & $\begin{array}{l}\text { ENTROPY } \\
\text { BTU/LB-R }\end{array}$ & $\begin{array}{l}\text { CV } \\
\text { BTU / LB }\end{array}$ & $\begin{array}{l}C P \\
-R\end{array}$ & $\begin{array}{l}\text { VELOCITY } \\
\text { OF SOUND } \\
\text { FT/SEC }\end{array}$ \\
\hline $\begin{array}{r}4.0 \\
5.0 \\
6.0 \\
7.0 \\
8.0 \\
9.0 \\
10.0 \\
11.0 \\
12.0 \\
13.0\end{array}$ & $\begin{array}{l}0.08593 \\
0.08655 \\
0.08720 \\
0.08807 \\
0.08915 \\
0.09044 \\
0.09185 \\
0.09336 \\
0.09499 \\
0.09677\end{array}$ & $\begin{array}{l}361.0 \\
391.0 \\
391.0 \\
381.0 \\
365.0 \\
348.0 \\
331.0 \\
314.0 \\
296.0 \\
279.0\end{array}$ & $\begin{array}{l}42.0 \\
30.6 \\
39.5 \\
48.1 \\
53.4 \\
55.8 \\
56.6 \\
56.5 \\
55.9 \\
55.0\end{array}$ & $\begin{array}{l}1.992 \\
2.165 \\
2.384 \\
2.560 \\
3.016 \\
3.476 \\
3.998 \\
4.531 \\
5.098 \\
5.701\end{array}$ & $\begin{array}{l}11.54 \\
11.78 \\
12.07 \\
12.44 \\
12.92 \\
13.52 \\
14.20 \\
14.90 \\
15.65 \\
16.45\end{array}$ & $\begin{array}{l}0.3072 \\
0.3712 \\
0.4307 \\
0.4910 \\
0.5549 \\
0.6232 \\
0.6926 \\
0.7597 \\
0.8256 \\
0.8905\end{array}$ & $\begin{array}{l}0.2719 \\
0.3050 \\
0.3432 \\
0.3877 \\
0.4447 \\
0.5080 \\
0.5286 \\
0.5487 \\
0.5705 \\
0.5917\end{array}$ & $\begin{array}{l}0.2987 \\
0.3216 \\
0.3769 \\
0.4487 \\
0.5366 \\
0.6303 \\
0.6799 \\
0.7296 \\
0.7824 \\
0.8359\end{array}$ & $\begin{array}{l}1355.0 \\
1381.0 \\
1410.0 \\
1428.0 \\
1429.0 \\
1414.0 \\
1405.0 \\
1390.0 \\
1371.0 \\
1351.0\end{array}$ \\
\hline $\begin{array}{l}14.0 \\
15.0 \\
16.0 \\
17.0 \\
18.0 \\
19.0 \\
20.0 \\
22.0 \\
24.0 \\
26.0\end{array}$ & $\begin{array}{l}0.09870 \\
0.1008 \\
0.1030 \\
0.1054 \\
0.1380 \\
0.1108 \\
0.1139 \\
0.1205 \\
0.1278 \\
0.1357\end{array}$ & $\begin{array}{l}263.0 \\
248.0 \\
233.0 \\
220.0 \\
208.0 \\
196.0 \\
185.0 \\
168.0 \\
155.0 \\
148.0\end{array}$ & $\begin{array}{l}53.8 \\
52.4 \\
50.9 \\
49.2 \\
47.5 \\
45.6 \\
43.6 \\
39.8 \\
36.1 \\
32.7\end{array}$ & $\begin{array}{r}6.340 \\
7.015 \\
7.724 \\
8.466 \\
9.239 \\
10.10 \\
10.99 \\
12.85 \\
14.76 \\
16.70\end{array}$ & $\begin{array}{l}17.31 \\
18.21 \\
19.17 \\
20.18 \\
21.24 \\
22.42 \\
23.64 \\
26.23 \\
28.96 \\
31.78\end{array}$ & $\begin{array}{l}0.9546 \\
1.018 \\
1.081 \\
1.143 \\
1.205 \\
1.269 \\
1.333 \\
1.458 \\
1.578 \\
1.692\end{array}$ & $\begin{array}{l}0.6113 \\
0.6292 \\
0.6451 \\
0.6594 \\
0.6720 \\
0.6847 \\
0.6949 \\
0.7101 \\
0.7204 \\
0.7275\end{array}$ & $\begin{array}{l}0.8892 \\
0.9420 \\
0.9939 \\
1.045 \\
1.094 \\
1.143 \\
1.188 \\
1.268 \\
1.331 \\
1.372\end{array}$ & $\begin{array}{l}1331.0 \\
1310.0 \\
1290.0 \\
1270.0 \\
1252.0 \\
1231.0 \\
1212.0 \\
1178.0 \\
1153.0 \\
1135.0\end{array}$ \\
\hline $\begin{array}{l}28.0 \\
36.0 \\
32.0 \\
34.0 \\
36.0 \\
38.0 \\
40.0\end{array}$ & $\begin{array}{l}0.1441 \\
0.1529 \\
0.1620 \\
0.1713 \\
0.1808 \\
0.1904 \\
0.2001\end{array}$ & $\begin{array}{l}144.0 \\
142.0 \\
143.0 \\
144.0 \\
147.0 \\
150.0 \\
154.0\end{array}$ & $\begin{array}{l}29.8 \\
27.3 \\
25.1 \\
23.2 \\
21.5 \\
20.0 \\
18.7\end{array}$ & $\begin{array}{l}18.58 \\
20.42 \\
22.26 \\
24.08 \\
25.89 \\
27.69 \\
29.47\end{array}$ & $\begin{array}{l}34.59 \\
37.41 \\
40.26 \\
43.12 \\
45.98 \\
48.84 \\
51.70\end{array}$ & $\begin{array}{l}1.797 \\
1.894 \\
1.986 \\
2.072 \\
2.154 \\
2.232 \\
2.305\end{array}$ & $\begin{array}{l}0.7333 \\
0.7383 \\
0.7426 \\
0.7460 \\
0.7488 \\
0.7512 \\
0.7530\end{array}$ & $\begin{array}{l}1.398 \\
1.417 \\
1.427 \\
1.432 \\
1.432 \\
1.430 \\
1.427\end{array}$ & $\begin{array}{l}1127.0 \\
1125.0 \\
1127.0 \\
1133.0 \\
1141.0 \\
1152.0 \\
1163.0\end{array}$ \\
\hline $\begin{array}{r}45.0 \\
50.0 \\
55.0 \\
60.0 \\
70.0 \\
80.0 \\
90.0 \\
100.0 \\
120.0 \\
140.0\end{array}$ & $\begin{array}{l}0.2245 \\
0.2490 \\
0.2735 \\
0.2978 \\
0.3460 \\
0.3937 \\
0.4437 \\
0.4873 \\
0.5796 \\
0.6739\end{array}$ & $\begin{array}{l}165.0 \\
178.0 \\
192.0 \\
206.0 \\
234.0 \\
263.0 \\
292.0 \\
320.0 \\
376.0 \\
432.0\end{array}$ & $\begin{array}{c}16.1 \\
14.1 \\
12.5 \\
11.2 \\
9.36 \\
8.03 \\
7.03 \\
6.26 \\
5.14 \\
4.36\end{array}$ & $\begin{array}{c}33.86 \\
38.16 \\
42.38 \\
46.54 \\
54.71 \\
62.73 \\
70.63 \\
78.45 \\
93.92 \\
109.2\end{array}$ & $\begin{array}{c}58.80 \\
65.82 \\
72.77 \\
79.63 \\
93.16 \\
106.5 \\
119.6 \\
132.6 \\
158.3 \\
183.8\end{array}$ & $\begin{array}{l}2.472 \\
2.620 \\
2.753 \\
2.872 \\
3.081 \\
3.258 \\
3.413 \\
3.550 \\
3.784 \\
3.981\end{array}$ & $\begin{array}{l}0.7563 \\
0.7580 \\
0.7589 \\
0.7593 \\
0.7591 \\
0.7584 \\
0.7575 \\
0.7566 \\
0.7550 \\
0.7537\end{array}$ & $\begin{array}{l}1.413 \\
1.397 \\
1.381 \\
1.366 \\
1.341 \\
1.321 \\
1.306 \\
1.295 \\
1.279 \\
1.268\end{array}$ & $\begin{array}{l}1196.0 \\
1233.0 \\
1270.0 \\
1309.0 \\
1384.0 \\
1457.0 \\
1526.0 \\
1593.0 \\
1718.0 \\
1835.0\end{array}$ \\
\hline $\begin{array}{l}160.0 \\
180.0 \\
200.0 \\
250.0 \\
300.0 \\
350.0 \\
400.0 \\
450.0 \\
500.0 \\
600.0\end{array}$ & $\begin{array}{l}0.7616 \\
0.8519 \\
0.9419 \\
1.166 \\
1.390 \\
1.613 \\
1.836 \\
2.059 \\
2.282 \\
2.728\end{array}$ & $\begin{array}{r}487.0 \\
541.0 \\
595.0 \\
730.0 \\
864.0 \\
1000.0 \\
1130.0 \\
1260.0 \\
1400.0 \\
1670.0\end{array}$ & $\begin{array}{l}3.80 \\
3.36 \\
3.02 \\
2.40 \\
2.00 \\
1.71 \\
1.50 \\
1.33 \\
1.20 \\
1.00\end{array}$ & $\begin{array}{l}124.5 \\
139.5 \\
154.7 \\
192.3 \\
229.8 \\
267.2 \\
304.5 \\
341.9 \\
379.2 \\
453.8\end{array}$ & $\begin{array}{l}209.1 \\
234.2 \\
259.3 \\
321.9 \\
384.2 \\
446.4 \\
508.5 \\
570.7 \\
632.7 \\
756.9\end{array}$ & $\begin{array}{l}4.150 \\
4.298 \\
4.430 \\
4.709 \\
4.936 \\
5.128 \\
5.294 \\
5.440 \\
5.571 \\
5.798\end{array}$ & $\begin{array}{l}0.7527 \\
0.7518 \\
0.7511 \\
0.7498 \\
0.7489 \\
0.7483 \\
0.7479 \\
0.7476 \\
0.7473 \\
0.7469\end{array}$ & $\begin{array}{l}1.261 \\
1.257 \\
1.253 \\
1.248 \\
1.245 \\
1.244 \\
1.243 \\
1.242 \\
1.242 \\
1.241\end{array}$ & $\begin{array}{l}1944.0 \\
2047.0 \\
2145.0 \\
2372.0 \\
2580.0 \\
2772.0 \\
2951.0 \\
3120.0 \\
3281.0 \\
3580.0\end{array}$ \\
\hline $\begin{array}{r}700.0 \\
860.0 \\
900.0 \\
1000.0 \\
1200.0 \\
1400.0 \\
1600.0 \\
1800.0 \\
2060.0 \\
2500.0\end{array}$ & $\begin{array}{r}3.174 \\
3.620 \\
4.066 \\
4.512 \\
5.404 \\
6.296 \\
7.188 \\
8.081 \\
8.973 \\
11.20\end{array}$ & $\begin{array}{l}1930.0 \\
2200.0 \\
2470.0 \\
2730.0 \\
3270.0 \\
3800.0 \\
4340.0 \\
4870.0 \\
5410.0 \\
6740.0\end{array}$ & $\begin{array}{l}0.855 \\
0.748 \\
0.665 \\
0.599 \\
0.499 \\
0.428 \\
0.374 \\
0.333 \\
0.300 \\
0.240\end{array}$ & $\begin{array}{r}528.4 \\
602.9 \\
677.4 \\
752.0 \\
901.0 \\
1050.0 \\
1199.0 \\
1348.0 \\
1497.0 \\
1870.0\end{array}$ & $\begin{array}{r}881.0 \\
1005.0 \\
1129.0 \\
1253.0 \\
1501.0 \\
1750.0 \\
1998.0 \\
2246.0 \\
2494.0 \\
3114.0\end{array}$ & $\begin{array}{l}5.989 \\
6.155 \\
6.301 \\
6.431 \\
6.658 \\
6.849 \\
7.015 \\
7.161 \\
7.292 \\
7.568\end{array}$ & $\begin{array}{l}0.7467 \\
0.7465 \\
0.7463 \\
0.7462 \\
0.7460 \\
0.7459 \\
0.7458 \\
0.7458 \\
0.7457 \\
0.7456\end{array}$ & $\begin{array}{l}1.241 \\
1.241 \\
1.241 \\
1.241 \\
1.241 \\
1.241 \\
1.241 \\
1.241 \\
1.241 \\
1.241\end{array}$ & $\begin{array}{l}3857.0 \\
4115.0 \\
4358.0 \\
4588.0 \\
5017.0 \\
5412.0 \\
5781.0 \\
6127.0 \\
6455.0 \\
7210.0\end{array}$ \\
\hline 3000.0 & 13.44 & 8080.0 & 0.200 & 2242.0 & 3735.0 & 7.795 & 0.7455 & 1.241 & 7893.0 \\
\hline
\end{tabular}

* tho-phase bounoary 
600 PSIA ISOBAR

\begin{tabular}{|c|c|c|c|c|c|c|c|c|c|c|}
\hline $\begin{array}{c}\text { TEMPERA TURE } \\
\text { OEG. R }\end{array}$ & $\begin{aligned} \text { DENSITY } \\
\\
\text { LB/CU FT }\end{aligned}$ & $\begin{array}{l}V(D H / O V)_{P} \\
\text { BTU/LB }\end{array}$ & $\begin{array}{l}V(D P / D U)_{V}- \\
\text { PSIA-CU FT/BTU }\end{array}$ & $\begin{array}{l}-V(D P / O V)_{T} \\
U \text { PSIA }\end{array}$ & $\begin{array}{l}\text { COVIOTISV } \\
\text { 1/OEG. R }\end{array}$ & $\begin{array}{l}\text { THERMAL } \\
\text { CONDUCTIVITY } \\
\text { BTU/FT-HR-R }\end{array}$ & $\begin{array}{r}\text { VISCOSITY } \\
\text { LB/FT }-S E C \\
\times \quad 10 E+6\end{array}$ & $\begin{array}{l}\text { THERMAL } \\
\text { OIFFUSIVITY } \\
\text { SQ FT/HR }\end{array}$ & $\begin{array}{l}\text { DIELECTRIC } \\
\text { CDNSTANT }\end{array}$ & $\begin{array}{l}\text { PRANDIL } \\
\text { NUMBER }\end{array}$ \\
\hline $\begin{array}{r}4.0 \\
5.0 \\
6.0 \\
7.0 \\
8.0 \\
9.0 \\
10.0 \\
11.00 \\
12.0 \\
13.0\end{array}$ & $\begin{array}{l}11.64 \\
11.55 \\
11.47 \\
11.35 \\
11.22 \\
11.06 \\
10.89 \\
10.71 \\
10.53 \\
10.33\end{array}$ & $\begin{array}{l}29.8 \\
47.5 \\
42.8 \\
40.3 \\
41.2 \\
43.4 \\
43.3 \\
43.3 \\
43.6 \\
43.8\end{array}$ & $\begin{array}{c}13.3 \\
8.67 \\
10.0 \\
10.9 \\
10.7 \\
9.94 \\
9.84 \\
9.62 \\
9.31 \\
8.99\end{array}$ & $\begin{array}{l}4200.0 \\
4510.0 \\
4480.0 \\
4320.0 \\
4100.0 \\
3840.0 \\
3610.0 \\
3360.0 \\
3120.0 \\
2880.0\end{array}$ & $\begin{array}{l}0.0100 \\
0.00677 \\
0.00881 \\
0.0111 \\
0.0130 \\
0.0145 \\
0.0157 \\
0.0168 \\
0.0179 \\
0.0191\end{array}$ & $\begin{array}{l}0.0118 \\
0.0146 \\
0.0160 \\
0.0173 \\
0.0186 \\
0.0199 \\
0.0210 \\
0.0220 \\
0.0228 \\
0.0236\end{array}$ & $\begin{array}{l}9.42 \\
8.60 \\
7.71 \\
6.90 \\
6.23 \\
5.68 \\
5.25 \\
4.90 \\
4.63 \\
4.40\end{array}$ & $\begin{array}{l}0.00338 \\
0.00393 \\
0.00369 \\
0.00339 \\
0.00309 \\
0.00285 \\
0.00284 \\
0.00281 \\
0.00277 \\
0.00273\end{array}$ & $\begin{array}{l}1.02095 \\
1.02097 \\
1.02098 \\
1.02100 \\
1.02101 \\
1.02102 \\
1.02101 \\
1.02100 \\
1.02098 \\
1.02094\end{array}$ & $\begin{array}{l}0.861 \\
0.683 \\
0.655 \\
0.645 \\
0.647 \\
0.649 \\
0.612 \\
0.585 \\
0.570 \\
0.562\end{array}$ \\
\hline $\begin{array}{l}14.0 \\
15.0 \\
16.0 \\
17.0 \\
18.0 \\
19.0 \\
20.0 \\
22.0 \\
24.0 \\
26.0\end{array}$ & $\begin{array}{l}10.13 \\
9.923 \\
9.707 \\
9.485 \\
9.257 \\
9.022 \\
8.783 \\
8.301 \\
7.825 \\
7.363\end{array}$ & $\begin{array}{l}44.0 \\
44.2 \\
44.2 \\
44.3 \\
44.3 \\
44.3 \\
44.3 \\
44.4 \\
44.8 \\
45.6\end{array}$ & $\begin{array}{l}8.68 \\
8.39 \\
8.12 \\
7.87 \\
7.63 \\
7.38 \\
7.15 \\
6.75 \\
6.41 \\
6.11\end{array}$ & $\begin{array}{l}2660.0 \\
2460.0 \\
2260.0 \\
2090.0 \\
1920.0 \\
1770.0 \\
1630.0 \\
1390.0 \\
1220.0 \\
1090.0\end{array}$ & $\begin{array}{l}0.0202 \\
0.0213 \\
0.0225 \\
0.0236 \\
0.0247 \\
0.0258 \\
0.0268 \\
0.0286 \\
0.0297 \\
0.0301\end{array}$ & $\begin{array}{l}0.0241 \\
0.0245 \\
0.0249 \\
0.0251 \\
0.0252 \\
0.0253 \\
0.0253 \\
0.0252 \\
0.0250 \\
0.0248\end{array}$ & $\begin{array}{l}4.21 \\
4.05 \\
3.92 \\
3.81 \\
3.71 \\
3.63 \\
3.56 \\
3.46 \\
3.39 \\
3.36\end{array}$ & $\begin{array}{l}0.00268 \\
0.00263 \\
0.00258 \\
0.00253 \\
0.00249 \\
0.00245 \\
0.00242 \\
0.00239 \\
0.00240 \\
0.00245\end{array}$ & $\begin{array}{l}1.02088 \\
1.02081 \\
1.02072 \\
1.02061 \\
1.02048 \\
1.02032 \\
1.02015 \\
1.01973 \\
1.01924 \\
1.01869\end{array}$ & $\begin{array}{l}0.559 \\
0.560 \\
0.564 \\
0.571 \\
0.580 \\
0.591 \\
0.603 \\
0.628 \\
0.651 \\
0.670\end{array}$ \\
\hline $\begin{array}{l}28.0 \\
30.0 \\
32.0 \\
34.0 \\
36.0 \\
38.0 \\
40.0\end{array}$ & $\begin{array}{l}6.939 \\
6.540 \\
6.173 \\
5.837 \\
5.531 \\
5.252 \\
4.998\end{array}$ & $\begin{array}{l}46.8 \\
48.4 \\
50.2 \\
52.1 \\
54.2 \\
56.5 \\
58.7\end{array}$ & $\begin{array}{l}5.85 \\
5.65 \\
5.47 \\
5.32 \\
5.19 \\
5.07 \\
4.97\end{array}$ & $\begin{array}{r}1000.0 \\
931.0 \\
881.0 \\
843.0 \\
813.0 \\
790.0 \\
771.0\end{array}$ & $\begin{array}{l}0.0299 \\
0.0293 \\
0.0285 \\
0.0275 \\
0.0264 \\
0.0253 \\
0.0243\end{array}$ & $\begin{array}{l}0.0246 \\
0.0244 \\
0.0243 \\
0.0243 \\
0.0243 \\
0.0244 \\
0.0245\end{array}$ & $\begin{array}{l}3.34 \\
3.34 \\
3.36 \\
3.38 \\
3.41 \\
3.45 \\
3.49\end{array}$ & $\begin{array}{l}0.00253 \\
0.00264 \\
0.00276 \\
0.00291 \\
0.00307 \\
0.00324 \\
0.00343\end{array}$ & $\begin{array}{l}1.01812 \\
1.01752 \\
1.01692 \\
1.01634 \\
1.01577 \\
1.01522 \\
1.01470\end{array}$ & $\begin{array}{l}0.685 \\
0.698 \\
0.709 \\
0.717 \\
0.724 \\
0.729 \\
0.732\end{array}$ \\
\hline $\begin{array}{r}45.0 \\
50.0 \\
55.0 \\
60.0 \\
70.0 \\
80.0 \\
90.0 \\
100.0 \\
120.0 \\
140.0\end{array}$ & $\begin{array}{l}4.454 \\
4.016 \\
3.656 \\
3.358 \\
2.890 \\
2.540 \\
2.269 \\
2.052 \\
1.725 \\
1.491\end{array}$ & $\begin{array}{r}64.7 \\
71.0 \\
77.3 \\
83.8 \\
96.8 \\
110.0 \\
123.0 \\
136.0 \\
162.0 \\
187.0\end{array}$ & $\begin{array}{l}4.77 \\
4.62 \\
4.51 \\
4.41 \\
4.27 \\
4.17 \\
4.09 \\
4.03 \\
3.94 \\
3.88\end{array}$ & $\begin{array}{l}737.0 \\
715.0 \\
700.0 \\
690.0 \\
677.0 \\
668.0 \\
662.0 \\
657.0 \\
649.0 \\
644.0\end{array}$ & $\begin{array}{l}0.0218 \\
0.0197 \\
0.0179 \\
0.0163 \\
0.0138 \\
0.0120 \\
0.0106 \\
0.00953 \\
0.00791 \\
0.00678\end{array}$ & $\begin{array}{l}0.0248 \\
0.0253 \\
0.0259 \\
0.0266 \\
0.0283 \\
0.0296 \\
0.0311 \\
0.0327 \\
0.0359 \\
0.0391\end{array}$ & $\begin{array}{l}3.60 \\
3.72 \\
3.85 \\
3.98 \\
4.25 \\
4.51 \\
4.77 \\
5.03 \\
5.52 \\
6.00\end{array}$ & $\begin{array}{l}0.00395 \\
0.00452 \\
0.00514 \\
0.00580 \\
0.00723 \\
0.00881 \\
0.0105 \\
0.0123 \\
0.0163 \\
0.0207\end{array}$ & $\begin{array}{l}1.01351 \\
1.01248 \\
1.01159 \\
1.01081 \\
1.00953 \\
1.00853 \\
1.00772 \\
1.00705 \\
1.00603 \\
1.00526\end{array}$ & $\begin{array}{l}0.737 \\
0.739 \\
0.738 \\
0.736 \\
0.731 \\
0.726 \\
0.721 \\
0.716 \\
0.708 \\
0.701\end{array}$ \\
\hline $\begin{array}{l}160.0 \\
180.0 \\
200.0 \\
250.0 \\
300.0 \\
350.0 \\
400.0 \\
450.0 \\
500.0 \\
600.0\end{array}$ & $\begin{array}{l}1.313 \\
1.174 \\
1.062 \\
0.8576 \\
0.7196 \\
0.06200 \\
0.5446 \\
0.4856 \\
0.4382 \\
0.3666\end{array}$ & $\begin{array}{l}212.0 \\
238.0 \\
263.0 \\
325.0 \\
387.0 \\
449.0 \\
511.0 \\
573.0 \\
635.0 \\
759.0\end{array}$ & $\begin{array}{l}3.84 \\
3.81 \\
3.78 \\
3.74 \\
3.71 \\
3.69 \\
3.68 \\
3.66 \\
3.666 \\
3.64\end{array}$ & $\begin{array}{l}639.0 \\
635.0 \\
632.0 \\
626.0 \\
622.0 \\
619.0 \\
616.0 \\
614.0 \\
613.0 \\
610.0\end{array}$ & $\begin{array}{l}0.00594 \\
0.00529 \\
0.00477 \\
0.00384 \\
0.00322 \\
0.00277 \\
0.00243 \\
0.00217 \\
0.00195 \\
0.00163\end{array}$ & $\begin{array}{l}0.0422 \\
0.0452 \\
0.0482 \\
0.0554 \\
0.0622 \\
0.0687 \\
0.0748 \\
0.0807 \\
0.0863 \\
0.0973\end{array}$ & $\begin{array}{l}6.46 \\
6.90 \\
7.21 \\
8.25 \\
9.24 \\
10.2 \\
11.1 \\
12.0 \\
12.8 \\
14.5\end{array}$ & $\begin{array}{l}0.0255 \\
0.0306 \\
0.0362 \\
0.0517 \\
0.0694 \\
0.0891 \\
0.111 \\
0.134 \\
0.159 \\
0.214\end{array}$ & $\begin{array}{l}1.00468 \\
1.00421 \\
1.00382 \\
1.00312 \\
1.00263 \\
1.00228 \\
1.00201 \\
1.00180 \\
1.00162 \\
1.00136\end{array}$ & $\begin{array}{l}0.696 \\
0.691 \\
0.675 \\
0.670 \\
0.666 \\
0.664 \\
0.663 \\
0.663 \\
0.664 \\
0.665\end{array}$ \\
\hline $\begin{array}{r}700.0 \\
800.0 \\
90000 \\
1000.0 \\
1200.0 \\
1400.0 \\
1600.0 \\
1800.0 \\
2000.0 \\
2500.0\end{array}$ & $\begin{array}{l}0.3151 \\
0.2763 \\
0.2460 \\
0.2216 \\
0.1851 \\
0.1588 \\
0.1391 \\
0.1238 \\
0.1114 \\
0.08925\end{array}$ & $\begin{array}{r}883.0 \\
1010.0 \\
1130.0 \\
1260.0 \\
1500.0 \\
1750.0 \\
2000.0 \\
2250.0 \\
2500.0 \\
3120.0\end{array}$ & $\begin{array}{l}3.64 \\
3.63 \\
3.62 \\
3.62 \\
3.62 \\
3.61 \\
3.61 \\
3.61 \\
3.61 \\
3.600\end{array}$ & $\begin{array}{l}609.0 \\
608.0 \\
607.0 \\
606.0 \\
605.0 \\
604.0 \\
603.0 \\
603.0 \\
602.0 \\
602.0\end{array}$ & $\begin{array}{l}0.00140 \\
0.00123 \\
0.00110 \\
0.000989 \\
0.000825 \\
0.000709 \\
0.000621 \\
0.000552 \\
0.000497 \\
0.000398\end{array}$ & $\begin{array}{l}0.108 \\
0.118 \\
0.128 \\
0.138 \\
0.157 \\
0.175 \\
0.192 \\
0.208 \\
0.224 \\
0.263\end{array}$ & $\begin{array}{l}16.1 \\
17.6 \\
19.1 \\
20.6 \\
23.3 \\
26.0 \\
28.6 \\
31.0 \\
33.4 \\
39.2\end{array}$ & $\begin{array}{l}0.276 \\
0.345 \\
0.420 \\
0.502 \\
0.682 \\
0.886 \\
1.11 \\
1.36 \\
1.62 \\
2.37\end{array}$ & $\begin{array}{l}1.00117 \\
1.00103 \\
1.00092 \\
1.00083 \\
1.00069 \\
1.00060 \\
1.00052 \\
1.00046 \\
1.00042 \\
1.00034\end{array}$ & $\begin{array}{l}0.665 \\
0.666 \\
0.666 \\
0.666 \\
0.666 \\
0.666 \\
0.666 \\
0.666 \\
0.666 \\
0.665\end{array}$ \\
\hline 3000.0 & 0.07443 & 3740.0 & 3.60 & 601.0 & 0.000332 & 0.299 & 44.6 & 3.24 & 1.00028 & 0.665 \\
\hline
\end{tabular}


700 PSIA ISOBAR

THERMOOYNAMIC PROPERTIES OF HELIUM 4

\begin{tabular}{|c|c|c|c|c|c|c|c|c|c|}
\hline $\begin{array}{c}\text { TEMPERATURE } \\
\text { OEG. R }\end{array}$ & $\begin{array}{l}\text { VOLUME } \\
\text { CU FT/LB }\end{array}$ & $\begin{array}{c}\text { ISOTHERM } \\
\text { DERIVATIVE } \\
\text { CU FT-PSIANL }\end{array}$ & $\begin{array}{l}\text { ISOCHORE } \\
\text { OERIVATIVE } \\
\text { PSIA/R }\end{array}$ & $\begin{array}{l}\text { INTERNAL } \\
\text { ENERGY } \\
\text { BTU/LB }\end{array}$ & $\begin{array}{l}\text { ENTHALPY } \\
\text { BTU/LB }\end{array}$ & $\begin{array}{l}\text { ENTROPY } \\
\text { BTU/LB-R }\end{array}$ & $\begin{array}{l}\text { CV } \\
\text { BTU }\end{array}$ & , LB-R & $\begin{array}{l}\text { VELOCITY } \\
\text { OF SOUND } \\
\text { FT/SEC }\end{array}$ \\
\hline $\begin{array}{r}5.0 \\
6.0 \\
7.0 \\
8.0 \\
9.0 \\
10.0 \\
11.0 \\
12.0 \\
13.0\end{array}$ & $\begin{array}{l}0.08472 \\
0.08537 \\
0.08617 \\
0.08715 \\
0.08828 \\
0.08952 \\
0.09082 \\
0.09223 \\
0.09375\end{array}$ & $\begin{array}{l}419.0 \\
426.0 \\
419.0 \\
406.0 \\
390.0 \\
374.0 \\
357.0 \\
340.0 \\
323.0\end{array}$ & $\begin{array}{l}36.7 \\
42.7 \\
50.4 \\
55.4 \\
57.8 \\
58.7 \\
58.7 \\
58.3 \\
57.5\end{array}$ & $\begin{array}{l}2.316 \\
2.512 \\
2.762 \\
3.094 \\
3.531 \\
4.029 \\
4.536 \\
5.076 \\
5.651\end{array}$ & $\begin{array}{l}13.30 \\
13.58 \\
13.93 \\
14.39 \\
14.97 \\
15.63 \\
16.31 \\
17.03 \\
17.80\end{array}$ & $\begin{array}{l}0.3571 \\
0.4154 \\
0.4731 \\
0.5346 \\
0.6005 \\
0.6676 \\
0.7326 \\
0.7962 \\
0.8587\end{array}$ & $\begin{array}{l}0.3031 \\
0.3282 \\
0.3728 \\
0.4327 \\
0.4989 \\
0.5215 \\
0.5431 \\
0.5659 \\
0.5877\end{array}$ & $\begin{array}{l}0.3244 \\
0.3628 \\
0.4311 \\
0.5178 \\
0.6103 \\
0.6581 \\
0.7053 \\
0.7549 \\
0.8048\end{array}$ & $\begin{array}{l}1441.0 \\
1477.0 \\
1499.0 \\
1500.0 \\
1487.0 \\
1479.0 \\
1466.0 \\
1449.0 \\
1431.0\end{array}$ \\
\hline $\begin{array}{l}14.0 \\
15.0 \\
16.0 \\
17.0 \\
18.0 \\
19.0 \\
20.0 \\
22.0 \\
24.0 \\
26.0\end{array}$ & $\begin{array}{l}0.09537 \\
0.09712 \\
0.09898 \\
0.1010 \\
0.1031 \\
0.1054 \\
0.1078 \\
0.1131 \\
0.1189 \\
0.1252\end{array}$ & $\begin{array}{l}306.0 \\
291.0 \\
276.0 \\
262.0 \\
249.0 \\
237.0 \\
225.0 \\
205.0 \\
189.0 \\
178.0\end{array}$ & $\begin{array}{l}56.5 \\
55.4 \\
54.1 \\
52.6 \\
51.1 \\
49.3 \\
47.5 \\
44.0 \\
40.5 \\
37.1\end{array}$ & $\begin{array}{c}6.259 \\
6.900 \\
7.573 \\
8.277 \\
9.009 \\
9.834 \\
10.69 \\
12.46 \\
14.30 \\
16.19\end{array}$ & $\begin{array}{l}18.62 \\
19.49 \\
20.40 \\
21.37 \\
22.37 \\
23.49 \\
24.66 \\
27.12 \\
29.71 \\
32.42\end{array}$ & $\begin{array}{l}0.9203 \\
0.9812 \\
1.041 \\
1.101 \\
1.159 \\
1.221 \\
1.282 \\
1.400 \\
1.515 \\
1.624\end{array}$ & $\begin{array}{l}0.6078 \\
0.6260 \\
0.6422 \\
0.6567 \\
0.6696 \\
0.6824 \\
0.5929 \\
0.7088 \\
0.7197 \\
0.7274\end{array}$ & $\begin{array}{l}0.8541 \\
0.9025 \\
0.9498 \\
0.996 \\
1.041 \\
1.084 \\
1.125 \\
1.200 \\
1.263 \\
1.311\end{array}$ & $\begin{array}{l}1412.0 \\
1393.0 \\
1375.0 \\
1357.0 \\
1339.0 \\
1319.0 \\
1301.0 \\
1268.0 \\
1240.0 \\
1219.0\end{array}$ \\
\hline $\begin{array}{l}28.0 \\
30.0 \\
32.0 \\
34.0 \\
36.0 \\
38.0 \\
40.0\end{array}$ & $\begin{array}{l}0.1320 \\
0.1371 \\
0.1465 \\
0.1542 \\
0.1620 \\
0.1700 \\
0.1781\end{array}$ & $\begin{array}{l}171.0 \\
167.0 \\
165.0 \\
165.0 \\
166.0 \\
168.0 \\
171.0\end{array}$ & $\begin{array}{l}34.1 \\
31.4 \\
29.0 \\
26.9 \\
25.1 \\
23.4 \\
21.9\end{array}$ & $\begin{array}{l}18.03 \\
19.83 \\
21.64 \\
23.44 \\
25.24 \\
27.03 \\
28.81\end{array}$ & $\begin{array}{l}35.14 \\
37.86 \\
40.63 \\
43.43 \\
46.25 \\
49.07 \\
51.90\end{array}$ & $\begin{array}{l}1.725 \\
1.819 \\
1.908 \\
1.993 \\
2.074 \\
2.150 \\
2.223\end{array}$ & $\begin{array}{l}0.7333 \\
0.7384 \\
0.7426 \\
0.7460 \\
0.7489 \\
0.7512 \\
0.7532\end{array}$ & $\begin{array}{l}1.347 \\
1.375 \\
1.393 \\
1.405 \\
1.411 \\
1.414 \\
1.414\end{array}$ & $\begin{array}{l}1206.0 \\
1200.0 \\
1198.0 \\
1199.0 \\
1204.0 \\
1211.0 \\
1219.0\end{array}$ \\
\hline $\begin{array}{r}45.0 \\
50.0 \\
55.0 \\
60.0 \\
70.0 \\
80.0 \\
90.0 \\
100.0 \\
120.0 \\
140.0\end{array}$ & $\begin{array}{l}0.1987 \\
0.2194 \\
0.2402 \\
0.2610 \\
0.3023 \\
0.3432 \\
0.3837 \\
0.4238 \\
0.5031 \\
0.5816\end{array}$ & $\begin{array}{l}180.0 \\
191.0 \\
204.0 \\
217.0 \\
245.0 \\
273.0 \\
302.0 \\
330.0 \\
386.0 \\
442.0\end{array}$ & $\begin{array}{c}18.9 \\
16.6 \\
14.7 \\
13.2 \\
11.0 \\
9.43 \\
8.25 \\
7.34 \\
6.02 \\
5.10\end{array}$ & $\begin{array}{c}33.21 \\
37.53 \\
41.78 \\
45.97 \\
54.20 \\
62.28 \\
70.24 \\
78.11 \\
93.65 \\
109.0\end{array}$ & $\begin{array}{c}58.96 \\
65.97 \\
72.92 \\
79.81 \\
93.39 \\
106.8 \\
120.0 \\
133.0 \\
158.9 \\
184.4\end{array}$ & $\begin{array}{l}2.389 \\
2.537 \\
2.669 \\
2.789 \\
2.998 \\
3.177 \\
3.333 \\
3.470 \\
3.706 \\
3.903\end{array}$ & $\begin{array}{l}0.7567 \\
0.7588 \\
0.7600 \\
0.7605 \\
0.7606 \\
0.7600 \\
0.7592 \\
0.7583 \\
0.7566 \\
0.7551\end{array}$ & $\begin{array}{l}1.408 \\
1.397 \\
1.384 \\
1.371 \\
1.347 \\
1.328 \\
1.313 \\
1.301 \\
1.283 \\
1.272\end{array}$ & $\begin{array}{l}1246.0 \\
1278.0 \\
1312.0 \\
1347.0 \\
1418.0 \\
1487.0 \\
1555.0 \\
1619.0 \\
1742.0 \\
1857.0\end{array}$ \\
\hline $\begin{array}{l}160.0 \\
180.0 \\
200.0 \\
250.0 \\
300.0 \\
350.0 \\
400.0 \\
450.0 \\
500.0 \\
600.0\end{array}$ & $\begin{array}{l}0.6595 \\
0.7370 \\
0.8142 \\
1.006 \\
1.198 \\
1.389 \\
1.581 \\
1.772 \\
1.963 \\
2.345\end{array}$ & $\begin{array}{r}497.0 \\
551.0 \\
606.0 \\
740.0 \\
874.0 \\
1010.0 \\
1140.0 \\
1270.0 \\
1410.0 \\
1670.0\end{array}$ & $\begin{array}{l}4.44 \\
3.93 \\
3.52 \\
2.80 \\
2.33 \\
2.00 \\
1.75 \\
1.55 \\
1.40 \\
1.16\end{array}$ & $\begin{array}{l}124.3 \\
139.5 \\
154.6 \\
192.3 \\
229.8 \\
267.2 \\
304.6 \\
342.0 \\
379.3 \\
453.9\end{array}$ & $\begin{array}{l}209.8 \\
235.0 \\
260.1 \\
322.7 \\
385.1 \\
447.3 \\
509.5 \\
571.6 \\
633.7 \\
757.9\end{array}$ & $\begin{array}{l}4.072 \\
4.221 \\
4.353 \\
4.632 \\
4.860 \\
5.052 \\
5.218 \\
5.364 \\
5.495 \\
5.721\end{array}$ & $\begin{array}{l}0.7539 \\
0.7529 \\
0.7521 \\
0.7506 \\
0.7497 \\
0.7490 \\
0.7484 \\
0.7481 \\
0.7477 \\
0.7473\end{array}$ & $\begin{array}{l}1.264 \\
1.259 \\
1.255 \\
1.249 \\
1.246 \\
1.244 \\
1.243 \\
1.242 \\
1.242 \\
1.241\end{array}$ & $\begin{array}{l}1964.0 \\
2066.0 \\
2163.0 \\
2389.0 \\
2594.0 \\
2785.0 \\
2963.0 \\
3131.0 \\
3291.0 \\
3589.0\end{array}$ \\
\hline $\begin{array}{r}700.0 \\
800.0 \\
900.0 \\
1000.0 \\
1200.0 \\
1400.0 \\
1600.0 \\
1800.0 \\
2000.0 \\
2500.0\end{array}$ & $\begin{array}{l}2.727 \\
3.109 \\
3.491 \\
3.873 \\
4.638 \\
5.402 \\
6.167 \\
6.932 \\
7.696 \\
9.609\end{array}$ & $\begin{array}{l}1940.0 \\
2210.0 \\
2470.0 \\
2740.0 \\
3280.0 \\
3810.0 \\
4340.0 \\
4880.0 \\
5410.0 \\
6750.0\end{array}$ & $\begin{array}{l}1.00 \\
0.873 \\
0.776 \\
0.698 \\
0.582 \\
0.499 \\
0.437 \\
0.388 \\
0.349 \\
0.280\end{array}$ & $\begin{array}{r}528.5 \\
603.1 \\
677.6 \\
752.1 \\
901.2 \\
1050.0 \\
1199.0 \\
1348.0 \\
1497.0 \\
1870.0\end{array}$ & $\begin{array}{r}882.0 \\
1006.0 \\
1130.0 \\
1254.0 \\
1502.0 \\
1750.0 \\
1999.0 \\
2247.0 \\
2495.0 \\
3115.0\end{array}$ & $\begin{array}{l}5.913 \\
6.078 \\
6.224 \\
6.355 \\
6.581 \\
6.773 \\
6.938 \\
7.084 \\
7.215 \\
7.492\end{array}$ & $\begin{array}{l}0.7470 \\
0.7468 \\
0.7466 \\
0.7465 \\
0.7463 \\
0.7461 \\
0.7460 \\
0.7459 \\
0.7459 \\
0.7457\end{array}$ & $\begin{array}{l}1.241 \\
1.241 \\
1.241 \\
1.241 \\
1.241 \\
1.241 \\
1.241 \\
1.241 \\
1.241 \\
1.241\end{array}$ & $\begin{array}{l}3865.0 \\
4122.0 \\
4365.0 \\
4594.0 \\
5023.0 \\
5417.0 \\
5785.0 \\
6131.0 \\
6458.0 \\
7213.0\end{array}$ \\
\hline 3000.0 & 11.52 & 8090.0 & 0.233 & 2242.0 & 3736.0 & 7.718 & 0.7457 & 1.241 & 7895.0 \\
\hline
\end{tabular}

- tho-pHase boundary 
7NO PSIA ISOBAR

\begin{tabular}{|c|c|c|c|c|c|c|c|c|c|c|}
\hline TEMPERATUR & E DENSITY & $V(\mathrm{OH} / \mathrm{OV})_{\mathrm{P}}$ & $v(D P / D U)_{v}-$ & $-V(D P / O V)_{T}$ & $(O V / O T)_{P} / V$ & $\begin{array}{c}\text { THERMAL } \\
\text { CONOUCTIVITY }\end{array}$ & VISCOSITY & $\begin{array}{c}\text { THERMAL } \\
\text { OIFFUSIVITY }\end{array}$ & $\begin{array}{l}\text { OIELECTRIC } \\
\text { CONSTANT }\end{array}$ & $\begin{array}{l}\text { PRANOTL } \\
\text { NUMBER }\end{array}$ \\
\hline DEG. $R$ & LB/CU FT & ATU/LB & PSIA-CU FT/BTU & PSI A & 1/OEG. R & BTU/FT-HR-R & $\begin{array}{r}\text { LB/FT-SEC } \\
\times 10 E+6\end{array}$ & $S Q F T / H R$ & & \\
\hline $\begin{array}{r}5.0 \\
6.0 \\
7.0 \\
8.0 \\
9.0 \\
10.0 \\
11.0 \\
12.0 \\
13.0\end{array}$ & $\begin{array}{l}11.80 \\
11.71 \\
11.60 \\
11.48 \\
11.33 \\
11.17 \\
11.01 \\
10.84 \\
10.67\end{array}$ & $\begin{array}{l}43.7 \\
42.4 \\
41.6 \\
43.5 \\
46.6 \\
46.9 \\
47.2 \\
47.7 \\
48.1\end{array}$ & $\begin{array}{c}10.3 \\
11.1 \\
11.7 \\
11.2 \\
10.2 \\
10.1 \\
9.82 \\
9.50 \\
9.18\end{array}$ & $\begin{array}{l}4940.0 \\
4990.0 \\
4870.0 \\
4660.0 \\
4420.0 \\
4180.0 \\
3930.0 \\
3680.0 \\
3440.0\end{array}$ & $\begin{array}{l}0.00742 \\
0.00855 \\
0.0104 \\
0.0119 \\
0.01131 \\
0.0140 \\
0.0149 \\
0.0158 \\
0.0167\end{array}$ & $\begin{array}{l}0.0151 \\
0.0166 \\
0.0179 \\
0.0193 \\
0.0206 \\
0.0219 \\
0.0229 \\
0.0239 \\
0.0247\end{array}$ & $\begin{array}{c}10.0 \\
8.78 \\
7.78 \\
6.97 \\
6.31 \\
5.80 \\
5.40 \\
5.07 \\
4.81\end{array}$ & $\begin{array}{l}0.00395 \\
0.00390 \\
0.00358 \\
0.00325 \\
0.00299 \\
0.00297 \\
0.00295 \\
0.00292 \\
0.00287\end{array}$ & $\begin{array}{l}1.02091 \\
1.02093 \\
1.02096 \\
1.02098 \\
1.02100 \\
1.02101 \\
1.02102 \\
1.02101 \\
1.02100\end{array}$ & $\begin{array}{l}0.768 \\
0.693 \\
0.674 \\
0.672 \\
0.672 \\
0.628 \\
0.597 \\
0.577 \\
0.564\end{array}$ \\
\hline $\begin{array}{l}14.0 \\
15.0 \\
16.0 \\
17.0 \\
18.0 \\
19.0 \\
20.0 \\
22.0 \\
24.0 \\
26.0\end{array}$ & $\begin{array}{c}10.49 \\
10.30 \\
10.10 \\
9.903 \\
9.699 \\
9.488 \\
9.275 \\
8.843 \\
8.410 \\
7.985\end{array}$ & $\begin{array}{l}48.5 \\
48.8 \\
49.0 \\
49.1 \\
49.2 \\
49.3 \\
49.4 \\
49.5 \\
49.7 \\
50.2\end{array}$ & $\begin{array}{l}8.87 \\
8.59 \\
8.33 \\
8.09 \\
7.87 \\
7.61 \\
7.39 \\
7.01 \\
6.68 \\
6.39\end{array}$ & $\begin{array}{l}3210.0 \\
2990.0 \\
2790.0 \\
2590.0 \\
2410.0 \\
2240.0 \\
2090.0 \\
1810.0 \\
1590.0 \\
1420.0\end{array}$ & $\begin{array}{l}0.0176 \\
0.0185 \\
0.0194 \\
0.0203 \\
0.0212 \\
0.0220 \\
0.0228 \\
0.0243 \\
0.0254 \\
0.0261\end{array}$ & $\begin{array}{l}0.0253 \\
0.0258 \\
0.0262 \\
0.0265 \\
0.0267 \\
0.0268 \\
0.0269 \\
0.0269 \\
0.0267 \\
0.0266\end{array}$ & $\begin{array}{l}4.59 \\
4.40 \\
4.25 \\
4.12 \\
4.01 \\
3.92 \\
3.84 \\
3.71 \\
3.63 \\
3.58\end{array}$ & $\begin{array}{l}0.00283 \\
0.00278 \\
0.00273 \\
0.00269 \\
0.00265 \\
0.00261 \\
0.00258 \\
0.00253 \\
0.00252 \\
0.00254\end{array}$ & $\begin{array}{l}1.02097 \\
1.02093 \\
1.02087 \\
1.02080 \\
1.02072 \\
1.02061 \\
1.02049 \\
1.02019 \\
1.01983 \\
1.01941\end{array}$ & $\begin{array}{l}0.557 \\
0.554 \\
0.554 \\
0.557 \\
0.563 \\
0.569 \\
0.578 \\
0.597 \\
0.617 \\
0.636\end{array}$ \\
\hline $\begin{array}{l}28.0 \\
30.0 \\
32.0 \\
34.0 \\
36.0 \\
38.0 \\
40.0\end{array}$ & $\begin{array}{l}7.577 \\
7.189 \\
6.825 \\
6.485 \\
6.171 \\
5.881 \\
5.614\end{array}$ & $\begin{array}{l}51.2 \\
52.5 \\
54.0 \\
55.8 \\
57.7 \\
59.7 \\
61.9\end{array}$ & $\begin{array}{l}6.13 \\
5.92 \\
5.73 \\
5.57 \\
5.42 \\
5.30 \\
5.18\end{array}$ & $\begin{array}{r}1300.0 \\
1200.0 \\
1130.0 \\
1070.0 \\
1020.0 \\
989.0 \\
960.0\end{array}$ & $\begin{array}{l}0.0263 \\
0.0262 \\
0.0258 \\
0.0252 \\
0.0245 \\
0.0237 \\
0.0228\end{array}$ & $\begin{array}{l}0.0264 \\
0.0262 \\
0.0260 \\
0.0259 \\
0.0259 \\
0.0259 \\
0.0259\end{array}$ & $\begin{array}{l}3.55 \\
3.53 \\
3.54 \\
3.55 \\
3.57 \\
3.60 \\
3.63\end{array}$ & $\begin{array}{l}0.00258 \\
0.00265 \\
0.00274 \\
0.00285 \\
0.00297 \\
0.00311 \\
0.00326\end{array}$ & $\begin{array}{l}1.01895 \\
1.01846 \\
1.01795 \\
1.01744 \\
1.01692 \\
1.01642 \\
1.01593\end{array}$ & $\begin{array}{l}0.652 \\
0.668 \\
0.681 \\
0.692 \\
0.700 \\
0.708 \\
0.713\end{array}$ \\
\hline $\begin{array}{r}45.0 \\
50.0 \\
55.0 \\
60.0 \\
70.0 \\
80.0 \\
90.0 \\
100.0 \\
120.0 \\
140.0\end{array}$ & $\begin{array}{l}5.034 \\
4.557 \\
4.163 \\
3.831 \\
3.307 \\
2.913 \\
2.606 \\
2.360 \\
1.988 \\
1.719\end{array}$ & $\begin{array}{r}67.6 \\
73.6 \\
79.9 \\
86.2 \\
99.1 \\
112.0 \\
125.0 \\
138.0 \\
164.0 \\
189.0\end{array}$ & $\begin{array}{l}4.96 \\
4.79 \\
4.65 \\
4.54 \\
4.38 \\
4.26 \\
4.17 \\
4.10 \\
4.00 \\
3.93\end{array}$ & $\begin{array}{l}907.0 \\
873.0 \\
849.0 \\
832.0 \\
810.0 \\
796.0 \\
786.0 \\
779.0 \\
768.0 \\
760.0\end{array}$ & $\begin{array}{l}0.0208 \\
0.0190 \\
0.0173 \\
0.0159 \\
0.0136 \\
0.0118 \\
0.0105 \\
0.00942 \\
0.00783 \\
0.00672\end{array}$ & $\begin{array}{l}0.0261 \\
0.0265 \\
0.0270 \\
0.0276 \\
0.0289 \\
0.0303 \\
0.0318 \\
0.0334 \\
0.0365 \\
0.0396\end{array}$ & $\begin{array}{l}3.73 \\
3.84 \\
3.96 \\
4.09 \\
4.34 \\
4.60 \\
4.85 \\
5.10 \\
5.59 \\
6.06\end{array}$ & $\begin{array}{l}0.00369 \\
0.00417 \\
0.00469 \\
0.00525 \\
0.00648 \\
0.00784 \\
0.00931 \\
0.0109 \\
0.0143 \\
0.0181\end{array}$ & $\begin{array}{l}1.01478 \\
1.01375 \\
1.01283 \\
1.01203 \\
1.01067 \\
1.00960 \\
1.00872 \\
1.00799 \\
1.00685 \\
1.00601\end{array}$ & $\begin{array}{l}0.723 \\
0.728 \\
0.731 \\
0.731 \\
0.729 \\
0.725 \\
0.720 \\
0.716 \\
0.708 \\
0.701\end{array}$ \\
\hline $\begin{array}{l}160.0 \\
180.0 \\
200.0 \\
250.0 \\
300.0 \\
350.0 \\
400.0 \\
450.0 \\
500.0 \\
600.0\end{array}$ & $\begin{array}{l}1.516 \\
1.357 \\
1.228 \\
0.9936 \\
0.8346 \\
0.7197 \\
0.6326 \\
0.5644 \\
0.5095 \\
0.4265\end{array}$ & $\begin{array}{l}215.0 \\
240.0 \\
265.0 \\
328.0 \\
390.0 \\
452.0 \\
514.0 \\
576.0 \\
638.0 \\
762.0\end{array}$ & $\begin{array}{l}3.88 \\
3.84 \\
3.81 \\
3.76 \\
3.73 \\
3.70 \\
3.69 \\
3.68 \\
3.67 \\
3.65\end{array}$ & $\begin{array}{l}753.0 \\
748.0 \\
744.0 \\
735.0 \\
730.0 \\
725.0 \\
722.0 \\
719.0 \\
717.0 \\
714.0\end{array}$ & $\begin{array}{l}0.00589 \\
0.00525 \\
0.00474 \\
0.00381 \\
0.00326 \\
0.00275 \\
0.00242 \\
0.00216 \\
0.00195 \\
0.00163\end{array}$ & $\begin{array}{l}0.0426 \\
0.0450 \\
0.0486 \\
0.0557 \\
0.0625 \\
0.0690 \\
0.0751 \\
0.0810 \\
0.0865 \\
0.0974\end{array}$ & $\begin{array}{r}6.52 \\
6.96 \\
7.26 \\
8.30 \\
9.28 \\
10.2 \\
11.1 \\
12.0 \\
12.8 \\
14.5\end{array}$ & $\begin{array}{l}0.0222 \\
0.0267 \\
0.0315 \\
0.0449 \\
0.0601 \\
0.0770 \\
0.0955 \\
0.115 \\
0.137 \\
0.184\end{array}$ & $\begin{array}{l}1.00535 \\
1.03482 \\
1.00439 \\
1.00359 \\
1.00304 \\
1.00263 \\
1.00232 \\
1.00208 \\
1.00188 \\
1.00158\end{array}$ & $\begin{array}{l}0.696 \\
0.691 \\
0.675 \\
0.670 \\
0.656 \\
0.664 \\
0.663 \\
0.662 \\
0.663 \\
0.665\end{array}$ \\
\hline $\begin{array}{r}700.0 \\
800.0 \\
900.0 \\
1000.0 \\
1200.0 \\
1400.0 \\
1600.0 \\
1800.0 \\
2000.0 \\
2500.0\end{array}$ & $\begin{array}{l}0.3667 \\
0.3216 \\
0.2864 \\
0.2582 \\
0.2156 \\
0.1851 \\
0.1622 \\
0.1443 \\
0.1299 \\
0.1041\end{array}$ & $\begin{array}{r}886.0 \\
1010.0 \\
1130.0 \\
1260.0 \\
1510.0 \\
1750.0 \\
2000.0 \\
2250.0 \\
2500.0 \\
3120.0\end{array}$ & $\begin{array}{l}3.64 \\
3.63 \\
3.63 \\
3.62 \\
3.62 \\
3.61 \\
3.61 \\
3.61 \\
3.61 \\
3.60\end{array}$ & $\begin{array}{l}712.0 \\
710.0 \\
709.0 \\
708.0 \\
706.0 \\
705.0 \\
704.0 \\
704.0 \\
703.0 \\
702.0\end{array}$ & $\begin{array}{l}0.00140 \\
0.00123 \\
0.00109 \\
0.000987 \\
0.000824 \\
0.000708 \\
0.000620 \\
0.000552 \\
0.000497 \\
0.000398\end{array}$ & $\begin{array}{l}0.108 \\
0.118 \\
0.128 \\
0.138 \\
0.157 \\
0.175 \\
0.192 \\
0.208 \\
0.225 \\
0.263\end{array}$ & $\begin{array}{l}16.1 \\
17.6 \\
19.1 \\
20.6 \\
23.4 \\
26.0 \\
28.6 \\
31.1 \\
33.5 \\
39.2\end{array}$ & $\begin{array}{l}0.238 \\
0.297 \\
0.361 \\
0.431 \\
0.586 \\
0.760 \\
0.953 \\
1.16 \\
1.39 \\
2.04\end{array}$ & $\begin{array}{l}1.00136 \\
1.00120 \\
1.00107 \\
1.00096 \\
1.00081 \\
1.00069 \\
1.00061 \\
1.00054 \\
1.00049 \\
1.00039\end{array}$ & $\begin{array}{l}0.665 \\
0.665 \\
0.665 \\
0.665 \\
0.665 \\
0.665 \\
0.665 \\
0.665 \\
0.665 \\
0.665\end{array}$ \\
\hline 3000.0 & 0.08680 & 3740.0 & 3.60 & 702.0 & 0.000332 & 0.299 & 44.6 & 2.78 & 1.00033 & 0.665 \\
\hline
\end{tabular}




\begin{tabular}{|c|c|c|c|c|c|c|c|c|c|c|}
\hline $\begin{array}{c}\text { TEMPERAT } \\
\text { OEG. }\end{array}$ & $\begin{array}{l}\text { TURE } \\
\text { R }\end{array}$ & $\begin{array}{l}\text { VOLUME } \\
\text { CU FT/LB }\end{array}$ & $\begin{array}{l}\text { ISOTHERM } \\
\text { OERIVATIVE } \\
\text { CUFT-PSIA/LB }\end{array}$ & $\begin{array}{l}\text { ISOCHORE } \\
\text { OERIVATIVE } \\
\text { PSIA/R }\end{array}$ & $\begin{array}{l}\text { INTERNAL } \\
\text { ENERGY } \\
\text { BTU/LB }\end{array}$ & $\begin{array}{l}\text { ENTHALPY } \\
\text { BTU/LB }\end{array}$ & $\begin{array}{l}\text { ENTROPY } \\
\text { BTU/LB-R }\end{array}$ & $\begin{array}{l}\text { CV } \\
\text { BTU }\end{array}$ & $\begin{aligned} C P \\
\text { / LB }-R\end{aligned}$ & $\begin{array}{l}\text { VEL OCITY } \\
\text { OF SOUND } \\
\text { FT/SEC }\end{array}$ \\
\hline $\begin{array}{r}5.0 \\
6.0 \\
7.0 \\
8.0 \\
9.0 \\
16.0 \\
11.0 \\
12.0 \\
13.0\end{array}$ & & $\begin{array}{l}0.08306 \\
0.08374 \\
0.08450 \\
0.08540 \\
0.08643 \\
0.08753 \\
0.08869 \\
0.08993 \\
0.09125\end{array}$ & $\begin{array}{l}443.0 \\
458.0 \\
456.0 \\
445.0 \\
430.0 \\
415.0 \\
398.0 \\
381.0 \\
364.0\end{array}$ & $\begin{array}{l}45.1 \\
46.8 \\
53.1 \\
57.6 \\
59.8 \\
60.6 \\
60.6 \\
60.3 \\
59.7\end{array}$ & $\begin{array}{l}2.471 \\
2.650 \\
2.878 \\
3.189 \\
3.606 \\
4.084 \\
4.572 \\
5.090 \\
5.642\end{array}$ & $\begin{array}{l}14.78 \\
15.75 \\
15.40 \\
15.84 \\
16.41 \\
17.05 \\
17.71 \\
18.41 \\
19.16\end{array}$ & $\begin{array}{l}0.3423 \\
0.4006 \\
0.4566 \\
0.5162 \\
0.5803 \\
0.6457 \\
0.7089 \\
0.7707 \\
0.8314\end{array}$ & $\begin{array}{l}0.3094 \\
0.3172 \\
0.3605 \\
0.4227 \\
0.4912 \\
0.5156 \\
0.5384 \\
0.5619 \\
0.5843\end{array}$ & $\begin{array}{l}0.3388 \\
0.3544 \\
0.4178 \\
0.5032 \\
0.5945 \\
0.6409 \\
0.6862 \\
0.7334 \\
0.7805\end{array}$ & $\begin{array}{l}1498.0 \\
1540.0 \\
1564.0 \\
1566.0 \\
1553.0 \\
1547.0 \\
1534.0 \\
1518.0 \\
1501.0\end{array}$ \\
\hline $\begin{array}{l}14.0 \\
15.0 \\
16.0 \\
17.0 \\
18.0 \\
19.0 \\
20.0 \\
22.0 \\
24.0 \\
26.0\end{array}$ & & $\begin{array}{l}0.09266 \\
0.09417 \\
0.09577 \\
0.09747 \\
0.09927 \\
0.1012 \\
0.1032 \\
0.1076 \\
0.1124 \\
0.1176\end{array}$ & $\begin{array}{l}348.0 \\
332.0 \\
317.0 \\
303.0 \\
289.0 \\
276.0 \\
264.0 \\
242.0 \\
224.0 \\
210.0\end{array}$ & $\begin{array}{l}58.9 \\
57.9 \\
56.8 \\
55.5 \\
54.2 \\
52.5 \\
50.9 \\
47.5 \\
44.2 \\
41.0\end{array}$ & $\begin{array}{c}6.225 \\
6.840 \\
7.484 \\
8.158 \\
8.858 \\
9.653 \\
10.47 \\
12.19 \\
13.97 \\
15.81\end{array}$ & $\begin{array}{l}19.95 \\
20.79 \\
21.67 \\
22.60 \\
23.56 \\
24.65 \\
25.77 \\
28.13 \\
30.62 \\
33.23\end{array}$ & $\begin{array}{l}0.8911 \\
0.9499 \\
1.008 \\
1.065 \\
1.121 \\
1.181 \\
1.239 \\
1.353 \\
1.463 \\
1.569\end{array}$ & $\begin{array}{l}0.6048 \\
0.6232 \\
0.6397 \\
0.6544 \\
0.6675 \\
0.6805 \\
0.6912 \\
0.7077 \\
0.7193 \\
0.7275\end{array}$ & $\begin{array}{l}0.8268 \\
0.8721 \\
0.9162 \\
0.9592 \\
1.001 \\
1.040 \\
1.078 \\
1.148 \\
1.209 \\
1.260\end{array}$ & $\begin{array}{l}1484.0 \\
1467.0 \\
1450.0 \\
1433.0 \\
1417.0 \\
1398.0 \\
1381.0 \\
1349.0 \\
1321.0 \\
1298.0\end{array}$ \\
\hline $\begin{array}{l}28.0 \\
30.0 \\
32.0 \\
34.0 \\
36.0 \\
38.0 \\
40.0\end{array}$ & & $\begin{array}{l}0.1232 \\
0.1291 \\
0.1353 \\
0.1418 \\
0.1484 \\
0.1552 \\
0.1621\end{array}$ & $\begin{array}{l}200.0 \\
194.0 \\
189.0 \\
187.0 \\
187.0 \\
188.0 \\
189.0\end{array}$ & $\begin{array}{l}38.0 \\
35.3 \\
32.8 \\
30.5 \\
28.5 \\
26.7 \\
25.0\end{array}$ & $\begin{array}{l}17.61 \\
19.37 \\
21.14 \\
22.92 \\
24.70 \\
26.48 \\
28.25\end{array}$ & $\begin{array}{l}35.86 \\
38.49 \\
41.19 \\
43.92 \\
46.68 \\
49.47 \\
52.26\end{array}$ & $\begin{array}{l}1.667 \\
1.757 \\
1.844 \\
1.927 \\
2.006 \\
2.081 \\
2.153\end{array}$ & $\begin{array}{l}0.7337 \\
0.7388 \\
0.7430 \\
0.7464 \\
0.7492 \\
0.7516 \\
0.7535\end{array}$ & $\begin{array}{l}1.300 \\
1.334 \\
1.358 \\
1.375 \\
1.387 \\
1.394 \\
1.398\end{array}$ & $\begin{array}{l}1282.0 \\
1272.0 \\
1266.0 \\
1265.0 \\
1266.0 \\
1270.0 \\
1275.0\end{array}$ \\
\hline $\begin{array}{r}45.0 \\
50.0 \\
55.0 \\
60.0 \\
70.0 \\
80.0 \\
90.0 \\
100.0 \\
120.0 \\
140.0\end{array}$ & & $\begin{array}{l}0.1797 \\
0.1976 \\
0.2156 \\
0.2337 \\
0.2698 \\
0.3056 \\
0.3411 \\
0.3762 \\
0.4458 \\
0.5146\end{array}$ & $\begin{array}{l}196.0 \\
206.0 \\
218.0 \\
230.0 \\
256.0 \\
284.0 \\
312.0 \\
340.0 \\
396.0 \\
452.0\end{array}$ & $\begin{array}{l}21.6 \\
19.0 \\
16.9 \\
15.2 \\
12.7 \\
10.8 \\
9.48 \\
8.42 \\
6.90 \\
5.85\end{array}$ & $\begin{array}{r}32.64 \\
36.97 \\
41.24 \\
45.45 \\
53.73 \\
61.86 \\
59.86 \\
77.77 \\
93.39 \\
108.8\end{array}$ & $\begin{array}{r}59.26 \\
66.24 \\
73.18 \\
80.07 \\
93.70 \\
107.1 \\
120.4 \\
133.5 \\
159.4 \\
185.1\end{array}$ & $\begin{array}{l}2.318 \\
2.465 \\
2.597 \\
2.717 \\
2.927 \\
3.107 \\
3.263 \\
3.401 \\
3.637 \\
3.835\end{array}$ & $\begin{array}{l}0.7572 \\
0.7595 \\
0.7608 \\
0.7616 \\
0.7619 \\
0.7615 \\
0.7607 \\
0.7596 \\
0.7581 \\
0.7565\end{array}$ & $\begin{array}{l}1.399 \\
1.393 \\
1.384 \\
1.373 \\
1.352 \\
1.334 \\
1.319 \\
1.306 \\
1.288 \\
1.276\end{array}$ & $\begin{array}{l}1296.0 \\
1323.0 \\
1354.0 \\
1386.0 \\
1452.0 \\
1518.0 \\
1583.0 \\
1646.0 \\
1766.0 \\
1879.0\end{array}$ \\
\hline $\begin{array}{l}160.0 \\
180.0 \\
200.0 \\
250.0 \\
300.0 \\
350.0 \\
400.0 \\
450.0 \\
500.0 \\
600.0\end{array}$ & & $\begin{array}{l}0.5829 \\
0.6508 \\
0.7184 \\
0.8867 \\
1.054 \\
1.222 \\
1.389 \\
1.556 \\
1.723 \\
2.058\end{array}$ & $\begin{array}{r}507.0 \\
561.0 \\
616.0 \\
750.0 \\
884.0 \\
1020.0 \\
1150.0 \\
1280.0 \\
1420.0 \\
1680.0\end{array}$ & $\begin{array}{l}5.08 \\
4.49 \\
4.03 \\
3.21 \\
2.67 \\
2.28 \\
2.00 \\
1.77 \\
1.60 \\
1.33\end{array}$ & $\begin{array}{l}124.1 \\
139.4 \\
154.5 \\
192.2 \\
229.8 \\
267.3 \\
304.7 \\
342.0 \\
379.4 \\
454.0\end{array}$ & $\begin{array}{l}210.5 \\
235.8 \\
260.9 \\
323.6 \\
386.0 \\
448.3 \\
510.4 \\
572.6 \\
634.7 \\
758.8\end{array}$ & $\begin{array}{l}4.005 \\
4.154 \\
4.286 \\
4.566 \\
4.793 \\
4.985 \\
5.152 \\
5.298 \\
5.429 \\
5.655\end{array}$ & $\begin{array}{l}0.7552 \\
0.7541 \\
0.7532 \\
0.7515 \\
0.7504 \\
0.7496 \\
0.7490 \\
0.7485 \\
0.7482 \\
0.7477\end{array}$ & $\begin{array}{l}1.267 \\
1.261 \\
1.257 \\
1.250 \\
1.246 \\
1.244 \\
1.243 \\
1.242 \\
1.242 \\
1.241\end{array}$ & $\begin{array}{l}1985.0 \\
2086.0 \\
2182.0 \\
2405.0 \\
2609.0 \\
2798.0 \\
2975.0 \\
3142.0 \\
3301.0 \\
3599.0\end{array}$ \\
\hline $\begin{array}{r}700.0 \\
800.0 \\
900.0 \\
1000.0 \\
1200.0 \\
1400.0 \\
1600.0 \\
1800.0 \\
2000.0 \\
2500.0\end{array}$ & & $\begin{array}{l}2.392 \\
2.726 \\
3.060 \\
3.395 \\
4.063 \\
4.732 \\
5.401 \\
6.070 \\
6.739 \\
8.412\end{array}$ & $\begin{array}{l}1950.0 \\
2220.0 \\
2480.0 \\
2750.0 \\
3280.0 \\
3820.0 \\
4350.0 \\
4890.0 \\
5420.0 \\
6760.0\end{array}$ & $\begin{array}{l}1.14 \\
1.00 \\
0.886 \\
0.798 \\
0.665 \\
0.570 \\
0.499 \\
0.444 \\
0.399 \\
0.319\end{array}$ & $\begin{array}{r}528.6 \\
603.2 \\
677.8 \\
752.3 \\
901.4 \\
1050.0 \\
1199.0 \\
1348.0 \\
1498.0 \\
1870.0\end{array}$ & $\begin{array}{r}882.9 \\
1007.0 \\
1131.0 \\
1255.0 \\
1503.0 \\
1751.0 \\
2000.0 \\
2248.0 \\
2496.0 \\
3116.0\end{array}$ & $\begin{array}{l}5.846 \\
6.012 \\
6.158 \\
6.289 \\
6.515 \\
6.706 \\
6.872 \\
7.018 \\
7.149 \\
7.426\end{array}$ & $\begin{array}{l}0.7473 \\
0.7471 \\
0.7469 \\
0.7467 \\
0.7465 \\
0.7463 \\
0.7462 \\
0.7461 \\
0.7460 \\
0.7459\end{array}$ & $\begin{array}{l}1.241 \\
1.241 \\
1.241 \\
1.241 \\
1.241 \\
1.241 \\
1.241 \\
1.241 \\
1.241 \\
1.241\end{array}$ & $\begin{array}{l}3873.0 \\
4130.0 \\
4372.0 \\
4601.0 \\
5028.0 \\
5422.0 \\
5789.0 \\
6135.0 \\
6462.0 \\
7215.0\end{array}$ \\
\hline 3000.0 & & 10.08 & 8090.0 & 0.266 & 2243.0 & 3737.0 & 7.652 & 0.7458 & 1.241 & 7897.0 \\
\hline
\end{tabular}


600 PSIA ISOBAR

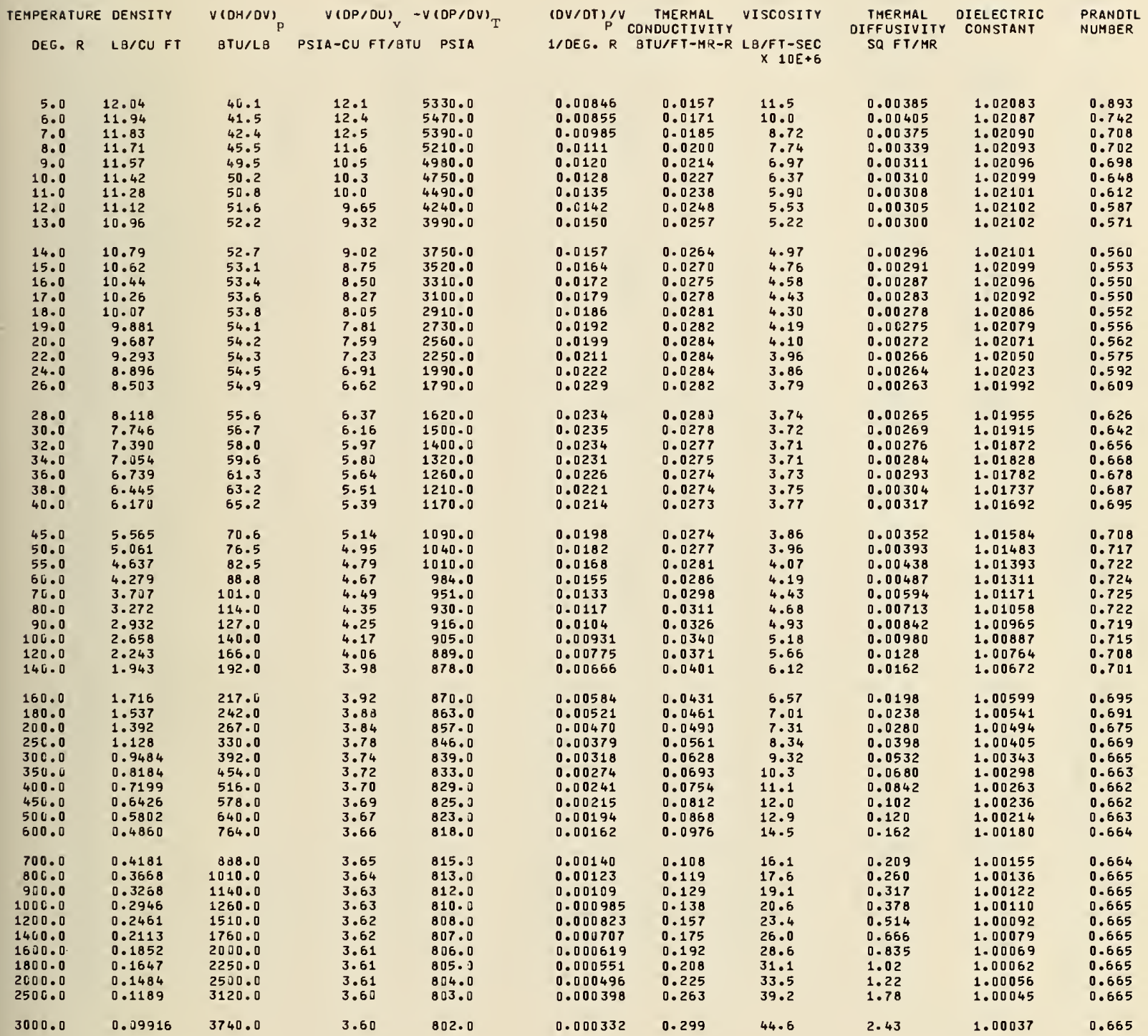




\begin{tabular}{|c|c|c|c|c|c|c|c|c|c|}
\hline $\begin{array}{c}\text { TEMPERATURE } \\
\text { OEG. R }\end{array}$ & $\begin{array}{l}\text { VOL UME } \\
\text { CU FT/LB }\end{array}$ & $\begin{array}{c}\text { I SOTHERM } \\
\text { OERI VATI VE } \\
\text { CU FT-PSIA/LB }\end{array}$ & $\begin{array}{l}\text { ISOCHORE } \\
\text { OERIVATIVE } \\
\text { PSIA/R }\end{array}$ & $\begin{array}{l}\text { INTERNAL } \\
\text { ENERGY } \\
\text { BTU/LB }\end{array}$ & $\begin{array}{l}\text { ENTHALPY } \\
\text { BTU/LB }\end{array}$ & $\begin{array}{l}\text { ENTROPY } \\
\text { BTU/LB-R }\end{array}$ & $\begin{array}{l}\text { CV } \\
\text { BTU }\end{array}$ & $\begin{array}{r}C P \\
\text {, LB }-R\end{array}$ & $\begin{array}{l}\text { VELOCITY } \\
\text { OF SOUNO } \\
\text { FT/SEC }\end{array}$ \\
\hline $\begin{array}{r}5.0 \\
6.0 \\
7.0 \\
8.0 \\
9.0 \\
10.0 \\
11.0 \\
12.0 \\
13.0\end{array}$ & $\begin{array}{l}0.08155 \\
0.08227 \\
0.08302 \\
0.08386 \\
0.08481 \\
0.38580 \\
0.08685 \\
0.08795 \\
0.08913\end{array}$ & $\begin{array}{l}463.0 \\
488.0 \\
490.0 \\
482.0 \\
468.0 \\
455.0 \\
438.0 \\
421.0 \\
404.0\end{array}$ & $\begin{array}{l}56.0 \\
51.9 \\
56.3 \\
59.9 \\
61.7 \\
62.3 \\
62.4 \\
62.1 \\
61.6\end{array}$ & $\begin{array}{l}2.627 \\
2.793 \\
3.003 \\
3.296 \\
3.697 \\
4.159 \\
4.630 \\
5.130 \\
5.663\end{array}$ & $\begin{array}{l}16.22 \\
16.50 \\
16.84 \\
17.27 \\
17.83 \\
18.46 \\
19.10 \\
19.79 \\
20.52\end{array}$ & $\begin{array}{l}0.3260 \\
0.3861 \\
0.4411 \\
0.4993 \\
0.5620 \\
0.6260 \\
0.6878 \\
0.7482 \\
0.8074\end{array}$ & $\begin{array}{l}0.3251 \\
0.3104 \\
0.3510 \\
0.4145 \\
0.4848 \\
0.5107 \\
0.5344 \\
0.5585 \\
0.5813\end{array}$ & $\begin{array}{l}0.3668 \\
0.3520 \\
0.4087 \\
0.4921 \\
0.5822 \\
0.6272 \\
0.6708 \\
0.7159 \\
0.7608\end{array}$ & $\begin{array}{l}1556.0 \\
1601.0 \\
1626.0 \\
1627.0 \\
1614.0 \\
1608.0 \\
1596.0 \\
1581.0 \\
1566.0\end{array}$ \\
\hline $\begin{array}{l}14.0 \\
15.0 \\
16.0 \\
17.0 \\
18.0 \\
19.0 \\
20.0 \\
22.0 \\
24.0 \\
26.0\end{array}$ & $\begin{array}{l}0.09038 \\
0.09170 \\
0.09311 \\
0.09459 \\
0.09616 \\
0.09783 \\
0.0996 \\
0.1033 \\
0.1074 \\
0.1118\end{array}$ & $\begin{array}{l}388.0 \\
372.0 \\
356.0 \\
342.0 \\
328.0 \\
315.0 \\
302.0 \\
279.0 \\
259.0 \\
243.0\end{array}$ & $\begin{array}{l}60.9 \\
60.1 \\
59.1 \\
58.0 \\
56.8 \\
55.3 \\
53.8 \\
50.6 \\
47.5 \\
44.4\end{array}$ & $\begin{array}{c}6.226 \\
6.819 \\
7.440 \\
8.089 \\
8.763 \\
9.535 \\
10.33 \\
11.99 \\
13.73 \\
15.52\end{array}$ & $\begin{array}{l}21.29 \\
22.10 \\
22.96 \\
23.85 \\
24.79 \\
25.84 \\
26.93 \\
29.21 \\
31.63 \\
34.15\end{array}$ & $\begin{array}{l}0.8655 \\
0.9227 \\
0.9789 \\
1.034 \\
1.089 \\
1.147 \\
1.203 \\
1.314 \\
1.420 \\
1.523\end{array}$ & $\begin{array}{l}0.6020 \\
0.6206 \\
0.6373 \\
0.6522 \\
0.6656 \\
0.6786 \\
0.6896 \\
0.7066 \\
0.7188 \\
0.7275\end{array}$ & $\begin{array}{l}0.8048 \\
0.8477 \\
0.8894 \\
0.9299 \\
0.9691 \\
1.006 \\
1.041 \\
1.107 \\
1.165 \\
1.216\end{array}$ & $\begin{array}{l}1550.0 \\
1534.0 \\
1518.0 \\
1502.0 \\
1487.0 \\
1470.0 \\
1453.0 \\
1422.0 \\
1395.0 \\
1371.0\end{array}$ \\
\hline $\begin{array}{l}28.0 \\
30.0 \\
32.0 \\
34.0 \\
36.0 \\
38.0 \\
46.0\end{array}$ & $\begin{array}{l}0.1165 \\
0.1215 \\
0.1268 \\
0.1323 \\
0.1380 \\
0.1439 \\
0.1499\end{array}$ & $\begin{array}{l}231.0 \\
222.0 \\
215.0 \\
211.0 \\
209.0 \\
209.0 \\
209.0\end{array}$ & $\begin{array}{l}41.5 \\
38.8 \\
36.2 \\
33.9 \\
31.8 \\
29.8 \\
28.1\end{array}$ & $\begin{array}{l}17.29 \\
19.00 \\
20.74 \\
22.49 \\
24.25 \\
26.01 \\
27.77\end{array}$ & $\begin{array}{l}36.70 \\
39.26 \\
41.88 \\
44.55 \\
47.26 \\
49.99 \\
52.74\end{array}$ & $\begin{array}{l}1.618 \\
1.706 \\
1.790 \\
1.871 \\
1.949 \\
2.022 \\
2.093\end{array}$ & $\begin{array}{l}0.7340 \\
0.7394 \\
0.7436 \\
0.7470 \\
0.7498 \\
0.7521 \\
0.7540\end{array}$ & $\begin{array}{l}1.259 \\
1.295 \\
1.324 \\
1.346 \\
1.362 \\
1.373 \\
1.380\end{array}$ & $\begin{array}{l}1353.0 \\
1341.0 \\
1333.0 \\
1328.0 \\
1327.0 \\
1328.0 \\
1331.0\end{array}$ \\
\hline $\begin{array}{r}45.0 \\
50.0 \\
55.0 \\
60.0 \\
70.0 \\
80.0 \\
90.0 \\
100.0 \\
120.0 \\
140.0\end{array}$ & $\begin{array}{l}0.1652 \\
0.1809 \\
0.1968 \\
0.2127 \\
0.2447 \\
0.2704 \\
0.3080 \\
0.3393 \\
0.4012 \\
0.4625\end{array}$ & $\begin{array}{l}214.0 \\
222.0 \\
232.0 \\
244.0 \\
269.0 \\
296.0 \\
323.0 \\
351.0 \\
407.0 \\
462.0\end{array}$ & $\begin{array}{l}24.3 \\
21.4 \\
19.1 \\
17.2 \\
14.3 \\
12.3 \\
10.7 \\
9.51 \\
7.78 \\
6.59\end{array}$ & $\begin{array}{r}32.14 \\
36.47 \\
40.75 \\
44.97 \\
53.29 \\
61.46 \\
69.50 \\
77.45 \\
93.14 \\
108.6\end{array}$ & $\begin{array}{c}59.67 \\
66.61 \\
73.54 \\
80.42 \\
94.06 \\
107.5 \\
120.8 \\
134.0 \\
160.0 \\
185.7\end{array}$ & $\begin{array}{l}2.256 \\
2.403 \\
2.534 \\
2.654 \\
2.865 \\
3.044 \\
3.201 \\
3.340 \\
3.577 \\
3.775\end{array}$ & $\begin{array}{l}0.7577 \\
0.7601 \\
0.7616 \\
0.7625 \\
0.7632 \\
0.7629 \\
0.7622 \\
0.7613 \\
0.7595 \\
0.7578\end{array}$ & $\begin{array}{l}1.388 \\
1.387 \\
1.381 \\
1.373 \\
1.355 \\
1.338 \\
1.323 \\
1.311 \\
1.292 \\
1.279\end{array}$ & $\begin{array}{l}1347.0 \\
1369.0 \\
1396.0 \\
1425.0 \\
1487.0 \\
1550.0 \\
1612.0 \\
1673.0 \\
1791.0 \\
1901.0\end{array}$ \\
\hline $\begin{array}{l}160.0 \\
180.0 \\
200.0 \\
250.0 \\
300.0 \\
350.0 \\
400.0 \\
450.0 \\
500.0 \\
600.0\end{array}$ & $\begin{array}{l}0.5233 \\
0.5837 \\
0.6439 \\
0.7936 \\
0.9427 \\
1.091 \\
1.240 \\
1.389 \\
1.537 \\
1.834\end{array}$ & $\begin{array}{r}517.0 \\
572.0 \\
626.0 \\
761.0 \\
894.0 \\
1030.0 \\
1160.0 \\
1290.0 \\
1430.0 \\
1690.0\end{array}$ & $\begin{array}{l}5.72 \\
5.06 \\
4.53 \\
3.61 \\
3.00 \\
2.57 \\
2.24 \\
1.99 \\
1.79 \\
1.49\end{array}$ & $\begin{array}{l}124.0 \\
139.2 \\
154.4 \\
192.2 \\
229.8 \\
267.3 \\
304.7 \\
342.1 \\
379.5 \\
454.1\end{array}$ & $\begin{array}{l}211.2 \\
236.5 \\
261.7 \\
324.5 \\
386.9 \\
449.2 \\
511.4 \\
573.5 \\
635.7 \\
759.8\end{array}$ & $\begin{array}{l}3.945 \\
4.095 \\
4.227 \\
4.507 \\
4.735 \\
4.927 \\
5.093 \\
5.240 \\
5.370 \\
5.597\end{array}$ & $\begin{array}{l}0.7564 \\
0.7552 \\
0.7542 \\
0.7524 \\
0.7511 \\
0.7502 \\
0.7495 \\
0.7490 \\
0.7486 \\
0.7481\end{array}$ & $\begin{array}{l}1.270 \\
1.263 \\
1.259 \\
1.251 \\
1.247 \\
1.245 \\
1.243 \\
1.243 \\
1.242 \\
1.241\end{array}$ & $\begin{array}{l}2005.0 \\
2105.0 \\
2200.0 \\
2420.0 \\
2623.0 \\
2811.0 \\
2987.0 \\
3154.0 \\
3312.0 \\
3608.0\end{array}$ \\
\hline $\begin{array}{r}700.0 \\
800.0 \\
900.0 \\
1000.0 \\
1200.0 \\
1400.0 \\
1600.0 \\
1800.0 \\
2000.0 \\
2500.0\end{array}$ & $\begin{array}{l}2.131 \\
2.428 \\
2.725 \\
3.022 \\
3.616 \\
4.211 \\
4.805 \\
5.400 \\
5.994 \\
7.481\end{array}$ & $\begin{array}{l}1960.0 \\
2230.0 \\
2490.0 \\
2760.0 \\
3290.0 \\
3830.0 \\
4360.0 \\
4890.0 \\
5430.0 \\
6760.0\end{array}$ & $\begin{array}{l}1.28 \\
1.12 \\
1.00 \\
0.897 \\
0.748 \\
0.641 \\
0.561 \\
0.499 \\
0.449 \\
0.359\end{array}$ & $\begin{array}{r}528.8 \\
603.3 \\
677.9 \\
752.5 \\
901.5 \\
1051.0 \\
1200.0 \\
1349.0 \\
1498.0 \\
1870.0\end{array}$ & $\begin{array}{r}883.9 \\
1008.0 \\
1132.0 \\
1256.0 \\
1504.0 \\
1752.0 \\
2000.0 \\
2249.0 \\
2497.0 \\
3117.0\end{array}$ & $\begin{array}{l}5.788 \\
5.954 \\
6.100 \\
6.231 \\
6.457 \\
6.648 \\
6.814 \\
6.960 \\
7.091 \\
7.367\end{array}$ & $\begin{array}{l}0.7477 \\
0.7474 \\
0.7471 \\
0.7470 \\
0.7467 \\
0.7465 \\
0.7464 \\
0.7463 \\
0.7462 \\
0.7460\end{array}$ & $\begin{array}{l}1.241 \\
1.241 \\
1.241 \\
1.241 \\
1.241 \\
1.241 \\
1.241 \\
1.241 \\
1.241 \\
1.241\end{array}$ & $\begin{array}{l}3881.0 \\
4137.0 \\
4378.0 \\
4607.0 \\
5033.0 \\
5427.0 \\
5793.0 \\
6138.0 \\
6465.0 \\
7218.0\end{array}$ \\
\hline 3000.0 & 8.960 & 8100.0 & 0.299 & 2243.0 & 3737.0 & 7.594 & 0.7459 & 1.241 & 7899.0 \\
\hline
\end{tabular}


90J PSIA ISOBAR

\begin{tabular}{|c|c|c|c|c|c|c|c|c|c|c|}
\hline TEMPERATURE & DENSITY & $V(D H / O V)_{P}$ & $V(O P / O U)_{\mathrm{v}}-v$ & $-V I O P / O V)_{T}$ & $(O V / O T) / V$ & $\begin{array}{c}\text { THERMAL } \\
\text { CONOUCTIVITY }\end{array}$ & VISCOSITY & $\begin{array}{c}\text { THERMAL } \\
\text { OIFFUSIVITY }\end{array}$ & $\begin{array}{l}\text { OIELECTRIC } \\
\text { CONSTANT }\end{array}$ & $\begin{array}{l}\text { PRANDTL } \\
\text { NUMBER }\end{array}$ \\
\hline DEG. $R$ & LB/CU FT & BTU/LB & PSIA-CU FT/BTU & PSIA & $1 / D E G . R$ & $9 T U / F T=H R-R$ & $\begin{array}{r}\text { LB/FT-SEC } \\
\times 10 E+6\end{array}$ & SQ FT/HR & & \\
\hline $\begin{array}{r}5.0 \\
6.0 \\
7.0 \\
8.0 \\
9.0 \\
10.0 \\
11.0 \\
12.0 \\
13.0\end{array}$ & $\begin{array}{l}12.26 \\
12.15 \\
12.05 \\
11.92 \\
11.79 \\
11.65 \\
11.51 \\
11.37 \\
11.22\end{array}$ & $\begin{array}{l}37.2 \\
40.2 \\
42.8 \\
47.2 \\
52.1 \\
53.3 \\
54.3 \\
55.2 \\
56.0\end{array}$ & $\begin{array}{c}14.1 \\
13.8 \\
13.3 \\
12.1 \\
10.8 \\
10.5 \\
10.1 \\
9.78 \\
9.45\end{array}$ & $\begin{array}{l}5680.0 \\
5930.0 \\
5900.0 \\
5740.0 \\
5520.0 \\
5300.0 \\
5040.0 \\
4790.0 \\
4540.0\end{array}$ & $\begin{array}{l}0.00987 \\
0.00876 \\
0.02954 \\
0.0104 \\
0.0112 \\
0.0118 \\
0.0124 \\
0.0130 \\
0.0136\end{array}$ & $\begin{array}{l}0.0162 \\
0.0177 \\
0.0191 \\
0.0206 \\
0.0221 \\
0.0234 \\
0.0247 \\
0.0257 \\
0.0267\end{array}$ & $\begin{array}{c}13.2 \\
11.2 \\
9.73 \\
8.57 \\
7.66 \\
6.96 \\
6.42 \\
5.99 \\
5.64\end{array}$ & $\begin{array}{l}0.00361 \\
0.00413 \\
0.00388 \\
0.00351 \\
0.00322 \\
0.00321 \\
0.00319 \\
0.00316 \\
0.00312\end{array}$ & $\begin{array}{l}1.02074 \\
1.02079 \\
1.02083 \\
1.02087 \\
1.02091 \\
1.02095 \\
1.02097 \\
1.02099 \\
1.02101\end{array}$ & $\begin{array}{l}1.07 \\
0.806 \\
0.749 \\
0.736 \\
0.727 \\
0.671 \\
0.629 \\
0.600 \\
0.579\end{array}$ \\
\hline $\begin{array}{l}14.0 \\
15.0 \\
16.0 \\
17.0 \\
18.0 \\
19.0 \\
26.0 \\
22.0 \\
24.0 \\
26.0\end{array}$ & $\begin{array}{c}11.06 \\
10.90 \\
10.74 \\
10.57 \\
10.40 \\
10.22 \\
10.04 \\
9.679 \\
9.312 \\
8.946\end{array}$ & $\begin{array}{l}56.7 \\
57.2 \\
57.6 \\
57.9 \\
58.2 \\
58.5 \\
58.7 \\
59.0 \\
59.2 \\
59.5\end{array}$ & $\begin{array}{l}9.15 \\
8.88 \\
8.64 \\
8.42 \\
8.21 \\
7.97 \\
7.76 \\
7.40 \\
7.10 \\
6.82\end{array}$ & $\begin{array}{l}4290.0 \\
4050.0 \\
3830.0 \\
3610.0 \\
3410.0 \\
3220.0 \\
3030.0 \\
2700.0 \\
2410.0 \\
2170.0\end{array}$ & $\begin{array}{l}0.0142 \\
0.0148 \\
0.0155 \\
0.0161 \\
0.0167 \\
0.0172 \\
0.0177 \\
0.0183 \\
0.0197 \\
0.0204\end{array}$ & $\begin{array}{l}0.0274 \\
0.0281 \\
0.0285 \\
0.0293 \\
0.0293 \\
0.0295 \\
0.0297 \\
0.0299 \\
0.0298 \\
0.0297\end{array}$ & $\begin{array}{l}5.35 \\
5.11 \\
4.91 \\
4.74 \\
4.60 \\
4.47 \\
4.37 \\
4.20 \\
4.08 \\
4.00\end{array}$ & $\begin{array}{l}0.00308 \\
0.00304 \\
0.00299 \\
0.00295 \\
0.00291 \\
0.00287 \\
0.00284 \\
0.00279 \\
0.00275 \\
0.00273\end{array}$ & $\begin{array}{l}1.02102 \\
1.02102 \\
1.02100 \\
1.02098 \\
1.02095 \\
1.02091 \\
1.02085 \\
1.02071 \\
1.02051 \\
1.02027\end{array}$ & $\begin{array}{l}0.565 \\
0.555 \\
0.550 \\
0.547 \\
0.547 \\
0.548 \\
0.551 \\
0.561 \\
0.574 \\
0.588\end{array}$ \\
\hline $\begin{array}{l}28.0 \\
30.0 \\
32.0 \\
34.0 \\
36.0 \\
38.0 \\
40.0\end{array}$ & $\begin{array}{l}8.583 \\
8.228 \\
7.885 \\
7.557 \\
7.245 \\
6.950 \\
6.673\end{array}$ & $\begin{array}{l}60.1 \\
61.0 \\
62.1 \\
63.5 \\
65.0 \\
66.8 \\
68.6\end{array}$ & $\begin{array}{l}6.58 \\
6.37 \\
6.18 \\
6.00 \\
5.84 \\
5.70 \\
5.58\end{array}$ & $\begin{array}{l}1980.0 \\
1820.0 \\
1700.3 \\
1600.0 \\
1520.0 \\
1450.0 \\
1390.0\end{array}$ & $\begin{array}{l}0.0210 \\
0.0212 \\
0.0213 \\
0.0212 \\
0.0209 \\
0.0206 \\
0.0201\end{array}$ & $\begin{array}{l}0.0296 \\
0.0294 \\
0.0292 \\
0.0290 \\
0.0299 \\
0.0288 \\
0.0287\end{array}$ & $\begin{array}{l}3.94 \\
3.90 \\
3.88 \\
3.87 \\
3.88 \\
3.89 \\
3.91\end{array}$ & $\begin{array}{l}0.00274 \\
0.00276 \\
0.00280 \\
0.00285 \\
0.00293 \\
0.00302 \\
0.00312\end{array}$ & $\begin{array}{l}1.01998 \\
1.01966 \\
1.01931 \\
1.01893 \\
1.01853 \\
1.01813 \\
1.01773\end{array}$ & $\begin{array}{l}0.604 \\
0.619 \\
0.634 \\
0.647 \\
0.658 \\
0.668 \\
0.677\end{array}$ \\
\hline $\begin{array}{r}45.0 \\
50.0 \\
55.0 \\
60.0 \\
70.0 \\
80.0 \\
90.0 \\
100.0 \\
120.0 \\
140.0\end{array}$ & $\begin{array}{l}6.054 \\
5.529 \\
5.083 \\
4.731 \\
4.087 \\
3.617 \\
3.247 \\
2.947 \\
2.492 \\
2.162\end{array}$ & $\begin{array}{r}73.8 \\
79.4 \\
85.3 \\
91.4 \\
104.0 \\
117.0 \\
130.0 \\
143.0 \\
168.0 \\
194.0\end{array}$ & $\begin{array}{l}5.31 \\
5.10 \\
4.93 \\
4.80 \\
4.59 \\
4.44 \\
4.33 \\
4.24 \\
4.11 \\
4.02\end{array}$ & $\begin{array}{l}1290.0 \\
1230.0 \\
1180.0 \\
1140.0 \\
1100.0 \\
1070.0 \\
1050.0 \\
1030.0 \\
1010.0 \\
1000.0\end{array}$ & $\begin{array}{l}0.0188 \\
0.0175 \\
0.0162 \\
0.0150 \\
0.0130 \\
0.0115 \\
0.0102 \\
0.00919 \\
0.00767 \\
0.00659\end{array}$ & $\begin{array}{l}0.0287 \\
0.0289 \\
0.0292 \\
0.0296 \\
0.0307 \\
0.0319 \\
0.0333 \\
0.0347 \\
0.0376 \\
0.0406\end{array}$ & $\begin{array}{l}3.98 \\
4.07 \\
4.18 \\
4.29 \\
4.53 \\
4.77 \\
5.01 \\
5.25 \\
5.72 \\
6.18\end{array}$ & $\begin{array}{l}0.00341 \\
0.00376 \\
0.00416 \\
0.00458 \\
0.00553 \\
0.00659 \\
0.00775 \\
0.00898 \\
0.0117 \\
0.0147\end{array}$ & $\begin{array}{l}1.01672 \\
1.01577 \\
1.01488 \\
1.01407 \\
1.01265 \\
1.01149 \\
1.01051 \\
1.00969 \\
1.00838 \\
1.00739\end{array}$ & $\begin{array}{l}0.694 \\
0.705 \\
0.712 \\
0.717 \\
0.720 \\
0.719 \\
0.717 \\
0.714 \\
0.707 \\
0.701\end{array}$ \\
\hline $\begin{array}{l}160.0 \\
180.0 \\
200.0 \\
250.0 \\
360.0 \\
356.0 \\
400.0 \\
450.0 \\
500.0 \\
600.0\end{array}$ & $\begin{array}{l}1.911 \\
1.713 \\
1.553 \\
1.263 \\
1.061 \\
0.9162 \\
0.8064 \\
0.7201 \\
0.6505 \\
0.5452\end{array}$ & $\begin{array}{l}219.0 \\
245.0 \\
270.0 \\
332.0 \\
395.0 \\
457.0 \\
519.0 \\
581.0 \\
643.0 \\
767.0\end{array}$ & $\begin{array}{l}3.96 \\
3.91 \\
3.87 \\
3.80 \\
3.76 \\
3.73 \\
3.71 \\
3.70 \\
3.68 \\
3.67\end{array}$ & $\begin{array}{l}988.0 \\
979.0 \\
972.0 \\
958.0 \\
949.0 \\
942.0 \\
936.0 \\
932.0 \\
929.0 \\
923.0\end{array}$ & $\begin{array}{l}0.00579 \\
0.00516 \\
0.00466 \\
0.00376 \\
0.00316 \\
0.00272 \\
0.00240 \\
0.00214 \\
0.00193 \\
0.00162\end{array}$ & $\begin{array}{l}0.0436 \\
0.0466 \\
0.0495 \\
0.0565 \\
0.0632 \\
0.0696 \\
0.0756 \\
0.0814 \\
0.0870 \\
0.0978\end{array}$ & $\begin{array}{c}6.63 \\
7.06 \\
7.36 \\
8.39 \\
9.36 \\
10.3 \\
11.2 \\
12.0 \\
12.9 \\
14.5\end{array}$ & $\begin{array}{l}0.0180 \\
0.0215 \\
0.0253 \\
0.0358 \\
0.0478 \\
0.0610 \\
0.0754 \\
0.0910 \\
0.108 \\
0.145\end{array}$ & $\begin{array}{l}1.00661 \\
1.00599 \\
1.00547 \\
1.00450 \\
1.00382 \\
1.00332 \\
1.00294 \\
1.00263 \\
1.00239 \\
1.00201\end{array}$ & $\begin{array}{l}0.695 \\
0.690 \\
0.674 \\
0.669 \\
0.665 \\
0.663 \\
0.661 \\
0.661 \\
0.662 \\
0.663\end{array}$ \\
\hline $\begin{array}{r}70 C .0 \\
80 C .0 \\
960.0 \\
10 C 0.0 \\
12 C 0.0 \\
146 C .0 \\
16 G 0.0 \\
1866.0 \\
2006.0 \\
2566.0\end{array}$ & $\begin{array}{l}0.4692 \\
0.4119 \\
0.3670 \\
0.3339 \\
0.2765 \\
0.2375 \\
0.2081 \\
0.1852 \\
0.1668 \\
0.1337\end{array}$ & $\begin{array}{r}891.0 \\
1010.0 \\
1140.0 \\
1260.0 \\
1510.0 \\
1760.0 \\
2010.0 \\
2250.0 \\
2500.0 \\
3120.0\end{array}$ & $\begin{array}{l}3.65 \\
3.64 \\
3.64 \\
3.63 \\
3.62 \\
3.62 \\
3.61 \\
3.61 \\
3.61 \\
3.63\end{array}$ & $\begin{array}{l}920.0 \\
917.0 \\
915.0 \\
913.0 \\
910.0 \\
909.0 \\
907.0 \\
906.0 \\
905.0 \\
904.0\end{array}$ & $\begin{array}{l}0.00139 \\
0.00122 \\
0.00109 \\
0.000983 \\
0.000822 \\
0.000706 \\
0.000619 \\
0.000551 \\
0.000496 \\
0.000398\end{array}$ & $\begin{array}{l}0.108 \\
0.119 \\
0.129 \\
0.138 \\
0.157 \\
0.175 \\
0.192 \\
0.209 \\
0.225 \\
0.263\end{array}$ & $\begin{array}{l}16.1 \\
17.7 \\
19.1 \\
20.6 \\
23.4 \\
26.0 \\
28.6 \\
31.1 \\
33.5 \\
39.2\end{array}$ & $\begin{array}{l}0.186 \\
0.232 \\
0.283 \\
0.337 \\
0.457 \\
0.593 \\
0.743 \\
0.908 \\
1.09 \\
1.59\end{array}$ & $\begin{array}{l}1.00174 \\
1.00153 \\
1.00136 \\
1.00123 \\
1.00103 \\
1.00089 \\
1.00078 \\
1.00069 \\
1.00063 \\
1.00050\end{array}$ & $\begin{array}{l}0.664 \\
0.664 \\
0.665 \\
0.665 \\
0.665 \\
0.665 \\
0.665 \\
0.665 \\
0.665 \\
0.665\end{array}$ \\
\hline 3000.0 & 0.1115 & 3740.0 & 3.60 & 903.0 & 0.000332 & 0.300 & 44.6 & 2.16 & 1.00342 & 0.665 \\
\hline
\end{tabular}

* two-phase goundary 


\begin{tabular}{|c|c|c|c|c|c|c|c|c|c|}
\hline $\begin{array}{c}\text { TEMPERATURE } \\
\text { OEG. R }\end{array}$ & $\begin{array}{l}\text { VOLUME } \\
\text { CU FT/LB }\end{array}$ & $\begin{array}{c}\text { ISOTHERM } \\
\text { OERIVATIVE } \\
\text { CU FT-PSIA/LB }\end{array}$ & $\begin{array}{l}\text { ISOCHORE } \\
\text { OERIVATIVE } \\
\text { PSIA / R }\end{array}$ & $\begin{array}{l}\text { INTERNAL } \\
\text { ENERGY } \\
\text { BTU/LB }\end{array}$ & $\begin{array}{l}\text { ENTHALPY } \\
\text { BTU/LB }\end{array}$ & $\begin{array}{l}\text { ENTROPY } \\
\text { BTU/LB-R }\end{array}$ & $\begin{array}{l}\text { CV } \\
\text { BTU / LB }\end{array}$ & $\begin{array}{l}C P \\
-R\end{array}$ & $\begin{array}{l}\text { VELOCITY } \\
\text { OF SOUNO } \\
\text { FT/SEC }\end{array}$ \\
\hline $\begin{array}{r}6.0 \\
7.0 \\
8.0 \\
9.0 \\
10.0 \\
11.0 \\
12.0 \\
13.0\end{array}$ & $\begin{array}{l}0.08094 \\
0.08168 \\
0.08248 \\
0.08336 \\
0.08428 \\
0.08523 \\
0.08623 \\
0.08729\end{array}$ & $\begin{array}{l}515.0 \\
522.0 \\
516.0 \\
505.0 \\
492.0 \\
476.0 \\
460.0 \\
443.0\end{array}$ & $\begin{array}{l}58.2 \\
59.9 \\
62.4 \\
63.6 \\
64.0 \\
64.0 \\
63.7 \\
63.3\end{array}$ & $\begin{array}{l}2.940 \\
3.134 \\
3.413 \\
3.800 \\
4.249 \\
4.705 \\
5.191 \\
5.707\end{array}$ & $\begin{array}{l}17.93 \\
18.26 \\
18.69 \\
19.24 \\
19.85 \\
20.49 \\
21.16 \\
21.87\end{array}$ & $\begin{array}{l}0.3715 \\
0.4263 \\
0.4836 \\
0.5452 \\
0.6081 \\
0.6688 \\
0.7280 \\
0.7859\end{array}$ & $\begin{array}{l}0.3082 \\
0.3441 \\
0.4083 \\
0.4798 \\
0.5067 \\
0.5311 \\
0.5556 \\
0.5786\end{array}$ & $\begin{array}{l}0.3560 \\
0.4037 \\
0.4842 \\
0.5726 \\
0.6162 \\
0.6582 \\
0.7015 \\
0.7445\end{array}$ & $\begin{array}{l}1660.0 \\
1684.0 \\
1684.0 \\
1671.0 \\
1665.0 \\
1653.0 \\
1639.0 \\
1625.0\end{array}$ \\
\hline $\begin{array}{l}14.0 \\
15.0 \\
16.0 \\
17.0 \\
18.0 \\
19.0 \\
20.0 \\
22.0 \\
24.0 \\
26.0\end{array}$ & $\begin{array}{l}0.08841 \\
0.08960 \\
0.09085 \\
0.09216 \\
0.09355 \\
0.09503 \\
0.09656 \\
0.0998 \\
0.1034 \\
0.1072\end{array}$ & $\begin{array}{l}426.0 \\
410.0 \\
395.0 \\
380.0 \\
366.0 \\
352.0 \\
339.0 \\
315.0 \\
294.0 \\
276.0\end{array}$ & $\begin{array}{l}62.7 \\
62.1 \\
61.2 \\
60.3 \\
59.2 \\
57.8 \\
56.3 \\
53.4 \\
50.4 \\
47.5\end{array}$ & $\begin{array}{c}6.253 \\
6.828 \\
7.430 \\
8.058 \\
8.711 \\
9.463 \\
10.24 \\
11.86 \\
13.56 \\
15.31\end{array}$ & $\begin{array}{l}22.62 \\
23.42 \\
24.25 \\
25.12 \\
26.03 \\
27.06 \\
28.12 \\
30.35 \\
32.69 \\
35.15\end{array}$ & $\begin{array}{l}0.8428 \\
0.8986 \\
0.9535 \\
1.007 \\
1.061 \\
1.117 \\
1.172 \\
1.280 \\
1.384 \\
1.483\end{array}$ & $\begin{array}{l}0.5994 \\
0.6182 \\
0.6350 \\
0.6501 \\
0.6636 \\
0.6768 \\
0.6879 \\
0.7054 \\
0.7182 \\
0.7274\end{array}$ & $\begin{array}{l}0.7865 \\
0.8275 \\
0.8673 \\
0.9059 \\
0.9432 \\
0.9780 \\
1.011 \\
1.073 \\
1.129 \\
1.179\end{array}$ & $\begin{array}{l}1610.0 \\
1595.0 \\
1580.0 \\
1566.0 \\
1552.0 \\
1535.0 \\
1520.0 \\
1490.0 \\
1463.0 \\
1439.0\end{array}$ \\
\hline $\begin{array}{l}28.0 \\
30.0 \\
32.0 \\
34.0 \\
36.0 \\
38.0 \\
40.0\end{array}$ & $\begin{array}{l}0.1112 \\
0.1156 \\
0.1202 \\
0.1249 \\
0.1299 \\
0.1350 \\
0.1403\end{array}$ & $\begin{array}{l}262.0 \\
251.0 \\
243.0 \\
237.0 \\
233.0 \\
231.0 \\
230.0\end{array}$ & $\begin{array}{l}44.6 \\
42.0 \\
39.4 \\
37.0 \\
34.8 \\
32.8 \\
30.9\end{array}$ & $\begin{array}{l}17.04 \\
18.71 \\
20.42 \\
22.14 \\
23.88 \\
25.62 \\
27.36\end{array}$ & $\begin{array}{l}37.63 \\
40.12 \\
42.67 \\
45.28 \\
47.93 \\
50.62 \\
53.34\end{array}$ & $\begin{array}{l}1.576 \\
1.661 \\
1.744 \\
1.823 \\
1.899 \\
1.971 \\
2.041\end{array}$ & $\begin{array}{l}0.7343 \\
0.7399 \\
0.7443 \\
0.7477 \\
0.7505 \\
0.7528 \\
0.7547\end{array}$ & $\begin{array}{l}1.222 \\
1.261 \\
1.292 \\
1.317 \\
1.336 \\
1.351 \\
1.362\end{array}$ & $\begin{array}{l}1421.0 \\
1407.0 \\
1397.0 \\
1390.0 \\
1386.0 \\
1385.0 \\
1386.0\end{array}$ \\
\hline $\begin{array}{r}45.0 \\
50.0 \\
55.0 \\
60.0 \\
70.0 \\
80.0 \\
90.0 \\
100.0 \\
120.0 \\
140.0\end{array}$ & $\begin{array}{l}0.1538 \\
0.1677 \\
0.1818 \\
0.1961 \\
0.2247 \\
0.2532 \\
0.2816 \\
0.3098 \\
0.3657 \\
0.4209\end{array}$ & $\begin{array}{l}232.0 \\
238.0 \\
247.0 \\
258.0 \\
282.0 \\
308.0 \\
335.0 \\
362.0 \\
418.0 \\
473.0\end{array}$ & $\begin{array}{l}27.0 \\
23.8 \\
21.3 \\
19.2 \\
16.0 \\
13.7 \\
11.9 \\
10.6 \\
8.67 \\
7.34\end{array}$ & $\begin{array}{c}31.71 \\
36.03 \\
40.31 \\
44.54 \\
52.88 \\
61.08 \\
69.17 \\
77.15 \\
92.90 \\
108.4\end{array}$ & $\begin{array}{c}60.19 \\
67.08 \\
73.97 \\
80.85 \\
94.49 \\
108.0 \\
121.3 \\
134.5 \\
160.6 \\
186.4\end{array}$ & $\begin{array}{l}2.202 \\
2.347 \\
2.479 \\
2.598 \\
2.809 \\
2.989 \\
3.146 \\
3.285 \\
3.523 \\
3.722\end{array}$ & $\begin{array}{l}0.7583 \\
0.7607 \\
0.7624 \\
0.7634 \\
0.7643 \\
0.7642 \\
0.7636 \\
0.7628 \\
0.7609 \\
0.7592\end{array}$ & $\begin{array}{l}1.376 \\
1.380 \\
1.377 \\
1.372 \\
1.357 \\
1.341 \\
1.327 \\
1.315 \\
1.295 \\
1.282\end{array}$ & $\begin{array}{l}1397.0 \\
1415.0 \\
1439.0 \\
1465.0 \\
1522.0 \\
1582.0 \\
1642.0 \\
1701.0 \\
1815.0 \\
1923.0\end{array}$ \\
\hline $\begin{array}{l}160.0 \\
180.0 \\
200.0 \\
250.0 \\
300.0 \\
350.0 \\
400.0 \\
450.0 \\
500.0 \\
600.0\end{array}$ & $\begin{array}{l}0.4756 \\
0.5301 \\
0.5843 \\
0.7190 \\
0.8532 \\
0.9872 \\
1.121 \\
1.255 \\
1.388 \\
1.655\end{array}$ & $\begin{array}{r}528.0 \\
582.0 \\
636.0 \\
771.0 \\
905.0 \\
1040.0 \\
1170.0 \\
1300.0 \\
1440.0 \\
1700.0\end{array}$ & $\begin{array}{l}6.37 \\
5.62 \\
5.04 \\
4.01 \\
3.33 \\
2.85 \\
2.49 \\
2.21 \\
1.99 \\
1.66\end{array}$ & $\begin{array}{l}123.8 \\
139.1 \\
154.3 \\
192.2 \\
229.8 \\
267.3 \\
304.8 \\
342.2 \\
379.6 \\
454.3\end{array}$ & $\begin{array}{l}211.9 \\
237.3 \\
262.5 \\
325.3 \\
387.8 \\
450.1 \\
512.4 \\
574.5 \\
636.6 \\
760.8\end{array}$ & $\begin{array}{l}3.892 \\
4.042 \\
4.175 \\
4.455 \\
4.683 \\
4.875 \\
5.041 \\
5.187 \\
5.318 \\
5.545\end{array}$ & $\begin{array}{l}0.7577 \\
0.7564 \\
0.7553 \\
0.7532 \\
0.7518 \\
0.7508 \\
0.7501 \\
0.7495 \\
0.7491 \\
0.7484\end{array}$ & $\begin{array}{l}1.272 \\
1.265 \\
1.260 \\
1.252 \\
1.248 \\
1.245 \\
1.244 \\
1.243 \\
1.242 \\
1.241\end{array}$ & $\begin{array}{l}2026.0 \\
2124.0 \\
2218.0 \\
2436.0 \\
2637.0 \\
2824.0 \\
2999.0 \\
3165.0 \\
3322.0 \\
3617.0\end{array}$ \\
\hline $\begin{array}{r}700.0 \\
800.0 \\
900.0 \\
1000.0 \\
1200.0 \\
1400.0 \\
1600.0 \\
1800.0 \\
2000.0 \\
2500.0\end{array}$ & $\begin{array}{l}1.923 \\
2.190 \\
2.457 \\
2.724 \\
3.259 \\
3.793 \\
4.328 \\
4.863 \\
5.398 \\
6.736\end{array}$ & $\begin{array}{l}1970.0 \\
2230.0 \\
2500.0 \\
2770.0 \\
3300.0 \\
3830.0 \\
4370.0 \\
4900.0 \\
5430.0 \\
6770.0\end{array}$ & $\begin{array}{l}1.42 \\
1.25 \\
1.11 \\
1.00 \\
0.831 \\
0.712 \\
0.623 \\
0.554 \\
0.499 \\
0.399\end{array}$ & $\begin{array}{r}528.9 \\
603.5 \\
678.1 \\
752.6 \\
901.7 \\
1051.0 \\
1200.0 \\
1349.0 \\
1498.0 \\
1871.0\end{array}$ & $\begin{array}{r}884.9 \\
1009.0 \\
1133.0 \\
1257.0 \\
1505.0 \\
1753.0 \\
2001.0 \\
2249.0 \\
2498.0 \\
3118.0\end{array}$ & $\begin{array}{l}5.736 \\
5.902 \\
6.048 \\
6.179 \\
6.405 \\
6.596 \\
6.762 \\
6.908 \\
7.038 \\
7.315\end{array}$ & $\begin{array}{l}0.7480 \\
0.7477 \\
0.7474 \\
0.7472 \\
0.7469 \\
0.7467 \\
0.7466 \\
0.7465 \\
0.7464 \\
0.7462\end{array}$ & $\begin{array}{l}1.241 \\
1.241 \\
1.241 \\
1.241 \\
1.240 \\
1.241 \\
1.241 \\
1.241 \\
1.241 \\
1.241\end{array}$ & $\begin{array}{l}3890.0 \\
4145.0 \\
4385.0 \\
4613.0 \\
5039.0 \\
5431.0 \\
5798.0 \\
6142.0 \\
6468.0 \\
7220.0\end{array}$ \\
\hline 3000.0 & 8.074 & 8100.0 & 0.333 & 2243.0 & 3736.0 & 7.541 & 0.7461 & 1.241 & 7901.0 \\
\hline
\end{tabular}


1000 PSIA ISOBAR

TEMPERATURE OENSITY V(OH/OV) V V(OP/OU) ${ }_{V}-V(O P / O V)_{T}$ DEG. R LB/CUFT BTU/LO PSIA-CUFT/BTU PSIA

\begin{tabular}{|c|c|c|c|c|}
\hline $\begin{array}{r}6.0 \\
7.0 \\
8.0 \\
9.0 \\
10.0 \\
11.0 \\
12.0 \\
13.0\end{array}$ & $\begin{array}{l}12.36 \\
12.24 \\
12.12 \\
12.00 \\
11.87 \\
11.73 \\
11.60 \\
11.46\end{array}$ & $\begin{array}{l}38.9 \\
43.0 \\
48.6 \\
54.5 \\
56.2 \\
57.5 \\
58.7 \\
59.7\end{array}$ & $\begin{array}{l}15.3 \\
14.2 \\
12.6 \\
11.1 \\
10.6 \\
10.3 \\
9.89 \\
9.55\end{array}$ & $\begin{array}{l}6360.0 \\
6390.0 \\
6260.0 \\
6060.0 \\
5840.0 \\
5590.0 \\
5330.0 \\
5070.0\end{array}$ \\
\hline $\begin{array}{l}14.0 \\
15.0 \\
16.0 \\
17.0 \\
18.0 \\
19.0 \\
20.0 \\
22.0 \\
24.0 \\
26.0\end{array}$ & $\begin{array}{l}11.31 \\
11.16 \\
11.01 \\
10.85 \\
10.69 \\
10.52 \\
10.36 \\
10.02 \\
9.675 \\
9.332\end{array}$ & $\begin{array}{l}60.4 \\
61.0 \\
61.5 \\
61.9 \\
62.3 \\
62.7 \\
63.0 \\
63.4 \\
63.7 \\
64.0\end{array}$ & $\begin{array}{l}9.25 \\
8.99 \\
8.76 \\
8.55 \\
8.35 \\
8.11 \\
7.91 \\
7.56 \\
7.26 \\
6.99\end{array}$ & $\begin{array}{l}4820.0 \\
4580.0 \\
4340.0 \\
4120.0 \\
3910.0 \\
3710.0 \\
3510.0 \\
3160.0 \\
2840.0 \\
2580.0\end{array}$ \\
\hline $\begin{array}{l}28.0 \\
30.0 \\
32.0 \\
34.0 \\
36.0 \\
38.0 \\
40.0\end{array}$ & $\begin{array}{l}8.990 \\
8.652 \\
8.322 \\
8.003 \\
7.698 \\
7.406 \\
7.129\end{array}$ & $\begin{array}{l}64.5 \\
65.2 \\
66.2 \\
67.4 \\
68.8 \\
70.4 \\
72.1\end{array}$ & $\begin{array}{l}6.76 \\
6.55 \\
6.36 \\
6.19 \\
6.03 \\
5.88 \\
5.75\end{array}$ & $\begin{array}{l}2350.0 \\
2170.0 \\
2020.0 \\
1930.0 \\
1790.0 \\
1710.0 \\
1640.0\end{array}$ \\
\hline $\begin{array}{r}45.0 \\
50.0 \\
55.0 \\
60.0 \\
70.0 \\
80.0 \\
90.0 \\
100.0 \\
120.0 \\
140.0\end{array}$ & $\begin{array}{l}6.503 \\
5.964 \\
5.500 \\
5.100 \\
4.451 \\
3.949 \\
3.551 \\
3.228 \\
2.735 \\
2.376\end{array}$ & $\begin{array}{r}77.0 \\
82.4 \\
88.2 \\
94.1 \\
107.0 \\
119.0 \\
132.0 \\
145.0 \\
171.0 \\
196.0\end{array}$ & $\begin{array}{l}5.47 \\
5.25 \\
5.07 \\
4.92 \\
4.70 \\
4.53 \\
4.41 \\
4.31 \\
4.17 \\
4.07\end{array}$ & $\begin{array}{l}1510.0 \\
1420.0 \\
1360.0 \\
1320.0 \\
1250.0 \\
1220.0 \\
1190.0 \\
1170.0 \\
1140.0 \\
1120.0\end{array}$ \\
\hline $\begin{array}{l}160.0 \\
180.0 \\
200.0 \\
250.0 \\
300.0 \\
350.0 \\
400.0 \\
450.0 \\
500.0 \\
600.0\end{array}$ & $\begin{array}{l}2.102 \\
1.887 \\
1.712 \\
1.391 \\
1.172 \\
1.313 \\
0.8921 \\
0.7971 \\
0.7204 \\
0.6041\end{array}$ & $\begin{array}{l}222.0 \\
247.0 \\
272.0 \\
335.0 \\
397.0 \\
459.0 \\
521.0 \\
583.0 \\
645.0 \\
769.0\end{array}$ & $\begin{array}{l}4.00 \\
3.94 \\
3.90 \\
3.83 \\
3.78 \\
3.75 \\
3.72 \\
3.71 \\
3.69 \\
3.67\end{array}$ & $\begin{array}{l}1110.0 \\
1130.0 \\
1090.0 \\
1070.0 \\
1060.0 \\
1050.0 \\
1040.0 \\
1040.0 \\
1040.0 \\
1030.0\end{array}$ \\
\hline $\begin{array}{r}700.0 \\
800.0 \\
900.0 \\
1000.0 \\
1200.0 \\
1400.0 \\
1600.0 \\
1800.0 \\
2000.0 \\
2500.0\end{array}$ & $\begin{array}{l}0.5202 \\
0.4567 \\
0.4070 \\
0.3671 \\
0.3 \cup 69 \\
0.2636 \\
0.2310 \\
0.2056 \\
0.1852 \\
0.1485\end{array}$ & $\begin{array}{r}893.0 \\
1020.0 \\
1140.0 \\
1260.0 \\
1510.0 \\
1760.0 \\
2010.0 \\
2260.0 \\
2500.0 \\
3120.0\end{array}$ & $\begin{array}{l}3.66 \\
3.65 \\
3.64 \\
3.63 \\
3.62 \\
3.62 \\
3.61 \\
3.61 \\
3.61 \\
3.60\end{array}$ & $\begin{array}{l}1020.0 \\
1020.0 \\
1020.0 \\
1020.0 \\
1010.0 \\
1010.0 \\
1010.0 \\
1010.0 \\
1010.0 \\
1000.0\end{array}$ \\
\hline $300 \mathrm{G} .0$ & 0.1239 & 3740.0 & 3.60 & 1000.0 \\
\hline
\end{tabular}

COV/OTI/V THERMAL VISCOSITY 1/OEG. R BTU/FT-HR-R LB/FT-SEC $\times 10 \mathrm{E}+6$
THERMAL OIELECTRIC DIFFUSIVITY CONSTANT SQ FT/HR

PRANOTL NUMBER

\begin{tabular}{|c|c|c|c|c|c|}
\hline $\begin{array}{l}0.00914 \\
0.00938 \\
0.0100 \\
0.0105 \\
0.0110 \\
0.0114 \\
0.0120 \\
0.0125\end{array}$ & $\begin{array}{l}0.0182 \\
0.0197 \\
0.0212 \\
0.0228 \\
0.0242 \\
0.0255 \\
0.0266 \\
0.0276\end{array}$ & $\begin{array}{c}12.7 \\
10.8 \\
9.44 \\
8.39 \\
7.58 \\
6.97 \\
6.47 \\
6.07\end{array}$ & $\begin{array}{l}0.00415 \\
0.00399 \\
0.00362 \\
0.00331 \\
0.00331 \\
0.00330 \\
0.00327 \\
0.00324\end{array}$ & $\begin{array}{l}1.02070 \\
1.02075 \\
1.02080 \\
1.02085 \\
1.02089 \\
1.02093 \\
1.02096 \\
1.02098\end{array}$ & $\begin{array}{l}0.890 \\
0.798 \\
0.775 \\
0.760 \\
0.696 \\
0.648 \\
0.614 \\
0.590\end{array}$ \\
\hline $\begin{array}{l}0.0130 \\
0.0136 \\
0.0141 \\
0.0146 \\
0.0151 \\
0.0156 \\
0.0160 \\
0.0169 \\
0.0177 \\
0.0184\end{array}$ & $\begin{array}{l}0.0284 \\
0.0291 \\
0.0297 \\
0.0301 \\
0.0305 \\
0.0308 \\
0.0310 \\
0.0312 \\
0.0312 \\
0.0312\end{array}$ & $\begin{array}{l}5.75 \\
5.47 \\
5.25 \\
5.05 \\
4.89 \\
4.75 \\
4.63 \\
4.44 \\
4.30 \\
4.20\end{array}$ & $\begin{array}{l}0.00320 \\
0.00315 \\
0.00311 \\
0.00307 \\
0.00303 \\
0.00299 \\
0.00296 \\
0.00290 \\
0.00286 \\
0.00283\end{array}$ & $\begin{array}{l}1.02100 \\
1.02101 \\
1.02102 \\
1.02101 \\
1.02100 \\
1.02098 \\
1.02094 \\
1.02084 \\
1.02070 \\
1.02052\end{array}$ & $\begin{array}{l}0.572 \\
0.560 \\
0.552 \\
0.547 \\
0.544 \\
0.543 \\
0.544 \\
0.550 \\
0.560 \\
0.572\end{array}$ \\
\hline $\begin{array}{l}0.0190 \\
0.0193 \\
0.0195 \\
0.0195 \\
0.0194 \\
0.0192 \\
0.0189\end{array}$ & $\begin{array}{l}0.0310 \\
0.0308 \\
0.0306 \\
0.0305 \\
0.0303 \\
0.0302 \\
0.0301\end{array}$ & $\begin{array}{l}4.13 \\
4.08 \\
4.05 \\
4.03 \\
4.03 \\
4.04 \\
4.05\end{array}$ & $\begin{array}{l}0.00282 \\
0.00283 \\
0.00285 \\
0.00289 \\
0.00294 \\
0.00301 \\
0.00310\end{array}$ & $\begin{array}{l}1.02030 \\
1.02004 \\
1.01975 \\
1.01943 \\
1.01910 \\
1.01874 \\
1.01838\end{array}$ & $\begin{array}{l}0.586 \\
0.601 \\
0.615 \\
0.628 \\
0.640 \\
0.651 \\
0.660\end{array}$ \\
\hline $\begin{array}{l}0.0179 \\
0.0167 \\
0.0156 \\
0.0146 \\
0.0127 \\
0.0112 \\
0.0100 \\
0.00907 \\
0.00759 \\
0.00653\end{array}$ & $\begin{array}{l}0.0299 \\
0.0300 \\
0.0302 \\
0.0306 \\
0.0315 \\
0.0327 \\
0.0341 \\
0.0354 \\
0.0382 \\
0.0412\end{array}$ & $\begin{array}{l}4.11 \\
4.19 \\
4.28 \\
4.39 \\
4.62 \\
4.85 \\
5.09 \\
5.32 \\
5.79 \\
6.24\end{array}$ & $\begin{array}{l}0.00335 \\
0.00365 \\
0.00399 \\
0.00437 \\
0.00522 \\
0.00617 \\
0.00721 \\
0.00833 \\
0.0108 \\
0.0135\end{array}$ & $\begin{array}{l}1.01746 \\
1.01656 \\
1.01571 \\
1.01492 \\
1.01351 \\
1.01232 \\
1.01131 \\
1.01046 \\
1.00909 \\
1.00804\end{array}$ & $\begin{array}{l}0.680 \\
0.693 \\
0.702 \\
0.709 \\
0.715 \\
0.716 \\
0.715 \\
0.712 \\
0.706 \\
0.700\end{array}$ \\
\hline $\begin{array}{l}0.00574 \\
0.00512 \\
0.00463 \\
0.00374 \\
0.00314 \\
0.00271 \\
0.00239 \\
0.00213 \\
0.00192 \\
0.00161\end{array}$ & $\begin{array}{l}0.0441 \\
0.0470 \\
0.0499 \\
0.0568 \\
0.0635 \\
0.0699 \\
0.0759 \\
0.0817 \\
0.0872 \\
0.0980\end{array}$ & $\begin{array}{r}6.69 \\
7.12 \\
7.41 \\
8.43 \\
9.39 \\
10.3 \\
11.2 \\
12.1 \\
12.9 \\
14.5\end{array}$ & $\begin{array}{l}0.0165 \\
0.0197 \\
0.0231 \\
0.0326 \\
0.0434 \\
0.0554 \\
0.0684 \\
0.0825 \\
0.0975 \\
0.131\end{array}$ & $\begin{array}{l}1.00721 \\
1.00654 \\
1.00598 \\
1.00493 \\
1.00420 \\
1.00366 \\
1.00324 \\
1.00291 \\
1.00264 \\
1.00222\end{array}$ & $\begin{array}{l}0.695 \\
0.690 \\
0.674 \\
0.668 \\
0.664 \\
0.662 \\
0.661 \\
0.661 \\
0.661 \\
0.663\end{array}$ \\
\hline $\begin{array}{l}0.00139 \\
0.00122 \\
0.00109 \\
0.000981 \\
0.000820 \\
0.000705 \\
0.000618 \\
0.000550 \\
0.000496 \\
0.000397\end{array}$ & $\begin{array}{l}0.109 \\
0.119 \\
0.129 \\
0.138 \\
0.157 \\
0.175 \\
0.192 \\
0.209 \\
0.225 \\
0.263\end{array}$ & $\begin{array}{l}16.1 \\
17.7 \\
19.2 \\
20.6 \\
23.4 \\
26.0 \\
28.6 \\
31.1 \\
33.5 \\
39.2\end{array}$ & $\begin{array}{l}0.168 \\
0.210 \\
0.255 \\
0.304 \\
0.413 \\
0.535 \\
0.670 \\
0.818 \\
0.978 \\
1.43\end{array}$ & $\begin{array}{l}1.00192 \\
1.00169 \\
1.00151 \\
1.00136 \\
1.00114 \\
1.00398 \\
1.00086 \\
1.00077 \\
1.00069 \\
1.00056\end{array}$ & $\begin{array}{l}0.664 \\
0.664 \\
0.664 \\
0.665 \\
0.665 \\
0.665 \\
0.665 \\
0.665 \\
0.665 \\
0.665\end{array}$ \\
\hline 0.000331 & 0.300 & 44.6 & 1.95 & 1.00047 & 0.665 \\
\hline
\end{tabular}

* two-phase bounoar 


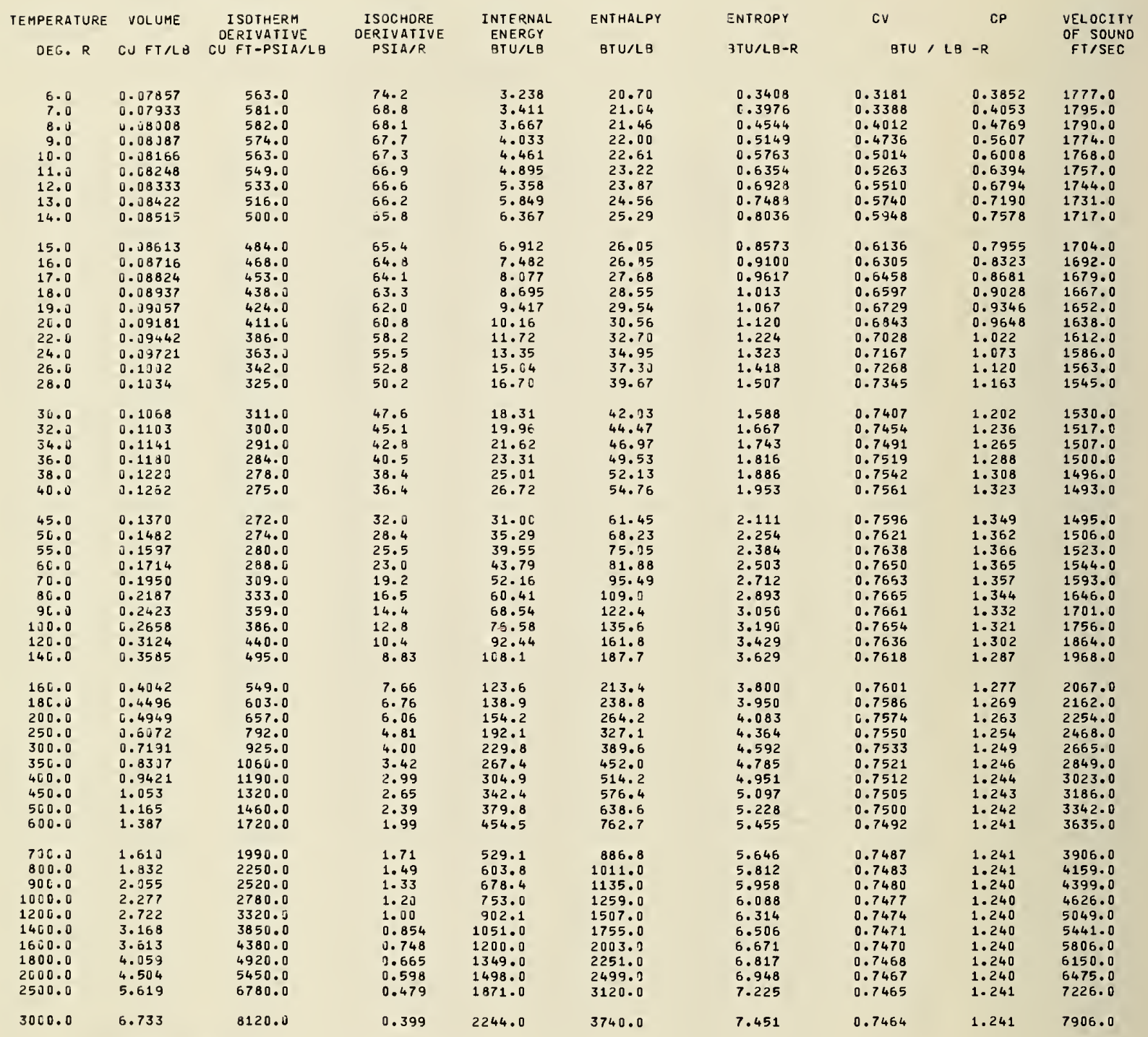


$120 J$ PSIA ISOBAR

TEMPERATURE DENSITY V(DH/OV) $V(D P / D U)_{V}-V(D P / O V) T$ DEG. $R$ LE/CUFT OTU/LB PSIA-CUFT/BTU PSIA

\begin{tabular}{|c|c|c|c|c|}
\hline 6.0 & 12.73 & 37.2 & 18.3 & 7160.0 \\
\hline 7.0 & 12.61 & 43.1 & 16.1 & 7330.0 \\
\hline 8.0 & 12.49 & 50.9 & 13.6 & 7260.0 \\
\hline 9.0 & 12.37 & 58.7 & 11.6 & 7100.0 \\
\hline 16.0 & 12.25 & 61.5 & 11.0 & 6900.0 \\
\hline 11.0 & 12.12 & 63.5 & 10.5 & 6650.0 \\
\hline 12.0 & 12. Ju & 65.2 & 10.1 & 6390.0 \\
\hline 13.0 & 11.87 & 66.6 & 9.72 & 6130.0 \\
\hline 14.0 & 11.74 & 67.6 & 9.42 & 5870.0 \\
\hline 15.0 & 11.61 & 68.4 & 9.17 & 5620.0 \\
\hline 16.0 & 11.47 & 69.0 & 8.95 & 5370.0 \\
\hline 17.0 & 11.33 & 69.5 & 8.76 & 5130.0 \\
\hline 18.0 & 11.19 & 70.0 & 8.57 & 4900.0 \\
\hline 19.0 & 11.04 & 70.6 & 8.35 & 4690.0 \\
\hline 20.0 & 10.89 & 71.1 & 8.15 & 4480.0 \\
\hline 22.0 & 10.59 & 71.7 & 7.82 & 4090.0 \\
\hline 24.0 & 10.29 & 72.2 & 7.53 & 3730.0 \\
\hline 26.0 & 9.98 & 72.5 & 7.28 & 3420.0 \\
\hline 28.0 & & 73.0 & 7.06 & 3150.0 \\
\hline 30.0 & 9.367 & 73.6 & 6.86 & 2910.0 \\
\hline 32.0 & 9.063 & 74.4 & 6.68 & 2720.0 \\
\hline 34.0 & 8.765 & 75.3 & 6.51 & 2550.0 \\
\hline 36.0 & 8.475 & 76.5 & 6.35 & 240000 \\
\hline 38.0 & 8.194 & 77.8 & 6.21 & 228000 \\
\hline 40.0 & 7.924 & 79.3 & 6.07 & 2180.0 \\
\hline 45.0 & 7.299 & 83.6 & 5.77 & 1980.0 \\
\hline 50.0 & 6.746 & 88.6 & 5.53 & 1850.0 \\
\hline 55.0 & 6.260 & 94.0 & 5.33 & 1750.0 \\
\hline 60.0 & 5.834 & 100.0 & 5.16 & 1680.0 \\
\hline 70.0 & 5.128 & 112.0 & 4.90 & 1590.0 \\
\hline$B C .0$ & 4.573 & 124.0 & 4.70 & 1520.0 \\
\hline 90.0 & 4.128 & 137.0 & 4.56 & 1480.0 \\
\hline 100.0 & 3.763 & 150.0 & 4.44 & 1456.0 \\
\hline 120.0 & 3.201 & 175.0 & 4.27 & 1410.0 \\
\hline 140.0 & 2.790 & 201.0 & 4.16 & 1380.0 \\
\hline 160.0 & 2.474 & 227.0 & 4.07 & 1360.0 \\
\hline $18 C .0$ & 2.224 & 252.0 & 4.01 & 1340.0 \\
\hline 200.0 & 2.021 & 277.0 & 3.96 & 1330.0 \\
\hline 250.0 & 1.647 & 340.0 & 3.87 & 1300.0 \\
\hline 300.0 & 1.391 & 402.0 & 3.81 & 1290.0 \\
\hline 350.0 & 1.204 & 464.0 & 3.78 & 1270.0 \\
\hline 400.0 & 1.062 & 526.0 & 3.75 & 1260.0 \\
\hline 450.0 & 0.9493 & 588.0 & 3.73 & 1260.0 \\
\hline 500.0 & 0.8586 & 650.0 & 3.71 & 1250.0 \\
\hline 600.0 & 0.7239 & 774.0 & 3.69 & 1240.0 \\
\hline 700.0 & & 898.0 & 3.67 & 1230.0 \\
\hline 800.0 & 0.5458 & 1020.0 & 3.66 & 1230.0 \\
\hline 900.0 & 0.4867 & 1150.0 & 3.65 & 1230.0 \\
\hline 1000.0 & 0.4391 & 1270.0 & 3.64 & 1220.0 \\
\hline $12 \cup 0.0$ & 0.3673 & 1520.0 & 3.63 & 1220.0 \\
\hline 1400.0 & 0.3157 & 1760.0 & 3.62 & 1220.0 \\
\hline 1600.0 & 0.2768 & 2010.0 & 3.62 & 1210.0 \\
\hline 1800.0 & 0.2464 & 2260.0 & 3.61 & 1210.0 \\
\hline 2000.0 & 0.2220 & 2510.0 & 3.61 & 1210.0 \\
\hline 2500.0 & 0.1780 & 3130.0 & 3.63 & 1210.0 \\
\hline 3006.0 & 0.1485 & 3750.0 & 3.60 & 1210.0 \\
\hline
\end{tabular}

COV/OT) NV THERMAL VISCDSITY 1/DEG. R BTU/FT-HR-R LB/FT-SEC $\times 10 E+6$

\begin{tabular}{|c|c|c|c|c|c|}
\hline $\begin{array}{l}0.0104 \\
0.00939 \\
0.00937 \\
0.00955 \\
0.00976 \\
0.0101 \\
0.0104 \\
0.0108 \\
0.0112\end{array}$ & $\begin{array}{l}0.0193 \\
0.0208 \\
0.0224 \\
0.0240 \\
0.0256 \\
0.0270 \\
0.0282 \\
0.0293 \\
0.0303\end{array}$ & $\begin{array}{r}15.9 \\
13.3 \\
11.4 \\
10.0 \\
8.91 \\
8.11 \\
7.48 \\
6.98 \\
6.56\end{array}$ & $\begin{array}{l}0.00394 \\
0.00407 \\
0.00377 \\
0.00347 \\
0.00348 \\
0.00348 \\
0.00346 \\
0.00344 \\
0.00340\end{array}$ & $\begin{array}{l}1.02050 \\
1.02057 \\
1.02064 \\
1.02070 \\
1.02075 \\
1.02080 \\
1.02085 \\
1.02089 \\
1.02092\end{array}$ & $\begin{array}{l}1.15 \\
0.931 \\
0.871 \\
0.837 \\
0.754 \\
0.692 \\
0.648 \\
0.616 \\
0.592\end{array}$ \\
\hline $\begin{array}{l}0.0116 \\
0.0121 \\
0.0125 \\
0.0129 \\
0.0132 \\
0.0136 \\
0.0142 \\
0.01149 \\
0.0154 \\
0.0159\end{array}$ & $\begin{array}{l}0.0311 \\
0.0317 \\
0.0323 \\
0.0327 \\
0.0330 \\
0.0333 \\
0.0336 \\
0.0338 \\
0.0338 \\
0.0337\end{array}$ & $\begin{array}{l}6.22 \\
5.93 \\
5.69 \\
5.49 \\
5.31 \\
5.16 \\
4.92 \\
4.74 \\
4.51 \\
4.51\end{array}$ & $\begin{array}{l}0.00336 \\
0.00332 \\
0.00328 \\
0.00324 \\
0.00320 \\
0.00317 \\
0.00311 \\
0.00306 \\
0.00302 \\
0.00299\end{array}$ & $\begin{array}{l}1.02095 \\
1.02098 \\
1.02100 \\
1.02101 \\
1.02102 \\
1.02101 \\
1.02099 \\
1.02093 \\
1.02083 \\
1.02370\end{array}$ & $\begin{array}{l}0.574 \\
0.561 \\
0.551 \\
0.545 \\
0.541 \\
0.538 \\
0.538 \\
0.542 \\
0.550 \\
0.560\end{array}$ \\
\hline $\begin{array}{l}0.0163 \\
0.0166 \\
0.0168 \\
0.0168 \\
0.0168 \\
0.0167\end{array}$ & $\begin{array}{l}0.0335 \\
0.0333 \\
0.0331 \\
0.0329 \\
0.0328 \\
0.0326\end{array}$ & $\begin{array}{l}4.43 \\
4.38 \\
4.35 \\
4.33 \\
4.32 \\
4.32\end{array}$ & $\begin{array}{l}0.00298 \\
0.00298 \\
0.00299 \\
0.00302 \\
0.00306 \\
0.00311\end{array}$ & $\begin{array}{l}1.02054 \\
1.02035 \\
1.02013 \\
1.01989 \\
1.01963 \\
1.01935\end{array}$ & $\begin{array}{l}0.573 \\
0.585 \\
0.598 \\
0.609 \\
0.621 \\
0.631\end{array}$ \\
\hline $\begin{array}{l}0.0161 \\
0.0154 \\
0.0145 \\
0.0137 \\
0.0121 \\
0.0108 \\
0.00972 \\
0.00882 \\
0.00742 \\
0.0064 C\end{array}$ & $\begin{array}{l}0.0323 \\
0.0323 \\
0.0323 \\
0.0326 \\
0.0333 \\
0.0343 \\
0.0354 \\
0.0367 \\
0.0394 \\
0.0422\end{array}$ & $\begin{array}{l}4.35 \\
4.41 \\
4.49 \\
4.59 \\
4.79 \\
5.01 \\
5.24 \\
5.46 \\
5.92 \\
6.36\end{array}$ & $\begin{array}{l}0.00328 \\
0.00351 \\
0.00378 \\
0.00409 \\
0.00478 \\
0.00557 \\
0.00644 \\
0.00738 \\
0.00945 \\
0.0118\end{array}$ & $\begin{array}{l}1.01861 \\
1.01784 \\
1.01707 \\
1.01633 \\
1.01497 \\
1.01378 \\
1.01275 \\
1.01186 \\
1.01039 \\
1.00925\end{array}$ & $\begin{array}{l}0.653 \\
0.670 \\
0.683 \\
0.692 \\
0.703 \\
0.708 \\
0.709 \\
0.708 \\
0.704 \\
0.698\end{array}$ \\
\hline $\begin{array}{l}0.00564 \\
0.00504 \\
0.00456 \\
0.00369 \\
0.00311 \\
0.00268 \\
0.00236 \\
0.00211 \\
0.00191 \\
0.00160\end{array}$ & $\begin{array}{l}0.0451 \\
0.0479 \\
0.0507 \\
0.0576 \\
0.0642 \\
0.0704 \\
0.0764 \\
0.0822 \\
0.0876 \\
0.0983\end{array}$ & $\begin{array}{l}6.80 \\
7.22 \\
7.51 \\
8.51 \\
9.47 \\
10.4 \\
11.3 \\
12.1 \\
12.9 \\
14.6\end{array}$ & $\begin{array}{l}0.0143 \\
0.0170 \\
0.0199 \\
0.0279 \\
0.0369 \\
0.0470 \\
0.0579 \\
0.0696 \\
0.0822 \\
0.110\end{array}$ & $\begin{array}{l}1.00833 \\
1.00758 \\
1.00696 \\
1.00577 \\
1.00493 \\
1.00431 \\
1.00382 \\
1.00344 \\
1.00312 \\
1.00264\end{array}$ & $\begin{array}{l}0.693 \\
0.689 \\
0.673 \\
0.667 \\
0.663 \\
0.661 \\
0.660 \\
0.659 \\
0.660 \\
0.662\end{array}$ \\
\hline $\begin{array}{l}0.00138 \\
0.00121 \\
0.00108 \\
0.000977 \\
0.000818 \\
0.000703 \\
0.000616 \\
0.000549 \\
0.000495 \\
0.000397\end{array}$ & $\begin{array}{l}0.109 \\
0.119 \\
0.129 \\
0.139 \\
0.157 \\
0.175 \\
0.192 \\
0.209 \\
0.225 \\
0.263\end{array}$ & $\begin{array}{l}16.2 \\
17.7 \\
19.2 \\
20.6 \\
23.4 \\
26.0 \\
28.6 \\
31.1 \\
33.5 \\
39.2\end{array}$ & $\begin{array}{l}0.141 \\
0.176 \\
0.214 \\
0.255 \\
0.345 \\
0.447 \\
0.560 \\
0.683 \\
0.816 \\
1.19\end{array}$ & $\begin{array}{l}1.00228 \\
1.00201 \\
1.00180 \\
1.00163 \\
1.00137 \\
1.00118 \\
1.00103 \\
1.00092 \\
1.00083 \\
1.00067\end{array}$ & $\begin{array}{l}0.663 \\
0.663 \\
0.664 \\
0.664 \\
0.664 \\
0.665 \\
0.665 \\
0.665 \\
0.665 \\
0.665\end{array}$ \\
\hline 0.000331 & 0.300 & 44.6 & 1.63 & 1.00056 & 0.665 \\
\hline
\end{tabular}

IHERMAL DIELECTRIC PRANDIL DIFFUSIVITY CDNSTANT NUMBER SQ FT/HR

NUMB 0.754

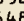

.574

551

541

.538

5

585

(

631

0.683

0.703

0.709

693 .673

.663

661 662

663

664

664

0.665

665

* two-phase goundary 


\begin{tabular}{|c|c|c|c|c|c|c|c|c|c|}
\hline $\begin{array}{c}\text { TEMPERATURE } \\
\text { OEG. R }\end{array}$ & $\begin{array}{l}\text { VOLUME } \\
\text { CU FT/LB }\end{array}$ & $\begin{array}{c}\text { ISOTHERM } \\
\text { OERIVATIVE } \\
\text { CU FT-PSIA/LB }\end{array}$ & $\begin{array}{l}\text { ISOCHORE } \\
\text { DERIVATIVE } \\
\text { PSIA/R }\end{array}$ & $\begin{array}{l}\text { INTERNAL } \\
\text { ENERGY } \\
\text { BTU/LB }\end{array}$ & $\begin{array}{l}\text { ENTHALPY } \\
\text { BTU/LB }\end{array}$ & $\begin{array}{l}\text { ENTROPY } \\
\text { BTU/LB-R }\end{array}$ & $\begin{array}{l}\text { CV } \\
\text { BTU }\end{array}$ & /LB $\quad C P$ & $\begin{array}{l}\text { VEL OCITY } \\
\text { OF SOUNO } \\
\text { FT/SEC }\end{array}$ \\
\hline $\begin{array}{r}7.0 \\
8.0 \\
9.0 \\
10.0 \\
11.0 \\
12.0 \\
13.0 \\
14.0\end{array}$ & $\begin{array}{l}0.07731 \\
0.07804 \\
0.07877 \\
0.07949 \\
0.08020 \\
0.08094 \\
0.08171 \\
0.08252\end{array}$ & $\begin{array}{l}635.0 \\
642.0 \\
638.0 \\
630.0 \\
616.0 \\
601.0 \\
586.0 \\
569.0\end{array}$ & $\begin{array}{l}80.0 \\
74.9 \\
72.3 \\
70.7 \\
69.7 \\
69.1 \\
68.7 \\
68.4\end{array}$ & $\begin{array}{l}3.698 \\
3.940 \\
4.292 \\
4.706 \\
5.124 \\
5.569 \\
6.040 \\
6.537\end{array}$ & $\begin{array}{l}23.74 \\
24.17 \\
24.71 \\
25.31 \\
25.92 \\
26.55 \\
27.22 \\
27.93\end{array}$ & $\begin{array}{l}0.3693 \\
0.4274 \\
0.4877 \\
0.5484 \\
0.6065 \\
0.6627 \\
0.7173 \\
0.7706\end{array}$ & $\begin{array}{l}0.3449 \\
0.4013 \\
0.4721 \\
0.4994 \\
0.5238 \\
0.5480 \\
0.5704 \\
0.5908\end{array}$ & $\begin{array}{l}0.4230 \\
0.4801 \\
0.5568 \\
0.5923 \\
0.6271 \\
0.6636 \\
0.7001 \\
0.7359\end{array}$ & $\begin{array}{l}1899.0 \\
1886.0 \\
1867.0 \\
1850.0 \\
1849.0 \\
1837.0 \\
1825.0 \\
1813.0\end{array}$ \\
\hline $\begin{array}{l}15.0 \\
16.0 \\
17.0 \\
18.0 \\
19.0 \\
20.0 \\
22.0 \\
24.0 \\
26.0 \\
28.0\end{array}$ & $\begin{array}{l}0.08335 \\
0.08423 \\
0.08514 \\
0.08610 \\
0.08711 \\
0.08816 \\
0.09034 \\
0.09265 \\
0.09509 \\
0.09769\end{array}$ & $\begin{array}{l}553.0 \\
538.0 \\
522.0 \\
507.0 \\
493.0 \\
480.0 \\
454.0 \\
430.0 \\
408.0 \\
389.0\end{array}$ & $\begin{array}{l}68.1 \\
67.7 \\
67.3 \\
66.7 \\
65.6 \\
64.5 \\
62.2 \\
59.8 \\
57.3 \\
54.9\end{array}$ & $\begin{array}{c}7.059 \\
7.605 \\
8.173 \\
8.763 \\
9.462 \\
10.18 \\
11.69 \\
13.27 \\
14.91 \\
16.52\end{array}$ & $\begin{array}{l}28.67 \\
29.44 \\
30.25 \\
31.98 \\
32.05 \\
33.04 \\
35.11 \\
37.29 \\
39.56 \\
41.84\end{array}$ & $\begin{array}{l}0.8227 \\
0.8737 \\
0.9237 \\
0.9727 \\
1.026 \\
1.077 \\
1.178 \\
1.274 \\
1.366 \\
1.452\end{array}$ & $\begin{array}{l}0.6093 \\
0.6261 \\
0.6415 \\
0.6556 \\
0.6688 \\
0.6804 \\
0.6998 \\
0.7147 \\
0.7257 \\
0.7341\end{array}$ & $\begin{array}{l}0.7711 \\
0.8055 \\
0.8392 \\
0.8720 \\
0.9017 \\
0.9301 \\
0.9833 \\
1.032 \\
1.076 \\
1.117\end{array}$ & $\begin{array}{l}1801.0 \\
1790.0 \\
1779.0 \\
1768.0 \\
1755.0 \\
1743.0 \\
1719.0 \\
1696.0 \\
1674.0 \\
1656.0\end{array}$ \\
\hline $\begin{array}{l}30.0 \\
32.0 \\
34.0 \\
36.0 \\
38.0 \\
40.0\end{array}$ & $\begin{array}{l}0.1005 \\
0.1034 \\
0.1064 \\
0.1096 \\
0.1129 \\
0.1163\end{array}$ & $\begin{array}{l}372.0 \\
358.0 \\
346.0 \\
337.0 \\
329.0 \\
323.0\end{array}$ & $\begin{array}{l}52.5 \\
50.1 \\
47.8 \\
45.5 \\
43.4 \\
41.3\end{array}$ & $\begin{array}{l}18.07 \\
19.65 \\
21.28 \\
22.92 \\
24.58 \\
26.25\end{array}$ & $\begin{array}{l}44.12 \\
46.46 \\
48.87 \\
51.34 \\
53.86 \\
56.41\end{array}$ & $\begin{array}{l}1.530 \\
1.606 \\
1.679 \\
1.749 \\
1.817 \\
1.883\end{array}$ & $\begin{array}{l}0.7410 \\
0.7462 \\
0.7502 \\
0.7532 \\
0.7556 \\
0.7575\end{array}$ & $\begin{array}{l}1.156 \\
1.190 \\
1.220 \\
1.246 \\
1.268 \\
1.287\end{array}$ & $\begin{array}{l}1640.0 \\
1627.0 \\
1616.0 \\
1607.0 \\
1600.0 \\
1595.0\end{array}$ \\
\hline $\begin{array}{r}45.0 \\
50.0 \\
55.0 \\
60.0 \\
70.0 \\
80.0 \\
90.0 \\
100.0 \\
120.0 \\
140.0\end{array}$ & $\begin{array}{l}0.1253 \\
0.1346 \\
0.1442 \\
0.1541 \\
0.1740 \\
0.1942 \\
0.2143 \\
0.2344 \\
0.2744 \\
0.3140\end{array}$ & $\begin{array}{l}314.0 \\
313.0 \\
315.0 \\
321.0 \\
339.0 \\
361.0 \\
385.0 \\
410.0 \\
463.0 \\
517.0\end{array}$ & $\begin{array}{l}36.7 \\
32.8 \\
29.5 \\
26.8 \\
22.5 \\
19.3 \\
16.9 \\
15.0 \\
12.2 \\
10.3\end{array}$ & $\begin{array}{r}30.47 \\
34.70 \\
38.94 \\
43.16 \\
51.54 \\
59.81 \\
67.99 \\
76.07 \\
92.02 \\
107.7\end{array}$ & $\begin{array}{c}62.94 \\
69.60 \\
76.33 \\
83.10 \\
96.65 \\
110.1 \\
123.5 \\
136.8 \\
163.1 \\
189.1\end{array}$ & $\begin{array}{l}2.036 \\
2.177 \\
2.305 \\
2.423 \\
2.632 \\
2.812 \\
2.970 \\
3.110 \\
3.350 \\
? .550\end{array}$ & $\begin{array}{l}0.7610 \\
0.7635 \\
0.7653 \\
0.7666 \\
0.7681 \\
0.7686 \\
0.7685 \\
0.7679 \\
0.7662 \\
0.7642\end{array}$ & $\begin{array}{l}1.321 \\
1.341 \\
1.351 \\
1.355 \\
1.353 \\
1.345 \\
1.335 \\
1.325 \\
1.306 \\
1.292\end{array}$ & $\begin{array}{l}1590.0 \\
1595.0 \\
1606.0 \\
1622.0 \\
1663.0 \\
1710.0 \\
1760.0 \\
1811.0 \\
1913.0 \\
2012.0\end{array}$ \\
\hline $\begin{array}{l}160.0 \\
180.0 \\
200.0 \\
250.0 \\
300.0 \\
350.0 \\
400.0 \\
450.0 \\
500.0 \\
600.0\end{array}$ & $\begin{array}{l}0.3532 \\
0.3922 \\
0.4310 \\
0.5274 \\
0.6233 \\
0.7188 \\
0.8143 \\
0.9096 \\
1.005 \\
1.196\end{array}$ & $\begin{array}{r}571.0 \\
625.0 \\
679.0 \\
812.0 \\
946.0 \\
1080.0 \\
1210.0 \\
1340.0 \\
1480.0 \\
1740.0\end{array}$ & $\begin{array}{l}8.95 \\
7.90 \\
7.07 \\
5.61 \\
4.66 \\
3.99 \\
3.48 \\
3.09 \\
2.78 \\
2.32\end{array}$ & $\begin{array}{l}123.3 \\
138.7 \\
154.0 \\
192.1 \\
229.9 \\
267.5 \\
305.0 \\
342.5 \\
380.0 \\
454.7\end{array}$ & $\begin{array}{l}214.9 \\
240.4 \\
265.8 \\
328.8 \\
391.4 \\
453.9 \\
516.1 \\
578.3 \\
640.5 \\
764.7\end{array}$ & $\begin{array}{l}3.722 \\
3.872 \\
4.006 \\
4.287 \\
4.516 \\
4.708 \\
4.875 \\
5.021 \\
5.152 \\
5.378\end{array}$ & $\begin{array}{l}0.7624 \\
0.7608 \\
0.7594 \\
0.7567 \\
0.7548 \\
0.7534 \\
0.7524 \\
0.7516 \\
0.7509 \\
0.7500\end{array}$ & $\begin{array}{l}1.281 \\
1.273 \\
1.266 \\
1.256 \\
1.250 \\
1.247 \\
1.245 \\
1.243 \\
1.242 \\
1.241\end{array}$ & $\begin{array}{l}2108.0 \\
2201.0 \\
2290.0 \\
2499.0 \\
2694.0 \\
2875.0 \\
3046.0 \\
3208.0 \\
3363.0 \\
3653.0\end{array}$ \\
\hline $\begin{array}{r}700.0 \\
800.0 \\
900.0 \\
1000.0 \\
1200.0 \\
1400.0 \\
1600.0 \\
1800.0 \\
2000.0 \\
2500.0\end{array}$ & $\begin{array}{l}1.386 \\
1.577 \\
1.767 \\
1.958 \\
2.339 \\
2.721 \\
3.103 \\
3.484 \\
3.866 \\
4.821\end{array}$ & $\begin{array}{l}2010.0 \\
2270.0 \\
2540.0 \\
2800.0 \\
3330.0 \\
3860.0 \\
4400.0 \\
4930.0 \\
5460.0 \\
6800.0\end{array}$ & $\begin{array}{l}1.99 \\
1.74 \\
1.55 \\
1.39 \\
1.16 \\
1.00 \\
0.872 \\
0.775 \\
0.698 \\
0.558\end{array}$ & $\begin{array}{r}529.4 \\
604.1 \\
678.7 \\
753.3 \\
902.5 \\
1052.0 \\
1201.0 \\
1350.0 \\
1499.0 \\
1872.0\end{array}$ & $\begin{array}{r}888.7 \\
1013.0 \\
1137.0 \\
1261.0 \\
1509.0 \\
1757.0 \\
2005.0 \\
2253.0 \\
2501.0 \\
3121.0\end{array}$ & $\begin{array}{l}5.570 \\
5.735 \\
5.881 \\
6.012 \\
6.238 \\
6.429 \\
6.595 \\
6.741 \\
6.872 \\
7.149\end{array}$ & $\begin{array}{l}0.7494 \\
0.7489 \\
0.7485 \\
0.7483 \\
0.7478 \\
0.7476 \\
0.7473 \\
0.7472 \\
0.7470 \\
0.7468\end{array}$ & $\begin{array}{l}1.241 \\
1.240 \\
1.240 \\
1.240 \\
1.240 \\
1.240 \\
1.240 \\
1.240 \\
1.240 \\
1.240\end{array}$ & $\begin{array}{l}3922.0 \\
4174.0 \\
4412.0 \\
4638.0 \\
5060.0 \\
5450.0 \\
5814.0 \\
6157.0 \\
6482.0 \\
7231.0\end{array}$ \\
\hline 3000.0 & 5.776 & 8130.0 & 0.465 & 2244.0 & 3742.0 & 7.375 & 0.7466 & 1.241 & 7910.0 \\
\hline
\end{tabular}


1400 PSIA ISOBAR

OEG. R LB/CUFT BTU/LE PSIA-CU FT/BTU PSIA
(OVIOT) $/ V$ THERMAL VISCOSITY

1/OEG. R GTU/FT-HR-R LA/FT-SEC
THERMAL DIELECTRIC DIFFUSIVITY CONSTANT

SQ FT/HR

CONSTAN
PRANDTL

NUMBER

\footnotetext{
FTh
}

\begin{tabular}{|c|c|c|c|c|c|c|c|}
\hline $\begin{array}{l}17.9 \\
14.6 \\
12.1 \\
11.3 \\
10.7 \\
10.2 \\
9.84 \\
9.55\end{array}$ & $\begin{array}{l}8210.0 \\
8230.0 \\
8100.0 \\
7920.0 \\
7690.0 \\
7430.0 \\
7170.0 \\
6900.0\end{array}$ & $\begin{array}{l}0.00974 \\
0.00910 \\
0.00893 \\
0.00893 \\
0.00907 \\
0.00930 \\
0.00959 \\
0.00991\end{array}$ & $\begin{array}{l}0.0219 \\
0.0236 \\
0.0253 \\
0.0269 \\
0.0284 \\
0.0298 \\
0.0310 \\
0.0320\end{array}$ & $\begin{array}{r}16.1 \\
13.6 \\
11.7 \\
10.4 \\
9.37 \\
8.57 \\
7.94 \\
7.43\end{array}$ & $\begin{array}{l}0.00400 \\
0.00383 \\
0.00357 \\
0.00361 \\
0.00363 \\
0.00363 \\
0.00361 \\
0.00359\end{array}$ & $\begin{array}{l}1.02037 \\
1.02045 \\
1.02052 \\
1.02059 \\
1.02065 \\
1.02070 \\
1.02075 \\
1.02080\end{array}$ & $\begin{array}{l}1.12 \\
1.00 \\
0.931 \\
0.823 \\
0.744 \\
0.688 \\
0.647 \\
0.615\end{array}$ \\
\hline $\begin{array}{l}9.32 \\
9.11 \\
8.93 \\
8.76 \\
8.54 \\
8.36 \\
8.03 \\
7.75 \\
7.51 \\
7.30\end{array}$ & $\begin{array}{l}6640.0 \\
6380.0 \\
6130.0 \\
5890.0 \\
5660.0 \\
5440.0 \\
5020.0 \\
4640.0 \\
4290.0 \\
3980.0\end{array}$ & $\begin{array}{l}0.0103 \\
0.0106 \\
0.0110 \\
0.0113 \\
0.0116 \\
0.0119 \\
0.0124 \\
0.0129 \\
0.0133 \\
0.0138\end{array}$ & $\begin{array}{l}0.0329 \\
0.0336 \\
0.0342 \\
0.0347 \\
0.0351 \\
0.0354 \\
0.0359 \\
0.0361 \\
0.0361 \\
0.0361\end{array}$ & $\begin{array}{l}7.00 \\
6.65 \\
6.35 \\
6.10 \\
5.88 \\
5.69 \\
5.39 \\
5.17 \\
5.01 \\
4.88\end{array}$ & $\begin{array}{l}0.00355 \\
0.00351 \\
0.00347 \\
0.00343 \\
0.00339 \\
0.00336 \\
0.00329 \\
0.00324 \\
0.00319 \\
0.00316\end{array}$ & $\begin{array}{l}1.02085 \\
1.02089 \\
1.02092 \\
1.02095 \\
1.02098 \\
1.02100 \\
1.02102 \\
1.02101 \\
1.02097 \\
1.02091\end{array}$ & $\begin{array}{l}0.592 \\
0.574 \\
0.561 \\
0.552 \\
0.544 \\
0.538 \\
0.533 \\
0.533 \\
0.537 \\
0.544\end{array}$ \\
\hline $\begin{array}{l}7.12 \\
6.94 \\
6.78 \\
6.63 \\
6.48 \\
6.34\end{array}$ & $\begin{array}{l}3710.0 \\
3470.0 \\
3260.0 \\
3070.0 \\
2910.0 \\
2780.0\end{array}$ & $\begin{array}{l}0.0142 \\
0.0145 \\
0.0147 \\
0.0148 \\
0.0149 \\
0.0149\end{array}$ & $\begin{array}{l}0.0360 \\
0.0358 \\
0.0356 \\
0.0354 \\
0.0352 \\
0.0350\end{array}$ & $\begin{array}{l}4.79 \\
4.71 \\
4.66 \\
4.62 \\
4.60 \\
4.59\end{array}$ & $\begin{array}{l}0.00313 \\
0.00311 \\
0.00311 \\
0.00311 \\
0.00313 \\
0.00317\end{array}$ & $\begin{array}{l}1.02082 \\
1.02070 \\
1.02056 \\
1.02039 \\
1.02020 \\
1.01999\end{array}$ & $\begin{array}{l}0.553 \\
0.564 \\
0.575 \\
0.586 \\
0.596 \\
0.507\end{array}$ \\
\hline $\begin{array}{l}6.04 \\
5.78 \\
5.57 \\
5.38 \\
5.09 \\
4.87 \\
4.71 \\
4.57 \\
4.33 \\
4.24\end{array}$ & $\begin{array}{l}2510.0 \\
2320.0 \\
2190.0 \\
2090.0 \\
1950.0 \\
1860.0 \\
1800.0 \\
1750.0 \\
1690.0 \\
1650.0\end{array}$ & $\begin{array}{l}0.0146 \\
0.0141 \\
0.0135 \\
0.0128 \\
0.0115 \\
0.0104 \\
0.00940 \\
0.00856 \\
0.00724 \\
0.00527\end{array}$ & $\begin{array}{l}0.0345 \\
0.0344 \\
0.0344 \\
0.0345 \\
0.0350 \\
0.0359 \\
0.0363 \\
0.0380 \\
0.0405 \\
0.0433\end{array}$ & $\begin{array}{l}4.59 \\
4.63 \\
4.70 \\
4.78 \\
4.96 \\
5.17 \\
5.39 \\
5.60 \\
6.04 \\
6.49\end{array}$ & $\begin{array}{l}0.00328 \\
0.00346 \\
0.00367 \\
0.00392 \\
0.00450 \\
0.00517 \\
0.00591 \\
0.00672 \\
0.00852 \\
0.0105\end{array}$ & $\begin{array}{l}1.01941 \\
1.01877 \\
1.01911 \\
1.01744 \\
1.01617 \\
1.01502 \\
1.01399 \\
1.01308 \\
1.01156 \\
1.01035\end{array}$ & $\begin{array}{l}0.630 \\
0.649 \\
0.665 \\
0.676 \\
0.691 \\
0.699 \\
0.703 \\
0.703 \\
0.701 \\
0.696\end{array}$ \\
\hline $\begin{array}{l}4.15 \\
4.07 \\
4.01 \\
3.91 \\
3.85 \\
3.80 \\
3.77 \\
3.75 \\
3.73 \\
3.70\end{array}$ & $\begin{array}{l}1620.0 \\
1590.0 \\
1570.0 \\
1540.0 \\
1520.0 \\
1500.0 \\
1490.0 \\
1480.0 \\
1470.0 \\
1460.0\end{array}$ & $\begin{array}{l}0.00554 \\
0.00496 \\
0.00449 \\
0.00364 \\
0.00307 \\
0.00266 \\
0.00234 \\
0.00210 \\
0.00190 \\
0.00159\end{array}$ & $\begin{array}{l}0.0463 \\
0.0488 \\
0.0515 \\
0.0583 \\
0.0649 \\
0.0710 \\
0.0773 \\
0.0825 \\
0.0881 \\
0.0987\end{array}$ & $\begin{array}{r}6.90 \\
7.32 \\
7.60 \\
8.59 \\
9.54 \\
10.4 \\
11.3 \\
12.2 \\
13.0 \\
14.6\end{array}$ & $\begin{array}{l}0.0127 \\
0.0150 \\
0.0175 \\
0.0245 \\
0.0323 \\
0.0410 \\
0.0504 \\
0.0605 \\
0.0712 \\
0.0950\end{array}$ & $\begin{array}{l}1.00936 \\
1.00855 \\
1.00787 \\
1.00657 \\
1.00564 \\
1.00494 \\
1.00439 \\
1.00395 \\
1.00360 \\
1.00304\end{array}$ & $\begin{array}{l}0.692 \\
0.687 \\
0.672 \\
0.666 \\
0.662 \\
0.660 \\
0.658 \\
0.658 \\
0.659 \\
0.660\end{array}$ \\
\hline $\begin{array}{l}3.68 \\
3.67 \\
3.65 \\
3.65 \\
3.63 \\
3.63 \\
3.62 \\
3.61 \\
3.61 \\
3.60\end{array}$ & $\begin{array}{l}1450.0 \\
1440.0 \\
1430.0 \\
1430.0 \\
1420.0 \\
1420.0 \\
1420.0 \\
1410.0 \\
1410.0 \\
1410.0\end{array}$ & $\begin{array}{l}0.00137 \\
0.00121 \\
0.00138 \\
0.000974 \\
0.000815 \\
0.000701 \\
0.000615 \\
0.000548 \\
0.000494 \\
0.000396\end{array}$ & $\begin{array}{l}0.109 \\
0.119 \\
0.129 \\
0.139 \\
0.157 \\
0.175 \\
0.192 \\
0.209 \\
0.225 \\
0.263\end{array}$ & $\begin{array}{l}16.2 \\
17.7 \\
19 . ? \\
20.6 \\
23.4 \\
26.1 \\
28.6 \\
31.1 \\
33.5 \\
39.2\end{array}$ & $\begin{array}{l}0.122 \\
0.152 \\
0.184 \\
0.219 \\
0.297 \\
0.384 \\
0.481 \\
0.587 \\
0.701 \\
1.02\end{array}$ & $\begin{array}{l}1.00264 \\
1.00233 \\
1.00208 \\
1.00189 \\
1.00158 \\
1.00137 \\
1.00120 \\
1.00107 \\
1.00097 \\
1.00078\end{array}$ & $\begin{array}{l}0.662 \\
0.662 \\
0.663 \\
0.663 \\
0.664 \\
0.664 \\
0.664 \\
0.665 \\
0.665 \\
0.665\end{array}$ \\
\hline 3.63 & 1410.0 & 0.000331 & 0.300 & 44.6 & 1.40 & 1.00065 & 0.665 \\
\hline
\end{tabular}

* tho-PHASE boundarY 
1600 PSIA ISOBAR

THERMOOYNAMIC PROPERTIES OF HELIUM 4

\begin{tabular}{|c|c|c|c|c|c|c|c|c|c|}
\hline $\begin{array}{c}\text { TEMPERATURE } \\
\text { OEG. R }\end{array}$ & $\begin{array}{l}\text { VOLUME } \\
\text { CU FT/LB }\end{array}$ & $\begin{array}{c}\text { ISOTHERM } \\
\text { DERIVATIVE } \\
\text { CU FT-PSIAILB }\end{array}$ & $\begin{array}{l}\text { ISOCHORE } \\
\text { OERIVATIVE } \\
\text { PSIA/R }\end{array}$ & $\begin{array}{l}\text { INTERNAL } \\
\text { ENERGY } \\
\text { BTU/LB }\end{array}$ & $\begin{array}{l}\text { ENTHALPY } \\
\text { BTU/LB }\end{array}$ & $\begin{array}{l}\text { ENTROPY } \\
\text { BTU/LB-R }\end{array}$ & $\begin{array}{l}\text { CV } \\
\text { RTU / LB }\end{array}$ & $\begin{array}{r}C P \\
-R\end{array}$ & $\begin{array}{l}\text { VEL OCITY } \\
\text { OF SOUNO } \\
\text { FT/SEC }\end{array}$ \\
\hline $\begin{array}{r}7.0 \\
8.0 \\
9.0 \\
10.0 \\
11.0 \\
12.0 \\
13.0 \\
14.0 \\
15.0\end{array}$ & $\begin{array}{l}0.07554 \\
0.07627 \\
0.07696 \\
0.07762 \\
0.07827 \\
0.07893 \\
0.07961 \\
0.08031 \\
0.08104\end{array}$ & $\begin{array}{l}683.0 \\
698.0 \\
698.0 \\
693.0 \\
681.0 \\
667.0 \\
651.0 \\
636.0 \\
620.0\end{array}$ & $\begin{array}{l}93.6 \\
82.8 \\
77.4 \\
74.3 \\
72.5 \\
71.5 \\
71.0 \\
70.7 \\
70.5\end{array}$ & $\begin{array}{l}3.987 \\
4.224 \\
4.569 \\
4.973 \\
5.379 \\
5.811 \\
6.268 \\
6.748 \\
7.251\end{array}$ & $\begin{array}{l}26.37 \\
26.82 \\
27.37 \\
27.97 \\
28.57 \\
29.20 \\
29.85 \\
30.54 \\
31.26\end{array}$ & $\begin{array}{l}0.3403 \\
0.4014 \\
0.4626 \\
0.5233 \\
0.5809 \\
0.6363 \\
0.6899 \\
0.7420 \\
0.7928\end{array}$ & $\begin{array}{l}0.3627 \\
0.4085 \\
0.4754 \\
0.5005 \\
0.5234 \\
0.5464 \\
0.5678 \\
0.5874 \\
0.6053\end{array}$ & $\begin{array}{l}0.4576 \\
0.4932 \\
0.5600 \\
0.5894 \\
0.6197 \\
0.6525 \\
0.6857 \\
0.7188 \\
0.7515\end{array}$ & $\begin{array}{l}1998.0 \\
1976.0 \\
1952.0 \\
1944.0 \\
1932.0 \\
1921.0 \\
1909.0 \\
1898.0 \\
1888.0\end{array}$ \\
\hline $\begin{array}{l}16.0 \\
17.0 \\
18.0 \\
19.0 \\
20.0 \\
22.0 \\
24.0 \\
26.0 \\
28.0 \\
30.0\end{array}$ & $\begin{array}{l}0.08181 \\
0.08260 \\
0.08343 \\
0.08431 \\
0.08522 \\
0.08709 \\
0.08907 \\
0.09115 \\
0.09335 \\
0.09568\end{array}$ & $\begin{array}{l}604.0 \\
589.0 \\
574.0 \\
559.0 \\
546.0 \\
519.0 \\
495.0 \\
472.0 \\
452.0 \\
433.0\end{array}$ & $\begin{array}{l}70.3 \\
70.0 \\
69.6 \\
68.7 \\
67.7 \\
65.7 \\
63.5 \\
61.2 \\
59.0 \\
56.7\end{array}$ & $\begin{array}{r}7.776 \\
8.323 \\
8.890 \\
9.570 \\
10.27 \\
11.74 \\
13.27 \\
14.88 \\
16.44 \\
17.95\end{array}$ & $\begin{array}{l}32.01 \\
32.80 \\
33.61 \\
34.55 \\
35.52 \\
37.54 \\
39.66 \\
41.88 \\
44.10 \\
46.30\end{array}$ & $\begin{array}{l}0.8424 \\
0.8911 \\
0.9387 \\
0.9905 \\
1.041 \\
1.139 \\
1.233 \\
1.323 \\
1.406 \\
1.482\end{array}$ & $\begin{array}{l}0.6218 \\
0.6371 \\
0.6513 \\
0.6644 \\
0.6762 \\
0.6964 \\
0.7124 \\
0.7241 \\
0.7332 \\
0.7409\end{array}$ & $\begin{array}{l}0.7839 \\
0.8158 \\
0.8471 \\
0.8753 \\
0.9024 \\
0.9533 \\
1.000 \\
1.041 \\
1.081 \\
1.119\end{array}$ & $\begin{array}{l}1878.0 \\
1869.0 \\
1859.0 \\
1848.0 \\
1836.0 \\
1815.0 \\
1794.0 \\
1773.0 \\
1756.0 \\
1741.0\end{array}$ \\
\hline $\begin{array}{l}32.0 \\
34.0 \\
36.0 \\
38.0 \\
40.0\end{array}$ & $\begin{array}{l}0.09814 \\
0.1007 \\
0.1034 \\
0.1062 \\
0.1090\end{array}$ & $\begin{array}{l}417.0 \\
403.0 \\
392.0 \\
382.0 \\
373.0\end{array}$ & $\begin{array}{l}54.5 \\
52.2 \\
50.0 \\
47.9 \\
45.8\end{array}$ & $\begin{array}{l}19.49 \\
21.07 \\
22.67 \\
24.28 \\
25.92\end{array}$ & $\begin{array}{l}48.57 \\
50.90 \\
53.30 \\
55.74 \\
58.23\end{array}$ & $\begin{array}{l}1.555 \\
1.626 \\
1.694 \\
1.760 \\
1.824\end{array}$ & $\begin{array}{l}0.7466 \\
0.7509 \\
0.7543 \\
0.7568 \\
0.7588\end{array}$ & $\begin{array}{l}1.153 \\
1.183 \\
1.210 \\
1.233 \\
1.254\end{array}$ & $\begin{array}{l}1728.0 \\
1716.0 \\
1706.0 \\
1697.0 \\
1691.0\end{array}$ \\
\hline $\begin{array}{r}45.0 \\
50.0 \\
55.0 \\
60.0 \\
70.0 \\
80.0 \\
90.0 \\
100.0 \\
120.0 \\
140.0\end{array}$ & $\begin{array}{l}0.1166 \\
0.1245 \\
0.1328 \\
0.1412 \\
0.1584 \\
0.1759 \\
0.1935 \\
0.2110 \\
0.2460 \\
0.2806\end{array}$ & $\begin{array}{l}360.0 \\
354.0 \\
353.0 \\
356.0 \\
370.0 \\
389.0 \\
412.0 \\
436.0 \\
487.0 \\
540.0\end{array}$ & $\begin{array}{l}41.1 \\
37.0 \\
33.4 \\
30.4 \\
25.6 \\
22.1 \\
19.3 \\
17.2 \\
14.0 \\
11.8\end{array}$ & $\begin{array}{c}30.06 \\
34.25 \\
38.45 \\
42.65 \\
51.01 \\
59.29 \\
67.49 \\
75.61 \\
91.63 \\
107.4\end{array}$ & $\begin{array}{l}64.60 \\
71.14 \\
77.78 \\
84.48 \\
97.95 \\
111.4 \\
124.8 \\
138.1 \\
164.5 \\
190.6\end{array}$ & $\begin{array}{l}1.974 \\
2.112 \\
2.238 \\
2.355 \\
2.563 \\
2.742 \\
2.900 \\
3.041 \\
3.281 \\
3.482\end{array}$ & $\begin{array}{l}0.7624 \\
0.7649 \\
0.7667 \\
0.7681 \\
0.7698 \\
0.7706 \\
0.7706 \\
0.7702 \\
0.7686 \\
0.7667\end{array}$ & $\begin{array}{l}1.294 \\
1.319 \\
1.335 \\
1.344 \\
1.348 \\
1.344 \\
1.336 \\
1.327 \\
1.310 \\
1.296\end{array}$ & $\begin{array}{l}1681.0 \\
1681.0 \\
1687.0 \\
1699.0 \\
1732.0 \\
1773.0 \\
1818.0 \\
1865.0 \\
1961.0 \\
2056.0\end{array}$ \\
\hline $\begin{array}{l}160.0 \\
180.0 \\
200.0 \\
250.0 \\
300.0 \\
350.0 \\
400.0 \\
450.0 \\
500.0 \\
600.0\end{array}$ & $\begin{array}{l}0.3150 \\
0.3491 \\
0.3831 \\
0.4675 \\
0.5514 \\
0.6350 \\
0.7184 \\
0.8018 \\
0.8852 \\
1.052\end{array}$ & $\begin{array}{r}594.0 \\
647.0 \\
700.0 \\
834.0 \\
966.0 \\
1100.0 \\
1230.0 \\
1360.0 \\
1490.0 \\
1760.0\end{array}$ & $\begin{array}{c}10.2 \\
9.04 \\
8.09 \\
6.41 \\
5.32 \\
4.55 \\
3.98 \\
3.53 \\
3.18 \\
2.65\end{array}$ & $\begin{array}{l}123.0 \\
138.5 \\
153.9 \\
192.0 \\
229.9 \\
267.6 \\
305.2 \\
342.7 \\
380.1 \\
455.0\end{array}$ & $\begin{array}{l}216.4 \\
242.0 \\
267.4 \\
330.5 \\
393.3 \\
455.7 \\
518.0 \\
580.3 \\
642.4 \\
766.6\end{array}$ & $\begin{array}{l}3.654 \\
3.805 \\
3.939 \\
4.221 \\
4.449 \\
4.642 \\
4.808 \\
4.955 \\
5.086 \\
5.312\end{array}$ & $\begin{array}{l}0.7648 \\
0.7630 \\
0.7615 \\
0.7585 \\
0.7563 \\
0.7547 \\
0.7535 \\
0.7526 \\
0.7519 \\
0.7508\end{array}$ & $\begin{array}{l}1.285 \\
1.276 \\
1.269 \\
1.258 \\
1.251 \\
1.248 \\
1.245 \\
1.244 \\
1.243 \\
1.241\end{array}$ & $\begin{array}{l}2149.0 \\
2239.0 \\
2325.0 \\
2531.0 \\
2721.0 \\
2900.0 \\
3069.0 \\
3230.0 \\
3383.0 \\
3671.0\end{array}$ \\
\hline $\begin{array}{r}700.0 \\
800.0 \\
900.0 \\
1000.0 \\
1200.0 \\
1400.0 \\
1600.0 \\
1800.0 \\
2000.0 \\
2500.0\end{array}$ & $\begin{array}{l}1.218 \\
1.385 \\
1.552 \\
1.718 \\
2.052 \\
2.386 \\
2.719 \\
3.053 \\
3.387 \\
4.222\end{array}$ & $\begin{array}{l}2020.0 \\
2290.0 \\
2550.0 \\
2820.0 \\
3350.0 \\
3880.0 \\
4410.0 \\
4940.0 \\
5480.0 \\
6810.0\end{array}$ & $\begin{array}{l}2.27 \\
1.99 \\
1.77 \\
1.59 \\
1.33 \\
1.14 \\
1.00 \\
0.885 \\
0.797 \\
0.638\end{array}$ & $\begin{array}{r}529.7 \\
604.4 \\
679.0 \\
753.6 \\
902.8 \\
1052.0 \\
1201.0 \\
1350.0 \\
1499.0 \\
1872.0\end{array}$ & $\begin{array}{r}890.7 \\
1015.0 \\
1139.0 \\
1263.0 \\
1511.0 \\
1759.0 \\
2007.0 \\
2255.0 \\
2503.0 \\
3123.0\end{array}$ & $\begin{array}{l}5.504 \\
5.669 \\
5.815 \\
5.946 \\
6.172 \\
6.363 \\
6.529 \\
6.575 \\
6.806 \\
7.082\end{array}$ & $\begin{array}{l}0.7501 \\
0.7495 \\
0.7491 \\
0.7488 \\
0.7483 \\
0.7480 \\
0.7477 \\
0.7475 \\
0.7474 \\
0.7471\end{array}$ & $\begin{array}{l}1.241 \\
1.240 \\
1.240 \\
1.240 \\
1.240 \\
1.240 \\
1.240 \\
1.240 \\
1.240 \\
1.240\end{array}$ & $\begin{array}{l}3938.0 \\
4188.0 \\
4425.0 \\
4650.0 \\
5071.0 \\
5459.0 \\
5822.0 \\
6164.0 \\
6488.0 \\
7236.0\end{array}$ \\
\hline 3000.0 & 5.058 & 8140.0 & 0.532 & 2245.0 & 3743.0 & 7.309 & 0.7469 & 1.240 & 7914.0 \\
\hline
\end{tabular}

* tho-phase bounoary 
THERMOPHYSICAL PROPERTIES OF HELIUM 4

1600 PSIA ISOBAR

\begin{tabular}{|c|c|c|c|c|c|c|c|c|c|c|}
\hline $\begin{array}{c}\text { TEMPERATURE } \\
\text { OEG. R }\end{array}$ & $\begin{array}{l}\text { E DENSITY } \\
\text { LB/CU FT }\end{array}$ & $\begin{array}{c}V(O H / O V)_{P} \\
8 T U / L 8\end{array}$ & $\begin{array}{l}V(D P / O U)_{V}- \\
P S I A-C U F T / B T U\end{array}$ & $\begin{array}{l}-V(O P / D V) T \\
U \text { PSIA }\end{array}$ & $\begin{array}{l}(D V / O T) / V \\
\text { 1/OEG. } R\end{array}$ & $\begin{array}{c}\text { THERMAL } \\
\text { CONOUCTIVITY } \\
\text { BTU/FT-HR-R }\end{array}$ & $\begin{array}{l}\text { VISCOSITY } \\
\text { LB/FT-SEC } \\
\times 10 E+6\end{array}$ & $\begin{array}{l}\text { THERMAL } \\
\text { OIFFUSIVITY } \\
\text { SO FT/HR }\end{array}$ & $\begin{array}{l}\text { DIELECTRIC } \\
\text { CONSTANT }\end{array}$ & $\begin{array}{l}\text { PRANOTL } \\
\text { NUMBER }\end{array}$ \\
\hline $\begin{array}{r}7.0 \\
8.0 \\
9.0 \\
10.0 \\
11.0 \\
12.0 \\
13.0 \\
14.0 \\
15.0\end{array}$ & $\begin{array}{l}13.24 \\
13.11 \\
12.99 \\
12.88 \\
12.78 \\
12.67 \\
12.56 \\
12.45 \\
12.34\end{array}$ & $\begin{array}{l}44.2 \\
54.5 \\
65.7 \\
70.8 \\
74.4 \\
77.1 \\
79.1 \\
80.5 \\
81.5\end{array}$ & $\begin{array}{c}19.5 \\
15.5 \\
12.5 \\
11.5 \\
10.8 \\
10.3 \\
9.95 \\
9.66 \\
9.43\end{array}$ & $\begin{array}{l}9040.0 \\
9150.0 \\
9070.0 \\
8920.0 \\
8700.0 \\
8450.0 \\
8180.0 \\
7910.0 \\
7650.0\end{array}$ & $\begin{array}{l}0.0104 \\
0.00905 \\
0.00853 \\
0.00833 \\
0.00833 \\
0.00846 \\
0.00867 \\
0.00893 \\
0.00922\end{array}$ & $\begin{array}{l}0.0229 \\
0.0247 \\
0.0264 \\
0.0282 \\
0.0298 \\
0.0312 \\
0.0325 \\
0.0336 \\
0.0346\end{array}$ & $\begin{array}{c}19.5 \\
16.1 \\
13.7 \\
12.0 \\
10.7 \\
9.75 \\
8.98 \\
8.35 \\
7.83\end{array}$ & $\begin{array}{l}0.00378 \\
0.00381 \\
0.00363 \\
0.00371 \\
0.00376 \\
0.00378 \\
0.00377 \\
0.00376 \\
0.00373\end{array}$ & $\begin{array}{l}1.02015 \\
1.02025 \\
1.02033 \\
1.02040 \\
1.02047 \\
1.02054 \\
1.02060 \\
1.02065 \\
1.02071\end{array}$ & $\begin{array}{l}1.40 \\
1.16 \\
1.05 \\
0.904 \\
0.804 \\
0.734 \\
0.682 \\
0.643 \\
0.613\end{array}$ \\
\hline $\begin{array}{l}16.0 \\
17.0 \\
18.0 \\
19.0 \\
20.0 \\
22.0 \\
24.0 \\
26.0 \\
28.0 \\
30.0\end{array}$ & $\begin{array}{l}12.22 \\
12.11 \\
11.99 \\
11.86 \\
11.74 \\
11.48 \\
11.23 \\
10.97 \\
10.71 \\
10.45\end{array}$ & $\begin{array}{l}82.4 \\
83.1 \\
83.7 \\
84.6 \\
85.3 \\
86.5 \\
87.5 \\
88.2 \\
88.7 \\
69.3\end{array}$ & $\begin{array}{l}9.24 \\
9.07 \\
8.92 \\
8.71 \\
8.54 \\
8.22 \\
7.94 \\
7.70 \\
7.51 \\
7.33\end{array}$ & $\begin{array}{l}7380.0 \\
7130.0 \\
6880.0 \\
6630.0 \\
6400.0 \\
5960.0 \\
5560.0 \\
5180.0 \\
4840.0 \\
4530.0\end{array}$ & $\begin{array}{l}0.00952 \\
0.00982 \\
0.0101 \\
0.0104 \\
0.0106 \\
0.0110 \\
0.0114 \\
0.0118 \\
0.0122 \\
0.0125\end{array}$ & $\begin{array}{l}0.0354 \\
0.0361 \\
0.0366 \\
0.0371 \\
0.0374 \\
0.0379 \\
0.0382 \\
0.0383 \\
0.0383 \\
0.0382\end{array}$ & $\begin{array}{l}7.40 \\
7.04 \\
6.74 \\
6.47 \\
6.24 \\
5.88 \\
5.61 \\
5.41 \\
5.25 \\
5.13\end{array}$ & $\begin{array}{l}0.00369 \\
0.00365 \\
0.00361 \\
0.00357 \\
0.00353 \\
0.00346 \\
0.00340 \\
0.00336 \\
0.00331 \\
0.00327\end{array}$ & $\begin{array}{l}1.02076 \\
1.02081 \\
1.02085 \\
1.02089 \\
1.02093 \\
1.02098 \\
1.02101 \\
1.02102 \\
1.02100 \\
1.02096\end{array}$ & $\begin{array}{l}0.591 \\
0.574 \\
0.561 \\
0.550 \\
0.542 \\
0.532 \\
0.529 \\
0.529 \\
0.533 \\
0.540\end{array}$ \\
\hline $\begin{array}{l}32.0 \\
34.0 \\
36.0 \\
38.0 \\
40.0\end{array}$ & $\begin{array}{c}10.19 \\
9.929 \\
9.672 \\
9.419 \\
9.171\end{array}$ & $\begin{array}{l}90.0 \\
90.7 \\
91.6 \\
92.6 \\
93.7\end{array}$ & $\begin{array}{l}7.16 \\
7.01 \\
6.86 \\
6.72 \\
6.58\end{array}$ & $\begin{array}{l}4250.0 \\
4010.0 \\
3790.0 \\
3590.0 \\
3420.0\end{array}$ & $\begin{array}{l}0.0128 \\
0.0130 \\
0.0132 \\
0.0133 \\
0.0134\end{array}$ & $\begin{array}{l}0.0381 \\
0.0379 \\
0.0377 \\
0.0375 \\
0.0373\end{array}$ & $\begin{array}{l}5.04 \\
4.96 \\
4.91 \\
4.87 \\
4.85\end{array}$ & $\begin{array}{l}0.00324 \\
0.00323 \\
0.00322 \\
0.00323 \\
0.00324\end{array}$ & $\begin{array}{l}1.02090 \\
1.02081 \\
1.02070 \\
1.02057 \\
1.02042\end{array}$ & $\begin{array}{l}0.549 \\
0.558 \\
0.567 \\
0.577 \\
0.587\end{array}$ \\
\hline $\begin{array}{r}45.0 \\
50.0 \\
55.0 \\
60.0 \\
70.0 \\
80.0 \\
90.0 \\
100.0 \\
120.0 \\
140.0\end{array}$ & $\begin{array}{l}8.578 \\
8.031 \\
7.533 \\
7.082 \\
6.311 \\
5.684 \\
5.168 \\
4.738 \\
4.066 \\
3.564\end{array}$ & $\begin{array}{r}97.2 \\
101.0 \\
106.0 \\
111.0 \\
123.0 \\
135.0 \\
147.0 \\
160.0 \\
185.0 \\
211.0\end{array}$ & $\begin{array}{l}6.28 \\
6.02 \\
5.79 \\
5.59 \\
5.28 \\
5.04 \\
4.85 \\
4.70 \\
4.48 \\
4.33\end{array}$ & $\begin{array}{l}3090.0 \\
2840.0 \\
2660.0 \\
2520.0 \\
2330.0 \\
2210.0 \\
2130.0 \\
2070.0 \\
1980.0 \\
1920.0\end{array}$ & $\begin{array}{l}0.0133 \\
0.0130 \\
0.0126 \\
0.0121 \\
0.0110 \\
0.0100 \\
0.00908 \\
0.00831 \\
0.00707 \\
0.00615\end{array}$ & $\begin{array}{l}0.0368 \\
0.0365 \\
0.0364 \\
0.0363 \\
0.0367 \\
0.0373 \\
0.0382 \\
0.0393 \\
0.0417 \\
0.0443\end{array}$ & $\begin{array}{l}4.83 \\
4.85 \\
4.90 \\
4.96 \\
5.13 \\
5.32 \\
5.53 \\
5.74 \\
6.17 \\
6.59\end{array}$ & $\begin{array}{l}0.00332 \\
0.00345 \\
0.00361 \\
0.00382 \\
0.00431 \\
0.00489 \\
0.00554 \\
0.00625 \\
0.00783 \\
0.00959\end{array}$ & $\begin{array}{l}1.01998 \\
1.01946 \\
1.01890 \\
1.01832 \\
1.01716 \\
1.01606 \\
1.01505 \\
1.01415 \\
1.01260 \\
1.01135\end{array}$ & $\begin{array}{l}0.611 \\
0.631 \\
0.647 \\
0.661 \\
0.679 \\
0.690 \\
0.696 \\
0.698 \\
0.697 \\
0.694\end{array}$ \\
\hline $\begin{array}{l}160.0 \\
180.0 \\
200.0 \\
250.0 \\
300.0 \\
350.0 \\
400.0 \\
450.0 \\
500.0 \\
600.0\end{array}$ & $\begin{array}{l}3.175 \\
2.864 \\
2.610 \\
2.139 \\
1.814 \\
1.575 \\
1.392 \\
1.247 \\
1.130 \\
0.9508\end{array}$ & $\begin{array}{l}236.0 \\
262.0 \\
287.0 \\
350.0 \\
412.0 \\
474.0 \\
536.0 \\
598.0 \\
660.0 \\
784.0\end{array}$ & $\begin{array}{l}4.22 \\
4.13 \\
4.07 \\
3.95 \\
3.88 \\
3.83 \\
3.79 \\
3.77 \\
3.74 \\
3.71\end{array}$ & $\begin{array}{l}1880.0 \\
1850.0 \\
1830.0 \\
1780.0 \\
1750.0 \\
1730.0 \\
1710.0 \\
1700.0 \\
1690.0 \\
1670.0\end{array}$ & $\begin{array}{l}0.00544 \\
0.00488 \\
0.00442 \\
0.00360 \\
0.00304 \\
0.00263 \\
0.00232 \\
0.00208 \\
0.00183 \\
0.00158\end{array}$ & $\begin{array}{l}0.0470 \\
0.0497 \\
0.0524 \\
0.0591 \\
0.0655 \\
0.0716 \\
0.0775 \\
0.0831 \\
0.0885 \\
0.0990\end{array}$ & $\begin{array}{c}7.01 \\
7.42 \\
7.69 \\
8.67 \\
9.61 \\
10.5 \\
11.4 \\
12.2 \\
13.0 \\
14.6\end{array}$ & $\begin{array}{l}0.0115 \\
0.0136 \\
0.0158 \\
0.0220 \\
0.0289 \\
0.0365 \\
0.0447 \\
0.0536 \\
0.0630 \\
0.0839\end{array}$ & $\begin{array}{l}1.01032 \\
1.00946 \\
1.00873 \\
1.00732 \\
1.00631 \\
1.00554 \\
1.00494 \\
1.00445 \\
1.00406 \\
1.00344\end{array}$ & $\begin{array}{l}0.690 \\
0.686 \\
0.671 \\
0.665 \\
0.661 \\
0.658 \\
0.657 \\
0.657 \\
0.657 \\
0.659\end{array}$ \\
\hline $\begin{array}{r}700.0 \\
800.0 \\
900.0 \\
1000.0 \\
1200.0 \\
1400.0 \\
1600.0 \\
1800.0 \\
2000.0 \\
2500.0\end{array}$ & $\begin{array}{l}0.8207 \\
0.7220 \\
0.6444 \\
0.5819 \\
0.4873 \\
0.4192 \\
0.3677 \\
0.3275 \\
0.2952 \\
0.2368\end{array}$ & $\begin{array}{r}907.0 \\
1030.0 \\
1150.0 \\
1280.0 \\
1530.0 \\
1770.0 \\
2020.0 \\
2270.0 \\
2520.0 \\
3140.0\end{array}$ & $\begin{array}{l}3.69 \\
3.67 \\
3.66 \\
3.65 \\
3.64 \\
3.63 \\
3.62 \\
3.62 \\
3.61 \\
3.61\end{array}$ & $\begin{array}{l}1660.0 \\
1650.0 \\
1650.0 \\
1640.0 \\
1630.0 \\
1630.0 \\
1620.0 \\
1620.0 \\
1620.0 \\
1610.0\end{array}$ & $\begin{array}{l}0.00137 \\
0.00120 \\
0.00107 \\
0.000970 \\
0.000813 \\
0.000699 \\
0.000614 \\
0.000547 \\
0.000493 \\
0.000396\end{array}$ & $\begin{array}{l}0.109 \\
0.120 \\
0.129 \\
0.139 \\
0.158 \\
0.175 \\
0.192 \\
0.209 \\
0.225 \\
0.263\end{array}$ & $\begin{array}{l}16.2 \\
17.7 \\
19.2 \\
20.6 \\
23.4 \\
26.1 \\
28.6 \\
31.1 \\
33.5 \\
39.2\end{array}$ & $\begin{array}{l}0.108 \\
0.134 \\
0.162 \\
0.193 \\
0.261 \\
0.337 \\
0.422 \\
0.514 \\
0.615 \\
0.897\end{array}$ & $\begin{array}{l}1.00299 \\
1.00264 \\
1.00237 \\
1.00214 \\
1.00180 \\
1.00155 \\
1.00137 \\
1.00122 \\
1.00110 \\
1.00089\end{array}$ & $\begin{array}{l}0.661 \\
0.662 \\
0.662 \\
0.663 \\
0.664 \\
0.664 \\
0.664 \\
0.664 \\
0.664 \\
0.665\end{array}$ \\
\hline 3000.0 & 0.1977 & 3750.0 & 3.60 & 1610.0 & 0.000330 & 0.300 & 44.6 & 1.22 & 1.00074 & 0.665 \\
\hline
\end{tabular}

- THO-PHASE BOUNDARY 


\begin{tabular}{|c|c|c|c|c|c|c|c|c|c|}
\hline TEMPERATURE & $\begin{array}{l}\text { VOL UME } \\
\text { CU FT/LB }\end{array}$ & $\begin{array}{c}\text { ISOTHERM } \\
\text { OERIVATIVE } \\
\text { CU FT -PSIA/LB }\end{array}$ & $\begin{array}{l}\text { ISOCHORE } \\
\text { OERIVATIVE } \\
\text { PSIA/R }\end{array}$ & $\begin{array}{l}\text { INTERNAL } \\
\text { ENEREY } \\
\text { BTU/LP }\end{array}$ & RTU/Le & $\begin{array}{l}\text { ENTROPY } \\
\text { BTU/L8-R }\end{array}$ & $\begin{array}{l}\text { CV } \\
\text { BTU / LB }\end{array}$ & $-R$ & $\begin{array}{l}\text { VELOCITY } \\
\text { OF SOUNO } \\
\text { FT/SEC }\end{array}$ \\
\hline $\begin{array}{r}7.0 \\
8.0 \\
9.0 \\
10.0 \\
11.0 \\
12.0 \\
13.0 \\
14.0 \\
15.0\end{array}$ & $\begin{array}{l}0.07395 \\
0.07470 \\
0.07537 \\
0.07599 \\
0.07658 \\
0.07718 \\
0.07779 \\
0.07842 \\
0.07907\end{array}$ & $\begin{array}{l}727.0 \\
750.0 \\
755.0 \\
752.0 \\
742.0 \\
729.0 \\
715.0 \\
699.0 \\
683.0\end{array}$ & $\begin{array}{r}110.0 \\
91.9 \\
83.1 \\
73.1 \\
75.3 \\
73.8 \\
73.0 \\
72.7 \\
72.6\end{array}$ & $\begin{array}{l}4.274 \\
4.514 \\
4.856 \\
5.255 \\
5.655 \\
6.077 \\
6.522 \\
6.988 \\
7.476\end{array}$ & $\begin{array}{l}28.92 \\
29.41 \\
29.98 \\
30.58 \\
31.18 \\
31.80 \\
32.45 \\
33.13 \\
33.83\end{array}$ & $\begin{array}{l}0.3100 \\
0.3759 \\
0.4390 \\
0.5003 \\
0.5578 \\
0.6127 \\
0.6655 \\
0.7167 \\
0.7665\end{array}$ & $\begin{array}{l}0.3928 \\
0.4229 \\
0.4831 \\
0.5046 \\
0.5251 \\
0.5461 \\
0.5661 \\
0.5845 \\
0.6017\end{array}$ & $\begin{array}{l}0.5102 \\
0.5160 \\
0.5696 \\
0.5914 \\
0.6164 \\
0.5450 \\
0.6748 \\
0.7051 \\
0.7355\end{array}$ & $\begin{array}{l}2091.0 \\
2059.0 \\
2031.0 \\
2021.0 \\
2009.0 \\
1997.0 \\
1986.0 \\
1976.0 \\
1967.0\end{array}$ \\
\hline $\begin{array}{l}16.0 \\
17.0 \\
18.0 \\
19.0 \\
20.0 \\
22.0 \\
24.0 \\
26.0 \\
28.0 \\
30.0\end{array}$ & $\begin{array}{l}0.07975 \\
0.08046 \\
0.08119 \\
0.08197 \\
0.08276 \\
0.08442 \\
0.08615 \\
0.08796 \\
0.08986 \\
0.09188\end{array}$ & $\begin{array}{l}668.0 \\
652.0 \\
637.0 \\
623.0 \\
609.0 \\
583.0 \\
558.0 \\
535.0 \\
513.0 \\
494.0\end{array}$ & $\begin{array}{l}72.5 \\
72.4 \\
72.2 \\
71.4 \\
70.6 \\
68.8 \\
66.8 \\
64.7 \\
62.6 \\
60.5\end{array}$ & $\begin{array}{c}7.983 \\
8.512 \\
9.060 \\
9.723 \\
10.41 \\
11.84 \\
13.34 \\
14.91 \\
16.44 \\
17.90\end{array}$ & $\begin{array}{l}34.57 \\
35.33 \\
36.12 \\
37.04 \\
37.99 \\
39.97 \\
42.06 \\
44.23 \\
46.79 \\
48.53\end{array}$ & $\begin{array}{l}0.8150 \\
0.8625 \\
0.9089 \\
0.9597 \\
1.009 \\
1.106 \\
1.198 \\
1.286 \\
1.367 \\
1.440\end{array}$ & $\begin{array}{l}0.6177 \\
0.6327 \\
0.6468 \\
0.6599 \\
0.6718 \\
0.6927 \\
0.7097 \\
0.7222 \\
0.7320 \\
0.7403\end{array}$ & $\begin{array}{l}0.7659 \\
0.7963 \\
0.8263 \\
0.8533 \\
0.8794 \\
0.9287 \\
0.9735 \\
1.013 \\
1.052 \\
1.089\end{array}$ & $\begin{array}{l}1959.0 \\
1950.0 \\
1942.0 \\
1932.0 \\
1922.0 \\
1902.0 \\
1883.0 \\
1864.0 \\
1848.0 \\
1834.0\end{array}$ \\
\hline $\begin{array}{l}32.0 \\
34.0 \\
36.0 \\
38.0 \\
40.0\end{array}$ & $\begin{array}{l}0.09400 \\
0.09622 \\
0.09852 \\
0.1009 \\
0.1034\end{array}$ & $\begin{array}{l}476.0 \\
461.0 \\
447.0 \\
435.0 \\
425.0\end{array}$ & $\begin{array}{l}58.4 \\
56.2 \\
54.1 \\
52.0 \\
49.9\end{array}$ & $\begin{array}{l}19.41 \\
20.94 \\
22.51 \\
24.09 \\
25.69\end{array}$ & $\begin{array}{l}50.74 \\
53.01 \\
55.35 \\
57.73 \\
60.15\end{array}$ & $\begin{array}{l}1.512 \\
1.581 \\
1.647 \\
1.712 \\
1.774\end{array}$ & $\begin{array}{l}0.7466 \\
0.7514 \\
0.7550 \\
0.7577 \\
0.7599\end{array}$ & $\begin{array}{l}1.122 \\
1.152 \\
1.179 \\
1.203 \\
1.224\end{array}$ & $\begin{array}{l}1820.0 \\
1808.0 \\
1798.0 \\
1789.0 \\
1781.0\end{array}$ \\
\hline $\begin{array}{r}45.0 \\
50.0 \\
55.0 \\
60.0 \\
70.0 \\
80.0 \\
90.0 \\
100.0 \\
120.0 \\
140.0\end{array}$ & $\begin{array}{l}0.1099 \\
0.1168 \\
0.1239 \\
0.1313 \\
0.1464 \\
0.1618 \\
0.1774 \\
0.1929 \\
0.2239 \\
0.2547\end{array}$ & $\begin{array}{l}407.0 \\
396.0 \\
392.0 \\
393.0 \\
402.0 \\
419.0 \\
440.0 \\
463.0 \\
512.0 \\
564.0\end{array}$ & $\begin{array}{l}45.1 \\
40.9 \\
37.2 \\
33.9 \\
28.7 \\
24.8 \\
21.7 \\
19.3 \\
15.8 \\
13.3\end{array}$ & $\begin{array}{c}29.76 \\
33.89 \\
38.05 \\
42.22 \\
50.56 \\
58.84 \\
67.05 \\
75.19 \\
91.27 \\
107.1\end{array}$ & $\begin{array}{c}66.39 \\
72.81 \\
79.35 \\
85.98 \\
99.36 \\
112.8 \\
126.2 \\
139.5 \\
165.9 \\
192.0\end{array}$ & $\begin{array}{l}1.921 \\
2.056 \\
2.181 \\
2.296 \\
2.502 \\
2.681 \\
2.839 \\
2.979 \\
3.220 \\
3.422\end{array}$ & $\begin{array}{l}0.7637 \\
0.7662 \\
0.7680 \\
0.7695 \\
0.7715 \\
0.7725 \\
0.7727 \\
0.7724 \\
0.7709 \\
0.7690\end{array}$ & $\begin{array}{l}1.268 \\
1.298 \\
1.318 \\
1.331 \\
1.342 \\
1.341 \\
1.336 \\
1.329 \\
1.313 \\
1.299\end{array}$ & $\begin{array}{l}1768.0 \\
1764.0 \\
1766.0 \\
1774.0 \\
1800.0 \\
1836.0 \\
1876.0 \\
1920.0 \\
2010.0 \\
2101.0\end{array}$ \\
\hline $\begin{array}{l}160.0 \\
180.0 \\
200.0 \\
250.0 \\
300.0 \\
350.0 \\
400.0 \\
450.0 \\
500.0 \\
600.0\end{array}$ & $\begin{array}{l}0.2853 \\
0.3156 \\
0.3459 \\
0.4209 \\
0.4954 \\
0.5697 \\
0.6439 \\
0.7179 \\
0.7920 \\
0.9400\end{array}$ & $\begin{array}{r}617.0 \\
669.0 \\
723.0 \\
855.0 \\
987.0 \\
1120.0 \\
1250.0 \\
1380.0 \\
1510.0 \\
1780.0\end{array}$ & $\begin{array}{c}11.5 \\
10.2 \\
9.10 \\
7.22 \\
5.98 \\
5.12 \\
4.47 \\
3.97 \\
3.57 \\
2.98\end{array}$ & $\begin{array}{l}122.8 \\
138.3 \\
153.8 \\
192.0 \\
229.9 \\
267.7 \\
305.3 \\
342.9 \\
380.3 \\
455.2\end{array}$ & $\begin{array}{l}217.9 \\
243.5 \\
269.0 \\
332.3 \\
395.1 \\
457.6 \\
519.9 \\
582.2 \\
644.3 \\
768.5\end{array}$ & $\begin{array}{l}7.594 \\
3.745 \\
3.880 \\
4.162 \\
4.391 \\
4.584 \\
4.750 \\
4.897 \\
5.028 \\
5.254\end{array}$ & $\begin{array}{l}0.7670 \\
0.7652 \\
0.7635 \\
0.7602 \\
0.7578 \\
0.7560 \\
0.7547 \\
0.7536 \\
0.7528 \\
0.7516\end{array}$ & $\begin{array}{l}1.288 \\
1.279 \\
1.272 \\
1.260 \\
1.253 \\
1.248 \\
1.246 \\
1.244 \\
1.243 \\
1.241\end{array}$ & $\begin{array}{l}2190.0 \\
2277.0 \\
2361.0 \\
2562.0 \\
2749.0 \\
2926.0 \\
3092.0 \\
3251.0 \\
3403.0 \\
3688.0\end{array}$ \\
\hline $\begin{array}{r}700.0 \\
800.0 \\
900.0 \\
1000.0 \\
1200.0 \\
1400.0 \\
1600.0 \\
1800.0 \\
2000.0 \\
2500.0\end{array}$ & $\begin{array}{l}1.088 \\
1.236 \\
1.384 \\
1.532 \\
1.828 \\
2.125 \\
2.421 \\
2.718 \\
3.015 \\
3.757\end{array}$ & $\begin{array}{l}2040.0 \\
2310.0 \\
2570.0 \\
2840.0 \\
3370.0 \\
3900.0 \\
4430.0 \\
4960.0 \\
5490.0 \\
6820.0\end{array}$ & $\begin{array}{l}2.55 \\
2.23 \\
1.99 \\
1.79 \\
1.49 \\
1.28 \\
1.12 \\
1.00 \\
0.896 \\
0.717\end{array}$ & $\begin{array}{r}530.0 \\
604.7 \\
679.3 \\
754.0 \\
903.2 \\
1052.0 \\
1202.0 \\
1351.0 \\
1500.0 \\
1873.0\end{array}$ & $\begin{array}{r}892.6 \\
1017.0 \\
1141.0 \\
1265.0 \\
1513.0 \\
1761.0 \\
2009.0 \\
2257.0 \\
2505.0 \\
3125.0\end{array}$ & $\begin{array}{l}5.446 \\
5.611 \\
5.757 \\
5.888 \\
6.114 \\
6.305 \\
6.471 \\
6.617 \\
6.747 \\
7.024\end{array}$ & $\begin{array}{l}0.7507 \\
0.7501 \\
0.7497 \\
0.7493 \\
0.7488 \\
0.7484 \\
0.7481 \\
0.7479 \\
0.7477 \\
0.7474\end{array}$ & $\begin{array}{l}1.241 \\
1.240 \\
1.240 \\
1.240 \\
1.240 \\
1.240 \\
1.240 \\
1.240 \\
1.240 \\
1.240\end{array}$ & $\begin{array}{l}3954.0 \\
4203.0 \\
4438.0 \\
4662.0 \\
5081.0 \\
5468.0 \\
5830.0 \\
6171.0 \\
6495.0 \\
7241.0\end{array}$ \\
\hline 3000.0 & 4.499 & 8150.0 & 0.598 & 2245.0 & 3745.0 & 7.250 & 0.7472 & 1.240 & 7918.0 \\
\hline
\end{tabular}


1800 PSIA ISOBAR

TEMPERATURE DENSITY V(OH/OV) VIOP/OU) $-V(O P / O V)_{\mathrm{P}}$ DEG. R LB/CUFT BTU/LB PSIA-CU FT/BTU PSIA

\begin{tabular}{|c|c|c|c|c|}
\hline $\begin{array}{r}7.0 \\
8.0 \\
9.0 \\
10.0 \\
11.0 \\
12.0 \\
13.0 \\
14.0 \\
15.0\end{array}$ & $\begin{array}{l}13.52 \\
13.39 \\
13.27 \\
13.16 \\
13.06 \\
12.96 \\
12.85 \\
12.75 \\
12.65\end{array}$ & $\begin{array}{l}45.7 \\
56.4 \\
68.7 \\
74.9 \\
79.3 \\
82.6 \\
84.9 \\
86.5 \\
87.6\end{array}$ & $\begin{array}{l}20.7 \\
16.2 \\
13.0 \\
11.8 \\
11.0 \\
10.4 \\
10.0 \\
9.75 \\
9.54\end{array}$ & $\begin{array}{r}9830.0 \\
10000.0 \\
10000.0 \\
9900.0 \\
9690.0 \\
9450.0 \\
9190.0 \\
8910.0 \\
8640.0\end{array}$ \\
\hline $\begin{array}{l}16.0 \\
17.0 \\
18.0 \\
19.0 \\
20.0 \\
22.0 \\
24.0 \\
26.0 \\
28.0 \\
30.0\end{array}$ & $\begin{array}{l}12.54 \\
12.43 \\
12.32 \\
12.20 \\
12.08 \\
11.85 \\
11.61 \\
11.37 \\
11.13 \\
10.88\end{array}$ & $\begin{array}{l}88.5 \\
89.2 \\
89.9 \\
90.8 \\
91.7 \\
93.2 \\
94.4 \\
95.3 \\
95.9 \\
96.6\end{array}$ & $\begin{array}{l}9.36 \\
9.20 \\
9.06 \\
8.87 \\
8.70 \\
8.39 \\
8.11 \\
7.87 \\
7.68 \\
7.51\end{array}$ & $\begin{array}{l}8370.0 \\
8110.0 \\
7850.0 \\
7600.0 \\
7360.0 \\
6900.0 \\
6480.0 \\
6080.0 \\
5710.0 \\
5370.0\end{array}$ \\
\hline $\begin{array}{l}32.0 \\
34.0 \\
36.0 \\
38.0 \\
40.0\end{array}$ & $\begin{array}{c}10.64 \\
10.39 \\
10.15 \\
9.909 \\
9.672\end{array}$ & $\begin{array}{r}97.3 \\
98.0 \\
98.8 \\
100.0 \\
101.0\end{array}$ & $\begin{array}{l}7.35 \\
7.20 \\
7.06 \\
6.92 \\
6.79\end{array}$ & $\begin{array}{l}5070.0 \\
4790.0 \\
4540.0 \\
4310.0 \\
4110.0\end{array}$ \\
\hline $\begin{array}{r}45.0 \\
50.0 \\
55.0 \\
60.0 \\
70.0 \\
80.0 \\
90.0 \\
100.0 \\
120.0 \\
140.0\end{array}$ & $\begin{array}{l}9.101 \\
8.565 \\
8.070 \\
7.617 \\
6.830 \\
6.179 \\
5.638 \\
5.184 \\
4.466 \\
3.926\end{array}$ & $\begin{array}{l}104.0 \\
108.0 \\
112.0 \\
117.0 \\
128.0 \\
140.0 \\
152.0 \\
165.0 \\
190.0 \\
216.0\end{array}$ & $\begin{array}{l}6.49 \\
6.23 \\
5.99 \\
5.79 \\
5.45 \\
5.19 \\
4.99 \\
4.83 \\
4.58 \\
4.42\end{array}$ & $\begin{array}{l}3700.0 \\
3390.0 \\
3160.0 \\
2990.0 \\
2750.0 \\
2590.0 \\
2480.0 \\
2400.0 \\
2290.0 \\
2210.0\end{array}$ \\
\hline $\begin{array}{l}160.0 \\
180.0 \\
200.0 \\
250.0 \\
300.0 \\
350.0 \\
400.0 \\
450.0 \\
500.0 \\
600.0\end{array}$ & $\begin{array}{l}3.505 \\
3.168 \\
2.891 \\
2.376 \\
2.019 \\
1.755 \\
1.553 \\
1.393 \\
1.263 \\
1.064\end{array}$ & $\begin{array}{l}241.0 \\
267.0 \\
292.0 \\
355.0 \\
417.0 \\
479.0 \\
541.0 \\
603.0 \\
665.0 \\
788.0\end{array}$ & $\begin{array}{l}4.29 \\
4.20 \\
4.12 \\
3.99 \\
3.91 \\
3.86 \\
3.82 \\
3.78 \\
3.76 \\
3.72\end{array}$ & $\begin{array}{l}2160.0 \\
2120.0 \\
2090.0 \\
2030.0 \\
1990.0 \\
1960.0 \\
1940.0 \\
1930.0 \\
1910.0 \\
1890.0\end{array}$ \\
\hline $\begin{array}{r}700.0 \\
600.0 \\
900.0 \\
1000.0 \\
1200.0 \\
1400.0 \\
1600.0 \\
1800.0 \\
2000.0 \\
2500.0\end{array}$ & $\begin{array}{l}0.9191 \\
0.8090 \\
0.7225 \\
0.6527 \\
0.5469 \\
0.4706 \\
0.4130 \\
0.3679 \\
0.3317 \\
0.2662\end{array}$ & $\begin{array}{r}912.0 \\
1040.0 \\
1160.0 \\
1280.0 \\
1530.0 \\
1780.0 \\
2020.0 \\
2270.0 \\
2520.0 \\
3140.0\end{array}$ & $\begin{array}{l}3.70 \\
3.68 \\
3.67 \\
3.66 \\
3.64 \\
3.63 \\
3.62 \\
3.62 \\
3.61 \\
3.61\end{array}$ & $\begin{array}{l}1880.0 \\
1870.0 \\
1860.0 \\
1850.0 \\
1840.0 \\
1830.0 \\
1830.0 \\
1820.0 \\
1820.0 \\
1820.0\end{array}$ \\
\hline 3000.0 & 0.2223 & 3760.0 & 3.60 & 1810.0 \\
\hline
\end{tabular}

COV/OTIN THERMAL VISCOSITY P CONDUCTIVITY

1/DEG. R BTU/FT-HR-R LB/FT-SEC $\times 10 E+6$

0.0112
0.00915
0.00829
0.00789
0.00777
0.00781
0.00795
0.00815
0.00840
0.00866
0.00892
0.00919
0.00939
0.00959
0.0100
0.0103
0.0106
0.0110
0.0113

0.0257

0.0276

0.0294

0.0311

0.0326

0.0340

0.0352

0.0362

0.0371

0.0378

0.0384
0.0389

0.0393

0.0399

0.0402

0.0404

0.0404

0.0404

0.0115

0.0117

0.0119

0.0121
0.0121

0.0402

0.0401

0.0398

0.0396
0.0394

0.0122

0.0120

0.0117

0.0105

0.00957

0.00877

0.00806

0.00690
0.00602

0.00534

0.00480

0.00436

0.00355

0.00261

0.00230

0.00206

0.00187

0.00157

0.0389

0.0385

0.0383

0.0382

0.0383

0.0388

0.0396

0.0406

0.0428

0.0479

0.0506

0.0532

0.0598

0.0661

0.0722

0.0780

0.0836

0.0889

0.0994

0.00136

0.00120

0.00107

0.000967

0.000810

0.000698

0.000612

0.000546

0.000395

0.110

0.120

0.130

0.139

0.158
0.175

0.193

0.209

0.225

0.000330

0.300

7.77

7.40

7.08
6.81

6.81
6.38

6.38

5.82

5.63

5.48

5.36

5.27

5.2

5.15
5.11

5.06
5.06

5.06

5.15

5.30

5.49

5.67

5.67
5.87

6.29
6.70

7.11

12.2

13.0

16.2

17.7
19.2

19.2
20.7

23.4

26.1

28.6

33.5

39.2

44.6

23.5
19.0
15.9
13.8
12.2
11.0
10.1
9.33
8.71
8.20

THERMAL DIELECTRIC IFFUSIVITY CONSTANT

SQ FT/HR

0.00347

0.00372

0.00365

0.00378

0.00386

0.00390

0.00392

0.00389

0.00386

0.00382

0.00378

0.00374

0.00370

0.00363

0.00356

0.00351

0.00346

0.00335

0.00333

0.00332

0.00333

0.00337

0.00346

0.00360

0.00376
0.00418

0.00469

0.00526

0.00589

0.00730

0.00889

0.0106

0.0125

0.0145

0.0200

0.0330

0.0403

0.0483

0.0567

0.0963

.119

0.145

0.172

0.233

0.301

0.376

0.458

0.547
0.798

1.09
1. 01991

1.02003

1. 02013

1.02028

1.02036

1.02042

1. 02049

1.02055

1. 02061

1.02067

1. 02072

1.02077
1.02082

1.02082

1.02096

1.02100

1.02102

1.02101

1.02099

1. 02095

1. 02089

1.02080
1.02070

1.02038

1. 01997

1.01900

1.91796

1. 01694

1.01597

1.01509
1.01354

1.01354
1.01226

1.01120

1.01030
1.00953

1. 00904

1.00695

1.00612

1.00547
1.00494

1.00494

1.00451

1.00333

1.00295

1. 01264

1.00240

1.00202

1.00174

1.00153

1.00123

1.00099

1.00083

1.80

1.37

1.00

0.873

0.785

0.721

0.637

0.610

0.589

0.573

0.559

0.549

0.535

0.52

0.527

0.531

0.538

0.545

0.554

0.562
0.571

0.594

0.614

0.632

0.647

0.681

0.681
0.688

0.688

0.692

0.694
0.691

0.688

0.684

0.669

0.664

0.660

0.657

0.656

0.656

0.656

0.650

0.661

0.662

0.662

0.665

0.664

0.664

0.664

0.665

- tho-phase boundary 


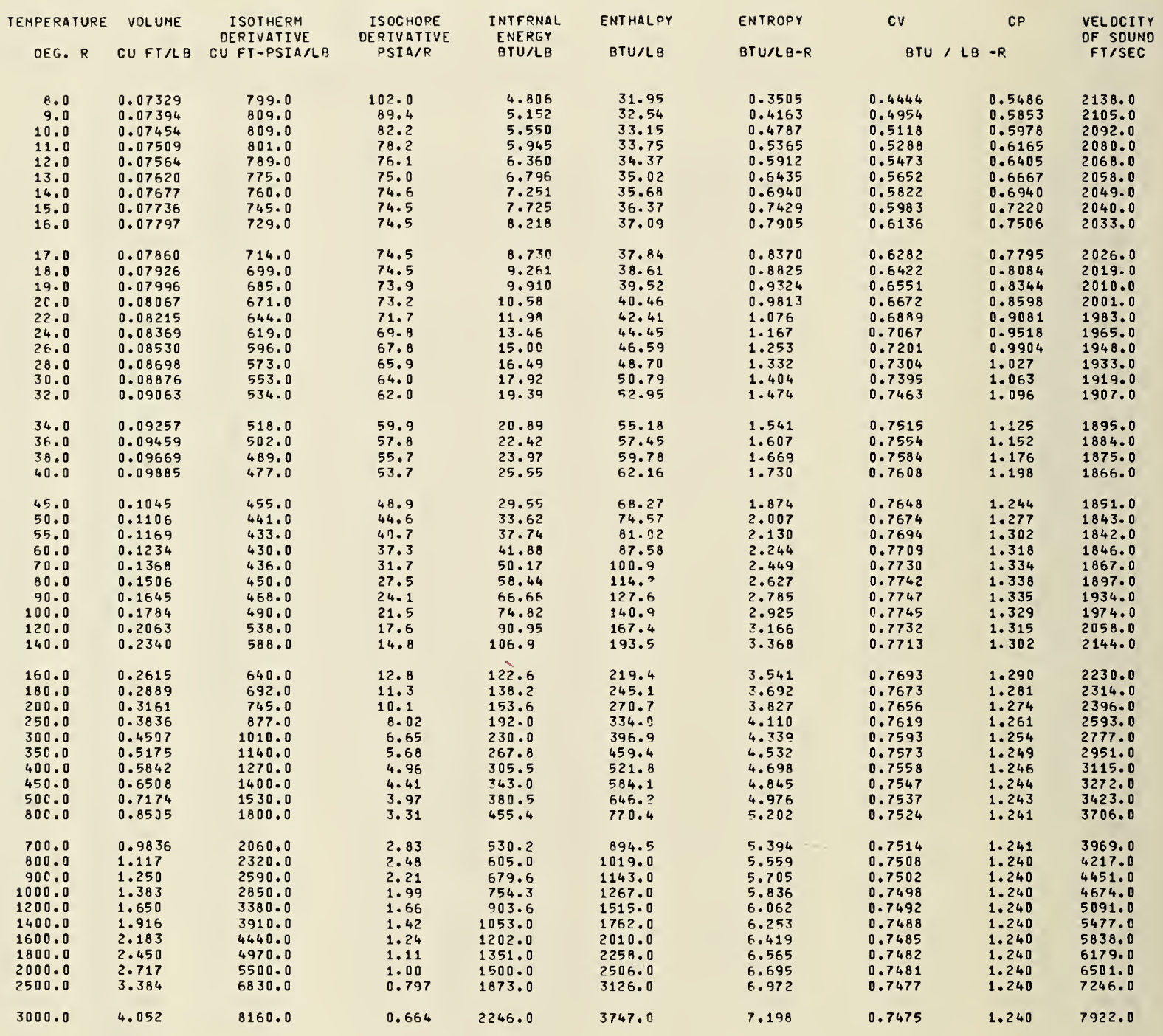


2000 PSIA ISOOAR

\begin{tabular}{|c|c|c|c|c|c|c|c|c|c|c|}
\hline TEMPERATURE & DENSITY & $V(\mathrm{DH} / \mathrm{OV})_{\mathrm{P}}$ & $V(D P / D U)_{v}-$ & $-V(D P / D V)_{T}$ & $(\mathrm{OV} / D T)_{\mathrm{p}} / \mathrm{V}$ & $\begin{array}{c}\text { THERMAL } \\
\text { CONDUCTIVITY }\end{array}$ & VISCOSITY & $\begin{array}{c}\text { THERMAL } \\
\text { DIFFUSIVITY }\end{array}$ & $\begin{array}{l}\text { DIELECTRIC } \\
\text { CONSTANT }\end{array}$ & $\begin{array}{l}\text { PRANDTL } \\
\text { NUMBER }\end{array}$ \\
\hline OEG. $R$ & LB/CU FT & BTU/LB & PSIA-CU FT/BTU & PSIA & 1/DEG. R & $B T U / F T-H R=R$ & $\begin{array}{r}\text { LB } / F T-S E C \\
\times 10 E+6\end{array}$ & SO FT/HR & & \\
\hline $\begin{array}{r}8.0 \\
9.0 \\
10.0 \\
11.0 \\
12.0 \\
13.0 \\
14.0 \\
15.0 \\
16.0\end{array}$ & $\begin{array}{l}13.64 \\
13.52 \\
13.42 \\
13.32 \\
13.22 \\
13.12 \\
13.03 \\
12.93 \\
12.83\end{array}$ & $\begin{array}{l}58.5 \\
71.7 \\
78.9 \\
84.1 \\
87.8 \\
90.4 \\
92.2 \\
93.4 \\
94.3\end{array}$ & $\begin{array}{c}16.9 \\
13.3 \\
12.0 \\
11.1 \\
10.5 \\
10.1 \\
9.83 \\
9.63 \\
9.46\end{array}$ & $\begin{array}{r}10900.0 \\
10900.0 \\
10900.0 \\
10700.0 \\
10400.0 \\
10200.0 \\
9900.0 \\
9630.0 \\
9350.0\end{array}$ & $\begin{array}{l}0.00938 \\
0.00816 \\
0.00758 \\
0.00733 \\
0.00729 \\
0.00737 \\
0.00753 \\
0.00773 \\
0.00796\end{array}$ & $\begin{array}{l}0.0268 \\
0.0287 \\
0.0306 \\
0.0324 \\
0.0340 \\
0.0354 \\
0.0367 \\
0.0378 \\
0.0387\end{array}$ & $\begin{array}{c}22.3 \\
18.4 \\
15.8 \\
13.9 \\
12.4 \\
11.3 \\
10.4 \\
9.65 \\
9.04\end{array}$ & $\begin{array}{l}0.00358 \\
0.00362 \\
0.00381 \\
0.00394 \\
0.00401 \\
0.00405 \\
0.00406 \\
0.00405 \\
0.00402\end{array}$ & $\begin{array}{l}1.01980 \\
1.01991 \\
1.02001 \\
1.02009 \\
1.02016 \\
1.02024 \\
1.02031 \\
1.02038 \\
1.02044\end{array}$ & $\begin{array}{l}1.64 \\
1.35 \\
1.11 \\
0.951 \\
0.843 \\
0.765 \\
0.707 \\
0.664 \\
0.631\end{array}$ \\
\hline $\begin{array}{l}17.0 \\
18.0 \\
19.0 \\
20.0 \\
22.0 \\
24.0 \\
26.0 \\
28.0 \\
30.0 \\
32.0\end{array}$ & $\begin{array}{l}12.72 \\
12.62 \\
12.51 \\
12.40 \\
12.17 \\
11.95 \\
11.72 \\
11.50 \\
11.27 \\
11.03\end{array}$ & $\begin{array}{r}95.0 \\
95.7 \\
96.7 \\
97.6 \\
99.4 \\
101.0 \\
102.0 \\
103.0 \\
104.0 \\
104.0\end{array}$ & $\begin{array}{l}9.32 \\
9.19 \\
9.02 \\
8.85 \\
8.55 \\
8.27 \\
8.03 \\
7.84 \\
7.68 \\
7.52\end{array}$ & $\begin{array}{l}9080.0 \\
8820.0 \\
8560.0 \\
8310.0 \\
7840.0 \\
7400.0 \\
6980.0 \\
6590.0 \\
6230.0 \\
5900.0\end{array}$ & $\begin{array}{l}0.00820 \\
0.00844 \\
0.00863 \\
0.00881 \\
0.00914 \\
0.00944 \\
0.00971 \\
0.0100 \\
0.0103 \\
0.0105\end{array}$ & $\begin{array}{l}0.0395 \\
0.0402 \\
0.0407 \\
0.0411 \\
0.0418 \\
0.0422 \\
0.0424 \\
0.0424 \\
0.0424 \\
0.0423\end{array}$ & $\begin{array}{l}8.53 \\
8.10 \\
7.73 \\
7.41 \\
6.90 \\
6.52 \\
6.23 \\
6.00 \\
5.82 \\
5.68\end{array}$ & $\begin{array}{l}0.00398 \\
0.00394 \\
0.00390 \\
0.00386 \\
0.00378 \\
0.00371 \\
0.00365 \\
0.00359 \\
0.00354 \\
0.00350\end{array}$ & $\begin{array}{l}1.02051 \\
1.02057 \\
1.02063 \\
1.02068 \\
1.02078 \\
1.02086 \\
1.02093 \\
1.02098 \\
1.02101 \\
1.02102\end{array}$ & $\begin{array}{l}0.606 \\
0.587 \\
0.570 \\
0.558 \\
0.540 \\
0.530 \\
0.524 \\
0.523 \\
0.526 \\
0.530\end{array}$ \\
\hline $\begin{array}{l}34.0 \\
36.0 \\
38.0 \\
40.0\end{array}$ & $\begin{array}{l}10.80 \\
10.57 \\
10.34 \\
10.12\end{array}$ & $\begin{array}{l}105.0 \\
106.0 \\
107.0 \\
108.0\end{array}$ & $\begin{array}{l}7.38 \\
7.24 \\
7.11 \\
6.98\end{array}$ & $\begin{array}{l}5590.0 \\
5310.0 \\
5060.0 \\
4830.0\end{array}$ & $\begin{array}{l}0.0107 \\
0.0109 \\
0.0110 \\
0.0111\end{array}$ & $\begin{array}{l}0.0421 \\
0.0419 \\
0.0417 \\
0.0414\end{array}$ & $\begin{array}{l}5.57 \\
5.49 \\
5.42 \\
5.37\end{array}$ & $\begin{array}{l}0.00346 \\
0.00344 \\
0.00342 \\
0.00342\end{array}$ & $\begin{array}{l}1.02101 \\
1.02098 \\
1.02094 \\
1.02088\end{array}$ & $\begin{array}{l}0.536 \\
0.543 \\
0.551 \\
0.559\end{array}$ \\
\hline $\begin{array}{r}45.0 \\
50.0 \\
55.0 \\
60.0 \\
70.0 \\
80.0 \\
90.0 \\
100.0 \\
120.0 \\
140.0\end{array}$ & $\begin{array}{l}9.565 \\
9.043 \\
8.555 \\
8.104 \\
7.308 \\
6.640 \\
6.079 \\
5.604 \\
4.847 \\
4.273\end{array}$ & $\begin{array}{l}111.0 \\
114.0 \\
118.0 \\
123.0 \\
134.0 \\
145.0 \\
158.0 \\
170.0 \\
195.0 \\
221.0\end{array}$ & $\begin{array}{l}6.68 \\
6.42 \\
6.18 \\
5.97 \\
5.62 \\
5.34 \\
5.12 \\
4.95 \\
4.68 \\
4.50\end{array}$ & $\begin{array}{l}4350.0 \\
3990.0 \\
3700.0 \\
3490.0 \\
3180.0 \\
2990.0 \\
2850.0 \\
2750.0 \\
2610.0 \\
2510.0\end{array}$ & $\begin{array}{l}0.0112 \\
0.0112 \\
0.0110 \\
0.0107 \\
0.0100 \\
0.00920 \\
0.00847 \\
0.00782 \\
0.00674 \\
0.00590\end{array}$ & $\begin{array}{l}0.0409 \\
0.0404 \\
0.0401 \\
0.0399 \\
0.0399 \\
0.0403 \\
0.0410 \\
0.0418 \\
0.0440 \\
0.0463\end{array}$ & $\begin{array}{l}5.29 \\
5.27 \\
5.29 \\
5.33 \\
5.46 \\
5.63 \\
5.81 \\
6.00 \\
6.40 \\
6.81\end{array}$ & $\begin{array}{l}0.00344 \\
0.00350 \\
0.00360 \\
0.00374 \\
0.00410 \\
0.00454 \\
0.00505 \\
0.00562 \\
0.00689 \\
0.00833\end{array}$ & $\begin{array}{l}1.02065 \\
1.02034 \\
1.01996 \\
1.01954 \\
1.01862 \\
1.01768 \\
1.01676 \\
1.01591 \\
1.01438 \\
1.01310\end{array}$ & $\begin{array}{l}0.580 \\
0.600 \\
0.618 \\
0.633 \\
0.657 \\
0.672 \\
0.681 \\
0.686 \\
0.690 \\
0.688\end{array}$ \\
\hline $\begin{array}{l}160.0 \\
18 C .0 \\
200.0 \\
250.0 \\
300.0 \\
350.0 \\
400.0 \\
450.0 \\
500.0 \\
600.0\end{array}$ & $\begin{array}{l}3.824 \\
3.462 \\
3.164 \\
2.607 \\
2.219 \\
1.932 \\
1.712 \\
1.537 \\
1.394 \\
1.176\end{array}$ & $\begin{array}{l}246.0 \\
272.0 \\
297.0 \\
360.0 \\
422.0 \\
484.0 \\
546.0 \\
608.0 \\
670.0 \\
793.0\end{array}$ & $\begin{array}{l}4.36 \\
4.26 \\
4.18 \\
4.04 \\
3.94 \\
3.88 \\
3.84 \\
3.80 \\
3.78 \\
3.74\end{array}$ & $\begin{array}{l}2450.0 \\
2400.0 \\
2360.0 \\
2290.0 \\
2240.0 \\
2200.0 \\
2180.0 \\
2150.0 \\
2140.0 \\
2110.0\end{array}$ & $\begin{array}{l}0.00524 \\
0.00472 \\
0.00429 \\
0.00351 \\
0.00297 \\
0.00258 \\
0.00228 \\
0.00205 \\
0.00186 \\
0.00156\end{array}$ & $\begin{array}{l}0.0489 \\
0.0515 \\
0.0541 \\
0.0605 \\
0.0668 \\
0.0728 \\
0.0786 \\
0.0841 \\
0.0893 \\
0.100\end{array}$ & $\begin{array}{r}7.21 \\
7.61 \\
7.87 \\
8.83 \\
9.74 \\
10.6 \\
11.5 \\
12.3 \\
13.1 \\
14.7\end{array}$ & $\begin{array}{l}0.00990 \\
0.0116 \\
0.0134 \\
0.0184 \\
0.0240 \\
0.0302 \\
0.0368 \\
0.0440 \\
0.0516 \\
0.0683\end{array}$ & $\begin{array}{l}1.01201 \\
1.01108 \\
1.01029 \\
1.00872 \\
1.00757 \\
1.00668 \\
1.00598 \\
1.00541 \\
1.00494 \\
1.00421\end{array}$ & $\begin{array}{l}0.685 \\
0.682 \\
0.668 \\
0.662 \\
0.658 \\
0.656 \\
0.654 \\
0.654 \\
0.655 \\
0.657\end{array}$ \\
\hline $\begin{array}{r}700.0 \\
800.0 \\
900.0 \\
1000.0 \\
1200.0 \\
1400.0 \\
1600.0 \\
1800.0 \\
2000.0 \\
2500.0\end{array}$ & $\begin{array}{l}1.017 \\
0.8954 \\
0.8000 \\
0.7230 \\
0.6062 \\
0.5218 \\
0.4581 \\
0.4082 \\
0.3681 \\
0.2955\end{array}$ & $\begin{array}{r}917.0 \\
1040.0 \\
1160.0 \\
1290.0 \\
1530.0 \\
1780.0 \\
2030.0 \\
2280.0 \\
2520.0 \\
3140.0\end{array}$ & $\begin{array}{l}3.71 \\
3.69 \\
3.68 \\
3.65 \\
3.65 \\
3.64 \\
3.63 \\
3.62 \\
3.61 \\
3.61\end{array}$ & $\begin{array}{l}2090.0 \\
2080.0 \\
2070.0 \\
2060.0 \\
2050.0 \\
2040.0 \\
2030.0 \\
2030.0 \\
2030.0 \\
2020.0\end{array}$ & $\begin{array}{l}0.00135 \\
0.00119 \\
0.00107 \\
0.000963 \\
0.000808 \\
0.000696 \\
0.000611 \\
0.000545 \\
0.000491 \\
0.000395\end{array}$ & $\begin{array}{l}0.110 \\
0.120 \\
0.130 \\
0.139 \\
0.158 \\
0.176 \\
0.193 \\
0.209 \\
0.225 \\
0.264\end{array}$ & $\begin{array}{l}16.2 \\
17.8 \\
19.2 \\
20.7 \\
23.4 \\
26.1 \\
28.6 \\
31.1 \\
33.5 \\
39.2\end{array}$ & $\begin{array}{l}0.0873 \\
0.108 \\
0.131 \\
0.156 \\
0.210 \\
0.271 \\
0.339 \\
0.413 \\
0.493 \\
0.719\end{array}$ & $\begin{array}{l}1.00367 \\
1.00325 \\
1.00292 \\
1.00264 \\
1.00223 \\
1.00193 \\
1.00170 \\
1.00151 \\
1.00137 \\
1.00110\end{array}$ & $\begin{array}{l}0.659 \\
0.660 \\
0.661 \\
0.662 \\
0.563 \\
0.663 \\
0.664 \\
0.664 \\
0.664 \\
0.664\end{array}$ \\
\hline 3000.0 & 0.2468 & 3760.0 & 3.60 & 2010.0 & 0.000330 & 0.300 & 44.6 & 0.980 & 1.00092 & 0.664 \\
\hline
\end{tabular}

* two-phase gounoary 


\begin{tabular}{|c|c|c|c|c|c|c|c|c|c|}
\hline $\begin{array}{c}\text { TFMPERATURE } \\
\text { OEG. R }\end{array}$ & $\begin{array}{l}\text { VOLUME } \\
\text { CU FT/L }\end{array}$ & $\begin{array}{c}\text { ISOTHERM } \\
\text { OERI VATIVE } \\
\text { CU FT-PSIA/LB }\end{array}$ & $\begin{array}{l}\text { ISOCHOPE } \\
\text { OERIVATIVE } \\
\text { PSIA/R }\end{array}$ & $\begin{array}{l}\text { INTERNAL } \\
\text { ENERGY } \\
\text { BTU/LB }\end{array}$ & $\begin{array}{l}\text { ENTHALPY } \\
\text { BTU/L8 }\end{array}$ & $\begin{array}{l}\text { ENTROPY } \\
\text { BTU/LB-R }\end{array}$ & $\begin{array}{l}\text { CV } \\
\text { BTU / L8 }\end{array}$ & $\begin{array}{r}C P \\
-R\end{array}$ & $\begin{array}{l}\text { VEL OCITY } \\
\text { OF SOUND } \\
\text { FT/SEC }\end{array}$ \\
\hline $\begin{array}{r}9.0 \\
10.0 \\
11.0 \\
12.0 \\
13.0 \\
14.0 \\
15.0 \\
16.0 \\
17.0\end{array}$ & $\begin{array}{l}0.07094 \\
0.07149 \\
0.07198 \\
0.07245 \\
0.07291 \\
0.07338 \\
0.07385 \\
0.07434 \\
0.07485\end{array}$ & $\begin{array}{l}935.0 \\
942.0 \\
939.0 \\
930.0 \\
918.0 \\
904.0 \\
889.0 \\
874.0 \\
860.0\end{array}$ & $\begin{array}{r}108.0 \\
94.0 \\
86.2 \\
81.9 \\
79.7 \\
78.8 \\
78.6 \\
78.8 \\
79.1\end{array}$ & $\begin{array}{l}5.907 \\
6.318 \\
6.714 \\
7.122 \\
7.544 \\
7.979 \\
8.428 \\
8.891 \\
9.370\end{array}$ & $\begin{array}{l}38.75 \\
39.41 \\
40.04 \\
40.66 \\
41.30 \\
41.95 \\
42.62 \\
43.31 \\
44.02\end{array}$ & $\begin{array}{l}0.3617 \\
0.4292 \\
0.4891 \\
0.5444 \\
0.5962 \\
0.6455 \\
0.6929 \\
0.7386 \\
0.7831\end{array}$ & $\begin{array}{l}0.5452 \\
0.5420 \\
0.5463 \\
0.5556 \\
0.5669 \\
0.5789 \\
0.5913 \\
0.5041 \\
0.5172\end{array}$ & $\begin{array}{l}0.6500 \\
0.6309 \\
0.6298 \\
0.6398 \\
0.6555 \\
0.6748 \\
0.6966 \\
0.7203 \\
0.7456\end{array}$ & $\begin{array}{l}2272.0 \\
2254.0 \\
2239.0 \\
2227.0 \\
2217.0 \\
2209.0 \\
2203.0 \\
2198.0 \\
2193.0\end{array}$ \\
\hline $\begin{array}{l}18.0 \\
19.0 \\
20.0 \\
22.0 \\
24.0 \\
26.0 \\
28.0 \\
30.0 \\
32.0 \\
34.0\end{array}$ & $\begin{array}{l}0.07538 \\
0.07593 \\
0.07651 \\
0.07768 \\
0.07891 \\
0.08017 \\
0.08148 \\
0.08286 \\
0.08429 \\
0.08579\end{array}$ & $\begin{array}{l}845.0 \\
830.0 \\
816.0 \\
790.0 \\
765.0 \\
741.0 \\
718.0 \\
697.0 \\
576.0 \\
657.0\end{array}$ & $\begin{array}{l}79.4 \\
79.2 \\
78.9 \\
77.9 \\
76.4 \\
74.7 \\
73.0 \\
71.4 \\
69.7 \\
67.8\end{array}$ & $\begin{array}{l}9.865 \\
10.48 \\
11.12 \\
12.46 \\
13.88 \\
15.36 \\
16.79 \\
18.15 \\
19.55 \\
20.98\end{array}$ & $\begin{array}{l}44.76 \\
45.64 \\
46.54 \\
48.43 \\
50.41 \\
52.47 \\
54.51 \\
56.51 \\
58.57 \\
60.69\end{array}$ & $\begin{array}{l}0.8265 \\
0.8749 \\
0.9222 \\
1.014 \\
1.102 \\
1.186 \\
1.262 \\
1.331 \\
1.397 \\
1.461\end{array}$ & $\begin{array}{l}0.6305 \\
0.6429 \\
0.6552 \\
0.6785 \\
0.6988 \\
0.7139 \\
0.7258 \\
0.7364 \\
0.7445 \\
0.7507\end{array}$ & $\begin{array}{l}0.7720 \\
0.7961 \\
0.8204 \\
0.8673 \\
0.9099 \\
0.9466 \\
0.9813 \\
1.016 \\
1.047 \\
1.075\end{array}$ & $\begin{array}{l}2189.0 \\
2182.0 \\
2176.0 \\
2162.0 \\
2148.0 \\
2134.0 \\
2121.0 \\
2110.0 \\
2099.0 \\
2088.0\end{array}$ \\
\hline $\begin{array}{l}36.0 \\
38.0 \\
40.0\end{array}$ & $\begin{array}{l}0.08733 \\
0.08893 \\
0.09057\end{array}$ & $\begin{array}{l}640.0 \\
624.0 \\
609.0\end{array}$ & $\begin{array}{l}65.9 \\
64.0 \\
62.0\end{array}$ & $\begin{array}{l}22.44 \\
23.92 \\
25.43\end{array}$ & $\begin{array}{l}62.87 \\
65.09 \\
67.36\end{array}$ & $\begin{array}{l}1.524 \\
1.584 \\
1.642\end{array}$ & $\begin{array}{l}0.7555 \\
0.7591 \\
0.7620\end{array}$ & $\begin{array}{l}1.100 \\
1.124 \\
1.146\end{array}$ & $\begin{array}{l}2078.0 \\
2068.0 \\
2060.0\end{array}$ \\
\hline $\begin{array}{r}45.0 \\
50.0 \\
55.0 \\
60.0 \\
70.0 \\
80.0 \\
90.0 \\
100.0 \\
120.0 \\
140.0\end{array}$ & $\begin{array}{l}0.09489 \\
0.09948 \\
0.1043 \\
0.1093 \\
0.1197 \\
0.1305 \\
0.1415 \\
0.1525 \\
0.1747 \\
0.1968\end{array}$ & $\begin{array}{l}579.0 \\
556.0 \\
540.0 \\
531.0 \\
525.0 \\
531.0 \\
544.0 \\
561.0 \\
604.0 \\
651.0\end{array}$ & $\begin{array}{l}57.3 \\
52.9 \\
43.8 \\
45.2 \\
38.9 \\
33.9 \\
29.9 \\
26.7 \\
21.9 \\
18.5\end{array}$ & $\begin{array}{c}29.28 \\
33.22 \\
37.23 \\
41.28 \\
49.46 \\
57.67 \\
65.88 \\
74.05 \\
90.25 \\
106.3\end{array}$ & $\begin{array}{c}73.21 \\
79.28 \\
85.51 \\
91.88 \\
104.9 \\
118.1 \\
131.4 \\
144.6 \\
171.1 \\
197.4\end{array}$ & $\begin{array}{l}1.780 \\
1.907 \\
2.026 \\
2.137 \\
2.337 \\
2.514 \\
2.670 \\
2.810 \\
3.051 \\
3.254\end{array}$ & $\begin{array}{l}0.7659 \\
0.7700 \\
0.7723 \\
0.7741 \\
0.7767 \\
0.7784 \\
0.7792 \\
0.7795 \\
0.7786 \\
0.7768\end{array}$ & $\begin{array}{l}1.193 \\
1.231 \\
1.261 \\
1.284 \\
1.313 \\
1.326 \\
1.329 \\
1.328 \\
1.318 \\
1.307\end{array}$ & $\begin{array}{l}2042.0 \\
2029.0 \\
2022.0 \\
2020.0 \\
2027.0 \\
2046.0 \\
2073.0 \\
2105.0 \\
2176.0 \\
2252.0\end{array}$ \\
\hline $\begin{array}{l}160.0 \\
180.0 \\
200.0 \\
250.0 \\
300.0 \\
350.0 \\
400.0 \\
450.0 \\
500.0 \\
600.0\end{array}$ & $\begin{array}{l}0.2188 \\
0.2407 \\
0.2624 \\
0.3164 \\
0.3701 \\
0.4235 \\
0.4768 \\
0.5300 \\
0.5831 \\
0.5894\end{array}$ & $\begin{array}{r}700.0 \\
751.0 \\
802.0 \\
932.0 \\
1050.0 \\
1190.0 \\
1320.0 \\
1450.0 \\
1580.0 \\
1840.0\end{array}$ & $\begin{array}{c}16.0 \\
14.1 \\
12.6 \\
10.0 \\
8.29 \\
7.99 \\
6.19 \\
5.50 \\
4.95 \\
4.12\end{array}$ & $\begin{array}{l}122.1 \\
137.8 \\
153.4 \\
191.9 \\
230.1 \\
268.1 \\
305.8 \\
343.5 \\
381.1 \\
456.1\end{array}$ & $\begin{array}{l}223.4 \\
249.2 \\
274.9 \\
338.4 \\
401.4 \\
464.1 \\
526.5 \\
588.8 \\
651.0 \\
775.2\end{array}$ & $\begin{array}{l}3.428 \\
3.580 \\
3.715 \\
3.998 \\
4.228 \\
4.421 \\
4.588 \\
4.735 \\
4.866 \\
5.092\end{array}$ & $\begin{array}{l}0.7747 \\
0.7726 \\
0.7705 \\
0.7663 \\
0.7631 \\
0.7606 \\
0.7588 \\
0.7573 \\
0.7561 \\
0.7544\end{array}$ & $\begin{array}{l}1.296 \\
1.287 \\
1.279 \\
1.265 \\
1.256 \\
1.251 \\
1.247 \\
1.245 \\
1.243 \\
1.241\end{array}$ & $\begin{array}{l}2330.0 \\
2407.0 \\
2484.0 \\
2669.0 \\
2845.0 \\
3012.0 \\
3172.0 \\
3325.0 \\
3471.0 \\
3749.0\end{array}$ \\
\hline $\begin{array}{r}700.0 \\
800.0 \\
900.0 \\
1000.0 \\
1200.0 \\
1400.0 \\
1600.0 \\
1800.0 \\
2000.0 \\
2500.0\end{array}$ & $\begin{array}{l}0.7957 \\
0.9021 \\
1.008 \\
1.115 \\
1.328 \\
1.541 \\
1.754 \\
1.957 \\
2.180 \\
2.714\end{array}$ & $\begin{array}{l}2110.0 \\
2370.0 \\
2630.0 \\
2890.0 \\
3420.0 \\
3950.0 \\
4480.0 \\
5010.0 \\
5540.0 \\
6870.0\end{array}$ & $\begin{array}{l}3.54 \\
3.09 \\
2.75 \\
2.48 \\
2.07 \\
1.77 \\
1.55 \\
1.38 \\
1.24 \\
0.995\end{array}$ & $\begin{array}{r}530.9 \\
605.7 \\
580.5 \\
755.2 \\
904.5 \\
1054.0 \\
1203.0 \\
1352.0 \\
1501.0 \\
1874.0\end{array}$ & $\begin{array}{r}899.3 \\
1023.0 \\
1147.0 \\
1271.0 \\
1519.0 \\
1767.0 \\
2015.0 \\
2263.0 \\
2511.0 \\
3131.0\end{array}$ & $\begin{array}{l}5.284 \\
5.449 \\
5.595 \\
5.726 \\
5.952 \\
6.143 \\
6.308 \\
6.454 \\
6.585 \\
6.862\end{array}$ & $\begin{array}{l}0.7532 \\
0.7523 \\
0.7517 \\
0.7511 \\
0.7504 \\
0.7498 \\
0.7494 \\
0.7491 \\
0.7489 \\
0.7485\end{array}$ & $\begin{array}{l}1.240 \\
1.240 \\
1.240 \\
1.240 \\
1.239 \\
1.240 \\
1.240 \\
1.240 \\
1.240 \\
1.240\end{array}$ & $\begin{array}{l}4008.0 \\
4252.0 \\
4483.0 \\
4704.0 \\
5117.0 \\
5500.0 \\
5858.0 \\
6195.0 \\
6517.0 \\
7259.0\end{array}$ \\
\hline 3000.0 & 3.248 & 8190.0 & 0.829 & 2247.0 & 3751.0 & 7.088 & 0.7482 & 1.240 & 7932.0 \\
\hline
\end{tabular}

- tho-phase bounoary 
THERMOPHYSICAL PROPERTIES DF HELIUM 4

2500 PSIA ISOBAR

\begin{tabular}{|c|c|c|c|c|c|c|c|c|c|c|}
\hline TEMPERATURE & DENSITY & $V(D M / D V)_{P}$ & $V(D P / D U)^{V}-$ & $-V(D P / D V)_{T}$ & (DV/DT) & $\begin{array}{c}\text { PHERMAL } \\
\text { CONDUCTIVITY }\end{array}$ & VISCDSITY & $\begin{array}{l}\text { THERMAL } \\
\text { DIFFUSIVITY }\end{array}$ & $\begin{array}{l}\text { DIELECTRIC } \\
\text { CONSTANT }\end{array}$ & $\begin{array}{l}\text { PRANDTL } \\
\text { NUMBER }\end{array}$ \\
\hline DEG. R & $\mathrm{LB} / \mathrm{CU} F \mathrm{~F}$ & BTU/LB & PSIA-CU FT/BTU & PSIA & 1/DEG. P & BTU/F T-HR-R & $\begin{array}{r}\text { LB/FT-SEC } \\
\times 10 E+6\end{array}$ & SO FT/HR & & \\
\hline $\begin{array}{r}9.0 \\
10.0 \\
11.0 \\
12.0 \\
13.0 \\
14.0 \\
15.0 \\
16.0 \\
17.0\end{array}$ & $\begin{array}{l}14.10 \\
13.99 \\
13.89 \\
13.80 \\
13.72 \\
13.63 \\
13.54 \\
13.45 \\
13.36\end{array}$ & $\begin{array}{r}79.2 \\
88.4 \\
95.3 \\
100.0 \\
103.0 \\
105.0 \\
107.0 \\
108.0 \\
108.0\end{array}$ & $\begin{array}{c}14.1 \\
12.4 \\
11.4 \\
10.7 \\
10.3 \\
10.0 \\
9.82 \\
9.69 \\
9.59\end{array}$ & $\begin{array}{l}13200.0 \\
13200.0 \\
13000.0 \\
12800.0 \\
12600.0 \\
12300.0 \\
12000.0 \\
11800.0 \\
11500.0\end{array}$ & $\begin{array}{l}0.00820 \\
0.00714 \\
0.00661 \\
0.00638 \\
0.00633 \\
0.00640 \\
0.00653 \\
0.00670 \\
0.00689\end{array}$ & $\begin{array}{l}0.0314 \\
0.0335 \\
0.0354 \\
0.0372 \\
0.0389 \\
0.0403 \\
0.0415 \\
0.0426 \\
0.0435\end{array}$ & $\begin{array}{l}26.2 \\
21.8 \\
18.7 \\
16.5 \\
14.7 \\
13.4 \\
12.3 \\
11.4 \\
10.6\end{array}$ & $\begin{array}{l}0.00343 \\
0.00379 \\
0.00405 \\
0.00422 \\
0.00432 \\
0.00438 \\
0.00440 \\
0.00440 \\
0.00437\end{array}$ & $\begin{array}{l}1.01935 \\
1.01947 \\
1.01957 \\
1.01965 \\
1.01974 \\
1.01982 \\
1.01990 \\
1.01998 \\
1.02005\end{array}$ & $\begin{array}{l}1.95 \\
1.48 \\
1.20 \\
1.02 \\
0.894 \\
0.806 \\
0.741 \\
0.692 \\
0.656\end{array}$ \\
\hline $\begin{array}{l}18.0 \\
19.0 \\
20.0 \\
22.0 \\
24.0 \\
26.0 \\
28.0 \\
30.0 \\
32.0 \\
34.0\end{array}$ & $\begin{array}{l}13.27 \\
13.17 \\
13.07 \\
12.87 \\
12.67 \\
12.47 \\
12.27 \\
12.07 \\
11.86 \\
11.66\end{array}$ & $\begin{array}{l}109.0 \\
110.0 \\
111.0 \\
113.0 \\
115.0 \\
117.0 \\
118.0 \\
120.0 \\
121.0 \\
121.0\end{array}$ & $\begin{array}{l}9.50 \\
9.35 \\
9.21 \\
8.91 \\
8.63 \\
8.38 \\
8.20 \\
8.04 \\
7.89 \\
7.75\end{array}$ & $\begin{array}{r}11200.0 \\
10900.0 \\
10700.0 \\
10200.0 \\
9700.0 \\
9250.0 \\
8820.0 \\
8410.0 \\
8020.0 \\
7660.0\end{array}$ & $\begin{array}{l}0.00709 \\
0.00724 \\
0.00739 \\
0.00766 \\
0.00788 \\
0.00807 \\
0.00828 \\
0.00849 \\
0.00868 \\
0.00885\end{array}$ & $\begin{array}{l}0.0443 \\
0.0449 \\
0.0454 \\
0.0462 \\
0.0467 \\
0.0469 \\
0.0471 \\
0.0471 \\
0.0470 \\
0.0468\end{array}$ & $\begin{array}{c}10.0 \\
9.46 \\
9.00 \\
8.27 \\
7.72 \\
7.30 \\
6.97 \\
6.71 \\
6.50 \\
6.34\end{array}$ & $\begin{array}{l}0.00432 \\
0.00428 \\
0.00424 \\
0.00414 \\
0.00405 \\
0.00398 \\
0.00391 \\
0.00384 \\
0.00378 \\
0.00374\end{array}$ & $\begin{array}{l}1.02013 \\
1.02020 \\
1.02028 \\
1.02041 \\
1.02053 \\
1.02064 \\
1.02074 \\
1.02082 \\
1.02089 \\
1.02094\end{array}$ & $\begin{array}{l}0.628 \\
0.604 \\
0.585 \\
0.559 \\
0.542 \\
0.530 \\
0.523 \\
0.521 \\
0.522 \\
0.524\end{array}$ \\
\hline $\begin{array}{l}36.0 \\
38.0 \\
40.0\end{array}$ & $\begin{array}{l}11.45 \\
11.25 \\
11.04\end{array}$ & $\begin{array}{l}122.0 \\
123.0 \\
124.0\end{array}$ & $\begin{array}{l}7.62 \\
7.49 \\
7.37\end{array}$ & $\begin{array}{l}7330.0 \\
7010.0 \\
6730.0\end{array}$ & $\begin{array}{l}0.00899 \\
0.00912 \\
0.00922\end{array}$ & $\begin{array}{l}0.0466 \\
0.0464 \\
0.0461\end{array}$ & $\begin{array}{l}6.20 \\
6.09 \\
6.01\end{array}$ & $\begin{array}{l}0.00370 \\
0.00367 \\
0.00365\end{array}$ & $\begin{array}{l}1.02098 \\
1.02101 \\
1.02102\end{array}$ & $\begin{array}{l}0.527 \\
0.532 \\
0.537\end{array}$ \\
\hline $\begin{array}{r}45.0 \\
50.0 \\
55.0 \\
60.0 \\
70.0 \\
80.0 \\
90.0 \\
100.0 \\
120.0 \\
140.0\end{array}$ & $\begin{array}{c}10.54 \\
10.05 \\
9.588 \\
9.149 \\
8.353 \\
7.664 \\
7.070 \\
6.557 \\
5.725 \\
5.081\end{array}$ & $\begin{array}{l}127.0 \\
130.0 \\
134.0 \\
138.0 \\
148.0 \\
159.0 \\
171.0 \\
183.0 \\
208.0 \\
233.0\end{array}$ & $\begin{array}{l}7.09 \\
6.83 \\
6.60 \\
6.38 \\
6.00 \\
5.69 \\
5.44 \\
5.23 \\
4.92 \\
4.70\end{array}$ & $\begin{array}{l}6100.0 \\
5590.0 \\
5180.0 \\
4860.0 \\
4380.0 \\
4070.0 \\
3840.0 \\
3680.0 \\
3460.0 \\
3310.0\end{array}$ & $\begin{array}{l}0.00940 \\
0.00947 \\
0.00943 \\
0.00930 \\
0.00888 \\
0.00834 \\
0.00779 \\
0.00726 \\
0.00634 \\
0.00560\end{array}$ & $\begin{array}{l}0.0455 \\
0.0449 \\
0.0445 \\
0.0441 \\
0.0438 \\
0.0439 \\
0.0443 \\
0.0450 \\
0.0467 \\
0.0489\end{array}$ & $\begin{array}{l}5.86 \\
5.79 \\
5.77 \\
5.78 \\
5.86 \\
5.99 \\
6.15 \\
6.32 \\
6.69 \\
7.07\end{array}$ & $\begin{array}{l}0.00362 \\
0.00363 \\
0.00368 \\
0.00376 \\
0.00400 \\
0.00432 \\
0.00472 \\
0.00516 \\
0.00619 \\
0.00736\end{array}$ & $\begin{array}{l}1.02098 \\
1.02086 \\
1.02066 \\
1.02041 \\
1.01978 \\
1.01906 \\
1.01830 \\
1.01755 \\
1.01613 \\
1.01488\end{array}$ & $\begin{array}{l}0.553 \\
0.571 \\
0.589 \\
0.605 \\
0.632 \\
0.651 \\
0.664 \\
0.672 \\
0.679 \\
0.681\end{array}$ \\
\hline $\begin{array}{l}160.0 \\
180.0 \\
200.0 \\
250.0 \\
300.0 \\
350.0 \\
400.0 \\
450.0 \\
500.0 \\
600.0\end{array}$ & $\begin{array}{l}4.571 \\
4.155 \\
3.811 \\
3.160 \\
2.702 \\
2.361 \\
2.098 \\
1.887 \\
1.715 \\
1.450\end{array}$ & $\begin{array}{l}259.0 \\
284.0 \\
309.0 \\
372.0 \\
434.0 \\
496.0 \\
558.0 \\
620.0 \\
682.0 \\
805.0\end{array}$ & $\begin{array}{l}4.53 \\
4.41 \\
4.31 \\
4.13 \\
4.02 \\
3.95 \\
3.89 \\
3.85 \\
3.82 \\
3.77\end{array}$ & $\begin{array}{l}3200.0 \\
3120.0 \\
3060.0 \\
2940.0 \\
2870.0 \\
2810.0 \\
2770.0 \\
2740.0 \\
2710.0 \\
2670.0\end{array}$ & $\begin{array}{l}0.00501 \\
0.00453 \\
0.00414 \\
0.00340 \\
0.00289 \\
0.00252 \\
0.00223 \\
0.00201 \\
0.001182 \\
0.00154\end{array}$ & $\begin{array}{l}0.0512 \\
0.0536 \\
0.0561 \\
0.0623 \\
0.0684 \\
0.0743 \\
0.0799 \\
0.0852 \\
0.0904 \\
0.101\end{array}$ & $\begin{array}{c}7.45 \\
7.83 \\
8.08 \\
9.01 \\
9.90 \\
10.8 \\
11.6 \\
12.4 \\
13.2 \\
14.7\end{array}$ & $\begin{array}{l}0.00864 \\
0.0100 \\
0.0115 \\
0.0156 \\
0.0201 \\
0.0251 \\
0.0305 \\
0.0363 \\
0.0424 \\
0.0559\end{array}$ & $\begin{array}{l}1.01378 \\
1.01282 \\
1.01198 \\
1.01028 \\
1.00900 \\
1.00800 \\
1.00719 \\
1.00654 \\
1.00599 \\
1.00513\end{array}$ & $\begin{array}{l}0.679 \\
0.676 \\
0.663 \\
0.658 \\
0.655 \\
0.652 \\
0.651 \\
0.651 \\
0.651 \\
0.654\end{array}$ \\
\hline $\begin{array}{r}700.0 \\
800.0 \\
900.0 \\
1000.0 \\
1200.0 \\
1400.0 \\
1600.0 \\
1800.0 \\
2000.0 \\
2500.0\end{array}$ & $\begin{array}{l}1.257 \\
1.109 \\
0.9916 \\
0.8970 \\
0.7532 \\
0.6490 \\
0.5702 \\
0.5084 \\
0.4586 \\
0.3685\end{array}$ & $\begin{array}{r}928.0 \\
1050.0 \\
1180.0 \\
1300.0 \\
1550.0 \\
1790.0 \\
2040.0 \\
2290.0 \\
2530.0 \\
3150.0\end{array}$ & $\begin{array}{l}3.73 \\
3.71 \\
3.69 \\
3.68 \\
3.66 \\
3.64 \\
3.63 \\
3.62 \\
3.62 \\
3.61\end{array}$ & $\begin{array}{l}2650.0 \\
2630.0 \\
2610.0 \\
2600.0 \\
2580.0 \\
2560.0 \\
2550.0 \\
2550.0 \\
2540.0 \\
2530.0\end{array}$ & $\begin{array}{l}0.00134 \\
0.00118 \\
0.00105 \\
0.000955 \\
0.000802 \\
0.000691 \\
0.000608 \\
0.000542 \\
0.000489 \\
0.000393\end{array}$ & $\begin{array}{l}0.111 \\
0.121 \\
0.131 \\
0.140 \\
0.158 \\
0.176 \\
0.193 \\
0.210 \\
0.226 \\
0.264\end{array}$ & $\begin{array}{l}16.3 \\
17.8 \\
19.3 \\
20.7 \\
23.5 \\
26.1 \\
28.7 \\
31.1 \\
33.5 \\
39.2\end{array}$ & $\begin{array}{l}0.0711 \\
0.0879 \\
0.106 \\
0.126 \\
0.170 \\
0.219 \\
0.273 \\
0.332 \\
0.397 \\
0.577\end{array}$ & $\begin{array}{l}1.00449 \\
1.00398 \\
1.00358 \\
1.00326 \\
1.00275 \\
1.00238 \\
1.00210 \\
1.00188 \\
1.00170 \\
1.00137\end{array}$ & $\begin{array}{l}0.656 \\
0.658 \\
0.659 \\
0.660 \\
0.662 \\
0.662 \\
0.663 \\
0.663 \\
0.664 \\
0.664\end{array}$ \\
\hline 3000.0 & 0.3079 & 3770.0 & 3.60 & 2520.0 & 0.000329 & 0.300 & 44.6 & 0.786 & 1.00115 & 0.664 \\
\hline
\end{tabular}

- two-phase boundary 
THERMOOYNAMIC PROPERTIES OF HELIUM 4

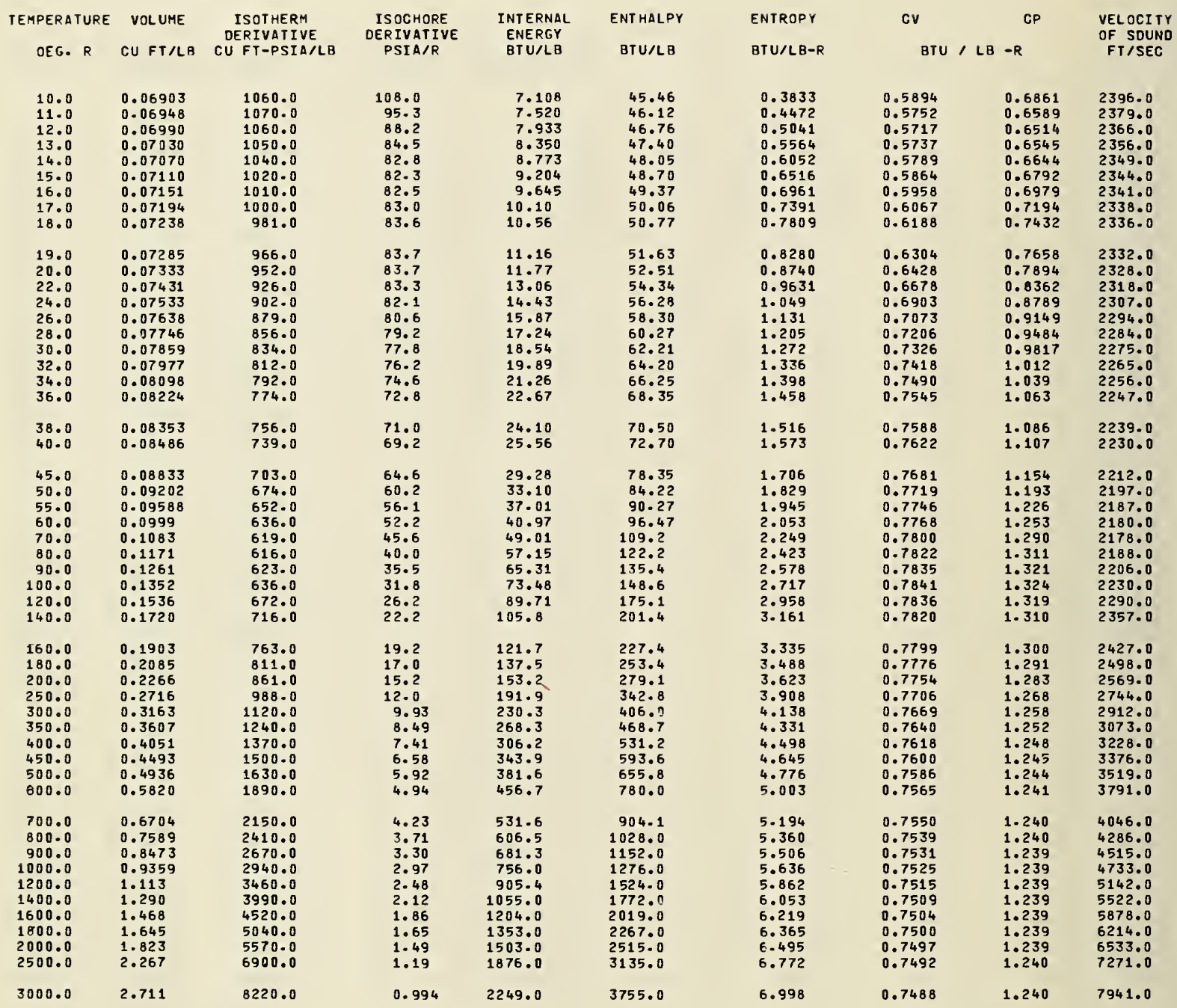


3000 PSIA ISOBAR

\begin{tabular}{|c|c|c|c|c|c|c|c|c|c|c|}
\hline $\begin{array}{c}\text { TENPERATURE } \\
\text { OEG. R }\end{array}$ & DENSITY & $\begin{array}{l}\text { V(OH/OV) } \\
\text { BTU/LB }\end{array}$ & $\begin{array}{l}V I O P / O U)_{V}-V \\
P S I A-C U F T / B T U\end{array}$ & ${ }_{\text {PSIA }}^{V(O P / O V)} T$ & $\begin{array}{l}\text { COV/OT RP } \\
\text { I/OEG. R }\end{array}$ & $\begin{array}{c}\text { THERHAL } \\
\text { CONDUCTIVITY } \\
\text { BTU/FT-HR-R }\end{array}$ & $\begin{array}{l}\text { VISCOSITY } \\
\text { LB/FT-SEC } \\
\times 10 E+6\end{array}$ & $\begin{array}{l}\text { THERHAL } \\
\text { OIFFUSIVITY } \\
\text { SQ FT/HR }\end{array}$ & $\begin{array}{l}\text { OIELECTRIC } \\
\text { CONSTANT }\end{array}$ & $\begin{array}{l}\text { PRANDTL } \\
\text { NUHBER }\end{array}$ \\
\hline $\begin{array}{l}10.0 \\
11.0 \\
12.0 \\
13.0 \\
14.0 \\
15.0 \\
16.0 \\
17.0 \\
18.0\end{array}$ & $\begin{array}{l}14.49 \\
14.39 \\
14.31 \\
14.22 \\
14.14 \\
14.06 \\
13.98 \\
13.90 \\
13.82\end{array}$ & $\begin{array}{r}97.9 \\
106.0 \\
112.0 \\
116.0 \\
118.0 \\
119.0 \\
120.0 \\
120.0 \\
120.0\end{array}$ & $\begin{array}{c}12.7 \\
11.5 \\
10.8 \\
10.4 \\
10.1 \\
10.0 \\
9.90 \\
9.84 \\
9.78\end{array}$ & $\begin{array}{l}15400.0 \\
15300.0 \\
15200.0 \\
14900.0 \\
14700.0 \\
14400.0 \\
14100.0 \\
13800.0 \\
13600.0\end{array}$ & $\begin{array}{l}0.00701 \\
0.00621 \\
0.00581 \\
0.00565 \\
0.00564 \\
0.00571 \\
0.00584 \\
0.00600 \\
0.00617\end{array}$ & $\begin{array}{l}0.0363 \\
0.0384 \\
0.0404 \\
0.0421 \\
0.0437 \\
0.0451 \\
0.0463 \\
0.0473 \\
0.0482\end{array}$ & $\begin{array}{l}29.5 \\
24.8 \\
21.4 \\
18.9 \\
16.9 \\
15.4 \\
14.1 \\
13.0 \\
12.2\end{array}$ & $\begin{array}{l}0.00365 \\
0.00405 \\
0.00433 \\
0.00453 \\
0.00465 \\
0.00472 \\
0.00475 \\
0.00473 \\
0.00469\end{array}$ & $\begin{array}{l}1.01891 \\
1.01902 \\
1.01912 \\
1.01921 \\
1.01930 \\
1.01939 \\
1.01947 \\
1.01956 \\
1.01964\end{array}$ & $\begin{array}{l}2.01 \\
1.53 \\
1.25 \\
1.06 \\
0.926 \\
0.833 \\
0.765 \\
0.714 \\
0.676\end{array}$ \\
\hline $\begin{array}{l}19.0 \\
20.0 \\
22.0 \\
24.0 \\
26.0 \\
28.0 \\
30.0 \\
32.0 \\
34.0 \\
36.0\end{array}$ & $\begin{array}{l}13.73 \\
13.64 \\
13.46 \\
13.27 \\
13.09 \\
12.91 \\
12.72 \\
12.54 \\
12.35 \\
12.16\end{array}$ & $\begin{array}{l}121.0 \\
122.0 \\
125.0 \\
128.0 \\
131.0 \\
132.0 \\
134.0 \\
135.0 \\
136.0 \\
137.0\end{array}$ & $\begin{array}{l}9.67 \\
9.55 \\
9.27 \\
8.96 \\
8.70 \\
8.51 \\
8.35 \\
8.20 \\
8.06 \\
7.93\end{array}$ & $\begin{array}{r}13300.0 \\
13000.0 \\
12500.0 \\
12000.0 \\
11500.0 \\
11000.0 \\
10600.0 \\
10200.0 \\
9790.0 \\
9410.0\end{array}$ & $\begin{array}{l}0.00631 \\
0.00645 \\
0.00668 \\
0.00686 \\
0.00701 \\
0.00717 \\
0.00733 \\
0.00749 \\
0.00762 \\
0.00774\end{array}$ & $\begin{array}{l}0.0489 \\
0.0495 \\
0.0503 \\
0.0509 \\
0.0512 \\
0.0514 \\
0.0514 \\
0.0513 \\
0.0511 \\
0.0509\end{array}$ & $\begin{array}{c}11.4 \\
10.8 \\
9.77 \\
9.02 \\
8.44 \\
7.99 \\
7.64 \\
7.35 \\
7.12 \\
6.93\end{array}$ & $\begin{array}{l}0.00465 \\
0.00459 \\
0.00447 \\
0.00436 \\
0.00427 \\
0.00419 \\
0.00411 \\
0.00404 \\
0.00399 \\
0.00394\end{array}$ & $\begin{array}{l}1.01973 \\
1.01981 \\
1.01997 \\
1.02012 \\
1.02026 \\
1.02039 \\
1.02050 \\
1.02061 \\
1.02070 \\
1.02079\end{array}$ & $\begin{array}{l}0.644 \\
0.620 \\
0.585 \\
0.561 \\
0.543 \\
0.531 \\
0.525 \\
0.522 \\
0.521 \\
0.521\end{array}$ \\
\hline $\begin{array}{l}38.0 \\
40.0\end{array}$ & $\begin{array}{l}11.97 \\
11.78\end{array}$ & $\begin{array}{l}138.0 \\
139.0\end{array}$ & $\begin{array}{l}7.81 \\
7.70\end{array}$ & $\begin{array}{l}9050.0 \\
8710.0\end{array}$ & $\begin{array}{l}0.00784 \\
0.00794\end{array}$ & $\begin{array}{l}0.0507 \\
0.0504\end{array}$ & $\begin{array}{l}6.78 \\
6.65\end{array}$ & $\begin{array}{l}0.00390 \\
0.00386\end{array}$ & $\begin{array}{l}1.02086 \\
1.02091\end{array}$ & $\begin{array}{l}0.523 \\
0.526\end{array}$ \\
\hline $\begin{array}{r}45.0 \\
50.0 \\
55.0 \\
60.0 \\
70.0 \\
80.0 \\
90.0 \\
100.0 \\
120.0 \\
140.0\end{array}$ & $\begin{array}{c}11.32 \\
10.87 \\
10.43 \\
10.01 \\
9.230 \\
8.538 \\
7.928 \\
7.394 \\
6.510 \\
5.814\end{array}$ & $\begin{array}{l}142.0 \\
145.0 \\
149.0 \\
153.0 \\
162.0 \\
172.0 \\
184.0 \\
196.0 \\
220.0 \\
246.0\end{array}$ & $\begin{array}{l}7.43 \\
7.18 \\
6.94 \\
6.72 \\
6.33 \\
6.00 \\
5.72 \\
5.49 \\
5.14 \\
4.88\end{array}$ & $\begin{array}{l}7960.0 \\
7330.0 \\
6800.0 \\
6370.0 \\
5710.0 \\
5260.0 \\
4940.0 \\
4700.0 \\
4380.0 \\
4160.0\end{array}$ & $\begin{array}{l}0.00811 \\
0.00821 \\
0.00824 \\
0.00820 \\
0.00797 \\
0.00761 \\
0.00719 \\
0.00677 \\
0.00599 \\
0.00533\end{array}$ & $\begin{array}{l}0.0497 \\
0.0491 \\
0.0485 \\
0.0481 \\
0.0475 \\
0.0473 \\
0.0475 \\
0.0480 \\
0.0494 \\
0.0513\end{array}$ & $\begin{array}{l}6.43 \\
6.30 \\
6.24 \\
6.21 \\
6.24 \\
6.34 \\
6.47 \\
6.62 \\
6.96 \\
7.32\end{array}$ & $\begin{array}{l}0.00381 \\
0.00379 \\
0.00380 \\
0.00383 \\
0.00399 \\
0.00423 \\
0.00454 \\
0.00490 \\
0.00576 \\
0.00674\end{array}$ & $\begin{array}{l}1.02100 \\
1.02101 \\
1.02096 \\
1.02084 \\
1.02046 \\
1.01995 \\
1.01935 \\
1.01873 \\
1.01747 \\
1.01630\end{array}$ & $\begin{array}{l}0.537 \\
0.552 \\
0.567 \\
0.583 \\
0.610 \\
0.632 \\
0.647 \\
0.658 \\
0.669 \\
0.672\end{array}$ \\
\hline $\begin{array}{l}160.0 \\
180.0 \\
200.0 \\
250.0 \\
300.0 \\
350.0 \\
400.0 \\
450.0 \\
500.0 \\
600.0\end{array}$ & $\begin{array}{l}5.255 \\
4.796 \\
4.412 \\
3.682 \\
3.162 \\
2.772 \\
2.469 \\
2.226 \\
2.026 \\
1.718\end{array}$ & $\begin{array}{l}271.0 \\
296.0 \\
322.0 \\
384.0 \\
447.0 \\
509.0 \\
571.0 \\
632.0 \\
694.0 \\
817.0\end{array}$ & $\begin{array}{l}4.69 \\
4.55 \\
4.43 \\
4.23 \\
4.10 \\
4.01 \\
3.94 \\
3.89 \\
3.85 \\
3.80\end{array}$ & $\begin{array}{l}4010.0 \\
3890.0 \\
3800.0 \\
3640.0 \\
3530.0 \\
3450.0 \\
3390.0 \\
3340.0 \\
3300.0 \\
3250.0\end{array}$ & $\begin{array}{l}0.00480 \\
0.00436 \\
0.00399 \\
0.00330 \\
0.00282 \\
0.00246 \\
0.00219 \\
0.00197 \\
0.00179 \\
0.00152\end{array}$ & $\begin{array}{l}0.0535 \\
0.0558 \\
0.0581 \\
0.0641 \\
0.0700 \\
0.0757 \\
0.0812 \\
0.0864 \\
0.0914 \\
0.101\end{array}$ & $\begin{array}{l}7.68 \\
8.05 \\
8.29 \\
9.19 \\
10.1 \\
10.9 \\
11.7 \\
12.5 \\
13.2 \\
14.8\end{array}$ & $\begin{array}{l}0.00783 \\
0.00901 \\
0.0103 \\
0.0137 \\
0.0176 \\
0.0218 \\
0.0263 \\
0.0312 \\
0.0363 \\
0.0476\end{array}$ & $\begin{array}{l}1.01523 \\
1.01427 \\
1.01342 \\
1.01165 \\
1.01028 \\
1.00920 \\
1.00831 \\
1.00759 \\
1.00697 \\
1.00600\end{array}$ & $\begin{array}{l}0.672 \\
0.671 \\
0.658 \\
0.654 \\
0.651 \\
0.649 \\
0.647 \\
0.647 \\
0.648 \\
0.651\end{array}$ \\
\hline $\begin{array}{r}700.0 \\
800.0 \\
900.0 \\
1000.0 \\
1200.0 \\
1400.0 \\
1600.0 \\
1800.0 \\
2000.0 \\
2500.0\end{array}$ & $\begin{array}{l}1.492 \\
1.318 \\
1.180 \\
1.069 \\
0.8984 \\
0.7750 \\
0.6813 \\
0.6078 \\
0.5486 \\
0.4411\end{array}$ & $\begin{array}{r}940.0 \\
1060.0 \\
1190.0 \\
1310.0 \\
1560.0 \\
1800.0 \\
2050.0 \\
2300.0 \\
2540.0 \\
3160.0\end{array}$ & $\begin{array}{l}3.76 \\
3.73 \\
3.71 \\
3.69 \\
3.67 \\
3.65 \\
3.64 \\
3.63 \\
3.62 \\
3.61\end{array}$ & $\begin{array}{l}3210.0 \\
3180.0 \\
3160.0 \\
3140.0 \\
3110.0 \\
3090.0 \\
3080.0 \\
3070.0 \\
3060.0 \\
3040.0\end{array}$ & $\begin{array}{l}0.00132 \\
0.00117 \\
0.00104 \\
0.000946 \\
0.0000966 \\
0.000687 \\
0.000604 \\
0.000539 \\
0.000487 \\
0.000392\end{array}$ & $\begin{array}{l}0.112 \\
0.121 \\
0.131 \\
0.141 \\
0.159 \\
0.176 \\
0.193 \\
0.210 \\
0.226 \\
0.264\end{array}$ & $\begin{array}{l}16.3 \\
17.9 \\
19.3 \\
20.8 \\
23.5 \\
26.2 \\
28.7 \\
31.2 \\
33.5 \\
39.3\end{array}$ & $\begin{array}{l}0.0603 \\
0.0744 \\
0.0897 \\
0.106 \\
0.143 \\
0.184 \\
0.229 \\
0.278 \\
0.332 \\
0.483\end{array}$ & $\begin{array}{l}1.00527 \\
1.00469 \\
1.00423 \\
1.00385 \\
1.00326 \\
1.00283 \\
1.00250 \\
1.00224 \\
1.00202 \\
1.00163\end{array}$ & $\begin{array}{l}0.654 \\
0.656 \\
0.658 \\
0.659 \\
0.660 \\
0.661 \\
0.662 \\
0.663 \\
0.663 \\
0.664\end{array}$ \\
\hline 3000.0 & 0.3688 & 3780.0 & 3.60 & 3030.0 & 0.000328 & 0.300 & 44.7 & 0.657 & 1.00137 & 0.664 \\
\hline
\end{tabular}

* tho-phase boundary 


\begin{tabular}{|c|c|c|c|c|c|c|c|c|c|}
\hline $\begin{array}{c}\text { TEMPERATURE } \\
\text { OEG. R }\end{array}$ & $\begin{array}{l}\text { VOLUME } \\
\text { CU FT/LB }\end{array}$ & $\begin{array}{c}\text { ISOTHERH } \\
\text { OERIVATIVE } \\
\text { CU FT-PSIA/LB }\end{array}$ & $\begin{array}{l}\text { ISOCHORE } \\
\text { OERIVATIVE } \\
\text { PSIA/R }\end{array}$ & $\begin{array}{l}\text { INTERNAL } \\
\text { ENERGY } \\
\text { BTU/LB }\end{array}$ & $\begin{array}{l}\text { ENTHALPY } \\
\text { ETU/LB }\end{array}$ & $\begin{array}{l}\text { ENTROPY } \\
\text { BTU/LE-R }\end{array}$ & $\begin{array}{l}\text { CV } \\
\text { eTU, LB. }\end{array}$ & $\begin{array}{l}C P \\
-R\end{array}$ & $\begin{array}{l}\text { VELOCITY } \\
\text { OF SOUND } \\
\text { FT/SEC }\end{array}$ \\
\hline $\begin{array}{l}11.0 \\
12.0 \\
13.0 \\
14.0 \\
15.0 \\
16.0 \\
17.0 \\
18.0 \\
19.0\end{array}$ & $\begin{array}{l}0.06740 \\
0.06779 \\
0.06815 \\
0.06850 \\
0.06885 \\
0.06921 \\
0.06957 \\
0.06995 \\
0.07036\end{array}$ & $\begin{array}{l}1190.0 \\
1180.0 \\
1180.0 \\
1160.0 \\
1150.0 \\
1140.0 \\
1120.0 \\
1110.0 \\
1090.0\end{array}$ & $\begin{array}{r}106.0 \\
95.0 \\
89.4 \\
86.6 \\
85.7 \\
85.8 \\
86.4 \\
87.3 \\
87.8\end{array}$ & $\begin{array}{c}8.344 \\
8.770 \\
9.190 \\
9.608 \\
10.03 \\
10.45 \\
10.88 \\
11.32 \\
11.90\end{array}$ & $\begin{array}{l}52.03 \\
52.70 \\
53.36 \\
54.00 \\
54.65 \\
55.30 \\
55.97 \\
56.66 \\
57.50\end{array}$ & $\begin{array}{l}0.4085 \\
0.4682 \\
0.5215 \\
0.5705 \\
0.6163 \\
0.5599 \\
0.7017 \\
0.7422 \\
0.7881\end{array}$ & $\begin{array}{l}0.6148 \\
0.5951 \\
0.5854 \\
0.5822 \\
0.5837 \\
0.5888 \\
0.5969 \\
0.6071 \\
0.6179\end{array}$ & $\begin{array}{l}0.7016 \\
0.6729 \\
0.6614 \\
0.6606 \\
0.6677 \\
0.6806 \\
0.6981 \\
0.7191 \\
0.7404\end{array}$ & $\begin{array}{l}2504.0 \\
2490.0 \\
2480.0 \\
2474.0 \\
2470.0 \\
2468.0 \\
2468.0 \\
2467.0 \\
2465.0\end{array}$ \\
\hline $\begin{array}{l}20.0 \\
22.0 \\
24.0 \\
26.0 \\
28.0 \\
30.0 \\
32.0 \\
34.0 \\
36.0 \\
38.0\end{array}$ & $\begin{array}{l}0.07077 \\
0.07162 \\
0.07250 \\
0.07341 \\
0.07433 \\
0.07530 \\
0.07630 \\
0.07733 \\
0.07838 \\
0.07947\end{array}$ & $\begin{array}{r}1080.0 \\
1060.0 \\
1030.0 \\
1010.0 \\
986.0 \\
964.0 \\
943.0 \\
923.0 \\
903.0 \\
884.0\end{array}$ & $\begin{array}{l}88.1 \\
88.2 \\
87.4 \\
86.0 \\
84.7 \\
83.5 \\
82.1 \\
80.6 \\
78.9 \\
77.2\end{array}$ & $\begin{array}{l}12.49 \\
13.73 \\
15.06 \\
16.46 \\
17.79 \\
19.05 \\
20.34 \\
21.67 \\
23.03 \\
24.42\end{array}$ & $\begin{array}{l}58.35 \\
60.15 \\
62.05 \\
64.03 \\
65.97 \\
67.85 \\
69.79 \\
71.79 \\
73.84 \\
75.93\end{array}$ & $\begin{array}{l}0.8330 \\
0.9202 \\
1.004 \\
1.085 \\
1.158 \\
1.223 \\
1.285 \\
1.346 \\
1.404 \\
1.461\end{array}$ & $\begin{array}{l}0.6302 \\
0.6569 \\
0.6816 \\
0.7005 \\
0.7151 \\
0.7284 \\
0.7387 \\
0.7467 \\
0.7529 \\
0.7579\end{array}$ & $\begin{array}{l}0.7635 \\
0.8109 \\
0.8545 \\
0.8906 \\
0.9234 \\
0.9561 \\
0.9853 \\
1.012 \\
1.035 \\
1.057\end{array}$ & $\begin{array}{l}2463.0 \\
2457.0 \\
2448.0 \\
2437.0 \\
2429.0 \\
2421.0 \\
2414.0 \\
2406.0 \\
2398.0 \\
2391.0\end{array}$ \\
\hline 40.0 & 0.08059 & 867.0 & 75.5 & 25.83 & 78.06 & 1.516 & 0.7618 & 1.078 & 2383.0 \\
\hline $\begin{array}{r}45.0 \\
50.0 \\
55.0 \\
60.0 \\
70.0 \\
80.0 \\
90.0 \\
100.0 \\
120.0 \\
140.0\end{array}$ & $\begin{array}{l}0.08350 \\
0.08658 \\
0.08980 \\
0.09316 \\
0.1002 \\
0.1076 \\
0.1152 \\
0.1229 \\
0.1386 \\
0.1543\end{array}$ & $\begin{array}{l}827.0 \\
794.0 \\
766.0 \\
745.0 \\
718.0 \\
706.0 \\
706.0 \\
714.0 \\
743.0 \\
782.0\end{array}$ & $\begin{array}{l}71.0 \\
66.7 \\
62.6 \\
58.7 \\
51.7 \\
45.8 \\
40.9 \\
36.8 \\
30.4 \\
25.8\end{array}$ & $\begin{array}{c}29.45 \\
33.18 \\
36.98 \\
40.86 \\
48.77 \\
56.82 \\
64.93 \\
73.06 \\
89.30 \\
105.4\end{array}$ & $\begin{array}{c}83.57 \\
89.29 \\
95.18 \\
101.2 \\
113.7 \\
126.5 \\
139.6 \\
152.7 \\
179.1 \\
205.4\end{array}$ & $\begin{array}{l}1.645 \\
1.766 \\
1.878 \\
1.983 \\
2.176 \\
2.347 \\
2.500 \\
2.539 \\
2.879 \\
3.082\end{array}$ & $\begin{array}{l}0.7686 \\
0.7731 \\
0.7764 \\
0.7790 \\
0.7870 \\
0.7857 \\
0.7875 \\
0.7884 \\
0.7885 \\
0.7871\end{array}$ & $\begin{array}{l}1.123 \\
1.162 \\
1.196 \\
1.225 \\
1.268 \\
1.295 \\
1.310 \\
1.318 \\
1.319 \\
1.312\end{array}$ & $\begin{array}{l}2366.0 \\
2351.0 \\
2339.0 \\
2329.0 \\
2320.0 \\
2322.0 \\
2333.0 \\
2351.0 \\
2400.0 \\
2458.0\end{array}$ \\
\hline $\begin{array}{l}160.0 \\
180.0 \\
200.0 \\
250.0 \\
300.0 \\
350.0 \\
400.0 \\
450.0 \\
500.0 \\
600.0\end{array}$ & $\begin{array}{l}0.1699 \\
0.1855 \\
0.2011 \\
0.2396 \\
0.2778 \\
0.3159 \\
0.3538 \\
0.3917 \\
0.4295 \\
0.5052\end{array}$ & $\begin{array}{r}826.0 \\
873.0 \\
921.0 \\
1040.0 \\
1170.0 \\
1300.0 \\
1420.0 \\
1550.0 \\
1680.0 \\
1940.0\end{array}$ & $\begin{array}{c}22.4 \\
19.7 \\
17.6 \\
14.0 \\
11.6 \\
9.87 \\
8.62 \\
7.66 \\
6.89 \\
5.74\end{array}$ & $\begin{array}{l}121.4 \\
137.3 \\
153.0 \\
192.0 \\
230.5 \\
268.6 \\
306.6 \\
344.4 \\
382.1 \\
457.3\end{array}$ & $\begin{array}{l}231.6 \\
257.5 \\
283.4 \\
347.3 \\
410.5 \\
473.4 \\
535.9 \\
598.3 \\
660.5 \\
784.8\end{array}$ & $\begin{array}{l}3.257 \\
3.410 \\
3.546 \\
3.831 \\
4.062 \\
4.255 \\
4.423 \\
4.569 \\
4.701 \\
4.927\end{array}$ & $\begin{array}{l}0.7850 \\
0.7826 \\
0.7803 \\
0.7750 \\
0.7707 \\
0.7674 \\
0.7648 \\
0.7627 \\
0.7611 \\
0.7586\end{array}$ & $\begin{array}{l}1.303 \\
1.294 \\
1.286 \\
1.271 \\
1.260 \\
1.254 \\
1.249 \\
1.246 \\
1.244 \\
1.241\end{array}$ & $\begin{array}{l}2521.0 \\
2586.0 \\
2652.0 \\
2817.0 \\
2977.0 \\
3133.0 \\
3283.0 \\
3427.0 \\
3567.0 \\
3833.0\end{array}$ \\
\hline $\begin{array}{r}700.0 \\
800.0 \\
900.0 \\
1000.0 \\
1200.0 \\
1400.0 \\
1600.0 \\
1800.0 \\
2000.0 \\
2500.0\end{array}$ & $\begin{array}{l}0.5808 \\
0.6565 \\
0.7322 \\
0.8080 \\
0.9596 \\
1.111 \\
1.263 \\
1.415 \\
1.567 \\
1.947\end{array}$ & $\begin{array}{l}2200.0 \\
2460.0 \\
2720.0 \\
2980.0 \\
3500.0 \\
4030.0 \\
4550.0 \\
5080.0 \\
5610.0 \\
6930.0\end{array}$ & $\begin{array}{l}4.93 \\
4.31 \\
3.84 \\
3.46 \\
2.88 \\
2.47 \\
2.17 \\
1.93 \\
1.74 \\
1.39\end{array}$ & $\begin{array}{r}532.4 \\
607.3 \\
682.1 \\
756.9 \\
906.4 \\
1056.0 \\
1205.0 \\
1354.0 \\
1504.0 \\
1877.0\end{array}$ & $\begin{array}{r}908.8 \\
1033.0 \\
1157.0 \\
1281.0 \\
1528.0 \\
1776.0 \\
2024.0 \\
2272.0 \\
2520.0 \\
3139.0\end{array}$ & $\begin{array}{l}5.118 \\
5.284 \\
5.430 \\
5.560 \\
5.786 \\
5.977 \\
6.143 \\
6.289 \\
6.419 \\
6.696\end{array}$ & $\begin{array}{l}0.7568 \\
0.7556 \\
0.7546 \\
0.7538 \\
0.7527 \\
0.7520 \\
0.7514 \\
0.7509 \\
0.7506 \\
0.7500\end{array}$ & $\begin{array}{l}1.240 \\
1.239 \\
1.239 \\
1.239 \\
1.239 \\
1.239 \\
1.239 \\
1.239 \\
1.239 \\
1.239\end{array}$ & $\begin{array}{l}4083.0 \\
4320.0 \\
4546.0 \\
4761.0 \\
5166.0 \\
5543.0 \\
5897.0 \\
6231.0 \\
6548.0 \\
7283.0\end{array}$ \\
\hline 3000.0 & 2.328 & 8250.0 & 1.16 & 2250.0 & 3759.0 & 6.922 & 0.7495 & 1.240 & 7951.0 \\
\hline
\end{tabular}


3500 PSIA ISOBAR

TEMPERATURE DENSITY V(OH/DV) $V(O P / D U)_{\mathrm{V}}-V(O P / D V)_{T}$

DEG. R LA/CUFT BTU/LB PSIA-CU FT/BTU PSIA

\begin{tabular}{|c|c|c|c|c|}
\hline $\begin{array}{l}11.0 \\
12.0 \\
13.0 \\
14.0 \\
15.0 \\
16.0 \\
17.0 \\
18.0 \\
19.0\end{array}$ & $\begin{array}{l}14.84 \\
14.75 \\
14.67 \\
14.60 \\
14.52 \\
14.45 \\
14.37 \\
14.30 \\
14.21\end{array}$ & $\begin{array}{l}117.0 \\
124.0 \\
128.0 \\
130.0 \\
130.0 \\
130.0 \\
131.0 \\
131.0 \\
131.0\end{array}$ & $\begin{array}{l}11.6 \\
10.8 \\
10.4 \\
10.2 \\
10.1 \\
10.1 \\
10.1 \\
10.1 \\
10.0\end{array}$ & $\begin{array}{l}17600.0 \\
17500.0 \\
17300.0 \\
17000.0 \\
16700.0 \\
16400.0 \\
16200.0 \\
15900.0 \\
15600.0\end{array}$ \\
\hline $\begin{array}{l}20.0 \\
22.0 \\
24.0 \\
26.0 \\
28.0 \\
30.0 \\
32.0 \\
34.0 \\
36.0 \\
38.0\end{array}$ & $\begin{array}{l}14.13 \\
13.96 \\
13.79 \\
13.62 \\
13.45 \\
13.28 \\
13.11 \\
12.93 \\
12.76 \\
12.58\end{array}$ & $\begin{array}{l}132.0 \\
135.0 \\
139.0 \\
142.0 \\
145.0 \\
147.0 \\
148.0 \\
150.0 \\
151.0 \\
152.0\end{array}$ & $\begin{array}{l}9.89 \\
9.61 \\
9.29 \\
9.01 \\
8.80 \\
8.63 \\
8.48 \\
8.34 \\
8.22 \\
8.10\end{array}$ & $\begin{array}{l}15300.0 \\
14700.0 \\
14200.0 \\
13700.0 \\
13300.0 \\
12800.0 \\
12400.0 \\
11900.0 \\
11500.0 \\
11100.0\end{array}$ \\
\hline 40.0 & 12.41 & 154.0 & 7.98 & 10800.0 \\
\hline $\begin{array}{r}45.0 \\
50.0 \\
55.0 \\
60.0 \\
70.0 \\
80.0 \\
90.0 \\
100.0 \\
120.0 \\
140.0\end{array}$ & $\begin{array}{l}11.98 \\
11.55 \\
11.14 \\
10.73 \\
9.98 \\
9.295 \\
8.682 \\
8.136 \\
7.217 \\
6.482\end{array}$ & $\begin{array}{l}157.0 \\
160.0 \\
163.0 \\
167.0 \\
176.0 \\
186.0 \\
197.0 \\
208.0 \\
233.0 \\
258.0\end{array}$ & $\begin{array}{l}7.72 \\
7.47 \\
7.24 \\
7.02 \\
6.62 \\
6.27 \\
5.98 \\
5.73 \\
5.34 \\
5.06\end{array}$ & $\begin{array}{l}9900.0 \\
9170.0 \\
8530.0 \\
8000.0 \\
7160.0 \\
6560.0 \\
6130.0 \\
5810.0 \\
5360.0 \\
5070.0\end{array}$ \\
\hline $\begin{array}{l}160.0 \\
180.0 \\
200.0 \\
250.0 \\
300.0 \\
350.0 \\
400.0 \\
450.0 \\
500.0 \\
600.0\end{array}$ & $\begin{array}{l}5.884 \\
5.390 \\
4.974 \\
4.174 \\
3.599 \\
3.166 \\
2.826 \\
2.553 \\
2.328 \\
1.979\end{array}$ & $\begin{array}{l}283.0 \\
309.0 \\
334.0 \\
397.0 \\
459.0 \\
521.0 \\
583.0 \\
645.0 \\
706.0 \\
829.0\end{array}$ & $\begin{array}{l}4.84 \\
4.68 \\
4.55 \\
4.32 \\
4.17 \\
4.06 \\
3.99 \\
3.93 \\
3.89 \\
3.82\end{array}$ & $\begin{array}{l}4860.0 \\
4710.0 \\
4580.0 \\
4360.0 \\
4210.0 \\
4110.0 \\
4030.0 \\
3960.0 \\
3910.0 \\
3840.0\end{array}$ \\
\hline $\begin{array}{r}700.0 \\
800.0 \\
900.0 \\
1000.0 \\
1200.0 \\
1400.0 \\
1600.0 \\
1800.0 \\
2000.0 \\
2500.0\end{array}$ & $\begin{array}{l}1.722 \\
1.523 \\
1.366 \\
1.238 \\
1.042 \\
0.8998 \\
0.7916 \\
0.7066 \\
0.6381 \\
0.5135\end{array}$ & $\begin{array}{r}952.0 \\
1070.0 \\
1200.0 \\
1320.0 \\
1570.0 \\
1810.0 \\
2060.0 \\
2310.0 \\
2550.0 \\
3170.0\end{array}$ & $\begin{array}{l}3.78 \\
3.75 \\
3.72 \\
3.70 \\
3.68 \\
3.66 \\
3.64 \\
3.63 \\
3.62 \\
3.61\end{array}$ & $\begin{array}{l}3780.0 \\
3740.0 \\
3710.0 \\
3690.0 \\
3650.0 \\
3620.0 \\
3600.0 \\
3590.0 \\
3580.0 \\
3560.0\end{array}$ \\
\hline 3000.0 & 0.4295 & 3790.0 & 3.60 & 3540.0 \\
\hline
\end{tabular}

* tho-phase goundary
IOVIOTIIV THERMAL VISCOSITY 1/OEG. P BTU/FT-HR-R LB/FT-SEC $\times 10 E+5$

THEPMAL DIELECTRIC IFFUSIVITY CONSTANT

PRANOTL SO FT/HR

$\begin{array}{llllll}0.00600 & 0.0413 & 32.5 & 0.00397 & 1.01846 & 1.99 \\ 0.00544 & 0.0434 & 27.6 & 0.00437 & 1.01857 & 1.54 \\ 0.00518 & 0.0453 & 24.0 & 0.00467 & 1.01867 & 1.26 \\ 0.00510 & 0.0471 & 21.2 & 0.00488 & 1.01877 & 1.07 \\ 0.00512 & 0.0486 & 19.0 & 0.00501 & 1.01886 & 0.941 \\ 0.00522 & 0.0499 & 17.3 & 0.00507 & 1.01895 & 0.848 \\ 0.00535 & 0.0510 & 15.8 & 0.00508 & 1.01904 & 0.781 \\ 0.00550 & 0.0519 & 14.7 & 0.00505 & 1.01913 & 0.730 \\ 0.00564 & 0.0527 & 13.6 & 0.00501 & 1.01922 & 0.590 \\ 0.00577 & 0.0533 & 12.8 & 0.00494 & 1.01932 & 0.659 \\ 0.00598 & 0.0543 & 11.4 & 0.00479 & 1.01949 & 0.515 \\ 0.00514 & 0.0549 & 10.4 & 0.00466 & 1.01966 & 0.585 \\ 0.00625 & 0.0552 & 9.67 & 0.00455 & 1.01982 & 0.561 \\ 0.00638 & 0.0554 & 9.08 & 0.00446 & 1.01997 & 0.545 \\ 0.00652 & 0.0554 & 8.61 & 0.00437 & 1.02012 & 0.535 \\ 0.00654 & 0.0553 & 8.23 & 0.00429 & 1.02025 & 0.528 \\ 0.00675 & 0.0552 & 7.93 & 0.00422 & 1.02037 & 0.523 \\ 0.00685 & 0.0549 & 7.58 & 0.00416 & 1.02048 & 0.521 \\ 0.00694 & 0.0547 & 7.47 & 0.00411 & 1.02059 & 0.520 \\ 0.00702 & 0.0544 & 7.30 & 0.00407 & 1.02068 & 0.521 \\ 0.00717 & 0.0537 & 7.00 & 0.00399 & 1.02085 & 0.527 \\ 0.00728 & 0.0529 & 5.81 & 0.00394 & 1.02097 & 0.538 \\ 0.00733 & 0.0523 & 6.70 & 0.00393 & 1.02101 & 0.551 \\ 0.00734 & 0.0518 & 6.64 & 0.00394 & 1.02100 & 0.566 \\ 0.00722 & 0.0510 & 6.62 & 0.00403 & 1.02083 & 0.593 \\ 0.00698 & 0.0506 & 6.58 & 0.00421 & 1.02050 & 0.615 \\ 0.00667 & 0.0506 & 6.79 & 0.00445 & 1.02007 & 0.632 \\ 0.00533 & 0.0509 & 6.92 & 0.00475 & 1.01957 & 0.545 \\ 0.00567 & 0.0521 & 7.22 & 0.00547 & 1.01850 & 0.659 \\ 0.00509 & 0.0537 & 7.56 & 0.00632 & 1.01743 & 0.664\end{array}$

0.00460

0.00419

0.00385

0.00320

0.00274

.00241

0.00214

0.00193

0.00150

0.0557

0.0579

0.0601

0.0659

0.0716

0.0771
0.0825

0.0876

0.0925

0.102

7.91

0.00727

1.01642

8.26

$8.4 \mathrm{~B}$

10.2

10.2
11.0

11.0
11.8

12.6

13.3
14.8

0.00830

0.00940

0.0124

0.0158

0.0194
0.0234

0.0275

0.0319

1.01550

1.01465

1.01286

1. 01144

1.01029
1.00935

1. 00856

1. 00790

1. 00683

0.00130

0.00103

0.112

16.4

0.000938

0.122

1. 00601

1. 00537

1.00485

1. 00442

1. 00376

0.141

.000790

0.177

$0.000601 \quad 0.194$

0.000485

0.174
0.210

20.8

23.5

26.2
28.7

28.7
31.2

33.6
39.3

1.00289

1.00259

1. 00234

1.00190

44.7

0.000327

0.300

0.564
0.666

0.653

0.650

0.647

0.645

0.644

0.644

0.644
0.648

0.652

0.654

0.656

0.657

0.659

0.661

0.66

0.662

0.652

0.553

0.654 


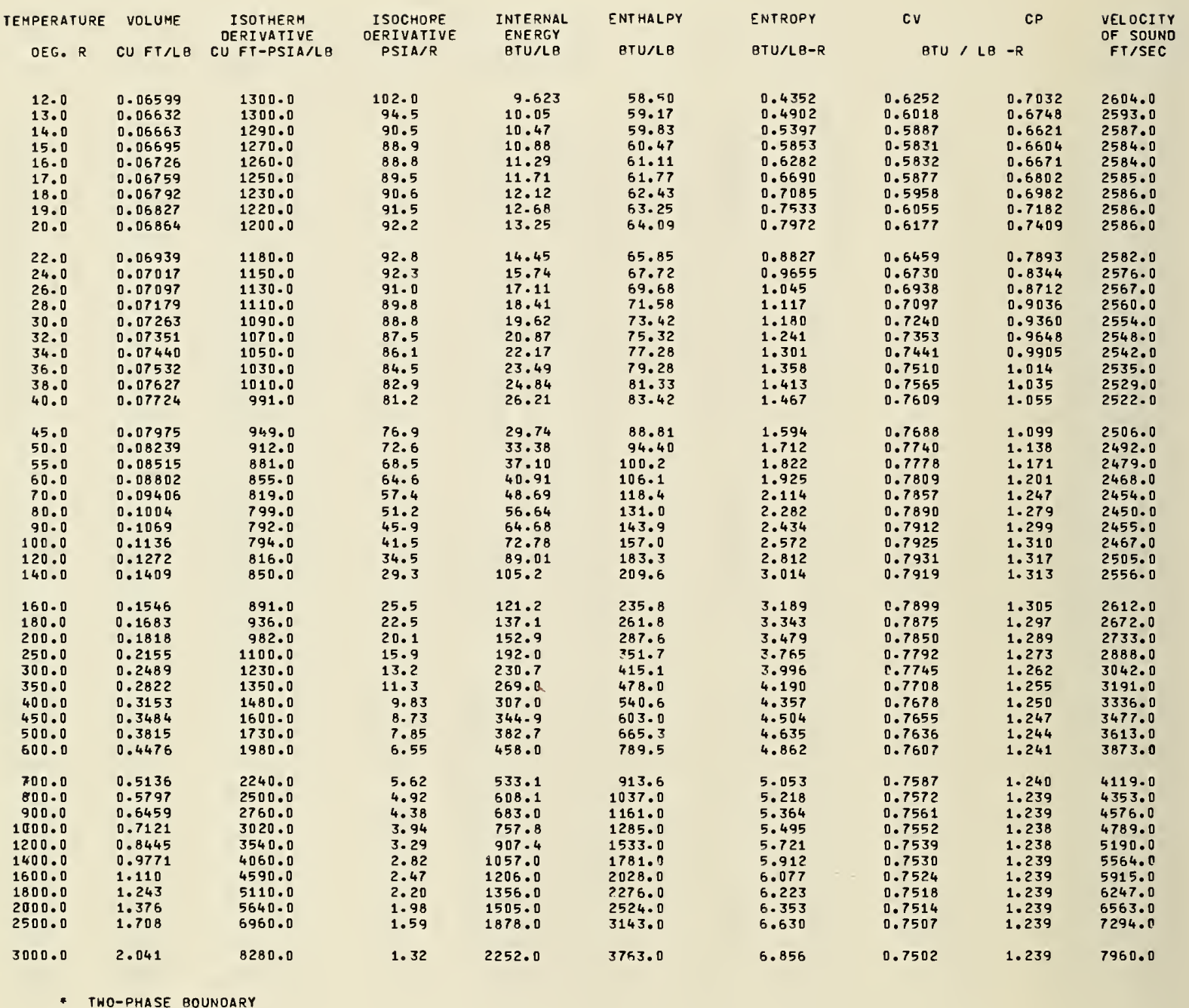


4000 PSIA ISOBAR

TEMPERATURE DENSITY V(OH/OV) ${ }_{P} \quad V(D P / O U)_{V}-V(O P / O V)_{T}$ DEg. $R$ LB/CUFT BTU/LB PSIA-CU FT/BTU PSIA

\begin{tabular}{|c|c|c|}
\hline $\begin{array}{l}12.0 \\
13.0 \\
14.0 \\
15.0 \\
16.0 \\
17.0 \\
18.0 \\
19.0 \\
20.0\end{array}$ & $\begin{array}{l}15.15 \\
15.08 \\
15.01 \\
14.94 \\
14.87 \\
14.80 \\
14.72 \\
14.65 \\
14.57\end{array}$ & $\begin{array}{l}135.0 \\
139.0 \\
141.0 \\
141.0 \\
141.0 \\
140.0 \\
140.0 \\
140.0 \\
141.0\end{array}$ \\
\hline $\begin{array}{l}22.0 \\
24.0 \\
26.0 \\
28.0 \\
30.0 \\
32.0 \\
34.0 \\
36.0 \\
38.0 \\
40.0\end{array}$ & $\begin{array}{l}14.41 \\
14.25 \\
14.09 \\
13.93 \\
13.77 \\
13.60 \\
13.44 \\
13.28 \\
13.11 \\
12.95\end{array}$ & $\begin{array}{l}144.0 \\
149.0 \\
153.0 \\
156.0 \\
158.0 \\
160.0 \\
162.0 \\
164.0 \\
165.0 \\
167.0\end{array}$ \\
\hline $\begin{array}{r}45.0 \\
50.0 \\
55.0 \\
60.0 \\
70.0 \\
80.0 \\
90.0 \\
100.0 \\
120.0 \\
140.0\end{array}$ & $\begin{array}{c}12.54 \\
12.14 \\
11.74 \\
11.36 \\
10.63 \\
9.96 \\
9.350 \\
8.809 \\
7.859 \\
7.095\end{array}$ & $\begin{array}{l}170.0 \\
173.0 \\
177.0 \\
181.0 \\
189.0 \\
199.0 \\
209.0 \\
221.0 \\
245.0 \\
270.0\end{array}$ \\
\hline $\begin{array}{l}160.0 \\
180.0 \\
200.0 \\
250.0 \\
300.0 \\
350.0 \\
400.0 \\
450.0 \\
500.0 \\
600.0\end{array}$ & $\begin{array}{l}6.467 \\
5.943 \\
5.499 \\
4.649 \\
4.017 \\
3.544 \\
3.171 \\
2.870 \\
2.621 \\
2.234\end{array}$ & $\begin{array}{l}295.0 \\
321.0 \\
346.0 \\
409.0 \\
472.0 \\
534.0 \\
595.0 \\
657.0 \\
718.0 \\
841.0\end{array}$ \\
\hline $\begin{array}{l}700.0 \\
800.0 \\
900.0 \\
1000.0 \\
200.0 \\
4000.0 \\
4800.0 \\
800.0 \\
8000.0 \\
500.0\end{array}$ & $\begin{array}{l}1.947 \\
1.725 \\
1.548 \\
1.404 \\
1.184 \\
1.023 \\
0.9010 \\
0.8047 \\
0.7270 \\
0.5855\end{array}$ & $\begin{array}{r}964.0 \\
1090.0 \\
1210.0 \\
1330.0 \\
1580.0 \\
1820.0 \\
2070.0 \\
2320.0 \\
2560.0 \\
3189.0\end{array}$ \\
\hline 000.0 & 0.4900 & 380000 \\
\hline
\end{tabular}

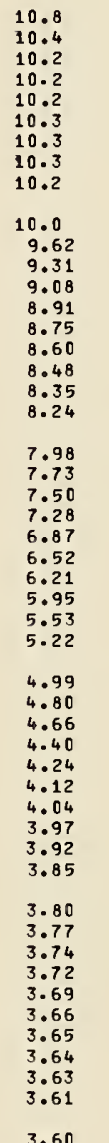

COV/OTISV THERMAL VISCOSITY 1/OEG. R BTU/FT-HR-R LB/FT-SEC $\times \quad 10 E+6$

$\begin{array}{ll}0.00519 & 0.0464 \\ 0.00484 & 0.0485 \\ 0.00469 & 0.0503 \\ 0.00468 & 0.0520 \\ 0.00474 & 0.0534 \\ 0.00486 & 0.0546 \\ 0.00499 & 0.0556 \\ 0.00513 & 0.0564 \\ 0.00526 & 0.0571 \\ 0.00546 & 0.0581 \\ 0.00561 & 0.0588 \\ 0.00570 & 0.0591 \\ 0.00580 & 0.0593 \\ 0.00592 & 0.0593 \\ 0.00602 & 0.0592 \\ 0.00611 & 0.0590 \\ 0.00619 & 0.0588 \\ 0.00626 & 0.0585 \\ 0.00633 & 0.0582 \\ & \\ 0.00647 & 0.0574 \\ 0.00656 & 0.0566 \\ 0.00662 & 0.0559 \\ 0.00665 & 0.0552 \\ 0.00659 & 0.0543 \\ 0.00643 & 0.0538 \\ 0.00620 & 0.0535 \\ 0.00593 & 0.0537 \\ 0.00538 & 0.0546 \\ 0.00486 & 0.0561 \\ 0.00442 & 0.0579 \\ 0.00404 & 0.0599 \\ 0.00372 & 0.0621 \\ 0.00311 & 0.0676 \\ 0.00268 & 0.0731 \\ 0.00235 & 0.0785 \\ 0.00210 & 0.0837 \\ 0.00190 & 0.0887 \\ 0.00173 & 0.0935 \\ 0.00148 & 0.103 \\ & \end{array}$

35.2

30.1

26.3

21.0
19.1

19.1
17.5

18100.0

17800.0

17500.0

17000.0

16500.0

15500.0

15000.0

14500.0

14100.0

13600.0

13200.0

11900.0

11100.0

10300.0

8710.0

7960.0

7410.0

6990.0

6410.0

5760.0

5560.0

5400.0

5110.0

4920.0

45800

4600.0

4530.0

4360.0

4310.0

4270.0

240.0

$416 \mathrm{C} .0$

4130.0

420.0

4100.0
4070.0

4060.0
16.2
15.1

13.3

12.0

11.0
10.2

9.64

9.64
9.16
8.77

8.45
8.18

8.18
7.97

7.57

7.32
7.16

7.16

7.06
6.99

7.01

7.09

7.20

7.48

7.79

8.12

8.46
8.67

9.52

10.3

11.1

11.9
12.7

13.4

0.00129

0.00114

0.00102

0.000785

0.000598

0.000534

0.000483

0.103

0.113

0.123
0.132

0.132
0.142

0.160

0.177

0.194

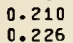

0.264

0.000326
THERMAL OIELECTRIC IFFUSIVITY CONSTANT SQ FT/HR

\begin{tabular}{|c|c|c|}
\hline $\begin{array}{l}0.00436 \\
0.00477 \\
0.00507 \\
0.00527 \\
0.00538 \\
0.00542 \\
0.00541 \\
0.00537 \\
0.00529\end{array}$ & $\begin{array}{l}1.01802 \\
1.01812 \\
1.01823 \\
1.01832 \\
1.01842 \\
1.01851 \\
1.01861 \\
1.01871 \\
1.01880\end{array}$ & $\begin{array}{l}1.92 \\
1.51 \\
1.25 \\
1.07 \\
0.944 \\
0.856 \\
0.791 \\
0.741 \\
0.703\end{array}$ \\
\hline $\begin{array}{l}0.00511 \\
0.00494 \\
0.00482 \\
0.00471 \\
0.00460 \\
0.00451 \\
0.00443 \\
0.00437 \\
0.00431 \\
0.00426\end{array}$ & $\begin{array}{l}1.01900 \\
1.01918 \\
1.01936 \\
1.01953 \\
1.01969 \\
1.01984 \\
1.01999 \\
1.02012 \\
1.02025 \\
1.02036\end{array}$ & $\begin{array}{l}0.650 \\
0.613 \\
0.584 \\
0.562 \\
0.548 \\
0.537 \\
0.530 \\
0.524 \\
0.521 \\
0.520\end{array}$ \\
\hline $\begin{array}{l}0.00416 \\
0.00410 \\
0.00406 \\
0.00405 \\
0.00410 \\
0.00422 \\
0.00441 \\
0.00466 \\
0.00528 \\
0.00602\end{array}$ & $\begin{array}{l}1.02061 \\
1.02080 \\
1.02092 \\
1.02100 \\
1.02099 \\
1.02082 \\
1.02053 \\
1.02016 \\
1.01928 \\
1.01833\end{array}$ & $\begin{array}{l}0.522 \\
0.529 \\
0.540 \\
0.552 \\
0.578 \\
0.500 \\
0.619 \\
0.632 \\
0.649 \\
0.657\end{array}$ \\
\hline $\begin{array}{l}0.00686 \\
0.00777 \\
0.00875 \\
0.0114 \\
0.0144 \\
0.0177 \\
0.0211 \\
0.0248 \\
0.0287 \\
0.0372\end{array}$ & $\begin{array}{l}1.01741 \\
1.01653 \\
1.01571 \\
1.01393 \\
1.01248 \\
1.01130 \\
1.01031 \\
1.00947 \\
1.00876 \\
1.00761\end{array}$ & $\begin{array}{l}0.659 \\
0.659 \\
0.649 \\
0.646 \\
0.643 \\
0.641 \\
0.640 \\
0.640 \\
0.641 \\
0.645\end{array}$ \\
\hline $\begin{array}{l}0.0469 \\
0.0575 \\
0.0690 \\
0.0814 \\
0.109 \\
0.140 \\
0.174 \\
0.211 \\
0.251 \\
0.364\end{array}$ & $\begin{array}{l}1.00673 \\
1.00602 \\
1.00545 \\
1.00498 \\
1.00424 \\
1.00369 \\
1.00327 \\
1.00293 \\
1.00266 \\
1.00216\end{array}$ & $\begin{array}{l}0.649 \\
0.652 \\
0.654 \\
0.656 \\
0.658 \\
0.660 \\
0.661 \\
0.661 \\
0.662 \\
0.663\end{array}$ \\
\hline
\end{tabular}

0.495

1.00181
PRANOTL NUMBER

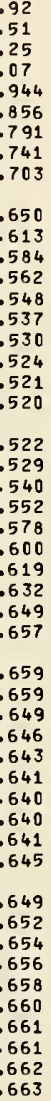

0.663

- Tho-PHASE boundary 


\begin{tabular}{|c|c|c|c|c|c|c|c|c|c|}
\hline $\begin{array}{c}\text { TEMPERATURE } \\
\text { OEG. R }\end{array}$ & $\begin{array}{l}\text { VOLUME } \\
\text { CU FT/LB }\end{array}$ & $\begin{array}{c}\text { I SOTHERM } \\
\text { OERIVATIVE } \\
\text { CU FT-PSIA L B }\end{array}$ & $\begin{array}{l}\text { ISOCHORE } \\
\text { OERIVATIVE } \\
\text { PSIA/R }\end{array}$ & $\begin{array}{l}\text { INTERNAL } \\
\text { ENERGY } \\
\text { BTU/LB }\end{array}$ & $\begin{array}{l}\text { ENTHALPY } \\
\text { ETU/LB }\end{array}$ & $\begin{array}{l}\text { ENTROPY } \\
\text { QTU/LA-R }\end{array}$ & $\begin{array}{l}\text { CV } \\
\text { RTU / LB }\end{array}$ & $\begin{array}{l}C P \\
-R\end{array}$ & $\begin{array}{l}\text { VEL OCITY } \\
\text { OF SOUNO } \\
\text { FT/SEC }\end{array}$ \\
\hline $\begin{array}{l}12.0 \\
13.0 \\
14.0 \\
15.0 \\
16.0 \\
17.0 \\
18.0 \\
19.0 \\
20.0 \\
22.0\end{array}$ & $\begin{array}{l}0.06442 \\
0.06473 \\
0.06502 \\
0.06530 \\
0.06559 \\
0.06588 \\
0.06618 \\
0.06649 \\
0.06682 \\
0.06750\end{array}$ & $\begin{array}{l}1410.0 \\
1410.0 \\
1400.0 \\
1390.0 \\
1380.0 \\
1360.0 \\
1350.0 \\
1330.0 \\
1320.0 \\
1300.0\end{array}$ & $\begin{array}{r}110.0 \\
100.0 \\
94.3 \\
92.1 \\
91.7 \\
92.4 \\
93.6 \\
94.9 \\
96.0 \\
97.2\end{array}$ & $\begin{array}{l}10.48 \\
10.93 \\
11.35 \\
11.76 \\
12.16 \\
12.55 \\
12.96 \\
13.49 \\
14.04 \\
15.20\end{array}$ & $\begin{array}{l}64.16 \\
64.87 \\
65.53 \\
65.17 \\
65.81 \\
67.45 \\
68.10 \\
68.90 \\
69.72 \\
71.45\end{array}$ & $\begin{array}{l}0.4043 \\
0.4616 \\
0.5120 \\
0.5576 \\
0.6000 \\
0.6400 \\
0.6785 \\
0.7223 \\
0.7653 \\
0.8492\end{array}$ & $\begin{array}{l}0.6617 \\
0.6226 \\
0.5982 \\
0.5845 \\
0.5788 \\
0.5793 \\
0.5849 \\
0.5933 \\
0.6054 \\
0.6350\end{array}$ & $\begin{array}{l}0.7410 \\
0.6938 \\
0.6679 \\
0.6567 \\
0.6566 \\
0.6649 \\
0.6797 \\
0.6982 \\
0.7207 \\
0.7703\end{array}$ & $\begin{array}{l}2709.0 \\
2698.0 \\
2692.0 \\
2689.0 \\
2690.0 \\
2692.0 \\
2695.0 \\
2697.0 \\
2699.0 \\
2698.0\end{array}$ \\
\hline $\begin{array}{l}24.0 \\
26.0 \\
28.0 \\
30.0 \\
32.0 \\
34.0 \\
36.0 \\
38.0 \\
40.0\end{array}$ & $\begin{array}{l}0.06820 \\
0.06892 \\
0.06965 \\
0.07041 \\
0.07119 \\
0.07199 \\
0.07280 \\
0.07364 \\
0.07450\end{array}$ & $\begin{array}{l}1270.0 \\
1250.0 \\
1230.0 \\
1210.0 \\
1190.0 \\
1170.0 \\
1150.0 \\
1130.0 \\
1110.0\end{array}$ & $\begin{array}{l}97.0 \\
95.8 \\
94.7 \\
93.7 \\
92.6 \\
91.2 \\
89.7 \\
88.1 \\
86.5\end{array}$ & $\begin{array}{l}16.46 \\
17.80 \\
19.06 \\
20.24 \\
21.46 \\
22.72 \\
24.01 \\
25.32 \\
26.66\end{array}$ & $\begin{array}{l}73.29 \\
75.23 \\
77.10 \\
78.91 \\
80.78 \\
82.70 \\
84.67 \\
86.68 \\
88.74\end{array}$ & $\begin{array}{l}0.9308 \\
1.010 \\
1.080 \\
1.142 \\
1.203 \\
1.261 \\
1.317 \\
1.372 \\
1.424\end{array}$ & $\begin{array}{l}0.6645 \\
0.6873 \\
0.7043 \\
0.7197 \\
0.7319 \\
0.7414 \\
0.7490 \\
0.7550 \\
0.7598\end{array}$ & $\begin{array}{l}0.8172 \\
0.8550 \\
0.8875 \\
0.9198 \\
0.9482 \\
0.9736 \\
0.996 \\
1.017 \\
1.037\end{array}$ & $\begin{array}{l}2693.0 \\
2686.0 \\
2680.0 \\
2676.0 \\
2671.0 \\
2665.0 \\
2661.0 \\
2656.0 \\
2650.0\end{array}$ \\
\hline $\begin{array}{r}45.0 \\
50.0 \\
55.0 \\
60.0 \\
70.0 \\
80.0 \\
90.0 \\
100.0 \\
120.0 \\
140.0\end{array}$ & $\begin{array}{l}0.07671 \\
0.07903 \\
0.08145 \\
0.08396 \\
0.08923 \\
0.09477 \\
0.1005 \\
0.1064 \\
0.1184 \\
0.1305\end{array}$ & $\begin{array}{r}1070.0 \\
1030.0 \\
995.0 \\
966.0 \\
922.0 \\
895.0 \\
881.0 \\
877.0 \\
891.0 \\
920.0\end{array}$ & $\begin{array}{l}82.3 \\
78.1 \\
74.0 \\
70.0 \\
62.7 \\
56.3 \\
50.8 \\
46.0 \\
38.4 \\
32.8\end{array}$ & $\begin{array}{c}30.11 \\
33.67 \\
37.33 \\
41.06 \\
48.73 \\
56.58 \\
64.56 \\
72.61 \\
88.80 \\
105.0\end{array}$ & $\begin{array}{c}94.03 \\
99.5 \\
105.2 \\
111.0 \\
123.1 \\
135.6 \\
148.3 \\
161.3 \\
187.5 \\
213.8\end{array}$ & $\begin{array}{l}1.549 \\
1.665 \\
1.773 \\
1.874 \\
2.060 \\
2.227 \\
2.377 \\
2.513 \\
2.752 \\
2.955\end{array}$ & $\begin{array}{l}0.7686 \\
0.7745 \\
0.7789 \\
0.7825 \\
0.7880 \\
0.7920 \\
0.7948 \\
0.7965 \\
0.7976 \\
0.7966\end{array}$ & $\begin{array}{l}1.080 \\
1.117 \\
1.151 \\
1.180 \\
1.229 \\
1.264 \\
1.288 \\
1.302 \\
1.314 \\
1.313\end{array}$ & $\begin{array}{l}2636.0 \\
2622.0 \\
2609.0 \\
2597.0 \\
2580.0 \\
2571.0 \\
2571.0 \\
2578.0 \\
2607.0 \\
2650.0\end{array}$ \\
\hline $\begin{array}{l}160.0 \\
180.0 \\
200.0 \\
250.0 \\
300.0 \\
350.0 \\
400.0 \\
450.0 \\
500.0 \\
600.0\end{array}$ & $\begin{array}{l}0.1427 \\
0.1548 \\
0.1669 \\
0.1968 \\
0.2265 \\
0.2560 \\
0.2854 \\
0.3147 \\
0.3441 \\
0.4027\end{array}$ & $\begin{array}{r}957.0 \\
1000.0 \\
1040.0 \\
1160.0 \\
1280.0 \\
1400.0 \\
1530.0 \\
1650.0 \\
1780.0 \\
2030.0\end{array}$ & $\begin{array}{l}28.5 \\
25.2 \\
22.5 \\
17.8 \\
14.8 \\
12.5 \\
11.0 \\
9.79 \\
8.81 \\
7.35\end{array}$ & $\begin{array}{l}121.1 \\
137.0 \\
152.9 \\
192.1 \\
230.9 \\
269.3 \\
307.5 \\
345.4 \\
363.3 \\
458.7\end{array}$ & $\begin{array}{l}240.0 \\
266.0 \\
291.9 \\
356.1 \\
419.6 \\
482.6 \\
545.3 \\
607.7 \\
670.0 \\
794.2\end{array}$ & $\begin{array}{l}3.130 \\
3.283 \\
3.420 \\
3.706 \\
3.938 \\
4.132 \\
4.299 \\
4.446 \\
4.578 \\
4.804\end{array}$ & $\begin{array}{l}0.7947 \\
0.7922 \\
0.7897 \\
0.7835 \\
0.7783 \\
0.7742 \\
0.7709 \\
0.7683 \\
0.7661 \\
0.7629\end{array}$ & $\begin{array}{l}1.307 \\
1.300 \\
1.292 \\
1.276 \\
1.264 \\
1.256 \\
1.251 \\
1.247 \\
1.244 \\
1.241\end{array}$ & $\begin{array}{l}2700.0 \\
2755.0 \\
2812.0 \\
2958.0 \\
3105.0 \\
3249.0 \\
3389.0 \\
3526.0 \\
3659.0 \\
3914.0\end{array}$ \\
\hline $\begin{array}{r}700.0 \\
800.0 \\
900.0 \\
1000.0 \\
1200.0 \\
1400.0 \\
1600.0 \\
1800.0 \\
2000.0 \\
2500.0\end{array}$ & $\begin{array}{l}0.4613 \\
0.5200 \\
0.5787 \\
0.6374 \\
0.7550 \\
0.8727 \\
0.9905 \\
1.108 \\
1.226 \\
1.522\end{array}$ & $\begin{array}{l}2290.0 \\
2540.0 \\
2800.0 \\
3060.0 \\
3580.0 \\
4100.0 \\
4620.0 \\
5150.0 \\
5670.0 \\
6990.0\end{array}$ & $\begin{array}{l}6.30 \\
5.52 \\
4.91 \\
4.43 \\
3.69 \\
3.17 \\
2.78 \\
2.47 \\
2.23 \\
1.78\end{array}$ & $\begin{array}{r}533.9 \\
608.9 \\
683.8 \\
758.7 \\
908.3 \\
1058.0 \\
1207.0 \\
1357.0 \\
1506.0 \\
1880.0\end{array}$ & $\begin{array}{r}918.3 \\
1042.0 \\
1166.0 \\
1290.0 \\
1537.0 \\
1785.0 \\
2033.0 \\
2280.0 \\
2528.0 \\
3148.0\end{array}$ & $\begin{array}{l}4.995 \\
5.161 \\
5.307 \\
5.437 \\
5.653 \\
5.854 \\
6.019 \\
6.165 \\
6.295 \\
6.572\end{array}$ & $\begin{array}{l}0.7606 \\
0.7589 \\
0.7576 \\
0.7566 \\
0.7551 \\
0.7541 \\
0.7533 \\
0.7528 \\
0.7523 \\
0.7515\end{array}$ & $\begin{array}{l}1.240 \\
1.239 \\
1.238 \\
1.238 \\
1.238 \\
1.238 \\
1.238 \\
1.239 \\
1.239 \\
1.239\end{array}$ & $\begin{array}{l}4155.0 \\
4386.0 \\
4606.0 \\
4816.0 \\
5214.0 \\
5585.0 \\
5934.0 \\
6264.0 \\
6578.0 \\
7306.0\end{array}$ \\
\hline 3000.0 & 1.817 & 8310.0 & 1.49 & 2253.0 & 3767.0 & 6.798 & 0.7509 & 1.239 & 7969.0 \\
\hline
\end{tabular}

- tWo-phase gounoarY 
4500 PSIA ISOBAR

\begin{tabular}{|c|c|c|c|c|c|c|c|c|c|c|}
\hline $\begin{array}{c}\text { TEMPERATURE } \\
\text { OEG. } R\end{array}$ & $\begin{array}{l}\text { E DENSITY } \\
\text { LB/CU FT }\end{array}$ & $\begin{array}{c}\text { V(OH/OV) } \\
\text { BTU/LB }\end{array}$ & $\begin{array}{l}V(D P / O U)_{V}- \\
\text { PSIA-CU FT/BTU }\end{array}$ & $\begin{array}{l}-V(O P / O V)_{T} T \\
U \text { PSIA }\end{array}$ & $\begin{array}{l}\text { COV/OT })_{\mathrm{P}}^{\prime V} \\
\text { I/DEG. R }\end{array}$ & $\begin{array}{c}\text { THERHAL } \\
\text { CONDUCTIVITY } \\
\text { BTU/FT-HR-R }\end{array}$ & $\begin{array}{l}\text { VISCOSITY } \\
\text { LB/F T-SEC } \\
\times \quad 10 E+6\end{array}$ & $\begin{array}{l}\text { THERMAL } \\
\text { OIFFUSIVITY } \\
\text { SQ FT/HR }\end{array}$ & $\begin{array}{l}\text { OIELECTRIC } \\
\text { CONSTANT }\end{array}$ & $\begin{array}{l}\text { PRANOTL } \\
\text { NUMBER }\end{array}$ \\
\hline $\begin{array}{l}12.0 \\
13.0 \\
14.0 \\
15.0 \\
16.0 \\
17.0 \\
18.0 \\
19.0 \\
20.0 \\
22.0\end{array}$ & $\begin{array}{l}15.52 \\
15.45 \\
15.38 \\
15.31 \\
15.25 \\
15.18 \\
15.11 \\
15.04 \\
14.97 \\
14.82\end{array}$ & $\begin{array}{l}148.0 \\
151.0 \\
153.0 \\
152.0 \\
150.0 \\
149.0 \\
148.0 \\
148.0 \\
148.0 \\
152.0\end{array}$ & $\begin{array}{l}10.7 \\
10.4 \\
10.3 \\
10.3 \\
10.4 \\
10.5 \\
10.6 \\
10.6 \\
10.6 \\
10.3\end{array}$ & $\begin{array}{l}22000.0 \\
21500.0 \\
21500.0 \\
21300.0 \\
21000.0 \\
20700.0 \\
20400.0 \\
20100.0 \\
19800.0 \\
19200.0\end{array}$ & $\begin{array}{l}0.00502 \\
0.00458 \\
0.00438 \\
0.00433 \\
0.00437 \\
0.00446 \\
0.00459 \\
0.00473 \\
0.00486 \\
0.00506\end{array}$ & $\begin{array}{l}0.0494 \\
0.0516 \\
0.0536 \\
0.0553 \\
0.0568 \\
0.0581 \\
0.0592 \\
0.0601 \\
0.0608 \\
0.0619\end{array}$ & $\begin{array}{l}44.6 \\
37.6 \\
32.4 \\
28.4 \\
25.3 \\
22.8 \\
20.8 \\
19.0 \\
17.6 \\
15.3\end{array}$ & $\begin{array}{l}0.00429 \\
0.00481 \\
0.00522 \\
0.00550 \\
0.00568 \\
0.00576 \\
0.00576 \\
0.00572 \\
0.00564 \\
0.00542\end{array}$ & $\begin{array}{l}1.01746 \\
1.01758 \\
1.01768 \\
1.01778 \\
1.01788 \\
1.01798 \\
1.01808 \\
1.01818 \\
1.01828 \\
1.01849\end{array}$ & $\begin{array}{l}2.41 \\
1.82 \\
1.45 \\
1.22 \\
1.05 \\
0.940 \\
0.858 \\
0.797 \\
0.751 \\
0.688\end{array}$ \\
\hline $\begin{array}{l}24.0 \\
26.0 \\
28.0 \\
30.0 \\
32.0 \\
34.0 \\
36.0 \\
38.0 \\
40.0\end{array}$ & $\begin{array}{l}14.66 \\
14.51 \\
14.36 \\
14.20 \\
14.05 \\
13.89 \\
13.74 \\
13.58 \\
13.42\end{array}$ & $\begin{array}{l}157.0 \\
162.0 \\
166.0 \\
169.0 \\
171.0 \\
173.0 \\
175.0 \\
177.0 \\
179.0\end{array}$ & $\begin{array}{c}10.0 \\
9.61 \\
9.36 \\
9.17 \\
9.00 \\
8.85 \\
8.72 \\
8.50 \\
8.48\end{array}$ & $\begin{array}{l}18700.0 \\
18200.0 \\
17700.0 \\
17200.0 \\
16700.0 \\
16200.0 \\
15800.0 \\
15300.0 \\
14900.0\end{array}$ & $\begin{array}{l}0.00520 \\
0.00528 \\
0.00536 \\
0.00546 \\
0.00554 \\
0.00562 \\
0.00568 \\
0.00575 \\
0.00580\end{array}$ & $\begin{array}{l}0.0626 \\
0.0629 \\
0.0631 \\
0.0631 \\
0.0630 \\
0.0628 \\
0.0625 \\
0.0622 \\
0.0618\end{array}$ & $\begin{array}{c}13.7 \\
12.5 \\
11.5 \\
10.7 \\
10.1 \\
9.65 \\
9.25 \\
8.92 \\
8.65\end{array}$ & $\begin{array}{l}0.00522 \\
0.00507 \\
0.00495 \\
0.00483 \\
0.00473 \\
0.00464 \\
0.00457 \\
0.00450 \\
0.00444\end{array}$ & $\begin{array}{l}1.01869 \\
1.01888 \\
1.01906 \\
1.01924 \\
1.01940 \\
1.01957 \\
1.01972 \\
1.01986 \\
1.02000\end{array}$ & $\begin{array}{l}0.644 \\
0.609 \\
0.582 \\
0.564 \\
0.550 \\
0.539 \\
0.531 \\
0.526 \\
0.522\end{array}$ \\
\hline $\begin{array}{r}45.0 \\
50.0 \\
55.0 \\
60.0 \\
70.0 \\
80.0 \\
90.0 \\
100.0 \\
120.0 \\
140.0\end{array}$ & $\begin{array}{c}13.04 \\
12.65 \\
12.28 \\
11.91 \\
11.21 \\
10.55 \\
9.949 \\
9.398 \\
8.445 \\
7.660\end{array}$ & $\begin{array}{l}183.0 \\
186.0 \\
190.0 \\
194.0 \\
202.0 \\
212.0 \\
222.0 \\
233.0 \\
257.0 \\
282.0\end{array}$ & $\begin{array}{l}8.21 \\
7.97 \\
7.72 \\
7.51 \\
7.10 \\
6.74 \\
6.42 \\
6.15 \\
5.70 \\
5.37\end{array}$ & $\begin{array}{r}13900.0 \\
13000.0 \\
12200.0 \\
11500.0 \\
10300.0 \\
9440.0 \\
8760.0 \\
8240.0 \\
7520.0 \\
7040.0\end{array}$ & $\begin{array}{l}\text { C.00591 } \\
C .00600 \\
0.00606 \\
0.00609 \\
c .00607 \\
0.00597 \\
0.00579 \\
0.00558 \\
0.00511 \\
r .00465\end{array}$ & $\begin{array}{l}0.0609 \\
0.0601 \\
0.0593 \\
0.0586 \\
0.0575 \\
0.0569 \\
0.0565 \\
0.0565 \\
0.0572 \\
0.0584\end{array}$ & $\begin{array}{l}8.15 \\
7.83 \\
7.61 \\
7.48 \\
7.35 \\
7.34 \\
7.39 \\
7.48 \\
7.73 \\
8.02\end{array}$ & $\begin{array}{l}0.00433 \\
0.00425 \\
0.00420 \\
0.00417 \\
0.00418 \\
0.00426 \\
0.00441 \\
0.00462 \\
0.00515 \\
0.00581\end{array}$ & $\begin{array}{l}1.02030 \\
1.02055 \\
1.02074 \\
1.02088 \\
1.02101 \\
1.02098 \\
1.02082 \\
1.02056 \\
1.01986 \\
1.01905\end{array}$ & $\begin{array}{l}0.520 \\
0.524 \\
0.532 \\
0.542 \\
0.566 \\
0.588 \\
0.506 \\
0.621 \\
0.640 \\
0.649\end{array}$ \\
\hline $\begin{array}{l}160.0 \\
180.0 \\
200.0 \\
250.0 \\
300.0 \\
350.0 \\
400.0 \\
450.0 \\
500.0 \\
600.0\end{array}$ & $\begin{array}{l}7.008 \\
6.460 \\
5.993 \\
5.082 \\
4.416 \\
3.907 \\
3.504 \\
3.177 \\
2.906 \\
2.483\end{array}$ & $\begin{array}{l}307.0 \\
333.0 \\
358.0 \\
421.0 \\
484.0 \\
546.0 \\
607.0 \\
669.0 \\
730.0 \\
853.0\end{array}$ & $\begin{array}{l}5.12 \\
4.92 \\
4.76 \\
4.48 \\
4.30 \\
4.17 \\
4.08 \\
4.01 \\
3.96 \\
3.88\end{array}$ & $\begin{array}{l}6710.0 \\
6450.0 \\
6250.0 \\
5900.0 \\
5660.0 \\
5490.0 \\
5350.0 \\
5250.0 \\
5170.0 \\
5050.0\end{array}$ & $\begin{array}{l}0.00425 \\
0.00390 \\
0.00361 \\
0.00303 \\
0.00261 \\
0.00230 \\
0.00206 \\
0.00186 \\
0.00170 \\
0.00146\end{array}$ & $\begin{array}{l}0.0601 \\
0.0620 \\
0.0640 \\
0.0693 \\
0.0747 \\
0.0799 \\
0.0850 \\
0.0899 \\
0.0946 \\
0.104\end{array}$ & $\begin{array}{l}8.33 \\
8.66 \\
8.85 \\
9.68 \\
10.5 \\
11.3 \\
12.0 \\
12.7 \\
13.5 \\
15.0\end{array}$ & $\begin{array}{l}0.00656 \\
0.00738 \\
0.00826 \\
0.0107 \\
0.0134 \\
0.0163 \\
0.0194 \\
0.0227 \\
0.0261 \\
0.0338\end{array}$ & $\begin{array}{l}1.01821 \\
1.01739 \\
1.01662 \\
1.01488 \\
1.01343 \\
1.01221 \\
1.01119 \\
1.01032 \\
1.00958 \\
1.00836\end{array}$ & $\begin{array}{l}0.653 \\
0.653 \\
0.644 \\
0.641 \\
0.639 \\
0.637 \\
0.636 \\
0.637 \\
0.637 \\
0.642\end{array}$ \\
\hline $\begin{array}{r}700.0 \\
800.0 \\
900.0 \\
1000.0 \\
1200.0 \\
1400.0 \\
1600.0 \\
1800.0 \\
2000.0 \\
2500.0\end{array}$ & $\begin{array}{l}2.168 \\
1.923 \\
1.728 \\
1.569 \\
1.325 \\
1.146 \\
1.010 \\
0.9022 \\
0.8154 \\
0.6572\end{array}$ & $\begin{array}{r}975.0 \\
1100.0 \\
1220.0 \\
1340.0 \\
1590.0 \\
1830.0 \\
2080.0 \\
2330.0 \\
2570.0 \\
3190.0\end{array}$ & $\begin{array}{l}3.62 \\
3.78 \\
3.75 \\
3.73 \\
3.69 \\
3.67 \\
3.65 \\
3.64 \\
3.63 \\
3.61\end{array}$ & $\begin{array}{l}4960.0 \\
4890.0 \\
4840.0 \\
4800.0 \\
4740.0 \\
4700.0 \\
4670.0 \\
4640.0 \\
4630.0 \\
4590.0\end{array}$ & $\begin{array}{l}0.00127 \\
0.00113 \\
0.00101 \\
0.000922 \\
0.000779 \\
0.000675 \\
0.000595 \\
0.000532 \\
0.000481 \\
0.000388\end{array}$ & $\begin{array}{l}0.114 \\
0.123 \\
0.133 \\
0.142 \\
0.160 \\
0.178 \\
0.194 \\
0.211 \\
0.227 \\
0.265\end{array}$ & $\begin{array}{l}16.5 \\
18.0 \\
19.5 \\
20.9 \\
23.6 \\
26.2 \\
28.8 \\
31.2 \\
33.6 \\
39.3\end{array}$ & $\begin{array}{l}0.0424 \\
0.0518 \\
0.0621 \\
0.0732 \\
0.0977 \\
0.125 \\
0.155 \\
0.189 \\
0.224 \\
0.325\end{array}$ & $\begin{array}{l}1.00741 \\
1.00665 \\
1.00603 \\
1.00552 \\
1.00471 \\
1.00411 \\
1.00365 \\
1.00327 \\
1.00297 \\
1.00241\end{array}$ & $\begin{array}{l}0.647 \\
0.650 \\
0.652 \\
0.654 \\
0.657 \\
0.659 \\
0.660 \\
0.661 \\
0.661 \\
0.662\end{array}$ \\
\hline 3000.0 & 0.5503 & 3810.0 & 3.60 & 4570.0 & 0.000325 & 0.301 & 44.7 & 0.441 & 1.00203 & 0.663 \\
\hline
\end{tabular}

* tho-pHase boundarY 
5000 PSIA ISOBAR

THERMOOYNAMIC PROPERTIES OF HELIUM 4

\begin{tabular}{|c|c|c|c|c|c|c|c|c|c|}
\hline $\begin{array}{c}\text { TEMPERATURE } \\
\text { OEG. R }\end{array}$ & $\begin{array}{l}\text { VOL UME } \\
\text { CU FT/LB }\end{array}$ & $\begin{array}{c}\text { ISOTHERM } \\
\text { OERIVATIVE } \\
\text { CU FT-PSIA/LB }\end{array}$ & $\begin{array}{l}\text { ISOCHORE } \\
\text { OERIVATIVE } \\
\text { PSIA/R }\end{array}$ & $\begin{array}{l}\text { INTERNAL } \\
\text { ENERGY } \\
\text { BTU/LA }\end{array}$ & $\begin{array}{l}\text { ENT HALPY } \\
\text { BTU/LB }\end{array}$ & $\begin{array}{l}\text { ENTROPY } \\
\text { BTU/LP R }\end{array}$ & $\begin{array}{l}\text { CV } \\
\text { RTU , LB }\end{array}$ & $\begin{array}{l}C P \\
-R\end{array}$ & $\begin{array}{l}\text { VEL OCITY } \\
\text { OF SOUNO } \\
\text { FT / SEC }\end{array}$ \\
\hline $\begin{array}{l}13.0 \\
14.0 \\
15.0 \\
16.0 \\
17.0 \\
18.0 \\
19.0 \\
20.0 \\
22.0 \\
24.0\end{array}$ & $\begin{array}{l}0.06333 \\
0.06360 \\
0.06386 \\
0.06412 \\
0.06438 \\
0.06465 \\
0.06494 \\
0.06523 \\
0.06585 \\
0.06650\end{array}$ & $\begin{array}{l}1520.0 \\
1510.0 \\
1500.0 \\
1490.0 \\
1480.0 \\
1460.0 \\
1450.0 \\
1430.0 \\
1410.0 \\
1390.0\end{array}$ & $\begin{array}{r}105.0 \\
98.2 \\
95.1 \\
94.3 \\
95.0 \\
96.4 \\
98.1 \\
100.0 \\
101.0 \\
102.0\end{array}$ & $\begin{array}{l}11.81 \\
12.24 \\
12.65 \\
13.04 \\
13.42 \\
13.81 \\
14.32 \\
14.86 \\
15.98 \\
17.20\end{array}$ & $\begin{array}{l}70.45 \\
71.13 \\
71.77 \\
72.41 \\
73.03 \\
73.67 \\
74.45 \\
75.25 \\
76.95 \\
78.77\end{array}$ & $\begin{array}{l}0.4349 \\
0.4865 \\
0.5324 \\
0.5745 \\
0.6139 \\
0.6515 \\
0.6944 \\
0.7364 \\
0.8188 \\
0.8994\end{array}$ & $\begin{array}{l}0.6474 \\
0.5105 \\
0.5877 \\
0.5756 \\
0.5717 \\
0.5743 \\
0.5813 \\
0.5931 \\
0.6243 \\
0.6561\end{array}$ & $\begin{array}{l}0.7177 \\
0.6773 \\
0.6559 \\
0.6484 \\
0.6515 \\
0.6629 \\
0.6799 \\
0.7021 \\
0.7532 \\
0.8022\end{array}$ & $\begin{array}{l}2795.0 \\
2788.0 \\
2786.0 \\
2787.0 \\
2791.0 \\
2796.0 \\
2800.0 \\
2804.0 \\
2806.0 \\
2803.0\end{array}$ \\
\hline $\begin{array}{l}26.0 \\
28.0 \\
30.0 \\
32.0 \\
34.0 \\
36.0 \\
38.0 \\
40.0\end{array}$ & $\begin{array}{l}0.06715 \\
0.06782 \\
0.06850 \\
0.06921 \\
0.06993 \\
0.07067 \\
0.07143 \\
0.07220\end{array}$ & $\begin{array}{l}1370.0 \\
1350.0 \\
1330.0 \\
1310.0 \\
1290.0 \\
1270.0 \\
1250.0 \\
1230.0\end{array}$ & $\begin{array}{r}100.0 \\
99.4 \\
98.5 \\
97.4 \\
96.1 \\
94.6 \\
93.1 \\
91.5\end{array}$ & $\begin{array}{l}18.51 \\
19.75 \\
20.90 \\
22.08 \\
23.31 \\
24.57 \\
25.86 \\
27.17\end{array}$ & $\begin{array}{l}80.69 \\
82.54 \\
84.32 \\
86.16 \\
88.06 \\
90.00 \\
91.99 \\
94.01\end{array}$ & $\begin{array}{l}0.9777 \\
1.047 \\
1.109 \\
1.168 \\
1.225 \\
1.281 \\
1.335 \\
1.387\end{array}$ & $\begin{array}{l}0.6811 \\
0.6992 \\
0.7155 \\
0.7285 \\
0.7387 \\
0.7468 \\
0.7533 \\
0.7586\end{array}$ & $\begin{array}{l}0.8414 \\
0.8741 \\
0.9063 \\
0.9346 \\
0.9597 \\
0.9823 \\
1.003 \\
1.022\end{array}$ & $\begin{array}{l}2797.0 \\
2792.0 \\
2789.0 \\
2786.0 \\
2782.0 \\
2778.0 \\
2773.0 \\
2768.0\end{array}$ \\
\hline $\begin{array}{r}45.0 \\
50.0 \\
55.0 \\
60.0 \\
70.0 \\
80.0 \\
90.0 \\
100.0 \\
120.0 \\
140.0\end{array}$ & $\begin{array}{l}0.07418 \\
0.07625 \\
0.07841 \\
0.08064 \\
0.08532 \\
0.09023 \\
0.09534 \\
0.1006 \\
0.1113 \\
0.1222\end{array}$ & $\begin{array}{r}1180.0 \\
1140.0 \\
1110.0 \\
1080.0 \\
1030.0 \\
992.0 \\
971.0 \\
962.0 \\
967.0 \\
990.0\end{array}$ & $\begin{array}{l}87.4 \\
83.2 \\
79.1 \\
75.1 \\
67.7 \\
61.1 \\
55.4 \\
50.4 \\
42.3 \\
36.2\end{array}$ & $\begin{array}{c}30.55 \\
34.04 \\
37.63 \\
41.30 \\
48.86 \\
56.62 \\
64.53 \\
72.53 \\
88.68 \\
104.9\end{array}$ & $\begin{array}{l}99.23 \\
104.6 \\
110.2 \\
116.0 \\
127.9 \\
140.2 \\
152.8 \\
165.7 \\
191.7 \\
218.0\end{array}$ & $\begin{array}{l}1.509 \\
1.623 \\
1.730 \\
1.830 \\
2.013 \\
2.177 \\
2.326 \\
2.461 \\
2.699 \\
2.901\end{array}$ & $\begin{array}{l}0.7682 \\
0.7748 \\
0.7798 \\
0.7838 \\
0.7902 \\
0.7948 \\
0.7981 \\
0.8002 \\
0.8019 \\
0.8012\end{array}$ & $\begin{array}{l}1.064 \\
1.101 \\
1.133 \\
1.163 \\
1.212 \\
1.249 \\
1.276 \\
1.294 \\
1.311 \\
1.313\end{array}$ & $\begin{array}{l}2756.0 \\
2743.0 \\
2731.0 \\
2719.0 \\
2699.0 \\
2687.0 \\
2682.0 \\
2684.0 \\
2705.0 \\
2741.0\end{array}$ \\
\hline $\begin{array}{l}160.0 \\
180.0 \\
200.0 \\
250.0 \\
300.0 \\
350.0 \\
400.0 \\
450.0 \\
500.0 \\
600.0\end{array}$ & $\begin{array}{l}0.1331 \\
0.1440 \\
0.1548 \\
0.1817 \\
0.2084 \\
0.2350 \\
0.2614 \\
0.2878 \\
0.3141 \\
0.3668\end{array}$ & $\begin{array}{l}1020.0 \\
1060.0 \\
1110.0 \\
1220.0 \\
1340.0 \\
1460.0 \\
1580.0 \\
1700.0 \\
1830.0 \\
2080.0\end{array}$ & $\begin{array}{l}31.5 \\
27.9 \\
25.0 \\
19.8 \\
16.4 \\
14.0 \\
12.2 \\
10.8 \\
9.76 \\
8.14\end{array}$ & $\begin{array}{l}121.0 \\
137.0 \\
152.9 \\
192.3 \\
231.1 \\
269.7 \\
307.9 \\
346.0 \\
383.9 \\
459.4\end{array}$ & $\begin{array}{l}244.2 \\
270.3 \\
296.3 \\
360.5 \\
424.1 \\
487.2 \\
549.9 \\
612.4 \\
674.7 \\
799.0\end{array}$ & $\begin{array}{l}3.076 \\
3.230 \\
3.367 \\
3.654 \\
3.886 \\
4.080 \\
4.248 \\
4.395 \\
4.526 \\
4.753\end{array}$ & $\begin{array}{l}0.7994 \\
0.7969 \\
0.7943 \\
0.7877 \\
0.7822 \\
0.7776 \\
0.7740 \\
0.7711 \\
0.7687 \\
0.7650\end{array}$ & $\begin{array}{l}1.308 \\
1.302 \\
1.294 \\
1.278 \\
1.266 \\
1.257 \\
1.252 \\
1.248 \\
1.245 \\
1.241\end{array}$ & $\begin{array}{l}2786.0 \\
2836.0 \\
2889.0 \\
3026.0 \\
3166.0 \\
3305.0 \\
3441.0 \\
3574.0 \\
3703.0 \\
3953.0\end{array}$ \\
\hline $\begin{array}{r}700.0 \\
800.0 \\
900.0 \\
1000.0 \\
1200.0 \\
1400.0 \\
1600.0 \\
1800.0 \\
2000.0 \\
2500.0\end{array}$ & $\begin{array}{l}0.4194 \\
0.4721 \\
0.5249 \\
0.5776 \\
0.6833 \\
0.7891 \\
0.8950 \\
1.001 \\
1.107 \\
1.373\end{array}$ & $\begin{array}{l}2330.0 \\
2590.0 \\
2840.0 \\
3100.0 \\
3620.0 \\
4140.0 \\
4660.0 \\
5180.0 \\
5710.0 \\
7020.0\end{array}$ & $\begin{array}{l}6.98 \\
6.12 \\
5.45 \\
4.91 \\
4.10 \\
3.52 \\
3.08 \\
2.74 \\
2.47 \\
1.98\end{array}$ & $\begin{array}{r}534.6 \\
609.7 \\
684.7 \\
759.6 \\
909.3 \\
1059.0 \\
1208.0 \\
1358.0 \\
1507.0 \\
1881.0\end{array}$ & $\begin{array}{r}923.0 \\
1047.0 \\
1171.0 \\
1294.0 \\
1542.0 \\
1790.0 \\
2037.0 \\
2285.0 \\
2532.0 \\
3152.0\end{array}$ & $\begin{array}{l}4.944 \\
5.109 \\
5.255 \\
5.386 \\
5.611 \\
5.802 \\
5.967 \\
6.113 \\
6.244 \\
6.520\end{array}$ & $\begin{array}{l}0.7625 \\
0.7606 \\
0.7591 \\
0.7580 \\
0.7563 \\
0.7552 \\
0.7543 \\
0.7537 \\
0.7532 \\
0.7522\end{array}$ & $\begin{array}{l}1.239 \\
1.238 \\
1.238 \\
1.238 \\
1.238 \\
1.238 \\
1.238 \\
1.238 \\
1.238 \\
1.239\end{array}$ & $\begin{array}{l}4191.0 \\
4418.0 \\
4635.0 \\
4843.0 \\
5237.0 \\
5605.0 \\
5952.0 \\
6280.0 \\
6592.0 \\
7317.0\end{array}$ \\
\hline 3000.0 & 1.638 & 8330.0 & 1.65 & 2254.0 & 3771.0 & 6.746 & 0.7516 & 1.239 & 7978.0 \\
\hline
\end{tabular}


5000 PSIA ISOBAR

\begin{tabular}{|c|c|c|c|c|c|c|c|c|c|c|}
\hline $\begin{array}{c}\text { TEMPERATURE } \\
\text { DEG. R }\end{array}$ & E DENSITY & $\begin{array}{c}V(D H / D V)_{P} \\
\text { BTU/LB }\end{array}$ & $\begin{array}{l}V(D P / D U)_{V}-V \\
\text { PSIA-CU FT/BTU }\end{array}$ & $\begin{array}{l}-V(D P / O V)_{T} \\
\text { PSIA }\end{array}$ & $\begin{array}{l}(D V / D T)_{\mathrm{P}} / V \\
1 / O E G . R\end{array}$ & $\begin{array}{c}\text { THERMAL } \\
\text { CDNDUCTIVITY } \\
\text { GTU/FT-HR-R }\end{array}$ & $\begin{array}{l}\text { VISCDSITY } \\
\text { LB/FT-SEC } \\
\times \quad 10 E+6\end{array}$ & $\begin{array}{l}\text { THERMAL } \\
\text { DIFFUSIVITY } \\
\text { SQ FT/HR }\end{array}$ & $\begin{array}{l}\text { DIELECTRIC } \\
\text { CDNSTANT }\end{array}$ & $\begin{array}{l}\text { PRANDTL } \\
\text { NUMBER }\end{array}$ \\
\hline $\begin{array}{l}13.0 \\
14.0 \\
15.0 \\
16.0 \\
17.0 \\
18.0 \\
19.0 \\
20.0 \\
22.0 \\
24.0\end{array}$ & $\begin{array}{l}15.79 \\
15.72 \\
15.66 \\
15.60 \\
15.53 \\
15.47 \\
15.40 \\
15.33 \\
15.19 \\
15.04\end{array}$ & $\begin{array}{l}164.0 \\
164.0 \\
162.0 \\
160.0 \\
157.0 \\
156.0 \\
155.0 \\
155.0 \\
159.0 \\
165.0\end{array}$ & $\begin{array}{l}10.3 \\
10.2 \\
10.3 \\
10.5 \\
10.7 \\
10.9 \\
11.0 \\
11.0 \\
10.7 \\
10.3\end{array}$ & $\begin{array}{l}24000.0 \\
23800.0 \\
23500.0 \\
23200 \cdot 0 \\
22900.0 \\
22600 \cdot 0 \\
22300.0 \\
22000.0 \\
21400.0 \\
20900.0\end{array}$ & $\begin{array}{l}0.00438 \\
0.00413 \\
0.00404 \\
0.00406 \\
0.00414 \\
0.00426 \\
0.00440 \\
0.00453 \\
0.00474 \\
0.00487\end{array}$ & $\begin{array}{l}0.0547 \\
0.0568 \\
0.0586 \\
0.0602 \\
0.0616 \\
0.0628 \\
0.0637 \\
0.0645 \\
0.0656 \\
0.0663\end{array}$ & $\begin{array}{l}46.6 \\
39.7 \\
34.4 \\
30.4 \\
27.1 \\
24.5 \\
22.3 \\
20.5 \\
17.6 \\
15.6\end{array}$ & $\begin{array}{l}0.00483 \\
0.00533 \\
0.00571 \\
0.00596 \\
0.00509 \\
0.00612 \\
0.00609 \\
0.00599 \\
0.00574 \\
0.00549\end{array}$ & $\begin{array}{l}1.01703 \\
1.01714 \\
1.01724 \\
1.01734 \\
1.01744 \\
1.01755 \\
1.01765 \\
1.01776 \\
1.01797 \\
1.01818\end{array}$ & $\begin{array}{l}2.20 \\
1.70 \\
1.39 \\
1.18 \\
1.03 \\
0.931 \\
0.857 \\
0.803 \\
0.729 \\
0.678\end{array}$ \\
\hline $\begin{array}{l}26.0 \\
28.0 \\
30.0 \\
32.0 \\
34.0 \\
36.0 \\
38.0 \\
40.0\end{array}$ & $\begin{array}{l}14.89 \\
14.75 \\
14.60 \\
14.45 \\
14.30 \\
14.15 \\
14.00 \\
13.85\end{array}$ & $\begin{array}{l}170.0 \\
175.0 \\
178.0 \\
181.0 \\
184.0 \\
186.0 \\
188.0 \\
190.0\end{array}$ & $\begin{array}{l}9.91 \\
9.64 \\
9.43 \\
9.25 \\
9.10 \\
8.96 \\
8.83 \\
8.71\end{array}$ & $\begin{array}{l}20400.0 \\
19900.0 \\
19400.0 \\
18900.0 \\
18400.0 \\
17900.0 \\
17500.0 \\
17000.0\end{array}$ & $\begin{array}{l}0.00494 \\
0.00501 \\
0.00509 \\
0.00516 \\
0.00523 \\
0.00528 \\
0.00533 \\
0.00538\end{array}$ & $\begin{array}{l}0.0667 \\
0.0668 \\
0.0668 \\
0.0666 \\
0.0664 \\
0.0661 \\
0.0657 \\
0.0653\end{array}$ & $\begin{array}{c}14.0 \\
12.8 \\
11.9 \\
11.2 \\
10.6 \\
10.1 \\
9.69 \\
9.36\end{array}$ & $\begin{array}{l}0.00532 \\
0.00518 \\
0.00505 \\
0.00493 \\
0.00484 \\
0.00475 \\
0.00468 \\
0.00462\end{array}$ & $\begin{array}{l}1.01838 \\
1.01858 \\
1.01877 \\
1.01895 \\
1.01913 \\
1.01929 \\
1.01945 \\
1.01961\end{array}$ & $\begin{array}{l}0.637 \\
0.605 \\
0.582 \\
0.564 \\
0.551 \\
0.540 \\
0.532 \\
0.527\end{array}$ \\
\hline $\begin{array}{r}45.0 \\
50.0 \\
55.0 \\
60.0 \\
70.0 \\
80.0 \\
90.0 \\
100.0 \\
120.0 \\
140.0\end{array}$ & $\begin{array}{c}13.48 \\
13.11 \\
12.75 \\
12.40 \\
11.72 \\
11.08 \\
10.49 \\
9.942 \\
8.984 \\
8.184\end{array}$ & $\begin{array}{l}194.0 \\
198.0 \\
202.0 \\
207.0 \\
215.0 \\
225.0 \\
235.0 \\
246.0 \\
269.0 \\
294.0\end{array}$ & $\begin{array}{l}8.44 \\
8.19 \\
7.95 \\
7.73 \\
7.31 \\
6.94 \\
6.61 \\
6.33 \\
5.87 \\
5.52\end{array}$ & $\begin{array}{r}16000.0 \\
15000.0 \\
14100.0 \\
13300.0 \\
12000.0 \\
11000.0 \\
10200.0 \\
9570.0 \\
8690.0 \\
8100.0\end{array}$ & $\begin{array}{l}0.00547 \\
0.00555 \\
0.00560 \\
0.00563 \\
0.00563 \\
0.00556 \\
0.00543 \\
0.00526 \\
0.00487 \\
0.00446\end{array}$ & $\begin{array}{l}0.0644 \\
0.0634 \\
0.0625 \\
0.0618 \\
0.0605 \\
0.0597 \\
0.0593 \\
0.0592 \\
0.0596 \\
0.0607\end{array}$ & $\begin{array}{l}8.74 \\
8.34 \\
8.07 \\
7.90 \\
7.71 \\
7.66 \\
7.69 \\
7.76 \\
7.98 \\
8.24\end{array}$ & $\begin{array}{l}0.00449 \\
0.00439 \\
0.00433 \\
0.00428 \\
0.00426 \\
0.00431 \\
0.00443 \\
0.00460 \\
0.00506 \\
0.00565\end{array}$ & $\begin{array}{l}1.01995 \\
1.02024 \\
1.02049 \\
1.02068 \\
1.02093 \\
1.02102 \\
1.02097 \\
1.02082 \\
1.02030 \\
1.01962\end{array}$ & $\begin{array}{l}0.520 \\
0.521 \\
0.527 \\
0.535 \\
0.556 \\
0.577 \\
0.596 \\
0.611 \\
0.631 \\
0.642\end{array}$ \\
\hline $\begin{array}{l}160.0 \\
180.0 \\
200.0 \\
250.0 \\
300.0 \\
350.0 \\
400.0 \\
450.0 \\
500.0 \\
600.0\end{array}$ & $\begin{array}{l}7.513 \\
6.945 \\
6.459 \\
5.502 \\
4.798 \\
4.256 \\
3.826 \\
3.475 \\
3.184 \\
2.727\end{array}$ & $\begin{array}{l}319.0 \\
345.0 \\
370.0 \\
434.0 \\
496.0 \\
558.0 \\
620.0 \\
681.0 \\
742.0 \\
865.0\end{array}$ & $\begin{array}{l}5.25 \\
5.03 \\
4.86 \\
4.56 \\
4.36 \\
4.23 \\
4.12 \\
4.05 \\
3.99 \\
3.90\end{array}$ & $\begin{array}{l}7690.0 \\
7380.0 \\
7140.0 \\
6710.0 \\
6420.0 \\
6210.0 \\
6050.0 \\
5920.0 \\
5820.0 \\
5670.0\end{array}$ & $\begin{array}{l}0.00410 \\
0.00377 \\
0.00349 \\
0.00295 \\
0.00255 \\
0.00225 \\
0.00202 \\
0.00183 \\
0.00168 \\
0.00144\end{array}$ & $\begin{array}{l}0.0622 \\
0.0640 \\
0.0659 \\
0.0710 \\
0.0762 \\
0.0813 \\
0.0862 \\
0.0910 \\
0.0956 \\
0.105\end{array}$ & $\begin{array}{c}8.54 \\
8.85 \\
9.03 \\
9.83 \\
10.6 \\
11.4 \\
12.1 \\
12.8 \\
13.5 \\
15.0\end{array}$ & $\begin{array}{l}0.00633 \\
0.00708 \\
0.00788 \\
0.0101 \\
0.0125 \\
0.0152 \\
0.0180 \\
0.0210 \\
0.0241 \\
0.0310\end{array}$ & $\begin{array}{l}1.01888 \\
1.01812 \\
1.01739 \\
1.01571 \\
1.01428 \\
1.01306 \\
1.01201 \\
1.01112 \\
1.01034 \\
1.00907\end{array}$ & $\begin{array}{l}0.646 \\
0.648 \\
0.639 \\
0.637 \\
0.635 \\
0.633 \\
0.633 \\
0.633 \\
0.634 \\
0.639\end{array}$ \\
\hline $\begin{array}{r}700.0 \\
800.0 \\
900.0 \\
1000.0 \\
1200.0 \\
1400.0 \\
1600.0 \\
1800.0 \\
2000.0 \\
2500.0\end{array}$ & $\begin{array}{l}2.384 \\
2.118 \\
1.905 \\
1.731 \\
1.463 \\
1.267 \\
1.117 \\
0.999 \\
0.9033 \\
0.7286\end{array}$ & $\begin{array}{r}987.0 \\
1110.0 \\
1230.0 \\
1350.0 \\
1600.0 \\
1850.0 \\
2090.0 \\
2340.0 \\
2580.0 \\
3200.0\end{array}$ & $\begin{array}{l}3.84 \\
3.80 \\
3.77 \\
3.74 \\
3.70 \\
3.68 \\
3.66 \\
3.64 \\
3.63 \\
3.61\end{array}$ & $\begin{array}{l}5560.0 \\
5480.0 \\
5420.0 \\
5370.0 \\
5300.0 \\
5240.0 \\
5210.0 \\
5180.0 \\
5150.0 \\
5110.0\end{array}$ & $\begin{array}{l}0.00126 \\
0.00112 \\
0.00101 \\
0.000914 \\
0.000774 \\
0.000671 \\
0.000592 \\
0.000530 \\
0.000479 \\
0.000387\end{array}$ & $\begin{array}{l}0.115 \\
0.124 \\
0.133 \\
0.143 \\
0.161 \\
0.178 \\
0.195 \\
0.211 \\
0.227 \\
0.265\end{array}$ & $\begin{array}{l}16.5 \\
18.0 \\
19.5 \\
20.9 \\
23.5 \\
26.3 \\
28.8 \\
31.2 \\
33.6 \\
39.3\end{array}$ & $\begin{array}{l}0.0388 \\
0.0473 \\
0.0566 \\
0.0666 \\
0.0887 \\
0.113 \\
0.141 \\
0.171 \\
0.203 \\
0.294\end{array}$ & $\begin{array}{l}1.00806 \\
1.00726 \\
1.00660 \\
1.00604 \\
1.00517 \\
1.00452 \\
1.00401 \\
1.00361 \\
1.00328 \\
1.00266\end{array}$ & $\begin{array}{l}0.644 \\
0.648 \\
0.651 \\
0.653 \\
0.656 \\
0.658 \\
0.659 \\
0.660 \\
0.661 \\
0.662\end{array}$ \\
\hline 3000.0 & 0.6104 & 3820.0 & 3.60 & 5090.0 & 0.000324 & 0.301 & 44.7 & 0.398 & 1.00224 & 0.663 \\
\hline
\end{tabular}

* thD-phase goundary 
6000 PSIA ISOBAR

THERHOOYNAMIC PROPERTIES OF HELIUH 4

\begin{tabular}{|c|c|c|c|c|c|c|c|c|c|}
\hline $\begin{array}{c}\text { TEHPERATURE } \\
\text { OEG. R }\end{array}$ & $\begin{array}{l}\text { VOLUHE } \\
\text { CU FT/LB }\end{array}$ & $\begin{array}{c}\text { ISOTHERM } \\
\text { OERIVATIVE } \\
\text { CU FT-PSIA/LB }\end{array}$ & $\begin{array}{l}\text { ISOCHORE } \\
\text { OERIVATIVE } \\
\text { PSIA/R }\end{array}$ & $\begin{array}{l}\text { INTERNAL } \\
\text { ENERGY } \\
\text { BTU/LA }\end{array}$ & $\begin{array}{l}\text { ENTHALPY } \\
\text { RTU/LB }\end{array}$ & $\begin{array}{l}\text { ENTROPY } \\
\text { BTU/LR-R }\end{array}$ & $\begin{array}{l}\text { CV } \\
\text { RTU }\end{array}$ &,$\quad L B=R$ & $\begin{array}{l}\text { VEL OCITY } \\
\text { OF SOUNO } \\
\text { FT/SEC }\end{array}$ \\
\hline $\begin{array}{l}15.0 \\
16.0 \\
17.0 \\
18.0 \\
19.0 \\
20.0 \\
22.0 \\
24.0 \\
26.0\end{array}$ & $\begin{array}{l}0.06142 \\
0.06164 \\
0.06186 \\
0.06209 \\
0.06233 \\
0.06258 \\
0.06311 \\
0.06366 \\
0.06422\end{array}$ & $\begin{array}{l}1720.0 \\
1700.0 \\
1690.0 \\
1680.0 \\
1660.0 \\
1650.0 \\
1620.0 \\
1600.0 \\
1590.0\end{array}$ & $\begin{array}{r}101.0 \\
99.0 \\
100.0 \\
101.0 \\
104.0 \\
106.0 \\
109.0 \\
110.0 \\
109.0\end{array}$ & $\begin{array}{l}14.46 \\
14.84 \\
15.21 \\
15.56 \\
16.04 \\
16.53 \\
17.58 \\
18.74 \\
20.01\end{array}$ & $\begin{array}{l}82.70 \\
83.33 \\
83.94 \\
84.54 \\
85.29 \\
86.06 \\
87.70 \\
89.47 \\
91.36\end{array}$ & $\begin{array}{l}0.4880 \\
0.5298 \\
0.5682 \\
0.6042 \\
0.6453 \\
0.6855 \\
0.7650 \\
0.8437 \\
0.9208\end{array}$ & $\begin{array}{l}0.5994 \\
0.5727 \\
0.5586 \\
0.5543 \\
0.5581 \\
0.5692 \\
0.6032 \\
0.6400 \\
0.6693\end{array}$ & $\begin{array}{l}0.6612 \\
0.6374 \\
0.6292 \\
0.6328 \\
0.6468 \\
0.6684 \\
0.7227 \\
0.7766 \\
0.8193\end{array}$ & $\begin{array}{l}2962.0 \\
2964.0 \\
2970.0 \\
2978.0 \\
2987.0 \\
2994.0 \\
3003.0 \\
3003.0 \\
2999.0\end{array}$ \\
\hline $\begin{array}{l}28.0 \\
30.0 \\
32.0 \\
34.0 \\
36.0 \\
38.0 \\
40.0\end{array}$ & $\begin{array}{l}0.06480 \\
0.06538 \\
0.06598 \\
0.06660 \\
0.06722 \\
0.06786 \\
0.06850\end{array}$ & $\begin{array}{l}1570.0 \\
1550.0 \\
1530.0 \\
1510.0 \\
1490.0 \\
1470.0 \\
1450.0\end{array}$ & $\begin{array}{l}108.0 \\
108.0 \\
107.0 \\
105.0 \\
104.0 \\
102.0 \\
101.0\end{array}$ & $\begin{array}{l}21.19 \\
22.28 \\
23.41 \\
24.58 \\
25.79 \\
27.02 \\
28.29\end{array}$ & $\begin{array}{c}93.18 \\
94.92 \\
96.72 \\
98.57 \\
100.5 \\
102.4 \\
104.4\end{array}$ & $\begin{array}{l}0.9892 \\
1.049 \\
1.107 \\
1.163 \\
1.218 \\
1.270 \\
1.321\end{array}$ & $\begin{array}{l}0.6898 \\
0.7077 \\
0.7220 \\
0.7334 \\
0.7425 \\
0.7499 \\
0.7560\end{array}$ & $\begin{array}{l}0.8529 \\
0.8852 \\
0.9135 \\
0.9384 \\
0.9606 \\
0.9807 \\
0.999\end{array}$ & $\begin{array}{l}2996.0 \\
2995.0 \\
2993.0 \\
2992.0 \\
2989.0 \\
2986.0 \\
2983.0\end{array}$ \\
\hline $\begin{array}{r}45.0 \\
50.0 \\
55.0 \\
60.0 \\
70.0 \\
80.0 \\
90.0 \\
100.0 \\
120.0 \\
140.0\end{array}$ & $\begin{array}{l}0.07016 \\
0.07188 \\
0.07366 \\
0.07549 \\
0.07931 \\
0.08332 \\
0.08748 \\
0.09176 \\
0.1006 \\
0.1096\end{array}$ & $\begin{array}{l}1410.0 \\
1370.0 \\
1330.0 \\
1290.0 \\
1230.0 \\
1190.0 \\
1160.0 \\
1140.0 \\
1120.0 \\
1130.0\end{array}$ & $\begin{array}{l}96.7 \\
92.6 \\
88.4 \\
84.4 \\
76.9 \\
70.0 \\
63.9 \\
58.5 \\
49.6 \\
42.7\end{array}$ & $\begin{array}{c}31.54 \\
34.92 \\
38.40 \\
41.97 \\
49.33 \\
56.97 \\
64.71 \\
72.61 \\
88.64 \\
104.8\end{array}$ & $\begin{array}{l}109.5 \\
114.8 \\
120.2 \\
125.9 \\
137.4 \\
149.5 \\
161.9 \\
174.6 \\
200.4 \\
226.5\end{array}$ & $\begin{array}{l}1.441 \\
1.552 \\
1.656 \\
1.754 \\
1.933 \\
2.093 \\
2.239 \\
2.373 \\
2.608 \\
2.810\end{array}$ & $\begin{array}{l}0.7672 \\
0.7751 \\
0.7811 \\
0.7861 \\
0.7940 \\
0.7999 \\
0.8042 \\
0.8072 \\
0.8101 \\
0.8101\end{array}$ & $\begin{array}{l}1.040 \\
1.075 \\
1.106 \\
1.135 \\
1.184 \\
1.224 \\
1.254 \\
1.277 \\
1.302 \\
1.310\end{array}$ & $\begin{array}{l}2974.0 \\
2964.0 \\
2953.0 \\
2941.0 \\
2920.0 \\
2903.0 \\
2891.0 \\
2886.0 \\
2892.0 \\
2915.0\end{array}$ \\
\hline $\begin{array}{l}160.0 \\
180.0 \\
200.0 \\
250.0 \\
300.0 \\
350.0 \\
400.0 \\
450.0 \\
500.0 \\
600.0\end{array}$ & $\begin{array}{l}0.1186 \\
0.1277 \\
0.1367 \\
0.1591 \\
0.1813 \\
0.2034 \\
0.2253 \\
0.2472 \\
0.2691 \\
0.3128\end{array}$ & $\begin{array}{l}1160.0 \\
1190.0 \\
1230.0 \\
1340.0 \\
1450.0 \\
1570.0 \\
1690.0 \\
1810.0 \\
1930.0 \\
2170.0\end{array}$ & $\begin{array}{l}37.3 \\
33.1 \\
29.7 \\
23.5 \\
19.5 \\
16.7 \\
14.6 \\
12.9 \\
11.6 \\
9.71\end{array}$ & $\begin{array}{l}120.9 \\
137.0 \\
153.0 \\
192.6 \\
231.7 \\
270.4 \\
308.9 \\
347.1 \\
385.1 \\
460.8\end{array}$ & $\begin{array}{l}252.7 \\
278.9 \\
304.9 \\
369.4 \\
433.1 \\
496.4 \\
559.2 \\
621.7 \\
684.1 \\
808.3\end{array}$ & $\begin{array}{l}2.985 \\
3.138 \\
3.276 \\
3.564 \\
3.796 \\
3.991 \\
4.159 \\
4.306 \\
4.437 \\
4.664\end{array}$ & $\begin{array}{l}0.8085 \\
0.8060 \\
0.8032 \\
0.7961 \\
0.7898 \\
0.7845 \\
0.7802 \\
0.7767 \\
0.7738 \\
0.7694\end{array}$ & $\begin{array}{l}1.309 \\
1.305 \\
1.298 \\
1.282 \\
1.269 \\
1.260 \\
1.253 \\
1.249 \\
1.245 \\
1.241\end{array}$ & $\begin{array}{l}2949.0 \\
2990.0 \\
3035.0 \\
3157.0 \\
3285.0 \\
3414.0 \\
3542.0 \\
3667.0 \\
3791.0 \\
4030.0\end{array}$ \\
\hline $\begin{array}{r}700.0 \\
800.0 \\
900.0 \\
1000.0 \\
1200.0 \\
1400.0 \\
1600.0 \\
1800.0 \\
2000.0 \\
2500.0\end{array}$ & $\begin{array}{l}0.3565 \\
0.4003 \\
0.4441 \\
0.4879 \\
0.5757 \\
0.6637 \\
0.7517 \\
0.8398 \\
0.9280 \\
1.149\end{array}$ & $\begin{array}{l}2420.0 \\
2670.0 \\
2930.0 \\
3180.0 \\
3690.0 \\
4210.0 \\
4730.0 \\
5250.0 \\
5770.0 \\
7080.0\end{array}$ & $\begin{array}{l}8.34 \\
7.31 \\
6.50 \\
5.86 \\
4.90 \\
4.21 \\
3.69 \\
3.28 \\
2.96 \\
2.37\end{array}$ & $\begin{array}{r}536.2 \\
611.4 \\
686.5 \\
761.5 \\
911.3 \\
1061.0 \\
1211.0 \\
1360.0 \\
1510.0 \\
1884.0\end{array}$ & $\begin{array}{r}932.3 \\
1056.0 \\
1180.0 \\
1304.0 \\
1551.0 \\
1798.0 \\
2046.0 \\
2293.0 \\
2541.0 \\
3160.0\end{array}$ & $\begin{array}{l}4.855 \\
5.021 \\
5.166 \\
5.297 \\
5.522 \\
5.713 \\
5.878 \\
6.024 \\
6.154 \\
6.430\end{array}$ & $\begin{array}{l}0.7663 \\
0.7640 \\
0.7622 \\
0.7608 \\
0.7587 \\
0.7573 \\
0.7563 \\
0.7555 \\
0.7549 \\
0.7538\end{array}$ & $\begin{array}{l}1.239 \\
1.238 \\
1.237 \\
1.237 \\
1.237 \\
1.237 \\
1.237 \\
1.238 \\
1.238 \\
1.238\end{array}$ & $\begin{array}{l}4260.0 \\
4480.0 \\
4692.0 \\
4896.0 \\
5282.0 \\
5645.0 \\
5987.0 \\
6311.0 \\
6620.0 \\
7339.0\end{array}$ \\
\hline 3000.0 & 1.370 & 8390.0 & 1.98 & 2257.0 & 3779.0 & 6.656 & 0.7530 & 1.239 & 7995.0 \\
\hline
\end{tabular}

- tho-phase bounoary 
6000 PSIA ISOBAR

TEMPERATURE OENSITY V(OH/OV) $V$ VIDP/OU/V $-V(O P / O V)_{T}$ OEG. R LB/CUFT BTU/LB PSIA-CU FT/RTU PEIA

\begin{tabular}{|c|c|c|c|c|c|c|c|c|c|c|}
\hline $\begin{array}{l}15.0 \\
16.0 \\
17.0 \\
18.0 \\
19.0 \\
20.0 \\
22.0 \\
24.0 \\
26.0\end{array}$ & $\begin{array}{l}16.28 \\
16.22 \\
16.17 \\
16.11 \\
16.04 \\
15.98 \\
15.85 \\
15.71 \\
15.57\end{array}$ & $\begin{array}{l}184.0 \\
178.0 \\
173.0 \\
169.0 \\
166.0 \\
166.0 \\
170.0 \\
177.0 \\
185.0\end{array}$ & $\begin{array}{l}10.3 \\
10.7 \\
11.0 \\
11.3 \\
11.6 \\
11.7 \\
11.4 \\
11.0 \\
10.5\end{array}$ & $\begin{array}{l}28000.0 \\
27600.0 \\
27300.0 \\
27000.0 \\
26700.0 \\
26300.0 \\
25700.0 \\
25200.0 \\
24700.0\end{array}$ & $\begin{array}{l}0.00360 \\
0.00358 \\
0.00364 \\
0.00375 \\
0.00389 \\
0.00403 \\
0.00425 \\
0.00438 \\
0.00443\end{array}$ & $\begin{array}{l}0.0652 \\
0.0670 \\
0.0685 \\
0.0698 \\
0.0709 \\
0.0717 \\
0.0729 \\
0.0736 \\
0.0740\end{array}$ & $\begin{array}{l}49.8 \\
43.1 \\
37.9 \\
33.7 \\
30.3 \\
27.4 \\
23.0 \\
19.9 \\
17.6\end{array}$ & $\begin{array}{l}0.00606 \\
0.00648 \\
0.00674 \\
0.00685 \\
0.00683 \\
0.00672 \\
0.00637 \\
0.00603 \\
0.00580\end{array}$ & $\begin{array}{l}1.01617 \\
1.01627 \\
1.01638 \\
1.01648 \\
1.01659 \\
1.01670 \\
1.01693 \\
1.01716 \\
1.01738\end{array}$ & $\begin{array}{l}1.82 \\
1.48 \\
1.25 \\
1.10 \\
0.994 \\
0.920 \\
0.822 \\
0.756 \\
0.702\end{array}$ \\
\hline $\begin{array}{l}28.0 \\
30.0 \\
32.0 \\
34.0 \\
36.0 \\
38.0 \\
40.0\end{array}$ & $\begin{array}{l}15.43 \\
15.29 \\
15.16 \\
15.02 \\
14.88 \\
14.74 \\
14.60\end{array}$ & $\begin{array}{l}190.0 \\
195.0 \\
199.0 \\
202.0 \\
205.0 \\
208.0 \\
210.0\end{array}$ & $\begin{array}{c}10.2 \\
9.94 \\
9.74 \\
9.56 \\
9.41 \\
9.27 \\
9.14\end{array}$ & $\begin{array}{l}24200.0 \\
23700.0 \\
23200.0 \\
22700.0 \\
22200.0 \\
21700.0 \\
21200.0\end{array}$ & $\begin{array}{l}0.00448 \\
0.00454 \\
0.00460 \\
0.00464 \\
0.00468 \\
0.00472 \\
0.00475\end{array}$ & $\begin{array}{l}0.0740 \\
0.0739 \\
0.0737 \\
0.0734 \\
0.0730 \\
0.0725 \\
0.0721\end{array}$ & $\begin{array}{l}15.9 \\
14.5 \\
13.4 \\
12.6 \\
11.9 \\
11.3 \\
10.8\end{array}$ & $\begin{array}{l}0.00563 \\
0.00546 \\
0.00532 \\
0.00521 \\
0.00511 \\
0.00502 \\
0.00494\end{array}$ & $\begin{array}{l}1.01760 \\
1.01781 \\
1.01802 \\
1.01821 \\
1.01841 \\
1.01859 \\
1.01877\end{array}$ & $\begin{array}{l}0.658 \\
0.625 \\
0.600 \\
0.579 \\
0.563 \\
0.551 \\
0.541\end{array}$ \\
\hline $\begin{array}{r}45.0 \\
50.0 \\
55.0 \\
60.0 \\
70.0 \\
80.0 \\
90.0 \\
100.0 \\
120.0 \\
140.0\end{array}$ & $\begin{array}{c}14.25 \\
13.91 \\
13.58 \\
13.25 \\
12.61 \\
12.00 \\
11.43 \\
10.90 \\
9.943 \\
9.127\end{array}$ & $\begin{array}{l}216.0 \\
221.0 \\
226.0 \\
230.0 \\
240.0 \\
249.0 \\
260.0 \\
270.0 \\
293.0 \\
318.0\end{array}$ & $\begin{array}{l}8.85 \\
8.59 \\
8.34 \\
8.11 \\
7.68 \\
7.29 \\
6.95 \\
6.65 \\
6.16 \\
5.77\end{array}$ & $\begin{array}{l}20100.0 \\
19000.0 \\
16000.0 \\
17100.0 \\
15600.0 \\
14300.0 \\
13200.0 \\
12400.0 \\
11200.0 \\
10400.0\end{array}$ & $\begin{array}{l}0.00482 \\
0.00487 \\
0.00490 \\
0.00493 \\
0.00494 \\
0.00491 \\
0.00483 \\
0.00472 \\
0.00444 \\
0.00412\end{array}$ & $\begin{array}{l}0.0709 \\
0.0698 \\
0.0687 \\
0.0678 \\
0.0663 \\
0.0653 \\
0.0646 \\
0.0643 \\
0.0644 \\
0.0651\end{array}$ & $\begin{array}{l}10.0 \\
9.39 \\
9.00 \\
8.73 \\
8.42 \\
8.29 \\
8.26 \\
8.29 \\
8.45 \\
8.68\end{array}$ & $\begin{array}{l}0.00479 \\
0.00467 \\
0.00458 \\
0.00451 \\
0.00444 \\
0.00444 \\
0.00451 \\
0.00462 \\
0.00497 \\
0.00545\end{array}$ & $\begin{array}{l}1.01918 \\
1.01955 \\
1.01987 \\
1.02014 \\
1.02057 \\
1.02085 \\
1.02099 \\
1.02102 \\
1.02082 \\
1.02040\end{array}$ & $\begin{array}{l}0.526 \\
0.521 \\
0.521 \\
0.526 \\
0.541 \\
0.560 \\
0.577 \\
0.593 \\
0.615 \\
0.628\end{array}$ \\
\hline $\begin{array}{l}160.0 \\
180.0 \\
200.0 \\
250.0 \\
300.0 \\
350.0 \\
400.0 \\
450.0 \\
500.0 \\
600.0\end{array}$ & $\begin{array}{l}8.431 \\
7.833 \\
7.316 \\
6.285 \\
5.516 \\
4.918 \\
4.438 \\
4.045 \\
3.716 \\
3.197\end{array}$ & $\begin{array}{l}343.0 \\
368.0 \\
394.0 \\
457.0 \\
520.0 \\
582.0 \\
644.0 \\
705.0 \\
766.0 \\
888.0\end{array}$ & $\begin{array}{l}5.48 \\
5.24 \\
5.05 \\
4.70 \\
4.48 \\
4.32 \\
4.21 \\
4.12 \\
4.05 \\
3.95\end{array}$ & $\begin{array}{l}9770.0 \\
9340.0 \\
9000.0 \\
8400.0 \\
8000.0 \\
7700.0 \\
7480.0 \\
7310.0 \\
7160.0 \\
6950.0\end{array}$ & $\begin{array}{l}0.00382 \\
0.00354 \\
0.00330 \\
0.00280 \\
C .00244 \\
0.00216 \\
0.00195 \\
0.00177 \\
0.00163 \\
0.00140\end{array}$ & $\begin{array}{l}0.0664 \\
0.0679 \\
0.0696 \\
0.0743 \\
0.0792 \\
0.0840 \\
0.0887 \\
0.0933 \\
0.0976 \\
0.107\end{array}$ & $\begin{array}{l}8.94 \\
9.22 \\
9.38 \\
10.1 \\
10.9 \\
11.6 \\
12.3 \\
13.0 \\
13.7 \\
15.1\end{array}$ & $\begin{array}{l}0.00601 \\
0.00664 \\
0.00733 \\
0.00922 \\
0.0113 \\
0.0136 \\
0.0160 \\
0.0185 \\
0.0211 \\
0.0269\end{array}$ & $\begin{array}{l}1.01985 \\
1.01925 \\
1.01863 \\
1.01711 \\
1.01574 \\
1.01453 \\
1.01348 \\
1.01255 \\
1.01174 \\
1.01038\end{array}$ & $\begin{array}{l}0.635 \\
0.637 \\
0.630 \\
0.629 \\
0.627 \\
0.626 \\
0.626 \\
0.626 \\
0.627 \\
0.633\end{array}$ \\
\hline $\begin{array}{r}700.0 \\
800.0 \\
900.0 \\
1000.0 \\
1200.0 \\
1400.0 \\
1600.0 \\
1800.0 \\
2000.0 \\
2500.0\end{array}$ & $\begin{array}{l}2.805 \\
2.498 \\
2.252 \\
2.050 \\
1.737 \\
1.507 \\
1.330 \\
1.191 \\
1.078 \\
0.8705\end{array}$ & $\begin{array}{l}1010.0 \\
1130.0 \\
1250.0 \\
1380.0 \\
1620.0 \\
1870.0 \\
2110.0 \\
2360.0 \\
2600.0 \\
3220.0\end{array}$ & $\begin{array}{l}3.88 \\
3.83 \\
3.79 \\
3.76 \\
3.72 \\
3.69 \\
3.66 \\
3.65 \\
3.63 \\
3.61\end{array}$ & $\begin{array}{l}6800.0 \\
6580.0 \\
6590.0 \\
6520.0 \\
6420.0 \\
6340.0 \\
6290.0 \\
6250.0 \\
6220.0 \\
6160.0\end{array}$ & $\begin{array}{l}0.00123 \\
0.00109 \\
0.000987 \\
0.000899 \\
0.000763 \\
0.000663 \\
0.000586 \\
0.000525 \\
0.000475 \\
0.000385\end{array}$ & $\begin{array}{l}0.116 \\
0.125 \\
0.135 \\
0.144 \\
0.162 \\
0.179 \\
0.195 \\
0.212 \\
0.227 \\
0.265\end{array}$ & $\begin{array}{l}16.6 \\
18.1 \\
19.6 \\
21.0 \\
23.7 \\
26.3 \\
28.8 \\
31.3 \\
33.7 \\
39.4\end{array}$ & $\begin{array}{l}0.0334 \\
0.0406 \\
0.0483 \\
0.0567 \\
0.0752 \\
0.0959 \\
0.119 \\
0.144 \\
0.170 \\
0.246\end{array}$ & $\begin{array}{l}1.00929 \\
1.00040 \\
1.00767 \\
1.00705 \\
1.00606 \\
1.00532 \\
1.00473 \\
1.00426 \\
1.00388 \\
1.00316\end{array}$ & $\begin{array}{l}0.639 \\
0.644 \\
0.647 \\
0.650 \\
0.654 \\
0.656 \\
0.658 \\
0.659 \\
0.660 \\
0.661\end{array}$ \\
\hline 3000.0 & 0.7300 & 3840.0 & 3.60 & 6120.0 & 0.000323 & 0.301 & 44.7 & 0.333 & 1.00267 & 0.662 \\
\hline
\end{tabular}

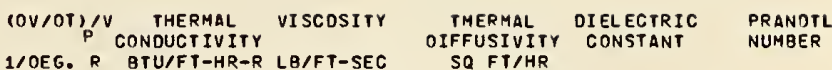
$\times \quad 10 E+6$ SQ FT/HR

* tho-phase boundary 
7000 PSIA ISOBAR

THERMOOYNAMIC PROPERTIES OF HELIUM 4

\begin{tabular}{|c|c|c|c|c|c|c|c|c|c|}
\hline $\begin{array}{c}\text { TEHPERATURE } \\
\text { OEG. R }\end{array}$ & $\begin{array}{l}\text { VOLUME } \\
\text { CU FT/LB }\end{array}$ & $\begin{array}{c}\text { ISOTHERH } \\
\text { DERIVATIVE } \\
\text { CU FT - PSIA/LB }\end{array}$ & $\begin{array}{l}\text { ISOCHORE } \\
\text { OERIVATIVE } \\
\text { PSIA/R }\end{array}$ & $\begin{array}{l}\text { INTERNAL } \\
\text { ENERGY } \\
\text { BTU/LB }\end{array}$ & $\begin{array}{l}\text { ENTHALPY } \\
\text { BTU/LB }\end{array}$ & $\begin{array}{l}\text { ENTROPY } \\
\text { BTU/LB-R }\end{array}$ & $\begin{array}{l}\text { CV } \\
\text { QTU / LA }\end{array}$ & $\begin{array}{l}C P \\
-R\end{array}$ & $\begin{array}{l}\text { VEL OCITY } \\
\text { OF SOUNO } \\
\text { FT/SEC }\end{array}$ \\
\hline $\begin{array}{l}16.0 \\
17.0 \\
18.0 \\
19.0 \\
20.0 \\
22.0 \\
24.0 \\
26.0 \\
28.0 \\
30.0\end{array}$ & $\begin{array}{l}0.05960 \\
0.05980 \\
0.05999 \\
0.05020 \\
0.06042 \\
0.06088 \\
0.06137 \\
0.06187 \\
0.06238 \\
0.06289\end{array}$ & $\begin{array}{l}1910.0 \\
1890.0 \\
1880.0 \\
1860.0 \\
1850.0 \\
1830.0 \\
1810.0 \\
1790.0 \\
1780.0 \\
1760.0\end{array}$ & $\begin{array}{l}103.0 \\
103.0 \\
105.0 \\
109.0 \\
112.0 \\
117.0 \\
119.0 \\
118.0 \\
117.0 \\
116.0\end{array}$ & $\begin{array}{l}16.67 \\
17.02 \\
17.35 \\
17.80 \\
18.25 \\
19.23 \\
20.32 \\
21.54 \\
22.69 \\
23.72\end{array}$ & $\begin{array}{c}93.93 \\
94.53 \\
95.11 \\
95.83 \\
96.56 \\
98.14 \\
99.9 \\
101.7 \\
103.5 \\
105.2\end{array}$ & $\begin{array}{l}0.4915 \\
0.5291 \\
0.5639 \\
0.6031 \\
0.6416 \\
0.7182 \\
0.7950 \\
0.8713 \\
0.9387 \\
0.998\end{array}$ & $\begin{array}{l}0.5736 \\
0.5478 \\
0.5355 \\
0.5356 \\
0.5457 \\
0.5825 \\
0.6246 \\
0.6586 \\
0.6814 \\
0.7006\end{array}$ & $\begin{array}{l}0.6317 \\
0.6108 \\
0.6058 \\
0.6164 \\
0.6374 \\
0.6953 \\
0.7548 \\
0.8018 \\
0.8368 \\
0.8694\end{array}$ & $\begin{array}{l}3121.0 \\
3128.0 \\
3138.0 \\
3152.0 \\
3164.0 \\
3179.0 \\
3183.0 \\
3181.0 \\
3180.0 \\
3180.0\end{array}$ \\
\hline $\begin{array}{l}32.0 \\
34.0 \\
36.0 \\
38.0 \\
40.0\end{array}$ & $\begin{array}{l}0.06342 \\
0.06396 \\
0.06450 \\
0.06506 \\
0.06562\end{array}$ & $\begin{array}{l}1740.0 \\
1720.0 \\
1700.0 \\
1690.0 \\
1670.0\end{array}$ & $\begin{array}{l}115.0 \\
114.0 \\
113.0 \\
111.0 \\
110.0\end{array}$ & $\begin{array}{l}24.81 \\
25.93 \\
27.09 \\
28.28 \\
29.50\end{array}$ & $\begin{array}{l}107.0 \\
108.8 \\
110.7 \\
112.6 \\
114.6\end{array}$ & $\begin{array}{l}1.055 \\
1.110 \\
1.163 \\
1.215 \\
1.265\end{array}$ & $\begin{array}{l}0.7160 \\
0.7285 \\
0.7385 \\
0.7467 \\
0.7535\end{array}$ & $\begin{array}{l}0.8978 \\
0.9227 \\
0.9448 \\
0.9647 \\
0.9828\end{array}$ & $\begin{array}{l}3180.0 \\
3179.0 \\
3178.0 \\
3177.0 \\
3175.0\end{array}$ \\
\hline $\begin{array}{r}45.0 \\
50.0 \\
55.0 \\
60.0 \\
70.0 \\
80.0 \\
90.0 \\
100.0 \\
120.0 \\
140.0\end{array}$ & $\begin{array}{l}0.06706 \\
0.06854 \\
0.07006 \\
0.07162 \\
0.07486 \\
0.07825 \\
0.08175 \\
0.08536 \\
0.09281 \\
0.1004\end{array}$ & $\begin{array}{l}1630.0 \\
1580.0 \\
1540.0 \\
1510.0 \\
1440.0 \\
1390.0 \\
1350.0 \\
1320.0 \\
1290.0 \\
1280.0\end{array}$ & $\begin{array}{r}105.0 \\
101.0 \\
97.0 \\
92.9 \\
85.2 \\
78.1 \\
71.7 \\
66.0 \\
55.4 \\
48.9\end{array}$ & $\begin{array}{c}32.66 \\
35.94 \\
39.32 \\
42.80 \\
50.00 \\
57.46 \\
65.11 \\
72.92 \\
88.83 \\
104.9\end{array}$ & $\begin{array}{l}119.6 \\
124.8 \\
130.1 \\
135.6 \\
147.0 \\
158.9 \\
171.1 \\
183.6 \\
209.1 \\
235.1\end{array}$ & $\begin{array}{l}1.383 \\
1.492 \\
1.595 \\
1.690 \\
1.866 \\
2.024 \\
2.168 \\
2.299 \\
2.532 \\
2.733\end{array}$ & $\begin{array}{l}0.7662 \\
0.7751 \\
0.7821 \\
0.7879 \\
0.7973 \\
0.8044 \\
0.8098 \\
0.8137 \\
0.8178 \\
0.8185\end{array}$ & $\begin{array}{l}1.022 \\
1.057 \\
1.087 \\
1.115 \\
1.163 \\
1.204 \\
1.236 \\
1.261 \\
1.292 \\
1.306\end{array}$ & $\begin{array}{l}3169.0 \\
3162.0 \\
3152.0 \\
3142.0 \\
3120.0 \\
3100.0 \\
3084.0 \\
3074.0 \\
3068.0 \\
3079.0\end{array}$ \\
\hline $\begin{array}{l}160.0 \\
180.0 \\
200.0 \\
250.0 \\
300.0 \\
350.0 \\
400.0 \\
450.0 \\
500.0 \\
600.0\end{array}$ & $\begin{array}{l}0.1082 \\
0.1159 \\
0.1236 \\
0.1428 \\
0.1618 \\
0.1807 \\
0.1995 \\
0.2182 \\
0.2369 \\
0.2742\end{array}$ & $\begin{array}{l}1300.0 \\
1320.0 \\
1360.0 \\
1450.0 \\
1560.0 \\
1680.0 \\
1790.0 \\
1910.0 \\
2030.0 \\
2270.0\end{array}$ & $\begin{array}{l}42.9 \\
38.1 \\
34.2 \\
27.2 \\
22.6 \\
19.3 \\
16.9 \\
15.0 \\
13.5 \\
11.3\end{array}$ & $\begin{array}{l}121.1 \\
137.2 \\
153.3 \\
193.1 \\
232.4 \\
271.3 \\
309.9 \\
348.2 \\
386.4 \\
462.3\end{array}$ & $\begin{array}{l}261.3 \\
287.4 \\
313.5 \\
378.2 \\
442.1 \\
505.5 \\
568.4 \\
631.0 \\
693.4 \\
317.7\end{array}$ & $\begin{array}{l}2.907 \\
3.061 \\
3.199 \\
3.487 \\
3.720 \\
3.916 \\
4.084 \\
4.231 \\
4.363 \\
4.589\end{array}$ & $\begin{array}{l}0.8172 \\
0.8149 \\
0.8120 \\
0.8043 \\
0.7973 \\
0.7914 \\
0.7864 \\
0.7824 \\
0.7790 \\
0.7739\end{array}$ & $\begin{array}{l}1.309 \\
1.306 \\
1.301 \\
1.286 \\
1.272 \\
1.262 \\
1.255 \\
1.250 \\
1.246 \\
1.241\end{array}$ & $\begin{array}{l}3103.0 \\
3135.0 \\
3173.0 \\
3282.0 \\
3399.0 \\
3518.0 \\
3638.0 \\
3757.0 \\
3875.0 \\
4105.0\end{array}$ \\
\hline $\begin{array}{r}700.0 \\
800.0 \\
900.0 \\
1000.0 \\
1200.0 \\
1400.0 \\
1600.0 \\
1800.0 \\
2000.0 \\
2500.0\end{array}$ & $\begin{array}{l}0.3115 \\
0.3489 \\
0.3863 \\
0.4238 \\
0.4988 \\
0.5740 \\
0.6492 \\
0.7246 \\
0.8001 \\
0.9889\end{array}$ & $\begin{array}{l}2510.0 \\
2760.0 \\
3010.0 \\
3260.0 \\
3770.0 \\
4280.0 \\
4800.0 \\
5310.0 \\
5830.0 \\
7130.0\end{array}$ & $\begin{array}{l}9.67 \\
8.48 \\
7.55 \\
6.81 \\
5.69 \\
4.89 \\
4.29 \\
3.82 \\
3.44 \\
2.76\end{array}$ & $\begin{array}{r}537.8 \\
613.2 \\
688.4 \\
763.4 \\
913.4 \\
1063.0 \\
1213.0 \\
1363.0 \\
1512.0 \\
1886.0\end{array}$ & $\begin{array}{r}941.6 \\
1065.0 \\
1189.0 \\
1 \geq 13.0 \\
1560.0 \\
1807.0 \\
2055.0 \\
2302.0 \\
2549.0 \\
3168.0\end{array}$ & $\begin{array}{l}4.780 \\
4.946 \\
5.091 \\
5.222 \\
5.447 \\
5.638 \\
5.803 \\
5.948 \\
6.079 \\
6.355\end{array}$ & $\begin{array}{l}0.7702 \\
0.7674 \\
0.7653 \\
0.7636 \\
0.7612 \\
0.7595 \\
0.7583 \\
0.7574 \\
0.7566 \\
0.7553\end{array}$ & $\begin{array}{l}1.238 \\
1.237 \\
1.237 \\
1.236 \\
1.236 \\
1.236 \\
1.237 \\
1.237 \\
1.237 \\
1.238\end{array}$ & $\begin{array}{l}4327.0 \\
4541.0 \\
4747.0 \\
4946.0 \\
5326.0 \\
5683.0 \\
6020.0 \\
6341.0 \\
6647.0 \\
7360.0\end{array}$ \\
\hline 3000.0 & 1.178 & 8440.0 & 2.30 & 2260.0 & 3787.0 & $E .5 B 1$ & 0.7544 & 1.238 & 8011.0 \\
\hline
\end{tabular}

* tho-PHASE BOUNOARY 
7000 PSIA ISOBAR

\begin{tabular}{|c|c|c|c|c|c|c|c|c|c|c|}
\hline $\begin{array}{c}\text { TEMPERATURE } \\
\text { OEG. R }\end{array}$ & $\begin{array}{l}\text { DENSITY } \\
\text { LE/CU FT }\end{array}$ & $\begin{array}{c}V(D H / O V)_{P} \\
\text { BTU/LB }\end{array}$ & $\begin{array}{l}V(O P / O U)_{V}- \\
\text { PSIA-CU FT/OTU }\end{array}$ & $\begin{array}{l}V(O P / O V)_{T} \\
\text { PSIA }\end{array}$ & $\begin{array}{l}(O \mathrm{~V} / O T)_{\mathrm{P}} / \mathrm{V} \\
1 / O E G \cdot \mathrm{R}\end{array}$ & $\begin{array}{c}\text { THERHAL } \\
\text { CONDUCTIVITY } \\
\text { OTU/F T-HR-R }\end{array}$ & $\begin{array}{l}\text { VISCOSITY } \\
\begin{array}{r}\text { LB/FT-SEC } \\
\times \quad 10 E+6\end{array}\end{array}$ & $\begin{array}{l}\text { THERHAL } \\
\text { OIFFUSIVITY } \\
\text { SQ FT/HR }\end{array}$ & $\begin{array}{l}\text { OIELECTRIC } \\
\text { CONSTANT }\end{array}$ & $\begin{array}{l}\text { PRANOTL } \\
\text { NUMBER }\end{array}$ \\
\hline $\begin{array}{l}16.0 \\
17.0 \\
18.0 \\
19.0 \\
20.0 \\
22.0 \\
24.0 \\
26.0 \\
28.0 \\
30.0\end{array}$ & $\begin{array}{l}16.78 \\
16.72 \\
16.67 \\
16.61 \\
16.55 \\
16.43 \\
16.29 \\
16.16 \\
16.03 \\
15.90\end{array}$ & $\begin{array}{l}197.0 \\
188.0 \\
181.0 \\
176.0 \\
174.0 \\
179.0 \\
188.0 \\
197.0 \\
204.0 \\
209.0\end{array}$ & $\begin{array}{l}10.7 \\
11.2 \\
11.8 \\
12.2 \\
12.4 \\
12.2 \\
11.7 \\
11.1 \\
10.7 \\
10.4\end{array}$ & $\begin{array}{l}32000.0 \\
31700.0 \\
31300.0 \\
31000.0 \\
30600.0 \\
30000.0 \\
29500.0 \\
29000.0 \\
28500.0 \\
28000.0\end{array}$ & $\begin{array}{l}0.00321 \\
0.00325 \\
0.00335 \\
0.00351 \\
0.00366 \\
0.00389 \\
0.00402 \\
0.00407 \\
0.00411 \\
0.00416\end{array}$ & $\begin{array}{l}0.0738 \\
0.0754 \\
0.0769 \\
0.0781 \\
0.0789 \\
0.0802 \\
0.0808 \\
0.0811 \\
0.0811 \\
0.0810\end{array}$ & $\begin{array}{l}60.2 \\
52.2 \\
45.7 \\
40.5 \\
36.2 \\
29.7 \\
25.2 \\
21.8 \\
19.4 \\
17.5\end{array}$ & $\begin{array}{l}0.00696 \\
0.00739 \\
0.00761 \\
0.00762 \\
0.00748 \\
0.00702 \\
0.00657 \\
0.00626 \\
0.00605 \\
0.00586\end{array}$ & $\begin{array}{l}1.01521 \\
1.01532 \\
1.01543 \\
1.01554 \\
1.01566 \\
1.01590 \\
1.01614 \\
1.01638 \\
1.01661 \\
1.01684\end{array}$ & $\begin{array}{l}1.86 \\
1.52 \\
1.30 \\
1.15 \\
1.05 \\
0.928 \\
0.846 \\
0.777 \\
0.719 \\
0.676\end{array}$ \\
\hline $\begin{array}{l}32.0 \\
34.0 \\
36.0 \\
38.0 \\
40.0\end{array}$ & $\begin{array}{l}15.77 \\
15.64 \\
15.50 \\
15.37 \\
15.24\end{array}$ & $\begin{array}{l}214.0 \\
218.0 \\
222.0 \\
225.0 \\
228.0\end{array}$ & $\begin{array}{c}10.2 \\
10.0 \\
9.84 \\
9.68 \\
9.54\end{array}$ & $\begin{array}{l}27500.0 \\
26900.0 \\
26400.0 \\
25900.0 \\
25400.0\end{array}$ & $\begin{array}{l}0.00420 \\
0.00423 \\
0.00426 \\
0.00429 \\
0.00431\end{array}$ & $\begin{array}{l}0.0806 \\
0.0802 \\
0.0797 \\
0.0791 \\
0.0786\end{array}$ & $\begin{array}{l}16.0 \\
14.8 \\
13.9 \\
13.1 \\
12.5\end{array}$ & $\begin{array}{l}0.00569 \\
0.00556 \\
0.00544 \\
0.00534 \\
0.00525\end{array}$ & $\begin{array}{l}1.01706 \\
1.01728 \\
1.01749 \\
1.01770 \\
1.01789\end{array}$ & $\begin{array}{l}0.642 \\
0.614 \\
0.592 \\
0.575 \\
0.561\end{array}$ \\
\hline $\begin{array}{r}45.0 \\
50.0 \\
55.0 \\
60.0 \\
70.0 \\
80.0 \\
90.0 \\
100.0 \\
120.0 \\
140.0\end{array}$ & $\begin{array}{r}14.91 \\
14.59 \\
14.27 \\
13.96 \\
13.36 \\
12.78 \\
12.23 \\
11.71 \\
10.77 \\
9.96\end{array}$ & $\begin{array}{l}235.0 \\
241.0 \\
247.0 \\
252.0 \\
263.0 \\
273.0 \\
284.0 \\
294.0 \\
317.0 \\
341.0\end{array}$ & $\begin{array}{l}9.23 \\
8.95 \\
8.69 \\
8.45 \\
8.00 \\
7.60 \\
7.24 \\
6.93 \\
6.41 \\
6.00\end{array}$ & $\begin{array}{l}24200.0 \\
23100.0 \\
22000.0 \\
21000.0 \\
19200.0 \\
17700.0 \\
16500.0 \\
15400.0 \\
13900.0 \\
12800.0\end{array}$ & $\begin{array}{l}0.00435 \\
0.00438 \\
0.00440 \\
0.00442 \\
0.00443 \\
0.00441 \\
0.00436 \\
0.00428 \\
0.00407 \\
0.00383\end{array}$ & $\begin{array}{l}0.0772 \\
0.0758 \\
0.0746 \\
0.0735 \\
0.0718 \\
0.0705 \\
0.0697 \\
0.0692 \\
0.0689 \\
0.0694\end{array}$ & $\begin{array}{c}11.3 \\
10.5 \\
9.95 \\
9.57 \\
9.13 \\
8.91 \\
8.82 \\
8.81 \\
8.91 \\
9.09\end{array}$ & $\begin{array}{l}0.00506 \\
0.00492 \\
0.00481 \\
0.00472 \\
0.00462 \\
0.00458 \\
0.00461 \\
0.00468 \\
0.00495 \\
0.00534\end{array}$ & $\begin{array}{l}1.01836 \\
1.01878 \\
1.01916 \\
1.01949 \\
1.02005 \\
1.02047 \\
1.02076 \\
1.02093 \\
1.02101 \\
1.02082\end{array}$ & $\begin{array}{l}0.537 \\
0.526 \\
0.522 \\
0.523 \\
0.533 \\
0.548 \\
0.563 \\
0.578 \\
0.501 \\
0.516\end{array}$ \\
\hline $\begin{array}{l}160.0 \\
180.0 \\
200.0 \\
250.0 \\
300.0 \\
350.0 \\
400.0 \\
450.0 \\
500.0 \\
600.0\end{array}$ & $\begin{array}{l}9.246 \\
8.629 \\
8.089 \\
7.002 \\
6.180 \\
5.535 \\
5.014 \\
4.584 \\
4.222 \\
3.647\end{array}$ & $\begin{array}{l}366.0 \\
391.0 \\
417.0 \\
481.0 \\
544.0 \\
606.0 \\
668.0 \\
729.0 \\
790.0 \\
912.0\end{array}$ & $\begin{array}{l}5.68 \\
5.42 \\
5.21 \\
4.84 \\
4.58 \\
4.41 \\
4.28 \\
4.18 \\
4.10 \\
3.99\end{array}$ & $\begin{array}{r}12000.0 \\
11400.0 \\
11000.0 \\
10200.0 \\
9660.0 \\
9270.0 \\
8980.0 \\
8750.0 \\
8560.0 \\
8270.0\end{array}$ & $\begin{array}{l}0.00357 \\
0.00334 \\
0.00312 \\
0.00267 \\
0.00234 \\
0.00208 \\
0.00188 \\
0.00171 \\
0.00158 \\
0.00136\end{array}$ & $\begin{array}{l}0.0704 \\
0.0717 \\
0.0732 \\
0.0776 \\
0.0821 \\
0.0867 \\
0.0912 \\
0.0955 \\
0.100 \\
0.108\end{array}$ & $\begin{array}{c}9.32 \\
9.57 \\
9.71 \\
10.4 \\
11.1 \\
11.8 \\
12.5 \\
13.1 \\
13.8 \\
15.2\end{array}$ & $\begin{array}{l}0.00582 \\
0.00636 \\
0.00696 \\
0.00862 \\
0.0104 \\
0.0124 \\
0.0145 \\
0.0167 \\
0.0189 \\
0.0239\end{array}$ & $\begin{array}{l}1.02047 \\
1.02002 \\
1.01952 \\
1.01821 \\
1.01694 \\
1.01578 \\
1.01474 \\
1.01381 \\
1.01297 \\
1.01156\end{array}$ & $\begin{array}{l}0.624 \\
0.628 \\
0.621 \\
0.621 \\
0.620 \\
0.619 \\
0.619 \\
0.619 \\
0.620 \\
0.627\end{array}$ \\
\hline $\begin{array}{r}700.0 \\
800.0 \\
900.0 \\
1000.0 \\
1200.0 \\
1400.0 \\
1600.0 \\
1600.0 \\
2000.0 \\
2500.0\end{array}$ & $\begin{array}{l}3.210 \\
2.866 \\
2.589 \\
2.360 \\
2.005 \\
1.742 \\
1.540 \\
1.380 \\
1.250 \\
1.011\end{array}$ & $\begin{array}{l}1030.0 \\
1150.0 \\
1280.0 \\
1400.0 \\
1640.0 \\
1890.0 \\
2130.0 \\
2380.0 \\
2620.0 \\
3240.0\end{array}$ & $\begin{array}{l}3.91 \\
3.86 \\
3.81 \\
3.78 \\
3.73 \\
3.70 \\
3.67 \\
3.65 \\
3.64 \\
3.61\end{array}$ & $\begin{array}{l}8070.0 \\
7910.0 \\
7790.0 \\
7700.0 \\
7560.0 \\
7460.0 \\
7390.0 \\
7330.0 \\
7290.0 \\
7210.0\end{array}$ & $\begin{array}{l}0.00120 \\
0.00107 \\
0.000969 \\
0.000884 \\
0.000753 \\
0.000655 \\
0.000580 \\
0.000520 \\
0.000472 \\
0.000382\end{array}$ & $\begin{array}{l}0.118 \\
0.127 \\
0.136 \\
0.145 \\
0.162 \\
0.179 \\
0.196 \\
0.212 \\
0.228 \\
0.266\end{array}$ & $\begin{array}{l}16.7 \\
18.2 \\
19.6 \\
21.0 \\
23.8 \\
26.4 \\
28.9 \\
31.3 \\
33.7 \\
39.4\end{array}$ & $\begin{array}{l}0.0296 \\
0.0357 \\
0.0424 \\
0.0496 \\
0.0655 \\
0.0833 \\
0.103 \\
0.124 \\
0.147 \\
0.212\end{array}$ & $\begin{array}{l}1.01041 \\
1.00946 \\
1.00867 \\
1.00799 \\
1.00691 \\
1.00608 \\
1.00543 \\
1.00490 \\
1.00446 \\
1.00365\end{array}$ & $\begin{array}{l}0.634 \\
0.640 \\
0.644 \\
0.647 \\
0.651 \\
0.654 \\
0.656 \\
0.658 \\
0.659 \\
0.660\end{array}$ \\
\hline 3000.0 & 0.8489 & 3850.0 & 3.59 & 7170.0 & C.000321 & 0.302 & 44.8 & 0.287 & 1.00309 & 0.661 \\
\hline
\end{tabular}

* two-PHASE goundary 
BOOO PSIA ISOBAR

THERMODYNAMIC PROPERTIES OF HELIUM 4

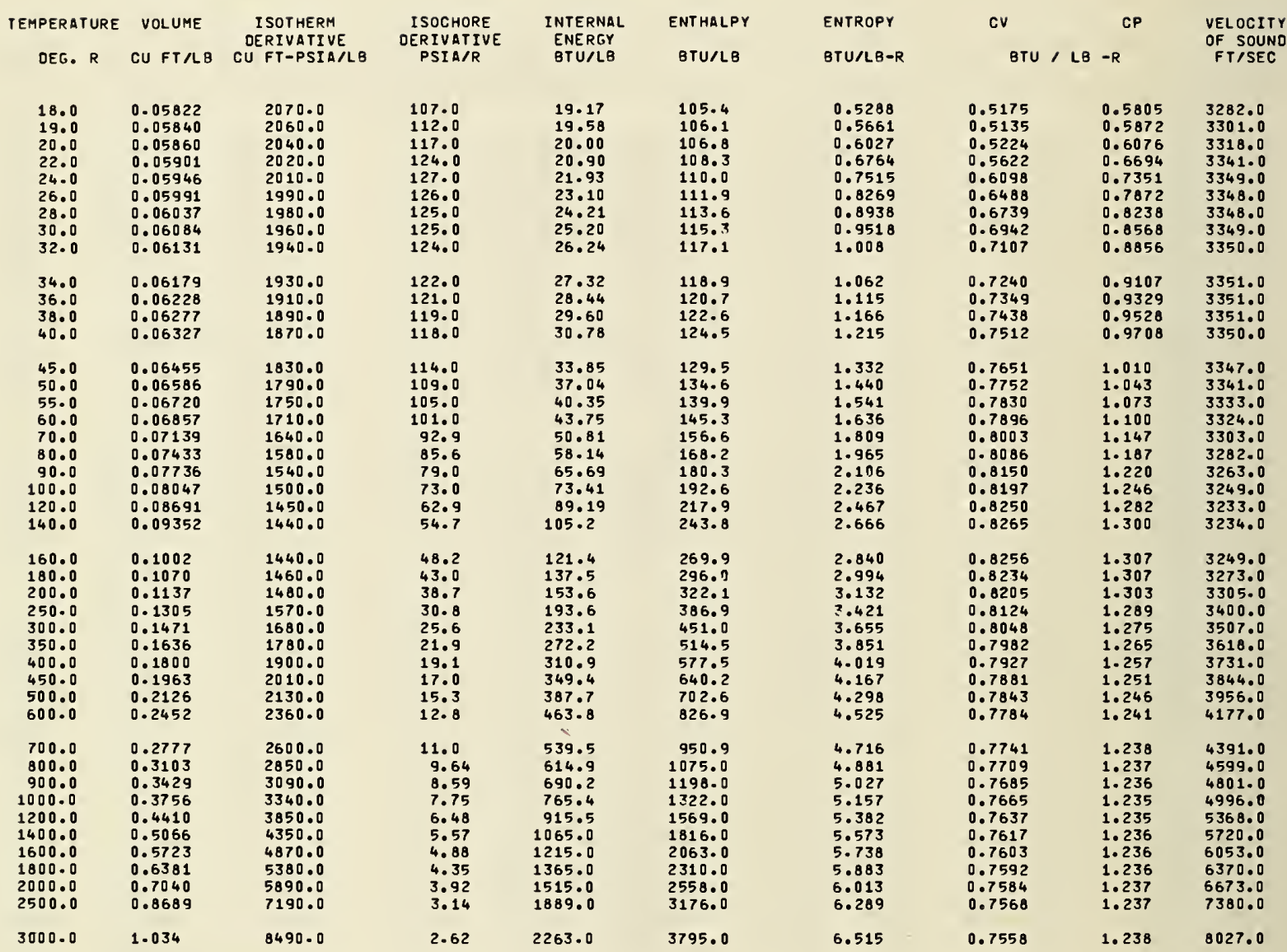


8000 PSIA ISOBAR

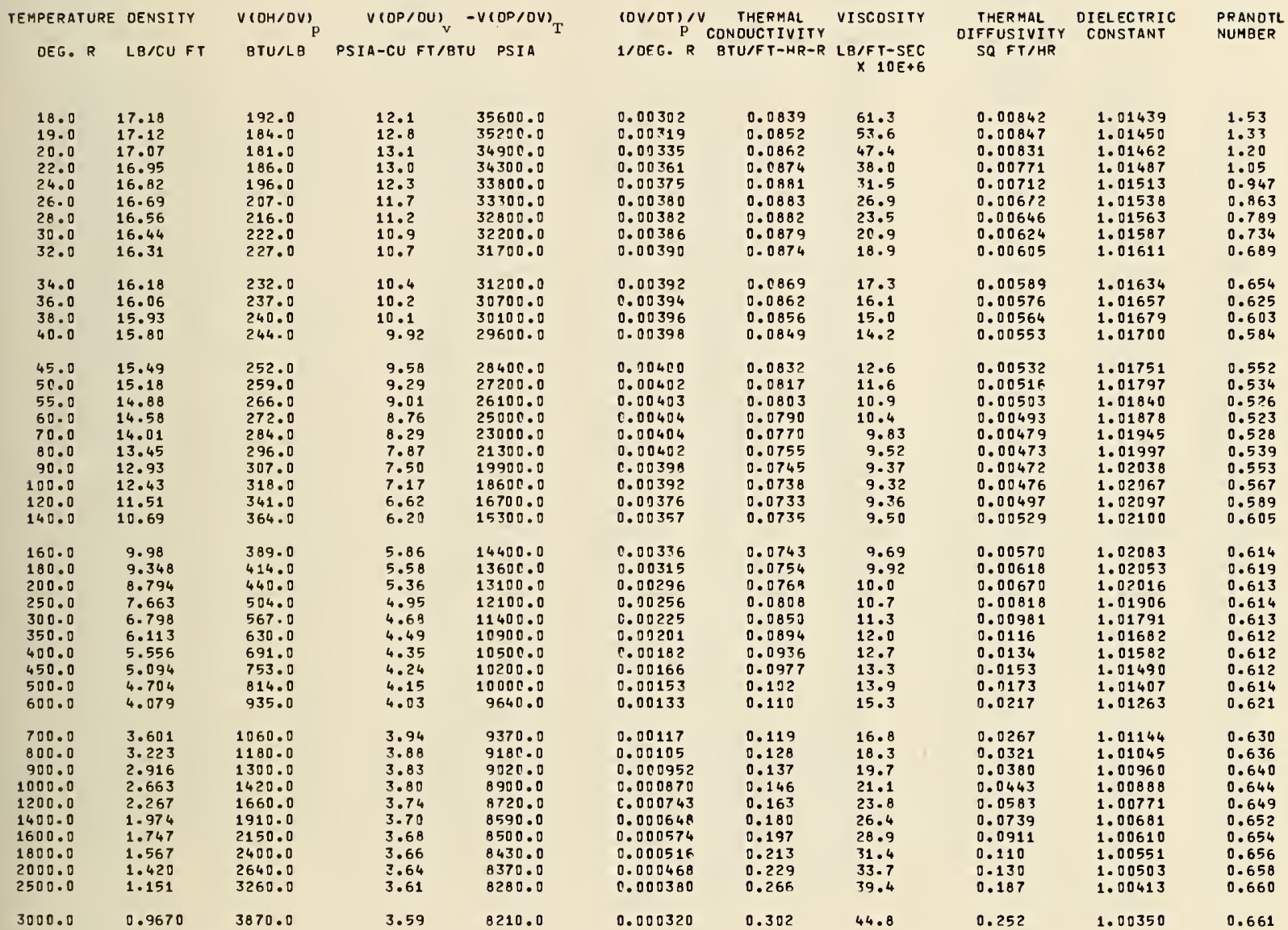

* tho-pHaSe goUndary 
9000 PSIA ISOBAR

THERMOOYNAMIC PROPERTIES OF HELIUM 4

\begin{tabular}{|c|c|c|c|c|c|c|c|c|c|}
\hline $\begin{array}{c}\text { TEMPERATURE } \\
\text { OEG. R }\end{array}$ & $\begin{array}{l}\text { VOLUME } \\
\text { CU FT/LB }\end{array}$ & $\begin{array}{c}\text { ISOTHERM } \\
\text { OERIVATIVE } \\
\text { CU FT-PSIA/LB }\end{array}$ & $\begin{array}{l}\text { ISOCHORE } \\
\text { OERIVATIVE } \\
\text { PSIA/R }\end{array}$ & $\begin{array}{l}\text { INTERNAL } \\
\text { ENERGY } \\
\text { BTU/LB }\end{array}$ & $\begin{array}{l}\text { ENTHALPY } \\
\text { BTU/LB }\end{array}$ & $\begin{array}{l}\text { ENTROPY } \\
\text { BTU/LB-R }\end{array}$ & $\begin{array}{l}\text { CV } \\
\text { BTU }\end{array}$ & /LB ${ }^{C P}$ & $\begin{array}{l}\text { VELOCITY } \\
\text { OF SOUND } \\
\text { FT/SEC }\end{array}$ \\
\hline $\begin{array}{l}19.0 \\
20.0 \\
22.0 \\
24.0 \\
26.0 \\
28.0 \\
30.0 \\
32.0 \\
34.0\end{array}$ & $\begin{array}{l}0.05686 \\
0.05703 \\
0.05741 \\
0.05782 \\
0.05824 \\
0.05867 \\
0.05909 \\
0.05952 \\
0.05996\end{array}$ & $\begin{array}{l}2240.0 \\
2230.0 \\
2210.0 \\
2200.0 \\
2190.0 \\
2180.0 \\
2160.0 \\
2140.0 \\
2120.0\end{array}$ & $\begin{array}{l}115.0 \\
121.0 \\
130.0 \\
134.0 \\
134.0 \\
133.0 \\
133.0 \\
132.0 \\
130.0\end{array}$ & $\begin{array}{l}21.39 \\
21.77 \\
22.58 \\
23.55 \\
24.68 \\
25.74 \\
26.70 \\
27.70 \\
28.74\end{array}$ & $\begin{array}{l}116.1 \\
116.8 \\
118.3 \\
119.9 \\
121.7 \\
123.5 \\
125.8 \\
126.9 \\
128.7\end{array}$ & $\begin{array}{l}0.5333 \\
0.5681 \\
0.6387 \\
0.7120 \\
0.7866 \\
0.8530 \\
0.9102 \\
0.9658 \\
1.020\end{array}$ & $\begin{array}{l}0.4914 \\
0.4990 \\
0.5417 \\
0.5952 \\
0.6396 \\
0.6672 \\
0.6885 \\
0.7058 \\
0.7200\end{array}$ & $\begin{array}{l}0.5582 \\
0.5778 \\
0.6439 \\
0.7164 \\
0.7741 \\
0.8128 \\
0.8464 \\
0.8756 \\
0.9011\end{array}$ & $\begin{array}{l}3436.0 \\
3459.0 \\
3489.0 \\
3502.0 \\
3504.0 \\
3504.0 \\
3506.0 \\
3507.0 \\
3509.0\end{array}$ \\
\hline $\begin{array}{l}36.0 \\
38.0 \\
40.0\end{array}$ & $\begin{array}{l}0.06041 \\
0.06085 \\
0.06131\end{array}$ & $\begin{array}{l}2110.0 \\
2090.0 \\
2070.0\end{array}$ & $\begin{array}{l}129.0 \\
127.0 \\
126.0\end{array}$ & $\begin{array}{l}29.83 \\
30.95 \\
32.10\end{array}$ & $\begin{array}{l}130.5 \\
132.4 \\
134.3\end{array}$ & $\begin{array}{l}1.072 \\
1.122 \\
1.171\end{array}$ & $\begin{array}{l}0.7316 \\
0.7411 \\
0.7491\end{array}$ & $\begin{array}{l}0.9235 \\
0.9435 \\
0.9615\end{array}$ & $\begin{array}{l}3510.0 \\
3510.0 \\
3511.0\end{array}$ \\
\hline $\begin{array}{r}45.0 \\
50.0 \\
55.0 \\
60.0 \\
70.0 \\
80.0 \\
90.0 \\
100.0 \\
120.0 \\
140.0\end{array}$ & $\begin{array}{l}0.06246 \\
0.06364 \\
0.06484 \\
0.06607 \\
0.06858 \\
0.07118 \\
0.07385 \\
0.07659 \\
0.08225 \\
0.08807\end{array}$ & $\begin{array}{l}2030.0 \\
1990.0 \\
1950.0 \\
1910.0 \\
1840.0 \\
1780.0 \\
1720.0 \\
1680.0 \\
1620.0 \\
1590.0\end{array}$ & $\begin{array}{r}121.0 \\
117.0 \\
113.0 \\
108.0 \\
100.0 \\
92.6 \\
85.7 \\
79.5 \\
68.9 \\
60.3\end{array}$ & $\begin{array}{r}35.08 \\
38.20 \\
41.44 \\
44.78 \\
51.71 \\
58.93 \\
66.39 \\
74.03 \\
89.69 \\
105.7\end{array}$ & $\begin{array}{l}139.2 \\
144.3 \\
149.5 \\
154.9 \\
166.0 \\
177.6 \\
189.5 \\
201.7 \\
226.8 \\
252.5\end{array}$ & $\begin{array}{l}1.287 \\
1.394 \\
1.494 \\
1.587 \\
1.759 \\
1.913 \\
2.053 \\
2.182 \\
2.410 \\
2.608\end{array}$ & $\begin{array}{l}0.7643 \\
0.7753 \\
0.7839 \\
0.7912 \\
0.8032 \\
0.8126 \\
0.8199 \\
0.8254 \\
0.8320 \\
0.8342\end{array}$ & $\begin{array}{l}1.000 \\
1.033 \\
1.062 \\
1.088 \\
1.135 \\
1.174 \\
1.207 \\
1.234 \\
1.272 \\
1.294\end{array}$ & $\begin{array}{l}3509.0 \\
3506.0 \\
3499.0 \\
3491.0 \\
3472.0 \\
3450.0 \\
3430.0 \\
3412.0 \\
3389.0 \\
3381.0\end{array}$ \\
\hline $\begin{array}{l}160.0 \\
180.0 \\
200.0 \\
250.0 \\
300.0 \\
350.0 \\
400.0 \\
450.0 \\
500.0 \\
600.0\end{array}$ & $\begin{array}{l}0.09399 \\
0.1000 \\
0.1059 \\
0.1208 \\
0.1356 \\
0.1502 \\
0.1647 \\
0.1792 \\
0.1937 \\
0.2225\end{array}$ & $\begin{array}{l}1580.0 \\
1590.0 \\
1610.0 \\
1690.0 \\
1790.0 \\
1890.0 \\
2000.0 \\
2110.0 \\
2220.0 \\
2460.0\end{array}$ & $\begin{array}{l}53.3 \\
47.6 \\
43.0 \\
34.3 \\
28.6 \\
24.4 \\
21.4 \\
19.0 \\
17.1 \\
14.3\end{array}$ & $\begin{array}{l}121.8 \\
138.0 \\
154.1 \\
194.2 \\
233.9 \\
273.1 \\
312.0 \\
350.6 \\
389.0 \\
465.3\end{array}$ & $\begin{array}{l}278.5 \\
304.6 \\
330.7 \\
395.6 \\
459.8 \\
523.5 \\
586.6 \\
649.3 \\
711.8 \\
836.2\end{array}$ & $\begin{array}{l}2.782 \\
2.936 \\
3.073 \\
3.363 \\
3.597 \\
3.793 \\
3.962 \\
4.110 \\
4.241 \\
4.468\end{array}$ & $\begin{array}{l}0.8338 \\
0.8317 \\
0.8289 \\
0.8204 \\
0.8122 \\
0.8050 \\
0.7989 \\
0.7939 \\
0.7896 \\
0.7830\end{array}$ & $\begin{array}{l}1.304 \\
1.306 \\
1.304 \\
1.292 \\
1.278 \\
1.267 \\
1.258 \\
1.252 \\
1.247 \\
1.241\end{array}$ & $\begin{array}{l}3388.0 \\
3405.0 \\
3430.0 \\
3512.0 \\
3610.0 \\
3714.0 \\
3820.0 \\
3927.0 \\
4034.0 \\
4246.0\end{array}$ \\
\hline $\begin{array}{r}700.0 \\
800.0 \\
900.0 \\
1000.0 \\
1200.0 \\
1400.0 \\
1600.0 \\
1800.0 \\
2000.0 \\
2500.0\end{array}$ & $\begin{array}{l}0.2513 \\
0.2802 \\
0.3091 \\
0.3380 \\
0.3960 \\
0.4542 \\
0.5125 \\
0.5708 \\
0.6292 \\
0.7756\end{array}$ & $\begin{array}{l}2690.0 \\
2930.0 \\
3180.0 \\
3420.0 \\
3920.0 \\
4420.0 \\
4930.0 \\
5440.0 \\
5960.0 \\
7250.0\end{array}$ & $\begin{array}{l}12.3 \\
10.8 \\
9.61 \\
8.67 \\
7.26 \\
6.24 \\
5.48 \\
4.88 \\
4.40 \\
3.53\end{array}$ & $\begin{array}{r}541.2 \\
616.8 \\
692.1 \\
767.4 \\
917.6 \\
1068.0 \\
1218.0 \\
1367.0 \\
1517.0 \\
1892.0\end{array}$ & $\begin{array}{r}960.1 \\
1084.0 \\
1207.0 \\
1331.0 \\
1578.0 \\
1825.0 \\
2072.0 \\
2319.0 \\
2566.0 \\
3184.0\end{array}$ & $\begin{array}{l}4.659 \\
4.824 \\
4.970 \\
5.100 \\
5.325 \\
5.515 \\
5.680 \\
5.826 \\
5.956 \\
6.232\end{array}$ & $\begin{array}{l}0.7781 \\
0.7745 \\
0.7717 \\
0.7695 \\
0.7662 \\
0.7640 \\
0.7623 \\
0.7611 \\
0.7601 \\
0.7584\end{array}$ & $\begin{array}{l}1.238 \\
1.236 \\
1.235 \\
1.235 \\
1.235 \\
1.235 \\
1.235 \\
1.236 \\
1.236 \\
1.237\end{array}$ & $\begin{array}{l}4454.0 \\
4656.0 \\
4852.0 \\
5043.0 \\
5409.0 \\
5755.0 \\
6084.0 \\
6398.0 \\
6698.0 \\
7399.0\end{array}$ \\
\hline 3000.0 & 0.9222 & 8540.0 & 2.95 & 2266.0 & 3803.0 & 6.457 & 0.7572 & 1.237 & 8042.0 \\
\hline
\end{tabular}

- tho-pHaSe boUnOARY 
THERMOPHYSICAL PROPERTIES OF HELIUM 4

9000 PSIA ISOBAR

TENPERATURE DENSITY V(OH/OV) VIOP/OU) ${ }_{V}-V(O P / O V)_{T}$ OEG. R LB/CUFT BTULLB PSIA-CUFT/BTU PSIA

\begin{tabular}{|c|c|c|c|c|}
\hline $\begin{array}{l}19.0 \\
20.0 \\
22.0 \\
24.0 \\
26.0 \\
28.0 \\
30.0 \\
32.0 \\
34.0\end{array}$ & $\begin{array}{l}17.59 \\
17.53 \\
17.42 \\
17.29 \\
17.17 \\
17.05 \\
16.92 \\
16.80 \\
16.68\end{array}$ & $\begin{array}{l}192.0 \\
187.0 \\
191.0 \\
203.0 \\
217.0 \\
226.0 \\
233.0 \\
239.0 \\
245.0\end{array}$ & $\begin{array}{l}13.3 \\
13.8 \\
13.7 \\
13.0 \\
12.2 \\
11.7 \\
11.4 \\
11.1 \\
10.9\end{array}$ & $\begin{array}{l}39500.0 \\
39100.0 \\
38500.0 \\
38000.0 \\
37600.0 \\
37100.0 \\
36500.0 \\
36000.0 \\
35400.0\end{array}$ \\
\hline $\begin{array}{l}36.0 \\
38.0 \\
40.0\end{array}$ & $\begin{array}{l}16.55 \\
16.43 \\
16.31\end{array}$ & $\begin{array}{l}250.0 \\
254.0 \\
259.0\end{array}$ & $\begin{array}{l}10.6 \\
10.5 \\
10.3\end{array}$ & $\begin{array}{l}34900.0 \\
34300.0 \\
33800.0\end{array}$ \\
\hline $\begin{array}{r}45.0 \\
50.0 \\
55.0 \\
60.0 \\
70.0 \\
80.0 \\
90.0 \\
100.0 \\
120.0 \\
140.0\end{array}$ & $\begin{array}{l}16.01 \\
15.71 \\
15.42 \\
15.14 \\
14.58 \\
14.05 \\
13.54 \\
13.06 \\
12.16 \\
11.35\end{array}$ & $\begin{array}{l}268.0 \\
276.0 \\
284.0 \\
291.0 \\
304.0 \\
317.0 \\
329.0 \\
341.0 \\
364.0 \\
388.0\end{array}$ & $\begin{array}{l}9.92 \\
9.60 \\
9.31 \\
9.04 \\
6.55 \\
8.11 \\
7.72 \\
7.38 \\
6.81 \\
6.37\end{array}$ & $\begin{array}{l}32500.0 \\
31300.0 \\
30100.0 \\
29000.0 \\
26900.0 \\
25000.0 \\
23400.0 \\
21900.0 \\
19700.0 \\
18100.0\end{array}$ \\
\hline $\begin{array}{l}160.0 \\
180.0 \\
200.0 \\
250.0 \\
300.0 \\
350.0 \\
400.0 \\
450.0 \\
500.0 \\
600.0\end{array}$ & $\begin{array}{c}10.64 \\
10.00 \\
9.440 \\
8.277 \\
7.376 \\
6.657 \\
6.070 \\
5.580 \\
5.163 \\
4.494\end{array}$ & $\begin{array}{l}412.0 \\
437.0 \\
462.0 \\
526.0 \\
590.0 \\
653.0 \\
715.0 \\
776.0 \\
837.0 \\
958.0\end{array}$ & $\begin{array}{l}6.01 \\
5.73 \\
5.49 \\
5.06 \\
4.77 \\
4.56 \\
4.41 \\
4.29 \\
4.20 \\
4.06\end{array}$ & $\begin{array}{l}16900.0 \\
15900.0 \\
15200.0 \\
14000.0 \\
13200.0 \\
12600.0 \\
12100.0 \\
11800.0 \\
11500.0 \\
11000.0\end{array}$ \\
\hline $\begin{array}{r}700.0 \\
800.0 \\
900.0 \\
1000.0 \\
1200.0 \\
1400.0 \\
1600.0 \\
1800.0 \\
2000.0 \\
2500.0\end{array}$ & $\begin{array}{l}3.979 \\
3.569 \\
3.235 \\
2.958 \\
2.525 \\
2.202 \\
1.951 \\
1.752 \\
1.589 \\
1.289\end{array}$ & $\begin{array}{l}1080.0 \\
1200.0 \\
1320.0 \\
1440.0 \\
1680.0 \\
1930.0 \\
2170.0 \\
2420.0 \\
2660.0 \\
3270.0\end{array}$ & $\begin{array}{l}3.97 \\
3.90 \\
3.85 \\
3.81 \\
3.75 \\
3.71 \\
3.68 \\
3.66 \\
3.64 \\
3.61\end{array}$ & $\begin{array}{r}10700.0 \\
10500.0 \\
10300.0 \\
10100.0 \\
9900.0 \\
9740.0 \\
9620.0 \\
9530.0 \\
9460.0 \\
9340.0\end{array}$ \\
\hline 3000.0 & 1.084 & 3890.0 & 3.59 & 9260.0 \\
\hline
\end{tabular}

(OV/OT) $/ V$ THERMAL VISCOSITY 1/OEG. R BTU/FT-HR-R LB/FT-SEC $\times 10 E+6$

\begin{tabular}{|c|}
\hline $\begin{array}{l}0.00291 \\
0.00309 \\
0.00337 \\
0.00352 \\
0.00357 \\
0.00359 \\
0.00363 \\
0.00366 \\
0.00368\end{array}$ \\
\hline $\begin{array}{l}0.00370 \\
0.00371 \\
0.00372\end{array}$ \\
\hline $\begin{array}{l}0.00373 \\
0.00374 \\
0.00374 \\
0.00374 \\
0.00373 \\
0.00370 \\
0.00367 \\
0.00362 \\
0.00350 \\
0.00334\end{array}$ \\
\hline $\begin{array}{l}0.00316 \\
0.00299 \\
0.00282 \\
0.00245 \\
0.00217 \\
0.00194 \\
0.00176 \\
0.00161 \\
0.00149 \\
0.00130\end{array}$ \\
\hline $\begin{array}{l}0.00115 \\
0.00103 \\
0.000936 \\
0.000857 \\
0.000733 \\
0.000641 \\
0.000569 \\
0.000512 \\
0.000465 \\
0.000378\end{array}$ \\
\hline 0.000318 \\
\hline
\end{tabular}

\begin{tabular}{|c|c|}
\hline $\begin{array}{l}0.0924 \\
0.0934 \\
0.0947 \\
0.0953 \\
0.0954 \\
0.0952 \\
0.0947 \\
0.0941 \\
0.0935\end{array}$ & $\begin{array}{l}70.5 \\
61.5 \\
48.2 \\
39.2 \\
32.8 \\
28.2 \\
24.8 \\
22.2 \\
20.1\end{array}$ \\
\hline $\begin{array}{l}0.0927 \\
0.0919 \\
0.0911\end{array}$ & $\begin{array}{l}18.5 \\
17.2 \\
16.1\end{array}$ \\
\hline $\begin{array}{l}0.0892 \\
0.0874 \\
0.0857 \\
0.0843 \\
0.0820 \\
0.0803 \\
0.0791 \\
0.0783 \\
0.0775 \\
0.0775\end{array}$ & $\begin{array}{l}14.1 \\
12.8 \\
11.9 \\
11.3 \\
10.5 \\
10.1 \\
9.92 \\
9.81 \\
9.79 \\
9.89\end{array}$ \\
\hline $\begin{array}{l}0.0781 \\
0.0791 \\
0.0803 \\
0.0839 \\
0.0879 \\
0.0920 \\
0.0960 \\
0.100 \\
0.104 \\
0.112\end{array}$ & $\begin{array}{l}10.1 \\
10.3 \\
10.3 \\
10.9 \\
11.6 \\
12.2 \\
12.8 \\
13.4 \\
14.0 \\
15.4\end{array}$ \\
\hline $\begin{array}{l}0.120 \\
0.129 \\
0.138 \\
0.147 \\
0.164 \\
0.181 \\
0.197 \\
0.213 \\
0.229 \\
0.267\end{array}$ & $\begin{array}{l}16.9 \\
18.4 \\
19.8 \\
21.2 \\
23.9 \\
26.5 \\
29.0 \\
31.4 \\
33.8 \\
39.5\end{array}$ \\
\hline 0.302 & 44.8 \\
\hline
\end{tabular}

THERMAL DIFFUSIVITY
SQ FT/HR

IELECTRIC SQ FTR
PRANOTL NUMBER

\subsection{1} 0.00922

0.00845

0.01769

0.00717

0.00687

0.00661

0.00640

0.00606

0.00593
0.00581

0.00557

0.00538

0.00523

0.00512

0.00495

0.00487

0.00484

0.00486

0.00528

0.00563

0.00605

0.00652

0.00785

0.00932

0.0109

0.0126

0.0143

0.0161
0.0200

0.0245

0.0293

0.0346

0.0402

0.0527

0.0666

0.0819

0.0986

0.117

0.225
$1.01347 \quad 1.53$

$\begin{array}{ll}1.01359 & 1.37\end{array}$

1.01413

$1.01440 \quad 0.959$

$1.01466 \quad 0.868$

$1.01492 \quad 0.798$

$\begin{array}{ll}1.01517 & 0.743 \\ 1.01541 & 0.698\end{array}$

$1.01565 \quad 0.663$

$\begin{array}{ll}1.01588 & 0.634 \\ 1.01611 & 0.611\end{array}$

$1.01665 \quad 0.570$

$1.01715 \quad 0.546$

1.01804

$1.01879 \quad 0.525$

$\begin{array}{ll}1.01879 & 0.525 \\ 1.01940 & 0.534\end{array}$

$\begin{array}{ll}1.01940 & 0.534 \\ 1.01990 & 0.545\end{array}$

$\begin{array}{ll}1.01990 & 0.545 \\ 1.02029 & 0.557\end{array}$

$1.02079 \quad 0.579$

$1.02099 \quad 0.604$

$1.02084 \quad 0.610$

$1.02058 \quad 0.605$

0.607
1.01870

$1.01770 \quad 0.605$

$1.01675 \quad 0.605$

0.605

$1.01586 \quad 0.606$

$\begin{array}{ll}1.01504 & 0.607 \\ 1.01360 & 0.616\end{array}$

$1.01239 \quad 0.625$

$1.01136 \quad 0.632$

$1.01048 \quad 0.637$

1.009720 .641

$1.00848 \quad 0.646$

$1.00751 \quad 0.650$

$1.00674 \quad 0.653$

$1.00611 \quad 0.655$

$1.00559 \quad 0.656$

$1.00390 \quad 0.660$
$1.02100 \quad 0.595$

- tho-phase boundary 
10000 PSIA ISOBAR

THERMOOYNAMIC PROPERTIES CF HELIUM 4

\begin{tabular}{|c|c|c|c|c|c|c|c|c|c|}
\hline $\begin{array}{c}\text { TEMPERATURE } \\
\text { OEG. R }\end{array}$ & CU FT/LB & $\begin{array}{c}\text { ISOTHERM } \\
\text { OERIVATIVE } \\
\text { CU FT-PSIA/LB }\end{array}$ & $\begin{array}{l}\text { ISOCHORE } \\
\text { OERIVATIVE } \\
\text { PSIA/R }\end{array}$ & $\begin{array}{l}\text { INTERNAL } \\
\text { ENERGY } \\
\text { BTU/LE }\end{array}$ & $\begin{array}{l}\text { ENTHALPY } \\
\text { BTU/LE }\end{array}$ & $\begin{array}{l}\text { ENTROPY } \\
\text { BTU/LB-R }\end{array}$ & $\begin{array}{l}\text { CV } \\
\text { gTU / L9 }\end{array}$ & $\begin{array}{l}C P \\
-R\end{array}$ & $\begin{array}{l}\text { VEL OCITY } \\
\text { OF SOUNO } \\
\text { FT/SEC }\end{array}$ \\
\hline $\begin{array}{l}20.0 \\
22.0 \\
24.0 \\
26.0 \\
28.0 \\
30.0 \\
32.0 \\
34.0 \\
36.0 \\
38.0\end{array}$ & $\begin{array}{l}0.05566 \\
0.05601 \\
0.05640 \\
0.05680 \\
0.05719 \\
0.05758 \\
0.05798 \\
0.05838 \\
0.05879 \\
0.05920\end{array}$ & $\begin{array}{l}2410.0 \\
2390.0 \\
2390.0 \\
2380.0 \\
2370.0 \\
2350.0 \\
2330.0 \\
2310.0 \\
2300.0 \\
2280.0\end{array}$ & $\begin{array}{l}123.0 \\
135.0 \\
141.0 \\
142.0 \\
141.0 \\
140.0 \\
139.0 \\
138.0 \\
137.0 \\
135.0\end{array}$ & $\begin{array}{l}23.55 \\
24.28 \\
25.18 \\
26.26 \\
27.29 \\
28.21 \\
29.17 \\
30.18 \\
31.24 \\
32.32\end{array}$ & $\begin{array}{l}126.6 \\
128.0 \\
129.6 \\
131.4 \\
133.2 \\
134.8 \\
136.5 \\
138.3 \\
140.1 \\
142.0\end{array}$ & $\begin{array}{l}0.5369 \\
0.5344 \\
0.6757 \\
0.7496 \\
0.8155 \\
0.8721 \\
0.9271 \\
0.9805 \\
1.032 \\
1.082\end{array}$ & $\begin{array}{l}0.4751 \\
0.5210 \\
0.5807 \\
0.6309 \\
0.6611 \\
0.6832 \\
0.7014 \\
0.7164 \\
0.7286 \\
0.7388\end{array}$ & $\begin{array}{l}0.5475 \\
0.6180 \\
0.6979 \\
0.7619 \\
0.8030 \\
0.8373 \\
0.8671 \\
0.8930 \\
0.9158 \\
0.9360\end{array}$ & $\begin{array}{l}3587.0 \\
3627.0 \\
3645.0 \\
3649.0 \\
3651.0 \\
3653.0 \\
3654.0 \\
3656.0 \\
3658.0 \\
3659.0\end{array}$ \\
\hline 40.0 & 0.05962 & 2260.0 & 133.0 & 33.44 & 143.8 & 1.131 & 0.7473 & 0.9542 & 3660.0 \\
\hline $\begin{array}{r}45.0 \\
50.0 \\
55.0 \\
60.0 \\
70.0 \\
80.0 \\
90.0 \\
100.0 \\
120.0 \\
140.0\end{array}$ & $\begin{array}{l}0.06068 \\
0.06176 \\
0.06285 \\
0.06396 \\
0.06623 \\
0.06857 \\
0.07097 \\
0.07342 \\
0.07847 \\
0.08366\end{array}$ & $\begin{array}{l}2220.0 \\
2180.0 \\
2150.0 \\
2110.0 \\
2040.0 \\
1970.0 \\
1910.0 \\
1860.0 \\
1790.0 \\
1750.0\end{array}$ & $\begin{array}{r}129.0 \\
124.0 \\
120.0 \\
115.0 \\
107.0 \\
99.1 \\
92.1 \\
85.7 \\
74.6 \\
65.6\end{array}$ & $\begin{array}{c}36.36 \\
39.41 \\
42.58 \\
45.86 \\
52.69 \\
59.81 \\
67.18 \\
74.75 \\
90.31 \\
106.2\end{array}$ & $\begin{array}{l}148.7 \\
153.8 \\
159.0 \\
164.3 \\
175.3 \\
186.8 \\
198.6 \\
210.7 \\
235.6 \\
261.1\end{array}$ & $\begin{array}{l}1.245 \\
1.352 \\
1.451 \\
1.544 \\
1.714 \\
1.867 \\
2.006 \\
2.133 \\
2.360 \\
2.557\end{array}$ & $\begin{array}{l}0.7636 \\
0.7755 \\
0.7849 \\
0.7929 \\
0.8060 \\
0.8164 \\
0.8246 \\
0.8309 \\
0.8386 \\
0.8416\end{array}$ & $\begin{array}{l}0.9930 \\
1.026 \\
1.054 \\
1.080 \\
1.125 \\
1.164 \\
1.197 \\
1.224 \\
1.264 \\
1.288\end{array}$ & $\begin{array}{l}3660.0 \\
3658.0 \\
3653.0 \\
3646.0 \\
3628.0 \\
3607.0 \\
3585.0 \\
3566.0 \\
3536.0 \\
3521.0\end{array}$ \\
\hline $\begin{array}{l}160.0 \\
180.0 \\
200.0 \\
250.0 \\
300.0 \\
350.0 \\
400.0 \\
450.0 \\
500.0 \\
600.0\end{array}$ & $\begin{array}{l}0.08895 \\
0.09429 \\
0.0996 \\
0.1130 \\
0.1263 \\
0.1394 \\
0.1525 \\
0.1655 \\
0.1785 \\
0.2043\end{array}$ & $\begin{array}{l}1730.0 \\
1730.0 \\
1740.0 \\
1810.0 \\
1900.0 \\
2000.0 \\
2100.0 \\
2210.0 \\
2320.0 \\
2550.0\end{array}$ & $\begin{array}{l}58.2 \\
52.1 \\
47.1 \\
37.8 \\
31.4 \\
26.9 \\
23.6 \\
21.0 \\
18.9 \\
15.8\end{array}$ & $\begin{array}{l}122.3 \\
138.5 \\
154.7 \\
194.9 \\
234.7 \\
274.1 \\
313.2 \\
351.9 \\
390.4 \\
466.9\end{array}$ & $\begin{array}{l}287.0 \\
313.1 \\
339.2 \\
404.2 \\
458.6 \\
532.3 \\
595.6 \\
658.4 \\
720.9 \\
845.3\end{array}$ & $\begin{array}{l}2.730 \\
2.883 \\
3.021 \\
3.311 \\
3.546 \\
3.742 \\
3.911 \\
4.059 \\
4.191 \\
4.418\end{array}$ & $\begin{array}{l}0.8416 \\
0.8398 \\
0.8370 \\
0.8282 \\
0.8195 \\
0.8118 \\
0.8052 \\
0.7996 \\
0.7949 \\
0.7875\end{array}$ & $\begin{array}{l}1.300 \\
1.305 \\
1.305 \\
1.294 \\
1.281 \\
1.269 \\
1.260 \\
1.253 \\
1.248 \\
1.241\end{array}$ & $\begin{array}{l}3520.0 \\
3530.0 \\
3549.0 \\
3620.0 \\
3708.0 \\
3805.0 \\
3905.0 \\
4007.0 \\
4110.0 \\
4314.0\end{array}$ \\
\hline $\begin{array}{r}700.0 \\
800.0 \\
900.0 \\
1000.0 \\
1200.0 \\
1400.0 \\
1600.0 \\
1800.0 \\
2000.0 \\
2500.0\end{array}$ & $\begin{array}{l}0.2302 \\
0.2561 \\
0.2820 \\
0.3080 \\
0.3600 \\
0.4122 \\
0.4645 \\
0.5169 \\
0.5694 \\
0.7098\end{array}$ & $\begin{array}{l}2780.0 \\
3020.0 \\
3260.0 \\
3500.0 \\
3990.0 \\
4490.0 \\
5000.0 \\
5500.0 \\
6020.0 \\
7300.0\end{array}$ & $\begin{array}{c}13.6 \\
11.9 \\
10.5 \\
9.59 \\
8.03 \\
6.91 \\
6.06 \\
5.40 \\
4.87 \\
3.91\end{array}$ & $\begin{array}{r}542.9 \\
618.6 \\
694.1 \\
769.4 \\
919.8 \\
1070.0 \\
1220.0 \\
1370.0 \\
1520.0 \\
1894.0\end{array}$ & $\begin{array}{r}969.2 \\
1093.0 \\
1216.0 \\
1340.0 \\
1586.0 \\
1833.0 \\
2080.0 \\
2327.0 \\
2574.0 \\
3192.0\end{array}$ & $\begin{array}{l}4.609 \\
4.774 \\
4.919 \\
5.049 \\
5.274 \\
5.464 \\
5.629 \\
5.775 \\
5.905 \\
6.181\end{array}$ & $\begin{array}{l}0.7821 \\
0.7781 \\
0.7749 \\
0.7724 \\
0.7688 \\
0.7662 \\
0.7644 \\
0.7630 \\
0.7619 \\
0.7599\end{array}$ & $\begin{array}{l}1.237 \\
1.235 \\
1.234 \\
1.234 \\
1.234 \\
1.234 \\
1.235 \\
1.235 \\
1.236 \\
1.236\end{array}$ & $\begin{array}{l}4514.0 \\
4711.0 \\
4903.0 \\
5089.0 \\
5448.0 \\
5790.0 \\
6115.0 \\
6425.0 \\
6722.0 \\
7417.0\end{array}$ \\
\hline 3000.0 & 0.8325 & 8590.0 & 3.27 & 2269.0 & 3811.0 & 6.406 & 0.7586 & 1.237 & 8056.0 \\
\hline
\end{tabular}

- two-phase gounoary 
10000 PSIA ISORAR

\begin{tabular}{|c|c|c|c|c|c|c|c|c|c|c|}
\hline $\begin{array}{l}\text { TEMPE RA TURE } \\
\text { OEG. R }\end{array}$ & $\begin{array}{l}\text { DENSITY } \\
\text { LB/CU FT }\end{array}$ & $\begin{array}{l}V(O H / O V)_{P} \\
\text { BTU/L9 }\end{array}$ & $\begin{array}{l}V \text { VIOP/OU) } \\
\text { PSIA-CU FT/BTU }\end{array}$ & $\begin{array}{l}-V(O P / D V)_{T} \\
U \text { PSIA }\end{array}$ & $\begin{array}{l}\text { (DV/OT) }{ }_{P}^{\prime V} \\
\text { 1/DEG. } R\end{array}$ & $\begin{array}{c}\text { THERMAL } \\
\text { CONDUCTIVITY } \\
\text { BTU/FT-HR-R }\end{array}$ & $\begin{array}{l}\text { VISCOSITY } \\
\text { LB/FT-SEC } \\
\times 10 E+6\end{array}$ & $\begin{array}{l}\text { THERMAL } \\
\text { OIFFUSIVITY } \\
\text { SQ FT/HR }\end{array}$ & $\begin{array}{l}\text { DIELECTRIC } \\
\text { CONSTANT }\end{array}$ & $\begin{array}{l}\text { PRANOTL } \\
\text { NUMGER }\end{array}$ \\
\hline $\begin{array}{l}20.0 \\
22.0 \\
24.0 \\
26.0 \\
28.0 \\
30.0 \\
32.0 \\
34.0 \\
36.0 \\
38.0\end{array}$ & $\begin{array}{l}17.97 \\
17.85 \\
17.73 \\
17.61 \\
17.49 \\
17.37 \\
17.25 \\
17.13 \\
17.01 \\
16.89\end{array}$ & $\begin{array}{l}192.0 \\
196.0 \\
210.0 \\
225.0 \\
236.0 \\
244.0 \\
250.0 \\
256.0 \\
262.0 \\
267.0\end{array}$ & $\begin{array}{l}14.5 \\
14.5 \\
13.7 \\
12.8 \\
12.2 \\
11.8 \\
11.5 \\
11.3 \\
11.0 \\
10.8\end{array}$ & $\begin{array}{l}43300.0 \\
42700.0 \\
42300.0 \\
41900.0 \\
41400.0 \\
40800.0 \\
40200.0 \\
39600.0 \\
39100.0 \\
38500.0\end{array}$ & $\begin{array}{l}0.00285 \\
0.00315 \\
0.00332 \\
0.00338 \\
0.00340 \\
0.00344 \\
0.00346 \\
0.00348 \\
0.00350 \\
0.00351\end{array}$ & $\begin{array}{l}0.101 \\
0.102 \\
0.103 \\
0.102 \\
0.102 \\
0.102 \\
0.101 \\
0.100 \\
0.0991 \\
0.0982\end{array}$ & $\begin{array}{l}79.3 \\
60.8 \\
48.4 \\
39.8 \\
33.8 \\
29.3 \\
25.9 \\
23.3 \\
21.2 \\
19.5\end{array}$ & $\begin{array}{l}0.0102 \\
0.00925 \\
0.00829 \\
0.00764 \\
0.00727 \\
0.00699 \\
0.00674 \\
0.00654 \\
0.00636 \\
0.00621\end{array}$ & $\begin{array}{l}1.01258 \\
1.01285 \\
1.01314 \\
1.01343 \\
1.01370 \\
1.01397 \\
1.01423 \\
1.01449 \\
1.01474 \\
1.01498\end{array}$ & $\begin{array}{l}1.55 \\
1.33 \\
1.19 \\
1.07 \\
0.955 \\
0.869 \\
0.801 \\
0.747 \\
0.704 \\
0.669\end{array}$ \\
\hline 40.0 & 16.77 & 272.0 & 10.6 & 38000.0 & 0.00351 & 0.0973 & 18.1 & 0.00608 & 1.01522 & 0.641 \\
\hline $\begin{array}{r}45.0 \\
50.0 \\
55.0 \\
60.0 \\
70.0 \\
80.0 \\
90.0 \\
100.0 \\
120.0 \\
140.0\end{array}$ & $\begin{array}{l}16.48 \\
16.19 \\
15.91 \\
15.63 \\
15.10 \\
14.58 \\
14.09 \\
13.62 \\
12.74 \\
11.95\end{array}$ & $\begin{array}{l}282.0 \\
292.0 \\
300.0 \\
308.0 \\
323.0 \\
337.0 \\
350.0 \\
363.0 \\
387.0 \\
411.0\end{array}$ & $\begin{array}{c}10.2 \\
9.91 \\
9.59 \\
9.31 \\
8.79 \\
8.33 \\
7.92 \\
7.57 \\
6.98 \\
6.52\end{array}$ & $\begin{array}{l}36700.0 \\
35400.0 \\
34100.0 \\
32900.0 \\
30700.0 \\
28700.0 \\
26900.0 \\
25400.0 \\
22800.0 \\
20900.0\end{array}$ & $\begin{array}{l}0.00352 \\
0.00352 \\
0.00351 \\
0.00350 \\
0.00348 \\
0.00345 \\
0.00342 \\
0.00338 \\
0.00327 \\
0.00314\end{array}$ & $\begin{array}{l}0.0950 \\
0.0929 \\
0.0911 \\
0.0895 \\
0.0868 \\
0.0849 \\
0.0835 \\
0.0825 \\
0.0815 \\
0.0814\end{array}$ & $\begin{array}{l}15.7 \\
14.1 \\
13.0 \\
12.2 \\
11.3 \\
10.7 \\
10.5 \\
10.3 \\
10.2 \\
10.3\end{array}$ & $\begin{array}{l}0.00581 \\
0.00560 \\
0.00543 \\
0.00550 \\
0.00511 \\
0.00500 \\
0.00495 \\
0.00495 \\
0.00506 \\
0.00529\end{array}$ & $\begin{array}{l}1.01579 \\
1.01633 \\
1.01682 \\
1.01728 \\
1.01810 \\
1.01879 \\
1.01936 \\
1.01983 \\
1.02049 \\
1.02086\end{array}$ & $\begin{array}{l}0.590 \\
0.559 \\
0.541 \\
0.531 \\
0.525 \\
0.530 \\
0.539 \\
0.550 \\
0.570 \\
0.586\end{array}$ \\
\hline $\begin{array}{l}160.0 \\
180.0 \\
200.0 \\
250.0 \\
300.0 \\
350.0 \\
400.0 \\
450.0 \\
500.0 \\
600.0\end{array}$ & $\begin{array}{c}11.24 \\
10.61 \\
10.04 \\
8.848 \\
7.919 \\
7.172 \\
6.557 \\
6.042 \\
5.603 \\
4.894\end{array}$ & $\begin{array}{l}435.0 \\
460.0 \\
485.0 \\
549.0 \\
612.0 \\
675.0 \\
737.0 \\
799.0 \\
860.0 \\
981.0\end{array}$ & $\begin{array}{l}6.15 \\
5.85 \\
5.61 \\
5.15 \\
4.85 \\
4.63 \\
4.46 \\
4.34 \\
4.24 \\
4.10\end{array}$ & $\begin{array}{l}19500.0 \\
18400.0 \\
17500.0 \\
16000.0 \\
15000.0 \\
14300.0 \\
13800.0 \\
13400.0 \\
13000.0 \\
12500.0\end{array}$ & $\begin{array}{l}0.00299 \\
0.00284 \\
0.00269 \\
0.00236 \\
0.00209 \\
0.00186 \\
0.00171 \\
0.00157 \\
0.00145 \\
0.00127\end{array}$ & $\begin{array}{l}0.0818 \\
0.0826 \\
0.0837 \\
0.0870 \\
0.0907 \\
0.0945 \\
0.0983 \\
0.102 \\
0.106 \\
0.113\end{array}$ & $\begin{array}{l}10.4 \\
10.6 \\
10.7 \\
11.2 \\
11.8 \\
12.4 \\
13.0 \\
13.6 \\
14.1 \\
15.5\end{array}$ & $\begin{array}{l}0.00560 \\
0.00597 \\
0.00639 \\
0.00760 \\
0.00894 \\
0.0104 \\
0.0119 \\
0.0135 \\
0.0151 \\
0.0187\end{array}$ & $\begin{array}{l}1.02101 \\
1.02099 \\
1.02085 \\
1.02020 \\
1.01934 \\
1.01844 \\
1.01755 \\
1.01670 \\
1.01591 \\
1.01448\end{array}$ & $\begin{array}{l}0.596 \\
0.602 \\
0.598 \\
0.601 \\
0.600 \\
0.599 \\
0.599 \\
0.600 \\
0.601 \\
0.610\end{array}$ \\
\hline $\begin{array}{r}700.0 \\
800.0 \\
900.0 \\
1000.0 \\
1200.0 \\
1400.0 \\
1600.0 \\
1800.0 \\
2000.0 \\
2500.0\end{array}$ & $\begin{array}{l}4.344 \\
3.905 \\
3.546 \\
3.247 \\
2.778 \\
2.426 \\
2.153 \\
1.935 \\
1.756 \\
1.427\end{array}$ & $\begin{array}{l}1100.0 \\
1220.0 \\
1340.0 \\
1460.0 \\
1700.0 \\
1950.0 \\
2190.0 \\
2430.0 \\
2680.0 \\
3290.0\end{array}$ & $\begin{array}{l}4.00 \\
3.92 \\
3.87 \\
3.82 \\
3.76 \\
3.72 \\
3.68 \\
3.66 \\
3.64 \\
3.61\end{array}$ & $\begin{array}{l}12100.0 \\
11800.0 \\
11600.0 \\
11400.0 \\
11100.0 \\
10900.0 \\
10800.0 \\
10600.0 \\
10600.0 \\
10400.0\end{array}$ & $\begin{array}{l}0.00112 \\
0.00101 \\
0.000920 \\
0.000844 \\
0.000724 \\
0.000634 \\
0.000564 \\
0.000507 \\
0.000461 \\
0.000376\end{array}$ & $\begin{array}{l}0.122 \\
0.131 \\
0.130 \\
0.148 \\
0.165 \\
0.182 \\
0.198 \\
0.214 \\
0.230 \\
0.267\end{array}$ & $\begin{array}{l}17.0 \\
18.4 \\
19.9 \\
21.2 \\
23.0 \\
26.5 \\
29.0 \\
31.5 \\
33.8 \\
39.5\end{array}$ & $\begin{array}{l}0.0227 \\
0.0271 \\
0.0318 \\
0.0369 \\
0.0482 \\
0.0607 \\
0.0745 \\
0.0896 \\
0.106 \\
0.151\end{array}$ & $\begin{array}{l}1.01326 \\
1.01221 \\
1.01130 \\
1.01051 \\
1.00921 \\
1.00819 \\
1.00736 \\
1.00669 \\
1.00612 \\
1.00505\end{array}$ & $\begin{array}{l}0.620 \\
0.628 \\
0.633 \\
0.638 \\
0.644 \\
0.648 \\
0.651 \\
0.654 \\
0.655 \\
0.658\end{array}$ \\
\hline 3000.0 & 1.201 & 3910.0 & 3.59 & 10300.0 & 0.000317 & 0.303 & 44.9 & 0.204 & 1.00430 & 0.660 \\
\hline
\end{tabular}

- tho-phase boundary 
15000 PSIA ISOBAR

THERMOOYNAMIC PROPERTIFS OF HELIUM 4

\begin{tabular}{|c|c|c|c|c|c|c|c|c|c|}
\hline TEMPERATURE & VOLUME & $\begin{array}{l}\text { I SOTHERM } \\
\text { OERIVATIVE }\end{array}$ & $\begin{array}{l}\text { ISOCHORE } \\
\text { OERIVATIVE }\end{array}$ & $\begin{array}{l}\text { INTERNAL } \\
\text { ENERGY }\end{array}$ & ENTHAL PY & ENTROPY & $\mathrm{CV}$ & $C P$ & $\begin{array}{l}\text { VELOCITY } \\
\text { OF SOUNO }\end{array}$ \\
\hline DEG. $R$ & CU FT/LB & CU FT-PSIA/LB & PSIA/R & BTU/LB & BTU/LA & BTU/LA-R & BTU, LB & $-R$ & FT/SEC \\
\hline $\begin{array}{l}26.0 \\
28.0 \\
30.0 \\
32.0 \\
34.0 \\
36.0 \\
38.0 \\
40.0\end{array}$ & $\begin{array}{l}0.05160 \\
0.05191 \\
0.05219 \\
0.05248 \\
0.05277 \\
0.05307 \\
0.05337 \\
0.05368\end{array}$ & $\begin{array}{l}3310.0 \\
3310.0 \\
3270.0 \\
3240.0 \\
3220.0 \\
3190.0 \\
3170.0 \\
3150.0\end{array}$ & $\begin{array}{l}168.0 \\
170.0 \\
171.0 \\
171.0 \\
171.0 \\
170.0 \\
169.0 \\
167.0\end{array}$ & $\begin{array}{l}34.18 \\
35.04 \\
35.80 \\
36.63 \\
37.50 \\
38.42 \\
39.38 \\
40.37\end{array}$ & $\begin{array}{l}177.5 \\
179.2 \\
180.9 \\
182.4 \\
184.1 \\
185.8 \\
187.6 \\
189.5\end{array}$ & $\begin{array}{l}0.6001 \\
0.6636 \\
0.7170 \\
0.7694 \\
0.8206 \\
0.8705 \\
0.9191 \\
c .9662\end{array}$ & $\begin{array}{l}0.5863 \\
0.6323 \\
0.6591 \\
0.6816 \\
0.7003 \\
0.7160 \\
0.7292 \\
0.7403\end{array}$ & $\begin{array}{l}0.6957 \\
0.7544 \\
0.7944 \\
0.8293 \\
0.8596 \\
0.8861 \\
0.9093 \\
0.9299\end{array}$ & $\begin{array}{l}4267.0 \\
4275.0 \\
4275.0 \\
4276.0 \\
4277.0 \\
4279.0 \\
4281.0 \\
4283.0\end{array}$ \\
\hline $\begin{array}{r}45.0 \\
50.0 \\
55.0 \\
60.0 \\
70.0 \\
80.0 \\
90.0 \\
100.0 \\
120.0 \\
140.0\end{array}$ & $\begin{array}{l}0.05445 \\
0.05523 \\
0.05601 \\
0.05680 \\
0.05839 \\
0.06000 \\
0.06161 \\
0.06324 \\
0.06654 \\
0.06990\end{array}$ & $\begin{array}{l}3110.0 \\
3070.0 \\
3040.0 \\
3000.0 \\
2930.0 \\
2870.0 \\
2800.0 \\
2740.0 \\
2640.0 \\
2550.0\end{array}$ & $\begin{array}{r}163.0 \\
158.0 \\
152.0 \\
147.0 \\
137.0 \\
128.0 \\
123.0 \\
112.0 \\
99.2 \\
88.5\end{array}$ & $\begin{array}{c}42.99 \\
45.78 \\
48.70 \\
51.75 \\
58.17 \\
64.95 \\
72.01 \\
79.32 \\
94.49 \\
110.2\end{array}$ & $\begin{array}{l}194.2 \\
199.2 \\
204.3 \\
209.5 \\
220.4 \\
231.6 \\
243.1 \\
255.0 \\
279.3 \\
304.3\end{array}$ & $\begin{array}{l}1.078 \\
1.183 \\
1.280 \\
1.371 \\
1.538 \\
1.688 \\
1.824 \\
1.949 \\
2.170 \\
2.363\end{array}$ & $\begin{array}{l}0.7621 \\
0.7782 \\
0.7911 \\
0.8020 \\
0.8201 \\
0.8347 \\
0.8465 \\
0.8559 \\
0.8686 \\
0.8752\end{array}$ & $\begin{array}{l}0.9726 \\
1.007 \\
1.036 \\
1.061 \\
1.104 \\
1.140 \\
1.170 \\
1.196 \\
1.236 \\
1.264\end{array}$ & $\begin{array}{l}4288.0 \\
4291.0 \\
4291.0 \\
4288.0 \\
4276.0 \\
4258.0 \\
4237.0 \\
4214.0 \\
4169.0 \\
4131.0\end{array}$ \\
\hline $\begin{array}{l}160.0 \\
180.0 \\
200.0 \\
250.0 \\
300.0 \\
350.0 \\
400.0 \\
450.0 \\
500.0 \\
600.0\end{array}$ & $\begin{array}{l}0.07331 \\
0.07676 \\
0.08025 \\
0.08901 \\
0.09777 \\
0.1065 \\
0.1151 \\
0.1237 \\
0.1323 \\
0.1493\end{array}$ & $\begin{array}{l}2490.0 \\
2450.0 \\
2420.0 \\
2410.0 \\
2450.0 \\
2520.0 \\
2610.0 \\
2700.0 \\
2800.0 \\
3000.0\end{array}$ & $\begin{array}{l}79.5 \\
72.0 \\
65.6 \\
53.4 \\
44.9 \\
38.6 \\
33.9 \\
30.2 \\
27.3 \\
22.9\end{array}$ & $\begin{array}{l}126.2 \\
142.3 \\
158.6 \\
199.3 \\
239.7 \\
279.8 \\
319.5 \\
358.8 \\
397.9 \\
475.3\end{array}$ & $\begin{array}{l}329.8 \\
355.6 \\
381.5 \\
446.5 \\
511.3 \\
575.6 \\
639.3 \\
702.5 \\
765.3 \\
890.0\end{array}$ & $\begin{array}{l}2.533 \\
2.685 \\
2.321 \\
3.112 \\
3.348 \\
3.546 \\
3.716 \\
3.865 \\
3.997 \\
4.225\end{array}$ & $\begin{array}{l}0.8776 \\
0.8771 \\
0.8749 \\
0.8656 \\
0.8550 \\
0.8449 \\
0.8359 \\
0.8282 \\
0.8215 \\
0.8107\end{array}$ & $\begin{array}{l}1.282 \\
1.293 \\
1.299 \\
1.300 \\
1.291 \\
1.279 \\
1.269 \\
1.260 \\
1.253 \\
1.243\end{array}$ & $\begin{array}{l}4104.0 \\
4087.0 \\
4080.0 \\
4095.0 \\
4143.0 \\
4208.0 \\
4282.0 \\
4362.0 \\
4445.0 \\
4616.0\end{array}$ \\
\hline $\begin{array}{r}700.0 \\
800.0 \\
900.0 \\
1000.0 \\
1200.0 \\
1400.0 \\
1600.0 \\
1800.0 \\
2000.0 \\
2500.0\end{array}$ & $\begin{array}{l}0.1663 \\
0.1832 \\
0.2502 \\
0.2173 \\
0.2514 \\
0.2858 \\
0.3202 \\
0.3547 \\
0.3894 \\
0.4762\end{array}$ & $\begin{array}{l}3210.0 \\
3430.0 \\
3650.0 \\
3880.0 \\
4350.0 \\
4820.0 \\
5310.0 \\
5800.0 \\
6300.0 \\
7550.0\end{array}$ & $\begin{array}{c}19.7 \\
17.4 \\
15.5 \\
14.0 \\
11.8 \\
10.2 \\
8.93 \\
7.97 \\
7.20 \\
5.79\end{array}$ & $\begin{array}{r}552.1 \\
628.4 \\
704.4 \\
780.1 \\
931.2 \\
1082.0 \\
1232.0 \\
1383.0 \\
1533.0 \\
1909.0\end{array}$ & $\begin{array}{l}1014.0 \\
1137.0 \\
1261.0 \\
1384.0 \\
1630.0 \\
1876.0 \\
2122.0 \\
2368.0 \\
2614.0 \\
3231.0\end{array}$ & $\begin{array}{l}4.416 \\
4.581 \\
4.726 \\
4.855 \\
5.080 \\
5.259 \\
5.434 \\
5.579 \\
5.709 \\
5.984\end{array}$ & $\begin{array}{l}0.8026 \\
0.7964 \\
0.7915 \\
0.7876 \\
0.7818 \\
0.7778 \\
0.7748 \\
0.7726 \\
0.7708 \\
0.7677\end{array}$ & $\begin{array}{l}1.236 \\
1.233 \\
1.231 \\
1.230 \\
1.230 \\
1.230 \\
1.231 \\
1.232 \\
1.233 \\
1.234\end{array}$ & $\begin{array}{l}4788.0 \\
4960.0 \\
5131.0 \\
5299.0 \\
5628.0 \\
5945.0 \\
6251.0 \\
6545.0 \\
6829.0 \\
7498.0\end{array}$ \\
\hline 3000.0 & 0.5632 & 8820.0 & 4.85 & 2284.0 & 3848.0 & 6.209 & 0.7657 & 1.235 & 8117.0 \\
\hline
\end{tabular}


15000 PSIA ISOBAR

TEMPERATURE DENSITY V(OH/OV) $V$ VIOP/DU) $-V(D P / D V)_{T}$

OEG. R LB/CUFT BTU/LA PSIA-CU FT/BTU PSIA

\begin{tabular}{|c|c|c|c|c|}
\hline $\begin{array}{l}26.0 \\
28.0 \\
30.0 \\
32.0 \\
34.0 \\
36.0 \\
38.0 \\
40.0\end{array}$ & $\begin{array}{l}19.38 \\
19.26 \\
19.16 \\
19.05 \\
18.95 \\
18.84 \\
18.74 \\
18.63\end{array}$ & $\begin{array}{l}266.0 \\
283.0 \\
291.0 \\
299.0 \\
307.0 \\
314.0 \\
320.0 \\
326.0\end{array}$ & $\begin{array}{l}14.8 \\
14.0 \\
13.5 \\
13.2 \\
12.9 \\
12.6 \\
12.4 \\
12.1\end{array}$ & $\begin{array}{l}64200.0 \\
63700.0 \\
62700.0 \\
61800.0 \\
61000.0 \\
60200.0 \\
59400.0 \\
58700.0\end{array}$ \\
\hline $\begin{array}{r}45.0 \\
50.0 \\
55.0 \\
60.0 \\
70.0 \\
80.0 \\
90.0 \\
100.0 \\
120.0 \\
140.0\end{array}$ & $\begin{array}{l}18.37 \\
18.11 \\
17.85 \\
17.60 \\
17.13 \\
16.67 \\
16.23 \\
15.81 \\
15.03 \\
14.31\end{array}$ & $\begin{array}{l}341.0 \\
355.0 \\
368.0 \\
381.0 \\
404.0 \\
425.0 \\
445.0 \\
462.0 \\
494.0 \\
521.0\end{array}$ & $\begin{array}{c}11.6 \\
11.2 \\
10.8 \\
10.4 \\
9.77 \\
9.20 \\
8.71 \\
8.29 \\
7.50 \\
7.06\end{array}$ & $\begin{array}{l}57100.0 \\
55500.0 \\
54200.0 \\
52800.0 \\
50200.0 \\
47800.0 \\
45500.0 \\
43400.0 \\
39500.0 \\
36500.0\end{array}$ \\
\hline $\begin{array}{l}160.0 \\
180.0 \\
200.0 \\
250.0 \\
300.0 \\
350.0 \\
400.0 \\
450.0 \\
500.0 \\
600.0\end{array}$ & $\begin{array}{c}13.64 \\
13.03 \\
12.46 \\
11.23 \\
10.23 \\
9.391 \\
8.686 \\
8.083 \\
7.560 \\
6.698\end{array}$ & $\begin{array}{r}547.0 \\
572.0 \\
597.0 \\
659.0 \\
722.0 \\
785.0 \\
848.0 \\
910.0 \\
971.0 \\
1090.0\end{array}$ & $\begin{array}{l}6.64 \\
6.30 \\
6.02 \\
5.49 \\
5.13 \\
4.87 \\
4.67 \\
4.52 \\
4.39 \\
4.21\end{array}$ & $\begin{array}{l}33900.0 \\
31900.0 \\
30200.0 \\
27100.0 \\
25100.0 \\
23700.0 \\
22700.0 \\
21800.0 \\
21100.0 \\
20100.0\end{array}$ \\
\hline $\begin{array}{l}700.0 \\
800.0 \\
900.0 \\
1000.0 \\
1200.0 \\
1400.0 \\
1600.0 \\
1800.0 \\
2000.0 \\
2500.0\end{array}$ & $\begin{array}{l}6.014 \\
5.457 \\
4.994 \\
4.603 \\
3.977 \\
3.499 \\
3.123 \\
2.819 \\
2.568 \\
2.100\end{array}$ & $\begin{array}{l}1210.0 \\
1330.0 \\
1450.0 \\
1570.0 \\
1810.0 \\
2050.0 \\
2290.0 \\
2530.0 \\
2770.0 \\
3380.0\end{array}$ & $\begin{array}{l}4.09 \\
3.99 \\
3.92 \\
3.67 \\
3.79 \\
3.73 \\
3.69 \\
3.65 \\
3.64 \\
3.59\end{array}$ & $\begin{array}{l}19300.0 \\
18700.0 \\
18200.0 \\
17900.0 \\
17300.0 \\
16900.0 \\
16600.0 \\
16400.0 \\
16200.0 \\
15900.0\end{array}$ \\
\hline 3000.0 & 1.775 & 3990.0 & 3.57 & 15700.0 \\
\hline
\end{tabular}

(OV/OT) ${ }_{\mathrm{P}}$ CONDUCTIVITY VISCOSITY 1/OEG. $R$ BTU/FT $-H R-R$ LB/FT-SEC

$\begin{array}{ll}0.00262 & 0.139 \\ 0.00267 & 0.137 \\ 0.00273 & 0.136 \\ 0.00277 & 0.134 \\ 0.00280 & 0.133 \\ 0.00283 & 0.131 \\ 0.00284 & 0.129 \\ 0.00285 & 0.128 \\ 0.00285 & 0.124 \\ 0.00284 & 0.120 \\ 0.00281 & 0.117 \\ 0.00279 & 0.114 \\ 0.00273 & 0.110 \\ 0.00268 & 0.106 \\ 0.00263 & 0.104 \\ 0.00259 & 0.102 \\ 0.00250 & 0.100 \\ 0.00242 & 0.0993\end{array}$

97.4

97.4

76.8
62.9

62.9
52.8

52.8
45.3

45.3
39.5

35.1

31.5

25.4
21.5

19.0

15.1

13.9

13.2

12.7

12.1

0.00234

0.00226

0.00218

c. 00197

0.00179

C. 00150

0.00139

0.00129

0.00114

c. 00102

P. 000927

0.000850

c.000681

0.000602

0.000539

0.000487

c. 000365

0.000310
0.0991

0.0993

0.100

0.102

0.104

0.107

0.112

0.112

0.115

0.129

0.137

0.145

0.153

0.170

0.186

0.202

0.217

0.232

0.305
12.1

12.2
12.1

12.5

12.9

13.3

13.7

14.2

14.6

14.6
15.9

17.4

18.8
20.2

21.5
24.2

26.8

29.2

31.7

34.0

39.5

45.0
THERMAL DIELECTRIC DIFFUSIVITY CONSTANT

SQ FT/HR

0.0103
0.00945
0.00893
0.00851
0.00815
0.00785
0.00760
0.00737
0.00692

1.00883

1.00916

1.00946

1. 00975

1. 01005

1.01034

1.01062
1.01091

1.01159

1.01224

1.01286

1.01343

1.01449

1.01543

1.01626

1.01626
1.01699

1.01699
1.01820

1.01820
1.01912

1. 01981

1.02031
1.02065

1.02101

1. 02091

1. 02056

1.02007

1.01952

1.01893

1.01893
1.01776

0.0122

0.0174

0.0204

0.0236

0.0271

0.0347

0.0431

0.0524

0.0625

0.0734

1. 01665

1. 01563

1.01469

1. 01385

1. 01239

1. 01118

1. 01018

1.00933

1.00861

0.139

1.00619
PRANDTL

NUMBER

1.76

1.52

1.32

1.17

1.05
0.962

0.887

0.827

0.719

0.651

0.606

0.577

0.546

0.534

0.532

0.535

0.545
0.556

0.565

0.571

0.569

0.573

0.573

0.572

0.572

0.573

0.574

0.598

0.508

0.616

0.522

0.632

0.638

0.647

0.647

0.649

0.656

- two-phase bDundary 


\begin{tabular}{c|c|}
\hline $\begin{array}{c}\text { U.S. DEPT. OF COMM. } \\
\text { BIBLIOGRAPHIC DATA } \\
\text { SHEET }\end{array}$ & $\begin{array}{c}\text { I. PUBLICATION OR REPORT NO. } \\
\text { NBS TN-622 }\end{array}$ \\
\hline
\end{tabular}

Thermophysical Properties of Helium 4 from 4 to 3000

TITLE AND SUBTITLE
Thermophysical Properties of
with Pressures to 15000 PSIA

5. Publication Date

\begin{tabular}{l}
\hline AUTHOR(s) \\
Robert D. McCarty \\
\hline PERF ORMING ORGANIZATION NAME AND ADDRESS \\
NATIONAL BUREAU OF STANDARDS, Boulde $r$ Labs. \\
DEPARTMENT OF COMMERCE \\
Boulder, Colorado 80302
\end{tabular}

1. Sponsoring Organization Name and Address

National Aeronautics and Space Administration

Manned Spacecraft Center

Houston, Texas 77058

8. Performing Organization

6. Performing Organization Code

10. Project/Task/Work Unit No.

2750426

11. Contract/Grant No.

SUPPLEMENTARY NOTES

ABSTRACT (A 200-word or less factual summary of most significant information. If document includes a significant bibliography or literature survey, mention it here.)

Tables of thermophysical properties of helium 4 are presented for temperatures from 4 to 3000 Rankine for pressures to $15000 \mathrm{psia}$. The tables include, entropy, enthalpy, internal energy, density, volume, speed of sound, specific heat, thermal conductivity, viscosity, thermal diffusivity, Prandtl number and the dielectric constant for 74 isobars. Also included in the isobaric tables are quantities of special utility in heat transfer calculations: $(\partial \mathrm{P} / \partial \mathrm{V})_{\mathrm{T}},(\partial \mathrm{P} / \partial \mathrm{T})_{\rho}$, $\mathrm{V}(\partial \mathrm{H} / \partial \mathrm{V})_{\mathrm{P}}, \mathrm{V}(\partial \mathrm{P} / \partial \mathrm{U})_{\mathrm{V}},-\mathrm{V}(\partial \mathrm{P} / \partial \mathrm{V})_{\mathrm{T}}, 1 / \mathrm{V}(\partial \mathrm{V} / \partial \mathrm{T})_{\mathrm{P}}$

In addition to the isobaric tables, tables for the saturated vapor and liquid are given which include all of the above properties, plus the surface tension. Tables for the PpT of the freezing liquid, P P T of the lambda line, index of refraction and the derived Joule-Thomson inversion curve are also presented.

7. KEY WORDS (Alphabetical or der, separated by semicolons)

Density; dielectric constant; enthalpy; entropy; equation of state; fixed points; heat transfer coefficients; helium 4; index of refraction; Joule-Thomson coefficient; lambda line; latent heat; melting point; Prandtl number; specific heats; speed of sound; surface tension; thermal conductivity; thermal diffusivity; vapor pressure; viscosity; volume.

\section{AVAILABILITY STATEMENT}

X] UNL IMIT ED.

FOR OFFICIAL DISTRIBUTION. DO NOT RELEASE TO NTIS.

\begin{tabular}{|l|c|}
\hline $\begin{array}{l}\text { 19. SECURITY CLASS } \\
\text { (THIS REPURT) }\end{array}$ & 146 \\
UNCL ASSIFIED & NO. OF PAGES \\
\hline $\begin{array}{l}\text { 20. SECUR ITY CLASS } \\
\text { (THIS PAGE) }\end{array}$ & 22. Price \\
UNCL ASSIFIED & $\$ 1.25$ \\
\hline
\end{tabular}

USCOMM-DC $66244 . P 71$ 

PERIODICALS

JOURNAL OF RESEARCH reports National Bureau of Standards research and development in physics, mathematics, and chemistry. Comprehensive scientific papers give complete details of the work, including laboratory data, experimental procedures, and theoretical and mathematical analyses. Illustrated with photographs, drawings, and charts. Includes listings of other NBS papers as issued.

Published in two sections, available separately:

\section{- Physics and Chemistry}

Papers of interest primarily to scientists working in these fields. This section covers a broad range of physical and chemical research, with major emphasis on standards of physical measurement, fundamental constants, and properties of matter. Issued six times a year. Annual subscription: Domestic, $\$ 9.50 ; \$ 2.25$ additional for foreign mailing.

\section{- Mathematical Sciences}

Studies and compilations designed mainly for the mathematician and theoretical physicist. Topics in mathematical statistics, theory of experiment design, numerical analysis, theoretical physics and chemistry, logical design and programming of computers and computer systems. Short numerical tables. Issued quarterly. Annual subscription: Domestic, \$5.00; $\$ 1.25$ additional for foreign mailing.

\section{TECHNICAL NEWS BULLETIN}

The best single source of information concerning the Bureau's measurement, research, developmental, cooperative, and publication activities, this monthly publication is designed for the industry-oriented individual whose daily work involves intimate contact with science and technology-for engineers, chemists, physicists, research managers, product-development managers, and company executives. Includes listing of all NBS papers as issued. Annual subscription: Domestic, $\$ 3.00 ; \$ 1.00$ additional for foreign mailing.

\section{Bibliographic Subscription Services}

The following current-awareness and literaturesurvey bibliographies are issued periodically by the Bureau: Cryogenic Data Center Current Awareness Service (weekly), Liquefied Natural Gas (quarterly), Superconducting Devices and Materials (quarterly), and Electromagnetic Metrology Current Awareness Service (monthly). Available only from NBS Boulder Laboratories. Ordering and cost information may be obtained from the Program Information Office, National Bureau of Standards, Boulder, Colorado 80302.

\section{NONPERTODICALS}

Applied Mathematics Series. Mathematical tables, manuals, and studies.

Building Science Series. Research results, test methods, and performance criteria of building materials, components, systems, and structures.

Handbooks. Recommended codes of engineering and industrial practice (including safety codes) developed in cooperation with interested industries, professional organizations, and regulatory bodies.

Special Publications. Proceedings of NBS conferences, bibliographies, annual reports, wall charts, pamphlets, etc.

Monographs. Major contributions to the technical literature on various subjects related to the Bureau's scientific and technical activities.

\section{National Standard Reference Data Series.} NSRDS provides quantitative data on the physical and chemical properties of materials, compiled from the world's literature and critically evaluated.

Product Standards. Provide requirements for sizes, types, quality, and methods for testing various industrial products. These standards are developed cooperatively with interested Government and industry groups and provide the basis for common understanding of product characteristics for both buyers and sellers. Their use is voluntary.

Technical Notes. This series consists of communications and reports (covering both other-agency and NBS-sponsored work) of limited or transitory interest.

Federal Information Processing Standards Publications. This series is the official publication within the Federal Government for information on standards adopted and promulgated under the Public Law 89-306, and Bureau of the Budget Circular A-86 entitled, Standardization of Data Elements and Codes in Data Systems.

Consumer Information Series. Practical information, based on NBS research and experience, covering areas of interest to the consumer. Easily understandable language and illustrations provide useful background knowledge for shopping in today's technological marketplace.

\section{CATALOGS OF NBS PUBLICATIONS}

NBS Special Publication 305, Publications of the NBS. 1966-1967. When ordering, include Catalog No. C13.10:305. Price $\$ 2.00 ; 50$ cents additional for foreign mailing.

NBS Special Publication 305, Supplement 1, Publications of the NBS, 1968-1969. When ordering, include Catalog No. C13.10:305/Suppl. 1. Price $\$ 4.50 ; \$ 1.25$ additional for foreign mailing.

NBS Special Publication 305, Supplement 2, Publications of the NBS, 1970. When ordering, include Catalog No. C13.10:305/Suppl. 2. Price $\$ 3.25$; 85 cents additional for foreign mailing.

Order NBS publications (except Bibliographic Subscription Services) from: Superintendent of Documents, Government Printing Office, Washington, D.C. 20402. 
U.S. DEPARTMENT OF COMMERCE

National Bureau of Standards

Washington, D.C. 20234

POSTAGE ANO FEES PAIO

OFFICIAL BUSINESS

Penalty for Private Use, $\$ 300$
U.S. DEPARTMENT OF COMMERCE

215 